
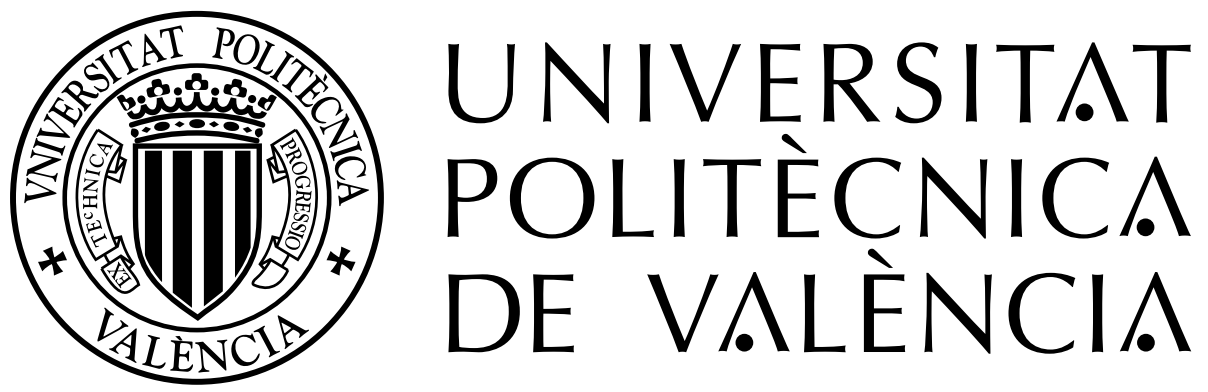

Departamento de Máquinas y Motores Térmicos

DOCTORAL THESIS:

"Experimental characterization and mean line modelling of twin-entry and dual-volute turbines working under different admission conditions with steady flow"

Presented by: Mr. Vishnu Samala

Supervised by: Dr. José Ramón Serrano Cruz

in fulfillment of the requirements for the degree of

Doctor of Philosophy 

Doctoral Thesis

"Experimental characterization and mean line modelling of twin-entry and dual-volute turbines working under different admission conditions with steady flow"

Presented by: Mr. Vishnu Samala

Supervised by: Dr. José Ramón Serrano Cruz

\section{THESIS EXAMINERS}

Dr. Nicholas C. Baines

Dr. Silvia Marelli

Dr. Francisco Vera García

DEFENSE COMMITTEE

Chairman: Dr. José Galindo Lucas

Secretary: Dr. Miguel Angel Reyes Belmonte

Member: Dr. Nicholas C. Baines

Valencia, 01 October 2020 



\begin{abstract}
Despite the importance of radial in-flow twin-entry and dual-volute turbines for turbocharged engines, their characteristic maps and fully predictive modelling using 1D gas dynamic codes are not well established yet. The complexity of the un-steady flow and the unequal admission of these turbines, when operating with pulses of engine exhaust gas, make them a challenging system. Mainly due to the unequal flow admission, an additional degree of freedom is introduced to well-known single entry vanned or vaneless turbines. Moreover, the addition of the second inlet to the turbine volute brings extra complexity in determining the steady-state turbine performance parameters under unequal admission conditions. This thesis has a main novelty, which is a simple procedure for characterizing experimentally and elaborating characteristic maps of these turbines with unequal flow conditions. This method of analysis allows easy interpolating within the proposed distinctive maps or simple convincing models for calculating and extrapolating full performance parameters of twin-entry and dual-volute turbines.
\end{abstract}

Here are also described two innovative 0D mean-line models that require a minimum quantity of experimental data for calibrating both: i.e. the mass flow parameter model and the isentropic efficiency model. Both models are predictive either in partial or unequal flow admission conditions using as inputs: the mass flow ratio and the total temperature ratio between the branches; the blade speed ratio and expansion ratio in each branch. These six inputs are generally instantaneously provided by 1D gas-dynamics codes. Therefore, the novelty of the model is its ability to be used in a quasi-steady way for twin and dual-volute turbines performance prediction. This can be achieved instantaneously as turbines are calculated under pulsating and uneven flow conditions at turbocharged engines.

Furthermore, a methodology for characterizing the discharge coefficient of a wastegate and scroll connection valve in a gas stand is shown. For estimating the gas flow over the same in one-dimensional models, an empirical model is correlated and validated. Finally, an optimal map of discharge coefficient has been drawn out through the interpolation method. This map can be integrated into the full one-dimensional turbocharged engine model system, in order to calculate the actual mass flow through the wastegate and scroll connection valves.

Finally, the models have been fully validated by coupling them with one-dimensional modelling software and simulated both the gas stand and the whole engine measured points. On the one hand, the validation results from the gas stand simulation show that the model can predict well all steady flow variables. On the other hand, the validation results from the whole engine simulation show that the model is able to produce all the full load engine variables like air mass flow and brake torque in a reasonable degree of agreement with the experimental data. 
Keywords: Turbocharger; Twin-entry radial turbines; Dual-volute radial turbines; unequal and partial flow admission; quasi-steady models; adiabatic efficiency model; reduced mass flow model; waste-gate, discharge coefficient. 


\section{Resumen}

A pesar de la importancia de las turbinas radiales de doble entrada y doble voluta en el flujo para motores turbo alimentados, sus mapas característicos y su modelado totalmente predictivo utilizando códigos dinámicos de gas 1D aún no están bien establecidos. La complejidad del flujo no estacionario y la admisión desigual de estas turbinas, cuando funcionan con pulsos de gases de escape del motor, las convierte en un sistema desafiante. Principalmente debido a la admisión de flujo desigual, se introduce un grado adicional de libertad con respecto a las turbinas conocidas como de una sola entrada con o sin álabes en el estator. Además, la adición de la segunda entrada a la voluta de la turbina aporta una complejidad adicional para determinar los parámetros de rendimiento de la turbina en estado estacionario y en condiciones de admisión desiguales. Esta tesis tiene como novedad principal un procedimiento simple para caracterizar experimentalmente y elaborar mapas característicos de estas turbinas con condiciones de flujo desiguales. Este método de análisis permite interpolar fácilmente dentro de los mapas distintivos propuestos o ajustar modelos simples y convincentes para calcular y extrapolar parámetros de rendimiento completo de turbinas de doble entrada y doble voluta.

También hemos descrito aquí, dos modelos innovadores de línea media 0D que requieren una cantidad mńima de datos experimentales para calibrar ambos: es decir, el modelo de parámetros de flujo másico y el modelo de eficiencia isentrópica. Ambos modelos son predictivos en condiciones de admisión de flujo parcial o desigual utilizando como entradas: la relación de flujo másico entre ramas; la relación de temperatura total entre ramas; la relación de velocidad de álabe a chorro en cada rama y la relación de presión en cada rama. Estas cinco entradas generalmente son proporcionadas instantáneamente por códigos de dinámica de gas 1D. Por lo tanto, la novedad del modelo es su capacidad de ser utilizado de manera casi constante para la predicción del rendimiento de las turbinas de doble entrada y de doble voluta. Esto se puede lograr instantáneamente ya que las turbinas se calculan en condiciones de flujo pulsante y desigual en motores turbo alimentados.

Además, se muestra una metodología para caracterizar el coeficiente de descarga de una válvula de alivio de presión. Para estimar el flujo de gas por la válvula de alivio en modelos unidimensionales, se correlaciona y valida un modelo empírico. Finalmente, se ha elaborado un mapa óptimo del coeficiente de descarga a través del método de interpolación, que puede integrarse en el sistema de modelo de motor turbo alimentado completo unidimensional, para calcular el flujo másico real a través de la válvula de descarga y las válvulas de conexión de desplazamiento.

Finalmente, los modelos han sido completamente validados al acoplarlos con un software de modelado unidimensional que simula tanto el banco de gas como el motor completo. Por un lado, los resultados de las validaciones del banco de gas muestran que el modelo puede predecir bien todas las variables de flujo estacionario. Por otro 
lado, los resultados de la validación de todo el motor muestran que el modelo es capaz de producir todas las variables del motor a plena carga como el flujo de masa de aire y el par de frenado con un buen grado de acuerdo con los datos experimentales. 


\section{Resum}

Malgrat la importáncia de les turbines radials amb doble entrada i de doble voluta per als motors turboalimentats, els seus mapes característics i el seu model completament predictiu mitjançant codis dinámics de gas 1D encara no estan ben establerts. La complexitat del flux constant i l'admissió desigual d'aquestes turbines, quan funcionen amb polsos de gas d'escapament del motor, les converteixen en un sistema difícil. Principalment a causa de la admissió de flux desigual, s'introdueix un grau addicional de llibertat a les conegudes turbines vendes o d'entrada d'una sola entrada. A més, l'addició de la segona entrada a la voluta de la turbina aporta una complexitat addicional per determinar els parámetres de rendiment de la turbina en estat estacionari en condicions d'admissió desigual. Aquesta tesi té com a novetat principal un procediment senzill per caracteritzar experimentalment i elaborar mapes característics d'aquestes turbines amb condicions de cabal desigual. Aquest métode d'análisi permet interpolar fácilment dins dels mapes distintius proposats o models senzills convincents per calcular i extrapolar parámetres de rendiment complet de les turbines d'entrada doble i de doble voluta.

Aquí també hem descrit dos models innovadors de línia mitjana 0D que requereixen una quantitat mínima de dades experimentals per calibrar tots dos: és a dir, el model de parámetre de flux massiu i el model d'eficiéncia isentròpica. Els dos models són predictius en condicions d'admissió de flux parcial o desigual utilitzant com a entrada: la proporció de flux entre les branques; la relació total de la temperatura entre les branques; la relació velocitat fulla-raig a cada branca i la proporció de pressió a cada branca. Aquests cinc inputs generalment es proporcionen de manera instantánia mitjançant codis de dinámica de gas 1D. Per tant, la novetat del model és la seva capacitat d'utilitzar-se d'una manera quasi constant per a la predicció del rendiment de les turbines bessones i de doble voluta. Es pot aconseguir de forma instantánia, ja que les turbines es calculen en condicions de flux pulsatòries i desiguals en motors turboalimentats.

A més, es mostra una metodologia per a caracteritzar el coeficient de descárrega d'una válvula de connexió per canals i desplaçaments en un suport de gas. Per estimar el flux de gas sobre el mateix en models unidimensionals, es correlaciona i valida un model empíric. Finalment, s'ha elaborat un mapa òptim de coeficient de descárrega mitjançant el métode d'interpolació, que pot integrar-se al sistema de model turboalimentat complet del motor turbo, per calcular el cabal de massa real a través de les válvules de connexió de desguás i desplaçament.

Finalment, els models s'han validat completament combinant-los amb un programari de modelatge unidimensional que simula tant el suport de gas com el motor sencer. D'una banda, els resultats de les validacions de l'estand de gas demostren que el model és capaç de predir bé totes les variables de flux constant. D'altra banda, els resultats de validació del motor complet demostren que el model és capaç de produir 
totes les variables del motor de cárrega completa, com ara el flux de massa d'aire i el pare de fre d'una bona manera amb les dades experimentals. 


\section{List of publications from the author of this thesis manuscript.}

The respondent is the co-author of the publications mentioned in this section, with his supervisor joint with other doctors of the I.U. CMT-Motores Térmicos (CMT). The origin of these publications were the research activities performed along with the candidate's doctorate, which was supervised by Prof. José Ramón Serrano Cruz, oriented and discussed with the other co-authors of the papers. The respondent performed experimental activities, results post-processing, analyzing and developed the models presented in the publications. Discussion of results and methodologies were done in collaboration with his supervisor as well as with the co-authors. The contribution of every author to the publications are included in the contributions description section of every publication. Laboratories, material and human resources of UPV and CMT were used to fulfill the Ph.D. works

The results of all these works have been compiled, ordered, completed, and further discussed in the present thesis manuscript. All parts, analyses, or conclusions from these or whatever other publications, have been appropriately referred along with the paper. Nevertheless, for the sake of readiness and to protect the thesis writing style, the publications of this list may not have been specifically cited every time, as part of the contents, figures and discussions have been directly imposed from my publications. This disclaimer corrects, compensates, and justifies the fact that being the $\mathrm{PhD}$ candidate, the experimental work of my thesis and the origin of the innovation component have resulted in the publications mentioned in this section, therefore compiling my thesis.

The $\mathrm{PhD}$ candidate is always the last signer of the CMT members in every publication since the protocol among CMT members is signing by seniority order, first the doctors, and last the $\mathrm{PhD}$ candidate. 


\section{Publication in Journal}

[1] J. R. Serrano, F. J. Arnau, T. Andrés, and V. Samala. "Experimental procedure for the characterization of turbocharger's waste-gate discharge coefficient". Advances in Mechanical Engineering 9.(10) (2017). ISSN: 16878140. DOI: $10.1177 / 1687814017728242$

[2] J. R. Serrano, P. Olmeda, F. J. Arnau, and V. Samala. "A holistic methodology to correct heat transfer and bearing friction losses from hot turbocharger maps in order to obtain adiabatic efficiency of the turbomachinery". International Journal of Engine Research (2019). ISSN: 20413149. DOI: 10.1177/ 1468087419834194

[3] J. R. Serrano, F. J. Arnau, L. M. Gracía-Cuevas, V. Samala, and L. Smith. "Experimental approach for the characterization and performance analysis of twin entry radial-inflow turbines in a gas stand and with different flow admission conditions". Applied Thermal Engineering (2019), p. 113737. ISSN: 1359-4311. DOI: $10.1016 / \mathrm{J}$. APPLTHERMALENG . 2019.113737

[4] J. R. Serrano, F. J. Arnau, L. M. García-Cuevas, and V. Samala. "A Robust Adiabatic Model for a Quasi-Steady Prediction of Far-Off Non-Measured Performance in Vaneless Twin-Entry or Dual-Volute Radial Turbines". Applied Sciences 10.(6) (2020), p. 1955. DOI: 10.3390/app10061955

\section{Publication in Congress}

[5] J. R. Serrano, F. J. Arnau, L. M. García-Cuevas, and V. Samala. "An experimental and modelling strategy for obtaining complete characteristic maps of twin-entry and dual-volute radial inflow turbines". In: Turbocharging Seminar 2019, Harbin , China. Nov. 10

[6] J. R. Serrano, F. J. Arnau, L. M. García-Cuevas, V. Samala, G. Stephane, and B. Samuel. "An experimental and modelling strategy for obtaining complete characteristic maps of dual-volute radial inflow turbines". In: ASME Turbo Expo 2020, London, England. Accepted for Publication (Recommended to Journal). June 2020 


\section{Acknowledgments}

It is an enjoyable journey of the last five years at the CMT-Motores Térmicos. This work would not have been completed without motivation and help from CMTMotores Térmicos management.

Firstly, I would like to express my sincere gratitude to my supervisor Prof. José Ramón Serrano Cruz, for his endless support, immense patience, motivation, and guidance throughout my $\mathrm{PhD}$. I owe it to him for the successful completion of my thesis, not only with his insightful comments and encouragement but also for the tough question that I was poised to widen my research from various perspectives. I could not have imagined having a better environment and advisor like him.

I also would like to thank Dr. Luis Miguel for his continuous help and guidance throughout these years. I have learned a lot from him. My gratitude also goes to Dr. Francisco Arnau, for his excellent advice and his help.

I cannot list by name here all the technical staff of the CMT-Motores Térmicos and the departmental workshop. They have contributed to modifying, updating and maintaining the test rig in a way or the other. I express my sincere appreciation to all of them, especially to Miguel Ortiz and Rafael Carrascosa, they both have helped in the building of the turbo test rig and also in the experiments.

I want to thank all my office mates, past and present over the last five years, especially Lele, Artem, Javi, Enrique and Lukas also Maria, Bárbara, Roberto, Alex and David. The PhD experience would have been rather different without these people.

Last but not least, I could not have finished this work without the support of my family: my parents and my siblings for having their faith and supporting me spiritually throughout. They are dearest in the heart and a great source of motivation.

I want to thank all my friends Santu, Flora, Sushma, Nishit, Anmol and Sanjeev whose faith on me have given positive energy to end this journey.

Special thanks go to my partner Birlipta, who has stood by me the whole time and helped me in a lot of ways for finishing this thesis. 

"You can't cross the sea merely by standing and staring at the water."

Rabindranath Tagore. 



\section{Contents}

1 Introduction 1

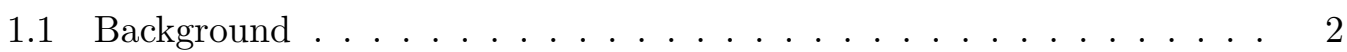

1.2 Motivation ...................... 4

1.3 Objectives .......................... 6

1.4 Thesis outline . . . . . . . . . . . . . . . 6

2 Literature review $\quad 9$

2.1 Introduction . . . . . . . . . . . . . . . . . . . . . 10

2.2 Experimental characterization . . . . . . . . . . . . . . 10

2.3 Turbine performance data . . . . . . . . . . . . . 12

2.4 Overview of double-entry turbine performance . . . . . . . . . . 15

2.5 Radial turbine modelling . . . . . . . . . . . . . . . . 16

2.6 Previous work . . . . . . . . . . . . . . . . . . 21

3 Measurement at different mass flow ratios in the double-entry $\begin{array}{ll}\text { turbines } & 35\end{array}$

3.1 Introduction . . . . . . . . . . . . . . . 36

3.2 Turbocharger gas stand for double-entry turbines . . . . . . . . . . . 36

3.3 Test methodology . . . . . . . . . . . . . . . . . 45

3.4 Quality of the measurements . . . . . . . . . . . . . . 51

3.5 Double-entry turbine performance parameters . . . . . . . . . . 55

4 Mass flow and efficiency models for double-entry turbines $\quad 71$

4.1 Introduction . . . . . . . . . . . . . . . . . . . 72

4.2 Reduced mass flow model for double-entry turbines . . . . . . . . . . 72

4.3 Efficiency fitting using VGT model . . . . . . . . . . . . . . . . . 85

4.4 Development of actual efficiency model for double-entry turbines . . . 90

4.5 Extrapolation of turbine performance parameters . . . . . . . . . . 107

4.6 Extrapolation of turbine performance parameters to non-measured MFRs . . . . . . . . . . . . . . . . . . . . . 114

5 Characterization of wastegate and scroll connection valves in double$\begin{array}{lr}\text { entry turbines } & 119\end{array}$

5.1 Introduction . . . . . . . . . . . . . . . . . . . . . . 120

5.2 Wastegate characterization . . . . . . . . . . . . . 120

5.3 Scroll connection valve characterization . . . . . . . . . . . . . 134

6 Heat transfer characterization $\quad 149$

6.1 Introduction . . . . . . . . . . . . . . . . . . 150

xvii 
6.2 Refined thermohydraulic tests . . . . . . . . . . . . . . 150

6.3 General correlation for heat transfer . . . . . . . . . . . . . . 154

6.4 Model validation using gas stand simulations . . . . . . . . . . . . 162

6.5 Heat transfer in double entry turbines . . . . . . . . . . . . 165

7 Double entry turbine model validation in $1 \mathrm{D}$ simulation software 169

7.1 Introduction . . . . . . . . . . . . . . . . . . . 170

7.2 Double entry turbine model quasi-steady adaptation . . . . . . . . 170

7.3 Double entry turbine modelling as a lookup table in GT-Power . . . . 171

7.4 Models validation in gas stand condition . . . . . . . . . . . . . 173

7.5 Models validation with engine conditions . . . . . . . . . . 182

$\begin{array}{llr}8 & \text { Conclusions and future works } & 209\end{array}$

8.1 Introduction . . . . . . . . . . . . . . . . . . 2 210

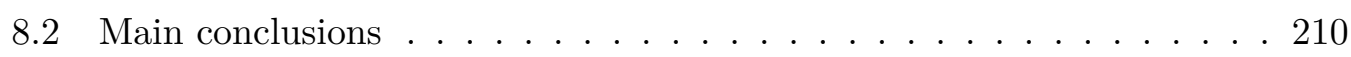

8.3 Future works . . . . . . . . . . . . . . . . . 218

$\begin{array}{ll}\text { Global bibliography } & 221\end{array}$ 


\section{List of Tables}

1.1 Turbocharger identity $\ldots \ldots \ldots \ldots \ldots$

3.1 Gas stand measurement equipments and their precision. . . . . . . . 43

3.2 Different flow admission conditions at the turbine inlets that were measured with T\#1TER turbine in the gas stand. . . . . . . . . . . . 46

3.3 Uncertainty of the performance parameters of T\#1TER . . . . . . . 55

4.1 Effective areas fitting coefficient values for T\#1TER and T\#2DVR . 82

4.2 Apparent efficiency fitting coefficients from Equation 4.18 for each turbine branch and MFR of T\#1TER. Every MFR map is fitted individually. . . . . . . . . . . . . . . . . . . . . . . . 89

4.3 Fitting coefficients of partial admission conditions of T\#1TER using apparent efficiency. . . . . . . . . . . . . . . . 92

4.4 Fitting coefficients of mixed turbine outlet temperature correction with new fitting function for T\#1TER . . . . . . . . . . . . . . . . 99

4.5 Final apparent efficiency fitting coefficients for three different doubleentry turbine types. Model constants for all three turbines are found in a global fitting using both branches together and all the MFRs data points . . . . . . . . . . . . . . . . . . . 104

4.6 Values of adjusted coefficient determination for both T\#1TER and T\#2DVR . . . . . . . . . . . . . . . 106

5.1 Quality of wastegate discharge coefficient model fitting for T\#2DVR and T\#4TER turbines . . . . . . . . . . . . . . . . 132

5.2 Quality of scroll connection valve discharge coefficient model fitting for T\#3DVM turbine . . . . . . . . . . . . . . . . . 146

6.1 Conductance and capacitance results from the thermohydraulic test bench . . . . . . . . . . . . . . . . 154

6.2 Main characteristics of the used turbocharger . . . . . . . . . . 156

6.3 Turbocharger material properties . . . . . . . . . . . . . . . . . 157

6.4 Capacitance model fitting values . . . . . . . . . . . . . . . . 158

6.5 Convective conductance model fitting parameter values. . . . . . . . 161

7.1 Engine main specifications . . . . . . . . . . . . . . . . . . . 184 



\section{List of Figures}

1.1 Difference in double-entry radial turbine geometries due to there designs; Twin-entry turbine (Left) and dual-volute turbine (Right) . . . 5

2.1 Turbine as single equivalent nozzle and stations distribution . . . . . . 22

2.2 Lumped heat transfer model for turbochargers [19] . . . . . . . . . . 27

2.3 Simplified schemes of a journal bearings (left) and a thrust bearing

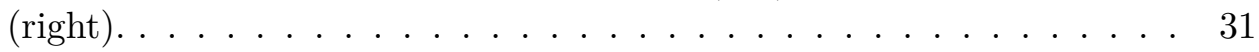

2.4 Schematic pressure distribution at the compressor and turbine wheels 31

2.5 Thermohydraulic test bench layout . . . . . . . . . . . 32

3.1 Hot and high flow test rig facility . . . . . . . . . . . 37

3.2 Combustion chamber front view(left) and flue gas pipe at the exit of combustion chamber(right) _. . . . . . . . . . . 39

3.3 Schematic layout of turbocharger gas stand for double-entry turbines . 40

3.4 Gas stand for testing the double-entry turbines at different flow admission conditions in steady flow situations. Mainly, showing the flow separation and controlling section in the gas stand. . . . . . . . . . 41

3.5 Showing the oil and water circuits used in the gas stand (a) Independent Oil system; (b) Independent water system . . . . . . . . . . . . . 42

3.6 Double-entry turbochargers setup in the gas stand. . . . . . . . . . 44

3.7 Turbocharger working in quasi-adiabatic and hot exposed conditions . 44

3.8 Comparison of MFR versus RMFR variables for both twin-entry and dual-volute turbines which are tested under quasi-adiabatic conditions $\quad 47$

3.9 Compressor map of T\#1TER in all admission cases . . . . . . . . 48

3.10 Twin-entry turbine inlet flow interaction map . . . . . . . . . . . . 49

3.11 Dual-Volute turbines inlet flow interaction map: T\#2DVR (Left) T\#3DVM

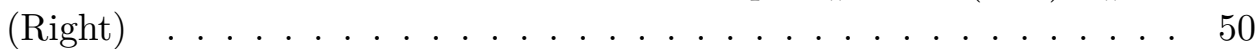

3.12 Turbine scroll pressure ratio with mass flow ratio . . . . . . . . . 50

3.13 Mass flow balance of T\#1TER turbocharger: (a)Turbine mass flow; (b)Compressor mass flow . . . . . . . . . . . . .

3.14 Power-based adiabatization approach for the turbine side: results shown for the T\#1TER at one Partial, full and unequal admission conditions (circle points represent with heat transfer, and diamond points represent without heat transfer $\ldots \ldots \ldots \ldots \ldots$

3.15 Power-based adiabatization approach for the compressor side: results shown for the T\#1TER at one Partial, full and unequal admission conditions (circle points represent with heat transfer, and diamond points represent without heat transfer $) \ldots \ldots \ldots . \ldots 53$ 
3.16 Turbocharger energy balance in all admission conditions: T\#1TER (left) and T\#2DVR (right) . . . . . . . . . . . . 54

3.17 Turbocharger absolute unbalance in all admission conditions: (a) shows the results for T\#1TER; (b) shows the results for T\#2DVR . . . . . . 56

3.18 Turbine map considering average inlet parameters of T\#1TER (left) and T\#2DVR (right) . . . . . . . . . . . . 57

3.19 Effective turbine efficiency with overall BSR for T\#1TER turbine . . 58

3.20 Relation between total isentropic power of turbine with compressor power in different flow admission conditions . . . . . . . . . . . 59

3.21 Effective turbine efficiency with overall BSR and MFR (Interpolated) for T\#1TER turbine . . . . . . . . . . . . . . . . 59

3.22 Turbine map considering double-entry turbines as two separate turbine (results shown for all the three turbochargers) . . . . . . . . . . 61

3.23 Enthalpy-entropy expansion process in double-entry turbines . . . . . 63

3.24 Flow at downstream of the turbine . . . . . . . . . . . . . . 64

$3.25 \mathrm{~K}$ type thermocouples at the turbine outlet measurement pipe . . . . . 64

3.26 Apparent efficiency maps considering double turbines as two separate turbine (results shown for all the three turbochargers) . . . . . . . 66

3.27 Turbine mass flow and apparent efficiency maps in the case of MFR = $0.2,0.8$ and 0.53 (results shown for twin-entry turbine) $\ldots \ldots$. . . 67

3.28 Apparent efficiency maps considering dual-volute turbines as two separate turbine (results shown for all the three turbochargers) . . . . .

4.1 Vaneless dual-entry turbines station distribution and two entries into two equivalent nozzles . . . . . . . . . . . . . . . 73

4.2 Geometrical description of vaneless turbine . . . . . . . . . . . 74

4.3 Geometrical description of twin-entry turbine (T\#1TER) . . . . . . 75

4.4 Geometrical description of Dual-Volute turbines (T\#2DVR and T\#3DVM) 77

4.5 Reduced mass flow coefficients obtained using the individual MFR fitting for both T\#1TER and T\#2DVR turbines. Each turbine entry map is fitted one by one. . . . . . . . . . . . . . . . . . 80

4.6 Reduced mass flow coefficient trends obtained using a global fitting procedure. Trends are imposed and all MFRs are fitted at the same time. The coefficients from individual MFR fittings are also shown for comparison. . . . . . . . . . . . . . .

4.7 Reduced mass flow coefficient trends obtained using a global fitting procedure for dual-volute mixed flow turbine (T\#3DVM). Trends are imposed and all MFRs are fitted at the same time. The coefficients from global fitting procedure for T\#2DVR turbine are also shown for comparison. . . . . . . . . . . . . . . .

4.8 Reduced mass flow modelled versus experimental results for T\#1TER, T\#2DVR, and T\#3DVM turbochargers using the global fitting constants found for the respective turbine type. . . . . . . . . . 8 
4.9 Geometrical description for calculating the flow angle in vaneless twinentry/dual-volute turbines. . . . . . . . . . . . 86

4.10 Rotor inlet flow angles at different flow admission conditions for Shroud and Hub branches of T\#1TER . . . . . . . . . . . . 87

4.11 T\#1TER turbine apparent efficiency modelled versus experimental using VGT efficiency model . . . . . . . . . . . . . . . 88

4.12 Double entry turbines expansion process in partial admission conditions 91

4.13 Extrapolation of partial admission conditions for T\#TER (MFR 0 and

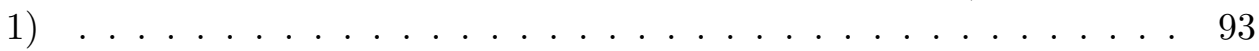

4.14 Estimated turbine outlet temperatures using extrapolated maps of MFR 0 and 1 for T\#1TER . . . . . . . . . . . . . . . 94

4.15 Estimated mixed turbine outlet temperature for full and unequal flows using extrapolated maps of MFR 0 and 1 for T\#1TER . . . . . . . 95

4.16 Error prediction in all admission conditions using the extreme flow maps for T\#1TER. (a) Mixed turbine outlet temperature error with MFR, (b) Mixed turbine outlet temperature error with $M F R_{x}^{\prime} \ldots \ldots$

4.17 Analysis of T\#1TER turbine outlet temperature error with different parameters in order to find an a correction function to reduce the error. (a) Error with $M F R_{x}$ and same reduced turbine speeds, (b) Error with reduced turbine speed of each branch and $M F R_{x}$, (c) Error with the BSR of each branch and $M F R_{x}$, (d) Error with same reduced turbine speed for different $M F R_{x}$ and BSR. . . . . . . . . . . . 97

4.18 Mixed turbine outlet temperature measured versus modelled for T\#1TER 99

4.19 Turbine apparent efficiency modelled versus experimental results for T\#1TER, T\#2DVR, and T\#3DVM . . . . . . . . . . 105

4.20 Procedure for extrapolating the reduced mass flow and apparent efficiency of double-entry turbines . . . . . . . . . . . . . . 108

4.21 Twin-entry turbine (T\#1TER) reduced mass flow and apparent efficiency extrapolation for shroud and hub branches using all maps fitting coefficients. . . . . . . . . . . . . . . . . 109

4.22 Cont . . . . . . . . . . . . . . . . . . . . 111

4.22 Dual-volute turbine (T\#2DVR) reduced mass flow and apparent efficiency extrapolation for LV and SV volutes using only the filled points fitting coefficients. . . . . . . . . . . . . . 112

4.23 Cont. . . . . . . . . . . . . . . . . . . . . 113

4.23 Dual-volute turbine (T\#3DVM) reduced mass flow and apparent efficiency extrapolation for LV and SV volutes using only 3 MFRs $(0,0.5$

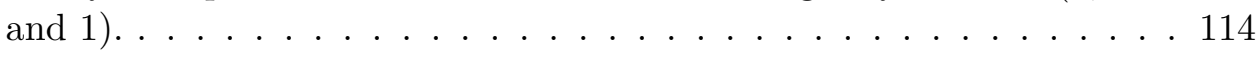

4.24 Prediction of model results using 3 maps of twin-entry radial inflow turbine during the fitting procedure . . . . . . . . . . . 115

4.25 Cont. . . . . . . . . . . . . . . . . . . . 115 
4.25 Twin-entry turbine (T\#1TER) reduced mass flow and apparent efficiency extrapolation for shroud and hub branches using only 3 MFRs $(0,1$ and 0.5$)$ model fitting constants. . . . . . . . . . . 116

4.26 Prediction of model results using 3 maps of dual-volute radial inflow turbine during the fitting procedure . . . . . . . . . . 117

4.27 Dual-volute turbine (T\#2DVR) reduced mass flow and apparent efficiency extrapolation for long and short volutes using only 3 MFRs ( 0 , 1 and 0.5$)$ model fitting constants . . . . . . . . . . . 118

5.1 Schematic layout of turbocharger gas stand . . . . . . . . . . . . 121

5.2 Test method for characterizing wastegate valve experimentally in a gas stand . . . . . . . . . . . . . . . . . . . 121

5.3 Turbine map with the points that measured when the wastegate valve is mechanically closed. (a) Twin-entry turbine with a given engine full load points, (b) Dual-volute turbine for a selected expansion ratios. . . 122

5.4 Electronic wastegate actuator . . . . . . . . . . . . . . 123

5.5 T\#4TER turbine stepper motor calibration curve for different tests . 124

5.6 T\#4TER turbine stepper motor current intensity for the points near the wastegate valve closing position . . . . . . . . . . . . . 125

5.7 Compressor map with the points tested at wastegate closed and at different opening positions. (a) T\#4TER compressor map with the given engine full load points, (b) T\#2DVR compressor map with the selected expansion ratios. . . . . . . . . . . . . . . . 126

5.8 Turbine mass flow diagram of an internal wastegate with the wastegate valve in the closed and open position. . . . . . . . . . . . . . 126

5.9 Turbine map with points tested when the wastegate is mechanically closed and with the several wastegate openings. (a) T\#4TER turbine map with the given engine full load points, (b) T\#2DVR turbine map with the selected expansion ratios. . . . . . . . . . . . . . 127

5.10 Experimentally estimated reduced mass flow through the wastegate section (legends refers to closed wastegate expansion ratio), (a) Wastegate flow of T\#4TER turbine, (b) Wastegate flow of T\#2DVR turbine (mass flow values are normalized with the maximum experimental WG flow $\ldots \ldots \ldots$. . . . . . . . . . . . . . . 127

5.11 Reduced mass flow in the wastegate section with the wastegate opening percentage (legends refers to closed wastegate expansion ratio), (a) T\#4TER turbine results. (b) T\#2DVR turbine results (mass flow values are normalized with the maximum experimental WG flow). . . 128

5.12 Experimental discharge coefficient values with the wastegate opening percentage (legends refers to closed wastegate expansion ratio), (a) T\#4TER turbine results, (b) T\#2DVR turbine results (actual discharge coefficient values are shown instead of normalized) $\ldots . . .131$ 
5.13 Wastegate valve discharge coefficient modelled as a function of turbine expansion ratio and wastegate opening position. (a) T\#4TER turbine predictions. (b) T\#2DVR turbine predictions. . . . . . . . . . 133

5.14 Modelled discharge coefficient against measured discharge coefficient . 133

5.15 Modelled wastegate reduced mass flow against measured wastegate reduced mass flow . . . . . . . . . . . . . . . . . . . 134

5.16 Exhaust mass flow conditions in a dual-volute turbine when the scroll connection valve is present. . . . . . . . . . . . . . 135

5.17 Test methodology for characterizing the scroll connection valve in partial admission maps. . . . . . . . . . . . . . . . . . . 136

5.18 T\#3DVM Partial admission maps tested with SCV closed and with different SCV openings. (a) SCV opening when all flow is in long volute branch, (b) SCV opening when all the flow is in short volute branch. . . . . . . . . . . . . . . . . 136

5.19 T\#3DVM compressor map with SCV closed and with different SCV opening tested points. (a) SCV opening when all flow is in long volute branch, (b) SCV opening when all the flow is in short volute branch. . 137

5.20 Showing the procedure for estimating the new SCV closed mass flow with the SCV opened test conditions. Once it is estimated, the actual SCV flow can be calculated. . . . . . . . . . . . . . . . . 139

5.21 Extrapolated T\#3DVM turbine map when main flow is passed through the long volute branch. Showing with new and old SCV closed mass flows for comparison. . . . . . . . . . . . . . . . . . . 140

5.22 Variation in turbine reduced speed for recovering the same expansion ratio in the active branch when the SCV is opened. Left: Long volute branch testing with SCV opening. Right: Short volute branch testing with SCV opening. . . . . . . . . . . . . . . . . . . . . 141

5.23 Extrapolated T\#3DVM turbine map when main flow is passed through the short volute branch. Showing with new and old SCV closed mass flows and there difference.

5.24 Actual mass flow through the SCV segment at different SCV openings. (a) Long volute branch testing with SCV opening, (b) Short volute branch testing with SCV opening. . . . . . . . . . . . . . . . . 142

5.25 Change in MFR with respective to the SCV opening and the flow direction to each side through SCV. Left: Main flow in the long volute branch with SCV opening. Right: Main flow in the short volute branch with SCV opening. . . . . . . . . . . . . . . . . . . . . 143

5.26 Actual mass flow against the ideal mass flow for SCV valve. (a) SCV flow LV to SV. (b) SCV flow SV to LV. . . . . . . . . . . . . . . . 144

5.27 Variation of SCV discharge coefficient values with scroll pressure ratio. (a) Flow passing from LV to SV, (b) Flow passing from SV to LV. . . 145

5.28 Modelled discharge coefficient of scroll connection valve. (a) For flow passing from LV to SV. (b) For flow passing from SV to LV. . . . . . . 146 
5.29 Comparison of modelled discharge coefficient values against experimental discharge coefficient values in all the SCV openings. (a) For flow passing from LV to SV. (b) For flow passing from SV to LV. . . . 147

5.30 Comparison of modelled SCV reduced mass flow values against experimental SCV reduced mass flow values in all the SCV openings. Left: For flow passing from LV to SV. Right: For flow passing from SV to LV.147

6.1 Node temperature evolution in whole test, both steady and transient test are performed at once. . . . . . . . . . . . . . . . . 151

6.2 A criteria of lower variation than $0.01^{\circ} \mathrm{C}$ per minute in turbocharger wall temperature is used to ensure that steady state conditions are achieved. . . . . . . . . . . . . . . . . . . 151

6.3 Showing thermocouple instrumentation on the turbocharger wall . . . 152

6.4 Node temperatures from steady state tests; (a) When the hot oil in turbine element; (b) When the hot oil in compressor element. . . . . . 153

6.5 Heat unbalance in steady state tests; (a) When the hot oil in turbine element; (b) When the hot oil in compressor element. . . . . . . . . 153

6.6 Capacitance fitting for different turbochargers . . . . . . . . . . 158

6.7 Simplification of turbocharger geometry $[65] \ldots \ldots$. . . . . . . . 159

6.8 Estimating an effective area where contact is taking place between the two metal nodes . . . . . . . . . . . . . . . . . . . 160

6.9 Conductive conductance fitting for different turbochargers . . . . . . 162

6.10 Comparison of normalized node temperature distribution between the experimental and CMT-TCM model output . . . . . . . . . . . . 163

6.11 Predictions of outlet temperatures from both CMT-TCM and GTTCM models; Left: Comparison of compressor outlet temperature; Right: Comparison of turbine outlet temperature. . . . . . . . . . . 164

6.12 Predictions of outlet temperatures from both CMT-TCM and GTTCM models; Left: Comparison of compressor outlet temperature; Right: Comparison of turbine outlet temperature. . . . . . . . . . . 165

6.13 Overview of the calculation methodology of heat transfer in a turbocharger to obtain the adiabatic efficiency. . . . . . . . . . 166

6.14 Overview of estimating the heat transfer in double entry turbines during the engine calculations. . . . . . . . . . . . . . . 167

7.1 Double entry turbine model computational domain . . . . . . . . . 171

7.2 Concept of modelling double entry turbine using look up table method in GT-power . . . . . . . . . . . . . . . . . 172

7.3 Dummy VGT position as a function of MFR . . . . . . . . . 173

7.4 Double entry turbocharger model as gas stand in GT-Power simulation software . . . . . . . . . . . . . . . . . . . . 174 
7.5 Gas stand simulation validation parameters; CMT-DETCM versus experimental values of T\#2DVR dual volute turbocharger; (a) Mass flow ratio, (b) Turbocharger speed, (c) Compressor mass flow and (d) Turbine outlet mass flow. . . . . . . . . . . . . . . . . . . . 177

7.6 Gas stand simulation validation parameters; GT-DETCM versus experimental values of T\#2DVR dual volute turbocharger. (a) Mass flow ratio, (b) Turbocharger speed, (c) Compressor mass flow and (d) Turbine outlet mass flow. . . . . . . . . . . . . . . . . . . . 178

7.7 Gas stand simulation validation parameters; CMT-DETCM versus experimental values of T\#2DVR dual volute turbocharger; (a) Compressor outlet temperature, (b) Mixed turbine outlet temperature . . . . . 179

7.8 Gas stand simulation validation parameters; GT-DETCM versus experimental values of T\#2DVR dual volute turbocharger; (a) Compressor outlet temperature, (b) Mixed turbine outlet temperature . . . . . 180

7.9 T\#2DVR turbine outlet estimation in each branch using CMT-DETCM turbo model (a) Outlet temperatures in full and unequal admissions,

(b) Outlet temperatures in partial admission conditions . . . . . . . . 180

7.10 T\#2DVR turbine outlet estimation in each branch using GT-DETCM turbo model (a) Outlet temperatures in full and unequal admissions,

(b) Outlet temperatures in partial admission conditions . . . . . . . . 182

7.11 The layout of the engine test cell and the sensor instrumentation . . . 183

7.12 Pressure and temperature sensor instrumentation on the exhaust line of the engine. . . . . . . . . . . . . . . . . 185

7.13 Dual volute turbine connection with the engine cylinders. . . . . . . 185

7.14 Wastegate and SCV flow areas with the cylindrical valve opening. . . 186

7.15 Dual volute mixed flow turbine engine test results at full load steady conditions. . . . . . . . . . . . . . . . 188

7.16 Exhaust manifold heat transfer multipliers in single entry and dual volute turbines during the engine simulations; Left: Single entry; Right: Dual-Volute. . . . . . . . . . . . . . . . . . . . 191

7.17 Methodology for simulating when the wastegate flow area is the same 192

7.18 Working of the designed control system when the wastegate flow area is same and the SCV valve is opened. . . . . . . . . . . . . . . 193

7.19 SCV opened inlet manifold boost pressure from the PID controller . . 194

7.20 CMT-DETCM and GT-DETCM model wastegate positions and the estimated discharge coefficient values. . . . . . . . . . . . . . . . 194

7.21 CMT-DETCM and GT-DETCM models SCV positions and the estimated discharge coefficient values. . . . . . . . . . . . . . . . . 195

7.22 Validation parameters of SCV opened simulation; Outputs from CMTDETCM and GT-DETCM were compared against the experimental data at different engine speeds. (a) Turbocharger speed; (b) Compressor outlet temperature; (c) Compressor outlet pressure; (d) Inlet manifold temperature. . . . . . . . . . . . . . . . . . 196 
7.23 Validation parameters of SCV opened simulation; Outputs from CMTDETCM ans GT-DETCM were compared against the experimental data at different engine speeds. (a) Air mass flow; (b) Brake torque;

(c) Turbine inlet pressure in LV; (d) Turbine inlet pressure in SV.

7.24 Validation parameters of SCV opened simulation; Outputs from CMTDETCM and GT-DETCM were compared against the experimental data at different engine speeds. (a) Turbine inlet temperature LV; (b) Turbine inlet temperature SV; (c) Turbine outlet temperature; (d) Turbine outlet pressure. . . . . . . . . . . . . . . . . . . 198

7.25 Methodology for simulating when the wastegate flow area is unequal with cylindrical opening angle . . . . . . . . . . . . . . . . 200

7.26 Working of the designed control system when the wastegate flow area is unequal and the SCV is closed . . . . . . . . . . . . . . . . . 201

7.27 Inlet manifold boost pressure from the PID controller . . . . . . . . . 202

7.28 CMT-DETCM and GT-DETCM models wastegate positions and there discharge coefficient values when the SCV is closed. (a) and (c) are the results from CMT-DETCM model; (b) and (d) are the results from GT-DETCM model. . . . . . . . . . . . . . . . . . . . . . . . 202

7.29 Validation parameters of SCV closed simulations; Outputs from CMTDETCM and GT-DETCM were compared against the experimental data at different engine speeds. (a) Turbocharger speed; (b) Compressor outlet temperature; (c) Compressor outlet pressure; (d) Inlet manifold temperature. . . . . . . . . . . . . . . . . 204

7.30 Validation parameters of SCV closed simulation; Outputs from CMTDETCM ang GT-DETCM were compared against the experimental data at different engine speeds. (a) Air mass flow; (b) Brake torque; (c) Turbine inlet pressure in LV; (d) Turbine inlet pressure in SV. . . 205

7.31 Validation parameters of SCV closed simulation; Outputs from CMTDETCM ang GT-DETCM were compared against the experimental data at different engine speeds. (a) Turbine inlet temperature LV; (b) Turbine inlet temperature SV; (c) Turbine outlet temperature; (d) Turbine outlet pressure. . . . . . . . . . . . . . . . 206 


\section{List of symbols}

\section{Latin characters}

\begin{tabular}{|c|c|c|}
\hline A & Area & $\mathrm{m}$ \\
\hline$B h$ & Blade height & $\mathrm{m}$ \\
\hline$c_{p}$ & Specific heat capacity & $\mathrm{J} \mathrm{kg}^{-1} \mathrm{~K}^{-1}$ \\
\hline$C_{d}$ & Discharge coefficient & - \\
\hline$C_{s s}$ & Isentropic jet velocity & $\mathrm{ms}^{-1}$ \\
\hline$C$ & Thermal Capacitance & $\mathrm{J} \cdot \mathrm{K}^{-1}$ \\
\hline$c$ & Specific heat capacity & $\mathrm{J} \cdot \mathrm{K}^{-1}$ \\
\hline$C_{s}$ & Contact surface average diameter & $\mathrm{m}$ \\
\hline$D$ & Diameter & $\mathrm{m}$ \\
\hline$d^{\prime}$ & Apparent efficiency fitting coefficient & - \\
\hline$D V$ & Dual-Volute & - \\
\hline$i, j, k$ & Generic codes & - \\
\hline$K$ & Conductance & $\mathrm{W} \cdot \mathrm{K}^{-1}$ \\
\hline$k$ & Conductivity & $\mathrm{W} \cdot \mathrm{m}^{-1} \cdot \mathrm{K}^{-1}$ \\
\hline$K$ & Efficiency equation coefficient (-) & \\
\hline$L$ & Length & $\mathrm{m}$ \\
\hline$m$ & Mass & $\mathrm{kg}$ \\
\hline$\dot{m}$ & Mass flow & $\mathrm{kg} \mathrm{s}^{-1}$ \\
\hline$\dot{m}^{*}$ & Reduced Mass Flow & $\frac{\mathrm{kg} \cdot \sqrt{\mathrm{K}}}{\mathrm{s} \cdot \mathrm{Pa}}$ \\
\hline M & Mach Number & \\
\hline$n$ & Rotational speed & $\mathrm{rpm}$ \\
\hline$p$ & Pressure & $\mathrm{Pa}$ \\
\hline$\dot{Q}$ & Heat flow & $\mathrm{W}$ \\
\hline $\mathrm{R}$ & Gas Constant & \\
\hline$r$ & Rotor radius & $\mathrm{m}$ \\
\hline$T$ & Temperature (K) & \\
\hline$c_{p}$ & Specific heat capacity & $\mathrm{J} \mathrm{kg}^{-1} \mathrm{~K}^{-1}$ \\
\hline$n$ & Rotational speed (rpm) & \\
\hline$p$ & Pressure $(\mathrm{Pa})$ & \\
\hline$T$ & Temperature (K) & \\
\hline$\dot{W}$ & Power & $\mathrm{W}$ \\
\hline$u$ & Blade tip speed & $\mathrm{ms}^{-1}$ \\
\hline$v$ & Absolute velocity & $\mathrm{ms}^{-1}$ \\
\hline$\Delta h_{M F R_{x}}^{S h / L V}$ & Apparent work Entry 1 & \\
\hline$\Delta h_{M F R_{x}}^{H / S V}$ & Apparent work Entry 2 & \\
\hline$\Delta h_{S h_{x} / L V_{x}}$ & Actual work Entry 1 & \\
\hline
\end{tabular}


$\Delta h_{H_{x} / S V_{x}} \quad$ Actual work Entry 2

$\Delta h_{S, S h_{x} / L V_{x}}$ Isentropic work Entry 1

$\Delta h_{S, H_{x} / S V_{x}} \quad$ Isentropic work Entry 2

\section{Greek characters}

$\begin{array}{lll}\alpha & \text { Absolute velocity angle } & \mathrm{rad} \\ \beta & \text { Relative velocity angle } & \mathrm{rad} \\ \gamma & \text { Heat capacity ratio } & \\ \sigma & \text { Corresponding blade-to-jet speed ratio } & \\ \rho & \text { Density } & \mathrm{kg} / \mathrm{m}^{3} \\ \theta & \text { Geometry fitting constant } & - \\ \eta_{M F R_{x}} & \text { Apparent turbine efficiency } & - \\ \eta & \text { Corresponding efficiency } & \\ \Pi & \text { Corresponding pressure ratio } & \end{array}$

\section{Sub- and Superscripts}

eff Refers to effective equivalent nozzle

exp Experimental value

C Compressor

C Compressor node

DV Dual-Volute

geom Geometry

$H \quad$ Refers to turbocharger housing

$H 1 \quad$ Housing node close to the turbine

$H 2$ Central housing node

$H 3 \quad$ Housing node close to the compressor

$H \quad$ Hub

$i \quad$ Discriminates $S h$ from $H$ in $T E$ or $L V$ from $S V$ in $D V$

$j \quad$ Refers to $T E$ or to $D V$ turbine

$k \quad$ Refers to flow in each direction

$m$ Mechanical

LV $\quad$ Long Volute

mod Model value

$m \quad$ Mean value

$\mathrm{Neq} \quad$ Refers to equivalent nozzle

pred Predicted value

red Refers to reduced variables

$S V \quad$ Short Volute

Sh Shroud

T Turbine node 
TE Twin-Entry

$t / s \quad$ Total-to-static

$T, s \quad$ Turbine isentropic

$T$ Turbine

$0 \quad$ Turbine inlet static states

ot Turbine inlet total conditions

4 Turbine outlet static states

04 Turbine outlet total conditions

$4 s \quad$ Turbine outlet isentropic conditions

$4 m$ Average value at the rotor outlet

\section{Acronyms}

$\begin{array}{ll}\text { BEVs } & \text { Battery Electric Vehicles } \\ \text { BSR } & \text { Blade Speed Ratio } \\ \text { DVR } & \text { Dual-volute Radial inflow turbine } \\ \text { DVM } & \text { Dual-volute Mixed inflow turbine } \\ \text { EGR } & \text { Exhaust Gas Recirculation (-) } \\ \text { ETE } & \text { Effective Turbine Efficiency (-) } \\ \text { ECU } & \text { Electronic Control Unit } \\ \text { ICE } & \text { Internal Combustion Engine } \\ \text { ILS } & \text { Independent Lubrication System } \\ \text { ICS } & \text { Independent Cooling System } \\ \text { MFP } & \text { Mass Flow Parameter } \\ \text { MFR } & \text { Mass Flow Ratio } \\ \text { MMA } & \text { Mixed Momentum Approach } \\ \text { OEM } & \text { Original Equipment Manufacturer } \\ \text { RMFR } & \text { Reduced Mass Flow Ratio } \\ \text { RMSE } & \text { Root Mean Square Error } \\ \text { SCV } & \text { Scroll Connection Valve } \\ \text { SSE } & \text { Sum of the Square due to Error } \\ \text { TTA } & \text { Two Turbine Approach } \\ \text { TER } & \text { Twin-Entry Radial inflow turbine } \\ \text { VGT } & \text { Variable Geometry Turbine } \\ \text { VNT } & \text { Variable Nozzle Turbine } \\ \text { VEMOD } & \text { Virtual Engine Model } \\ \text { WG } & \text { Wastegate }\end{array}$





\section{Chapter 1}

\section{Introduction}

\begin{tabular}{|c|c|c|}
\hline \multicolumn{3}{|l|}{ Contents } \\
\hline 1.1 & $\ldots \ldots \ldots \ldots \ldots$ & 2 \\
\hline 1.2 & $\ldots \ldots \ldots \ldots \ldots \ldots \ldots$ & 4 \\
\hline 1.3 & Objectives & 6 \\
\hline 1.4 & Thesis outline & 6 \\
\hline & 1.4.1 Turbocharger identities. . & 8 \\
\hline
\end{tabular}




\section{$1.1 \quad$ Background}

The invention of internal combustion engines (ICE), either in the form of spark ignition (SI) or compression ignition (CI) was a benefit to the transportation sector for its efficiency from many decades. Today, on the road, there are more than one billion cars and, internal combustion engines power over 99 per cent of them. Over the years, the primary goal of engine researchers and automotive manufacturers was to reduce vehicle emissions and also to be more fuel-efficient. Indeed, significant advances have been made over the last few decades, and the ICE technologies are significantly transparent and more efficient. However, the emission scandals like diesel-gate have threatened the ability and left a large stain on this technology. Besides, with the growing interest in global environmental issues, the automotive manufacturers are facing increasing challenges to reduce the gaseous emissions [7] coming from the ICE. Moreover, also to meet the strict emission legislation year by year [8]. Satisfying these more tightening regulations cost-effectively is the most crucial challenge for automotive makers nowadays.

Despite the rapid growth of electric car sales in recent years, one should note that the battery electric vehicles (BEV) have heavy $\mathrm{CO}_{2}$ footprints when analysed from cradle-to-grave $[9,10]$. Additionally, considering and targeting to more extensive use of electric drive powertrain in future still requires the development of the appropriate grid and charging infrastructure. It also needs the essential improvements in battery energy density for having long-range drives. These elements are crucial for effective market penetration of electric vehicles, including enhancements in cost-effectiveness as well as technology.

Roadmaps have been drawn to anticipate the potential automotive technology trends by 2050 [11]. It was predicted that a mix of solutions would characterize future mobility. The plug-in hybrid powertrain and small capacity turbocharged engines will play a significant part of the passenger cars need in decades ahead [11, 12]. Further, the electric vehicles will also play important roles by 2050 , depending on buyer acceptance (e.g. cost) and high energy density battery technologies. Moreover, alternative fuels will also be an essential part of the energy roadmap. It is foreseen that the ICE will be active until 2050, by representing a primary role as generating the power or for powering the vehicle itself, even in a fully electrified powertrain arrangement. Because of this, there is prominent importance in developing the thermal efficiency of internal combustion engines without a significant increase in the purchase and operating costs in the short-to-medium terms. These goals can be obtained through further downsizing and boosting, advancing in the combustion, after-treatment and also by partial electrification in the form of hybridization.

In order to compete with an electric powertrain, automotive engineers are developing new internal combustion engines to be environmentally friendly. At the same time, keeping the vehicle performance and also having sufficiently attractive fuel con- 
sumption to satisfy the customers' requirement. Over the years, automotive OEMs are increasingly going in for their engines downsizing. It has been proofed to be an efficient way in reducing the engine $\mathrm{CO}_{2}$ emission with promising cost-to-benefit ratio compared with many other advanced ICE technologies [13].

Turbocharging is one of the foremost ways of downsizing and has been enforced predominantly to diesel engines. Even though, the technology has also been used to other engine types such as the homogeneous charge compression ignition engine [14], and also to throttle loss recovery on the gasoline engine [15]. It had been used on passenger car as a means to increase the output power of the baseline engines. Turbocharged gasoline engines were restricted by its combustion system, due to knocking issues. Lately, turbocharged gasoline engines earned renewed attention because of the need for lower fuel consumption and the use of direct injection system, which enhances the combustion behaviour and knock resistance. The traditional single entry turbine is widely used in a turbocharger, due to its more straightforward construction. Moreover, it gave many advantages in terms of fuel economy and emission reduction with the previous and current regulations. However, concerning the future legal requirements for low emissions (Euro 6d Full and Euro 7 regulations) with fuel efficiency, there is still need and potential for optimization.

Typically, a turbocharger turbine always confronts with a high pulsating flow coming from the internal combustion engines [16]. In large multi-cylinder engines, the dynamic operation of the turbocharger is profoundly is affected by the pulsations. Typically, the pulsations were often dampened to make the pressure at the turbine inlet as constant as possible. This dampened effect can be adjusted by increasing the volume of the exhaust manifold and configuring the turbine with a single-entry housing. This method, referred to as constant pressure turbocharging, ensures a continuous flow over the turbine wheel. But, it does not maximize the use of available exhaust gas energy due to the dampening of pressure peaks.

Turbine configuration that utilizes the fluctuating behaviour of the exhaust flow tends to have a turbine with multiple inlets; as in the double-entry turbines. The exhaust manifolds feeding the multiple inlets are divided, and depending on the engine configuration, they carry the exhaust from a couple of cylinders each. In a 4-cylinder engine configuration, each entry of the turbine is fed by the two cylinders. In a 6-cylinder engine, each entry is fed by three cylinders etc. One of the advantages of inlet separation, besides the maximized exhaust energy utilization, is the improved gas exchange by avoiding an overlap of the pressure pulses. Separation of pulse coming from the engine exhaust and minimizing the interferences between the cylinders during the exhaust process is vital for achieving enhanced rated power and low-end-torque targets in highly boosted four-cylinder engines.

Nowadays, automotive manufacturers are focusing on a wide range of engine operating conditions which are different from traditional full load operation. For achieving an optimum matching between the turbocharger and internal combustion engines, au- 
tomotive manufacturers are relying on one-dimensional engine cycle simulation tools. These tools are helpful to predict and study the effect of various parameters on engine performance. 1D simulation codes make possible the calculation of gas dynamics engine behaviour at low computational costs. Furthermore, the method also shows a critical approach to simulate the unsteady performance of the turbine [17].

Moreover, the exhaust system is essential for meeting the emissions limits across the operating range of the engine. As asserted in [18], during the engine part loads and transient conditions of the internal combustion engines, the turbocharger turbine works at off-design conditions. It is not viable to capture this behaviour by the standard turbine maps provided by the manufacturers. Because they only offer a narrow range of data that can measure in the gas stand. Therefore, the turbocharger turbine models should be capable of simulating real-life conditions. So that the prediction of exhaust gas mass flow, the pressure drop across the turbine and an energy transfer to the compressor are essential.

In last few years several dissertations have been performed concerning the experimentation and modelling of single entry vaned and vaneless turbines at CMT-Motores Térmicos of Universitat Politécnica de Valéncia. The thesis of Reyes-Belmonte [19] deals with the problems related to the internal heat transfers and extrapolation modelling of turbine maps and in a physical methodology. Dombrovsky [20], further improved the models of Reyes-Belmonte [19] to enhance engine simulation using commercial 1D tools like GT-POWER ${ }^{T M}$ and also studied the effects of external heat transfers in turbochargers. Inhestern [21] focused his study at the extreme off-design working conditions of radial turbines experimentally. Further, CFD analysis was performed to understand better the flow phenomena occurring in this kind of turbine. From CFD analysis, Inhestern developed different types of loss models to have an accuracy extrapolation in adiabatic turbine efficiency maps.

\subsection{Motivation}

The pulsating energy of the exhaust gases coming from the internal combustion engines is unsteady. As a result, the components downstream of the engine, such as the turbocharger operates in the unsteady flow zone. Due to this, radial inflow turbines with two entries are designed, and it can be generally called as double-entry turbines. They are of two types one is twin-entry (where the turbine scroll is meridionally divided, as shown in Figure 1.1(a)) and another is dual-volute (turbine scroll is circumferentially divided, as shown in Figure 1.1(b)).

Twin-entry and dual-volute radial inflow turbines are key technologies for avoiding interferences in the exhaust process of turbocharged engine cylinders. Four-cylinder petrol engines with high valve overlap periods and, whatever turbocharged engine with more than four cylinders blowing to the same entry of a turbine will suffer 

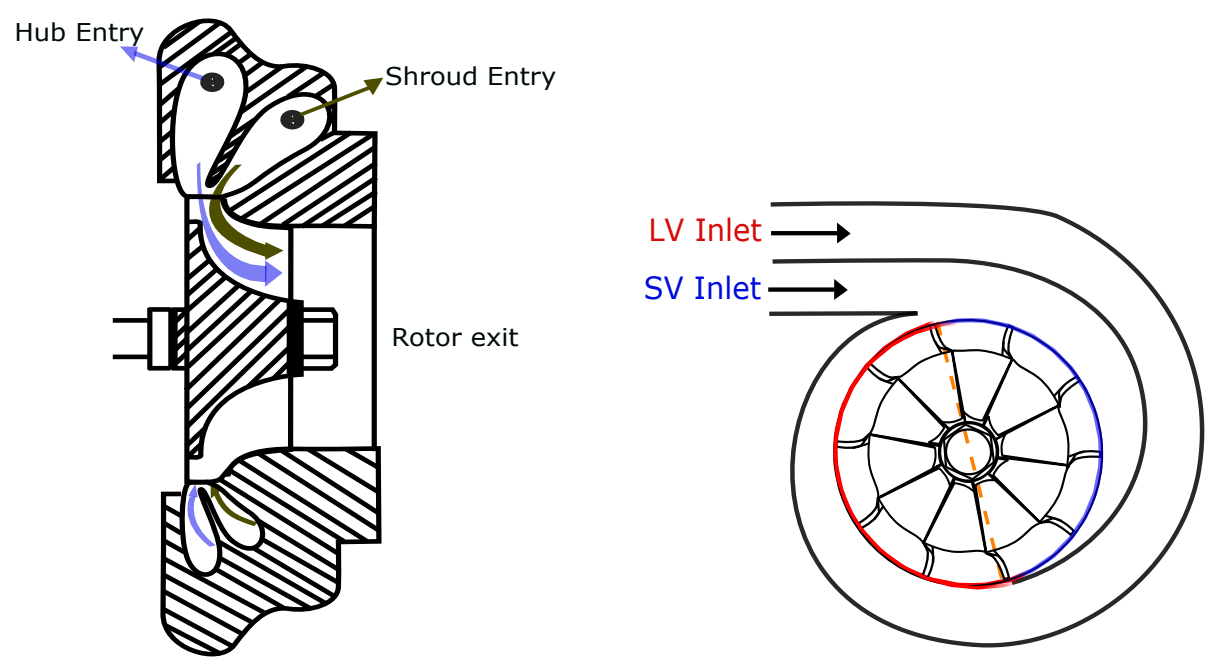

Figure 1.1: Difference in double-entry radial turbine geometries due to there designs; Twin-entry turbine (Left) and dual-volute turbine (Right)

from severe interferences in the exhaust process. Twin and dual-volute radial inflow turbines allow separating exhaust process of consecutive firing cylinders getting significant advantages in pumping losses and volumetric efficiency.

Even there are good examples in the literature about how to model single entry radial inflow turbines in turbocharged engines and with the one-dimensional gasdynamic codes. The corresponding twin-entry and dual-volute turbine modelling procedures are not predictive enough for calculating the entire gas exchange process of ICEs considering unsteady flow; the whole engine map (load and speed) and engine load and speed transients. One of the main reasons is the complexity of the unsteady exhaust gas, blown from the engine cylinders, makes it a challenging system. It is mainly due to the unequal flow admission conditions, which generates an additional degree of freedom concerning the well-known single entry vaned or vaneless turbines.

Quasi-steady modelling of turbine efficiency with the unsteady flow is a wellestablished procedure for radial inflow turbines in one-dimensional gas-dynamic codes. But in the case of double-entry turbines, the absence of adequate and completeenough characteristic maps do not allow this technique to be used. Many experimental studies were carried out to examine the performances of double-entry turbines working under different admission conditions. However, there is no clear information in the literature about how to determine the characteristic maps of this turbine types so that it gives particular information at different flow admission conditions. Besides, the knowledge of double-entry turbine behaviour is still inadequate, leading to an unsatisfactory performance prediction with the one-dimensional and zero-dimensional tools available. Therefore, it remains a question: How to get and how to manage com- 
plete and reliable enough maps of twin-entry and dual-volute turbines, for modelling or interpolating them in quasi-steady way at one-dimensional gas-dynamics engine codes.

\subsection{Objectives}

The main objective of this thesis is to provide a way of measuring and computing the performance parameters of the double-entry turbines under different flow admission conditions. Therefore, the flow behaviour can be explained and will be useful for the development of the mean-line model for a double-entry turbine working under any unequal and partial flow admission conditions. Another objective is to show a procedure for characterizing the discharge coefficient of valves that are integrated into the turbine housing.

- Experimental procedure for measuring the double-entry turbines in a different mass flow admission conditions.

- Development of mean-line model that is able to extrapolate the performance maps of double-entry turbines at different flow admission conditions.

- Development of a methodology to characterize the wastegate and scroll connection valve for double-entry turbines.

- Model development for obtaining the general heat transfer properties like metal conductance for a variety of turbochargers.

- Integration of double-entry turbine model into whole engine model for validation.

\subsection{Thesis outline}

The main content of this thesis distributed into eight different chapters which are described below.

Chapter 1, is an introduction to the work presented in this thesis and will outline the problem being discussed. The main objectives of this thesis are included in this chapter.

Chapter 2, represents a review of the published literature concerning the work in this thesis. These will highlight the gaps in the current knowledge and will help to add perspective to the work which will be carried out in this thesis. The review will look at research into double-entry turbine steady flow measurement at different 
flow admission conditions and how the performance maps are represented. Various proposals for modelling a radial inflow turbine and double-entry turbines have been studied. Further, the importance of turbocharger heat transfer phenomena has been reviewed, too.

Chapter 3 will describe the test facility at CMT-Motores Térmicos, including detail of the steady flow measurements performed with double-entry turbines at various flow admission conditions in the quasi-adiabatic state. The uncertainty in the measurement of the individual variables and dimensional analysis of the turbine performance parameters based on the literature study and with a systematic way of computing will also be discussed.

Chapter 4 will describe the development of a mass flow-oriented model for extrapolation of reduced mass flow and adiabatic efficiency of double-entry radial inflow turbines under any unequal, full and partial admission conditions. The model validations with different geometry type of double-entry turbine are also shown. Further, model sensibility analysis is also performed using only a few flow admission maps, and their results are also discussed.

Chapter 5 represents an experimental method to characterize the discharge coefficient of the wastegate and scroll connection valve (SCV) of double-entry turbines in the gas stand. Further, an empirical model is presented for being able to predict the discharge coefficient of the wastegate and SCV and to be able to use in one-dimensional engine calculations.

Chapter 6 indicates a small contribution of the work towards heat transfer in turbochargers. Refined experiments were performed using one of the turbochargers from Table 1.1 in the thermohydraulic test bench. The experiments were performed using the methodology described in the [19]. Further, a new model for estimating the conductive conductances for a different geometry type of turbochargers is presented. Moreover, the model validation using one-dimensional gas stand simulations is shown.

Chapter 7 shows the validation of the double-entry turbine model in one-dimensional simulation software. The models presented in Chapter 4 were integrated into a GTPower ${ }^{\mathrm{TM}}$ environment using an external library. After the integration, the doubleentry turbocharger model is simulated and validated in the gas stand steady flow conditions. Finally, the double-entry turbocharger model is also coupled with the whole 1D calibrated engine model and performed the simulations and validated with a steady engine full load points obtained from the engine test rig.

Chapter 8 summarizes the main conclusions of this $\mathrm{PhD}$ dissertation according to the objective laid out in chapter 1 . Moreover, the suggestions for future work that could further improve the models are presented. 


\subsubsection{Turbocharger identities}

In this thesis work, four different types of double-entry turbocharger units, in size and configuration is used. Table 1.1 shows the main characteristics of those turbochargers, and their names are defined; in which ' $R$ ' refers to a Radial inflow turbine and ' $M$ ' refers to a Mixed inflow turbine. From now on, the type of turbocharger will be referred with the given name.

Table 1.1: Turbocharger identity

\begin{tabular}{ccccc}
\hline Turbocharger & T\#1TER & T\#2DVR & T\#3DVM & T\#4TER \\
\hline Turbine type & Twin-Entry & Dual-Volute & Dual-Volute & Twin-Entry \\
Engine type & Petrol & Petrol & Petrol & Petrol \\
Gas stand test & Yes & Yes & Yes & Yes \\
Engine test & No & No & Yes & No \\
Water cooled & Yes & Yes & Yes & Yes \\
Wastegate & Yes & Yes & Yes & Yes \\
SCV & No & No & Yes & No \\
\hline
\end{tabular}

Moreover, twin-entry and dual-volute turbines have different geometries due to their designs. It is made merely by the type of flow division, as explained below:

- Twin-entry turbine: The scroll has a single wall around the entire perimeter of the turbine housing. This way, each entry admits the exhaust gas over the whole circumference of the turbine wheel. The entry closer to the turbine outlet is named as Shroud (Sh), and the entry which is closer to the bearing housing side is called Hub $(\mathrm{H})$. The same is shown in Figure 1.1(a).

- Dual-volute turbine: The scroll is divided in a way that each scroll admits the exhaust gas at a separate section of the rotor. The turbine scroll which has a longer volute is named as Long Volute (LV), and the scroll which has a shorter volute is named as Short Volute (SV), as shown in Figure 1.1(b).

From now on, the respective turbocharger turbine entries/scroll are referred to the named ones. 


\section{Chapter 2}

\section{Literature review}

\section{Contents}

2.1 Introduction . . . . . . . . . . . . . . . . . 10

2.2 Experimental characterization . . . . . . . . . . . . 10

2.3 Turbine performance data $\ldots \ldots \ldots \ldots \ldots$

2.4 Overview of double-entry turbine performance . . . . . . . . . 15

2.5 Radial turbine modelling . . . . . . . . . . . . . . . 16

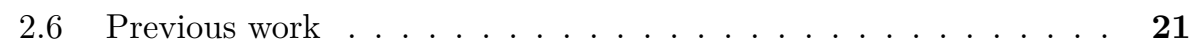

2.6.1 Description of VGT flow oriented model . . . . . . . . 22

2.6.1.1 Mass flow model . . . . . . . . . . . . . 22

2.6.1.2 Efficiency model . . . . . . . . . . . . . . . . . . 24

2.6.2 Heat transfer and mechanical losses model description . . 26

2.6.3 Thermohydraulic test bench . . . . . . . . . . . . . 31

2.6.3.1 Test bench description . . . . . . . . . . . 31

2.6.3.2 Test methodology . . . . . . . . . . . . . . 32 


\subsection{Introduction}

In recent year, automotive OEMs are seeing much interest in using the double-entry turbines. Especially for four-cylinder turbocharged petrol engines with wide valves overlap period in their timing diagram or six-cylinder compression ignition engines [22]. Some engine manufacturers choose a twin-entry turbine, and others go for a dual-volute turbine. The comparison of performance parameters between these two types of double-entry turbines has been extensively discussed since Pischinger and Wuüsche [23] and until more recently, such as in work by Romagnoli et al. [24].

The characterization of double-entry turbines is more difficult when compared to single entry turbines. It is due to having two separate branches at the turbine inlet (Sh/LV and H/SV) and one common branch at the turbine outlet. There is a need for standards concerning the measurements of double-entry turbochargers and the characterization of the individual entries.

In this chapter, a literature review of the most relevant works about the doubleentry radial inflow turbocharging modelling focused on the turbine performance and its prediction is presented.

\subsection{Experimental characterization}

Generally, single entry turbocharger performance maps are obtained using the compressor as a brake for the turbine. This way, the range of points measured for each iso-speed line is quite narrow; as the compressor has minimal braking range due to the choke and surge conditions. Low mass flow parameters are obtained for low rotational speeds, and high mass flow rate parameters are obtained for high rotational speeds. The compressor drains a high amount of power at higher rotational speeds.

The standard measurement of the double-entry turbine can be performed as with single entry turbine. That is, connecting the branch downstream of the combustion chamber to both inlet volutes upstream of the turbine and allowing the flow. This way, the turbocharger test bench remain without any changes to the standard test bench. When the design of turbine volutes is symmetrical, the mass flow rates in each turbine inlet will be identical. Therefore, the admission of the turbine rotor is equal. When the turbine volutes are asymmetrical, the unequal flow admission occurs. The level of flow admission at the turbine rotor naturally happens without the control of any systems. The turbine maps are provided in identical form as single entry turbine without the information about the conditions in each branch.

Many researchers concluded that the availability of the double-entry turbine steady flow maps under the same admission conditions only is not sufficient. In almost every engine operation conditions, the twin-entry or dual-volute turbines op- 
erate under different flow admission conditions due to the pulsating exhaust gas natures coming from the engine. Further, the staggered firing order of the engine cylinders also leads to different inlet pressure and temperature levels in the entries of twin/dual-volute turbines. Therefore, the flow condition is very different from the steady flow with regular admission, which is measured on the standard turbocharger gas stand.

The flow conditions between the entries of a double-entry radial inflow turbine can be divided into three different categories as follows:

- Full/equal admission: Having the same mass flow rate in each entry of the turbine.

- Unequal admission: Having different inlet temperature, pressure and mass flow levels between the entries of a double-entry radial inflow turbine.

- Partial admission: In a condition when the mass flow rate in one of the turbine entries drops to zero. It is considered as extreme partial admission conditions.

However, one should note that the extreme partial admission condition, i.e., zero flow in one of the branches, is not likely to appear under conventional engine operating conditions. Many researchers have also reported that the engine exhausts pulsatile flows significantly influence the performance of a double-entry turbine-powered turbocharger $[18,25,26]$. Therefore, to accurately characterize and model these turbines, the measurement at different flow admission conditions is needed.

In the early 1970s, Pischinger and Wünsche set up a unique turbocharger test rig for characterizing the twin-entry turbines under partial and full admission conditions. The gas stand set up was with a single combustion chamber. The tests are performed by allowing mass flow into two entries to obtain the full admission map. Later, only in each entry at once to obtain the partial admission maps.

Romagnoli et al. [27] studied the swallowing capacity of the twin-entry turbine experimentally under different levels of unequal and partial admission conditions. They tested the turbine at unequal admission conditions by keeping the constant pressure ratio in one branch and, letting the other branch free to vary and to meet the required operating state. For this, a specific turbocharger testbed with separated exhaust branches upstream of the turbine was adopted. Therefore, it was possible to influence the pressure and mass flow rates in each branch independently. The test was performed at two turbine rotational speeds, and it was concluded that the turbine swallowing capacity under unequal admission is linked to the full admission cases. Based on the test outcomes, a way to ascertain the swallowing capacity under partial admission given the full admission map is presented.

The aim of the FEV project extended turbine mapping [28] was an extensive measurement of twin-entry turbines under different flow admission conditions at the 
turbine inlets. The first generation of the testbed was equipped with the single combustion chamber, and the flow was divided into separated branches. Due to the specific heat losses, the turbine inlet temperature conditions are varied. In the second generation testbed, two independent combustion chambers were fitted that is, one for each entry of the turbine. It made feasibility to control the pressure, temperature and mass flow rates independently in each branch. For determining the flow conditions in the turbine, a definition of mass flow ratio with the reduced mass flow parameters (RMFR) has been proposed, as shown in Equation 2.1. RMFR is a ratio of the reduced mass flow of one branch to the total reduced mass flows in the other two branches of the turbine. The outcomes of the measurement on the testbed with two combustion chambers are discussed in [29].

$$
R M F R=\frac{\dot{m}_{r e d, S h / L V}}{\dot{m}_{r e d, S h / L V}+\dot{m}_{H / S V}}
$$

Nevertheless, using the RMFR definition adds complexity to the testing campaign. Moreover, the reduced mass flow of the turbine monotonously increases with the Mach number until the flow gets choked. Therefore, adding two Mach numbers (from different branches) in the denominator does not give a clear physical meaning to RMFR parameter. In addition to the increasing testing complexity, the RMFR definition does not prevent obtaining the same values of the ratio for many different and sometimes non-physical Mach number combinations. The described test methodology is very time-consuming and expensive by under the needful wide ranges of pressure ratios, blade speed ratios (BSR) and level of admission to obtain sufficient data for the creation of steady flow maps.

The similar test methodology was presented by Brinkert et al. [30]. Their aim of the work was to identify the features of asymmetric turbine scrolls under full, unequal and partial flow admission conditions. The standard steady flow maps describing the influences of sections on the overall parameters are discussed.

\subsection{Turbine performance data}

The gas stand measured variables are used to define the performance characteristics of the turbine. They are typically documented into a mass flow map and efficiency map. The mass flow map is composed of two main variables: total-to-static expansion ratio and reduced mass flow, as shown in the equations 2.2 and 2.3 respectively.

$$
\Pi_{0 t, 4}=\frac{p_{0 t}}{p_{04}}
$$




$$
\dot{m}_{\text {red }}=\frac{\dot{m} \cdot \sqrt{T_{0 t}}}{p_{0 t}}
$$

Whereas, the efficiency map is composed of a total-to-static efficiency either with a blade speed ratio (BSR) or total-to-static expansion ratio. The blade speed ratio is defined as the ratio between the blade tip speed and the isentropic velocity of a nozzle, as shown in Equation 2.4.

$$
\sigma=\frac{2 \cdot \pi \cdot n \cdot r_{3}}{\sqrt{2 \cdot c_{p} \cdot T_{0 t} \cdot\left[1-\left(\frac{1}{\Pi_{(0 t, 4)}}\right)^{(\gamma-1 / \gamma)}\right]}}
$$

Based on the fundamental definition of efficiency, the traditional way to calculate the total-to-static turbine efficiency $\eta_{t / s}$ (which is also called as adiabatic efficiency) is based on the ratio between turbine mechanical power and its isentropic power as shown in Equation 2.5.

$$
\eta_{t / s}=\frac{\dot{W}_{T}}{\dot{W}_{s}}=\frac{T_{0 t}-T_{04}}{T_{0 t} \cdot\left[1-\left(\frac{1}{\Pi_{0 t, 4}}\right)^{\left(\frac{\gamma-1}{\gamma}\right)}\right]}
$$

The mechanical efficiency $\eta_{m}$ can be computed by the ratio of compressor power to turbine power, as shown in Equation 2.6.

$$
\eta_{m}=\frac{\dot{m}_{C} \cdot c_{p}^{c} \cdot\left(T_{02}-T_{01}\right)}{\dot{m}_{T} \cdot c_{p}^{t} \cdot\left(T_{0 t}-T_{04}\right)}=\frac{\dot{W}_{C}}{\dot{W}_{T}}
$$

The product of mechanical efficiency and turbine adiabatic efficiency $\left(\eta_{t / s} \cdot \eta_{m}\right)$ gives the fraction of mechanical compressor power to isentropic turbine power, as shown in Equation 2.7. This ratio is called effective turbine efficiency (ETE), and it is commonly used by the turbocharger manufacturers to determine the efficiency of the turbine.

$$
\eta_{t / s} \cdot \eta_{m}=\frac{\dot{W}_{T}}{\dot{W}_{s}} \cdot \frac{\dot{W}_{C}}{\dot{W}_{T}}=\frac{\dot{W}_{C}}{\dot{W}_{\text {Tisen }}}=\eta_{E T E}
$$

In both mass flow and efficiency maps of the turbine, the data points are grouped by a reduced turbine speed parameter shown in Equation 2.8 .

$$
n_{\text {red }}=\frac{n}{\sqrt{T_{0 t}}}
$$


Romagnoli et al.[31] proposed a new definition of mass flow parameter (MFP) to study flow performance parameters of double-entry turbines. The MFP is calculated considering the contribution of each entry on the overall flow capacity, as shown in Equation 2.9. Whereas, for the expansion ratio, as shown in the Equation 2.10. In this way, all the admission conditions can be explained by only one turbine flow parameter, representing the total flow of the turbocharger turbine. In the case of full and unequal admission conditions, the expansion ratio is calculated as an average value between the turbine inlets, as shown in Equation 2.11. Whereas, for the inlet temperature a mass-weighted average value is considered according to Equation 2.12 $[31,32]$. For partial admission data, as the flow is only into one of the branches; therefore, the flow parameters and expansion ratio is determined using the conditions of flowing entry only [31].

$$
\begin{gathered}
\dot{m}_{r e d}=\frac{\left(\dot{m}_{S h / L V}+\dot{m}_{H / S V}\right) \cdot \sqrt{T_{0 t}^{a v g}}}{p_{0 t}^{a v g}} \\
\Pi_{t / s}=\frac{p_{0 t}^{a v g}}{p_{4}} \\
p_{0 t}^{a v g}=\frac{p_{0 t}^{S h / L V}+p_{0 t}^{H / S V}}{2} \\
T_{0 t}^{a v g}=\left(\frac{\dot{m}_{S h / L V}}{\dot{m}_{S h / L V}+\dot{m}_{H / S V}}\right) \cdot T_{0 t}^{S h / L V}+\left(\frac{\dot{m}_{H / S V}}{\dot{m}_{S h / L V}+\dot{m}_{H / S V}}\right) \cdot T_{0 t}^{H / S V}
\end{gathered}
$$

Few authors $[33,34,35]$ suggested that, if twin-entry and dual-volute turbines are modelled as in the view of two turbine approach (TTA) attached to a single compressor, then the effective turbine efficiency can be calculated as the ratio between the compressor power to the sum of two individual isentropic turbine powers of the individual turbine flows; as shown in the Equation 2.13.

$$
\eta_{E T E}^{T T A}=\frac{\dot{W}_{C}}{\dot{W}_{s}^{S h / L V}+\dot{W}_{s}^{H / S V}}
$$

where the isentropic conditions are estimated based on their individual inlet conditions as shown in equations 2.14 and 2.15 .

$$
\dot{W}_{s}^{S h / L V}=\dot{m}_{T}^{S h / L V} \cdot c_{p}^{S h / L V} \cdot\left[T_{0 t}^{S h / L V} \cdot\left(1-\left(\frac{1}{\Pi_{0 t, 4}^{S h / L V}}\right)^{\left(\frac{\gamma-1}{\gamma}\right)}\right)\right]
$$




$$
\dot{W}_{s}^{H / S V}=\dot{m}_{T}^{H / S V} \cdot c_{p}^{H / S V} \cdot\left[T_{0 t}^{H / S V} \cdot\left(1-\left(\frac{1}{\Pi_{0 t, 4}^{H / S V}}\right)^{\left(\frac{\gamma-1}{\gamma}\right)}\right)\right]
$$

In order to plot the effective turbine efficiency (TTA) with a blade speed ratio (BSR) values, an overall BSR definition is suggested as shown in the Equation 2.16

$$
\sigma_{\text {overall }}=\frac{\pi \cdot D_{t} \cdot n}{\sqrt{2\left(\frac{\dot{W}_{s}^{S h / L V}+\dot{W}_{s}^{H / S V}}{\dot{m}_{T}^{\text {total }}}\right)}}
$$

In the twin-entry turbines, the vaneless space is common for both shroud and hub branches. Many researchers assume that the flow of the independent inlets undergoes mixing before entering the turbine wheel. Accordingly, if one calculates the efficiency of the turbine, then the isentropic enthalpy differences have to be taken from the mixing state (i.e. at rotor inlet) to a common outlet. In this view, Holger et al.[35] suggested a definition called mixed momentum approach (MMA) as shown in Equation 2.17. It considers mixing spouting velocity $\left(c_{s, m i x}\right)$ which is obtained by mass averaging the two individual spouting velocities $c_{s, S h}$ and $c_{s, H}$ [35]

$$
\eta_{E T E}^{M M A}=\frac{\dot{W}_{C}}{\left(\frac{1}{\dot{m}_{T}}\right) \cdot\left(\frac{1}{2}\right) \cdot\left(\dot{m}_{S h} \cdot c_{s, S h}+\dot{m}_{H} \cdot c_{s, H}\right)^{2}}
$$

Similarly, Holger et al. [35], also suggested the approach of mixing volumes to estimate the mixed-flow blade speed ratio values in all the flow admission conditions. The authors compared the results from TTA and MMA approaches. They concluded that the same efficiency values were obtained in the case of full and partial admission conditions. But, the MMA approach showed the different efficiency values for unequal admissions.

\subsection{Overview of double-entry turbine performance}

Pischinger and Wunsche [23] were the first researchers who investigated the flow characteristics and the efficiencies of the radial twin-entry turbine in comparison with a dual volute turbine. The comparison was made under the steady flow at partial and full admission conditions. The conclusions of their studies pointed out that the efficiency losses at partial admission conditions are significant. Still, it mainly depends upon the turbine speed and pressure levels. They further concluded that 
twin-entry turbines have better efficiency when the flow conditions are the same in both turbine branches.

Dale and Watson [36] continued the work done by the Pischinger and Wunsche [23] on the twin-entry turbine by testing it with both equal and unequal admission. The effect of unequal admission on turbine performance was such that the best efficiency was found when the mass flow rate is higher in shroud side entry than in hub entry. Further indicated that the efficiency varies with different unequal admission conditions. The same outcome was presented later by Capobianco and Gambarotta [37] and also investigated by comparing the single-entry to a twin-entry turbine. They noticed that the efficiency of the twin-entry turbine is around $7 \%$ less under full admission conditions.

Hajilouy et al. [38] presented a numerical and experimental investigation of the performance and internal flow field characteristics of a twin-entry radial inflow turbine at full and partial admission conditions. The experimental performance results showed that maximum efficiency occurs at similar admission conditions for a considered range. Whereas, the lowest efficiency is observed when the entire flow is in the hub side branch.

Romagnoli et al. [27] reported the performance results obtained for three different turbine configurations. The turbine which was tested in their study was based on the existing nozzle less commercial turbine. They modified this nozzle less turbine into a variable geometry single-entry turbine and twin-entry turbine. The steady-state experiments conducted on twin-entry turbine under full admission at vane angle of $60^{\circ}$ showed that the influence of the divider is not essential concerning efficiency. However, at a vane angle between $40^{\circ}$ and $50^{\circ}$, a notable drop in efficiency was reported. Rajoo et al. [39] discussed the performance of the same modified twin-entry turbine examined under unsteady flow situations. They concluded that the swallowing capacity during full admission was varying between the two entries. The shroud side branch was more pressurized than the hub side branch. Chiong et al. [40] studied the accuracy of pulse flow model prediction. They pointed out that partial admission performance should consider during model characterization to have a significant pulse flow prediction quality. Further, it was concluded that twin-entry turbine is operating at unequal admission mode instead of full admission mode. Throughout the in-phase pulse flow conditions, they tested.

\subsection{Radial turbine modelling}

Mainly turbocharger turbine models are based on steady flow maps. By providing information about the mass flow rate and isentropic efficiency, and it solves the system of equations by assuming a quasi-steady behaviour. Whereas, in the case of doubleentry turbines, only the steady flow map under full admission condition (where the 
flow is the same in both entries of the turbine) is not enough for the simulation purposes. In reality, the exhaust pulses feeding each entry of the turbine will be timed so that they are out of phase with each other. As a result, double-entry turbines spends little time in full admission and majority of the time with unequal flows in their entries. Therefore, the turbine maps should also cover the necessary flow conditions like unequal and partial admissions between their entries.

Moreover, the addition of the second inlet to the turbine volute brings extra complexity in determining the steady-state turbine performance parameters. Numerous researchers were able to succeed in modelling the extrapolation tools for conventional single entry fixed and variable geometry turbines [41, 42, 43, 44, 45]. Some of them are more physical and were able to calculate the losses generated in the turbine while extrapolating the efficiency maps to off-design conditions [46].

Radial turbines modelling are made with various levels of complexity, having both interpolation and extrapolation capabilities. It can appear in the form of empirical models, semi-empirical models and physically based mean-line models.

The easiest way to modelling a radial turbine is to treat it as a map interpolating black box. In this point of view, the turbine behaviour can be obtained from interpolating the turbine map. The interpolation can be arranged with various levels of complexity, from simple linear interpolation with variables like turbine speed and expansion ratio to more difficult transfinite interpolators. This type of models are called empirical models. The drawback with the empirical models is that it does not allow numerical extrapolation abilities.

Semi-empirical models approximate the performance parameters of turbine by simple functions such as polynomials. These models have some success in both mass flow rate [47] and efficiency [48]. These type of models are useful when high-speed computations are needed, such as in real-time simulations. The extrapolation capabilities of these semi-empirical models are somewhat limited, as they are not based on sound physical principles.

Eriksson et al. [49] presented a simple empirical model for estimating the mass flow rate parameter of the turbine as a function of the expansion ratio and two parameters, $a_{1}$ and $b_{2}$ :

$$
\dot{m}_{\text {red }}=a_{1} \cdot \sqrt{1-\Pi_{0 t, 4}^{b_{2}}}
$$

Eriksson [50] also formulated a simple empirical model for turbine efficiency, using a quadratic function in blade speed ratio $(\sigma)$ and depending on the maximum turbine efficiency $\left(\eta_{t / s, \max }\right)$, the blade-to-jet speed ratio for maximum efficiency $\left(\sigma_{\max }\right)$ and 
a fitting parameter:

$$
\eta_{t / s}=\eta_{t / s, \max } \cdot\left[1-a_{1} \cdot\left(\sigma-\sigma_{\max }\right)^{2}\right]
$$

Fang et al. [47] developed a mass flow model for VGT turbines, and it depends on four parameters per VGT position. It assumes that all the curves for different speeds collapse:

$$
\dot{m}_{r e d}=a_{1}+a_{2} \cdot e^{a_{3} \cdot \omega \cdot\left(\Pi_{t / s}-1\right)}
$$

Fang et al. [48] also developed the turbine efficiency model, using the definition of total-to-static turbine efficiency. This model takes advantage of physical relations combined with empirical trend. All the fitting parameters change when VGT position is changed.

$$
\eta_{t / s}=a_{1}+\frac{\ln \Pi_{0 t, 4}}{\Pi_{0 t, 4}^{\frac{\gamma-1}{\gamma}}}-1 \cdot\left(a_{2}+a_{3} \cdot \sigma+a_{4} \cdot \sigma^{2}\right)
$$

A method that is currently used by a large fraction of the engine manufacturers is that of GT-Power ${ }^{T M}$, which relates the reduced mass flow rate of the turbine with the blade speed ratio $(\sigma)$ and four fitting parameters:

$$
\dot{m}_{\text {red }}=a_{1} \cdot\left[a_{2}+\left(1-a_{2}\right) \cdot\left(a_{3} \cdot \sigma\right)^{a_{4}}\right]
$$

where $a_{1}$ relates to the mass flow rate parameter at maximum efficiency, $a_{2}$ relates to reduced reduced mass flow rate at a blade-to-jet speed ratio equal to zero, $a_{3}$ is the inverse of the blade-to-jet-speed ratio at peak efficiency and $a_{4}$ is an adjusting parameter that varies between 2 to 4 . Turbine efficiency is modelled as:

$$
\eta_{t / s}=\eta_{t / s, \max } \cdot\left[1-\left(1-a_{3} \cdot \sigma\right)^{a_{5}}\right]
$$

where $a_{5}$ is the adjusting parameter that varies between 1.4 and 2.2 .

To increase the reliability even more in the extrapolations of turbine performance parameters, mean line models are needed. Mean-line models distinguish different sections of the turbine to calculate the flow angles and define losses through sub-models to be able to calculate the needed values finally. The extrapolation of this models are purely based on the physical principle rather than the numerical extrapolation. 
Watson and Janota [18] proposed to model the turbine using a single equivalent nozzle element. It varies the geometry depending on the operating point of the turbine. It provides excellent results for small expansion ratios but, at high expansion ratios typical of radial turbines, the equivalent nozzle became choked. Payri et al. [51] suggested that the turbine can be modelled using two nozzles in series connected by an internal plenum. The first and second nozzle represents the stator and rotor respectively, and their effective area were obtained by assuming that half the expansion ratio will happen in the stator and the other half in the rotor.

Jensen et al. [52] suggested a turbine model as a single ideal nozzle and modifying its effective throat section $\left(A_{e f f}\right)$ using a correlation:

$$
\begin{gathered}
\dot{m}_{r e d}=A_{e f f} \cdot \Pi_{0 t, 4}^{\frac{-1}{\gamma}} \cdot \sqrt{\frac{2 \cdot \gamma}{R \cdot(\gamma-1) \cdot\left(1-\Pi_{0 t, 4}^{\frac{1-\gamma}{\gamma}}\right)}} \\
A_{e f f}=a_{1}+a_{2} \cdot \Pi_{0 t, 4}+a_{3} \cdot n_{r e d}+a_{4} \cdot \Pi_{0 t, 4} \cdot n_{r e d}
\end{gathered}
$$

It produces the sonic flow in the nozzle throat when the turbine expansion ratio is greater than one and, maintains the mass flow parameter equal to that choking conditions. Jensen et al. [52] also proposed a semi-empirical model for turbine efficiency:

$$
\eta_{t / s}=a_{1}+a_{2} \cdot n_{\text {red }}+\left(a_{3}+a_{4} \cdot n_{\text {red }}\right) \cdot \sigma+\left(a_{5}+a_{6}\right) \cdot \sigma^{2}
$$

Serrano et al. [53] presented another correlation for $A_{\text {eff }}$ of Equation 2.24 by considering a linear trend between the effective area and the blade-to-jet speed ratio $(\sigma)$ :

$$
A_{e f f}=a_{1}+a_{2} \cdot \sigma
$$

A set of parameters were able to obtain for each iso-speed line and also for each vane position when working with VGT.

Serrano et al. [54] suggested a two-nozzle model, each one solved using Equation 2.24, with an intermediary plenum. The effective area of each nozzle was computed using semi-empirical correlations by taking into account the VGT position. A further development of the two nozzle mode is presented in the Ph.D. dissertation by Reyes-Belmonte [19]. The modelling approach uses two expansions in series to compute an equivalent single nozzle effective section. 
There are many methods on mean-line modelling of the twin-entry turbines which are available in the literature. However, at partial and unequal admission conditions, the prediction of efficiency from the model seems to be very difficult to obtain [55].

Hajilouy Benisi et al. [56] developed a twin-entry turbine model by modifying the one-dimensional equations, which are used to analyze the flow in single entry turbine. They obtained mean flow parameters along a mean streamline on the inlet and exit of each section, using continuity, momentum, energy and state equations and the second law of thermodynamics. Further, they divided the turbine flow passage into five components, including inlet passage, volute casing, interspace, incidence and rotor. The flow is modelled in each section with imposing loss equations as described in [56]. By doing this, some predicted results were obtained. However, the authors pointed out that, calculated efficiency and the mass flow are overestimated. In the case of partial admission conditions, there was a remarkable difference between predicted and experimental data.

Costall et al. [57] developed a model for twin-entry turbines and solved using a gas dynamics code. They analyzed the pulse flow performance of a twin-entry turbine under unequal and full admission conditions and, suggested that for full admission flow states, the twin-entry turbine can be modelled as a simplified single entry model, whereas for unequal flows a more analyzable model is necessary. This indicates that the double-entry turbine models should have the capability to reproduce the conditions in all the flow admission conditions.

Moreover, the complete unequal admission maps are not always available because many gas stands are not capable of operating in those conditions. Addressing this, Romagnoli et al. [24] proposed a map-based method for predicting the partial and unequal admission flows in double-entry turbines (both twin and dual) from a given full admission map. Based on the experimental data, they proposed two correlations, one for twin entry and another for a dual volute turbine. They conclude that the twin entry turbine characteristic agreed reasonably well with their approach and, the correlation with the performance of a dual volute turbine was less satisfactory.

Newton et al. [58] proposed a method for extrapolating from full admission map of the dual-volute turbine to obtain the unequal admission performances of both efficiency and mass flow parameters. The model is dependent on three constants; one of them is used for predicting the efficiency ratio between the unequal and full admission data points. The other two are used for estimating the mass flow ratio parameter. The values of the constants are determined by using the minimal amount of unequal admission flow data at different turbine speeds. They found good agreements between the predicted and experimental results. However, their analysis was restricted to one dual-volute geometry turbine. Not sure whether the same results can be found for another similar turbocharger without new extensive experimental campaigns needed. Moreover, the model also requires a significant number of experimental data for finding the values of constants. Chiong et al. [40] studied the pulse flow modelling of the 
twin-scroll turbine under pulse flow operating conditions. They pointed out that the overall performance prediction of the model can be enhanced by taking into account the partial admission characteristics during modelling.

Fredriksson et al. [59] proposed a mean-line model for asymmetrical twin-entry turbine by specifying the different flow conditions at each turbine inlet and static pressure at the outlet of a turbine. Their methodology is to solve each turbine passage from inlet to the splitter location separately. Further, from the volute splitter to the rotor inlet, two streams mix into one uniform flow. The model also considers the swirl energy loss from the divider wall to the rotor inlet in the form of pressure loss. The results demonstrated that the mass flow parameters are well predicted for full and partial admission flow conditions. In the case of efficiencies, they were over-predicted at low turbo speeds and pressure ratios.

Macek et al. [60] proposed a more complex model for twin-entry turbine using a loss parameters calibration criteria. The modelling was based on approximation of real physics. Palenschat et al. [61] implemented three significant changes in the single-entry model of Romagnoli and Martinez-Botas [42], for predicting the variables of an asymmetrical twin-entry turbine. Firstly, by adding the second entry model having the calculation procedure being identical to the single entry model. Secondly, the inlet duct was modelled one for each of the entry. It is done to resemble the flow passage from the turbine inlet to the volute tongue. In the end, the interspace model is considered to obtain the resulting flow angle and the flow properties after the mixing. The final model has been validated with three flow conditions, i.e., equal pressure, equal mass flow and unequal flow admissions. They concluded that the performance of the model is best at equal-pressure conditions and, becomes worse as flow imbalance between the branches is increasing. From the root mean square analysis, they showed that the model was able to predict all the parameters within $10 \%$ error. This error was calculated concerning the experimental data for similar pressure conditions. Whereas, in the case of unequal admission flows the difference in mass flow parameter between the model and experimental is about $20 \%$.

The current thesis demonstrates the methodology for modelling both twin-entry and dual-volute turbines to extrapolate the performance maps into off-design conditions for all the flow admission conditions. The modelling approach is the same for both types of turbines, and it is mainly based on systematizing the characteristic maps of the turbine, which is distinctly explained in chapter 3 .

\subsection{Previous work}

In the present thesis, the development steps of some of the equations used for doubleentry turbine modelling and heat transfer characterization are based on the $\mathrm{PhD}$ dissertations of Reyes-Belmonte [19] and Dombrovsky [20]. Therefore, to follow better 
the logic behind the development of models described in this thesis, in the following sections, a summary of previous works is added. This summary will guide the readers to better understand the origin of the equations used.

\subsubsection{Description of VGT flow oriented model}

\subsubsection{Mass flow model}

Procedure for extrapolating the turbine mass flow parameter is mainly based on the definition of the sub-critical mass flow parameter through the orifice of a single isentropic nozzle given by Equation 2.28 [18].

$$
\dot{m}_{\mathrm{red}}=A_{\mathrm{eff}} \cdot \sqrt{\frac{\gamma}{R}} \cdot\left(\frac{1}{\Pi_{0 t, 4}}\right)^{\frac{1}{\gamma}} \cdot \sqrt{\frac{2}{\gamma-1} \cdot\left[1-\left(\frac{1}{\Pi_{0 t, 4}}\right)^{\frac{\gamma-1}{\gamma}}\right]}
$$

The procedure developed by $[62,63]$ for modelling the turbine mass flow parameter is mainly based on viewing the turbine as a single equivalent nozzle that covers from station 0 to 4 of the radial turbine as shown in the Figure 2.1.

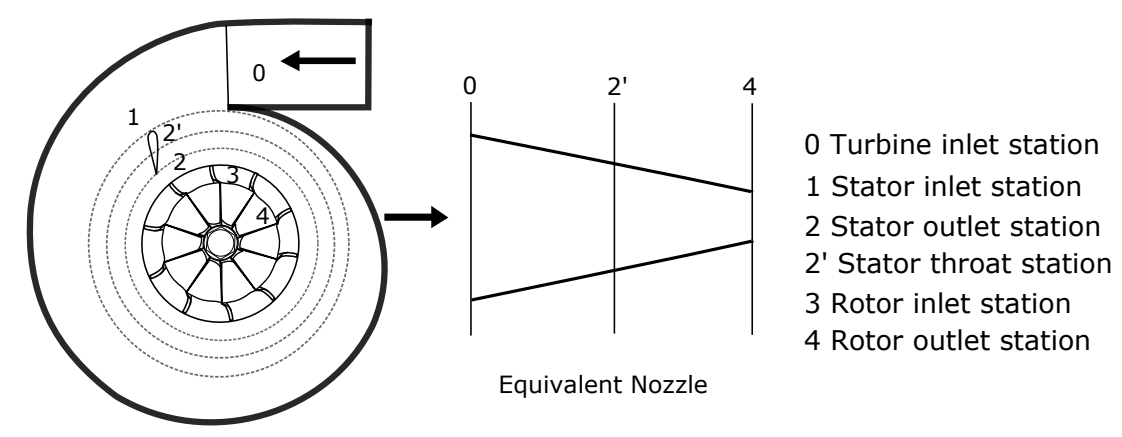

Figure 2.1: Turbine as single equivalent nozzle and stations distribution

The primary assumption of this model is the behavior of the turbine is quasisteady throughout the nozzle simulating the turbine; from both the thermodynamic and fluid dynamics perspective. The fundamental approach followed by Serrano et al. [63] is calculating for every turbine operating point an equivalent nozzle flow area $\left(A_{\text {eff }}\right)$ that represents the whole turbine expansion. Accordingly, this equivalent nozzle flow areas $\left(A_{\text {eff }}\right)$ should allow calculating the mass flow parameter for the entire operative range of the turbine; thus it is possible to plot the whole mass flow curve. To do so, first, the continuity equation was applied to stator, rotor and equivalent nozzle as shown in Equation 2.29. Later using the velocity definition and solving 
the mass flow, an expression for the equivalent nozzle area through stator and rotor combined, as shown in Equation 2.30 is obtained.

$$
\begin{gathered}
\dot{m}=A_{2^{\prime}} \rho_{2^{\prime}} v_{2^{\prime}}=A_{4} \rho_{4} v_{4}=A_{N e q} \rho_{4} v_{N e q} \\
A_{N e q}=A_{4} \sqrt{\frac{1+\left(\frac{u_{4}}{v_{N e q}}\right)^{2}-\left(\frac{u_{3}}{v_{N e q}}\right)^{2}+\left(\frac{w_{3}}{v_{N e q}}\right)^{2}}{\left(\frac{A_{2^{\prime}}}{A^{2}}\right)^{2}}}
\end{gathered}
$$

Further, Equation 2.30 is simplified by using the diameters of the turbine rotor to group the terms $u_{3}$ and $u_{4}$ and with the definition of total to static turbine efficiency to introduce the isentropic velocity $\left(c_{s s}\right)$ to replace the equivalent nozzle velocity $\left(v_{N e q}\right)$. Then, Equation 2.31 can be obtained [63].

$$
A_{N e q}=A_{4} \sqrt{\frac{1+\frac{\sigma^{2}}{\eta_{t s}}\left[\left(\frac{D_{4}}{D_{3}}\right)-1\right]+\frac{b}{\eta_{t s}}}{\left(\frac{A_{4}}{A_{3}}\right)^{2}\left(\frac{\rho_{4}}{\rho_{3}}\right)^{2}+1}}
$$

In order to find an expression for the density ratio shown in Equation 2.31, an additional hypothesis is done by considering that the efficiency of the rotor is equal to turbine total-to-static efficiency as described in the work of Serrano et al.[63]. Considering all these, a new expression for the throat area of the equivalent nozzle is obtained as shown in Equation 2.31. This final expression depends on the information available in a standard turbocharger map, some main geometrical parameters that are easily measurable and the corresponding fitting coefficients $(a, b, c$ and $d)$. This area changes for every turbine operating point [62]. As a result, it is possible to get a full range of equivalent nozzle effective flow area for each rack position in a VGT [63].

$$
A_{\mathrm{eff}}=\frac{a \cdot A_{4}^{\text {geom }} \cdot \sqrt{1+\frac{\sigma^{2}\left[\left(\frac{D_{4 m}}{D_{3}}\right)^{2}-1\right]+b}{\bar{\eta}_{t s}}}}{\sqrt{1+\left(c \cdot \frac{A_{4}^{\text {geom }}}{A_{2^{\prime}}^{\text {geor }}}\right)^{2} \cdot \frac{\left(\frac{1}{\Pi_{2^{\prime}, 4}}\right)^{2}}{\left(1-\eta_{t s} \cdot\left(1-\left(\frac{1}{\Pi_{2^{\prime}, 4}}\right)^{\frac{\gamma-1}{\gamma}}\right)\right)^{2}}}}
$$

Where $A_{2^{\prime}}^{\text {geom }}$ is the geometrical throat section of the stator vanes and can be calculated for any VGT as described in [63]. The rotor outlet geometrical area $\left(A_{4}^{\text {geom }}\right)$ 
is calculated by taking the arithmetic diameter between the turbine rotor shroud $\left(D_{4}\right)$ and rotor hub $\left(D_{n u t}\right)$ diameters as shown in Equation 2.33 .

$$
A_{4}^{\text {geom }}=\pi \cdot\left(\frac{D_{4}^{2}-D_{n u t}^{2}}{4}\right)
$$

Whereas in Equation 2.32, $D_{3}$ turbine rotor diameter and $D_{4 m}$ is the mean diameter between turbine rotor shroud $\left(D_{4}\right)$ and rotor hub $\left(D_{n u t}\right)$ diameters, $\eta_{t s}$ represents the turbine total-static adiabatic efficiency, $\sigma$ is the blade to jet speed ratio calculated using the Equation 2.34 and $\Pi_{2^{\prime}, 4}$ represents pressure ratio in the VGT rotor. $\Pi_{2^{\prime}, 4}$ is calculated from the total to static turbine pressure ratio by using a fitting constant ' $d$ ' as shown in Equation 2.35. It is obtained by making the hypothesis that the turbine stator pressure drop to the whole turbine total-to-static pressure drop ratio is constant for a VGT position [63]. A constant average value of turbine efficiency $\left(\eta_{t s}=\bar{\eta}_{t s}=0.8\right)$ was assumed relaying on ' $b$ ' coefficient to avoid the possible inconsistency during the extrapolations.

$$
\begin{gathered}
\sigma=\frac{2 \cdot \pi \cdot n \cdot r_{3}}{\sqrt{2 \cdot c_{p} \cdot T_{0 t} \cdot\left[1-\left(\frac{1}{\Pi_{0 t, 4}}\right)^{\frac{\gamma-1}{\gamma}}\right]}} \\
\Pi_{2^{\prime}, 4}=1+d \cdot\left(\Pi_{0 t, 4}-1\right)
\end{gathered}
$$

The coefficients ' $a$ ' and ' $c$ ' in Equation 2.32 are directly related to the rotor and stator discharge coefficient. The other two coefficients (' $b$ ' and ' $d$ ') comes from the theoretical considerations previously described; more details can be read in [45]. These four fitting parameters are calculated using regression analysis, with the help of a suppliers performance map and the turbocharger geometry data as input to the model. Once the equivalent nozzle (Equation 2.32) is known for every turbine operative point, the reduced mass flow parameter can be calculated using the definition of the sub-critical mass flow parameter through an orifice of single isentropic nozzle given by the Equation 2.28.

\subsubsection{Efficiency model}

The efficiency model presented in [63] is based on the use of the Euler equation of turbo-machinery for radial gas turbines and assuming constant meridional component velocities. By this, both assumptions in Equation 2.36, which represents the definition of the total to static adiabatic efficiency of the turbine, it is possible to express the numerator in the terms of velocities using Euler equation and turbine enthalpy drop 
as shown in Equation 2.37. The tangential velocities in the Equation 2.37 can be expressed in terms of meridional velocity using equations 2.38 and 2.39. Using these equations into Equation 2.36 and taking into account the isentropic evolution in the denominator, it is possible to express the total-static-turbine efficiency equation as shown in Equation 2.40.

$$
\begin{gathered}
\eta_{t / s}=\frac{T_{0 t}-T_{04}}{T_{0 t}-T_{4 s}} \\
\dot{W}=\dot{m} c_{p}\left(T_{0 t}-T_{04}\right)=\dot{m}\left(u_{3} c_{03}-u_{4} c_{04}\right) \\
c_{03}=c_{0} \tan \alpha_{3} \\
c_{04}=u_{3} \frac{r_{4}}{r_{3}}-c_{0} \tan \beta_{4} \\
\eta_{t / s}=\frac{u_{3} c_{0} \tan \alpha_{3}-\left[u_{3}\left(\frac{r_{4}}{r_{3}}\right)-c_{0} \tan \beta_{4}\right] u_{3}\left(\frac{r_{4}}{r_{3}}\right)}{c_{p} T_{0 t}\left(1-\left(\frac{1}{\Pi_{0 t, 4}}\right)^{\frac{\gamma-1}{\gamma}}\right)}
\end{gathered}
$$

Based on the definition of blade to jet speed ratio $(\sigma)$ as shown in the Equation 2.34, the Equation 2.41 is obtained for a constant tip speed maps.

$$
\eta_{t / s}=-2 \cdot\left(\frac{r_{4}}{r_{3}}\right)^{2} \cdot \sigma^{2}+2 \cdot \frac{A_{\mathrm{eff}}}{A_{0}^{\text {geom }}} \cdot\left(\tan \alpha_{3}+\frac{r_{4}}{r_{3}} \cdot \tan \beta_{4}\right)\left[\frac{1}{\Pi_{0 t, 4}}\right]^{\frac{1}{\gamma}} \cdot \sigma
$$

It is worth to highlight that the above equation is dependent on $A_{\text {eff }}$, and which can be calculated using the Equation 2.32. The rotor inlet flow angle $\left(\alpha_{3}\right)$ is estimated as a function of stator outlet flow angle $\left(\varphi_{2}^{\text {metal }}\right)$ as shown in the Equation $2.42[63$, $18]$

$$
\tan \alpha_{3}=z_{3}^{\text {geom }} \cdot \sin \varphi_{2}^{\text {metal }}
$$

Coefficient $z_{3}^{\text {geom }}$ is based on the geometry of VGT stator vanes and which was obtained theoretically as described in [63]. The final turbine efficiency equation has been lumped into $K_{i}$ terms as shown in the Equation 2.43. A global fitting parameter (to consider the flow deviation effects from 1D idealized stator and rotor channels 
geometry ' $z$ ' has been introduced in the $K_{2}^{\star}$ and it has six coefficients which mainly depend on the reduced turbine speed, blade to jet speed ratio and VGT position as shown in the Equation 2.46. The equations 2.44 and 2.47 are related to physical values from turbine geometry and from the turbine map as described in [63].

$$
\begin{gathered}
\eta_{t / s}=-K_{1} \cdot \sigma^{2}+K_{2}^{\star} \cdot\left(1-\frac{K_{3}}{\sigma^{2}}\right)^{\frac{1}{\gamma-1}} \cdot \sigma \\
K_{1}=2\left(\frac{r_{4}}{r_{3}}\right)^{2} \\
K_{2}^{\star}=2 \frac{A_{\mathrm{eff}}}{A_{0}^{\text {geom }}}\left(z \cdot z_{3}^{\text {geom }} \sin \left(\varphi_{2}^{\text {metal }}\right)+\sqrt{\frac{K_{1}}{2}} \tan \left(\beta_{4}^{\text {metal }}\right)\right) \\
z=-\left(a^{\prime} \cdot n_{\mathrm{red}}+b^{\prime}\right) \cdot \sigma+\left(c^{\prime} \cdot n_{\mathrm{red}}+d^{\prime} \cdot V G T^{2}+e^{\prime} \cdot V G T+f^{\prime}\right) \\
K_{3}=\frac{u_{3, \text { red }}^{2}}{2 c_{p}}
\end{gathered}
$$

\subsubsection{Heat transfer and mechanical losses model description}

The turbocharger thermal model used in this thesis is based on the electrical analogy. The hypothesis of one-dimensional temperature distribution and one-dimensional heat transfer allows considering the turbocharger as a thermal network consisting of a finite number of nodes. These nodes are linked to each other by thermal conductance. To take into account the thermal inertia during transient behaviour of the turbocharger; a thermal capacitance is associated with each node. Figure 2.2 shows the network of the lumped model. The simplification of turbocharger geometry into discrete nodes. Implies the assumption that the radial and circumferential temperature distribution in a cross-sectional area is negligible compared to the axial temperature one. It included the five metal nodes, and as many fluid nodes as the fluids in contact with the turbocharger:

- Node Gas = Exhaust gases moving the turbine

- Node $\mathrm{T}=$ Turbine case node

- Node H1 = Part of the housing placed near turbine case (turbine housing plate) 
- Node H2 = Central part of the housing

- Node H3 = Part of the housing placed near compressor case (compressor backplate)

- Node $\mathrm{C}=$ Compressor case node

- Node Air = Air passing through the compressor

- Node Oil = Lubricating oil node

- Node $\mathrm{W}=$ Cooling liquid node (only for cooled turbochargers)

- Node Ambient $=$ Ambient node for convective and radiative heat transfer

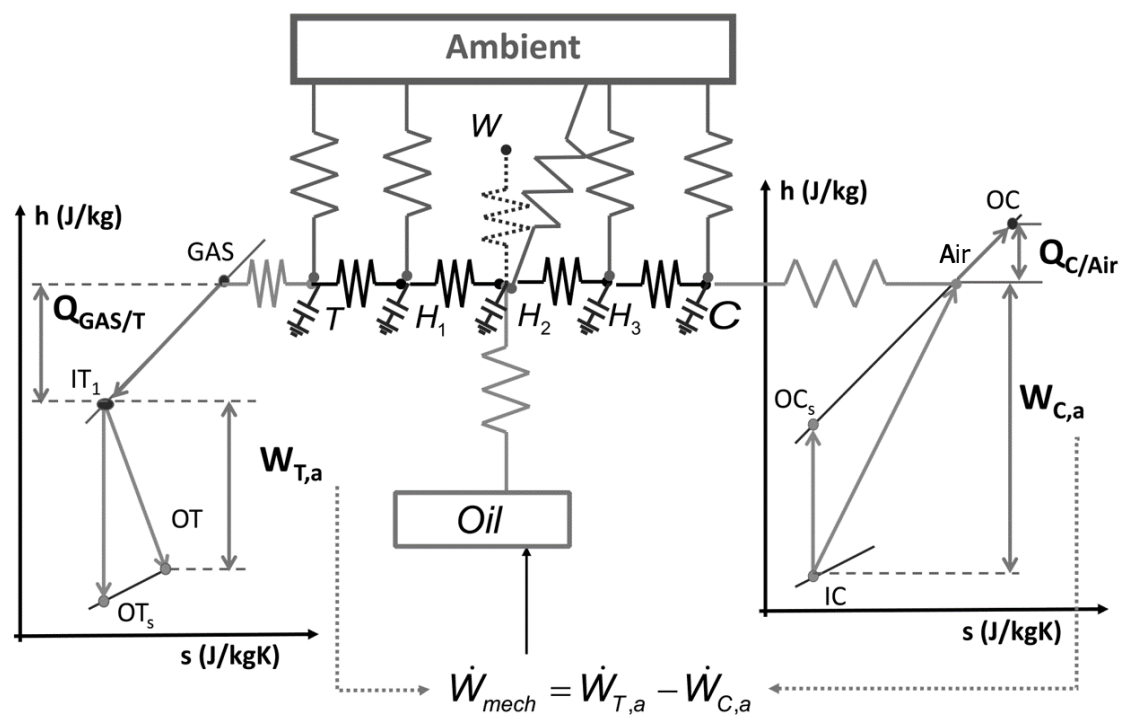

Figure 2.2: Lumped heat transfer model for turbochargers [19]

It is possible to subdivide all turbocharger heat fluxes into two main groups:

- External heat transfer: It includes heat transfer between metal nodes by radiation. It also considers heat transfer to the ambient by both convection (natural or forced) and radiation.

- Internal heat transfer: It includes heat transfer by conduction and heat transfer by convection between the metal nodes and the fluids circulating inside the turbocharger.

It is worth highlighting that thermal conductances and capacitances parameters are necessary to perform the calculations of the heat transfer model. In the previous 
work [64, 19], these parameters were measured for a given turbocharger unit, in a particular gas stand (called a thermohydraulic test bench) using the incompressible flow [19]. Similar tests were performed with T\#4TE petrol turbocharger from the Table 1.1. Details of the test methodology will be further discussed in the section 6.2. In order to save these experimental costs, a general correlation for both diesel and petrol turbochargers combined is proposed in this work in section 6.3. In the case of estimating the conductive conductances, the method is based on the previous experience [65]. However, the definition of calculating the area is refined with a physical meaning to be considered for different turbochargers. Whereas, for capacitance, the model proposed by Serrano et al. [65] has been used.

In previous works, the internal convections correlations are already developed by using the experimental data from a gas stand. In those tests, the turbocharger was thermally insulated from outside to avoid heat losses to the ambient [66]. The procedure is based on knowing the conductive conductances of a turbocharger from a thermohydraulic test bench. The internal convective conductances depend mainly on turbocharger geometry and operative conditions. They are usually calculated through Nusselt number, defined in Equation 2.48 (where $h$ represents a convective heat transfer coefficient, $D_{h}$ the hydraulic diameter and $k_{f}$ the thermal conductivity of the fluid). It physically represents the ratio between heat transfer by convection and the heat which would be transferred if the fluids were not in motion (i.e., only due to fluid conductivity).

$$
\begin{gathered}
N u_{D}=\frac{h \cdot D_{h}}{k_{f}} \\
N u_{D}=0.023 \cdot R e_{D}^{4 / 5} \cdot \operatorname{Pr}^{c} \\
N u_{D}=0.027 \cdot \operatorname{Re}_{D}^{4 / 5} \cdot \operatorname{Pr}^{1 / 3} \cdot\left(\frac{\mu}{\mu_{w}}\right)
\end{gathered}
$$

In smooth and long pipes, Nusselt number is usually determined by Dittus-Boelter or Sieder-Tate correlations (Equations 2.49 and Equation 2.50; where the subscript $w$ indicates that the property must be calculated at wall temperature). Turbocharger components geometry is more complicated than the one of a pipe. But, the experimental research campaigns have demonstrated that [66], introducing a new extra dimensionless number and changing constants coefficients and exponents, the correlations mentioned above give a good prediction for convective heat transfer coefficient calculation in turbochargers as well. The diameter of Nusselt number definition for turbocharger components is not the hydraulic diameter, as Equation 2.48 suggests, but an effective diameter $D_{\text {eff }}$ which changes for each studied element. Newton's law and Nusselt number definition lead to Equation 2.51, which allows calculating 
heat transfer between generic metal node $i$ and a generic fluid node $f$. As effective diameter and even effective length $L_{e f f}$, is a parameter which describes the geometry of considered element and changes for each one of them. Besides, the temperature difference $\Delta_{T}$ mutates its expression for each component. In previous work [66], these correlations were developed, and their constants were calibrated with different turbochargers. By these, the model can determine the proper convective heat fluxes for any operative conditions of the turbocharger.

$$
\dot{Q}_{f, i}=N u \cdot k_{f} \cdot \pi \cdot L_{e f f} \cdot \Delta_{T}
$$

In normal working operating conditions, turbine, compressor and housing temperatures are different between each other. Therefore, a heat transfer by radiation between them may occur. Furthermore, the turbocharger temperature is usually higher than the environment temperature. Then a heat transfer from a turbocharger surface to the ambient takes place. The ambient heat transfer includes both convection (forced and natural convection) and radiative heat transfer mechanisms. In order to determine the external convection and radiation, the turbocharger was tested in hot conditions without any thermal insulation to it in a gas stand [67]. For both radiative and convection heat transfer problems, the geometry of turbocharger is schematized through three cylinders of different diameters, as explained in [67]. Turbine and compressor thermal surfaces are constituted by three geometrical surfaces: external, cylindrical and internal one. The central housing is divided into three equal surfaces according to the lumped model network. In this way, the calculation of each required view factor is possible and performed by view factor algebra (i.e., reciprocity, closeness, and distribution). In radiative heat transfer, a view factor is the proportion of radiation leaving one surface, which is intercepted by a second surface. Once all the view factors have been characterized, all the heat transfers via radiations are calculated, making the hypothesis of grey surface and uniform temperature for each component. By considering two generic surfaces (1 and 2), the corresponding radiative heat transfer is determined by the Equation 2.52. Where $\sigma$ is the Stefan Boltzmann constant, $T_{1}$ and $T_{2}$ are the absolute temperatures, $\varepsilon_{1}$ and $\varepsilon_{2}$ are the total emissivities and $F_{1 \rightarrow 2}$ is the view factor of the considered surfaces.

$$
\dot{Q}_{r}=\frac{\sigma \cdot\left(T_{1}^{4}-T_{2}^{4}\right)}{\frac{1-\varepsilon_{1}}{A_{1} \cdot \varepsilon_{1}}+\frac{1}{A_{1} \cdot F_{1 \rightarrow 2}}+\frac{1-\varepsilon_{2}}{A_{2} \cdot \varepsilon_{2}}}
$$

Regarding the heat transfer to the ambient by convection, the geometric simplification is the same that radiative heat transfer issue. But only the cylindrical surfaces are taken into account. Three possible situations are considered: natural, forced and mixed convection. Their heat transfer convection coefficients were calculated by Nusselt number correlation of Churchill and Chu as explained in [67]. Both radiative and convection heat transfer models have been validated through a large number of 
experimental analysis and measurements before [67].

Mechanical losses models developed by Serrano et al [68] being studied into two different parts $\left(\dot{W}_{m}=\dot{W}_{j b}+\dot{W}_{t b}\right)$ in accordance with turbocharger geometrical characteristics. The bearings are studied with the simplified geometry, and their behaviour is analyzed by solving the Navier-Stokes equations and some simplifying assumptions. Oil behaviour is considered incompressible, and its flow through the bearing is deemed to be steady and constant on each section. Also, circumferentially symmetric. Furthermore, the body forces are neglected, and the film thickness is considered smaller than any other bearing part. Viscous stresses are considered comparable to the inertial forces of the fluid (small Reynolds number: $R e=\rho u_{c} / \mu$ ).

Solving Navier-Stokes equations in the journal bearing with those simplifying assumption, the corresponding friction losses are expressed by the Equation 2.53. As it is observed, those losses depend on shaft rotational speed $(N)$, oil viscosity $(\mu)$ at the average oil temperature $\left(\bar{T}_{\text {oil }}\right)$, geometrical parameters like journal bearing radius $\left(R_{j b}\right)$ and bearing length $\left(L_{j b}\right)$, oil film thickness $\left(h_{j b}\right)$ and a fitting parameter $k_{j b}$

$$
\dot{W}_{j b}=2 \pi R_{j b}^{3} k_{j b} \frac{L_{j b}}{h_{j b}} N^{2} \mu\left(\bar{T}_{o i l}\right)
$$

To the same extent, in the thrust bearing, friction losses may be expressed by the Equation 2.54 , where $k_{t b}$ is a fitting factor, $R_{t b, \max }, R_{t b, \min }$ and $\bar{R}_{t b}$ are maximum, minimum and average radius of the Figure $2.3, \varphi$ is a geometrical parameter defined by the Equation 2.55 and $k_{m}$ denotes the fraction of lubricating oil passing through the considered bearing. Finally, $F_{a t}$ is a term accounting the forces acting on the thrust bearing due to compressor and turbine pressure ratios difference. It is expressed by the Equation 2.56, referring to the Figure 2.4. Where $p_{2}^{\prime}$ and $p_{3}^{\prime}$ are the pressures at the outlet of the compressor wheel and the inlet of the turbine wheel respectively and, in the previous equations, $A_{\text {comp }}^{\prime}$ and $A_{\text {turb }}^{\prime}$ are the effective areas of the compressor and turbine wheels.

$$
\begin{gathered}
\dot{W}_{t b}=k_{t b} \pi\left(R_{t b, \text { max }}^{2}-R_{t b, \text { min }}^{2}\right) \bar{R}_{t b}^{2} \sqrt[3]{\left|\frac{F_{a t \rho}}{12 k_{m} \dot{m} \varphi \mu\left(\bar{T}_{\text {oil }}\right)}\right|} N^{2} \mu\left(\bar{T}_{o i l}\right) \\
\varphi=\frac{R_{t b, \text { max }}^{2}\left[\log \left(R_{t b, \text { max }}\right)-0.5\right]}{2}-\frac{R_{t b, \text { min }}^{2}\left[\log \left(R_{t b, \text { min }}\right)-0.5\right]}{2} \\
F_{a t}=\left|A_{c o m p}^{\prime} \frac{p_{1}-p_{2}}{4}-A_{t u r b}^{\prime} \frac{p_{3}^{\prime}-p_{4}}{2}\right|
\end{gathered}
$$




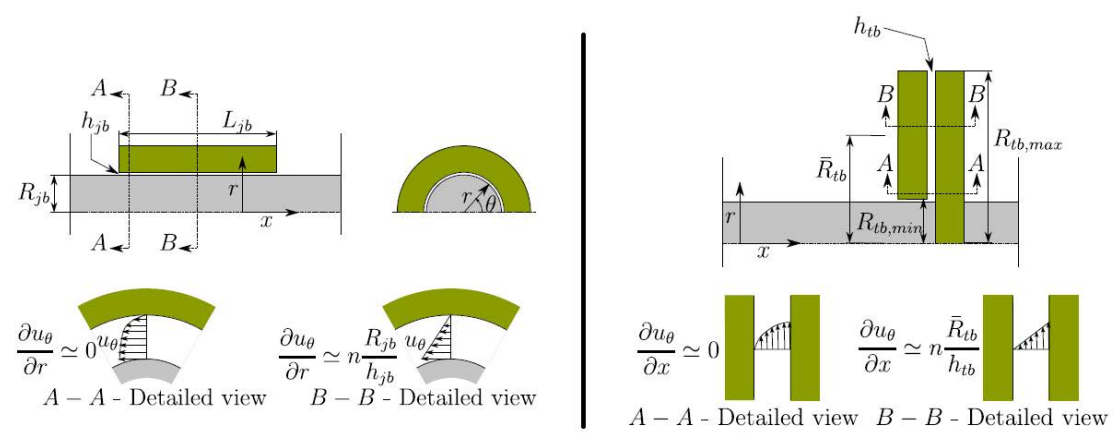

Figure 2.3: Simplified schemes of a journal bearings (left) and a thrust bearing (right).

[68]

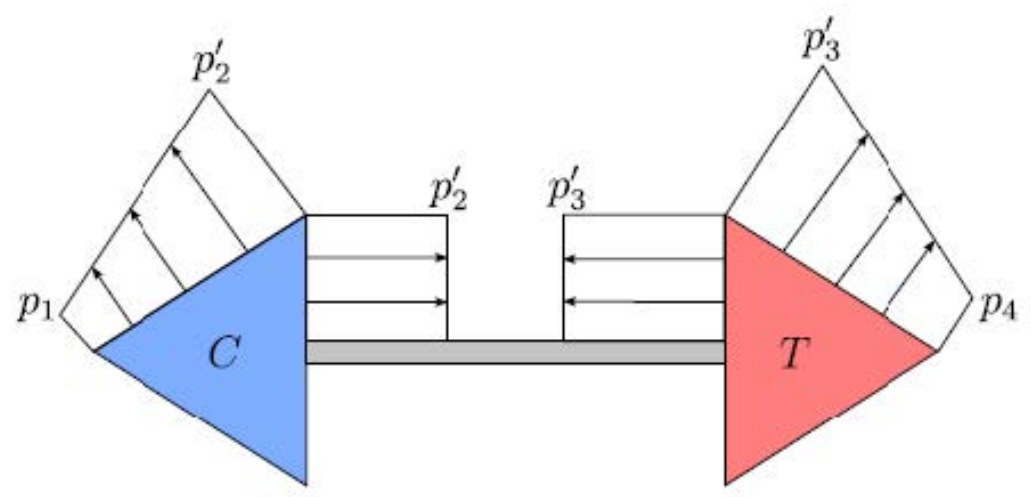

Figure 2.4: Schematic pressure distribution at the compressor and turbine wheels

[68]

\subsubsection{Thermohydraulic test bench}

In this section, a thermohydraulic test rig for determining the conductive conductance and capacitance of a turbocharger is described. Similar tests were performed with T\#4TER turbine, and the test results will be shown in section 6.2. In the following sections, the test rig description and test methodology are explained.

\subsubsection{Test bench description}

A schematic view of thermohydraulic test bench is shown in the Figure 2.5. The test bench has two different flow circuits. One is constituted by a high-temperature circuit 
which is connected to a heating system. Another one is a low-temperature circuit connected to a cooling system. The circulating fluid in both circuits is thermal oil. This fluid presents a lower heat transfer capacity compared to the other potentially usable liquids; for example, water. Other benefits of using the thermal oil are the simplicity to measure the flow and to detect possible leakages [19].

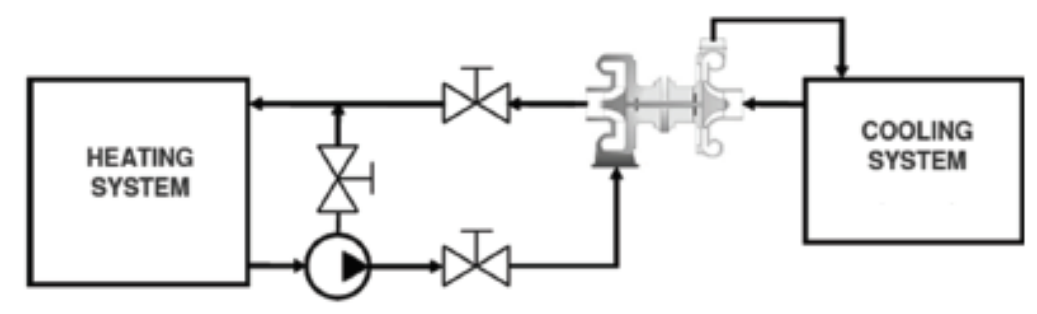

Figure 2.5: Thermohydraulic test bench layout

[19]

The turbocharger unit is placed in between hot and cold circuits. Its elements (turbine and compressor) are connected to those circuit systems, as shown in the Figure 2.5. Three electrical resistances integrate the heating system, and each one of them has a power of $12.5 \mathrm{~kW}$. Whereas, the cooling system is set up by an external heat exchanger. The heating system can deliver thermal oil up to $300^{\circ} \mathrm{C}$ and a volumetric flow up to $6.5 \mathrm{~m}^{3} / \mathrm{h}$. Manual valves and PID controllers were installed in both circuits, to maintain a constant flow and temperatures during the tests. Several probes were mounted on the test bench to measure the mass flow and temperatures at the inlet and outlet of both turbine and compressor. For measuring the fluid temperatures, thermoresistance type sensors were used. The oil flow is measured by two Coriolis mass flow meters (one for each circuit). To carry out the thermal conductive characterization of the turbocharger. The metal surface temperatures were measured in several points using the K-type thermocouples. The turbocharger is divided into five measurement planes corresponding to the five metal nodes of the lumped model, as shown in the Figure 2.2. On each measurement plane, at several azimuthal locations, few thermocouples were installed. It helps to check that the circumferential temperature distribution is negligible compared to the axial one (i.e. axial heat transfer hypothesis) [19].

\subsubsection{Test methodology}

The test is divided into two useful stage: steady state and transient state tests, both are required for turbocharger characterization. In both tests, the turbocharger is entirely thermal insulated, to avoid the heat transfer towards the environment. Further, the shaft is blocked to prevent mechanical power transfer between the turbine and compressor. No oil or water coolant was supplied to the corresponding lube 
ports. In the steady state test, in the hot circuit, hot thermal oil is heated up by the heating system and passed through turbocharger element (compressor or turbine) and consequently heats its case. In the cold circuit, cold thermal oil is passed through the compressor element. After a period of time, this will be heated up by heat coming from the hot oil circuit, which is transmitted through the housing and the shaft. Later, the thermal oil is passed through the cooling system in order to drain the thermal energy transferred by the hot circuit and, then keeps it inlet temperature constant. Transient state tests follows the steady state test. This consists of sudden removal of hot oil feeding the turbocharger element meanwhile, cold oil is passed at constant conditions through the other element. All the signals are recorded along the test until they are stabilized. 



\section{Chapter 3}

\section{Measurement at different mass flow ratios in the double-entry turbines}

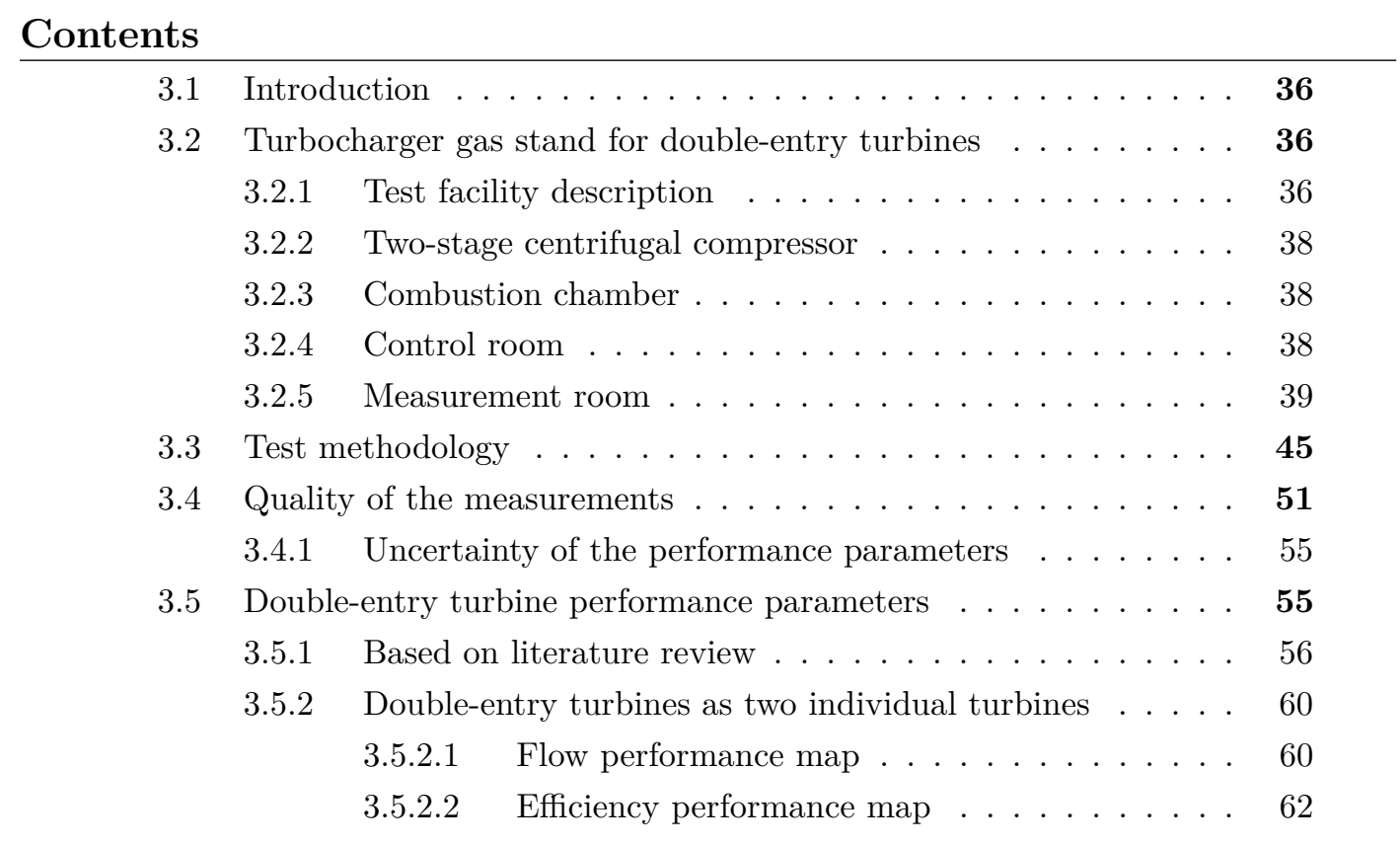




\subsection{Introduction}

In an internal combustion engine, double-entry turbine operates under unequal admission conditions by feeding the turbine with a different amount of flow in each entry for a majority of the time. Despite the impact on turbine performance, normal characteristic maps of these turbines are usually available only for full admission conditions. Therefore, firstly, this chapter is dedicated to explaining a way of measuring the double-entry turbines under different flow admission conditions in a gas stand, to characterize the mass flow and efficiency parameters. Secondly, a way of building the performance parameters of the double-entry turbine working under different flow admission conditions has been discussed. This way, the flow behaviour can be explained.

The works, analysis and ideas described in this chapter were the origins of publication number [3] from the publications list of the author of this thesis. In the sake of readiness and to protect the thesis writing style the publication number [3] from author's list of publications have not been specifically cited every time that ideas, figures or discussions contained in it are bring to this chapter again. This disclaimer corrects, compensates and justify this fact; being the Ph.D. candidate and the works of his thesis are the origin of the innovation component in the publication number [3] listed in the referred section.

\subsection{Turbocharger gas stand for double-entry turbines}

This section contains the description of an experimental test facility used for characterizing the double-entry turbochargers in a gas stand. At first, the turbocharger gas stand and its control system will be introduced. Later, the turbocharger setup and the test method will be presented.

\subsubsection{Test facility description}

The main experimental activities related to this thesis were performed in a specific test facility called hot and high flow gas stand, as shown in Figure 3.1, which is located in CMT-Motores Térmicos. The facility consists of four main areas: two-stage centrifugal compressor room, hot gas generator area, control cabin and the measuring chamber in which the instrumented turbocharger is located. In this test bench, the generation of hot gases sent into the turbine is realized through the coupling of a centrifugal compressor and a combustion chamber. 
(1) TWO - STAGE CENTRIFUGAL COMPRESSOR

(2) SETTLING TANK 1

(3) BLeEding VAlves

(4) SETTLING TANK 2

(5) COMBUSTION CHAMBER

(6) MEASUREMENTS ROOM

(7) ROOM EXTRACTOR

(8) CONTROL ROOM

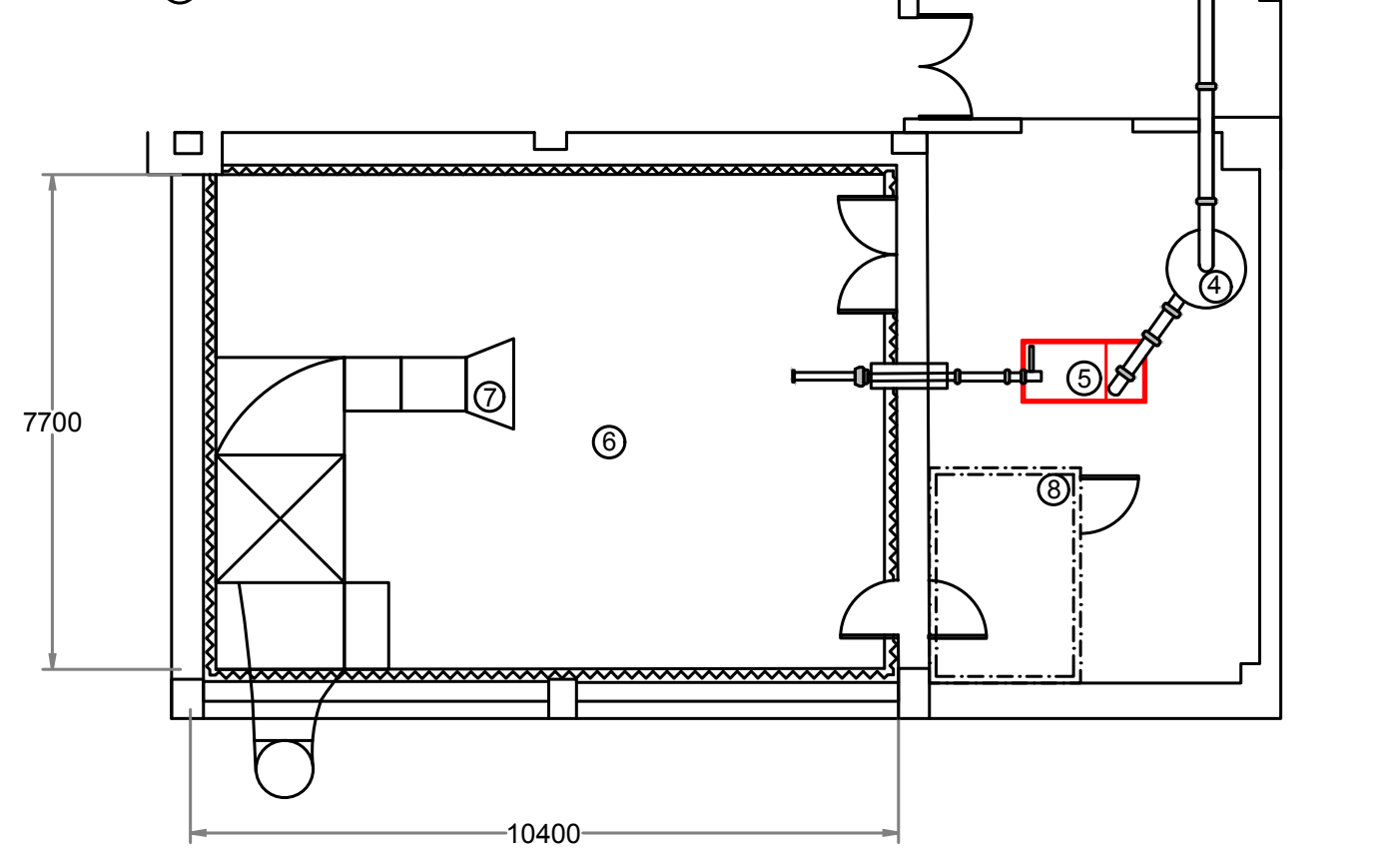

Figure 3.1: Hot and high flow test rig facility 


\subsubsection{Two-stage centrifugal compressor}

A two-stage centrifugal compressor is used as a gas supplier. This compressor is of the oil-free type and has an air cooler downstream of each stage (inter and after-cooler) and is driven by a $450 \mathrm{~kW}$ electric motor. The maximum gauge pressure it reaches is 5.30 bars and disposes of flow rates between 4400 and $7200 \mathrm{~m} 3 / \mathrm{h}$. A feedback regulator system intervenes on the regime of a rotation speed of the electric motor and the adjustment of valves always guarantee the same pressure in the tank downstream of the centrifugal compressor. On the line between the centrifugal compressor and the combustion chamber, there are two settling tanks for the storage of compressed air and three bleeding valves for the evacuation of any excess air. The second tank performs the task for accumulating a sufficient amount of air to turn off the combustion chamber gradually, in case the centrifugal compressor is stopped accidentally.

\subsubsection{Combustion chamber}

The combustion chamber is shown in the Figure 3.2 is fueled by diesel and is the innovative element of the hot and high flow test rig. It has the advantage of reaching the gas temperature up to $1200^{\circ} \mathrm{C}$ as compared to the traditional means hot gas generators (internal combustion engines or electric heaters). In fact, comparing with an electrical heaters, maximum air temperatures of about $600^{\circ} \mathrm{C}$ is only reached. Due to the temperature limitation of the cover, that wrap of electric wires is $680^{\circ} \mathrm{C}$. There are also other types of electrical heaters which can reach $1000^{\circ} \mathrm{C}$. However, it would be necessary to prevent all the water vapour present in the incoming air. Besides, work formed by a very thick tangle of electric wires, they introduce considerable pressure drops in the airflow. On the other hand, using large displacement internal combustion engines, which are fueled by Diesel or Petrol, the maximum temperatures that may reach is about $900^{\circ} \mathrm{C}$. Nevertheless, between the engine and turbocharger to be tested, a flow stabilization volume (to dampen the motor impulses) and relatively large ducts should be inserted to accommodate the measuring sensors.

Using the combustion chamber also has other advantages like getting the higher flow rate ranges $(0-2.15 \mathrm{~kg} / \mathrm{s})$, minor thermal inertia (on and off) and, greater precision in reaching the set temperature level. That also signifies less time required for the tests, from the possibility of realizing the thermal transients with the combustion chamber alone. All these features make the rig suitable for testing a wide variety of turbochargers, from the smallest to those for heavy-duty applications.

\subsubsection{Control room}

The control is adjacent to the measuring room, acoustically isolated and in total security. There are three computers in which the members can take the measurements, 

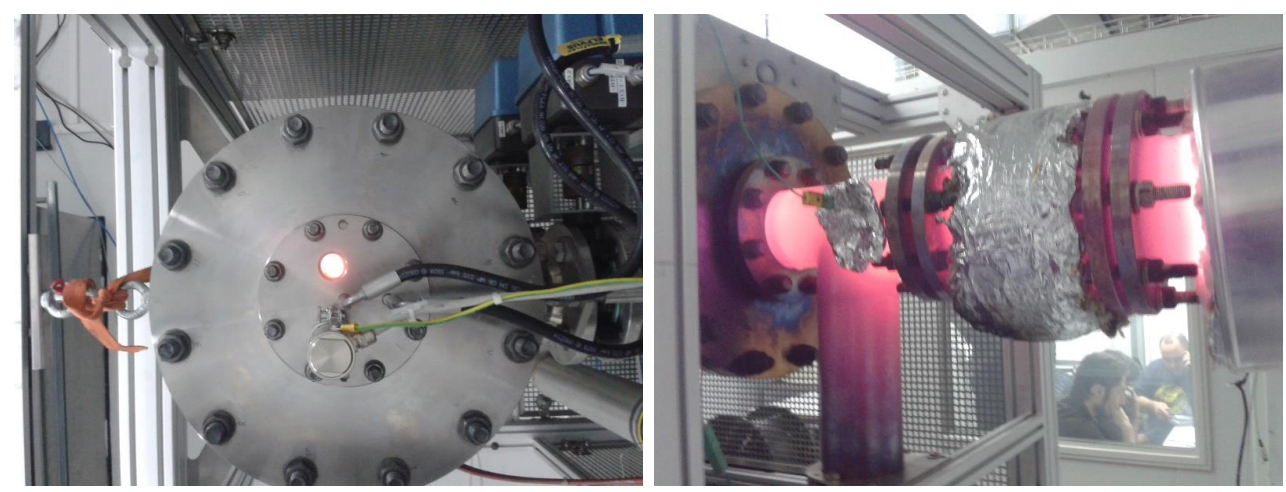

Figure 3.2: Combustion chamber front view(left) and flue gas pipe at the exit of combustion chamber(right)

and from them it is possible:

- To adjust the opening/closing of all the valves in the test bench.

- To adjust the pressure in the tank downstream of the two-stage centrifugal compressor.

- To set the temperature levels of the gas in the combustion chamber.

- To monitor and acquiring the values of all quantities measured by the sensors.

Before being able to start the data acquisition, it is necessary to reach the thermal balance of the metallic wall temperatures of the turbocharger. Therefore, one of the three computers (the data logger) is used only to observe the variations of the surface temperatures in the different points of the equipment The stability criteria was set to less than $0.5^{\circ} \mathrm{C}$ variation. Once all the wall temperatures are stable, it is possible to proceed and record these quantities in one minute of operation. In the same time interval, all the measurements coming from the test rig sensors are recorded with a second computer. The third computer is used for regulating and controlling the combustion chamber and the upstream centrifugal compressor. The control and data acquisition system is automatic and has been implemented by the CMT operators. This instrumentation is based on the National Instrument field point modules managed through a Labview interface.

\subsubsection{Measurement room}

In the measurement room, we find the system containing the turbocharger and measurement sensors. The room is acoustically insulated and has an air exchange system to guarantee the healthiness of the room inside. The test rig is divided into two 
circuits, one traversed by the exhaust gases, which is the turbine side and the other by the fresh air that is compressor side as shown in the Figure 3.3.

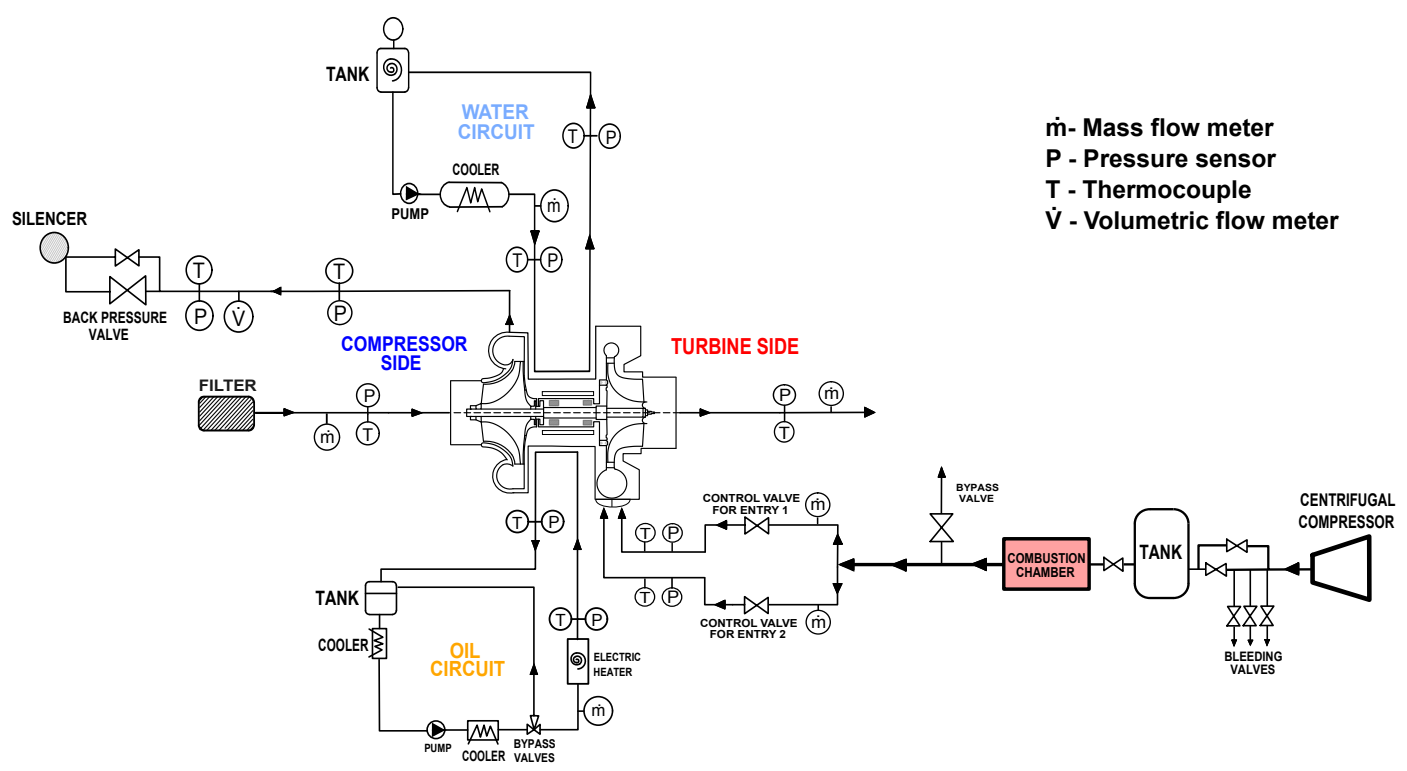

Figure 3.3: Schematic layout of turbocharger gas stand for double-entry turbines

Turbine side: The hot gases are coming from the combustion chamber and flow towards the turbine through a refractory metal pipe (which is extraordinarily resistant to heat up to $1100^{\circ} \mathrm{C}$ ). This pipe has a diameter of 125 millimetres and completely thermally insulated. Not all the gases produced by the combustion chamber are forced to pass through the turbine. For various reasons, it is necessary to provide a divergent branch from the main flow duct, which is parallel to the turbine circuit. In that branch, only a bypass valve, of the butterfly type is included, which helps to discharge the exhaust gases directly to the outside without passing them into the turbine.

The tests of double-entry turbines usually contain the measurement of both turbine inlets, i.e. full admission condition, and also a measurement of each turbine inlet while the other inlet held closed (partial admission). This kind of tests can be performed on a test bench designed for single entry turbines characterization, named as a standard gas stand test bench. Moreover, while testing the double-entry turbine at full admission conditions in a standard gas stand bench, only total mass flow rates are measured. Therefore, the mass flow distribution between the individual turbine entries is unknown and both uncontrolled under full admission conditions. For these reasons, the measurement room of hot and high flow test rig has been modified to investigate the twin-entry and dual-volute turbochargers under a variety of operational conditions based on the flow simulation of a turbocharger using compressed air. The main features are the possibility of generating the cold or hot flow in the 
double-entry turbine at different flow admission requirements.

As shown in Figure 3.3, downstream of the combustion chamber, the airflow is branched into two pipes, which are called as Turbine Entry 1 (for Sh/LV) and Turbine Entry 2 (for H/SV), referring to their positions shown in Figure 3.4. Each of these branches is instrumented with a V-cone type sensor for measuring the air mass flow rate and a control valve for varying the flow going into the branches. Further, the pressure and temperature sensors were also installed on each branch according to the SAE standards [69],[70] and, they are recorded in static conditions. This way, it allows testing the double-entry turbocharger with different flow admission conditions at the turbine inlets and able to record the flow, pressure and temperature in each branch. At the downstream of the turbine, a third V-cone sensor is placed for measuring the total mass flow exiting from the turbine, and also the pressure and temperature sensors were instrumented.

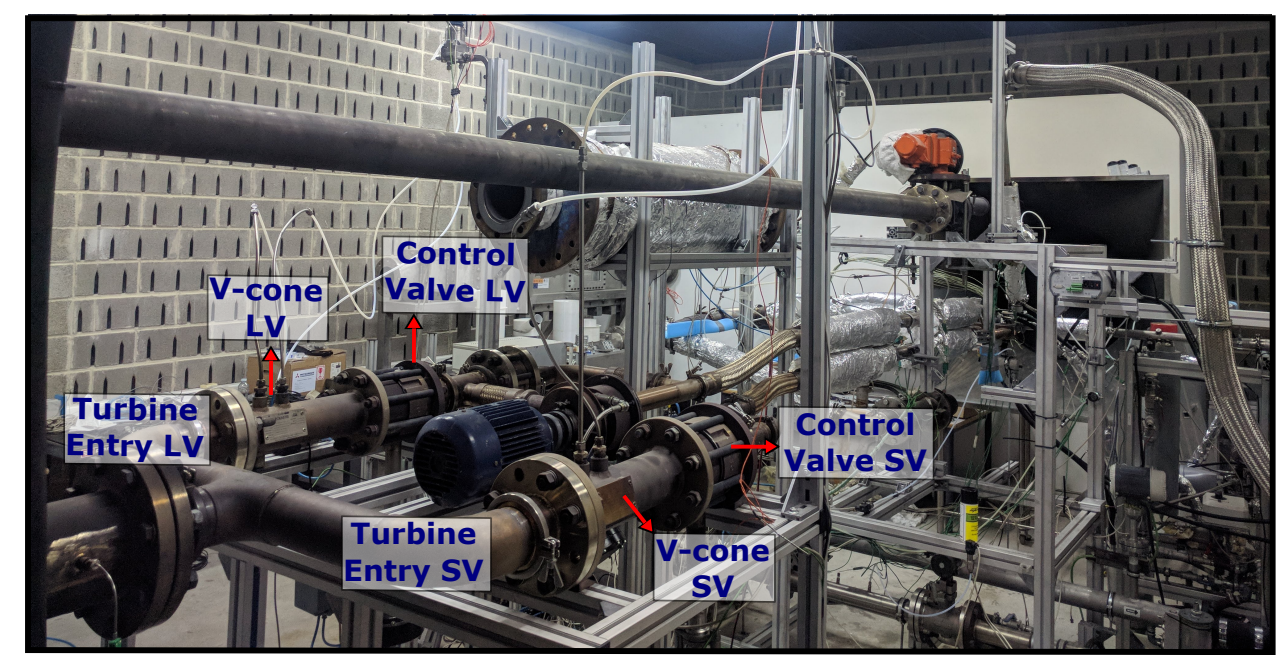

Figure 3.4: Gas stand for testing the double-entry turbines at different flow admission conditions in steady flow situations. Mainly, showing the flow separation and controlling section in the gas stand.

Compressor side: The compressor of the turbocharger takes air from the ambient. It passes through a filter first to purify the air from any impurities. Then it passes by a hot-plate type flow meter to measure the flow. At the downstream the turbocharger compressor, a vortex volumetric flow meter is placed to measure the airflow. Further, an electronic control back-pressure valve is placed to modify the pressure ratio of the compressor, i.e., to move from surge to choke conditions of the compressor. The silencer has the purpose of reducing the noise emission coming from the compressor; a membrane precedes the silencer for filtering air from any oil present. In fact, when the pressure at the compressor outlet drops (choke conditions), the lubricating oil can be pushed to flow directions. For the measurement of pressure and temperature, sensors were installed on the inlet and outlet pipes of a compressor according to SAE 
standards [69],[70] and, they are recorded in static conditions.

Oil and water circuits: Two independent circuits, as shown in the Figure 3.5 were used for the operation of lubricating the oil and water in the turbocharger. Both units include a tank, pump, heat exchanger (having water as cold fluid), an electric heater, and two feedback control systems (PID). These PIDs will ensure the temperature and pressure levels to which the user wants to desires the fluids in the central housing of the turbocharger. Gas stand equipment and their precision are shown in the Table 3.1 .

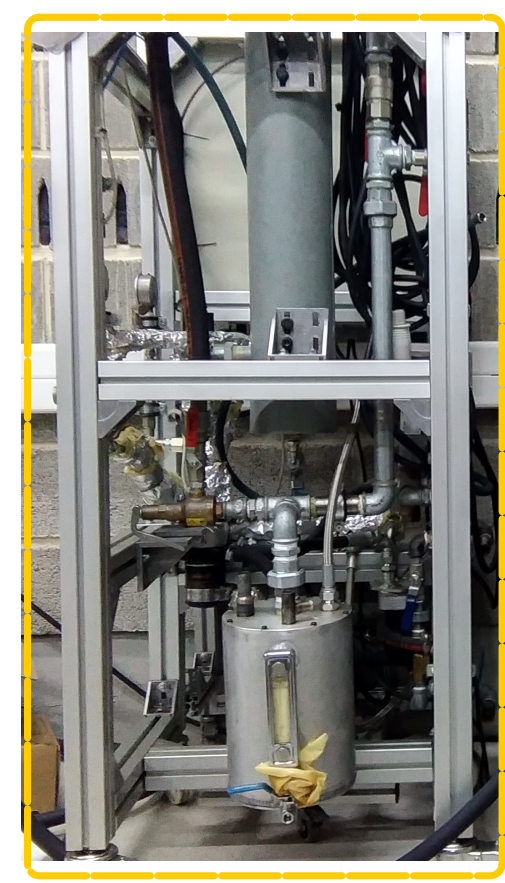

(a) Oil system

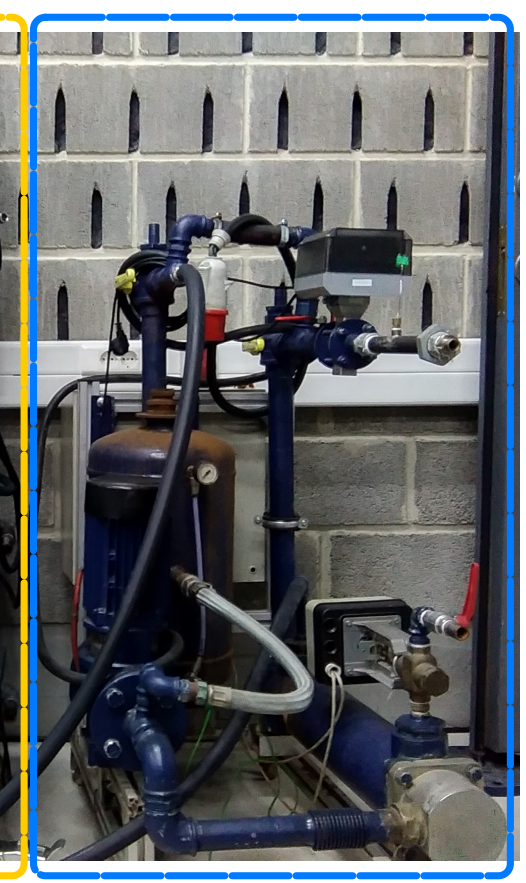

(b) Water system

Figure 3.5: Showing the oil and water circuits used in the gas stand (a) Independent Oil system; (b) Independent water system

At every inlet and outlet pipes of the turbine and compressor, 4 K-type thermocouples were instrumented and 4 pressure probes and their average is measured by the Piezoelectric sensor.

The temperatures measured by 4 K-type thermocouples per section, and their average value was used as the mean section temperature. The temperature probes that were used did have total temperature shields, so the measured temperature laid between the total and static temperature. A typical kinetic energy recovery factor $q$ 
0.5 was used:

$$
q=\frac{\widehat{T}-T}{T_{t}-T} \Rightarrow T=\widehat{T}-\frac{q}{2} \cdot \frac{v^{2}}{c_{p}}
$$

where $\widehat{T}$ is the measured temperature, $T$ is the static temperature, $T_{t}$ is the total temperature and $v$ is the mean flow velocity at the measurement section. The mean flow velocity was obtained as shown below:

$$
v=\frac{\dot{m}}{\rho \cdot A}=\frac{\dot{m} \cdot R \cdot T}{p \cdot A}
$$

where $\rho$ is the density, $p$ is the pressure, $\dot{m}$ and $A$ are the mass flow rate and area of the measurement section, respectively.

The total temperature $T_{0}$ and pressure $p_{0}$ were obtained as:

$$
\begin{gathered}
T_{t}=T+\frac{v^{2}}{2 \cdot c_{p}} \\
p_{t}=p \cdot\left(\frac{T_{t}}{T}\right)^{\frac{\gamma}{\gamma-1}}
\end{gathered}
$$

Table 3.1: Gas stand measurement equipments and their precision.

\begin{tabular}{cccc}
\hline Variable & Sensor types & Range & Typical Uncertainty \\
\hline Gas mass flow & V-cone and Thermal vortex & $45-1230 \mathrm{~kg} / \mathrm{hr}$ & $<1 \%$ of the measured values \\
Gas pressure & Piezoelectric & $0-5 \mathrm{bar}$ & $12.5 \mathrm{mbar}$ \\
Gas/metal temepratures & K-type thermocouples & $273-1500 \mathrm{~K}$ & $1.5 \mathrm{~K}$ \\
Oil pressure & Piezoelectric & $0-5 \mathrm{bar}$ & $12.5 \mathrm{mbar}$ \\
Oil mass flow & Coriolis & Few tens gr $/ \mathrm{s}$ & $2 \%$ of the measured values \\
Turbocharger speed & Inductive sensor & $<300 \mathrm{krpm}$ & $<500 \mathrm{rpm}$ \\
\hline
\end{tabular}

It is worth highlighting that the tests performed on this gas stand have been divided into two main groups, as shown below:

- Quasi-adiabatic tests: In this kind of tests, heat transfer and mechanical losses are decoupled, which is the primary objective of the turbocharger under study. The internal heat fluxes between the turbocharger elements are minimized by providing the lowest possible temperature gradient between its elements. It is done by maintaining temperatures of turbine inlet, oil inlet and compressor outlet at similar levels during the tests. The external heat transfers are minimized by insulating the outside surfaces of the turbocharger. Additionally, 
the inlet and outlet ducts are also insulated, as shown in Figure 3.6. From these tests, enthalpy variation in lubricating oil represents the turbocharger mechanical losses. The practical purpose of a quasi-adiabatic test program is thus to obtain an accurate measurement of the work transfer, as shown in Figure 3.7(a). The experimental information from this kind of test acts as the key tool for developing and modelling the turbine extrapolation models. Further, it also provides a better matching between the turbocharger and the engine.

- Hot exposed tests: Turbocharger manufacturers usually perform this test to obtain the characteristic maps of the turbine and compressor. The main difference with the previous test is that the turbocharger is not insulated externally and internal heat fluxes were also allowed. This type of tests will be useful to study the heat transfer problems in double-entry turbines. These involve all the possible heat transfer mechanisms: conduction, convection and radiation as shown in the Figure 3.7(b).

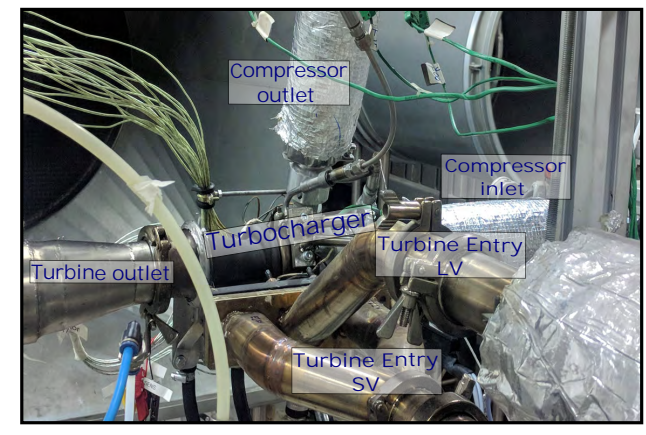

(a) Turbocharger in the gas stand before insulation

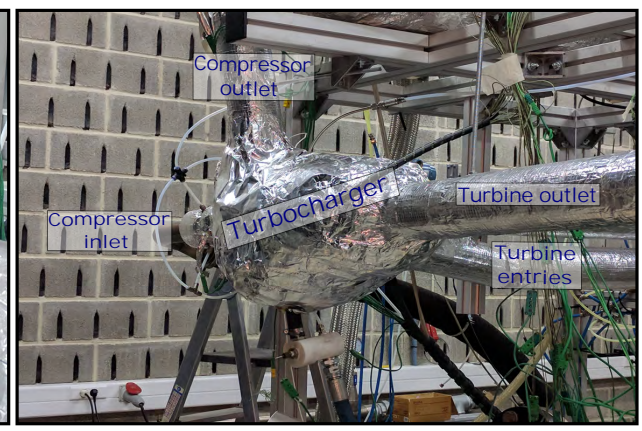

(b) Turbocharger in the gas stand after insulation

Figure 3.6: Double-entry turbochargers setup in the gas stand.

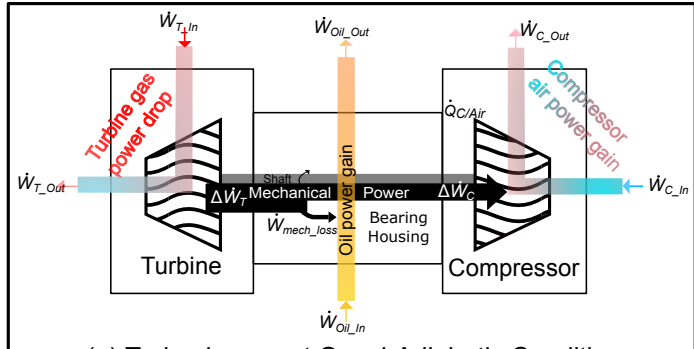

(a) Turbocharger at Qausi-Adiabatic Conditions

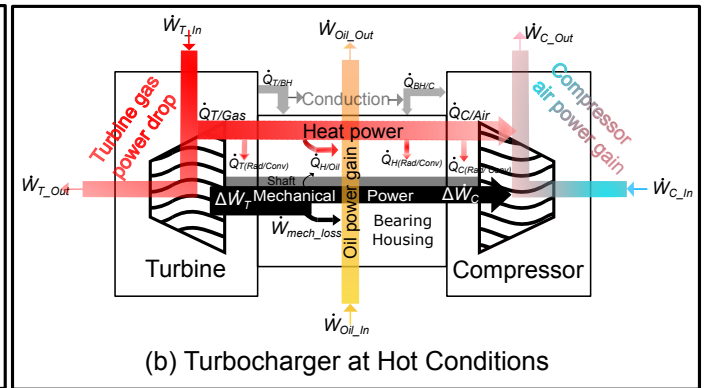

(b) Turbocharger at Hot Conditions

Figure 3.7: Turbocharger working in quasi-adiabatic and hot exposed conditions

Quasi-adiabatic tests were performed on the turbocharger T\#1TER (twin entry) and T\#2DVR (dual-volute) under different steady flow admission conditions with a turbine inlet temperature of around $363 \mathrm{~K}$. This temperature is achieved just by using the two-stage centrifugal compressor and there is not any other addition to the air 
flow. Whereas, the turbocharger T\#3DVM has been tested only in hot exposed tests at certain flow admission conditions with a turbine inlet temperature of around 673 K. For this, the combustion chamber is used to heat the air. It is worth highlighting that this thesis is mainly focused on developing the mean line modelling for doubleentry turbines using the adiabatic maps. Therefore, the heat transfer related issues for these turbochargers at hot conditions were not analyzed in this dissertation, and they will be discussed as in the future works. However, a methodology described in $[71,72]$ has been used to get the isentropic efficiencies of both turbine and compressor. The procedures will be explained in the further sections.

\subsection{Test methodology}

One of the main issues concerning the investigation of double-entry turbines is that of assessing the impact of each entry on the overall performance in steady-state under varying flow admission conditions. Moreover, in normal engine operation conditions, the double-entry turbines operate under different flow admission conditions due to the pulsating nature of exhaust gas coming from the engine. Therefore, the overall turbine performance will certainly depend on the mass flow distribution among each entry. Brinkert et al. [30] indicate that testing the twin-entry turbines with the scroll pressure ratio as only control parameter cannot guarantee equal flow conditions along one turbine speed line unless the turbine inlets have equal flow areas.

Therefore, the turbines used in this thesis were investigated under a range of different turbocharger speeds with varying actual mass flow conditions in Shroud/Long Volute and Hub/Short Volute branches. The actual mass flow rate at each entry of the turbine was controlled by two control valves, as shown in Figure 3.4. It is worth highlighting that the pressures and temperatures in each turbine were not explicitly controlled. But, they will change according to the flow situation in each turbine branch. However, this is likely to happen when the double-entry turbines work with the engine. Due to the exhaust pulsating flow nature, there will be a different inlet pressure and temperature, thereby the different masses in both branches. Consequently, this was one of the main goals of our research work: to develop simple and representative turbine testing procedures in a standard gas stand.

Table 3.2, reports the test conditions showing the flow rates between the inlets. The same compressor corrected speeds have been tested for every MFR. This imposition forces, indirectly, that the addition of mass flow in the two branches is similar for the same compressor corrected speed at different MFRs. These testing conditions are stated in three different categories, as follows:

- Partial admission: Passing flow through one of the turbine inlets by blocking the other one and vice versa. It is performed on the gas stand by blocking one 
of the inlets and employing the flow into an open inlet and vice versa.

- Equal/Full admission: Having the same mass flow rate in each entry of the turbine. It is possible to measure on the gas stand while setting both control valves to fully opened/adjusting the positions of the valves to maintain the same flow in both branches.

- Unequal admission: In the middle of two limits of partial and full admission cases, it is possible to measure the turbine with different flow admission points. These are the cases in an engine; twin-entry and dual-volute turbocharger operate by feeding the turbine with an unequal amount of flow in each entry of the turbine.

For distinctive allocation of flow conditions to the corresponding turbine characteristics, mass flow ratio (MFR) definition with actual mass flows has been used in this work. It is defined according to Equation 3.5, as the mass flow rate in the Shroud/Long Volute branch divided by the overall mass flow rate of the turbine.

$$
M F R=\frac{\dot{m}_{S h / L V}}{\dot{m}_{S h / L V}+\dot{m}_{H / S V}}
$$

Table 3.2: Different flow admission conditions at the turbine inlets that were measured with T\#1TER turbine in the gas stand.

\begin{tabular}{ccc}
\hline \multicolumn{3}{c}{ Test Matrix } \\
\hline \multirow{2}{*}{ MFR } & \multicolumn{2}{c}{ Flow split rate } \\
\cline { 2 - 3 } & Sh/H & H/H \\
\hline 0 & 0.00 & 1.00 \\
0.2 & 0.25 & 1.00 \\
0.31 & 0.50 & 1.00 \\
0.43 & 0.75 & 1.00 \\
\hline MFR & \multicolumn{2}{c}{ Flow split rate } \\
\cline { 2 - 3 } & Sh/Sh & H/Sh \\
\hline 0.53 & 1.00 & 0.89 \\
0.57 & 1.00 & 0.75 \\
0.67 & 1.00 & 0.50 \\
0.8 & 1.00 & 0.25 \\
1 & 1.00 & 0.00 \\
\hline
\end{tabular}

The MFR definition with the actual mass flows makes it simpler to test any double-entry turbine (twin-entry or dual-volute) in a standard gas stand. That is, it 
will have less parameters to control while testing the turbine as compared to $R M F R$ parameter that described in chapter 2. When testing the turbine using $R M F R$ at different constant flow admissions conditions, the turbine inlet temperature in each branch needs to be maintained constant through out the tests.

Moreover, the $M F R$ is proportional to the ratio of power in one branch to the total turbine power. Further, it keeps much of velocity ratio (Mach ratio) generalization effects that comes from $R M F R$ parameter $[28,29]$. It means that the ratio of Mach numbers is very similar to the ratio of mass flows. Therefore, both $M F R$ and $R M F R$ can be used for similar analysis. But, tests with constant $M F R$ is more comfortable and needs less control of the experiments. Even though the turbine characterization performed with $M F R$ led to small differences when compared to $R M F R$ parameter, as shown in Figure 3.8. At MFR equal to 0, 0.5 and 1, the difference between the $M F R$ and $R M F R$ is negligible. As the turbine inlet total pressure and the temperature is almost the same at both branches. The differences only appear at more unequal admission conditions, and they are small.
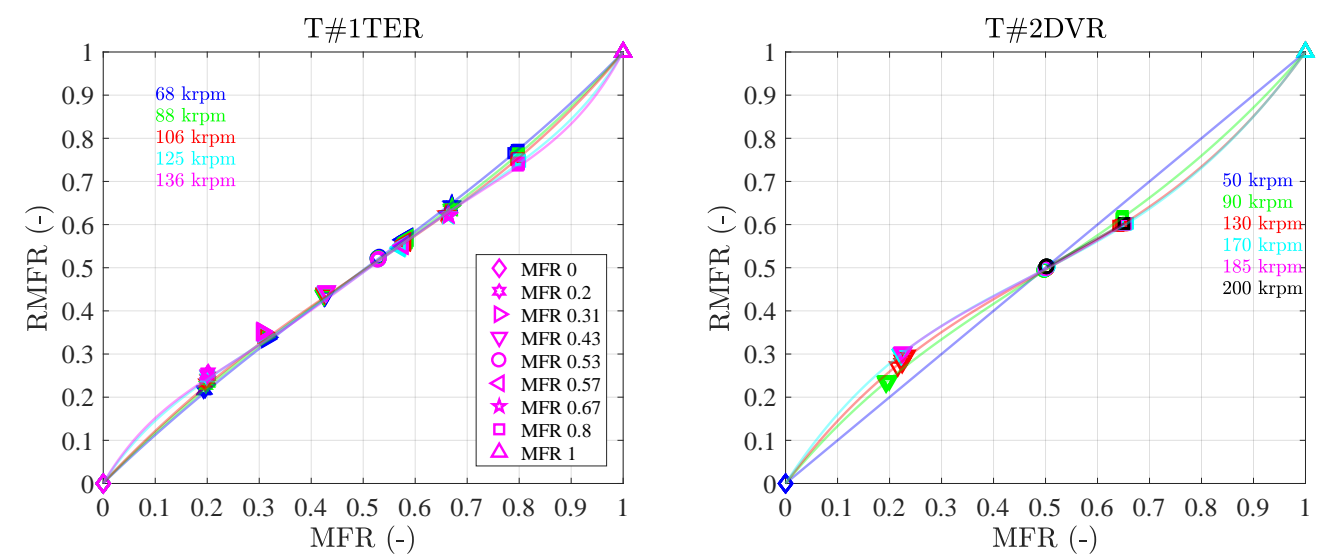

Figure 3.8: Comparison of MFR versus RMFR variables for both twin-entry and dual-volute turbines which are tested under quasi-adiabatic conditions

It is true that performing the tests with the $M F R$ definition; there will be a difference in the temperature and pressure at the turbine inlet and also, a small change in the reduced turbine speed of each branch. As mentioned before, it likely to happen when the double-entry turbines work with the engines due to their exhaust pulsating flow nature. Therefore, while testing the double-entry turbines with $M F R$ parameter, the turbine inlet pressure and temperature in each branch are not needed to be controlled. Only compressor speed and compressor performance (pressure ratio or mass flow) are regulated to be the same at different $M F R$, as shown in Figure 3.9. That way, the experiment is much more straightforward. Turbine inlet temperature can be constant or not, depending on heat transfer considerations. Turbine inlet pressure is a consequence of total turbine flow (to get the desired compressor power or compressor speed) and flows distribution (to get desired MFR). 
The measurements with different mass flow ratios at the T\#1TER turbine inlets are performed from $68 \mathrm{krpm}$ to $136 \mathrm{krpm}$. In the step of $18 \mathrm{krpm}$ in the compressor corrected speed and between compressor surge and choke conditions, as shown in Figure 3.9. It is worth to note that the different compressor corrected speed were maintained constant in all the tested MFRs. This way, the efficiency of the turbine is analyzed at different flow admission conditions at the turbine inlets.

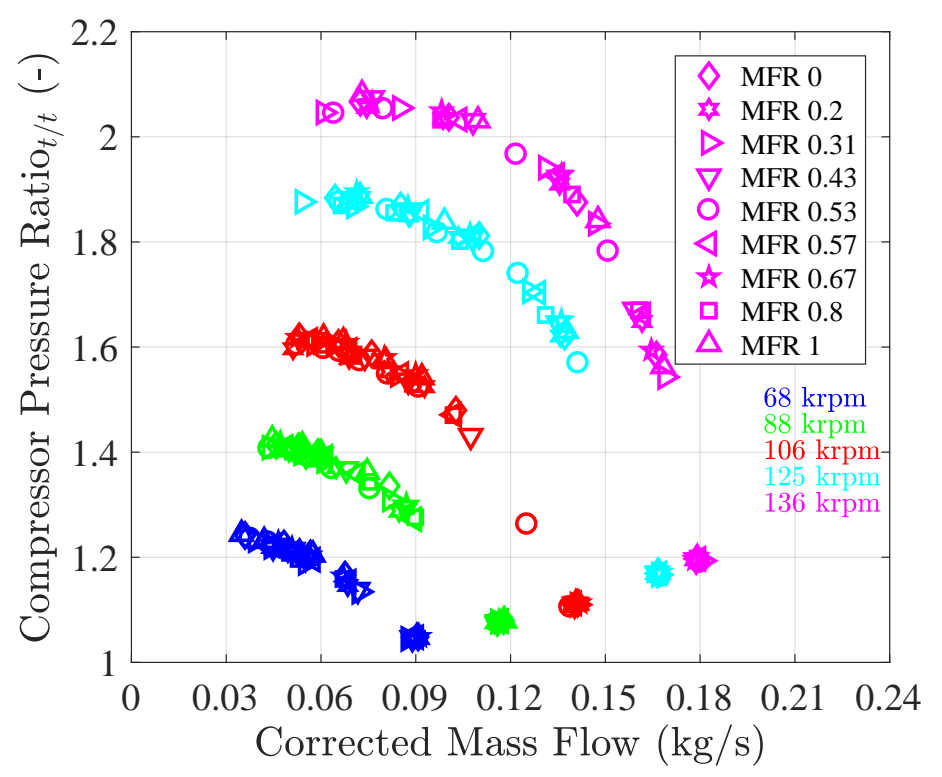

Figure 3.9: Compressor map of T\#1TER in all admission cases

As a result of being able to measure the turbine with different MFRs that has shown in the Table 3.2 in a gas stand, a flow interaction map can be created as shown in Figure 3.10. The flow interaction map shows the experimental turbine operating conditions corresponding to extreme situations for each entry; partial admission (MFR 0 and 1), and further equal and unequal mass flow states in Shroud/Long Volute and Hub/Short Volute branches (MFR 0.5, 0.43, 0.8,..). Figure 3.10 shows the results for the twin-entry turbine (T\#1TER), and it is observed that the maximum mass flow states in Shroud and Hub entries at partial admission (MFR $=0$ and $=1$ ) is very similar. Even at unequal and full admission conditions, the total flow given to the turbine is very comparable. Nevertheless, it can be noted that significant differences are found between the partial and rest of the admission conditions with respect to the total mass rate in the turbine. Indeed, the overall mass flow given to the twin-entry turbine to reach at higher compressor corrected speed is $20 \%$ lower in partial admission conditions, as shown in Figure 3.10. This indicates that the expansion ratio and, as will be shown later, also efficiency of the turbine changes with different mass flow ratio conditions at inlets. Since the total inlet mass flow rate to the turbine in each different admission conditions is changed accordingly in 
order to obtain as a result that, the corrected speed and operating conditions of the compressor are same in all turbine inlet admission cases, as shown in the Figure 3.9). This total mass flow is a function of the selected MFR (Figure 3.10).

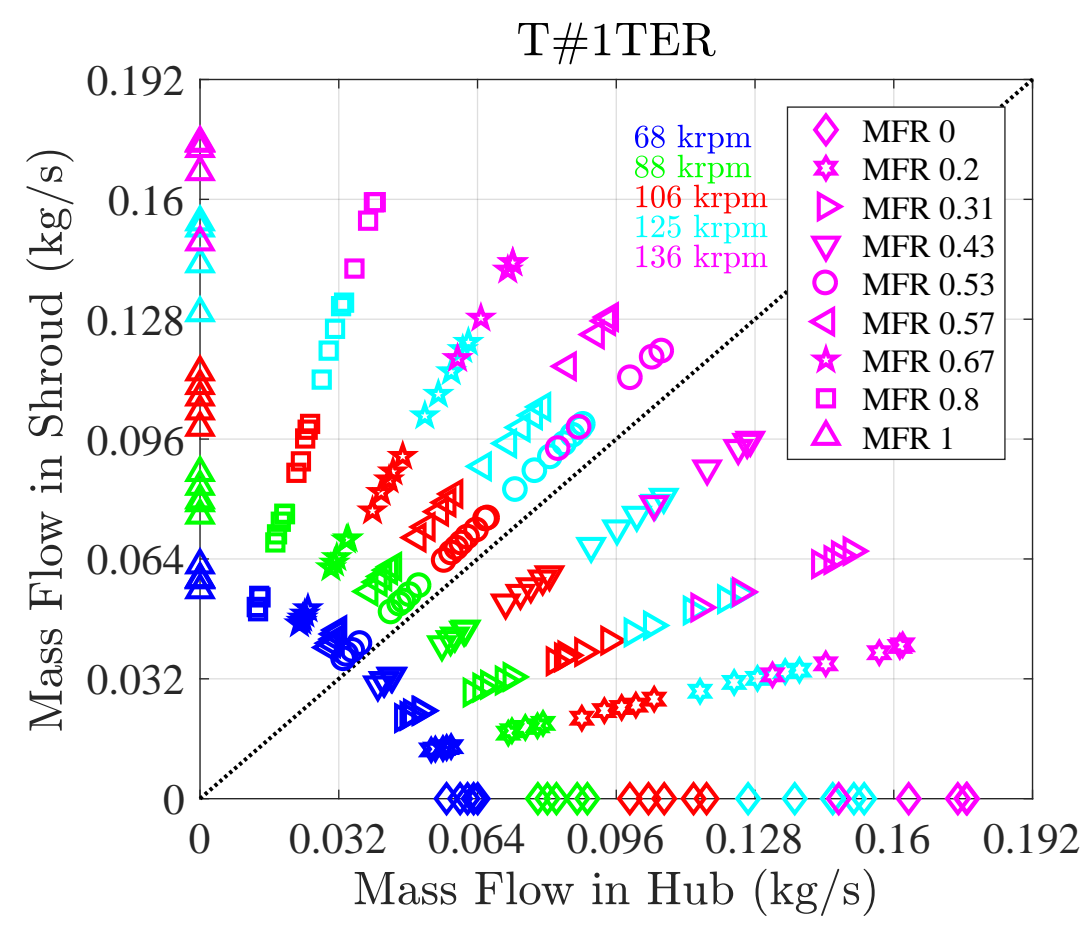

Figure 3.10: Twin-entry turbine inlet flow interaction map

The same methodology was applied to test the dual-volute turbocharges (T\#2DVR and T\#3DVM) as shown in Figure 3.11. For this turbochargers, only the full admission conditions were able to reach a corrected compressor speed of $200 \mathrm{krpm}$. In the case of partial admission conditions, the maximum speed that was able to reach is 170 $\mathrm{krpm}$, since there were needed too much pressure ratio to push the same total flow into the turbine. Moreover, the total mass flow given to the turbine T\#2DVR at 170 $\mathrm{krpm}$ is $15 \%$ lower in partial admission conditions (see Figure 3.11(Left)). The same outcome can be seen for the T\#3DVM turbocharger as well (see Figure 3.11(Right)).

Figure 3.12, shows the relation between scroll pressure ratio (shroud-to-hub for twin-entry and LV-to-SV for dual-volute) and mass flow ratio (MFR) for different flow admission conditions with turbine reduced speed. For a particular mass flow ratio between the zero and unity, the flow conditions in twin-entry and dual-volute inlet branch remain equal. That is, at $\mathrm{MFR}=0.5$, where the turbine works under full admission conditions. Therefore, the resulting scroll pressure ratio will be equal to unity and which can be seen in Figure 3.12. In our experiments, the position of two control valves located upstream of the turbine inlets is controlled by sending a signal from a computer and which is fixed for a given MFR during the entire test. 

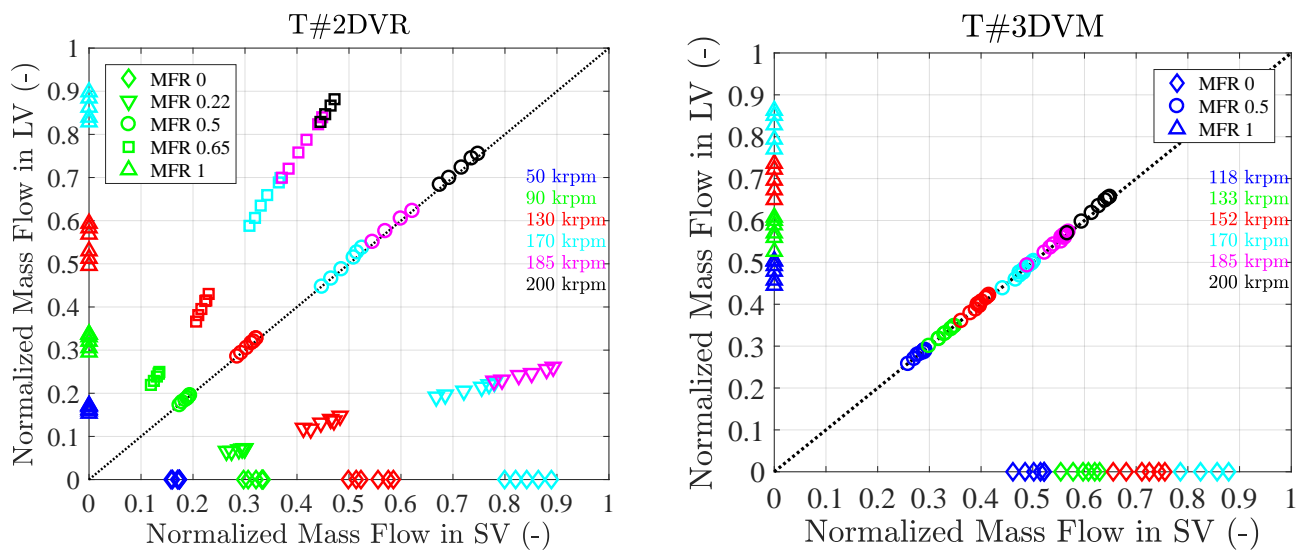

Figure 3.11: Dual-Volute turbines inlet flow interaction map: T\#2DVR (Left) T\#3DVM (Right)
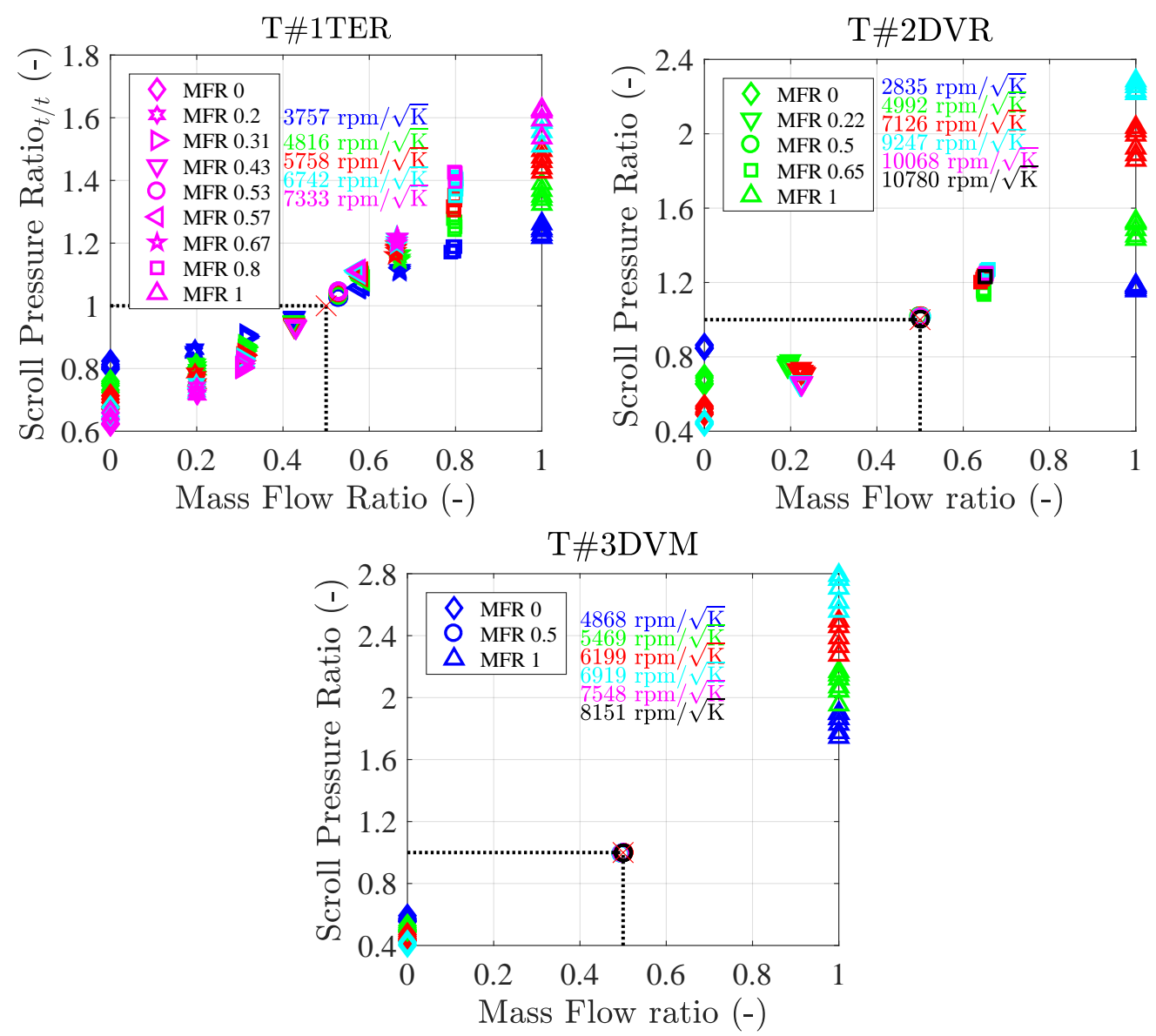

Figure 3.12: Turbine scroll pressure ratio with mass flow ratio 
While, measuring the twin-entry turbine (T\#1TER in Figure 3.12) at full admission condition, the position of control valve at the hub side branch has not been able to control precisely to $\mathrm{MFR}=0.5$. For this reason, the test completed at $\mathrm{MFR}=0.53$, which is very close to MFR 0.5 (Showed in the dotted lines of Figure 3.12). Moreover, the scroll pressure ratio is also almost close to unity.

It is worth highlighting that the scroll pressure ratio (SPR) is a function of MFR. Because, while testing the turbocharger in the gas stand, this parameter is not controlled but, it depends on the amount of flow in each turbine inlet. From Figure 3.12, it can also be noted that for an individual flow admission condition, the mass flow ratio is held constant for all measured turbine reduced speeds. Also, it can be checked that as Brinkert et al. [30] indicate, the scroll pressure ratio changes for same MFR as a function of the reduced speed of the turbine and the trend is inverted between MFR 0 and 1, it means that SPR grows as reduced speed goes down at MFR 0. But SPR grows with reduced speed at MFR 1 . Only at MFR $=0.5, \mathrm{SPR}$ is constant and equal to one for all reduced speeds.

Moreover, from Figure 3.12, it can also be observed that the scroll pressure ratio values between MFR 0 and 1 for different turbocharger speeds are inverted. For example, at MFR 0, the scroll pressure ratio values of lower turbocharger speed are higher as compared to the higher speeds. But, at MFR 1, the values are opposite for the same speeds. Only at $\mathrm{MFR}=0.5$, the scroll pressure ratio is constant and equal to one for all reduced speeds.

\subsection{Quality of the measurements}

In order to assess the quality of adiabatic measurements, it is essential to check the mass flow balance between the inlet and outlet of the turbine and compressor in contrast to the energy balance of the whole system. Because it shows relatively the importance of work transfer from the turbine to the compressor; in performing an energy balance of the system when the tests are performed in adiabatic/quasiadiabatic conditions. Moreover, the mass flow rate is one of the important variables to consider in estimating performance maps like reduced and corrected mass flow parameter of the turbine and compressor, respectively. Figure 3.13 shows the mass flow balance of the turbine and compressor for different mass flow ratios that were carried out in the gas stand. From Figure 3.13 (Left), it is clear that the total amount of mass flow given to the turbine in each different admission conditions is similar. As a result, the corrected speed of the compressor is not different, as shown in Figure 3.9. Further, Figure 3.13 demonstrates that there are no relevant leaks of flow from measurement pipes as the flow rate is balanced from inlet to outlet of the turbine and compressor. Also, the error is within the measurement difference of $\pm 3 \%$. 
(a) T\#1TER

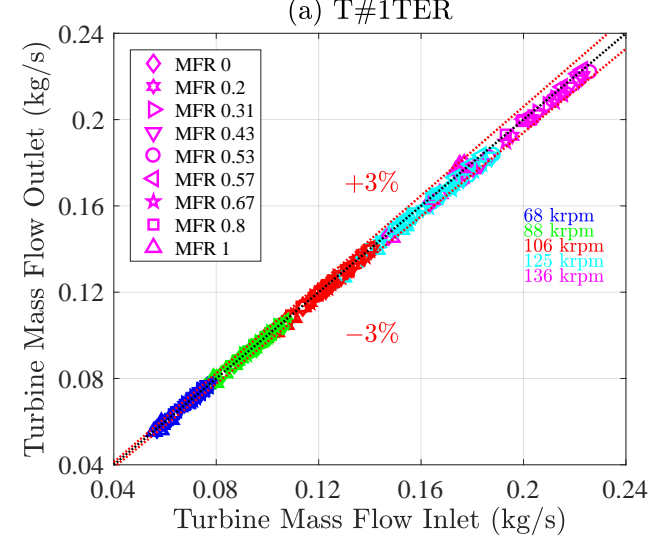

(b) T\#1TER

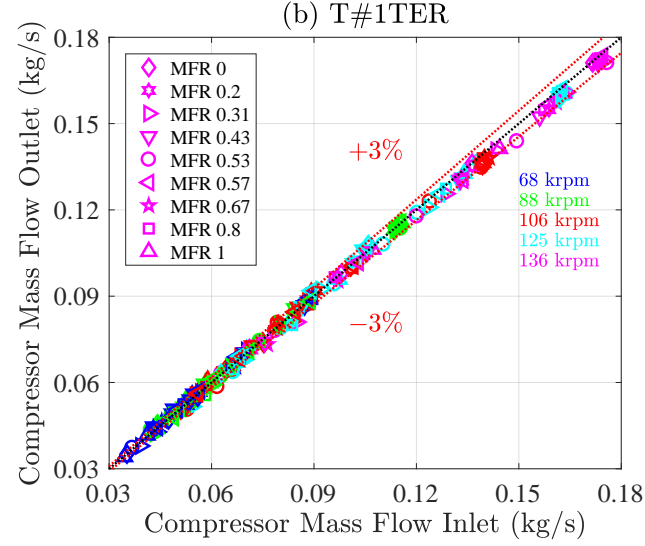

Figure 3.13: Mass flow balance of T\#1TER turbocharger: (a)Turbine mass flow; (b) Compressor mass flow

The procedure for testing the turbocharger at quasi-adiabatic conditions is by providing the lowest possible temperature gradients between its elements and also by minimizing the heat transfer internally and externally as shown in Figure 3.7(a). Zimmermann et al.[71], state that despite the significant insulation and controlling temperatures, remains a minor external and internal heat transfer. These minor heat losses are more significant at lower turbo speeds than at higher turbo speeds; even with the quasi-adiabatic tests. Similar was observed in our experiments at lower turbo speeds, and it is further shown in the following figures. At high speeds, the heat transfer effects on the compressor outlet temperature are almost negligible in comparison to mechanical power. Therefore, a heat transfer correction suggested by the Zimmermann et al. [71] is applied only to first three lower turbo speeds to obtain fully adiabatic results. The higher turbo speeds (125 and $136 \mathrm{krpm})$ are considered to be adiabatic enough with a relative unbalance of $\pm 3 \%$ as can be deduced from Figure 3.16. To determine whether the measurement is sufficiently adiabatic, the idea behind Zimmermann et al. [71] methodology was based on the power-based criterion. The approach is to identify a relatively constant heat transfer rate with the turbocharger speed. For this, a total turbine enthalpy drop is represented against the isentropic compressor power as shown Figure 3.14.

From the Figure 3.14, considering the points of maximum isentropic compressor power for first three-speed lines (filled points in the Figure 3.14), they fit into a straight line. Taking into consideration that, all the turbine power is used to be converted into compressor power and when the required compressor power reaches to zero (i.e., at zero speed), the turbine power has to be down to zero as well. This indicates the almost adiabatic measurements. Every deviation from zero at the axis ordinates is influenced by heat flows [71].

The same kind of approach is carried out on the measurement values of the com- 

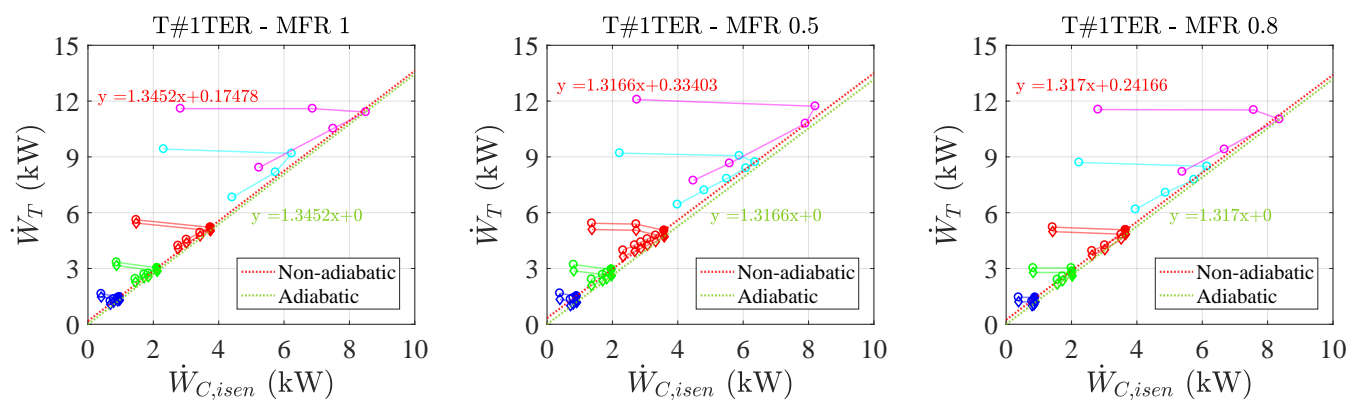

Figure 3.14: Power-based adiabatization approach for the turbine side: results shown for the T\#1TER at one Partial, full and unequal admission conditions (circle points represent with heat transfer, and diamond points represent without heat transfer)

pressor side, but by representing the effective compressor power against the isentropic compressor power as shown Figure 3.15.
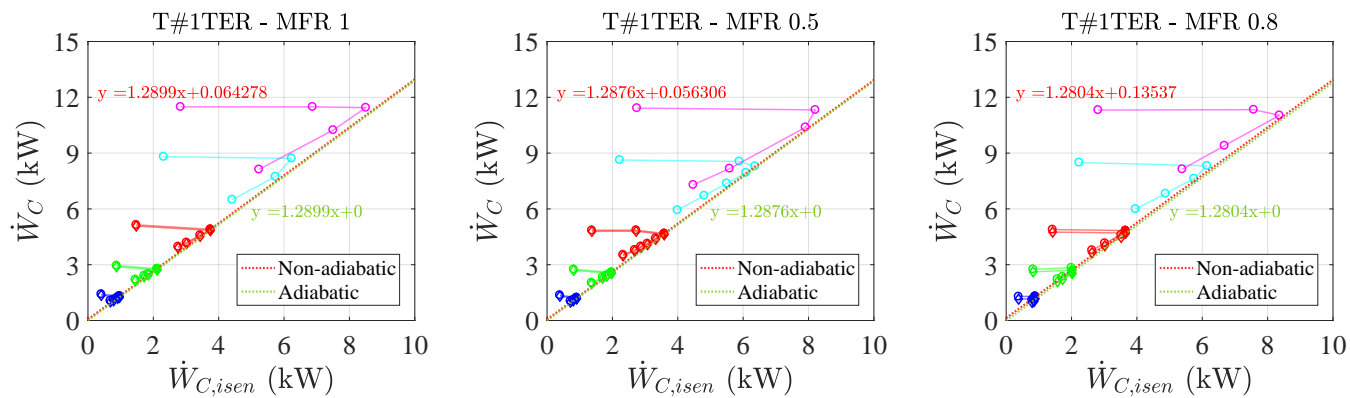

Figure 3.15: Power-based adiabatization approach for the compressor side: results shown for the T\#1TER at one Partial, full and unequal admission conditions (circle points represent with heat transfer, and diamond points represent without heat transfer)

Figure 3.16 shows the energy balance of T\#1TER and T\#2DVR turbochargers for different speed lines and MFRs. The energy balance is presented with and without including the minor heat losses that demonstrated in figures 3.14 and 3.15. The energy input is the total enthalpy drop in the turbine times turbine mass flow and the energy output is summation of power drop in the compressor air flow and in the oil flow. In quasi-adiabatic tests, the energy absorbed by the lubrication oil will mainly come from heat generated by the friction. Since tests were quasi-adiabatic, heat losses can usually be neglected [73]. Therefore, the mechanical losses are estimated by using the Equation 3.6.

$$
\dot{W}_{m l} \simeq \dot{m}_{\text {oil }} \cdot c \cdot \Delta T_{o i l}
$$

From Figure 3.16 (a) it can be observed that, at lower turbocharger speeds, a 
small fraction of heat transfer is present. It is represented as raw data processing (orange lines represents the different MFR data). Raw data processing represent the experimental data that are directly processed without any heat transfer corrections. After correcting the minor heat losses the explained before, it is evident that the lower turbocharger speeds were perfectly balanced (showed with different symbols for mentioned flow admission conditions). The error of energy balance is in between $\pm 0.5 \% \mathrm{~kW}$ from lower to higher turbocharger speeds. By assessing the overall energy balance of the turbocharger, it shows the significance of work transfer from the turbine to a compressor. It also signifies the accuracy of adiabatic efficiency calculations; as it depends on the ratio of isentropic enthalpy difference to the measured enthalpy difference between inlet and outlet stations of the turbine and compressor.

(a) T\#1TER

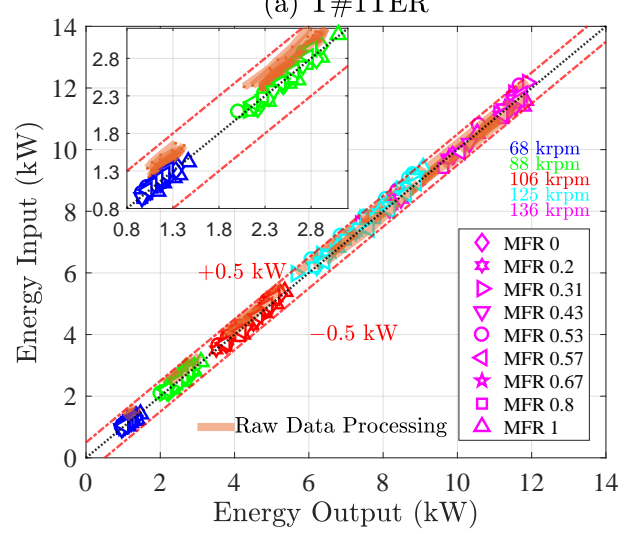

(b) T\#2DVR

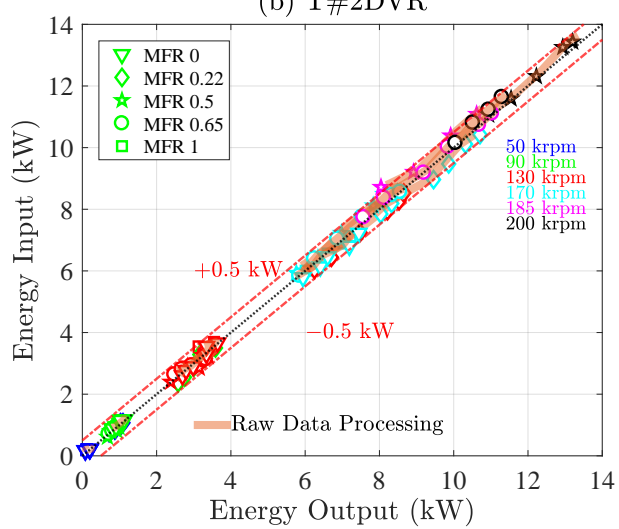

Figure 3.16: Turbocharger energy balance in all admission conditions: T\#1TER (left) and T\#2DVR (right)

The power-based adiabatization method is also applied to T\#2DVR turbocharger at only lower speeds. The outcomes of the energy balance before and after the correction of heat losses are shown in the Figure 3.16 (b). From the figure, it can be observed that the energy balance is in between $\pm 0.5 \% \mathrm{~kW}$ for all the turbocharger speeds.

It is worth highlighting that the turbocharger T\#3DVM is tested with high turbine inlet temperatures. That is, by allowing the internal and external heat transfer effects. The measurements were performed only with one full and two partial admission conditions. However, for modelling purpose, the performance data without heat transfer effects are needed. In this regards, the heat transfer correction suggested in $[71,72]$ and as shown in the Figures 3.14 and 3.15 is applied to obtain fully isentropic efficiency for both turbine and compressor. 


\subsubsection{Uncertainty of the performance parameters}

The uncertainty of the performance parameters was computed using the standard procedure described in [74] and applied in [75]. The mean and maximum values are presented in Table 3.3 .

Figure 3.17(a) shows the absolute unbalance for all the turbocharger speeds at mentioned flow conditions. It can be concluded that, after the heat transfer correction, the unbalance from lower to higher turbocharger speed is in between $\pm 1 \%$ to $\pm 3 \%$ referred to end of scale tests. Further, Figure 3.17(left) also shows the uncertainty of the unbalance and, as it can be seen, the vast majority of the points lies between the experimental uncertainty lines. Similar results can also be found for the dual-volute turbine (T\#2DVR) quasi-adiabatic tests (see Figure 3.17(b)).

Table 3.3: Uncertainty of the performance parameters of T\#1TER

\begin{tabular}{cccc}
\hline Parameter & Average value & Maximum value & Units \\
\hline \multicolumn{4}{c}{ Turbine } \\
\hline Mass flow ratio & 0.0017 & 0.0054 & - \\
$\dot{m}_{\text {red }}$ (total) & $4.88 \mathrm{E}-8$ & $8.94 \mathrm{E}-8$ & $\mathrm{~m} \mathrm{~s} \mathrm{~K}^{-0.5}$ \\
$\dot{m}_{\text {red }}$ (per branch) & $3.45 \mathrm{E}-8$ & $6.32 \mathrm{E}-8$ & $\mathrm{~m} \mathrm{~s} \mathrm{~K}^{-0.5}$ \\
Expansion ratio & 0.015 & 0.023 & - \\
$\eta_{t / s}$ & 0.020 & 0.049 & - \\
$\eta_{E T E}$ & 0.015 & 0.035 & - \\
$\sigma$ & 0.006 & 0.022 & - \\
Power & 95 & 216 & $\mathrm{~W}$ \\
\hline \multicolumn{5}{c}{ Compressor } \\
Pressure ratio & 0.885 & 1.686 & $\mathrm{~g} \mathrm{~s}^{-1}$ \\
Power & 95 & 0.009 & - \\
\hline
\end{tabular}

\subsection{Double-entry turbine performance parameters}

In the following sections, the impact of double-entry turbine flow and efficiency performance parameters in different admission conditions is shown. Firstly, the performance maps were calculated based on the literature review, and later a procedure for systematizing the maps has been discussed. 

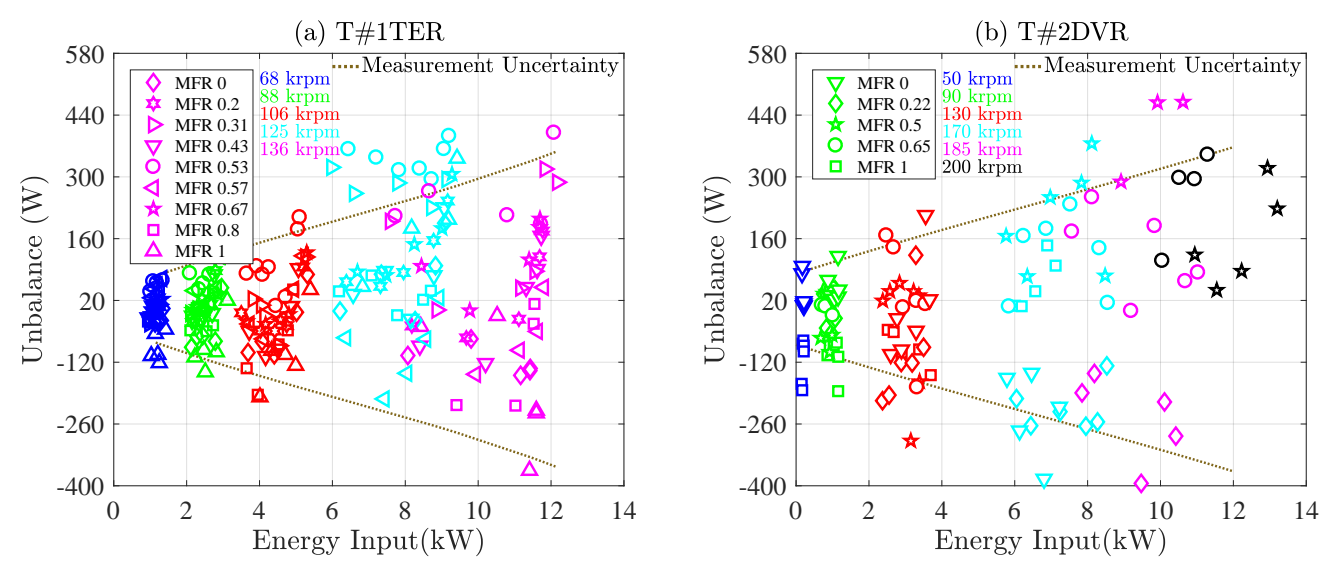

Figure 3.17: Turbocharger absolute unbalance in all admission conditions: (a) shows the results for T\#1TER; (b) shows the results for T\#2DVR

\subsubsection{Based on literature review}

Figure 3.18 shows the flow performance of twin-entry (T\#1TER) and dual-volute (T\#2DVR) turbine for different mass flow ratios with the proposed method of Romagnoli et al. [31]. It can be noted that the calculated reduced mass flow parameter is not alike for all flow admission conditions. The main significant difference is found between the partial and rest of the admissions. Indeed, the total mass flow given to the turbines while testing in partial admission is $20 \%$ lower for T\#1TER and $15 \%$ lower for T\#2DVR, when it is compared to the other full and unequal admission conditions. Nevertheless, for both turbines in full and unequal flow conditions have all very similar total flow among them. The same situation happens at partial admission conditions. That is, Shroud/Long Volute and Hub/Short Volute partial admission have both very similar flow for every turbocharger speed, as shown in Figure 3.10 and 3.11. Further, it is clear by observing Figure 3.13 (left) that, for every turbocharger speed, the quite similar total mass flow was delivered to the turbine at full and unequal admission conditions. But, the expression proposed by Romagnoli et al. [31] does not provide a convergence of mass flow parameter but shows a variety of values when used for all the flow admission conditions.

The diversity in reduced mass flow values for equal and unequal flow states shown in the Figure 3.18 is mainly due to the variations of inlet pressure and temperature at each entry of the turbine. Since the reduced mass flow parameter is the function of these both variables. The variations in pressure and temperature at each turbine entry are due to different flow situations in the branches. Although, this most likely happens when the double-entry turbine works with the engine. The conditions at the turbine inlets ( $\mathrm{Sh} / \mathrm{LV}$ and $\mathrm{H} / \mathrm{SV}$ ) vary due to the imbalance of flow coming from the engine [30]. Besides, representing both entries with one turbine flow parameter hides the mass flow distribution between their entries under unequal admission conditions. 

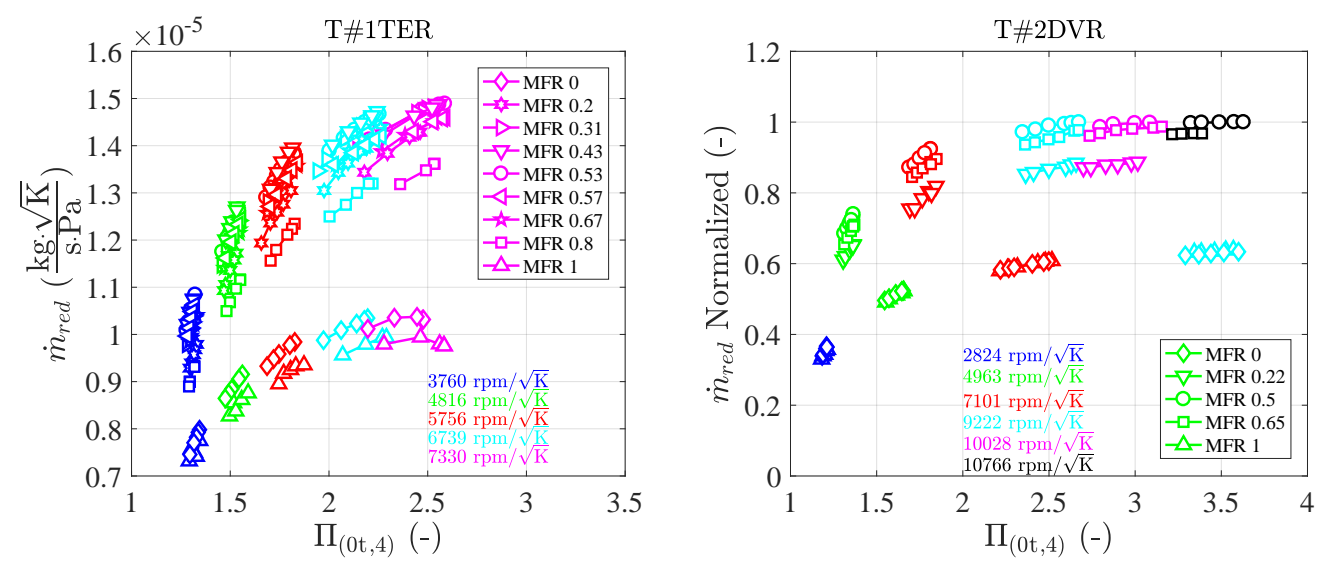

Figure 3.18: Turbine map considering average inlet parameters of T\#1TER (left) and T\#2DVR (right)

Therefore, it is not a viable solution to use a single map in the current doubleentry turbine models. The performance predictions of the turbine at the rest of the flow admission conditions can lead to unsatisfactory results when performing turbomatching/ doing engine simulations. Therefore, the overall performance of a turbine model will significantly depend on knowledge about mass flow distribution among the entries of double-entry turbines.

Figure 3.19, shows the effective turbine efficiency characteristics of a twin-entry turbine for different mass flow ratios that were tested in a gas stand. It is calculated using the Equation 2.13 (two turbine approach) as discussed in the chapter 2. For overall blade speed ratio of a turbine, Equation 2.16 has been used. It can be observed that the resulting turbine efficiency characteristics do not show any convincing information for further validating models or interpolating between these experimental data, even for simulating turbine performance under engine operative conditions.

Figure 3.20 shows the detail results about the relation between total isentropic power of the turbine to compressor power, tested at different mass flow ratio conditions. From the figure, it can be seen that compressor power shows a significant difference for the same isentropic power in the turbine. This difference can be noted when the flow is more in one branch than the other and depending on the turbocharger speed. Figure 3.20(a) shows for lower turbocharger speed of T\#1TER. It can be observed that sharing the equal flow in two branches gives better compressor power than at the rest of tested conditions. It is stated by noticing total turbine isentropic power of $3.9 \mathrm{~kW}$ (marked in dashed lines in Figure 3.20(a)) which is most similar in different flow admission conditions. However, as the turbocharger speed increases, the situation is changed, as shown in Figure 3.20(b). Having unequal flows at the turbine inlets shows better compressor power than passing the flow equally in both branches. It is stated by observing the total turbine isentropic power of 17.9 


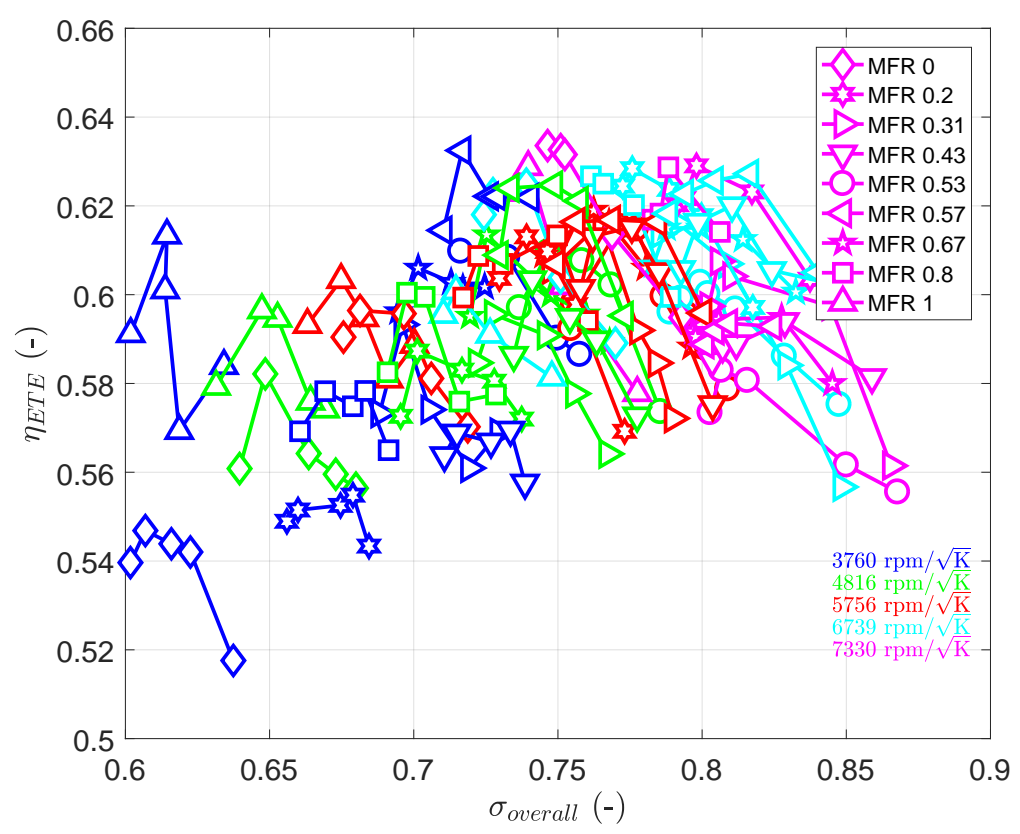

Figure 3.19: Effective turbine efficiency with overall BSR for T\#1TER turbine

$\mathrm{kW}$ (marked in dash lines in Figure 3.20(b)) in all the admission conditions. Therefore, not only MFR can be used to decide whether the efficiency would be higher or lower, but other parameters must be considered. From this, it can conclude that for the twin-entry turbine here tested, having more flow in the hub branch than shroud branch gives better compressor power at high turbocharger speed.

To better visualize the data of ETE at different flow admission conditions, twodimensional contour plot is preferred as shown in Figure 3.21. Inside this type of map, one can interpolate from the experimental data to show how a dependent variable such as efficiency varies with the mass flow ratios and overall blade to speed ratio. From Figure 3.21, it can be concluded that the peak efficiencies are mostly found when the turbine is working under unequal admission conditions (i.e., $\mathrm{MFR}=0.2$, $0.6,0.8)$. Further, in the BSR range of between 0.72 and 0.80 according to definition shown in Equation 2.16. It is also evident that the overall blade speed ratio is always higher under full and unequal admission conditions than partial admission. Due to considering the mass average isentropic power between the two branches shown in Equation 2.16. Nevertheless, the usability of this information is required an extensive test campaign. It is undesirable but true since not a physical model can be extracted from Figure 3.21. But, only interpolating between the efficiency valleys and efficiency peaks as a function of MFR, BSR and maybe also turbine reduced speed. Therefore, a new approach to analyze the data of efficiency maps for these turbines with all admission conditions would be worthy. 

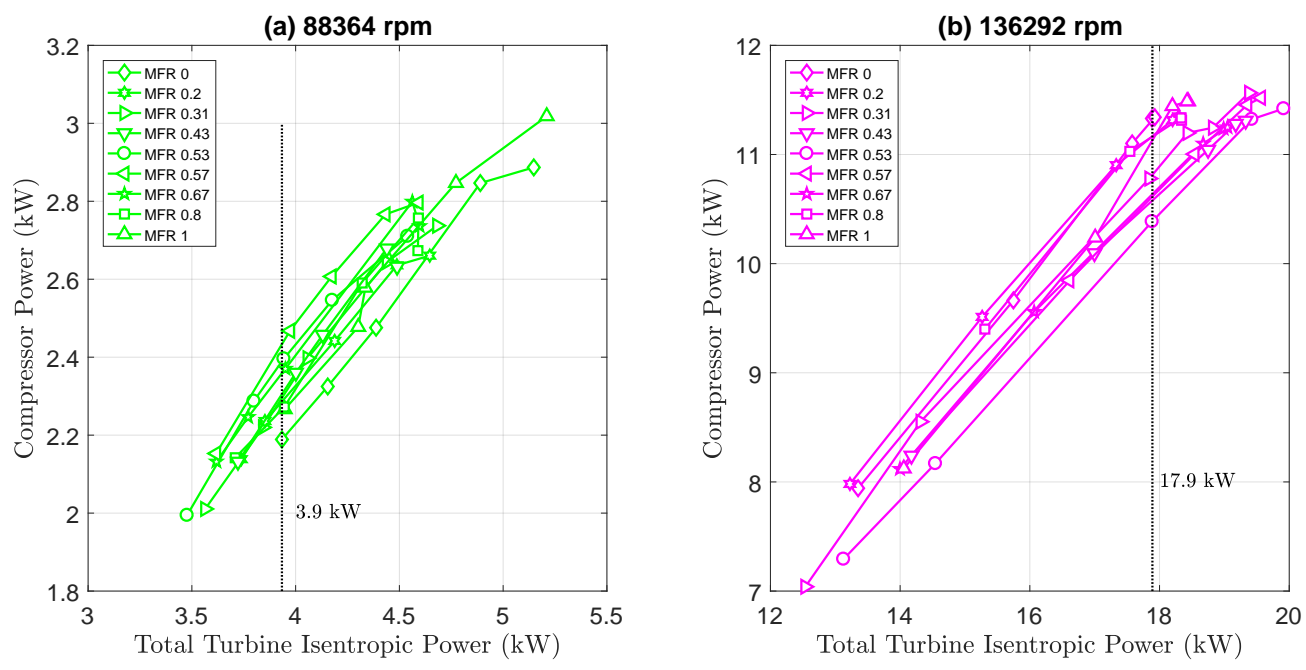

Figure 3.20: Relation between total isentropic power of turbine with compressor power in different flow admission conditions

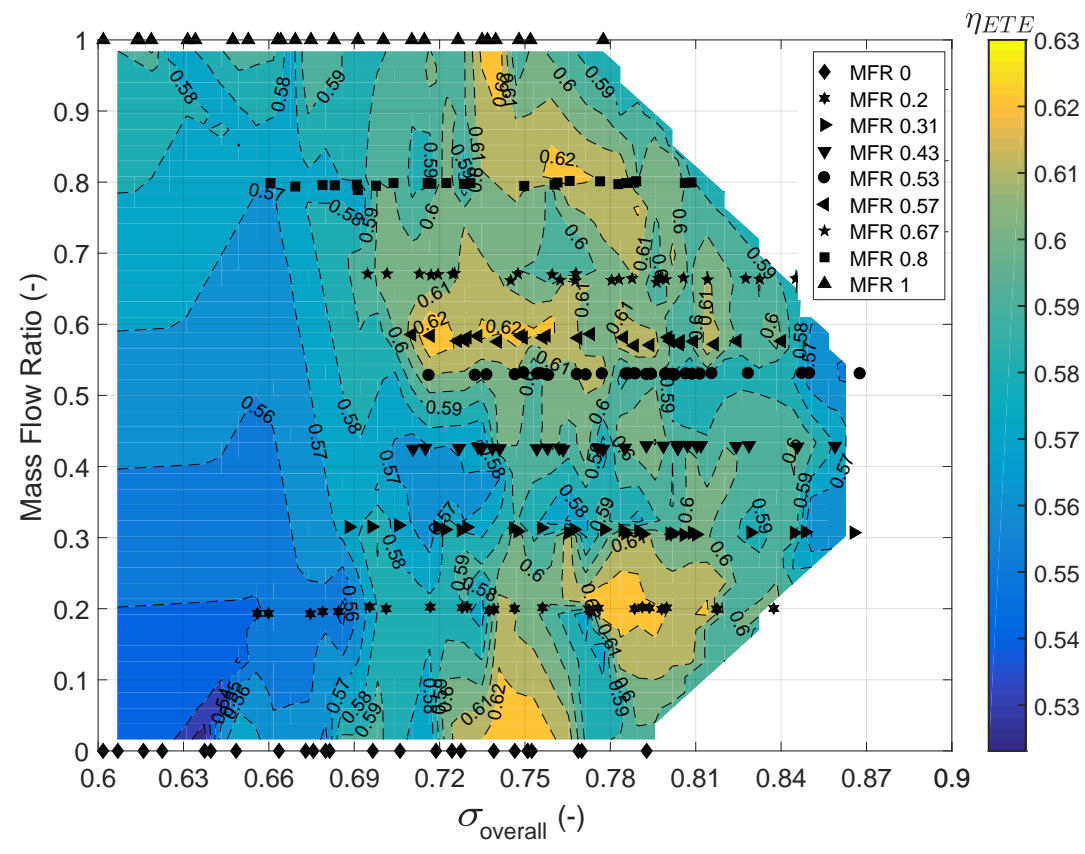

Figure 3.21: Effective turbine efficiency with overall BSR and MFR (Interpolated) for T\#1TER turbine 
Also analyzing Figures 3.20 and 3.21, one can deduce that the global BSR defined in Equation 2.16 is a key parameter for analyzing the global ETE. Much better than reduced speed or MFR, since looks more important for higher ETE being in the right range of BSR (0.72 to 0.8) than at full admission conditions. Being suggested for this type of global efficiency characterization, BSR and MFR can be used as independent variables.

\subsubsection{Double-entry turbines as two individual turbines}

\subsubsection{Flow performance map}

In double-entry turbochargers, if the mass flows entering into each entry of the turbine are similar. Then all of the conventional dimensionless performance parameters such as reduced mass flow, reduced speed, expansion ratio and blade speed ratio are identical to single-entry turbine [32]. However, once the flow conditions between entries vary, standard performance parameters need to be re-evaluated for considering each flow appropriately. Accordingly, assuming each inlet supplies to a separate section of the nozzle. Thus using average pressure ratio and temperature to analyze the results of a double-entry turbine is not suitable or significant. Therefore, it is proposed to treat each entry of the double-entry turbine as a separate turbine. Then compute the performance parameters independently using their corresponding turbine entry inlet conditions like pressure, temperature and mass flow. These parameters can be calculated using well-known equations as shown from 3.7-3.9. In the following equations, the term $i$ represents the generic code for turbine inlet branch.

$$
\begin{gathered}
n_{r e d, i}=\frac{n}{\sqrt{T_{0 t, i}}} \\
\Pi_{(t / s), i}=\frac{p_{0 t, i}}{p_{4}} \\
\dot{m}_{r e d, i}=\frac{\dot{m}_{i} \cdot \sqrt{T_{0 t, i}}}{p_{0 t, i}}
\end{gathered}
$$

Figure 3.22 show the resulting mass flow parameter of two separate turbines approach for different mass flow admission conditions. That is one for each inlet of the double-entry turbines. From the figure, it can be concluded that results show an explicit dependency of flow behaviour with mass flow ratio variations. In twinentry (Figure 3.22(a) and (b)) and also for both dual-volute turbines (Figure 3.22 (c)-(f)). In the twin-entry turbine, for an increasing mass flow ratio, the turbine flow parameter of the Shroud branch increases (as shown in Figure 3.22(a)) and hub branch decreases (as shown in Figure 3.22(b)). Whereas, in the case of dual-volute 

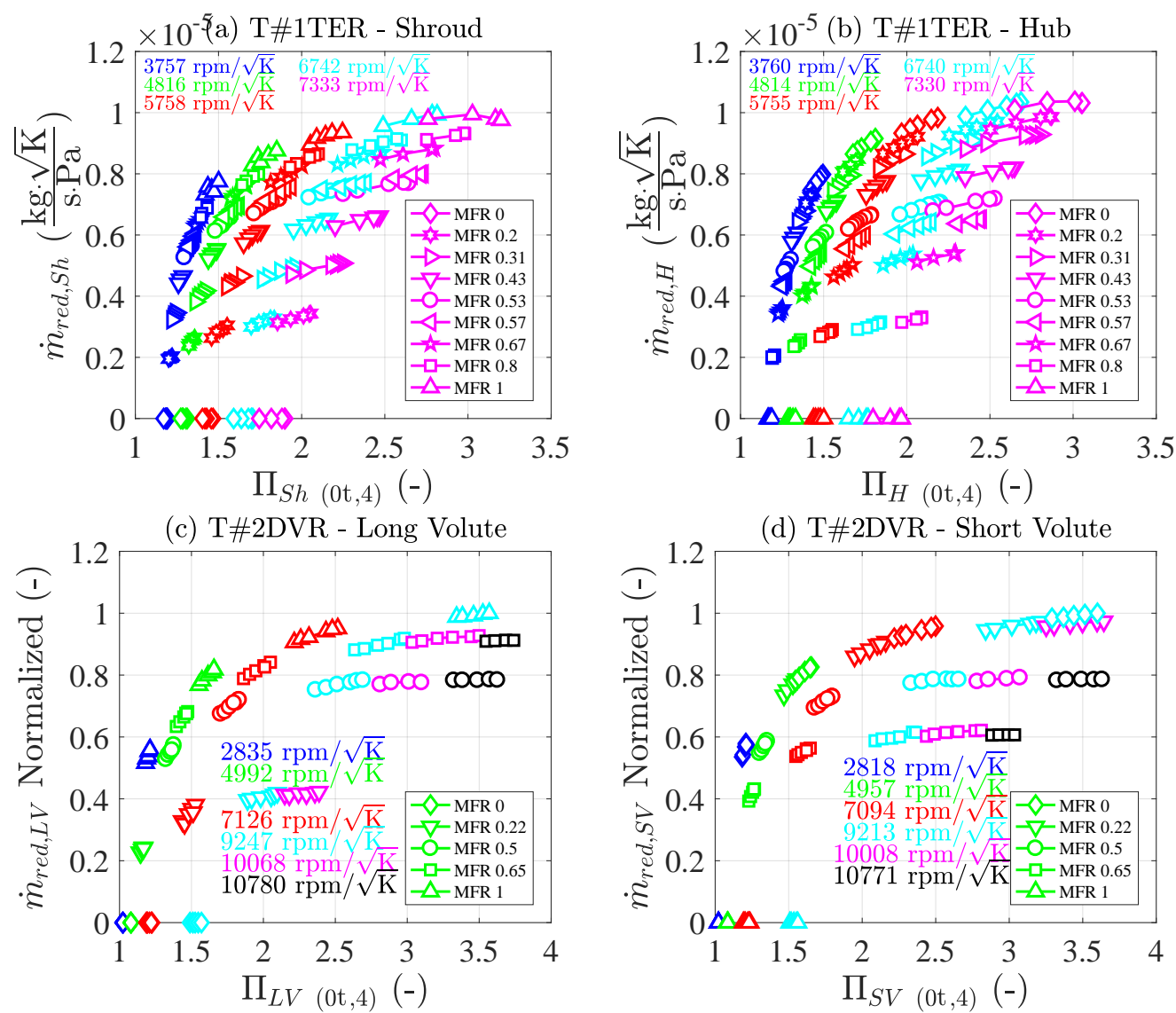

(e) T\#3DVM - Long Volute
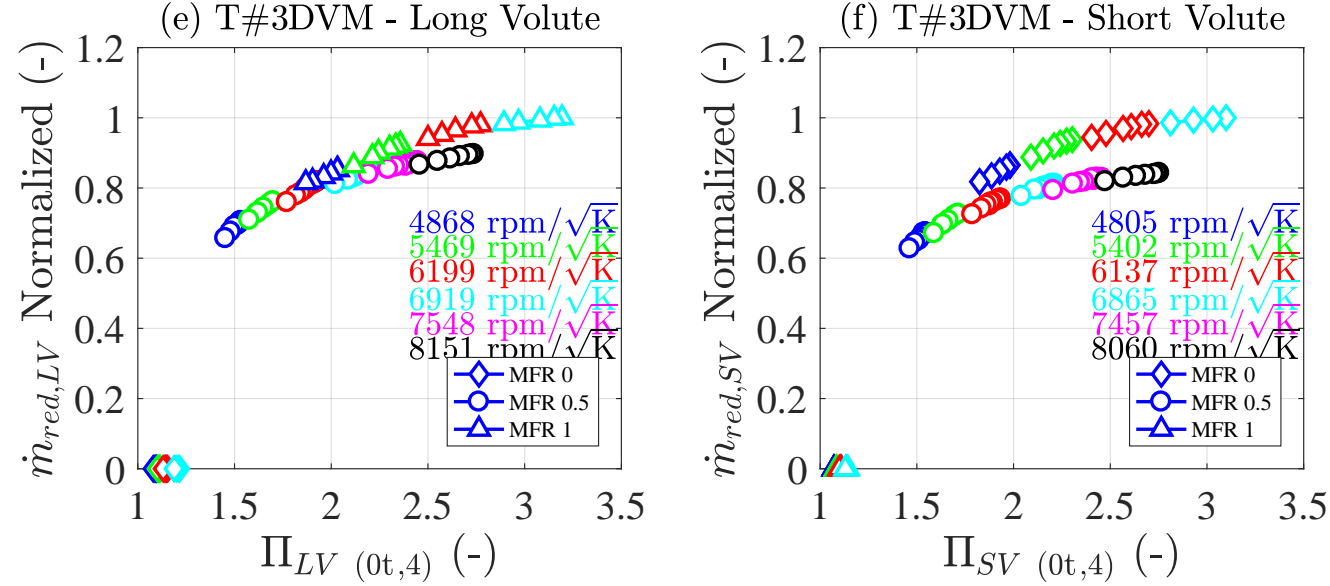

Figure 3.22: Turbine map considering double-entry turbines as two separate turbine (results shown for all the three turbochargers) 
turbines, there is a non-linear relation between the MFR and reduced mass flow. For instance, in the case of LV entry of T\#2DVR (Figure 3.22), there is very few difference between the reduced mass flow values at very high unequal $(\mathrm{MFR}=0.65)$ and partial admission conditions $(\mathrm{MFR}=1)$ but, big differences between low unequal $(\mathrm{MFR}=0.22)$ and full admission conditions $(\mathrm{MFR}=0.5)$. The same phenomenon is observed at SV entry of T\#2DVR (Figure 3.22(d)) but with the opposite trend. In the case of T\#3DVM LV entry (Figure 3.22(e)), the difference between the full and partial admission flows is very small as compared with SV entry of same turbocharger (Figure 3.22(f)).

The flow performance maps obtained for both twin-entry and dual-volute turbines using the method explained in this section provided reliable information of flow going into each turbine branch at different flow admission conditions. Furthermore, the resulting flow parameter at various mass flow ratios showed comparable characteristics similar to single-entry variable geometry turbine (VGT) [76].

\subsubsection{Efficiency performance map}

When the double-entry turbine is working in an engine, the inlet conditions are different in both branches. In this regard, it can be assumed that the power produced by the Shroud/Long Volute entry and Hub/Short Volute entry is different. Consequently, total-to-static turbine efficiency can be computed independently for turbine entry 1 and entry 2, as shown in Equation 3.10. The definition of total-to-static efficiency for two individual turbines is according to the enthalpy-entropy adiabatic expansion of the turbine shown in Figure 3.23. From now on, the efficiency defined in Equation 3.10 is called as apparent efficiency.

$$
\eta_{M F R_{x}(t / s)}^{i}=\frac{T_{0 t}^{i}-T_{04}^{M F R_{x}}}{T_{0 t}^{i}-T_{4 s}^{i}}
$$

The isentropic temperatures are also estimated individually for each entry of the double-entry turbines, as shown in the Equation 3.11.

$$
T_{4 s}^{i}=T_{0 t}^{i} \cdot\left(\frac{1}{\Pi_{i,(0 t, 4)}}\right)^{(\gamma-1 / \gamma)}
$$

For the accurate calculation of the isentropic efficiencies, the careful determination of the turbine outlet temperature is necessary. It is true that measuring the turbine outlet temperature in a gas stand; some difficulty arises due to the complex flow at downstream the turbine as shown in Figure 3.24. Baar et al. [77] proposed different techniques for obtaining reliable values of turbine outlet temperature measurements in the gas stand. They compared the isentropic efficiencies calculated using the different 


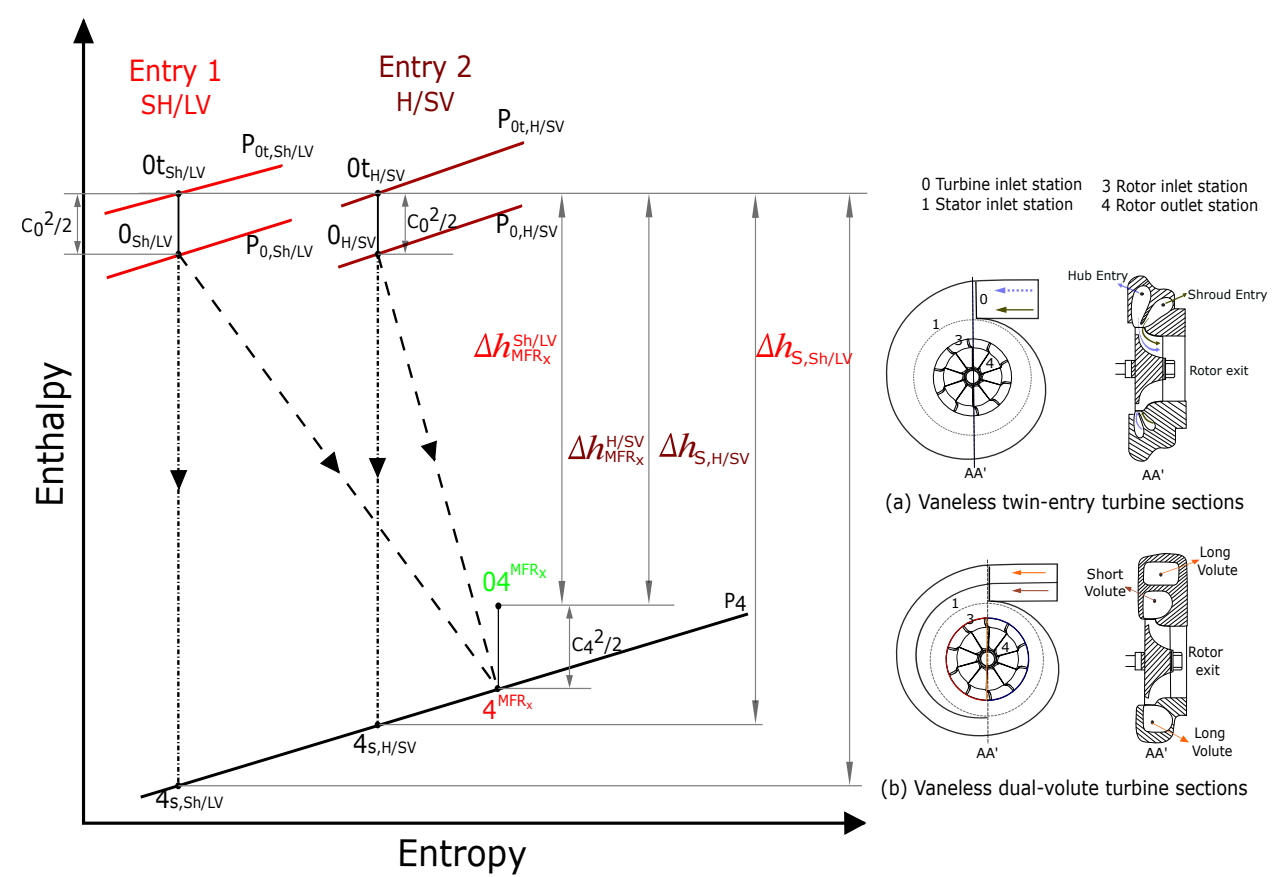

Figure 3.23: Enthalpy-entropy expansion process in double-entry turbines

approaches, and they were not conclusive enough to make the statement of using any particular technique. Though, with a mixed device approach for avoiding the complex flow at the turbine outlet showed the results very close to the SAE standard measurement method [77]. Therefore, it was decided to use four standard K type thermocouples at the turbine outlet measurement pipe, as shown in the Figure 3.25. Their average values were used for computing the apparent efficiencies. Moreover, the quality of the experiments was assured by checking the energy balance, as shown in Figure 3.16 after the correction of heat transfer and, this shows the measurements are reliable enough.

By considering the two entries of the double-entry turbine as an individual turbine, the available work from each branch is the enthalpy differences between their inlet and outlet condition of the turbine. However, the turbine outlet branch is common for both inlets. Accordingly, a mix of temperatures $\left(T_{04}^{M F R_{x}}\right)$ that is coming from the individual branches (see Figure 3.24) is the only possible temperature to be measured; even at quasi-adiabatic conditions. Therefore, it is a convenient method to assume the outlet stations in both branches are at equal temperature. Even logic says this fact cannot be accurate, and other experimental information is not available. But, the mass averaged temperature, which is mixed between entry 1 and entry 2 outlet flows (see Figure 3.23). As a consequence, the efficiency defined in the Equation 3.10 is called as 'Apparent Efficiency'. 


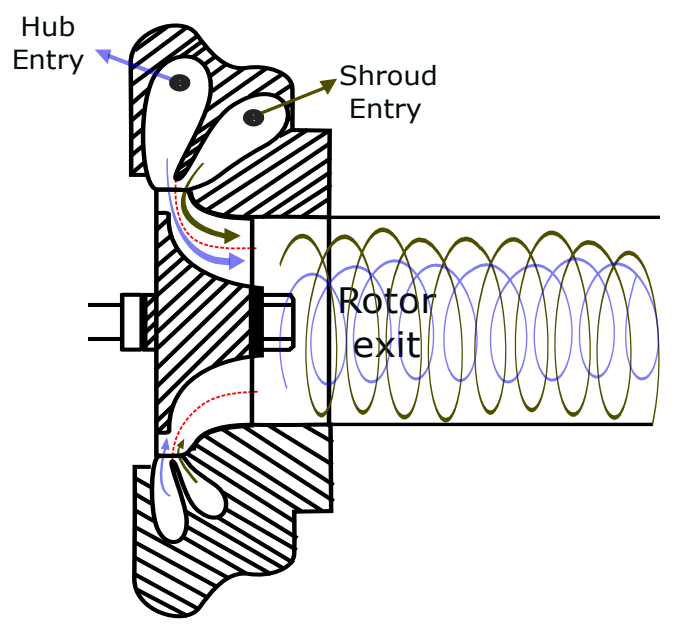

Figure 3.24: Flow at downstream of the turbine
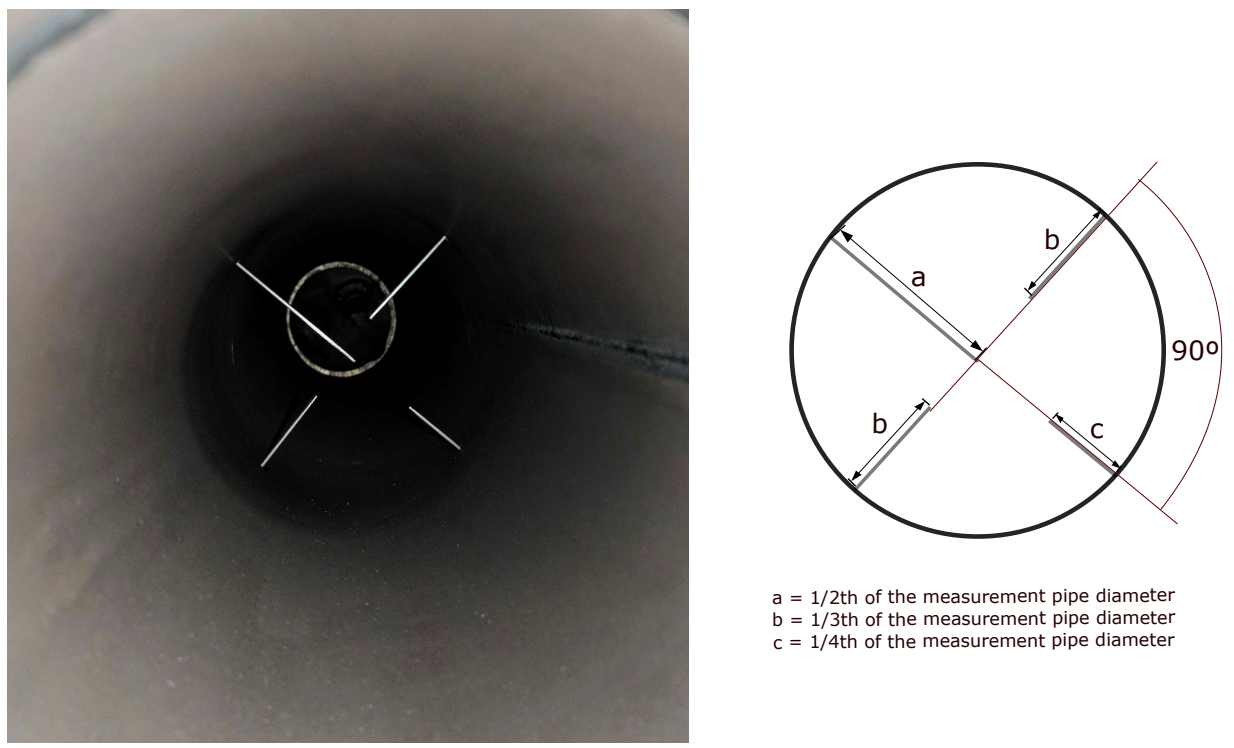

$a=1 / 2$ th of the measurement pipe diamete $b=1 / 3$ th of the measurement pipe diameter
$c=1 / 4$ th of the measurement pipe diameter

Figure 3.25: K type thermocouples at the turbine outlet measurement pipe 
The mechanical efficiency of the double-entry turbine $\left(\eta_{m}\right)$ can be calculated as a ratio between the mechanical compressor power to sum of individual turbine branch power as shown in Equation 3.12

$$
\begin{aligned}
\eta_{m}^{D E}= & \frac{\dot{m}_{C} \cdot c_{p}^{c} \cdot\left(T_{02}-T_{01}\right)}{\dot{m}_{T}^{S h / L V} \cdot c_{p}^{t} \cdot\left(T_{0 t}^{S h / L V}-T_{04}^{M F R_{x}}\right)+\dot{m}_{T}^{H / S V} \cdot c_{p}^{t} \cdot\left(T_{0 t}^{H / S V}-T_{04}^{M F R_{x}}\right)} \\
& =\frac{\dot{W}_{C}}{\dot{W}_{T}^{S h / L V}+\dot{W}_{T}^{H / S V}}
\end{aligned}
$$

It leads to the definition of effective turbine efficiency (ETE (TTA)), as mentioned before in Equation 2.13. It can be computed by taking the sum of apparent efficiencies of both branches and doing a weighted average with the rate of isentropic power in each branch to total isentropic power of the turbine $(\beta)$ and doing the product with mechanical efficiency $\left(\eta_{m}\right)$ as shown in Equation 3.13

$$
\begin{gathered}
\eta_{E T E}^{D E}=\left[\beta \cdot \eta_{M F R_{x}(t / s)}^{S h / L V}+(1-\beta) \cdot \eta_{M F R_{x}(t / s)}^{H / S V}\right] \cdot \eta_{m}^{D E} \\
\beta=\frac{\dot{W}_{T s}^{S h / L V}}{\dot{W}_{T s}^{S h / L V}+\dot{W}_{T s}^{H / S V}}
\end{gathered}
$$

It worth to note that the blade speed ratio (BSR) is also computed independently for each of double-entry turbine, as shown in Equation 3.15. However, in the case of a mixed-flow turbine (T\#3DVM), the Shroud and Hub wheel inlet diameters $\left(D_{3 s}\right.$ and $\left.D_{3 h}\right)$ are different due to their design. Therefore, calculating BSR in this turbine type, an equivalent inlet diameter which is defined as in Equation 3.16 is used [78].

$$
\begin{gathered}
\sigma_{i}=\frac{2 \cdot \pi \cdot n \cdot r_{3}}{\sqrt{2 \cdot c_{p, i} \cdot T_{0 t, i} \cdot\left[1-\left(\frac{1}{\Pi_{i,(0 t, 4)}}\right)^{\frac{\gamma-1}{\gamma}}\right]}} \\
D_{3}=\sqrt{\frac{D_{3 s}^{2}+D_{3 h}^{2}}{2}}
\end{gathered}
$$

Apparent efficiency results of twin-entry turbine

In this section, the results of twin-entry turbine efficiency in various mass flow ratios will be discussed. It is clear from the Figure 3.26 that, the major loss of efficiency has taken place when the twin-entry turbine is working at partial or highly unequal admission conditions (i.e., MFR $=0.2$ in Shroud and MFR $=0.8$ in Hub). 
Hajilouy-Benisi et al. [56] reported that in the case of twin-entry turbines when the flow is mainly or only fed into one entry. There will be essential backflows and mixing losses generation through the passage up to the trailing edge. From the figure, it can be observed that there are significant differences among iso-speeds (4 curves for each set of MFR) at each entry of apparent efficiencies. When they are at very low and high MFR numbers; i.e., at MFR $=0.2$ (yellow points in the Shroud) and MFR $=$ 0.8 (black points in Hub) respectively. One can see that $\mathrm{MFR}=0.67$ and 0.8 in $\mathrm{Hub}$ case and MFR $=0.31$ and 0.2 in Shroud case shows the best peak efficiencies. This effect is due to the apparent efficiency definition, which makes the branches with the lower flow to get benefit from the flow expansion (low $T_{4}$ ) generated by the branches with higher flow, as shown in Figure 3.23.
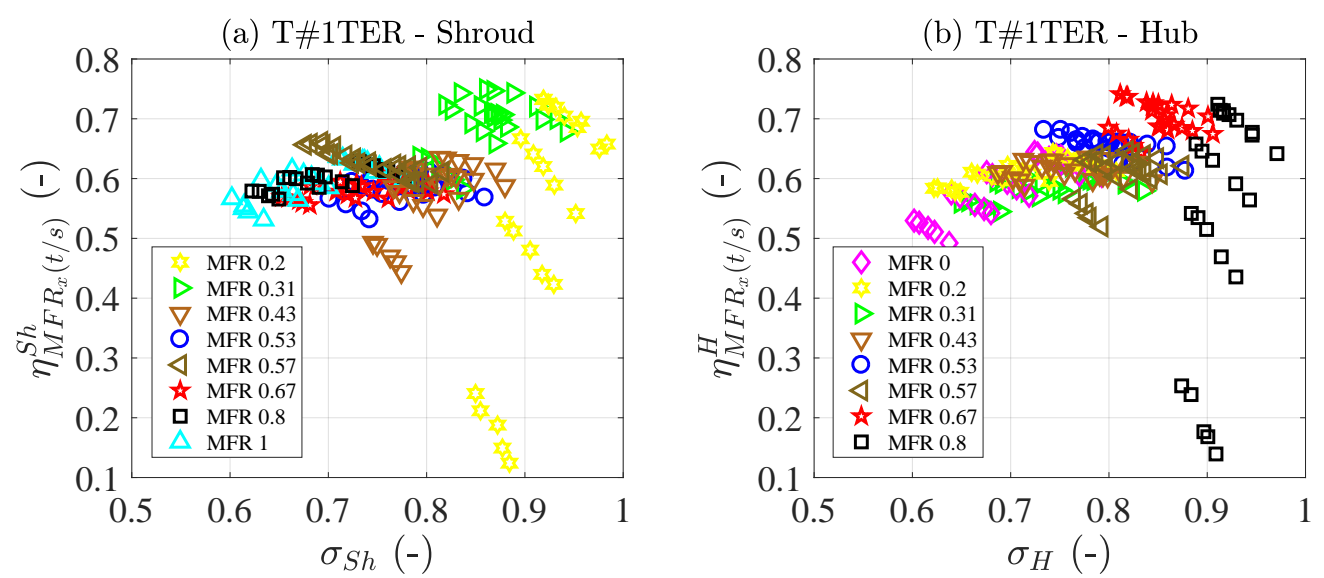

Figure 3.26: Apparent efficiency maps considering double turbines as two separate turbine (results shown for all the three turbochargers)

As a result, treating Shroud and Hub separately, two different efficiency maps can be obtained. These efficiency maps can be linked to the mass flow parameter maps. Looking at the Figure 3.27 (a) and (b), it clearly shows that the apparent efficiency is dependent on flow conditions in each branch. In these conditions, the hub efficiency is similar in the tested range of blade speed ratios, with different reduced turbine speeds, and also close to the maximum efficiency. It is also notable that the efficiency of shroud branch is higher than hub branch. Especially at higher reduced speeds and shows the significant difference between low and high speeds. However, this is only true as just apparent effects due to very low flow and expansion ratios. Because most of the flow expansion is produced by Hub branch and not by the Shroud one. The actual efficiency of Hub branch should be much lower than shown apparent efficiency. However, it must be reminded that in the gas stand during the experiments, measuring the turbine outlet temperature from each branch was not possible due to one common outlet branch. Similar results can be found (Figure 3.27 (c) and (d)) when the turbine is working at a mass flow ratio of 0.8 , but inverting Shroud by Hub situations. In this case, there is more flow in the Shroud branch than 

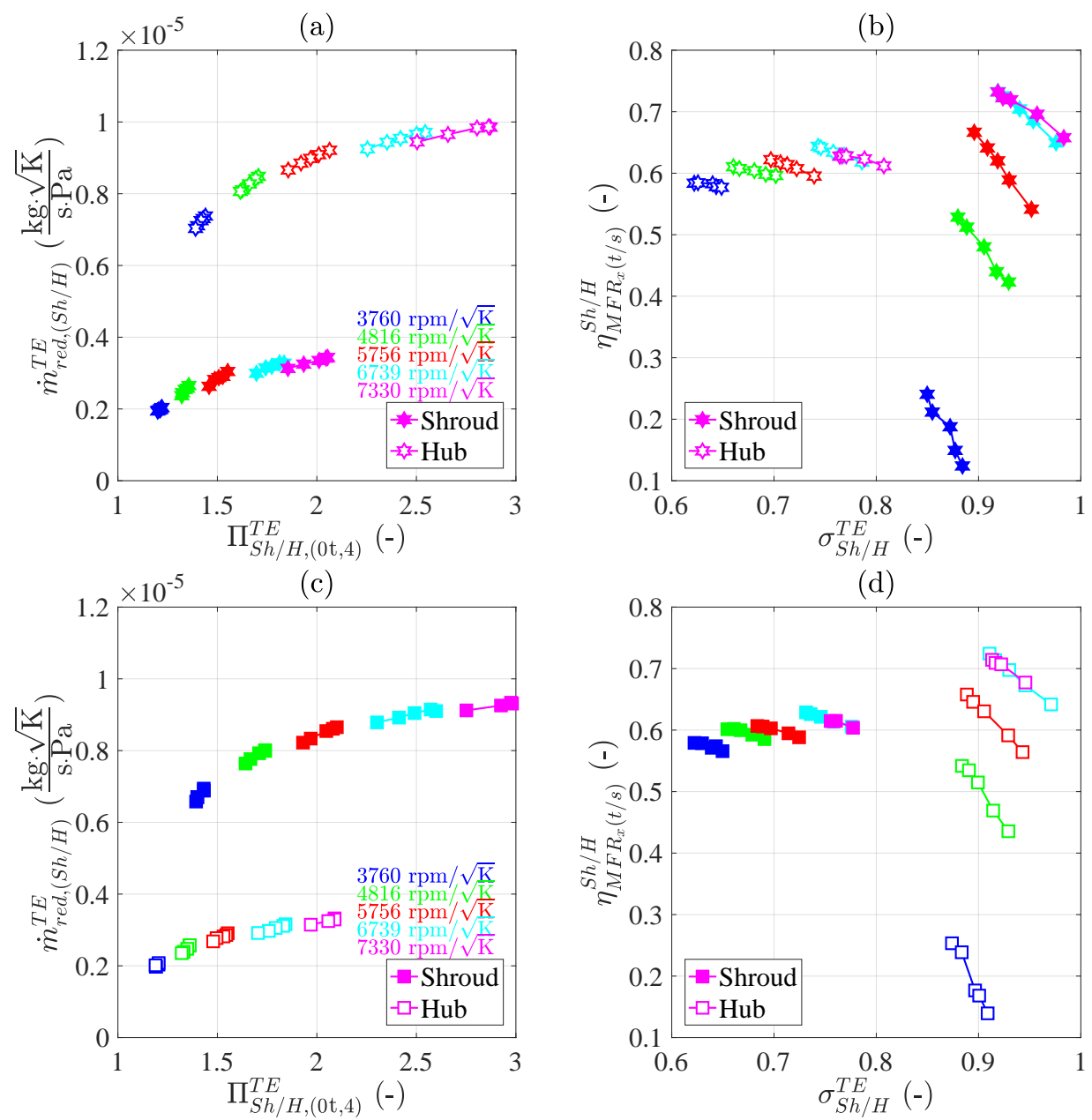

(d)
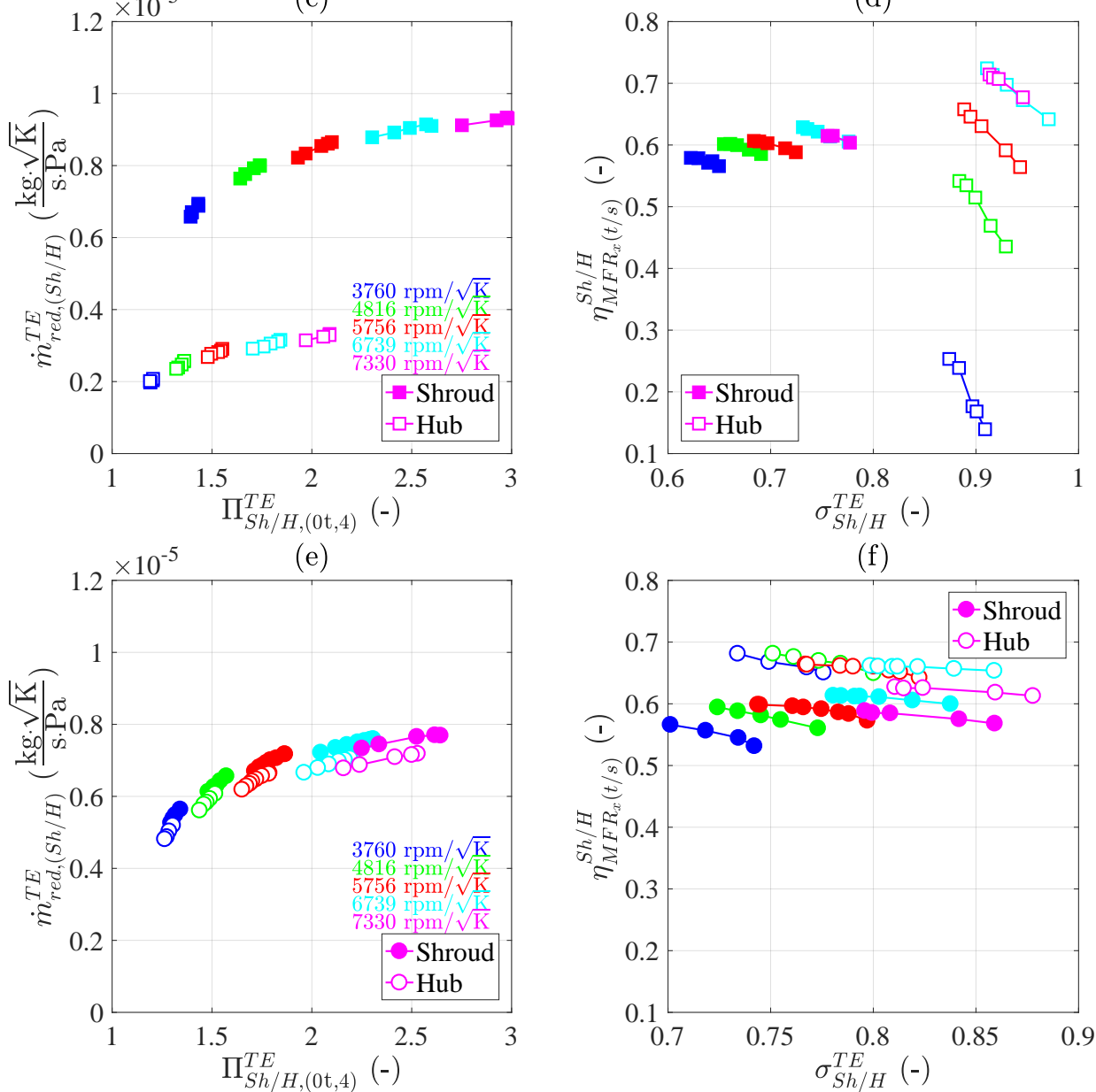

Figure 3.27: Turbine mass flow and apparent efficiency maps in the case of MFR $=0.2,0.8$ and 0.53 (results shown for twin-entry turbine) 
in the Hub branch.

Figure 3.27 (e) and (f) shows the outcomes of experimental results when the turbine is working at almost full admission conditions. Figure 3.27 (e) indicates that the Shroud and Hub branch have similar flow capacities. Indeed, turbine volutes are symmetrical. The small difference found in mass flows and expansion ratios is due to a lack of precision in fitting exactly full admission condition in the experiments. Aforementioned, the test was finished just closer to MFR 0.5. From Figure 3.27 (f) it is clear that peak efficiency points for Shroud and Hub branch are different. Despite having more flow in Shroud than Hub, the apparent maximum efficiencies are always found when the turbine is working with hub branch for all reduced speeds. The same can be seen in the case of unequal admission conditions when there is more flow in the Hub side. A possible explanation can say that having flow in Hub branch; there might be a fewer impact of tip leakage losses as compared to the Shroud branch. Also, one can think that at full admission 'Hub turbine' behaves more as radial inflow-turbine than the 'Shroud turbine'; being able the Hub to extract more work than the Shroud thanks to the higher inlet to outlet rotor radius.

\section{Apparent efficiency results of dual-volute turbines}

Figure 3.28 (a) and (b) shows the apparent efficiency results of both Long Volute (LV) and Short Volute (SV) branches of a dual-volute radial inflow turbine (T\#2DVR) tested at four different mass flow ratios. It can be observed that there are significant differences in apparent efficiencies between their branches. It is worth highlighting that the dual-volute turbine designs are made in such a way that each scroll admits the exhaust gas at a separate section of the rotor. Therefore, when dual-volute turbine works under partial admission conditions, it works like a windmill mode [18]. The non-flow part of the rotor uses the useful power provided in the flowing part. The dissipated work generates entropy, and so cause a reduction in the turbine efficiency at partial (i.e., at $\mathrm{MFR}=0$ and 1 ) than equal and unequal admission conditions. From Figure 3.28 (a) and (b) it is also notable that the apparent efficiency of the turbine decreases with the increasing flow capacity in each volute. That is, going from MFR $=0.5$ to 0.65 in $\mathrm{LV}$ branch, and from MFR $=0.5$ to 0.22 in SV branch. Indeed, when the MFR $=0.22$ and 0.65 , in $\mathrm{SV}$ and $\mathrm{LV}$ branches, the apparent efficiencies are shown higher values compared to other admission conditions. Aforementioned, this effect is due to the definition of apparent efficiency that is shown in Equation 3.10 and Figure 3.23. That makes the branches with the lower flow to get the benefit from the flow expansion (low $T_{4}$ ) generated by the branches with higher flow. 

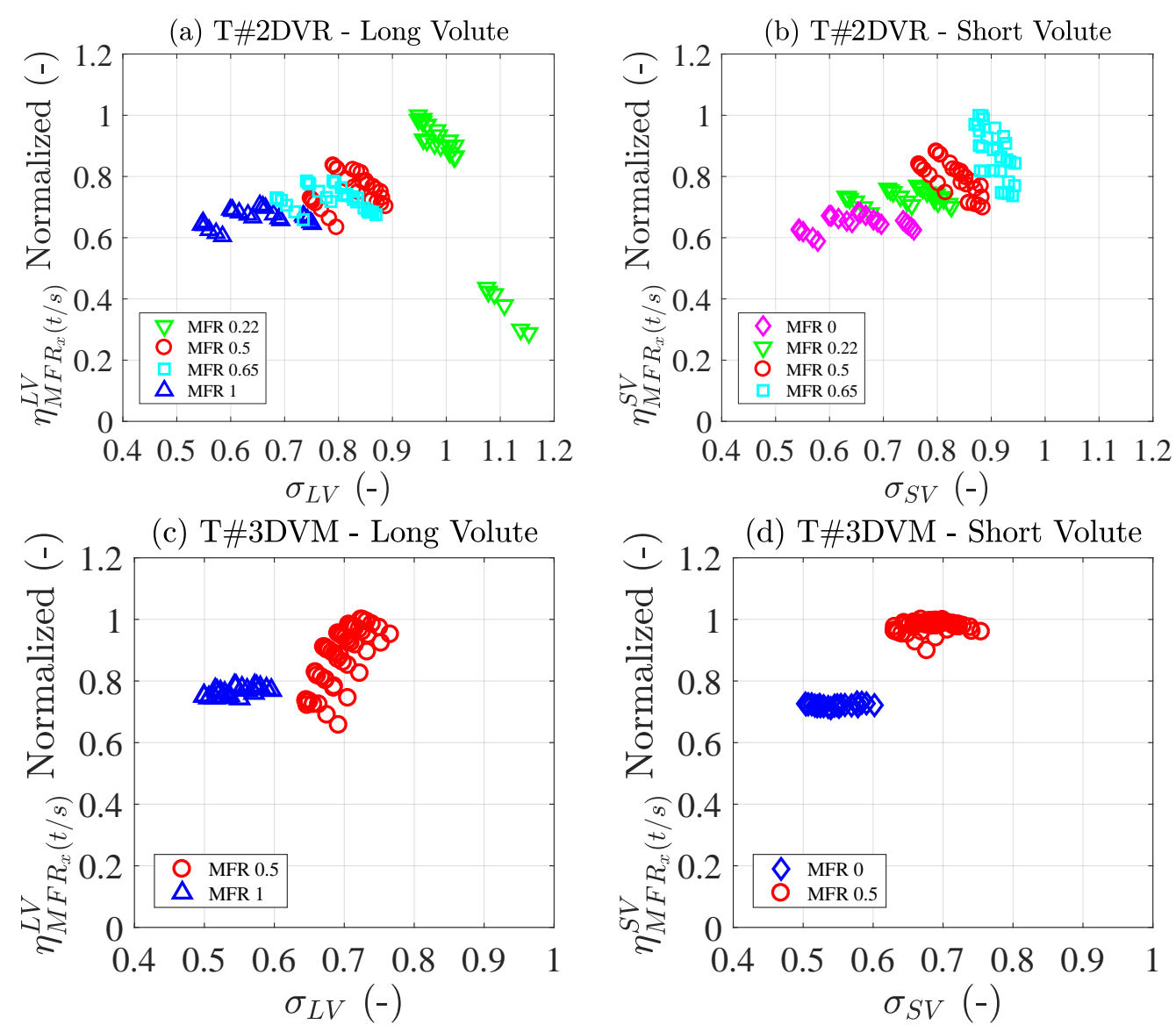

Figure 3.28: Apparent efficiency maps considering dual-volute turbines as two separate turbine (results shown for all the three turbochargers)

From the above all discussion, it can be concluded that the apparent efficiency definition, as shown in Equation 3.10, is useful to introduce order in the characterization of double-entry turbines. But, it does not necessary reflects which branch is extracting more efficiently the energy from the flow upstream of the turbine. Just in the case of partial (i.e., MFR $=0$ and 1$)$ and full $(\mathrm{MFR}=0.5)$ admission conditions, the apparent efficiency interpretation signifies the energy extraction of every branch reasonably. Moreover, the featured turbine apparent efficiency for two branches confirms that it depends on flow conditions in each branch. It appears that the flow resemblance is well represented with the mass flow ratio (MFR). Further, the variations of turbine inlet temperature and pressure according to flow in each branch are also captured.

The representation of double-entry turbine characteristics maps proposed in this chapter would be favourable for current turbocharger models [63]. In order to fit and be able to predict the conditions in off-design conditions. Besides, usage of this infor- 
mation can anticipate acceptable results in gas-dynamic codes. Further, the analysis in this chapter showed a solution for fitting or optimizing reliable one-dimensional double-entry turbine models. That is just by using the proposed way of elaborating the characteristics maps of twin-entry and dual-volute turbines. Then, the data in these maps can be used as the merit function to predict the behaviour of every turbine branch under all the tested MFR and BSR conditions. Alternatively, one can use the proposed arrangement for a quasi-steady interpolation of turbine efficiency and mass flow parameters. These could be done as a function of instantaneous mass flow ratio and expansion ratio or BSR when trying to model a twin-entry or dual-volute turbine. 


\section{Chapter 4}

\section{Mass flow and efficiency models for double-entry turbines}

\begin{tabular}{|c|c|c|c|}
\hline \multicolumn{4}{|l|}{ Contents } \\
\hline 4.1 & Introc & action $\ldots \ldots \ldots \ldots \ldots \ldots \ldots \ldots \ldots \ldots$ & 72 \\
\hline \multirow{5}{*}{4.2} & \multicolumn{3}{|c|}{ Reduced mass flow model for double-entry turbines $\ldots \ldots \ldots$. . } \\
\hline & 4.2 .1 & Twin-entry turbine geometry description . & 75 \\
\hline & 4.2 .2 & Dual-volute turbine geometry description & 76 \\
\hline & \multirow[t]{2}{*}{4.2 .3} & Effective area $\left(A_{e f f}\right)$ fitting as a function of MFR $\ldots$ & 77 \\
\hline & & 4.2.3.1 Coefficient analysis and calibration $\ldots \ldots \ldots$ & 78 \\
\hline 4.3 & \multicolumn{2}{|c|}{ Efficiency fitting using VGT model } & 85 \\
\hline \multirow[t]{4}{*}{4.4} & \multicolumn{3}{|c|}{ Development of actual efficiency model for double-entry turbines . $\mathbf{9 0}$} \\
\hline & 4.4 .1 & Mixed flow approach . . . . . . . . . . . . . . & 90 \\
\hline & 4.4 .2 & Mixed flow approach correction & 96 \\
\hline & 4.4 .3 & $\begin{array}{l}\text { From mixed flow corrected approach to apparent efficiency } \\
\text { merit function } \ldots \ldots \ldots \ldots \ldots \ldots \ldots \ldots\end{array}$ & 100 \\
\hline \multirow[t]{4}{*}{4.5} & \multicolumn{3}{|c|}{ Extrapolation of turbine performance parameters } \\
\hline & 4.5.1 & Twin-entry radial inflow turbine . . . . . . . . . . . . . & 107 \\
\hline & 4.5 .2 & Dual-volute radial inflow turbine $\ldots \ldots \ldots \ldots$ & 111 \\
\hline & 4.5 .3 & Dual-volute mixed inflow turbine $\ldots \ldots \ldots$ & 113 \\
\hline \multirow[t]{3}{*}{4.6} & \multicolumn{3}{|c|}{ Extrapolation of turbine performance parameters to non-measured } \\
\hline & 4.6 .1 & Twin-entry turbine using 3 MFRs . . . . . . . . . . . . & 114 \\
\hline & 4.6 .2 & Dual-volute radial inflow turbine using 3 MFRs . . . . . . & 117 \\
\hline
\end{tabular}




\subsection{Introduction}

The current chapter demonstrates the methodology for modelling both twin-entry and dual-volute turbines to extrapolate the performance maps into off-design conditions for all the flow admission conditions. The approach for modelling is the same for both types of turbines, and it is mainly based on systematizing the characteristic maps of the double-entry turbines, which is distinctly explained in the chapter 3 . It was concluded that mass flow parameters of both turbines showed comparable characteristics to any single-entry variable geometry turbine (VGT). Therefore, the VGT models of Payri et al. [62] and Serrano et al. [63] which is summarized in the subsection 2.6.1 of Chapter 2, are re-designed and adapted to work for double-entry turbines. First, the procedure of modelling the reduced mass flow parameters will be explained for both twin-entry and dual-volute turbines. In the case of apparent efficiency modelling; first, the re-design procedure is done based on data from twinentry turbine type. The results of its modelling are discussed by comparing with the experimental data. Later, the final developed model was applied to the other two dual-volute turbines for the validation.

The works, analysis and ideas described in this chapter were the origins of publication number [4] from the publications list of the author of this thesis. In the sake of readiness and to protect the thesis writing style the publication number [4] from author's list of publications have not been specifically cited every time that ideas, figures or discussions contained in it are bring to this chapter again. This disclaimer corrects, compensates and justify this fact; being the Ph.D. candidate and the works of his thesis the origin of the innovation component in the publication number [4] listed in the referred section.

\subsection{Reduced mass flow model for double-entry turbines}

The approach of considering the two entries as individual turbines, the resulting mass flow parameters showed an explicit dependency of the flow behaviour with the mass flow ratio (MFR, Equation 3.5); referring to the Figure 3.22 in chapter 3. Accordingly, the model presented by Serrano et al. [63], which is briefly described in the subsubsection 2.6.1.1 is re-designed to deal with twin-entry and dual-volute turbines. The approach is based on considering the two entries as two separate equivalent nozzles, as shown in Figure 4.1. Each equivalent nozzle will be as VGT, with its own set of maps depending on MFR instead of a VGT position. The flow passage is divided into several regions, as shown in Figure 4.1(a). The values of the effective area of two nozzles are calculated in the same way as in the case of a single entry VGT turbine. That is using individual characteristics curves of expansion ratio versus reduced mass flow rate shown in Figure 3.22 in subsubsection 3.5.2.1 for each branch. Considering the assumptions of two individual turbines, it is now possible 
to express the effective equivalent nozzle area for each turbine entry, as shown in Equation 4.1.
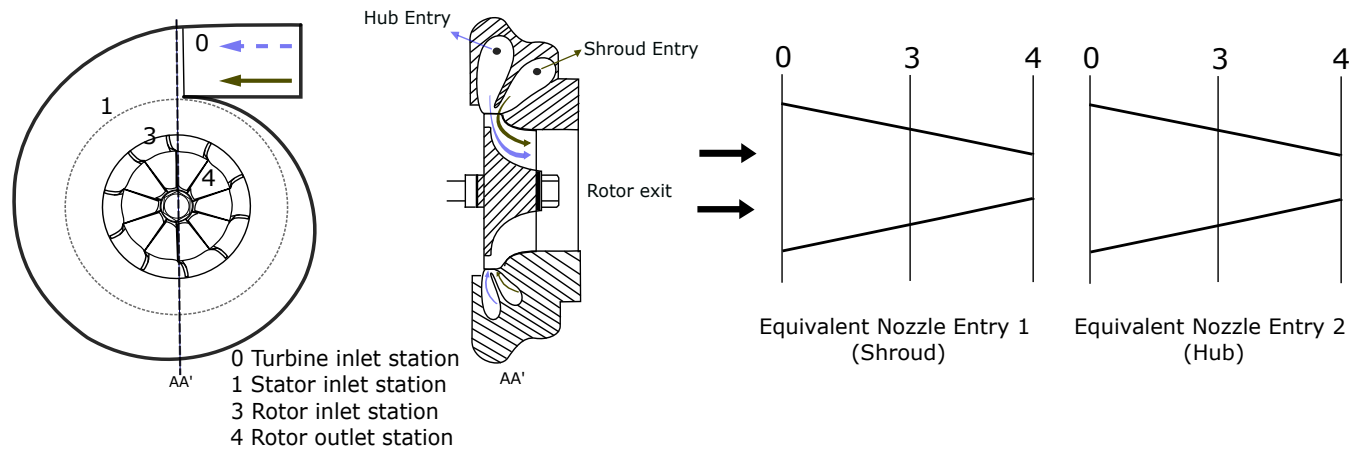

Equivalent Nozzle Entry 1

Equivalent Nozzle Entry 2 (Shroud)

(Hub)

(a) Vaneless twin-entry radial turbine stations distribution and modelling approach

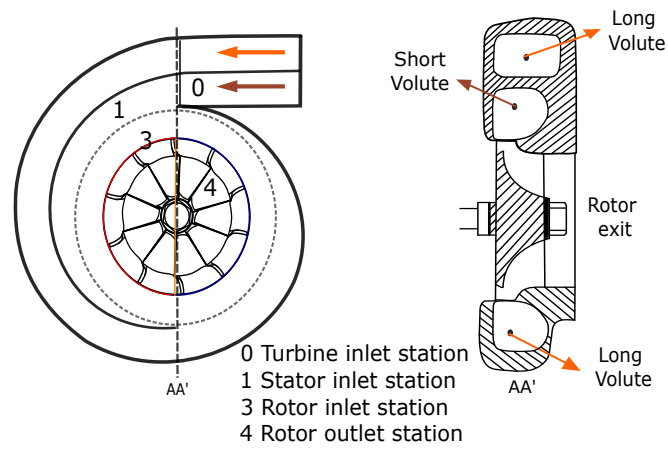

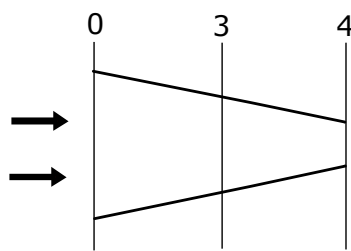

Equivalent Nozzle Entry 1 (Long Volute)

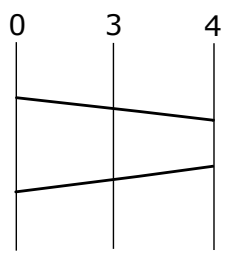

Equivalent Nozzle Entry 2 (Short Volute)

(b) Vaneless dual-volute radial turbine stations distribution and modelling approach

Figure 4.1: Vaneless dual-entry turbines station distribution and two entries into two equivalent nozzles

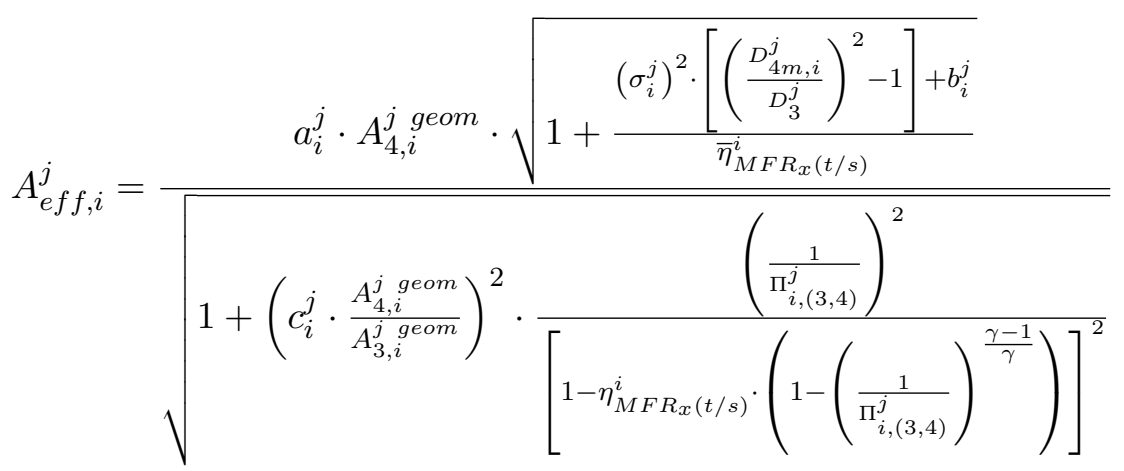

Whereas, $\Pi_{i,(3,4)}^{j}$ represents the pressure in the space between stator and rotor, 
which is estimated using the Equation 4.2 with $d_{i}^{j}$ as a fitting constant [63].

$$
\Pi_{i,(3,4)}^{j}=1+d_{i}^{j} \cdot\left(\Pi_{i,(0 t, 4)}^{j}-1\right)
$$

The terms $i$ and $j$ in the Equation 4.1 represent the generic codes for the turbine inlet (Shroud or Hub; Long Volute or Short Volute) and double-entry turbine type (twin-entry/dual-volute) respectively. Therefore for each turbine, there are two effective area equations (one for each turbine entry), which are dependent on their corresponding turbine entry geometries, the measured data of apparent efficiency $\left(\eta_{M F R_{x}(t / s)}^{i}\right)$, and the four fitting constants $\left(a_{i}^{j}, b_{i}^{j}, c_{i}^{j}\right.$ and $\left.d_{i}^{j}\right)$.

The effective area of the nozzle shown in Equation 4.1 is dependent on the stator and rotor outlet geometrical area. For VGT turbines, these areas are estimated based on the geometry of turbine and VGT positions as outlined in the subsubsection 2.6.1.1 and [63]. Nevertheless, twin-entry and dual-volute turbines used in this thesis are vaneless turbines. Therefore, the stator nozzle area is estimated using the turbine rotor diameter $\left(D_{3}\right)$ and blade height $(B h)$ as shown in Equation 4.3 (see Figure 4.2). In vaneless turbines, it is considered that the stator outlet area is the same as the rotor inlet area.

$$
A_{3}^{\text {geom }}=\pi \cdot D_{3} \cdot B h
$$

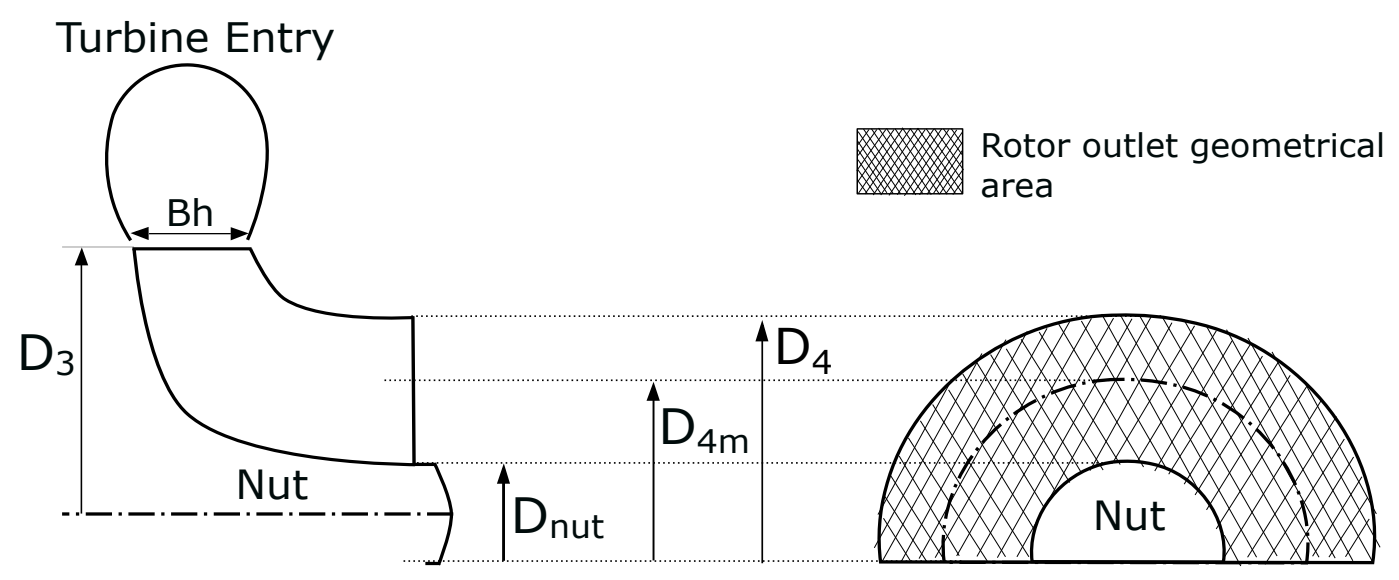

Figure 4.2: Geometrical description of vaneless turbine

For estimating the geometrical area at the rotor outlet, the Equation 2.33 can be used. Whereas in the Equation 4.1, $D_{4 m}$ is an arithmetic mean diameter between turbine rotor shroud $\left(D_{4}\right)$ and rotor hub $\left(D_{n u t}\right)$ diameters as shown in Equation 4.4 
(see Figure 4.2).

$$
D_{4 m}=\left(\frac{D_{4}+D_{n u t}}{2}\right)
$$

As mentioned in chapter 1, twin-entry and dual-volute turbines have different volute designs and geometries. Subsequently, the stator and rotor geometrical areas are estimated in different ways for every turbine type and, is further explained in the below sections. To have a clear explanation, firstly the method for determining the geometrical areas of the twin-entry turbine will be defined, and then for a dual-volute turbine.

\subsubsection{Twin-entry turbine geometry description}

In twin-entry turbines, the induction flow path from the turbine inlet to the leading edge of the rotor blades is divided into Shroud and Hub branches, as shown in Figure 4.3. Due to the assumption of two individual turbines, the blade height has been shared between two branches by splitting the actual value into half (as T\#1TER is symmetrical turbine). Subsequently, the stator area variation for two branches can be obtained, as shown in the Equation 4.5. In the case of asymmetric inlets, a proper fraction of blade height would be assigned for each turbine inlet.

$$
A_{3, i}^{T E \text { geom }}=\pi \cdot D_{3}^{T E} \cdot\left(\frac{B h^{T E}}{2}\right)
$$

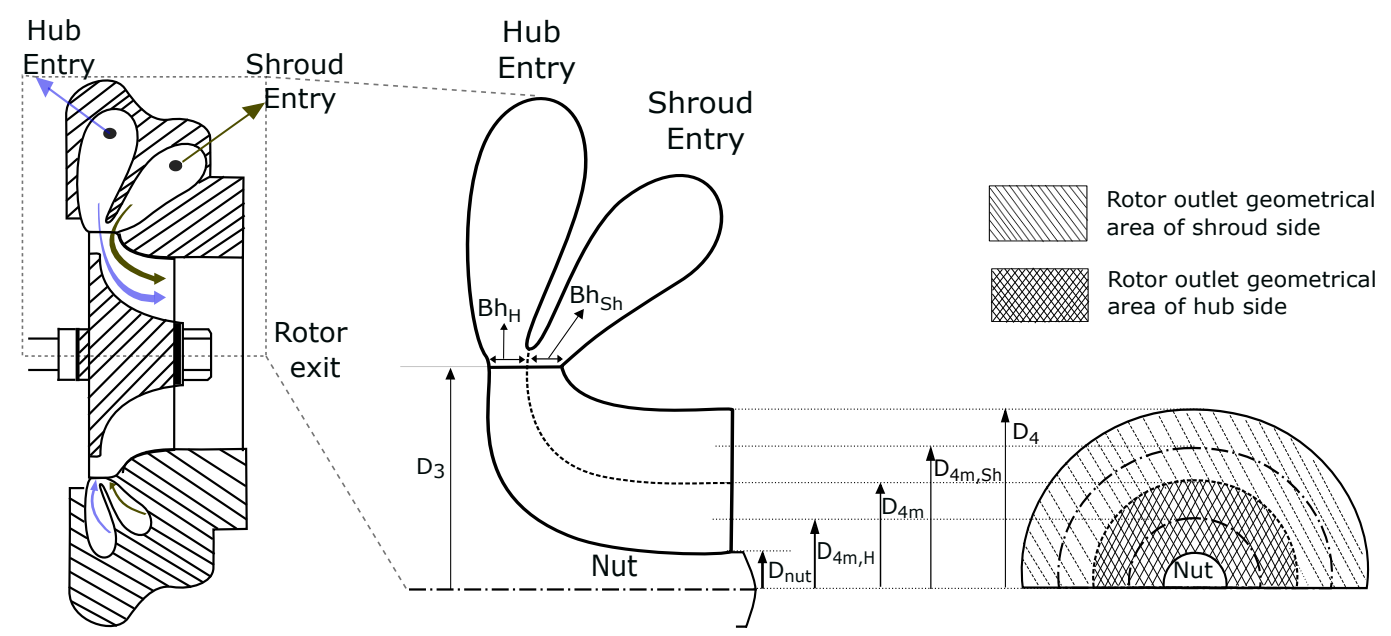

Figure 4.3: Geometrical description of twin-entry turbine (T\#1TER) 
For calculating the rotor outlet geometrical area $\left(A_{4, i}^{T E}\right.$ geom $)$ in the case of twinentry turbines, it is more relevant to consider the rotor outlet area for each branch in the view of two turbine approach modelling. In this regards, the area estimated using Equation 2.33 is shared equally between the shroud and hub branches, as shown in the Equation 4.6. By this, it is ensured that the mean-line $\left(D_{4 m}\right)$ divides the blade-to-blade duct into shroud and hub branches as shown in Figure 4.3. Once the geometrical area of each branch is known, it is possible to estimate the rotor outlet mean diameters of shroud $\left(D_{4 m, S h}\right)$ and hub $\left(D_{4 m, H}\right)$ branches by using the Equations 4.7 and 4.8 based on the Figure 4.3.

$$
\begin{gathered}
A_{4, i}^{T E \text { geom }}=\frac{A_{4}^{\text {geom }}}{2} \\
\frac{A_{4, S h}^{T E \text { geom }}}{2}=\pi \cdot\left(\frac{D_{4}^{2}-D_{4 m, S h}^{2}}{4}\right) \\
\frac{A_{4, H}^{T E \text { geom }}}{2}=\pi \cdot\left(\frac{D_{4 m, H}^{2}-D_{n u t}^{2}}{4}\right)
\end{gathered}
$$

The geometrical areas and diameters estimated from the Equations 4.5 to 4.8 are used in corresponding effective equivalent nozzle area $\left(A_{e f f, i}^{T E}\right)$ of each branch while fitting the model. It is worth highlighting that Equation 4.1 also depends on the apparent efficiency of each branch. For that, the apparent efficiency values obtained by applying Equation 4.22 to each are used.

\subsubsection{Dual-volute turbine geometry description}

In dual-volute turbines, the flow path is designed in a way that each entry feeds the separate section of the rotor. It is due to the different volute lengths. Based on these different flow paths, the stator and rotor geometrical areas are also estimated in different ways (see Figure 4.4). Doing this, it is appropriate to determine the mass flow parameter through long and short volutes entries of dual-volute turbines.

As the flow is expanding at different sections of the rotor, as shown in Figure 4.4, stator area is estimated by blade height $(B h)$ and half the wheel length $\left((\pi / 2) \cdot D_{3}\right)$ for each volute as shown in Equation 4.9. Similarly, the rotor outlet area of each volute is also determined by considering half the value of the entire area, which is estimated using the Equation 2.33 and Equation 4.10. It is due to the flow coming from each volute is not expanding through the entire circumference of the rotor. The rotor outlet diameter $\left(D_{4 m, i}^{D V}\right)$ is estimated in the same way as in vaneless turbines, 

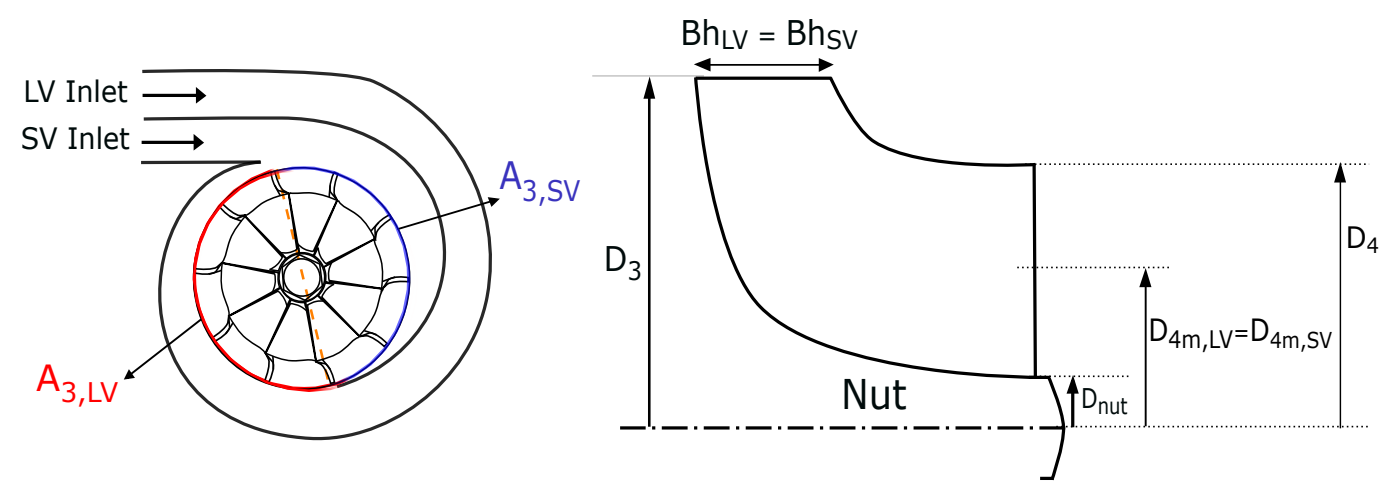

Figure 4.4: Geometrical description of Dual-Volute turbines (T\#2DVR and T\#3DVM)

as shown in Equation 4.4 .

$$
\begin{gathered}
A_{3, i}^{D V} \text { geom }=\pi \cdot\left(\frac{D_{3}}{2}\right) \cdot B h^{D V} \\
A_{4, i}^{D V \text { geom }}=\frac{A_{4}^{\text {geom }}}{2}
\end{gathered}
$$

Once all the geometric parameters are defined, the effective equivalent nozzle area (Equation 4.1) for each turbine entry of the double-entry turbine (T\#1TER, T\#2DVR and T\#3DVM) is known, and the reduced mass flow parameter in each branch can be calculated using the expression of flow through an orifice with isentropic expansion, as shown in the Equation 4.11.

$$
\dot{m}_{r e d, i}^{j}=A_{e f f, i}^{j} \cdot \sqrt{\frac{\gamma}{R}} \cdot\left(\frac{1}{\Pi_{i,(0 t, 4)}^{j}}\right)^{\frac{1}{\gamma}} \cdot \sqrt{\frac{2}{\gamma-1} \cdot\left[1-\left(\frac{1}{\Pi_{i,(0 t, 4)}^{j}}\right)^{\frac{\gamma-1}{\gamma}}\right]}
$$

\subsubsection{Effective area $\left(A_{e f f}\right)$ fitting as a function of MFR}

Using the experimental data of one twin-entry (T\#1TER) and one dual-volute (T\#2DVR) turbines that introduced in chapter 3. It is possible to study the behaviour of the fitting coefficients of effective equivalent nozzle area (Equation 4.1) for both entries and with different mass flow ratios (MFR). As the dual-volute mixed flow turbine is measured with few MFRs, therefore this turbine is used for the validation by imposing the found trends from other two turbines. 
Once the experimental reduced mass flow parameter of each entry of the doubleentry turbine is known, the experimental effective nozzle area $\left(A_{\text {eff,i }}^{j}\right)$ of the same can be calculated by using the Equation 4.11. Knowing all the geometrical parameters and experimental $A_{e f f, i}^{j}$, Equation 4.1 is then used to fit the values for $a_{i}^{j}, b_{i}^{j}, c_{i}^{j}$ and $d_{i}^{j}$ coefficients for the whole turbine map of each entry. The fitting is performed with a least square method, which minimizes the root mean square error between the modelled and experimental values. Accordingly, the coefficient values of Equation 4.1 are obtained. These values for each turbine entry of the turbocharger T\#1TER and T\#2DVR are plotted in Figure 4.5 versus the corresponding MFR. The analysis of individual map fitting coefficients of both entries of two turbochargers is detailed in subsubsection 4.2.3.1 to obtain some physical trends.

\subsubsection{Coefficient analysis and calibration}

As mentioned before, the fitting is performed as two individual VGTs with their corresponding turbine entry map data and, each MFR map of T\#1TER and T\#2DVR turbines are fitted separately to study the behavior of the coefficients as shown in Figure 4.5. It is worth highlighting that, according to MFR definition shown in Equation 3.5, the turbine mass flow of the Shroud/Long Volute entry increases and Hub/Short Volute decreases. The descriptions of each coefficient with the MFR are detailed below:

- Coefficient $a$ : It represents the discharge coefficient of the rotor, as shown in Equation 4.1 and Equation 4.12. The rotor effective flow area is variable with MFR, considering the amount of mass flow entering into each turbine entry. That is, growing as the mas flow grows, and this is true according to the definition of the discharge coefficient. Therefore it is regarded as some quadratic behaviour with MFR and the magnitude of this coefficient should be in between 0 and 1 . Therefore, the coefficient ' $a$ ' trends are exactly as expected.

$$
A_{4, i}^{j}=A_{4, i}^{j \text { geom }} \cdot a_{i}^{j}
$$

- Coefficient $b$ : It comes from the speed ratios obtained from velocity triangle speed ratio, and its order of magnitude can be estimated using Equation 4.13 as described in [63]. This coefficient showed the dependency with mass flow going into each turbine branch. More mass flow in a turbine branch gives higher ' $b$ ' values than with lower mass flows. This is due to higher radial velocities at rotor inlet, for same peripheral speed and expansion ratio values. This trend is clearly shown in the Figure 4.5(c) and (d) for both branches of every turbine 
type.

$$
\begin{aligned}
b= & \left(\frac{v_{0}}{C_{s s}}\right)^{2}+\left(\frac{w_{3}}{C_{s s}}\right)^{2}=\frac{A_{\mathrm{eff}}}{A_{0}} \cdot\left(\frac{1}{\Pi_{T}}\right)^{\left(\frac{1}{\gamma}\right)}+O\left[10^{-1}\right] \\
& =O\left[10^{-1}\right]+O\left[10^{-1}\right] \rightarrow O\left[10^{-1}\right] \leq b \leq O\left[10^{0}\right]
\end{aligned}
$$

- Coefficient $c$ : It signifies the relation between rotor and stator discharge coefficient (' $a$ ' and ' $C_{D s}$ ') as shown in Equation 4.14. As the effective flow area of the stator is variable with the mass flow ratio in the turbine; consequently, the coefficient value of ' $c$ ' is expected to increase. In addition, ' $c$ ' must justify always the Equation 4.14, i.e., as an effective section introduced through the discharge coefficient $\left(C_{D s}\right)$ should not be greater than unity [63].

$$
C_{D s}=\left(\frac{a}{c}\right) \leq 1
$$

- Coefficient $d$ : It is related to the pressure ratio in the rotor with respect to the total turbine pressure ratio [63]. It shows that the coefficient value decreases with more flow going into the branch and tends to 0.5. This explains that with a high amount of flow in the turbine branches, the pressure ratio in the rotor tends to be half of the total pressure ratio in the turbine. With low flow quantities, most of the expansion is occurring in the rotor. Both are the expected logic trends.

Summarizing, all the coefficients values and trends with MFR have physical meaning, as they are derived from theoretical considerations [63]. These coefficients showed the exactly expected trends with the various MFRs in both turbines, symmetrical twin-entry (T\#1TER) and asymmetrical dual-volute (T\#2DVR). By reviewing the fitting coefficients of each turbine entry of the two different radial in-flow types and for several MFRs in Figure 4.5, it is possible to impose those physical trends with MFR for each turbine entry: a linear trend for coefficients ' $b$ ', ' $c$ ' and ' $d$ ' and a quadratic behaviour for ' $a$ '. In this approach, the observed trends can be fitted using seven coefficients for each turbine entry. Theses coefficients can be adjusted using a non-linear fitting method for all MFRs of a given branch at the same time. This implies that one single fitting procedure and one set of parameters will be needed for each entry of the turbine to predict the reduced mass flow in all the flow admission conditions.

Further elaborating this conclusion, only measuring three MFRs is enough for fitting the whole range of turbine mass flow parameter. Any other non-measured MFRs can be predicted by using the three MFRs fitting coefficients. It is easy to think that the three most convenient MFRs to be measured in the gas stand are $\mathrm{MFR}=0, \mathrm{MFR}=1$ and, $\mathrm{MFR}=0.5$ (two partial and one full admission) as did for the dual-volute mixed in-flow turbine (T\#3DVM). 
(a) Shroud and Long Volute

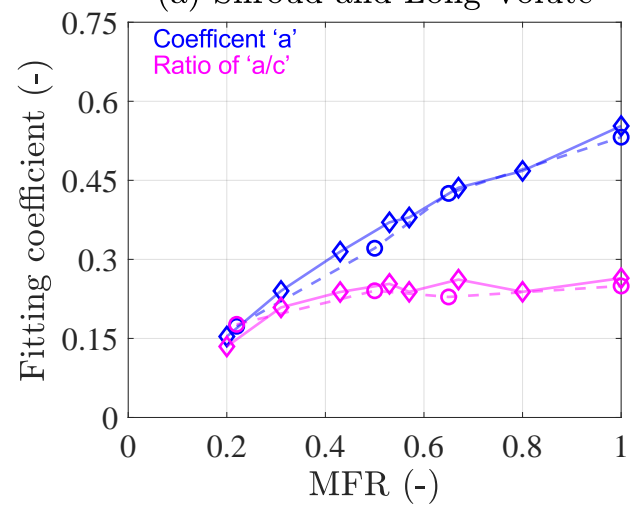

(c) Shroud and Long Volute

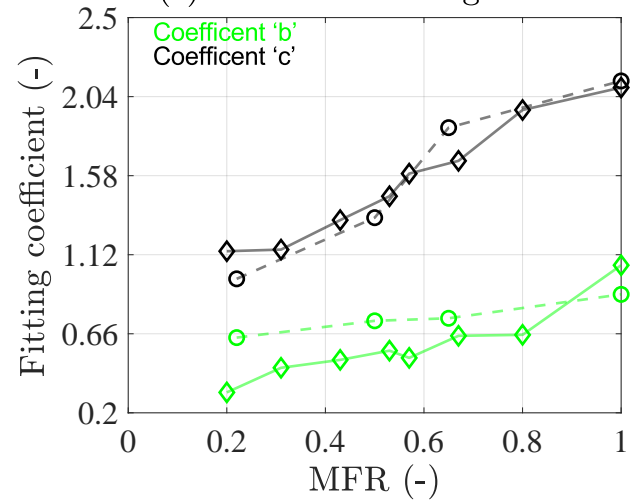

(e) Shroud and Long Volute

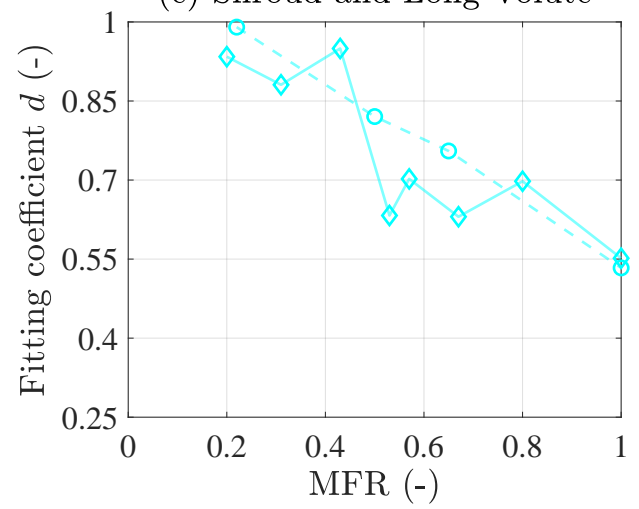

(b) Hub and Short Volute

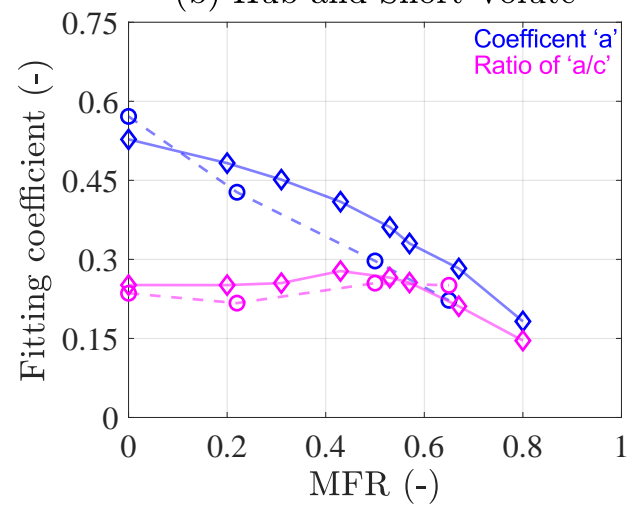

(d) Hub and Short Volute

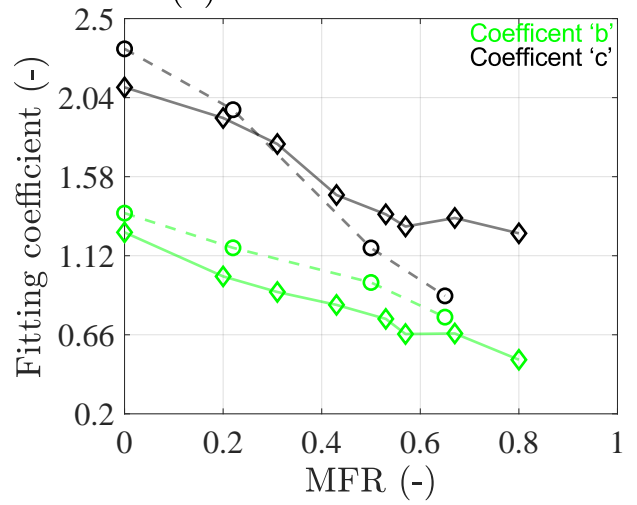

(f) Hub and Short Volute

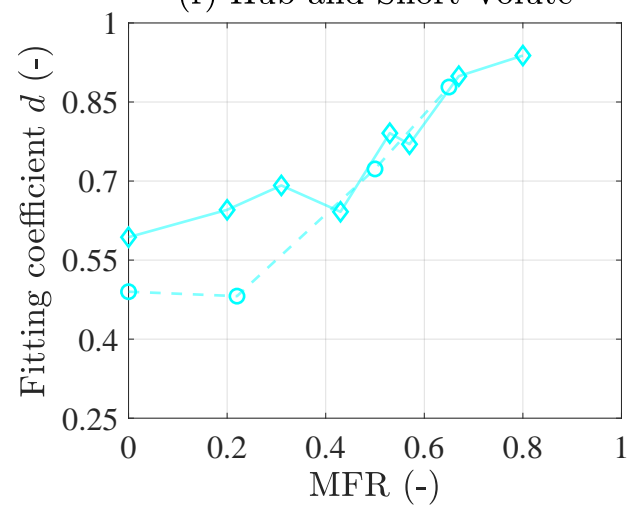

Figure 4.5: Reduced mass flow coefficients obtained using the individual MFR fitting for both T\#1TER and T\#2DVR turbines. Each turbine entry map is fitted one by one. 
(a) Shroud and Long Volute

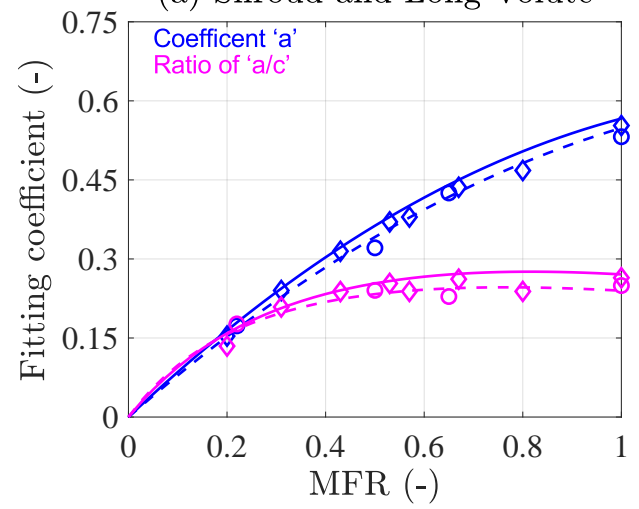

(c) Shroud and Long Volute

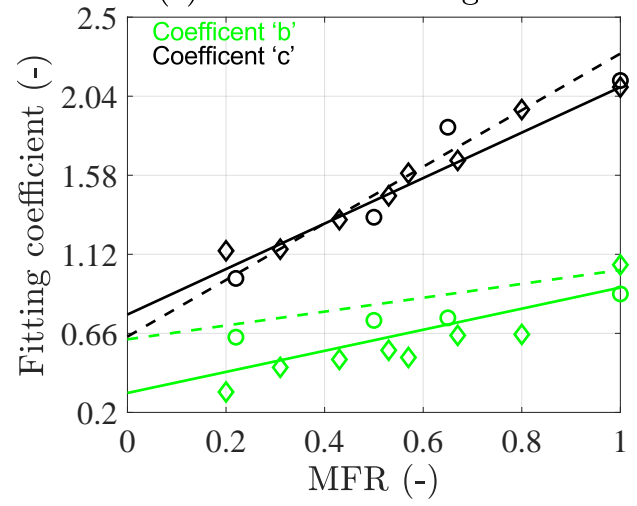

(e) Shroud and Long Volute

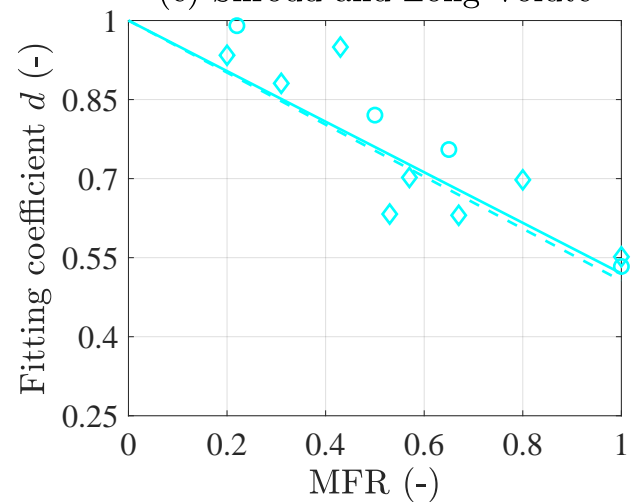

(b) Hub and Short Volute

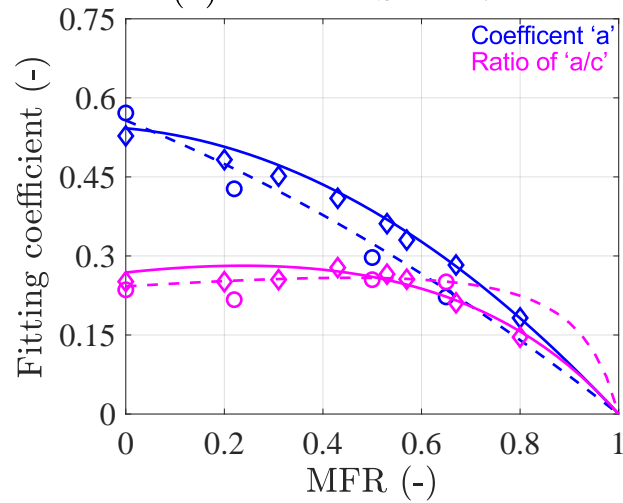

(d) Hub and Short Volute

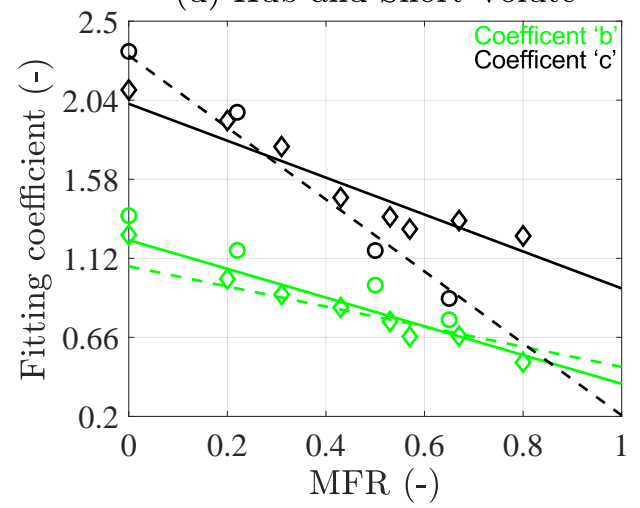

(f) Hub and Short Volute

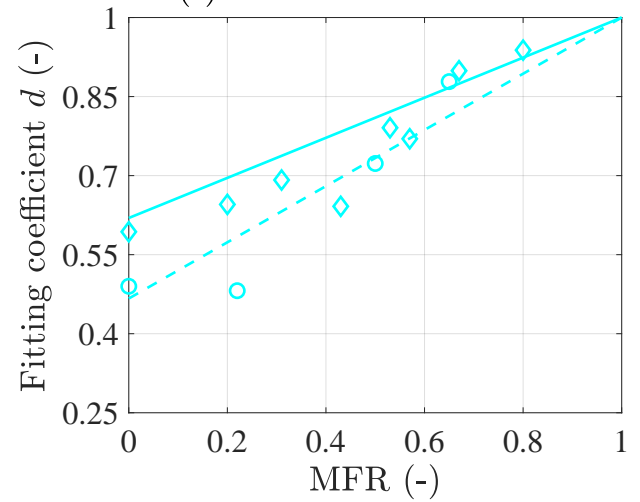

Figure 4.6: Reduced mass flow coefficient trends obtained using a global fitting procedure. Trends are imposed and all MFRs are fitted at the same time. The coefficients from individual MFR fittings are also shown for comparison. 
Figure 4.6 shows the obtained results of the coefficients from a global fitting with MFR after imposing the discussed quadratic and linear trends. The first conclusion emerging is that the obtained trends are very similar between the twin-entry (T\#1TER) and dual-volute (T\#DVR) turbines. It is an excellent result since initial values are mandatory for non-linear fitting procedures. Therefore, the same set of initial values can be used for fitting both types of double-entry turbines. In addition, being the tested turbines so different (twin-entry symmetric vs dual-volute asymmetric) one can assume that averaging for each branch parameters set obtained in this work (Table 4.1) is general enough to be used as initial values for the users in their respective model calculation of another turbine.

Table 4.1: Effective areas fitting coefficient values for T\#1TER and T\#2DVR

\begin{tabular}{cccccc}
\hline Reduced mass flow fitting & & T1\#TER & T2\#DVR & T1\#TER & T2\#DVR \\
\cline { 2 - 6 } coefficient & $a_{1}$ & -0.3192 & -0.2691 & -0.4586 & -0.1803 \\
$a_{i}^{j}=a_{1} \cdot M F R^{2}+a_{2} \cdot M F R+a_{3}$ & $a_{2}$ & 0.8856 & 0.8176 & -0.0837 & -0.3768 \\
& $a_{3}$ & 0 & 0 & $-\left(a_{1}+a_{2}\right)$ & $-\left(a_{1}+a_{2}\right)$ \\
$b_{i}^{j}=b_{1} \cdot M F R+b_{2}$ & $b_{1}$ & 0.6134 & 0.4037 & -0.8364 & -0.5848 \\
$c_{i}^{j}=c_{1} \cdot M F R+c_{2}$ & $b_{2}$ & 0.3130 & 0.625 & 1.2262 & 1.0732 \\
$d_{i}^{j}=d_{1} \cdot M F R+d_{2}$ & $c_{1}$ & 1.3234 & 1.6468 & -1.0741 & -2.0935 \\
& $c_{2}$ & 0.7695 & 0.6412 & 2.0191 & 2.2996 \\
& $d_{1}$ & -0.4799 & -0.4938 & 0.3804 & 0.5332 \\
& $d_{2}$ & 1 & 1 & $\left(1-d_{1}\right)$ & $\left(1-d_{1}\right)$ \\
\hline
\end{tabular}

In Figure 4.6, the difference observed between twin-entry (T\#1TER) and dualvolute (T\#2DVR) turbines can be well explained by the effect of the asymmetry. That is, T\#1TER is symmetric between shroud and hub entries and T\#2DVR turbines are not symmetric between their entries. From Figure 4.6, this effect is particularly evident in coefficients ' $a$ ', ' $c$ ' and ' $d$ ' and did not show much in ' $a / c$ '. While in the twin-entry turbine, the coefficient ' $a$ ' (representing the discharge coefficients) are quite symmetrical. This is not the case in the dual-volute turbine with coefficient ' $a$ ' at the short volute (Figure 4.6(b)). At the short volute, the coefficient ' $a$ ' shows lower values than at the long volute as the flow reduces (MFR increasing for the short volute) and coefficient ' $a / c$ ' (representing stator discharge coefficient) does not show this trend. Looking for asymmetry effects, one can think it is an effect of the volute tongue affecting much more to rotor outlet discharge coefficient than to stator outlet discharge coefficient.

Figure 4.7 shows the coefficient results of dual-volute mixed flow turbine (T\#3DVM) obtained from the global fitting procedure. The average values of each branch parameter from the Table 4.1 were used as initial values during the fitting procedure. The coefficients results of T\#3DVM are further compared with the T\#2DVR parameters obtained from the global fitting procedure (i.e. Figure 4.7). This will highlight the difference between the coefficient parameters for the same type of double-entry turbine. The effect of geometrical asymmetry can well explain the difference between 


\section{- $\ominus-T \# 2 D V R$ All Points Fitting $\rightarrow-\mathrm{T} \# 3 \mathrm{DVM}$ All Points Fitting}

(a) Long Volute

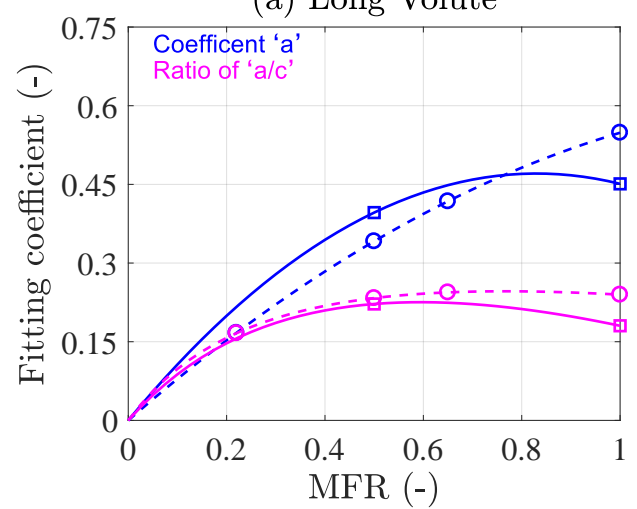

(b) Long Volute

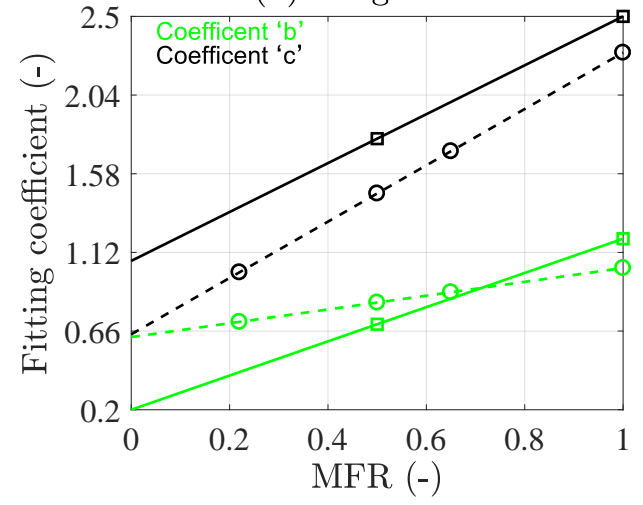

(e) Long Volute

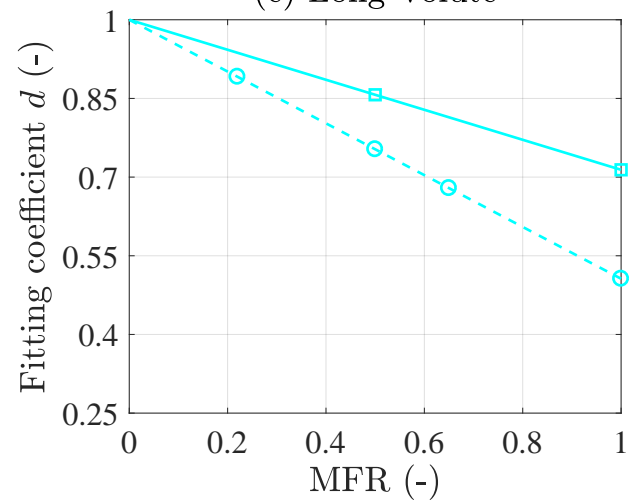

(a) Short Volute

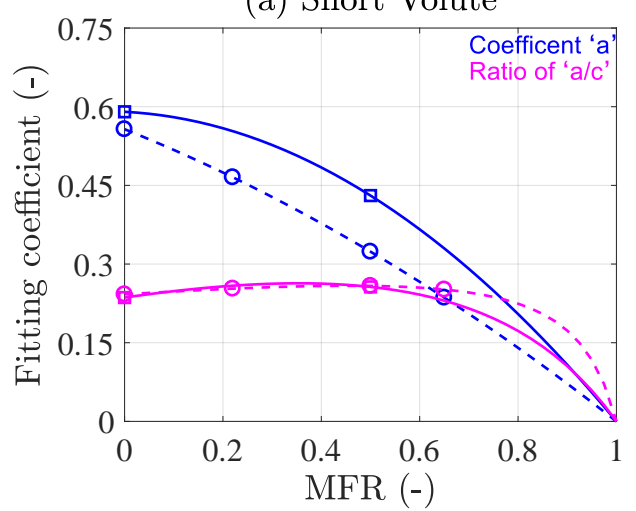

(d) Short Volute

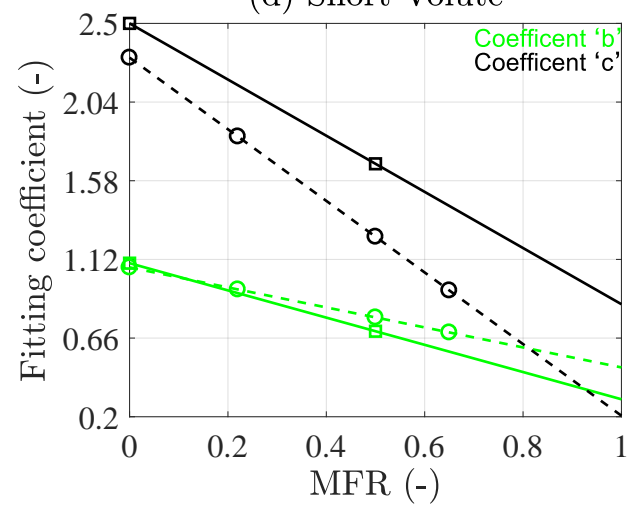

(f) Short Volute

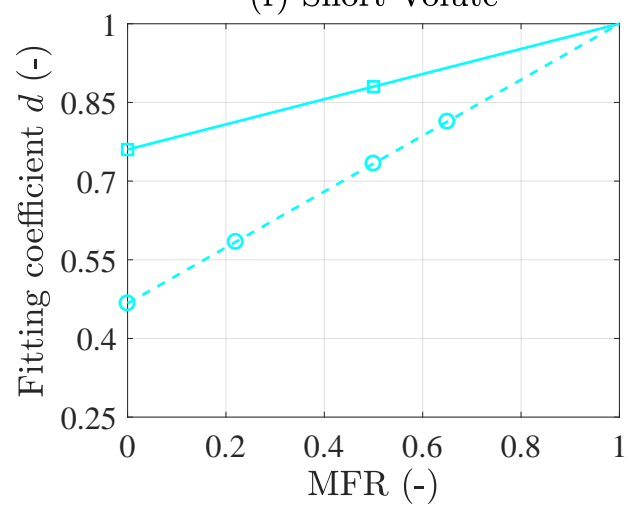

Figure 4.7: Reduced mass flow coefficient trends obtained using a global fitting procedure for dual-volute mixed flow turbine (T\#3DVM). Trends are imposed and all MFRs are fitted at the same time. The coefficients from global fitting procedure for T\#2DVR turbine are also shown for comparison. 
the values of the coefficients of LV and SV entries of T\#3DVM turbine.
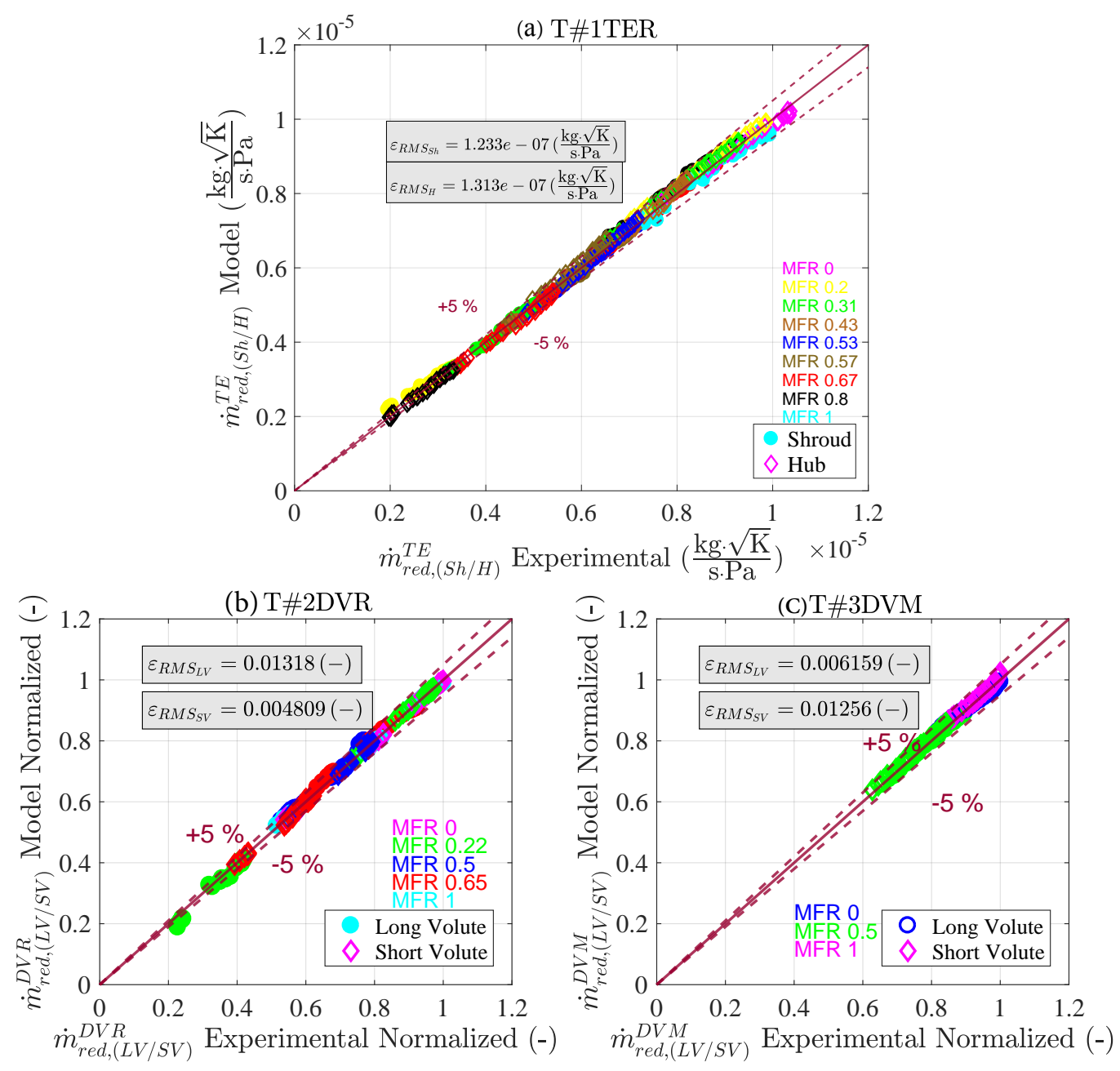

Figure 4.8: Reduced mass flow modelled versus experimental results for T\#1TER, T\#2DVR, and T\#3DVM turbochargers using the global fitting constants found for the respective turbine type.

Figure 4.8 shows the reduced mass flow model fitting results of the two entries for the twin-entry and two dual-volute turbines calculated with Equation 4.11 and the corresponding coefficients, which are obtained using the global fitting process previously described. In Figure 4.8, it can be observed that the model estimates the reduced mass flow with errors within $\pm 5 \%$ in both twin-entry and dual-volute turbines at all flow admission conditions. Figure 4.8(b) and (c) shows the root mean square error in terms of normalized flow parameter and, which is below $1.4 \%$ for long volute and below $0.5 \%$ for short volute respectively. Therefore, it can be considered that the both procedure explained above and the obtained coefficients, as initial fitting values can be valid for other twin-entry and dual-volute turbochargers. It is worth 
noticing the importance of having accurate enough initial values in the non-linear fitting procedure can assess the goodness of fit in the model.

The procedure for estimating a new turbine reduced mass flow map is as follows. Firstly, the seven initial values of fitting coefficients here proposed for each turbine inlet (seven for a Shroud and seven for a Hub or seven for Long Volute and seven for Short Volute, depending on turbine type) need to be provided for the non-linear fitting procedure. Values presented in Table 4.1 can be used as an example of initial values for non-linear fitting calculation. Further, the final values can be obtained with the corresponding effective area $\left(A_{e f f, i}^{j}\right)$, geometries, and turbine map data. After the fitting, the model can calculate the reduced mass flow for any non-measured MFR, turbine reduced speed and pressure ratio. Even for far-off non measured conditions by substituting the fitted $A_{e f f, i}^{j}$ in Equation 4.11.

\subsection{Efficiency fitting using VGT model}

In the case of apparent efficiency fitting, the model described in the subsubsection 2.6.1.2 and [63] is re-designed in a similar way to the reduced mass flow fitting procedure. The efficiency Equation 4.15 (from now on it is called an apparent efficiency) is fitted independently for each entry of double-entry turbine. Aforementioned, the turbochargers used in this thesis are vaneless turbines; accordingly, the VGT model is modified. The stator outlet flow angle $\left(\varphi_{2}^{\text {flow }}\right)$ is not guided by blades anymore as in VGT turbine model. Therefore, the $K_{2}^{\star}$ term is expressed, as shown in the Equation 4.17. Moreover, in $z_{i}^{j}$ function (Equation 4.18), the set of each turbine maps depending on the MFR were provided. It was a first and straight forward adaption of Equation 2.46 and the model explained in [63].

$$
\begin{aligned}
& \eta_{i,(t / s)}^{j}=-K_{1} \cdot\left(\sigma_{i}^{j}\right)^{2}+K_{2}^{\star} \cdot\left[1-\frac{K_{3}}{\left(\sigma_{i}^{j}\right)^{2}}\right]^{\frac{1}{\gamma-1}} \cdot\left(\sigma_{i}^{j}\right) \\
& K_{1}=2\left(\frac{r_{4, i}^{j}}{r_{3}^{j}}\right)^{2} \\
& K_{2}^{\star}=2 \frac{A_{\text {eff }, i}^{j}}{A_{0, i}^{j \text { geom }}}\left(z_{i}^{j} \cdot \tan \alpha_{3, i}^{j \text { flow }}+\sqrt{\frac{K_{1}}{2}} \cdot \tan \beta_{4, i}^{j \text { metal }}\right) \\
& z_{i}^{j}=-\left(a_{i}^{\prime j} \cdot n_{r e d, i}^{j}+b_{i}^{\prime j}\right) \cdot \sigma_{i}^{j}+\left(c_{i}^{\prime j} \cdot n_{r e d, i}^{j}+d_{i}^{\prime j} \cdot M F R^{j^{2}}+e_{i}^{\prime j} \cdot M F R^{j}+f_{i}^{\prime j}\right)
\end{aligned}
$$


Nevertheless, in vaneless turbines, the volute or casing will take over the function of flow guidance at the entry to a rotor in addition to flow distribution and acceleration [18]. Therefore, a physical definition based on geometry for a compressible flow has been considered, according to Watson and Janota [18]. This definition is used to calculate the rotor inlet gas angle $\left(\alpha_{3}^{\text {flow }}\right)$ for vaneless turbines, as shown in Equation 4.19.

$$
\tan \alpha_{3}^{\text {flow }}=\frac{r_{0} \cdot \rho_{3} \cdot 2 \pi \cdot B_{h} \cdot C_{D r 3}}{A_{0} \cdot \rho_{0} \cdot C_{D 0}}
$$

In the above equation, the term $A_{0}$ will be the area at the tongue of each branch, $B_{h}$ will be the blade height of each branch and, $r_{c}$ being a radius of the centroid of section $A_{0}$ as shown in Figure 4.9.

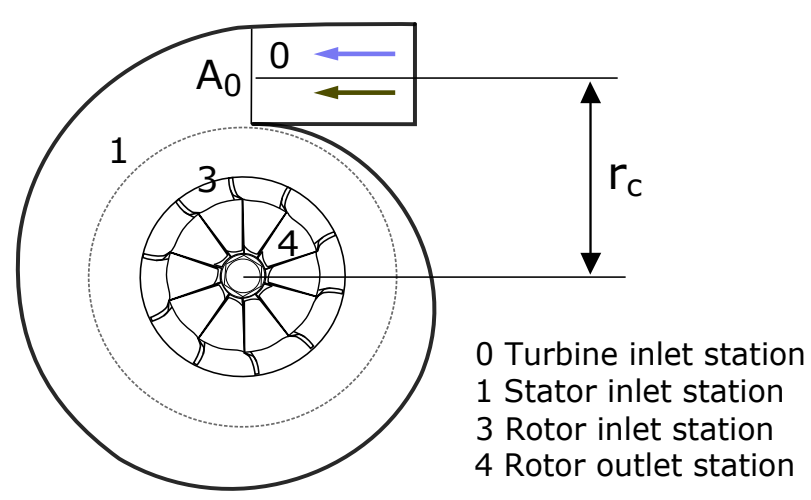

Figure 4.9: Geometrical description for calculating the flow angle in vaneless twin-entry/dual-volute turbines.

Just for calculating this flow angle, it was assumed that the discharge coefficient at the tongue $\left(C_{D 3}\right)$ (station 3 in Figure 4.9$)$ is similar to radial discharge coefficient $\left(C_{D r 3}\right)$. Therefore, their ratio is very close to unity. For estimating the density ratios shown in Equation 4.19, an iterative procedure should be used. In order to avoid this, an isentropic expansion between stations 0 and 3 was assumed. Then the density ratio can be expressed as a function of pressure ratio $\left(p_{3} / p_{0}\right)$. However, the pressure ratio between 0 to 3 station is not available in the turbine maps; thus, an additional assumption is made in this point for this particular purpose. Equation 4.2 can be used to express the isentropic expansion between station 0 and 3, as shown in Equation 4.20. It can be noted that the coefficient ' $d$ ' in Equation 4.20 comes from the reduced mass flow fitting, and it represents the expansion ratio in the rotor 
concerning the total turbine expansion ratio.

$$
\frac{\rho_{3}}{\rho_{0}}=\left(\frac{p_{3}}{p_{0}}\right)^{\frac{1}{\gamma}}=\left(\frac{1+d_{i}^{j} \cdot\left(\Pi_{i,(0 t, 4)}^{j}-1\right)}{\Pi_{i,(0 t, 4)}^{j}}\right)^{\frac{1}{\gamma}}
$$

Finally, the Equation 4.19 now can be expressed, as shown in Equation 4.21 for both branches. This expression should be calculated with their corresponding turbine entry geometry and expansion ratios according to the assumption of two turbines modelling. In this manner, the rotor inlet gas angle $\left(\alpha_{3}^{\text {flow }}\right)$ can be approximated for vaneless twin-entry or dual-volute turbines.

$$
\tan \alpha_{3, i}^{j \text { flow }}=\left(\frac{r_{c, i}^{j} \cdot 2 \pi \cdot B_{h, i}^{j}}{A_{0, i}^{j}}\right) \cdot\left(\frac{1+d_{i}^{j} \cdot\left(\Pi_{i,(0 t, 4)}^{j}-1\right)}{\Pi_{i,(0 t, 4)}^{j}}\right)^{\frac{1}{\gamma}}
$$

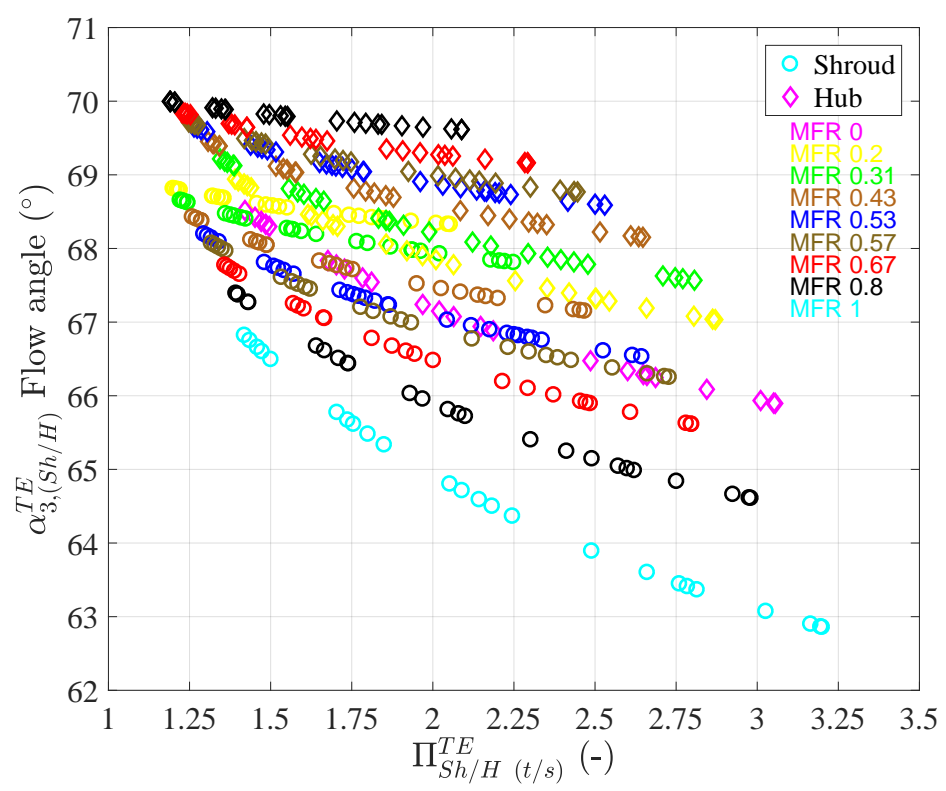

Figure 4.10: Rotor inlet flow angles at different flow admission conditions for Shroud and Hub branches of T\#1TER

Figure 4.10 illustrate the variation of rotor inlet flow angles with the total-to-static turbine expansion ratio of each turbine branch. Here, the results are corresponding to the twin-entry turbine T\#TER. From the Figure 4.10, it is clear that as the flow in Shroud and Hub branch is increasing, the flow angle becomes lower. At MFR = 0.2 (yellow circled points) and MFR $=0.8$ (black diamond points) conditions, the flow is lower in shroud and hub branches respectively. But, the flow angle values are 
higher and shows a continuous reduction of their values from lower to higher turbine expansion ratios.

It is noteworthy that the $K_{1}$ term in the Equation 4.16 is dependent on the ratio of rotor outlet radius of each branch $\left(r_{4, i}^{j}\right)$ and turbine wheel radius $\left(r_{3}^{j}\right)$. For this, the geometry simplifications of twin-entry and dual-volute turbines as demonstrated above for fitting the reduced mass flow model are also considered in fitting the apparent efficiency.

In this way, the apparent efficiency model has been fitted separately for each branch of the twin-entry turbine, using the data of the whole turbine map, respectively. This way, it will be able to analyze the behaviour of the coefficients for each turbine branch with MFR. Accordingly, a set of coefficients for each MFR of the individual twin-entry turbine branch are obtained, as shown in the Table 4.2. It is clear that the model does not show any benefit trends with mass flow ratio parameter (e.g., like the reduced mass flow fitting parameters). Except that coefficients ' $d_{i}^{\prime j}$, and ' $e_{i}^{\prime j}$, tends to zero in all the MFR for both branches. Moreover, the value of the coefficient ' $a_{i}^{\prime j}$, is close to zero for many MFRs.

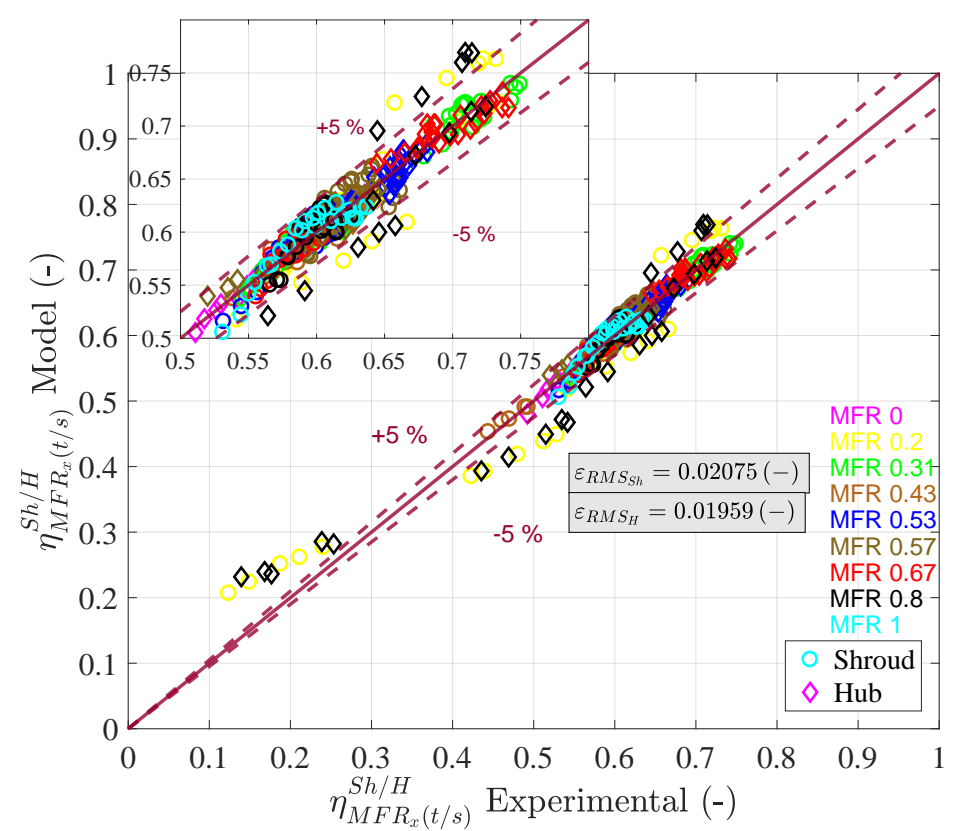

Figure 4.11: T\#1TER turbine apparent efficiency modelled versus experimental using VGT efficiency model

Figure 4.11 shows the model results of both branches at different MFR by using the coefficients from Table 4.2. The results are coherent with the experimental data of each branch with an average Root Mean Square Error (RMSE) of 0.02017 for both branches together (separate RMSE for each branch are shown in Figure 4.11). Furthermore, the model is also sufficient to extrapolate until off-design conditions of 
Table 4.2: Apparent efficiency fitting coefficients from Equation 4.18 for each turbine branch and MFR of T\#1TER. Every MFR map is fitted individually.

\begin{tabular}{|c|c|c|c|c|c|c|c|}
\hline$M F R_{x}$ & Branch & $a^{\prime T E}$ & $b^{\prime T E}$ & $c^{\prime T E}$ & $d^{\prime T E}$ & $e^{\prime T E}$ & $f^{\prime T E}$ \\
\hline \multirow{2}{*}{0} & Sh & - & - & - & - & - & - \\
\hline & $\mathrm{H}$ & 0.0205 & 1.4095 & 0.1379 & 0 & 0 & 0.6572 \\
\hline \multirow{2}{*}{0.2} & Sh & 0.1349 & 0.3864 & 0.4552 & 0 & 0 & $3.850 \mathrm{E}-14$ \\
\hline & $\mathrm{H}$ & $2.313 \mathrm{E}-14$ & 1.064 & 0.0854 & 0 & 0 & 0.6612 \\
\hline \multirow{2}{*}{0.31} & Sh & 0.1443 & 0.0180 & 0.2638 & 0 & 0 & 0.3116 \\
\hline & $\mathrm{H}$ & $2.238 \mathrm{E}-14$ & 0.9210 & 0.0768 & 0 & 0 & 0.6227 \\
\hline \multirow{2}{*}{0.43} & Sh & 0.1380 & 0.0501 & 0.2382 & 0 & 0 & 0 \\
\hline & $\mathrm{H}$ & $1.496 \mathrm{E}-13$ & 0.8709 & 0.0678 & 0 & 0 & 0.7276 \\
\hline \multirow{2}{*}{0.53} & Sh & $2.228 \mathrm{E}-14$ & 0.7188 & 0.1006 & 0 & 0 & 0.5424 \\
\hline & $\mathrm{H}$ & $3.994 \mathrm{E}-14$ & 0.7276 & 0.0551 & 0 & 0 & 0.8262 \\
\hline \multirow{2}{*}{0.57} & Sh & $2.220 \mathrm{E}-14$ & 0.7060 & 0.0814 & 0 & 0 & 0.6297 \\
\hline & $\mathrm{H}$ & 0.1508 & $4.441 \mathrm{E}-14$ & 0.2012 & 0 & 0 & 0.1183 \\
\hline \multirow{2}{*}{0.67} & Sh & $2.515 \mathrm{E}-14$ & 0.8564 & 0.1024 & 0 & 0 & 0.5150 \\
\hline & $\mathrm{H}$ & 0.1416 & $7.668 \mathrm{E}-11$ & 0.1990 & 0 & 0 & 0.3941 \\
\hline \multirow{2}{*}{0.8} & Sh & $2.869 \mathrm{E}-14$ & 1.0488 & 0.1160 & 0 & 0 & 0.5362 \\
\hline & $\mathrm{H}$ & 0.0205 & 0.5535 & 0.5070 & 0 & 0 & $5.038 \mathrm{E}-12$ \\
\hline \multirow{2}{*}{1} & Sh & $2.222 \mathrm{E}-14$ & 1.4958 & 0.1515 & 0 & 0 & 0.6301 \\
\hline & $\mathrm{H}$ & - & - & - & - & - & - \\
\hline
\end{tabular}


each turbine branch map with their corresponding MFR fitting constants from the Table 4.2. But, this model has a limitation that it is not potential to extrapolate to non-measured MFRs and reduced turbine speed as compared to the reduced mass flow model of double-entry turbines (section 4.2); this is because the coefficients do not show any apparent trends with the MFR. Consequently, in this thesis, the efficiency model has been improved to make it able to extrapolate to non-measured MFRs and speeds of twin-entry/dual-volute turbochargers. The procedure to refine the model is based on the analysis of twin-entry turbocharger experimental data.Figure 4.11 shows the model results of both branches at different MFR by using the coefficients from Table 4.2. The results are coherent with the experimental data of each branch with an average Root Mean Square Error (RMSE) of 0.02017 for both branches together (separate RMSE for each branch are shown in Figure 4.11). Furthermore, the model is also sufficient to extrapolate until off-design conditions of each turbine branch map with their corresponding MFR fitting constants from the Table 4.2. But, this model has a limitation that it is not potential to extrapolate to non-measured MFRs and reduced turbine speed as compared to the reduced mass flow model of double-entry turbines (section 4.2). This is because the coefficients do not show any apparent trends with the MFR. Consequently, in this thesis, the efficiency model has been improved to make it able to extrapolate to non-measured MFRs and speeds of twinentry/dual-volute turbochargers. The procedure to refine the model is based on the analysis of twin-entry turbocharger experimental data.

\subsection{Development of actual efficiency model for double- entry turbines}

\subsubsection{Mixed flow approach}

As stated in chapter 3, the assumption of two entries as an individual turbine, the apparent efficiency of each turbine branch is estimated with the enthalpy difference. This difference is between the turbine scroll inlet temperature and mix of temperature that is available at the outlet of a turbine, as shown in Equation 4.22. However, it can be assumed that, at the rotor outlet, temperature coming from the individual turbine branch can be different under full and unequal flow admission conditions. This is due to the different expansions of flow with their given efficiencies in each branch. These different temperatures coming from the branches will define actual efficiency processes shown with continuous lines in Figure 4.12; in contrast with apparent efficiency process drawn with dotted lines in Figure 4.12.

$$
\eta_{M F R_{x}(t / s)}^{i}=\frac{T_{0 t}^{i}-T_{04}^{M F R_{x}}}{T_{0 t}^{i}-T_{4 s}^{i}}
$$


Meanwhile, when the turbine is operating under partial admission conditions $(\mathrm{MFR}=0$ or 1$)$, the power produced by the turbine is only due to that working branch (entry 1 or 2 ). Besides, in these conditions, there is only one turbine outlet temperature and which can be measured in the gas stand. Therefore, the first hypothesis tested was that, is it able to calculate the turbine outlet temperature in all the admission conditions using only the extreme flow cases (MFR $=0$ and 1$)$ as two individual turbines?

To test the hypothesis, T\#1TER turbine fitting results from the Table 4.2 and coefficients which tend to zero $\left(a_{i}^{\prime T E}, d_{i}^{\prime T E}\right.$ and $\left.e_{i}^{\prime T E}\right)$ are avoided in the further analysis. Accordingly, only 3 fitting parameters $\left(b_{i}^{\prime T E}, c_{i}^{\prime T E}\right.$ and $\left.f_{i}^{\prime T E}\right)$ of each branch are now used as shown in Equation 4.23. These parameters are fitted again for the efficiency Equation 4.15 for only partial admission conditions (where we have only the actual efficiency of that branch).

$$
z_{i}^{T E}=-\left(b_{i}^{\prime T E} \cdot \sigma_{i}^{T E}\right)+\left(c_{i}^{\prime T E} \cdot n_{\text {red,i }}^{T E}\right)+f_{i}^{\prime T E}
$$

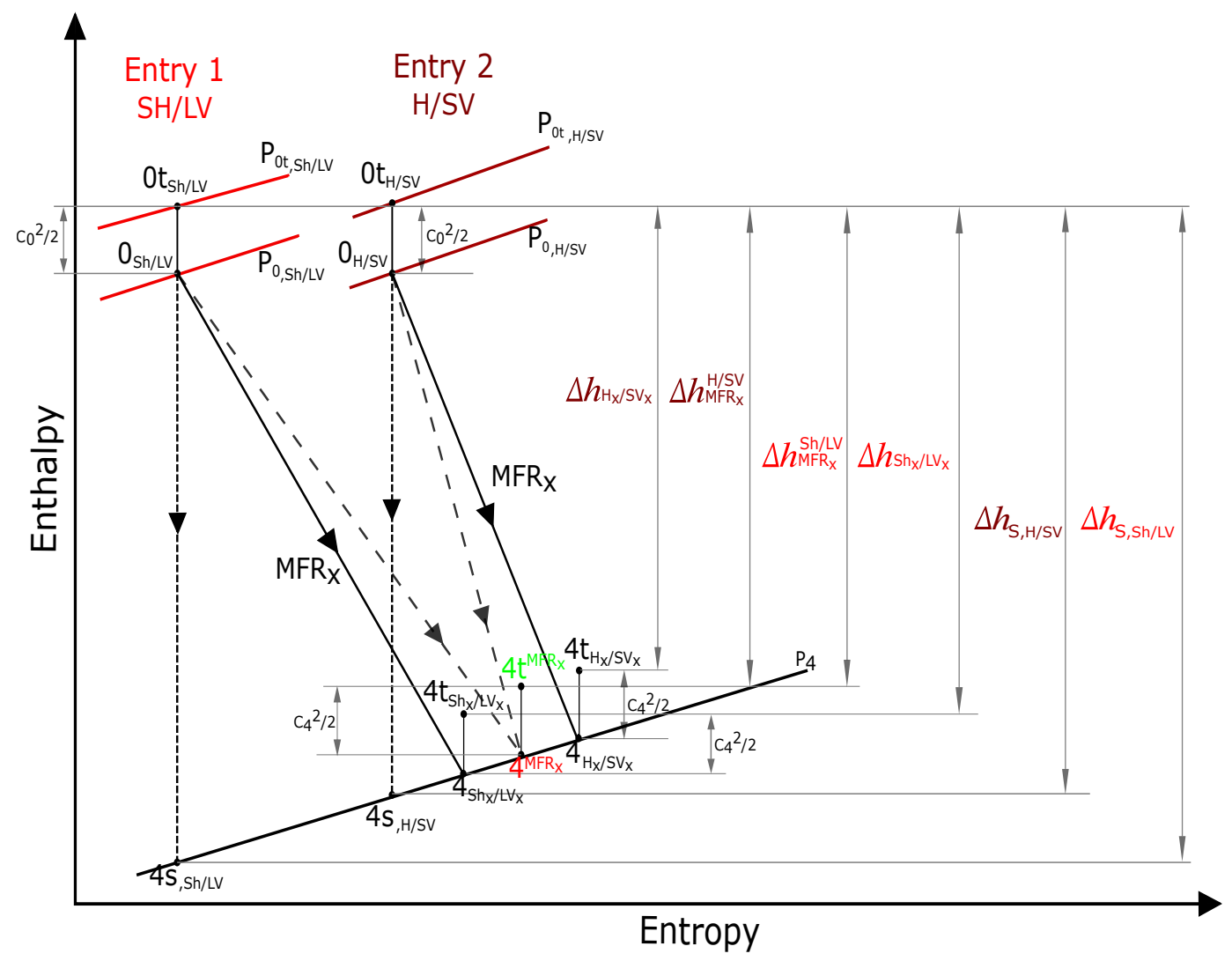

Figure 4.12: Double entry turbines expansion process in partial admission conditions 
Table 4.3: Fitting coefficients of partial admission conditions of T\#1TER using apparent efficiency.

\begin{tabular}{llll}
\hline$M F R_{x}$ & $b^{\prime T E}$ & $c^{\prime T E}$ & $f^{\prime T E}$ \\
\hline $0(\mathrm{H})$ & 1.4685 & 0.1493 & 0.6280 \\
$1(\mathrm{Sh})$ & 1.4278 & 0.1189 & 0.6895 \\
\hline
\end{tabular}

Figure 4.13, shows the extrapolation of extreme flow cases for shroud and hub branches of the twin-entry turbine (T\#1TER), using the individual fitting constants of MFR 0 and 1 from the Table 4.3 and combining them in Equation 4.23 for efficiency. In addition, the global fitting constants of effective equivalent nozzle area of each branch as shown in Figure 4.6 and in Table 4.1 are used for reduced mass flow parameter calculation. For extrapolating the partial admission maps, the methodology proposed by Payri et al. [62] has been followed. Equation 4.23 shows the parameter that accounts for average flow deviation from the calculated rotor inlet angle (Equation 4.21) and imposed metal angle at the rotor outlet [20]. These are the only 'losses correction' of Equation 4.15 model, apart from the discharge coefficients accounted by the flow model. Equation 4.23 shows how the 'average flow deviation' depends linearly on both reduced speed and blade to speed ratio. From Figure 4.13, it can be observed that the model prediction is good and also one can see clearly how the hub branch with a lower trimming (with higher rotor inlet to outlet radius ratio) shows a significant difference in mass flow parameter between different reduced speeds for a given expansion ratio. It means a higher work in the centrifugal forces field than the shroud branch with a lower radius ratio. Figure 4.13 also shows slightly higher peak efficiency at hub branch, probably due to the higher trimming (higher specific work) and the lower tip leakage losses when the flow is concentrated in hub branch than in shroud $[79,80]$.

For predicting the same turbine outlet temperatures as in experiments at different flow admission conditions, a look-up table is generated with the extrapolated efficiency, blade to speed ratio and reduced turbine speeds of MFR 0 and 1 separately, corresponds to hub and shroud entries in the turbine T\#1TER. Imagining, if every other flow admission condition (MFRs different than 0 or 1 ) were a quasi-steady mass average of what happens at partial admission conditions (MFR 0 and MFR 1). Then, it is possible to calculate the static turbine outlet temperature $\left(T_{4, i}^{T E}\right)$ using the Equation 4.24. Here, $\eta_{i, t / s}^{T E}$ is the estimated efficiency from the look-up table for all the MFRs and is estimated based on the input of BSR and reduced turbine speed.

$$
T_{4, i}^{T E}=T_{0 t, i}^{T E}\left[1-\eta_{i,(t / s)}^{T E}\left(1-\left(\Pi_{i,(0 t, 4)}^{T E}\right)^{\frac{1-\gamma}{\gamma}}\right)\right]-\frac{c_{4}^{2}}{2 c_{p}}
$$



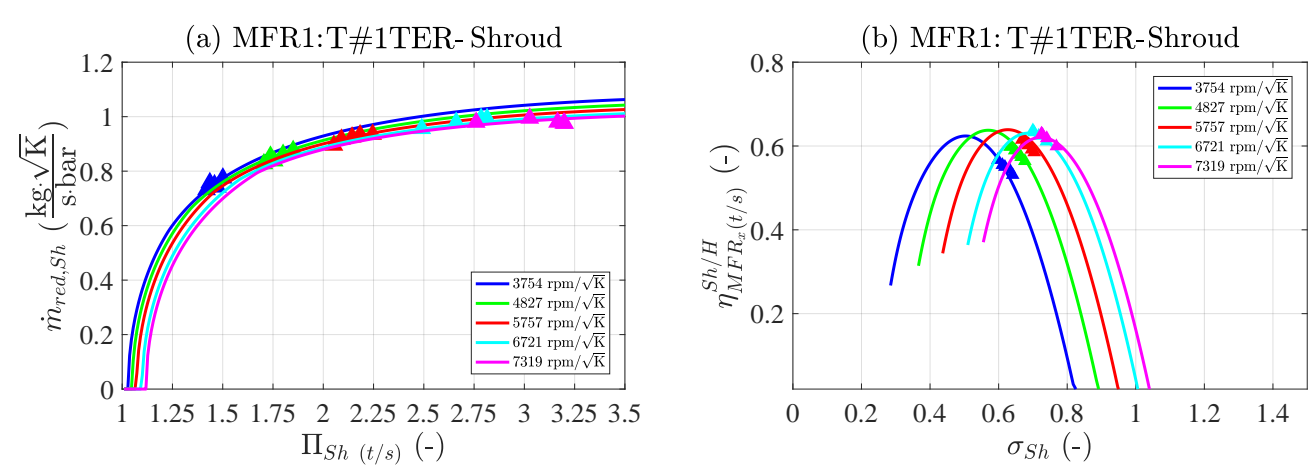

(c) MFR0:T\#1TER-Hub
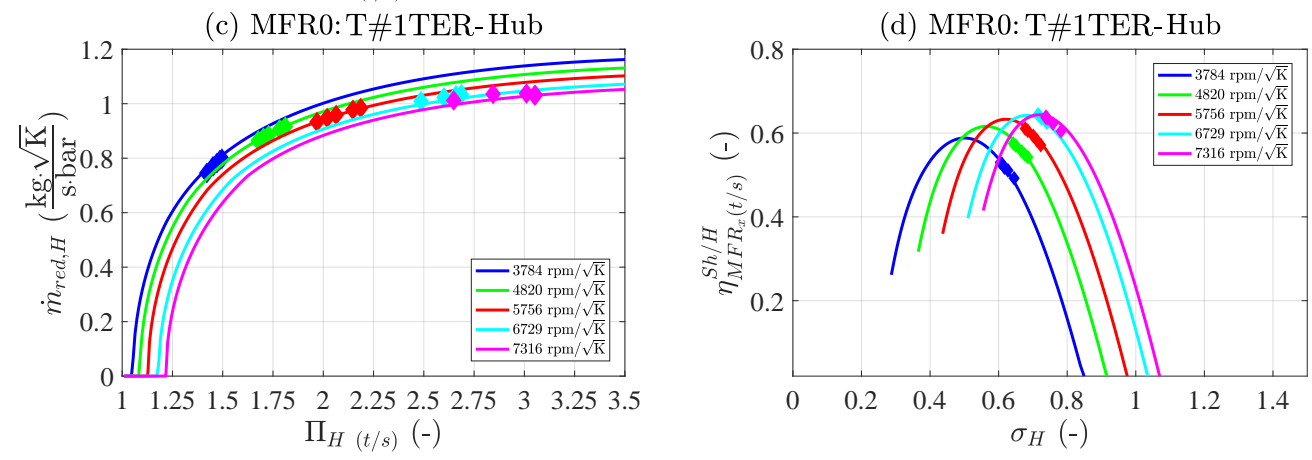

Figure 4.13: Extrapolation of partial admission conditions for T\#TER (MFR 0 and 1)

It is worth checking that, in the case of partial admission conditions, the estimated temperatures using the Equation 4.24 should be equal to the experimental values and, the same can be noticeable in the Figure 4.14. It is important to note that the temperatures are very symmetrical between MFR 0 and 1 partial admission cases in all the turbine reduced speeds.

In order to check the validity of this approach in other flow admission conditions, the efficiencies of each branch in full and unequal admission conditions were estimated using the look-up table; at the desired BSR and turbine reduced speed at those conditions. Finally, the mixed static turbine outlet temperature is calculated as a mass-weighted average between the individual turbine outlet temperature (Equation 4.24) as shown in the Equation 4.25. It is done because the flow expansion in both branches finishes with a common outlet station where the temperatures are mixed depending on the flow repartition. In fact, from the gas stand, the mixed turbine temperature is the only information available at the turbine outlet station throughout full and unequal flow admissions at the turbine inlet.

$$
T_{4}^{M F R_{x}}=T_{4, S h x}^{T E} \cdot M F R_{x}+T_{4, H x}^{T E} \cdot\left(1-M F R_{x}\right)
$$

From Figure 4.15, it can be observed that the prediction of temperatures using the mixed flow approach (Equation 4.25) is always higher for unequal and full admission 


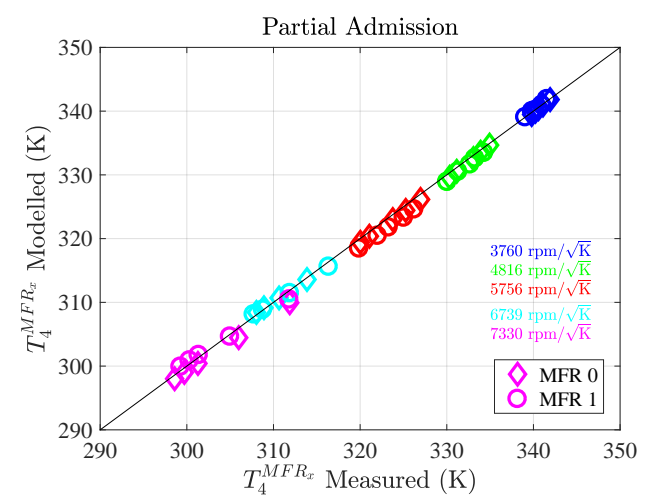

Figure 4.14: Estimated turbine outlet temperatures using extrapolated maps of MFR 0 and 1 for T\#1TER

flow conditions. Moreover, Figure 4.15 also shows an additional aspect that the prediction of temperatures are nearly symmetrical trend with the distance between actual $M F R_{x}$ and MFR $=0.5$ (see Figure $4.15 \mathrm{a}$, b and c) and also the error was maximum at $\mathrm{MFR}=0.5$. The difference between the estimated mixed temperature and experimental values were further investigated with $M F R_{x}$ and new parameter $M F R_{x}^{\prime}$ as shown in Equation 4.26; this is done to observe the symmetry with respect to the $\mathrm{MFR}=0.5$.

$$
M F R_{x}^{\prime}=\left|M F R_{x}-0.5\right|
$$

Figure 4.16 shows the temperature prediction error $\left(\Delta T_{4}^{M F R_{x}}\right)$ for all the MFRs and reduced turbine speeds with $M F R_{x}$ and $M F R_{x}^{\prime}$ parameters. Based on the Figure 4.16 (a) and (b), the following conclusion can be made:

- The error is substantially zero for both partial admission conditions, as there are no mixing of flows at the outlet branch of the turbine. Moreover, the deviation of the error in MFR 0 and 1 is quite symmetrical to MFR 0.5.

- The errors are always positive in the case of full and unequal admission flows. It is understood that the proposed mixed approach is always under predicting the apparent efficiency. The error is lower at highly uneven flows between branches and maximum around full admission. That is, increasing from low unbalanced flows (i.e., MFR 0.2 and 0.8) to having the same flows (MFR 0.5) in both branches.

- The parameter $M F R_{x}^{\prime}$ given in the Equation 4.26 shows a significance of turbine outlet temperature as demonstrated in Figure 4.16(b). In this figure, the error tends to be lower (efficiency lower) when new parameter $M F R_{x}^{\prime}$ is equal to 0.5 and higher (efficiency higher) when it is similar to zero. 

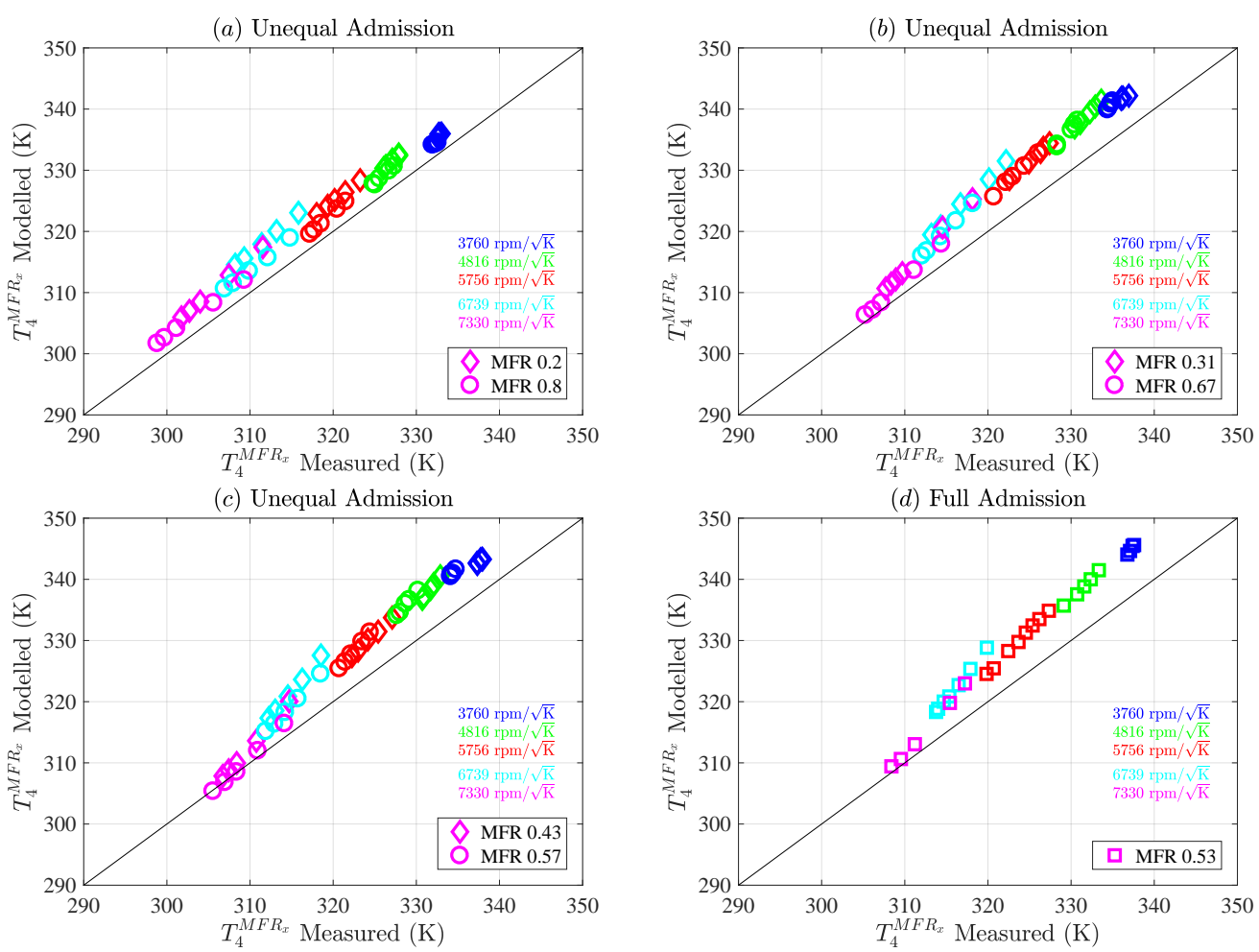

Figure 4.15: Estimated mixed turbine outlet temperature for full and unequal flows using extrapolated maps of MFR 0 and 1 for T\#1TER
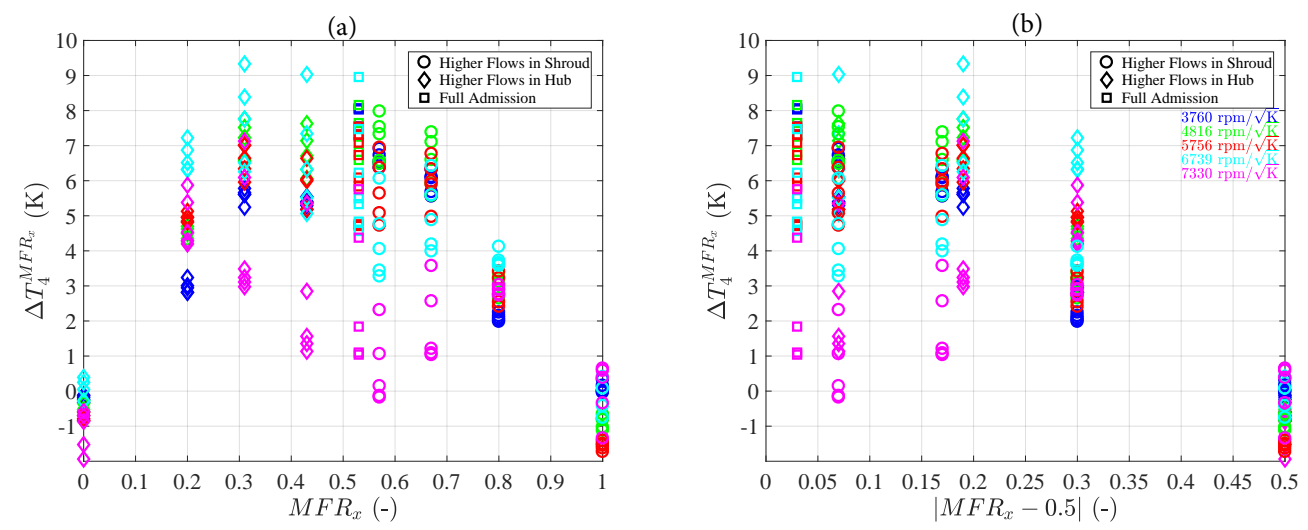

Figure 4.16: Error prediction in all admission conditions using the extreme flow maps for T\#1TER. (a) Mixed turbine outlet temperature error with MFR, (b) Mixed turbine outlet temperature error with $M F R_{x}^{\prime}$. 
- The reasoning for always positive errors can be explained as losses due to the sudden expansion of flow when the turbine is working only with one branch while the other is blocked. In these conditions, the flow also expands into a non-flow branch. As a consequence, this mixed flow approach gives the lower efficiency as compared to the double-entry turbine operating with two branches together at different flow admissions.

- Having the two turbines individually for each inlet with an extreme flow condition and predicting the situations of other flow admissions by a mixing approach always produces higher temperatures. The efficiency losses for the individual turbines are added through the mixing method and increase the entropy of the turbine.

- In adiabatic calculations, the outlet temperature represents more or less the efficiency; as a result, higher the temperature gives a lower performance. The best conditions are those where the $T_{4}^{M F R_{x}}$ error is always higher and which corresponds to having more flow in hub branch and also having same flow in both branches (according to the Figure 4.16). This is especially true at low turbocharger speeds (black color in Figure 4.16). Thus, it means that the losses between both branches of the twin-entry turbine are a maximum when there is no flow in one branch. As the flow conditions are changing from partial admission to full admission state, the efficiency is better.

From the former conclusions, it can be understood that, having a calibration function, it could be possible to reduce the error and obtain the same outlet temperature as in the experimental points in all flow conditions. Once the right mixed turbine outlet temperature is known, the model will have the capabilities of predicting the proper efficiency of each branch in all flow situations.

\subsubsection{Mixed flow approach correction}

Based on the outcome of mixed outlet temperatures in all the flow conditions that shown in subsection 4.4.1, it is clear that a fitting parameter can be feasible for adjusting the error $\left(\Delta T_{4}^{M F R_{x}}\right)$ generated by the mixed flow approach in the case of full and unequal flow admissions. In a way that is appropriate to reduce the error, more analysis was done with variables like a blade to speed ratio (BSR), $M F R_{x}$ and reduced turbine speed as shown in the Figure 4.17.

If the error is examined against $M F R_{x}$ parameter and turbine reduced speed as shown in Figure 4.17 (a) and (b) respectively. The error shows a comparatively parabolic trend with turbine reduced speeds and also, the error decreases from full admission flow to partial admission flow. Furthermore, if the error is observed with a blade speed ratios of each branch with a different $M F R_{x}$ as shown in Figure 4.17 (c). 
The error showed a logarithmic trend with $M F R_{x}$ parameter and also moderately linear as BSR grows. Besides, if the error is ascertained with the similar turbine reduced speed of all the flow admission conditions as shown in the Figure 4.17 (d). The Y-intercept values show a parabolic dependency with the same turbine reduced speed for various mass flow ratio conditions and blade speed ratios. Moreover, from Figure 4.16, it is clear that the observed trends can agree with $M F R_{x}^{\prime}$, as this parameter signified that the error is symmetric as shown in Figure 4.16 (b). One must bear in mind that the trend of the error in $T_{4, T E}^{M F R_{x}}$ is the same as the trend of any correction coefficient designed to increase the efficiency at full and unequal admission conditions, in order to reduce the said error.
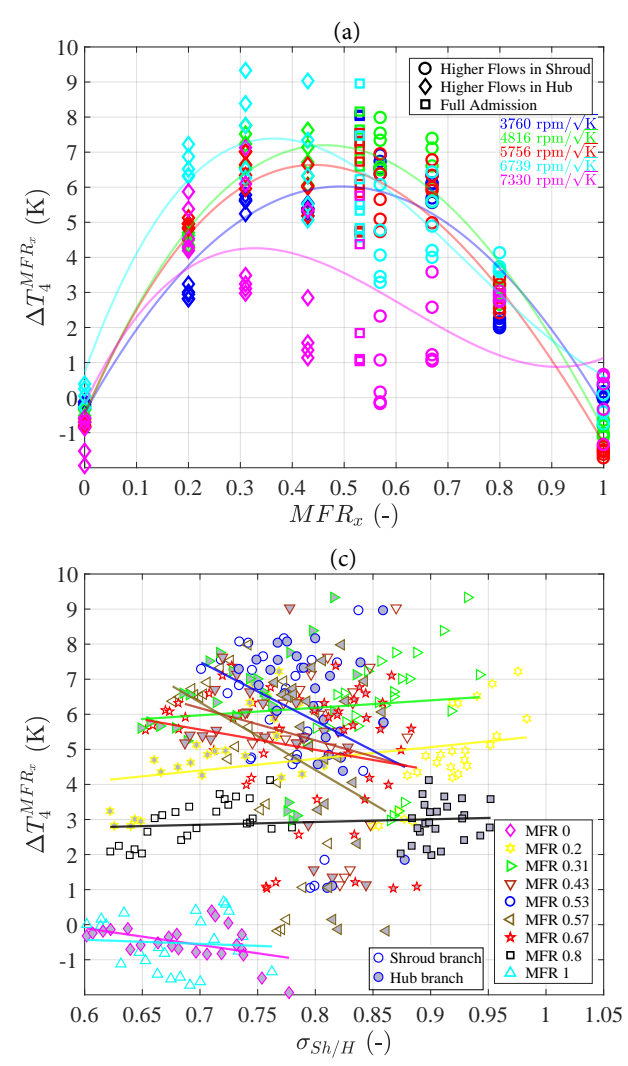

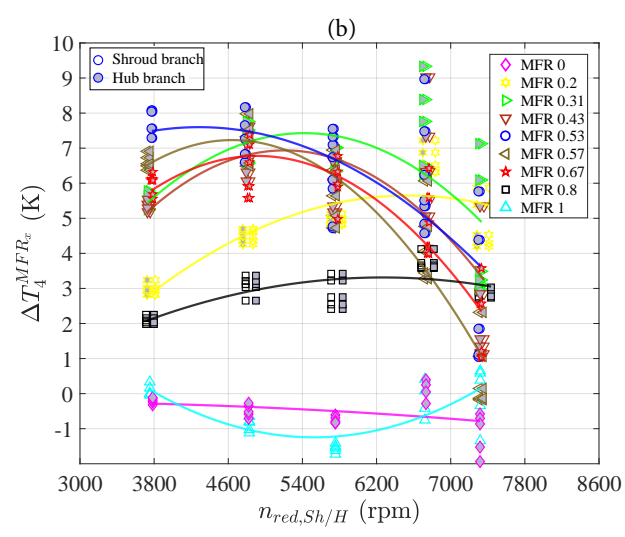

(d)

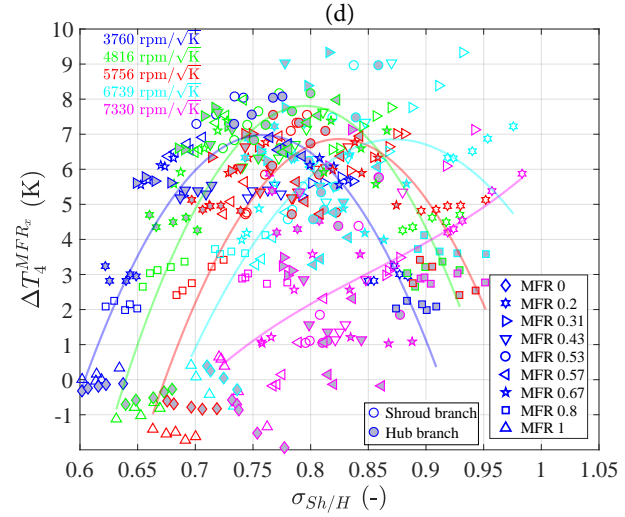

Figure 4.1\%: Analysis of T\#1TER turbine outlet temperature error with different parameters in order to find an a correction function to reduce the error. (a) Error with $M F R_{x}$ and same reduced turbine speeds, (b) Error with reduced turbine speed of each branch and $M F R_{x}$, (c) Error with the BSR of each branch and $M F R_{x}$, (d) Error with same reduced turbine speed for different $M F R_{x}$ and BSR.

By considering all these, new fitting function $\left(z^{\prime j}\right)$ is proposed, as shown in Equation 4.27 according to the noticed trends that are shown in Figure 4.17. Equation 4.23 is a term that will be used to correct the average flow deviations from model hypotheses at partial admission conditions, Equation 4.27, in order increase efficiency with 
respect to partial admission when the turbine is working at full and unequal flow conditions. In other words, to reduce the error in calculated values of $T_{4 T E}^{M F R_{x}}$ at the light of new findings in the flow-oriented efficiency model of Equation 4.15 (see Figure 4.12).

$$
\begin{aligned}
z^{\prime j}= & \left(M F R_{x}^{\prime}-0.5\right) \cdot \ln \left[\left(M F R_{x}^{\prime}\right)^{k_{0}^{j}}+k_{1}^{j} \cdot \sigma_{i}^{j}\right] \\
& \cdot\left[k_{2}^{j}+k_{3}^{j} \cdot \sigma_{i}^{j} \cdot n_{r e d, i}^{j}-\frac{k_{4}^{j}\left(n_{r e d, i}^{j}\right)^{2}}{\left(M F R_{x}^{\prime}+\sigma_{i}^{j}\right)}\right]
\end{aligned}
$$

In the correction function $z^{\prime j}$, five fitting coefficients are introduced and, they will be the same for both branches. It is worth noting that the term $z^{j}$ becomes zero at $M F R_{x}=0$ or $M F R_{x}=1$; since in these cases the positive error in temperature does not exist, and efficiency is not under-predicted. The proposed flow correction function $\left(z^{\prime j}\right)$ is added to the old $z_{i}^{j}$ function (Equation 4.23) as shown in Equation 4.28.

$$
z_{i}^{j}=-\left(b_{i}^{\prime j} \cdot \sigma_{i}^{j}\right)+\left(c_{i}^{\prime j} \cdot n_{r e d, i}^{j}\right)+f_{i}^{\prime j}+z^{\prime j}
$$

It is important to note that final $z_{i}^{j}$ functions (Equation 4.28) have 11 fitting coefficients in total for the two branches; which are three coefficients for each branch $\left(b_{i}^{\prime j}, c_{i}^{\prime j}\right.$ and $\left.f_{i}^{\prime j}\right)$ obtained from turbine map data of $M F R_{x}=0$ and $M F R_{x}=1$ and further kept constant in the mixed flow approach. The other five coefficients $\left(k_{0}^{j}, k_{1}^{j}, k_{2}^{j}, k_{3}^{j}\right.$ and $\left.k_{4}^{j}\right)$ of $z^{\prime j}$ are associated to correct the extra flow deviation effects (overestimated) generated by the mixed flow approach. Now, the Equation 4.28 will be used in Equation 4.17 and further it will also appear in Equation 4.24. As a result, the final $T_{4}^{M F R_{x}}$ (Equation 4.25) will be now calculated with the $z^{\prime j}$ for reducing generated losses of mixed flow approach (Equation 4.28) apart from partial admission conditions; this process is called corrected-mixed-flow approach.

Summarizing, all 11 fitting coefficients are globally fitted together for both branches using a non-linear fitting procedure by using all the partial, unequal and full admission data points of each branch of T\#1TER. It is worth highlighting that $z^{\prime j}$ will be equal to zero while fitting the partial admission conditions $\left(M F R_{x}=0\right.$ or 1$)$; consequently, the coefficients obtained while fitting the partial admission data should be similar to the coefficients shown in Table 4.3. Table 4.4 shows the values of 11 fitting coefficients obtained while minimizing the root mean square error between the modelled and experimental mixed turbine outlet temperature values.

Figure 4.18 shows the level of correlation between the mixed turbine outlet temperatures measured in the gas stand and modelled $T_{4}^{M F R_{x}}$ values for all the mass flow ratios data points in the map of the twin-entry turbine (T\#1TER). It can be 
Table 4.4: Fitting coefficients of mixed turbine outlet temperature correction with new fitting function for T\#1TER

\begin{tabular}{ccccccccccc}
\hline \multicolumn{3}{c}{$M F R_{1}(\mathrm{Sh})$} & \multicolumn{4}{c}{ Fitting function $\left(z^{\prime T E}\right)$} & \multicolumn{3}{c}{$M F R_{0}(\mathrm{H})$} \\
\hline$b_{S h}^{\prime T E}$ & $c_{S h}^{\prime T E}$ & $f_{S h}^{\prime T E}$ & $k_{0}^{T E}$ & $k_{1}^{T E}$ & $k_{2}^{T E}$ & $k_{3}^{T E}$ & $k_{4}^{T E}$ & $b_{H}^{\prime T E}$ & $c_{H}^{\prime T E}$ & $f_{H}^{\prime T E}$ \\
\hline 1.4685 & 0.1493 & 0.6280 & 4.9408 & 0.0330 & 0.1386 & 0.0495 & $5.135 \mathrm{E}-03$ & 1.4278 & 0.1189 & 0.6895 \\
\hline
\end{tabular}

observed that the modelled $T_{4}^{M F R_{x}}$ values show good accordance with the experimental points and, it is evidenced by the RMSE value of $1.3518(\mathrm{~K})$. By using the proposed fitting function $\left(z^{\prime j}\right)$ within the $K_{2}^{\star}$ term of the apparent efficiency equation (Equation 4.17), it is possible to obtain the mixed turbine outlet temperature of all the MFRs only with the 11 fitting parameters shown in the Table 4.4 and 14 flow coefficients shown in Table 4.1. The efficiency model is a flow-oriented model, and both (reduced mass flow and efficiency) must be fitted together. Once the mixed turbine outlet temperature values are predicted well, these values can be used to obtain the apparent efficiency of each branch.

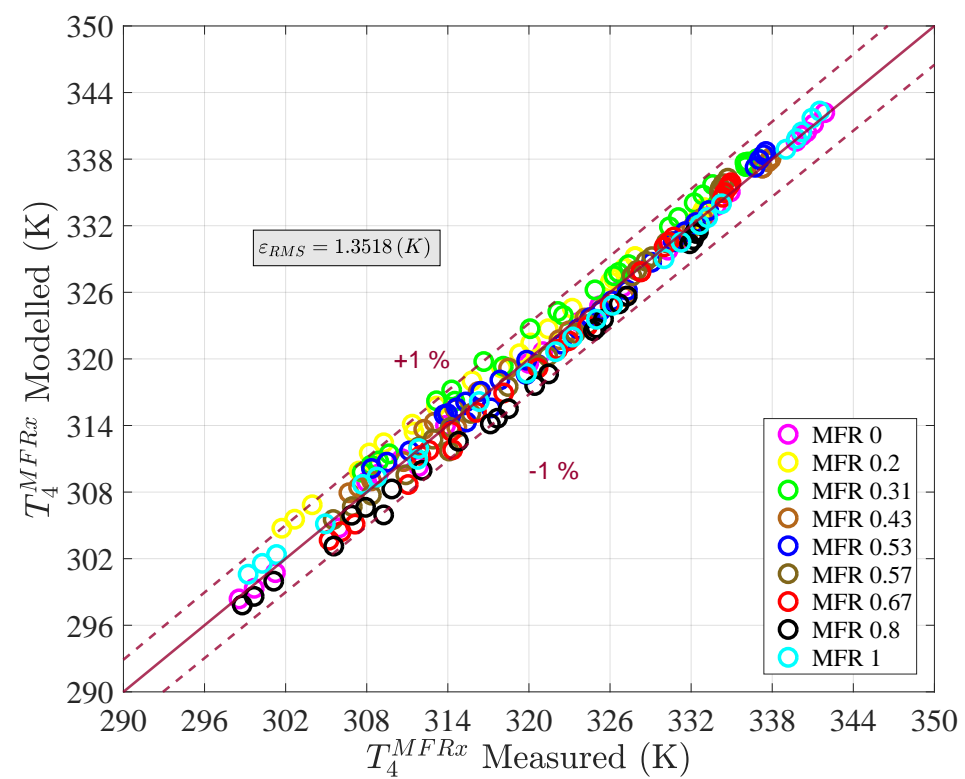

Figure 4.18: Mixed turbine outlet temperature measured versus modelled for T\#1TER 


\subsubsection{From mixed flow corrected approach to apparent efficiency merit function}

From subsection 4.4.2, it is clear that the mixed turbine outlet temperatures can be well predicted by adding the suggested calibrated function $\left(z^{\prime j}\right)$ in Equation 4.23 as shown in the Equation 4.28. Nevertheless, the big issue of the actual efficiency for applying the corrected-mixed-flow hypothesis is that it cannot be measured, but only the apparent efficiency can be measured. Because the individual turbine outlet temperatures coming from each branch are mixed in common outlet branch (according to Figure 4.12 showed with continues and dotted lines). Moreover, the double-entry turbine maps will be in terms of apparent efficiency either adiabatic or adiabatized from standard gas stand maps [2]. Consequently, it would not be possible to fit the proposed coefficients without objective experimental information and an adequate merit function. For this purpose, a relation must be established between the actual efficiencies calculated with the corrected-mixed-flow hypothesis and apparent efficiencies measured in the gas stand; instead of fitting with the mixed flow outlet temperatures measured in the gas stand. In this regards, inserting Equation 4.24 into Equation 4.25 , the following equation is obtained:

$$
\begin{aligned}
T_{4}^{M F R_{x}}= & M F R_{x} \cdot\left[T_{0 t, S h_{x}}^{T E}\left(1-\eta_{S h_{x}}^{T E}\left(1-\left(\Pi_{S h_{x},(0 t, 4)}^{T E}\right)^{\frac{1-\gamma}{\gamma}}\right)\right)-\frac{c_{4}^{2}}{2 c_{p}}\right]+ \\
& \left(1-M F R_{x}\right) \cdot\left[T_{0 t, H_{x}}^{T E}\left(1-\eta_{H_{x}}^{T E}\left(1-\left(\Pi_{H_{x},(0 t, 4)}^{T E}\right)^{\frac{1-\gamma}{\gamma}}\right)\right)-\frac{c_{4}^{2}}{2 c_{p}}\right]
\end{aligned}
$$

Further, inserting the Equation 4.29 into Equation 4.30 and rearranging the terms, mixed total temperature can be written as shown in Equation 4.31:

$$
\begin{gathered}
T_{04}^{M F R_{x}}=T_{4}^{M F R_{x}}+\frac{c_{4}^{2}}{2 c_{p}} \\
T_{04}^{M F R_{x}}=\left(M F R_{x}\right) \cdot T_{0 t, S h_{x}}^{T E}\left[1-\eta_{S h_{x}}^{T E}\left(1-\left(\Pi_{S h_{x},(0 t, 4)}^{T E}\right)\right]+\right. \\
\left(1-M F R_{x}\right) \cdot T_{0 t, H_{x}}^{T E}\left[1-\eta_{H_{x}}^{T E}\left(1-\left(\Pi_{H_{x},(0 t, 4)}^{T E}\right)^{\frac{1-\gamma}{\gamma}}\right)\right]- \\
\frac{c_{y}^{2}}{2 c_{p}}\left(M F R_{x}+1-\underline{M F R_{x}}\right)+\frac{c_{y}^{2}}{2 c_{p}}
\end{gathered}
$$

Recalling the definition of apparent efficiency (Equation 4.22) for both branches, 
and it can be written as follows:

$$
\begin{aligned}
\eta_{M F R_{x}(t / s)}^{S h_{x}} & =\frac{1-\frac{T_{04}^{M F R_{x}}}{T_{0 t, S h_{x}}^{T E}}}{\left(1-\left(\Pi_{S h_{x},(0 t, 4)}^{T E}\right)^{\frac{1-\gamma}{\gamma}}\right)} \\
\eta_{M F R_{x}(t / s)}^{H_{x}} & =\frac{1-\frac{T_{04}^{M F R_{x}}}{T_{0 t, H x}^{T E}}}{\left(1-\left(\Pi_{H_{x},(0 t, 4)}^{T E}\right)^{\frac{1-\gamma}{\gamma}}\right)}
\end{aligned}
$$

For easily simplifying the equations, the denominator terms of equations 4.32 and 4.33 is acknowledged as:

$$
\begin{aligned}
K_{\Pi}^{S h_{x}} & =\left(1-\left(\Pi_{S h_{x},(0 t, 4)}^{T E}\right)^{\frac{1-\gamma}{\gamma}}\right) \\
K_{\Pi}^{H_{x}} & =\left(1-\left(\Pi_{H_{x},(0 t, 4)}^{T E}\right)^{\frac{1-\gamma}{\gamma}}\right)
\end{aligned}
$$

Now, substituting the Equation 4.31 into Equation 4.32 and by continuing the simplification as shown below; the final apparent efficiency equation for Shroud/Long Volute branch can be obtained:

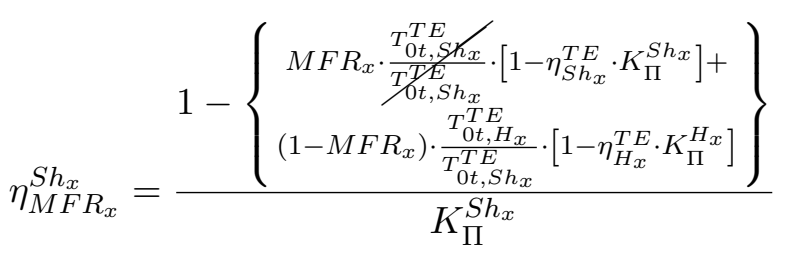

$$
\begin{aligned}
& \left(1-M F R_{x}\right)+M F R_{x} \cdot \eta_{S h_{x}}^{T E} \cdot K_{\Pi}^{S h_{x}}- \\
& =\frac{\left(1-M F R_{x}\right) \cdot \frac{T_{0 t}^{T E}}{T_{0 t}^{T E}, S h_{x}}+\left(1-M F R_{x}\right) \cdot \frac{T_{0 t}^{T E}}{T_{0 t, S H_{x}}^{T E}} \cdot \eta_{H_{x}}^{T E} \cdot K_{\Pi}^{H_{x}}}{K_{\Pi}^{S h_{x}}} \\
& \left(1-M F R_{x}\right) \cdot\left(1-\frac{T_{0 t, H_{x}}^{T E}}{T_{0 t, S h_{x}}^{T E}}\right)+M F R_{x} \cdot \eta_{S h_{x}}^{T E} \cdot K_{\Pi}^{S h_{x}}+ \\
& =\frac{\left(1-M F R_{x}\right) \cdot \eta_{H_{x}}^{T E} \cdot K_{\Pi}^{H x} \cdot \frac{T_{0 t, H_{x}}^{T E}}{T_{0 t, S h_{x}}^{T E}}}{K_{\Pi}^{S h_{x}}}
\end{aligned}
$$




$$
\begin{gathered}
=\frac{\left(1-M F R_{x}\right) \cdot\left[1+\left(\eta_{H_{x}}^{T E} \cdot K_{\Pi}^{H_{x}}-1\right) \cdot \frac{T_{0 t, H_{x}}^{T E}}{T_{0 t, S h_{x}}^{T E}}\right]+M F R_{x} \cdot \eta_{S h_{x}}^{T E} \cdot K_{\Pi}^{S h_{x}}}{K_{\Pi}^{S h_{x}}} \\
=\frac{1-M F R_{x}}{K_{\Pi}^{S h_{x}}}\left[1+\left(\eta_{H_{x}}^{T E} \cdot K_{\Pi}^{H_{x}}-1\right) \cdot \frac{T_{0 t, H_{x}}^{T E}}{T_{0 t, S h_{x}}^{T E}}\right]+M F R_{x} \cdot \eta_{S h_{x}}^{T E}
\end{gathered}
$$

Now re-substituting the $K_{\Pi}^{S h x}$ and $K_{\Pi}^{H x}$ terms in the Equation 4.36, the final apparent efficiency expression for Shroud/Long Volute branch is written as follows:

$$
\begin{aligned}
\eta_{M F R_{x}(t / s)}^{S h_{x} / L V_{x}}= & M F R_{x} \cdot \eta_{S h_{x} / L V_{x}}^{T E / D V}+\frac{1-M F R_{x}}{\left(1-\left(\Pi_{S h x / L V x,(0 t, 4)}^{T E / D V}\right)^{\frac{1-\gamma}{\gamma}}\right)} \\
& \cdot\left[1+\left(\eta_{H_{x} / S V_{x}}^{T E / D V}\left(1-\left(\Pi_{H x / S V x,(0 t, 4)}^{T E / D V}\right)^{\frac{1-\gamma}{\gamma}}\right)-1\right) \frac{T_{0 t, H x / S V x}^{T E / D V}}{T_{0 t, S h x / L V x}^{T E / D V}}\right]
\end{aligned}
$$

Similarly, the final apparent efficiency expression for Hub/Short Volute branch is obtained by substituting Equation 4.31 into Equation 4.33 and further continuing the similar simplification steps as shown for the Shroud/Long Volute branch.

$$
\begin{aligned}
& \eta_{M F R_{x}}^{H x}=\frac{1-\left\{\begin{array}{c}
M F R_{x} \cdot \frac{T_{0 t, S h_{x}}^{T E}}{T_{0 t, H_{x}}^{T E}} \cdot\left[1-\eta_{S h_{x}}^{T E} \cdot K_{\Pi}^{S h x}\right]+ \\
\left(1-M F R_{x}\right) \cdot \frac{T_{0 t, h_{x}}^{T E} / h_{x}}{T F_{t, H_{x}}}\left[1-\eta_{H_{x}}^{T E} \cdot K_{\Pi}^{H}\right]
\end{array}\right\}}{K_{\Pi}^{H_{x}}} \\
& 1-M F R_{x} \cdot \frac{T_{0 t, S h_{x}}^{T E}}{T_{0 t, H_{x}}^{T E}}+M F R_{x} \cdot \eta_{S h_{x}}^{T E} \cdot K_{\Pi}^{S h_{x}} \cdot \frac{T_{0 t, S h_{x}}^{T E}}{T_{0 t, H x}^{T E}}- \\
& =\frac{1+M F R_{x}+\left(1-M F R_{x}\right) \cdot \eta_{H_{x}}^{T E} \cdot K_{\Pi}^{H_{x}}}{K_{\Pi}^{H_{x}}} \\
& =\left(1-M F R_{x}\right) \cdot \eta_{H_{x}}^{T E}+\frac{M F R_{x}}{K_{\Pi}^{H_{x}}} \cdot\left[1+\left(\eta_{S h_{x}}^{T E} \cdot K_{\Pi}^{S h_{x}}-1\right) \cdot \frac{T_{0 t, S h_{x}}^{T E}}{T_{0 t, H_{x}}^{T E}}\right]
\end{aligned}
$$


Recalling the terms $K_{\Pi}^{S h x}$ and $K_{\Pi}^{H x}$ and substituting in Equation 4.4.3, the final apparent efficiency expression for Hub/Short Volute branch is written as follows:

$$
\begin{aligned}
\eta_{M F R_{x}(t / s)}^{H_{x} / S V_{x}}= & \left(1-M F R_{x}\right) \cdot \eta_{H_{x} / S V_{x}}^{T E / D V}+\frac{M F R_{x}}{\left(1-\left(\Pi_{H_{x} / S V_{x},(0 t, 4)}^{T E / D V}\right)^{\frac{1-\gamma}{\gamma}}\right)} \\
& \cdot\left[1+\left(\eta_{S h_{x} / L V_{x}}^{T E / D V}\left(1-\left(\Pi_{S h_{x} / L V_{x},(0 t, 4)}^{T E / D V}\right)^{\frac{1-\gamma}{\gamma}}\right)-1\right) \frac{T_{0 t, S h_{x} / L V_{x}}^{T E / D V}}{T_{0 t, H_{x} / S V_{x}}^{T E / D V}}\right]
\end{aligned}
$$

It is worth noting that the final formulations showed in equations 4.37 and 4.38 are a function of actual efficiencies (not apparent), expansion ratio and total inlet temperature of both turbine branches to follow the corrected-mixed-flow hypothesis to obtain the apparent efficiency measured in a gas stand.

If both branches have the same temperature at the turbine inlets (as could be the case in some gas stand tests), equations 4.37 and 4.38 can be significantly simplified into equations 4.39 and 4.40 as shown below:

$\eta_{M F R_{x}(t / s)}^{S h_{x} / L V_{x}}=M F R_{x} \cdot \eta_{S h_{x} / L V_{x}}^{T E / D V}+\left(1-M F R_{x}\right) \cdot \eta_{H_{x} / S V_{x}}^{T E / D V} \cdot \frac{\left(1-\left(\Pi_{H_{x} / S V_{x},(0 t, 4)}^{T E / D V}\right)^{\frac{1-\gamma}{\gamma}}\right)}{\left(1-\left(\Pi_{S h_{x} / L V_{x},(0 t, 4)}^{T E / D V}\right)^{\frac{1-\gamma}{\gamma}}\right)}$

$\eta_{M F R_{x}(t / s)}^{H_{x} / S V_{x}}=\left(1-M F R_{x}\right) \cdot \eta_{H_{x} / S V_{x}}^{T E / D V}+M F R_{x} \cdot \eta_{S h_{x} / L V_{x}}^{T E / D V} \cdot \frac{\left(1-\left(\Pi_{S h_{x} / L V_{x},(0 t, 4)}^{T E / D V}\right)^{\frac{1-\gamma}{\gamma}}\right)}{\left(1-\left(\Pi_{H_{x} / S V_{x},(0 t, 4)}^{T E / D V}\right)^{\frac{1-\gamma}{\gamma}}\right)}$

Whereas, $\eta_{S h_{x} / L V_{x}}^{T E / D V}$ and $\eta_{H_{x} / S V_{x}}^{T E / D V}$ are the actual efficiencies of the individual branches that comes from Equation 4.15 with corrected-mixed-flow hypothesis that is new $z_{i}^{j}$ fitting function (Equation 4.28) inside the $K_{2}^{\star}$ term. Now, the merit function finds the coefficient values based on apparent efficiencies (equations 4.37 and 4.38) since they are what can be experimentally obtained. The $z^{\prime j}$ coefficients are the same for the actual efficiencies of two branches $\left(\eta_{S h_{x} / L V_{x}}^{T E / D V}\right.$ and $\left.\eta_{H_{x} / S V_{x}}^{T E / D V}\right)$, as the mixed turbine outlet temperature is common for both apparent efficiency definitions as expressed 
beforehand. In summary, both apparent efficiency equations 4.37 and 4.38 have 11 fitting coefficients together, and they are globally fitted using a non-linear fitting procedure for a given double-entry turbine with the whole data points from the maps of the two branches (all available MFRs). For initial values, the fitting constants of mixed turbine outlet temperature model shown in Table 4.4 were used. A statically based function, as shown in Equation 4.41, is used for minimizing the overall root mean square error of the apparent efficiencies of both branches, and also not to have very different errors between them.

$$
\varepsilon_{R M S_{\text {overall }}}=\left(\varepsilon_{R M S_{\eta_{S h x}}}+\varepsilon_{R M S_{\eta_{H x}}}\right)+\left|\varepsilon_{R M S_{\eta_{S h x}}}-\varepsilon_{R M S_{\eta_{H x}}}\right|
$$

Table 4.5: Final apparent efficiency fitting coefficients for three different double-entry turbine types. Model constants for all three turbines are found in a global fitting using both branches together and all the MFRs data points

\begin{tabular}{cccccccccccc}
\hline Turbine & \multicolumn{3}{c}{$M F R_{1}$} & \multicolumn{3}{c}{ Fitting function $\left(z^{\prime}\right)$} \\
Type & $b_{S h / L V}^{\prime}$ & $c_{S h / L V}^{\prime}$ & $f_{S h / L V}^{\prime}$ & $k_{0}^{T E / D V}$ & $k_{1}^{T E / D V}$ & $k_{2}^{T E / D V}$ & $k_{3}^{T E / D V}$ & $k_{4}^{T E / D V}$ & $b_{H / S V}^{\prime}$ & $c_{H / S V}^{\prime}$ & $f_{H / S V}^{\prime}$ \\
\hline T\#1TER & 1.4471 & 0.1517 & 0.5767 & 4.0841 & 0.0753 & 0.2193 & 0.0582 & $6.721 \mathrm{E}-03$ & 1.4164 & 0.1250 & 0.6643 \\
\hline T\#2DVR & 1.3997 & 0.1041 & 0.9309 & 4.0735 & 0.0779 & 0.1862 & 0.0241 & $2.568 \mathrm{E}-03$ & 1.4074 & 0.1354 & 0.9088 \\
\hline T\#3DVM & 1.3733 & 0.0886 & 0.8391 & 3.9726 & 0.0816 & 0.0397 & 0.0438 & $1.671 \mathrm{E}-03$ & 1.3220 & 0.0613 & 0.6822 \\
\hline
\end{tabular}

In Figure 4.19(a) the level of correlation between the experimental and modelled apparent efficiency values (equations 4.37 and 4.38) of twin-entry turbine (T\#1TER) is shown. It can be observed that modelled efficiency values of Shroud and Hub branch are well predicted with the experimental data. In absolute terms, the obtained root mean square error is below $2 \%$ points for the T\#1TER. It is worth highlighting that all the partial, full and unequal flow apparent efficiency values are well fitted at the same time with 11 fitting coefficients as shown in Table 4.5. The final efficiency equations have been applied to the dual-volute radial inflow turbine (T\#2DVR) to validate the method outlined in the above sections. Figure 4.19(b) shows the agreement between the measured and predicted apparent efficiency of long and short volute branches of the dual-volute turbines using the equations 4.37 and 4.38. This plot shows partial, full, and few unequal admission data points are taken for all the different speed lines of both turbine inlets. However, all efficiency values are shown in Figure 4.19(b) have been normalized by the peak efficiency point of each branch. Later, the found fitting constants of T\#1DVR (all MFRs) shown in Table 4.5 are used as initial values in fitting procedure to fit the dual-volute mixed inflow turbine (T\#3DVM) using the 3 MFRs. These are only MFRs measured in the gas stand at hot exposed conditions. The adiabatized data (as explained in section 3.4) were used for the model. In relative terms, the obtained mean square error for both dual-volute turbines is below $3.5 \%$ of each branch. The model is able to capture all the apparent efficiency values of two branches of double-entry turbines at different MFRs and also by fitting with less number of MFR maps (only 5 MFRs in 
the case of T\#2DVR and 3 MFRs for T\#3DVM) it is able to produce good results.
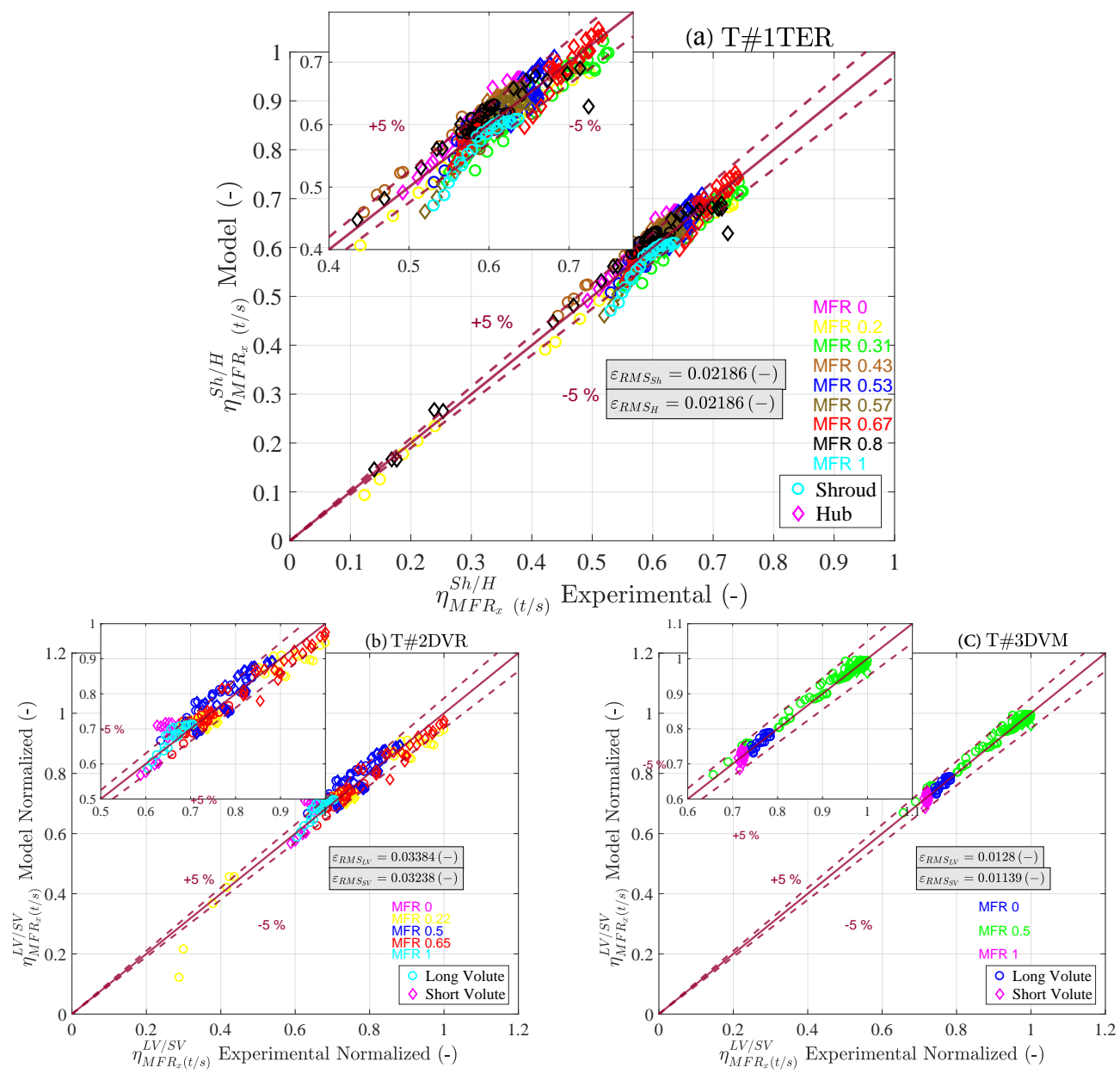

Figure 4.19: Turbine apparent efficiency modelled versus experimental results for T\#1TER, T\#2DVR, and T\#3DVM

The results showed in Figure 4.19 were obtained by also using the reduced mass flow model that described in section 4.2 , since the efficiency model is mass flow oriented, i.e., using the model outlined above with 11 fitting parameters for the efficiency and 14 parameters for the reduced mass flow. As they are in different models, the number of measured points needed for fitting both models is not the addition of all of them but the higher number, i.e. 14. Therefore, at least 14 points measured at three different MFRs (0, 0.5 and 1$)$ are needed for a global model fitting of the whole double-entry turbines.

The values of apparent efficiency model coefficients for all three double-entry turbines are summarized in Table 4.5. In which, $M F R_{0}$ and $M F R_{1}$ coefficients are applicable only for fitting the partial admission flows (i.e. having the flow only in 
one of the branches). Whereas, $z^{\prime}$ function coefficients acts as a correction factor to increase the efficiency when the model goes from partial to full admission flows. From these results, it can be concluded that the apparent efficiency model developed here can be used for both types of double-entry turbines, and it is not necessary to modify the fitting equations. Moreover, the coefficient values are similar for twin-entry and dual-volute turbines. Thus, a different calibration is not necessary, and their average values can be used as general initial values for fitting other double-entry turbines; what is valuable information for either twin-entry or dual-volute turbines simulation. It is worth noticing that, the importance of having accurate enough initial values in the non-linear fitting procedure, can assess the goodness of fit in the model. While fitting the apparent efficiency model, it is also essential to take into consideration the geometry simplifications for this type of turbines, as explained in section 4.2

Table 4.6 shows the values of correlation quality for both twin-entry and dualvolute turbines studied in these chapter and, which is calculated using the Equation 4.42 [81]. It is done to evaluate the predictive potential of the reduced mass flow and apparent efficiency models with there number of fitting coefficients.

$$
R_{a d j}^{2}=1-\frac{(n-1) \sum_{m=1}^{n}\left(V(m)_{\exp }-V(m)_{\text {pred }}\right)^{2}}{(n-c) \sum_{m=1}^{n}\left(V(m)_{\exp }-V(m)_{\text {mean }}\right)^{2}}
$$

Where in the Equation 4.42, $V_{\text {exp }}, V_{\text {pred }}$ and $V_{\text {mean }}$ are the experimental, predicted and mean experimental values respectively. Whereas, $n$ is the number of data points and $c$ is the number of fitting parameters in the model. From the $R_{a d j}^{2}$ adjusted values shown in the Table 4.6, it can be concluded that both models are well predicting the measured data, as adjusted coefficient determination values are closer to unity.

Table 4.6: Values of adjusted coefficient determination for both T\#1TER and T\#2DVR

\begin{tabular}{lcccc}
\hline \multirow{2}{*}{ Model } & \multicolumn{3}{c}{ R squared adjusted $R_{a d j}^{2}$} \\
\cline { 2 - 5 } & \multicolumn{2}{c}{ Twin Entry } & Dual Volute \\
\cline { 2 - 5 } & Shroud Branch & Hub Branch & Long Volute & Short Volute \\
\hline Reduced mass flow & 0.9963 & 0.9964 & 0.9952 & 0.9984 \\
Apparent efficiency & 0.9333 & 0.9287 & 0.9572 & 0.9319 \\
\hline
\end{tabular}




\subsection{Extrapolation of turbine performance parameters}

The methods for extrapolating the double-entry turbines performance parameters are based on the models developed in the section 4.2 and section 4.4. Both turbine branches should be extrapolated simultaneously since, the apparent efficiency model of each turbine branch shown in equations 4.39 and 4.40 depend on the expansion ratios, actual efficiency and total inlet temperature between there turbine inlet branches. Besides, the reduced mass flow and apparent efficiency equations of each turbine inlet branch are interrelated. As the apparent efficiency (Equations 4.39 and 4.40) appears in the effective equivalent nozzle area (Equation 4.1) of the reduced mass flow model and the Equation 4.1 appears in the apparent efficiency Equations 4.39 and 4.40. Therefore, a system with the effective equivalent nozzle area of reduced mass flow and the apparent efficiency equations of both turbine branches should be solved together using an iterative procedure for the extrapolation purpose.

In Figure 4.20, the procedure used for extrapolating the reduced flow and efficiency values of double-entry turbine models for both entries at the same time is presented. Following the flowchart, the extrapolation procedure starts with the input of the available map data of each turbine branch. By using this information, the effective equivalent nozzle area of each turbine branch can be solved from Equation 4.1 with the non-linear fitting procedure for finding the appropriate coefficient values (' $a$ ', ' $b$ ', ' $c$ ' and ' $d$ ') of each turbine branch. Later, using the fitted ' $d$ ' value, the next step will be estimating the rotor inlet flow angle with the help of turbine geometry and also data from the maps.

Later on, using the information of efficiency map and the estimated $A_{e f f, i}^{j}$ and $\tan \alpha_{33, i}^{j}$ flow values from the Equation 4.1 and Equation 4.21 of each turbine branch, the apparent efficiencies equations 4.37 and 4.38 are fitted together to find the 11 fitting parameters that are discussed in the subsection 4.4.3. After obtaining all the necessary coefficients for model and further keeping them constant, the system made of equations 4.1, 4.39 and 4.40 is solved together with an iterative procedure to obtain the extrapolated values of equivalent nozzle area and apparent efficiencies of each turbine inlet. Finally, by substituting the extrapolated equivalent nozzle area values $\left(A_{e f f, i}^{j}\right)$ in the Equation 4.11, the extrapolated reduced mass flow of each branches can be obtained.

\subsubsection{Twin-entry radial inflow turbine}

In this section, the extrapolation results of twin-entry turbines are discussed. For the extrapolation of reduced mass flow parameters, the coefficients from Table 4.1 which are graphically shown in the Figure 4.6 were used. Whereas in the case of apparent efficiency, the fitting coefficients shown in Table 4.5 were used. 


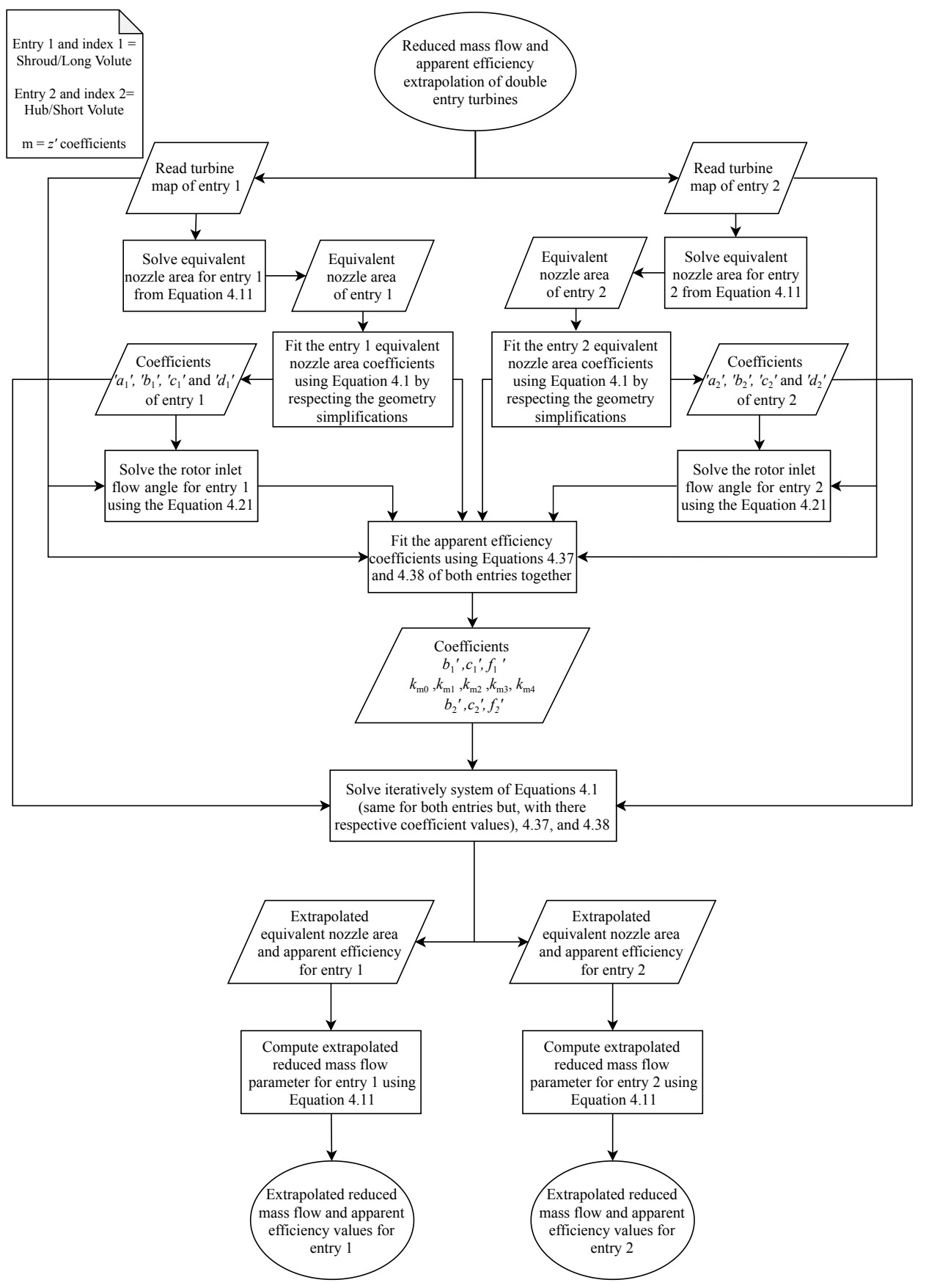

Figure 4.20: Procedure for extrapolating the reduced mass flow and apparent efficiency of double-entry turbines 


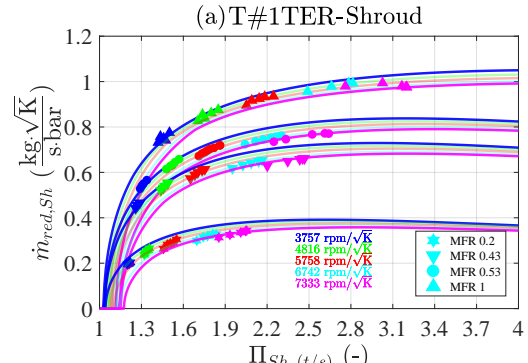

(c) MFR0.2:T\#1TER-Shroud

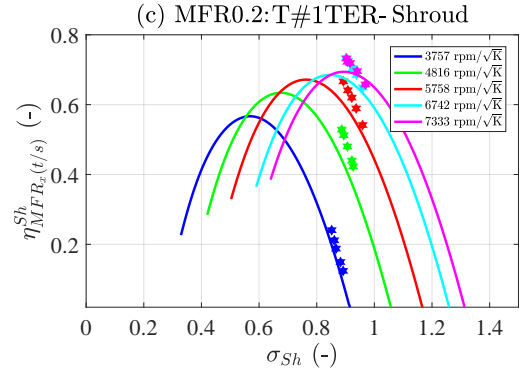

(e) MFR0.43:T\#1TER-Shroud
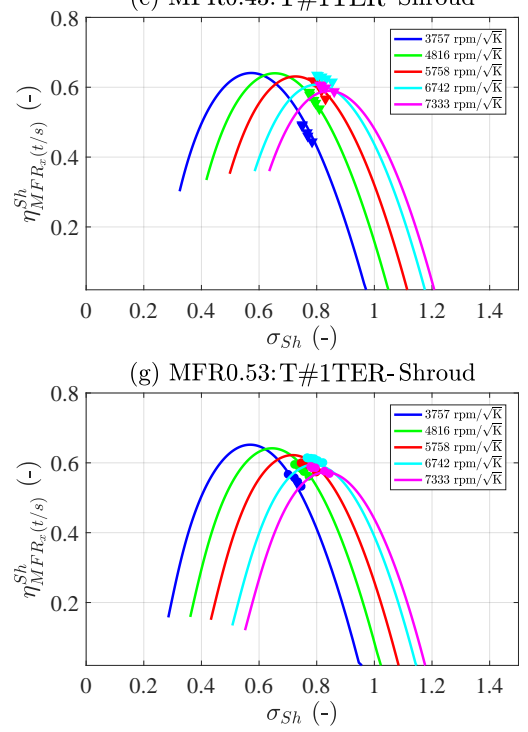

(i) MFR1:T\#1TER-Shroud

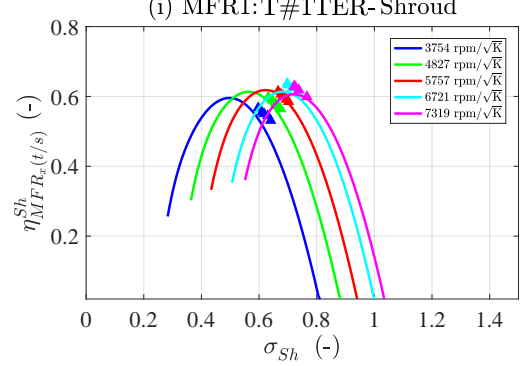

(b) T\#1TER-Hub

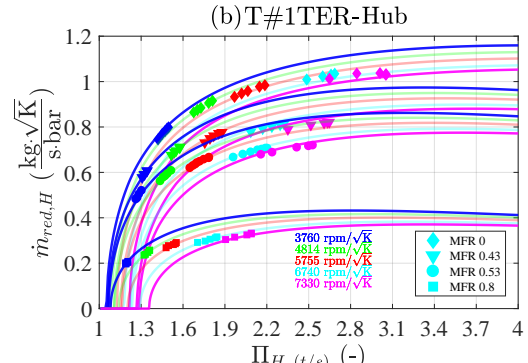

(d) MFR0.8:T\#1TER-Hub

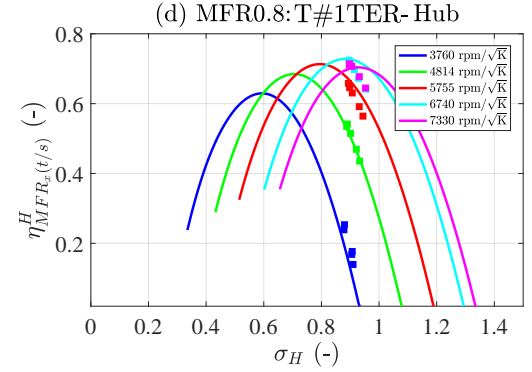

(f) MFR0.43:T\#1TER- Hub

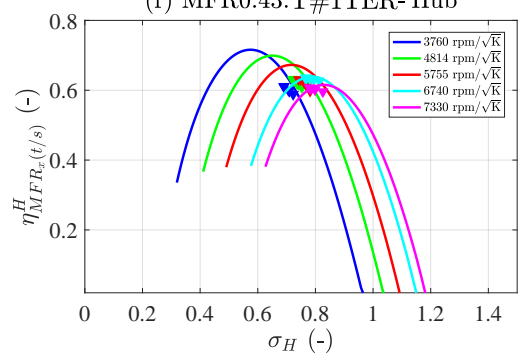

(h) MFR0.53:T\#1TER-Hub

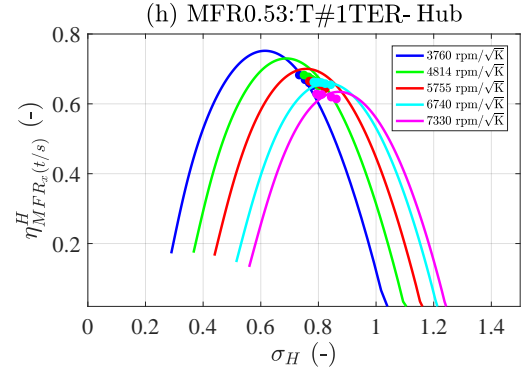

(j) MFR0:T\#1TER- Hub

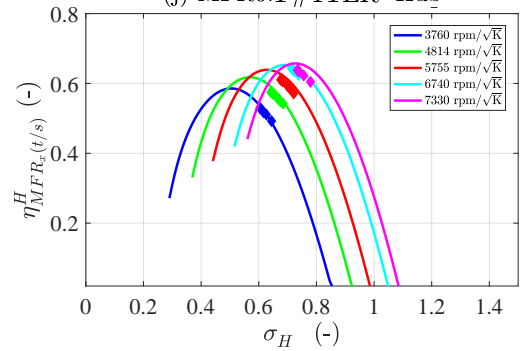

Figure 4.21: Twin-entry turbine (T\#1TER) reduced mass flow and apparent efficiency extrapolation for shroud and hub branches using all maps fitting coefficients. 
Figure 4.21 shows the model extrapolation results against experimental data for both reduced mass flow and apparent efficiency of T\#1TER in shroud and hub branches. In Figure 4.21, the results of some MFRs were disclosed; in which it includes the partial, full and unequal flow admission conditions in the turbine. From Figure 4.21, it is clear that the extrapolation produced by the model (solid lines) at both lower and higher turbine reduced speeds in all the shown MFRs are not only in considerable accordance with the experimental data (different symbol points) for both branches but producing expected and consistent results. The extrapolation results of reduced mass flow parameters of shroud and hub branches are shown in Figure 4.21(a) and (b). In the shroud branch, it is notable that there is a small distance between the reduced mass flow extrapolation lines at different reduced speeds and for a given pressure ratio. This effect is due to the lower rotor inlet to outlet radius ratio in shroud branch (see Figure 4.3). Therefore, this branch is acting like a high trimmed radial inflow turbine. However, this not the case in hub branch (Figure 4.21(b)), due to the higher rotor inlet to outlet radius ratio.

Figure 4.21(c) shows the extrapolation results of $\mathrm{MFR}=0.2$ where the flow conditions are lower in shroud entry and the model able to produce good accordance with the experimental data. However, it is notable that there are some differences found between the extrapolated and measured data at higher reduced speeds. Nevertheless, when the shroud branch is working at MFR $=0.2$, most of the flow expansion is produced by the hub branch and not by shroud one. Therefore, the actual efficiency of this branch should be much lower than shown apparent efficiency since it is getting the benefit of the lower mixed outlet temperature generated by the higher expansion in the other branch. Similar results can be seen in Figure 4.21(d) when the turbine is working at $\mathrm{MFR}=0.8$; in this case, there is more flow in the shroud than hub branch.

Figure 4.21(e) and (f) shows the extrapolation results of unequal admission conditions, where the hub branch is having more flow than the shroud one. For this case, the model is able to reproduce apparent efficiency values with good precision for both inlets at all reduced turbine speeds. Figure 4.21(g) and (h) shows the extrapolation results when the turbine is working at almost full admission conditions, and the model well captured the peak efficiency points of both branches. From the experimental data analysis that showed in the subsubsection 3.5.2.2, it was concluded that the maximum apparent efficiencies are always found when there is more flow in the hub side. The extrapolation results from the model are also showing the same effect, i.e., that the peak efficiencies of different reduced speeds can be seen with the higher flows in the hub branch. Moreover, the model extrapolations of having the flow only in a shroud or hub branch are also well captured by the model as it can be seen in Figure 4.21(i) and (j). The overall quality of the prediction is high and, both the reduced mass flow and apparent efficiency models able to bring out reasonable extrapolation in all the turbine reduced speeds in both branches. 


\subsubsection{Dual-volute radial inflow turbine}

In this section, the extrapolation results of the dual-volute radial inflow turbine are discussed. Figure 4.22 shows the extrapolation results of reduced mass flow against the expansion ratio and apparent efficiency against the blade speed ratio for both Long Volute (LV) and Short Volute (SV) entries of T\#2DVR. In the case of reduced mass flow extrapolation, Figure 4.22(a) and (b), the results were presented for certain MFRs where it includes the partial, full and unequal admission conditions in both volutes.

(a) T\#2DVR - Long Volute

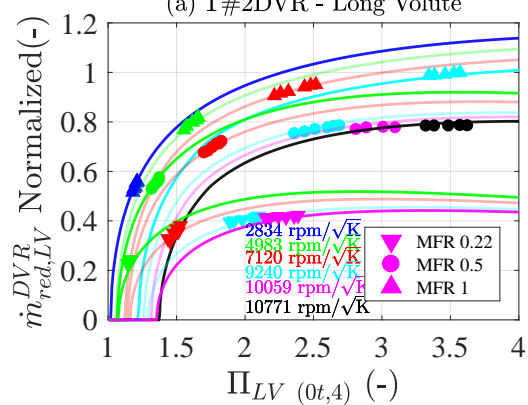

I 12 (c) MFR0.22: T\#2DVR - Long Volute

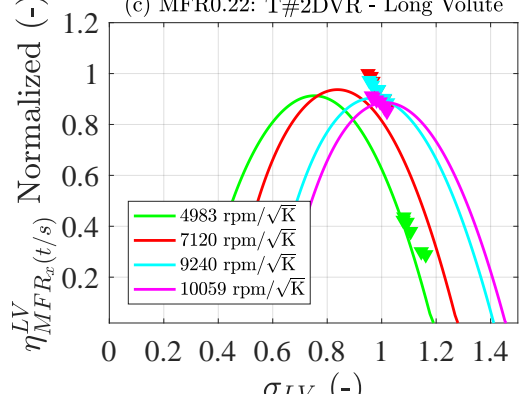

$\sigma_{L V}(-)$

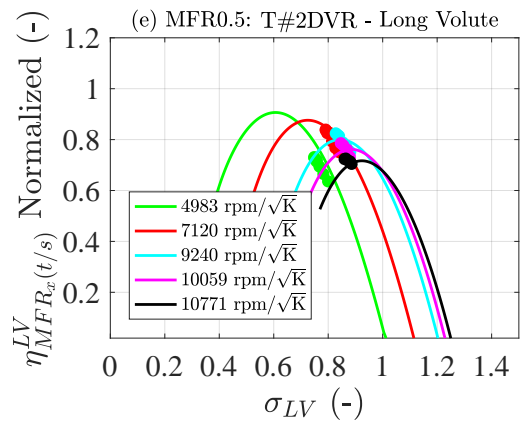

(b) T\#2DVR - Short Volute

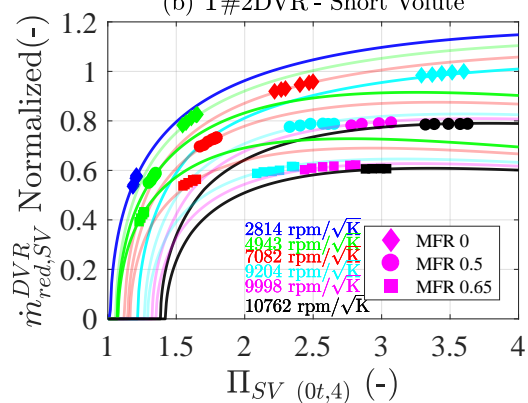

I 1 (d) MFR0.22: T\#2DVR - Short Volute
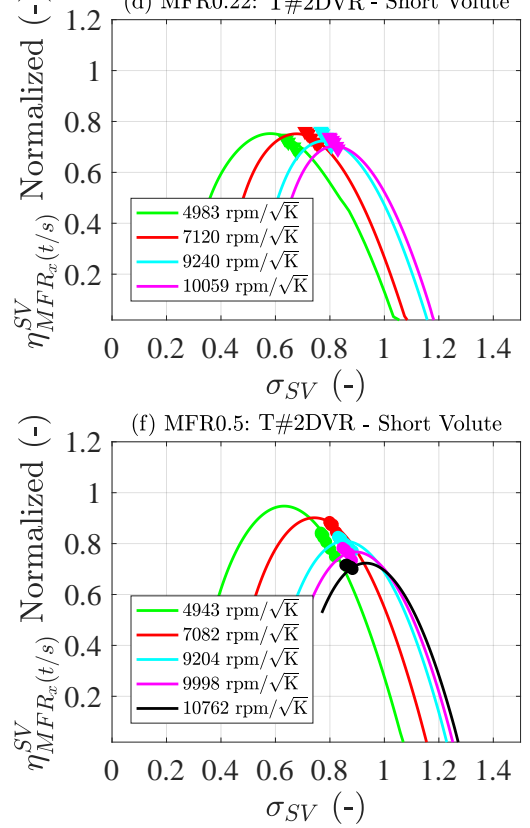

Figure 4.22: Cont 

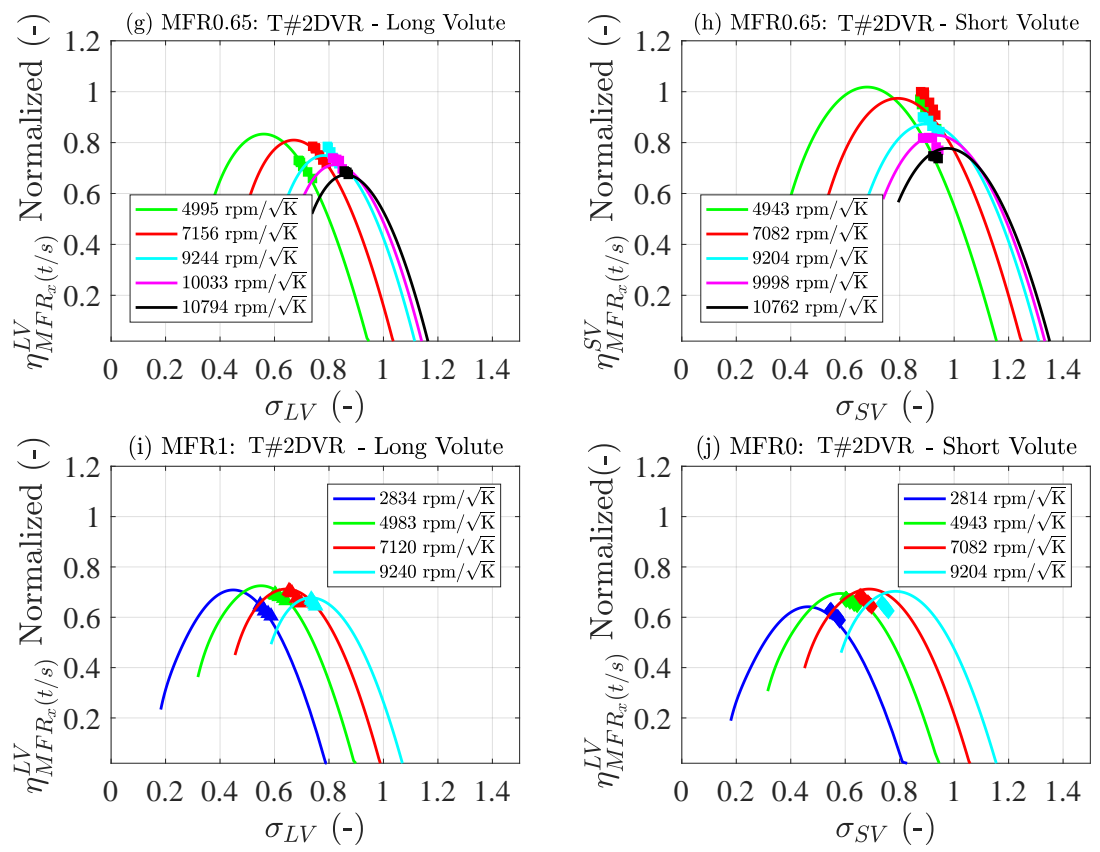

Figure 4.22: Dual-volute turbine (T\#2DVR) reduced mass flow and apparent efficiency extrapolation for LV and SV volutes using only the filled points fitting coefficients.

From the Figure 4.22(a) and (b), it can be seen that the agreements between the model extrapolation (lines) and the experimental data points (symbols) are effective. In Figure 4.22(a), small discrepancies are noteworthy in the extrapolation of $\mathrm{MFR}=0.22$ and $\mathrm{MFR}=1$ at lower and higher turbine reduced speeds. In the case of turbine apparent efficiency extrapolation, the results are presented for all the MFRs (see Figure 4.22(c) to (j)). The difference between the model and experimental data is maximum in the case of $\mathrm{MFR}=0.22$ in the long volute at higher reduced speeds. Aforementioned, the turbine inlets (LV/SV) with the lower flows (i.e., MFR $=0.2 / 0.8)$, their actual efficiencies will be different than the apparent efficiencies, as branches with lower flows getting the benefit of lower mixed turbine outlet temperature generated by the higher expansion in the other branch. Similar results can be found in Figure $4.22(\mathrm{~h})$ when the turbine is working at $\mathrm{MFR}=0.65$. In this case, there is more flow in the LV entry than SV entry, and the discrepancies are only seen at lower reduced turbine speeds. Moreover, in full admission (see Figure 4.22(e) and (f)) and partial admission (see Figure 4.22(i) and (j)) states, the agreements between the model extrapolation lines and experimental data points in both volutes is considerably well. 


\subsubsection{Dual-volute mixed inflow turbine}

Figure 4.23, shows the results of model extrapolation for turbine T\#3DVM up to nonmeasured data points. It is evident that for full $(\mathrm{MFR}=0.5)$ and partial admission cases $(\mathrm{MFR}=0$ and 1 ), the model extrapolation in reduced mass flow (Figure 4.23 (a), (c), (e) and (g)) and apparent efficiency (Figure 4.23 (b), (d), (f) and (h)) perfectly fits the experimental data, and extrapolates with coherence even with three MFRs $(=0,=1$ and $=0.5)$ that used for fitting the whole model. Because of there are not unequal admission measurements for this particular turbocharger. As a reminder: while fitting the turbine T\#3DVM, the obtained coefficients from T\#2DVR turbine are used as initial values in the non-linear fitting procedure.
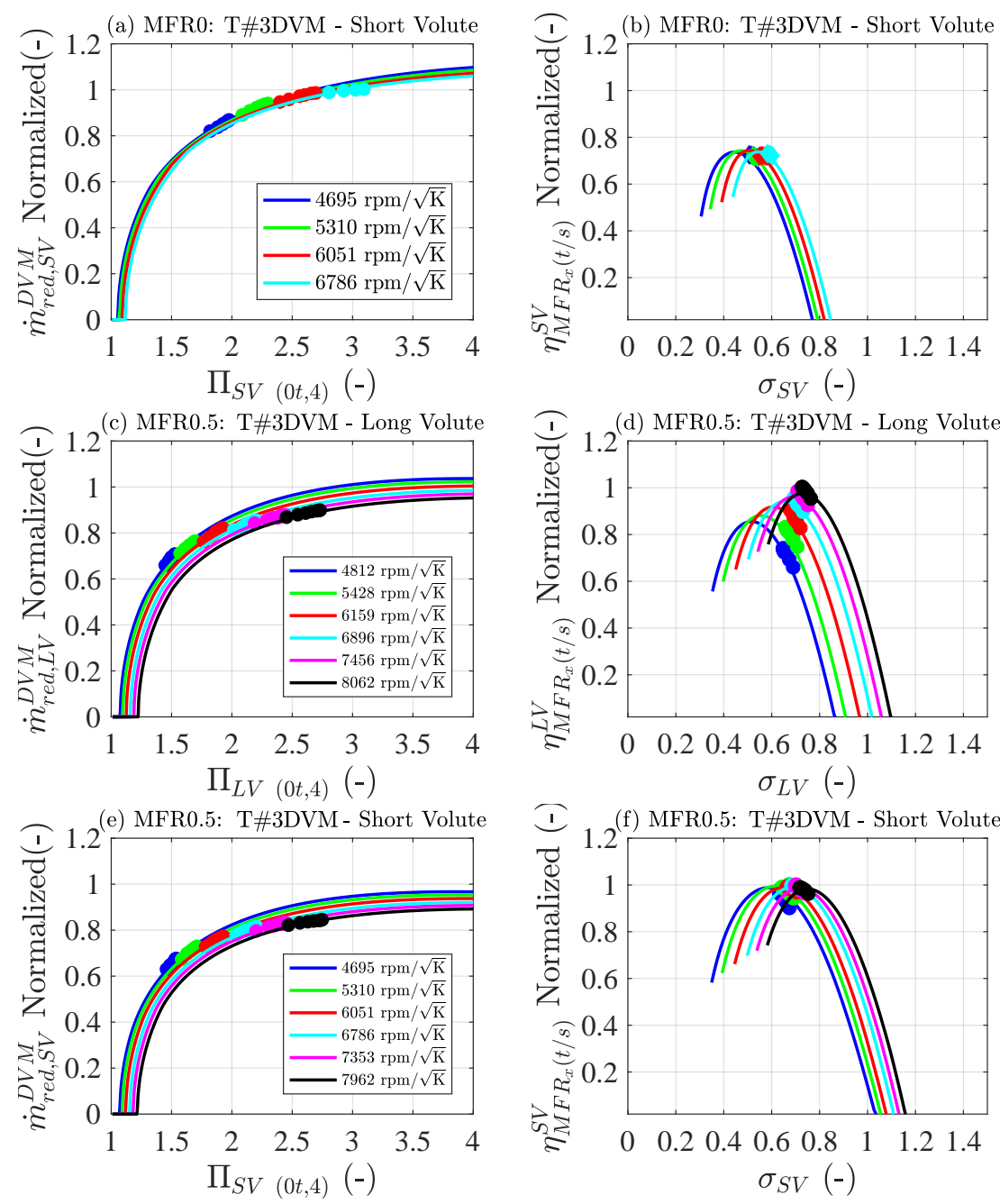

Figure 4.23: Cont. 

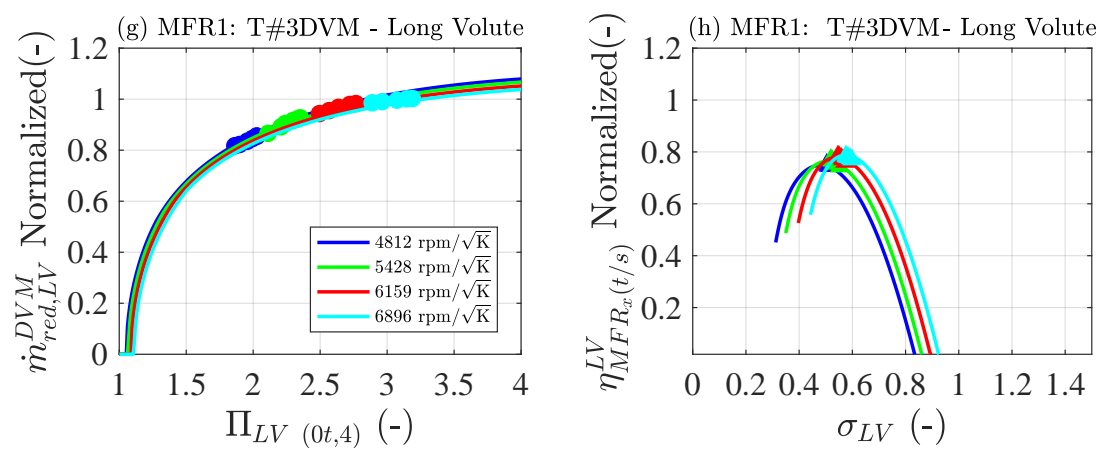

Figure 4.23: Dual-volute turbine (T\#3DVM) reduced mass flow and apparent efficiency extrapolation for LV and SV volutes using only 3 MFRs ( $0,0.5$ and 1$)$.

\subsection{Extrapolation of turbine performance parameters to non-measured MFRs}

It is worth introducing this section by noticing that the 25 constants are needed for fitting a double-entry turbine model: 14 constants, from which arise plots of Figure 4.6, for Equation 4.1 plus 11 constants, as in Table 4.5, for Equation 4.15. Moreover, fitting of both branches is done at the same time with all available flow conditions of the turbine. Therefore, one wonders: What is the minimum number of MFRs required for fitting the model? Also, if could it be fitted using only the three MFRs which are easiest to be measured $(\mathrm{MFR}=0,=1$ and $=0.5)$ and still predicting whatever another non-measured MFR? Accordingly, in this section, the prediction capabilities of reduced mass flow and actual adiabatic efficiency models are analyzed. That is, using only two partial and full admissions data for both twin-entry (T\#1TER) and dual-volute (T\#2DVR) radial inflow turbines.

\subsubsection{Twin-entry turbine using 3 MFRs}

In this section, the prediction results of reduced mass flow and apparent efficiency models for the T\#1TER turbine using 3 MFRs are discussed. A standard nonlinear fitting procedure is performed using the two partial, and full admission maps to find the fitting parameters of both models. Figure 4.24 shows a very high level of correlation between the experimental data and model predictions. It is worth to note once again that the MFRs $=0,=0.5,=1$ are the only MFRs used for fitting (highlighted as bold letters in Figure 4.24) and all the other MFRs are not, but just predicted by the models. Both the reduced mass flow and apparent efficiency model results using the all MFRs data (see Figure 4.8(a) and Figure 4.19(a)) and using only three MFRs (see Figure 4.24), the level of accuracy is not much different between them. 

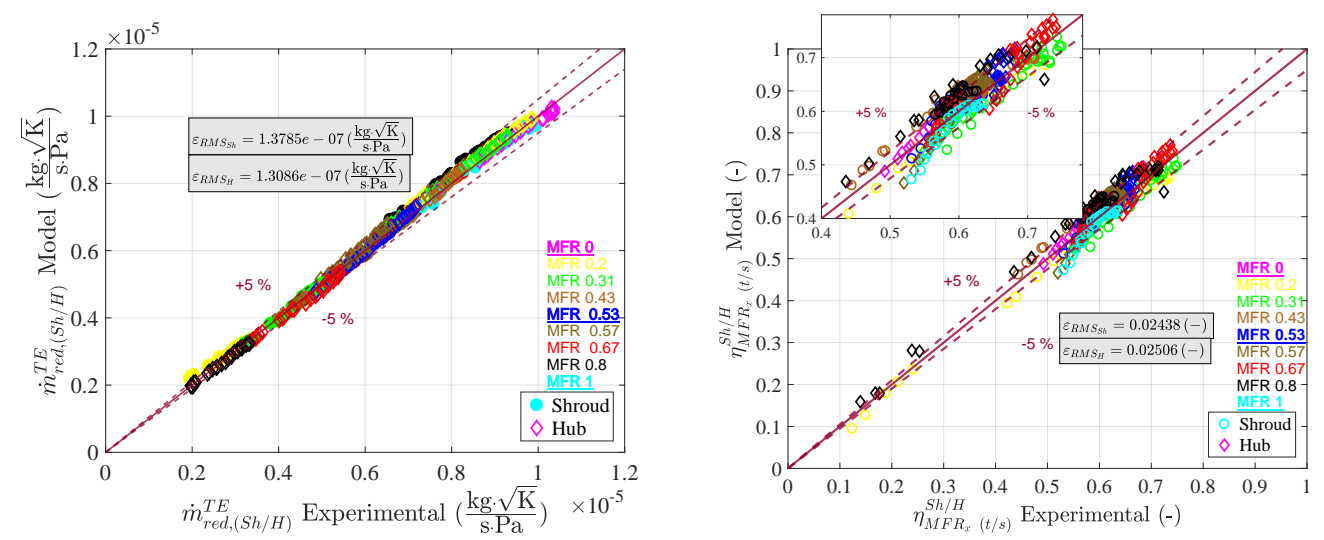

Figure 4.24: Prediction of model results using 3 maps of twin-entry radial inflow turbine during the fitting procedure

Figure 4.25, shows the results of model extrapolation for the twin-entry turbine up to non-measured data points (following the procedure shown in Figure 4.20). In Figure 4.25, the results of only some measured unequal admission conditions (not used for the model fitting) are disclosed. Which generally shows a good agreement with the experimental data points Figure 4.25(c) and (d) shows the apparent efficiency extrapolation results when the shroud entry turbine is working with the lower flow and hub with higher flow. The model can produce good accordance with the experimental data. However, at higher turbine reduced speeds, some error between the extrapolated and measured data is notable in shroud branch. Nevertheless, in these conditions, most of the flow expansion is produced by the hub branch. Accordingly, the actual efficiency of the shroud branch should be much lower than the shown apparent efficiency. Moreover, similar results can be seen in Figure 4.25(i) and $(\mathrm{j})$ when the turbine is working at $\mathrm{MFR}=0.8$. In this case, there is more flow in the shroud branch than hub branch. At other unequal admission conditions, i.e. for $\mathrm{MFR}=0.43$ and $=0.57$ shown in Figure 4.25 , the model is again able to predict the reduced mass flow and apparent efficiency values with good accuracy in both entries at all turbine reduced speeds.
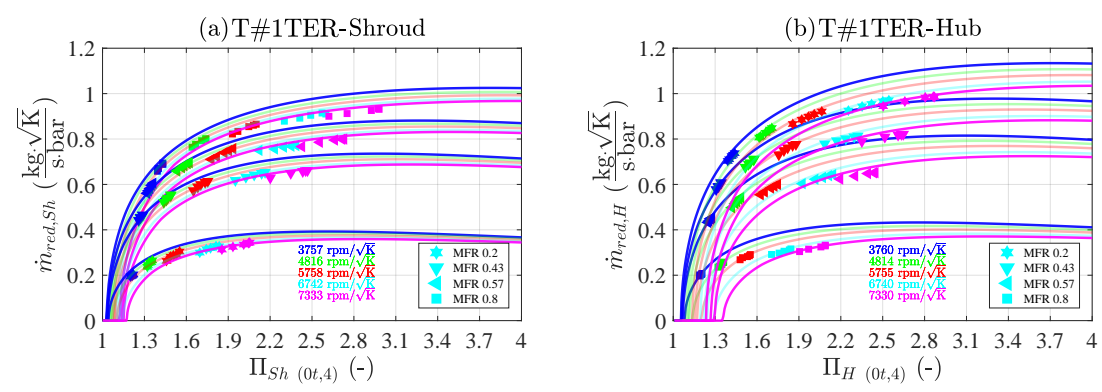

Figure 4.25: Cont. 
(c) MFR0.2:T\#1TER-Shroud

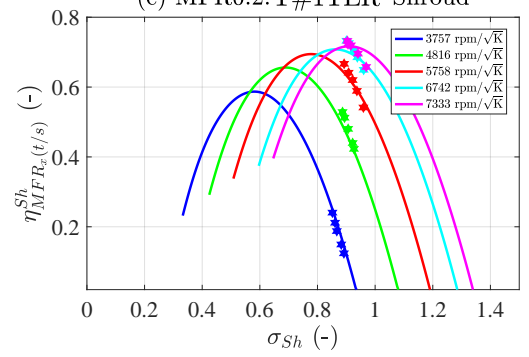

(e) MFR0.43: T\#1TER-Shroud

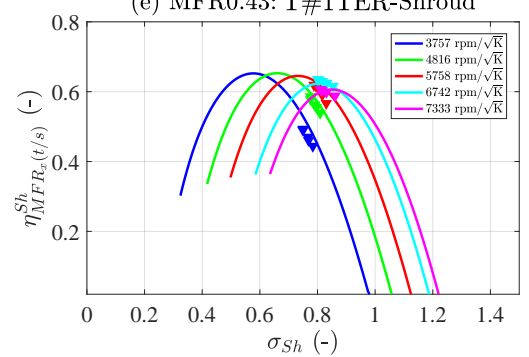

(g) MFR0.57: T\#1TER-Shroud

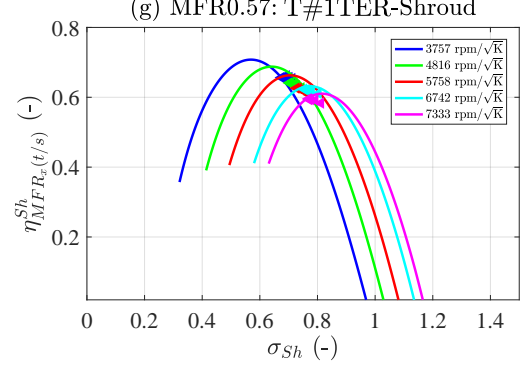

(i) MFR0.8: T\#1TER-Shroud

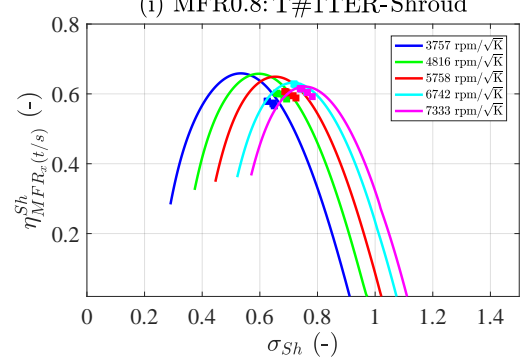

(d) MFR0.2: T\#1TER-Hub

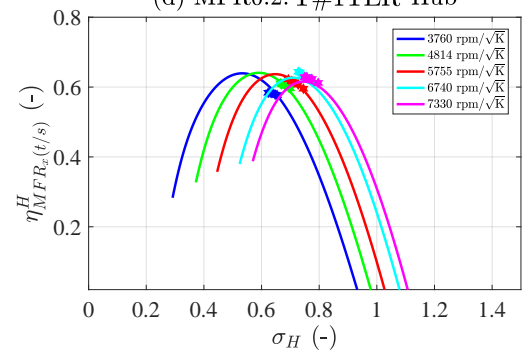

(f) MFR0.43: T\#1TER-Hub

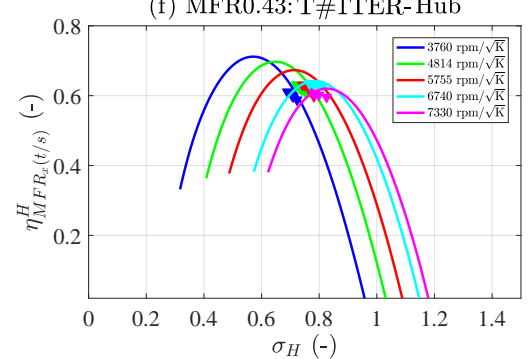

(h) MFR0.57: T\#1TER-Hub

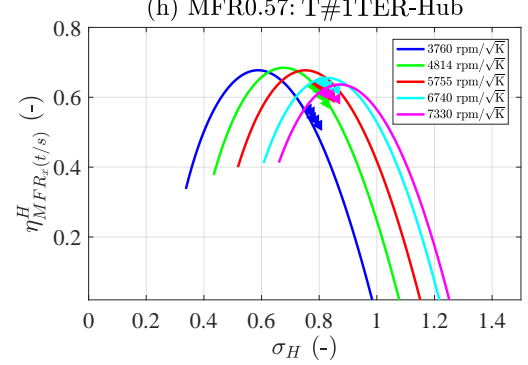

(j) MFR0.8: T\#1TER-Hub

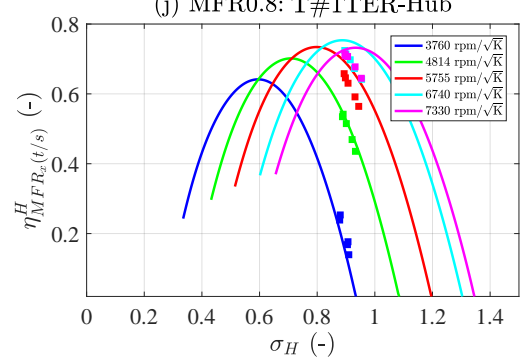

Figure 4.25: Twin-entry turbine (T\#1TER) reduced mass flow and apparent efficiency extrapolation for shroud and hub branches using only 3 MFRs $(0,1$ and 0.5$)$ model fitting constants. 


\subsubsection{Dual-volute radial inflow turbine using 3 MFRs}

In this section, the prediction results of the reduced mass flow and apparent efficiency models for dual-volute radial inflow turbine T\#2DVR using 3 MFRs are discussed. Again, the model is fitted with only 3 MFRs, which are highlighted as bold letters in the Figure 4.26, being predicted by the model the unequal admission conditions data points $(\mathrm{MFR}=0.22$ and $=0.65)$. Figure 4.26 shows the very high level of correlation between the experimental data and model predictions for unequal admission state data points. Moreover, both models with only three MFRs, the level of accuracy is not much different with the one fitted using all the MFRs (see Figure 4.8(b) and Figure 4.19(b)).
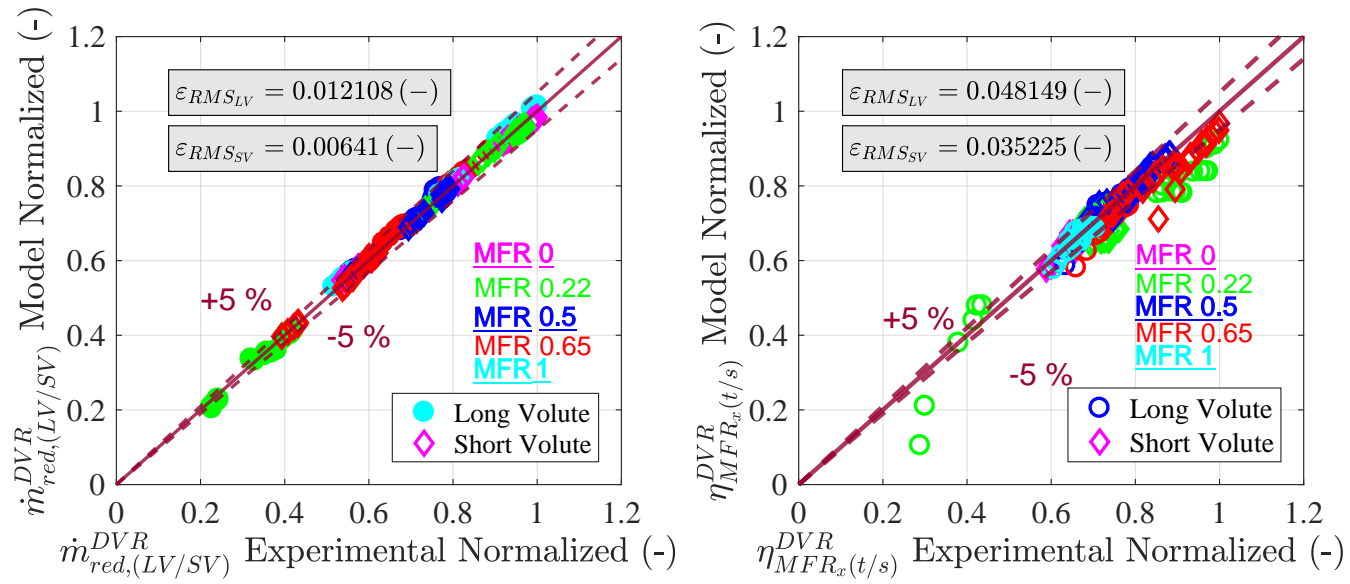

Figure 4.26: Prediction of model results using 3 maps of dual-volute radial inflow turbine during the fitting procedure

Figure 4.27, shows the model extrapolation results for the T\#2DVR turbine up to non-measured data points. In the Figure 4.27, the results of only unequal admission conditions (which are not used for the model fitting) are disclosed; showing the general agreements with the experimental data points. In the case of turbine apparent efficiency extrapolation (see Figure 4.27(c) to (f)) of unequal admission, on the one hand, big differences are found in between the experimental and model data at higher turbine reduced speeds in MFR $=0.22$ of both volutes of the turbine; as already shown in Figure 4.26(b). On the other hand, for $\mathrm{MFR}=0.65$ unequal admission condition, the significant error is only found at turbine reduced speed of $4943 \mathrm{rpmK}^{-0.5}$ in the short volute. The efficiency outcomes from the model for other speed lines for both volutes at $\mathrm{MFR}=0.65$ are well aligned with the experimental data points. 
(a) T\#2DVR - Long Volute

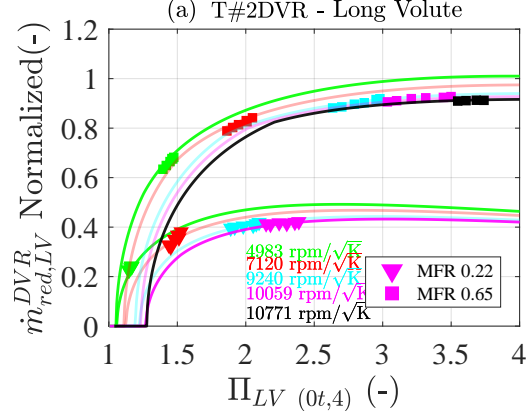

I 1.2 (c) MFR0.22: T\#2DVR - Long Volute



I 1.2 (e) MFR0.65: T\#2DVR - Long Volute

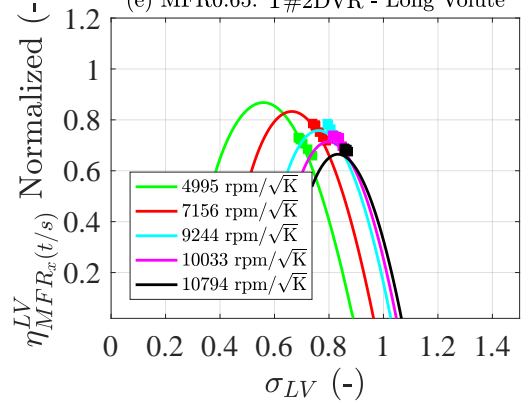

(b) T\#2DVR - Short Volute

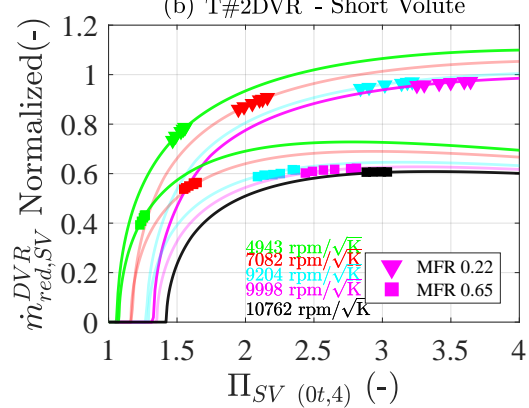

I 1.2 (d) MFR0.22: T\#2DVR - Short Volute

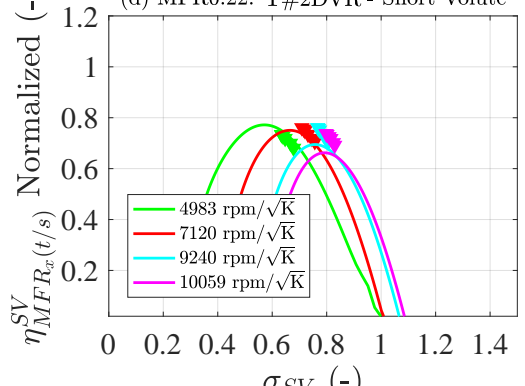

$\sigma_{S V}(-)$

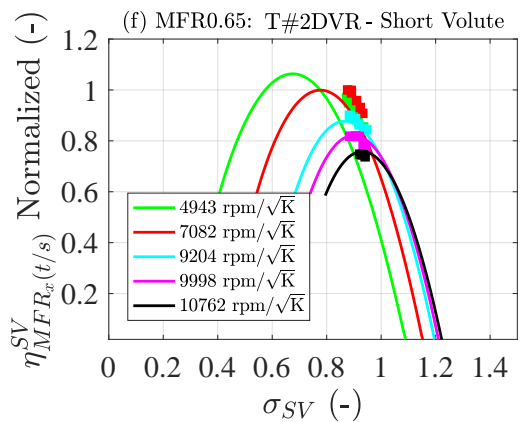

Figure 4.2\%: Dual-volute turbine (T\#2DVR) reduced mass flow and apparent efficiency extrapolation for long and short volutes using only 3 MFRs (0, 1 and 0.5$)$ model fitting constants

After observing the results of models using only three MFRs (0, 0.5 and 1) for different double-entry turbine geometries; it can be concluded that the general quality of predictions in reduced mass flow and apparent efficiency parameters is good enough for quasi-steady prediction of turbine efficiency working with pulsating flow from staggered cylinder outlets. Further, it is considered that the model can produce coherent and sensible enough extrapolations of the same parameters against MFR changes for any double-entry turbines. Moreover, the coefficients that are shown in the Table 4.5 (using all MFRs) for different geometries of double-entry turbines are similar. Further, they can be used as general initial values for a non-linear fitting procedure of other double-entry turbines, which is valuable information for any double-entry turbine simulation. 


\section{Chapter 5}

\section{Characterization of wastegate and scroll connection valves in double-entry turbines}

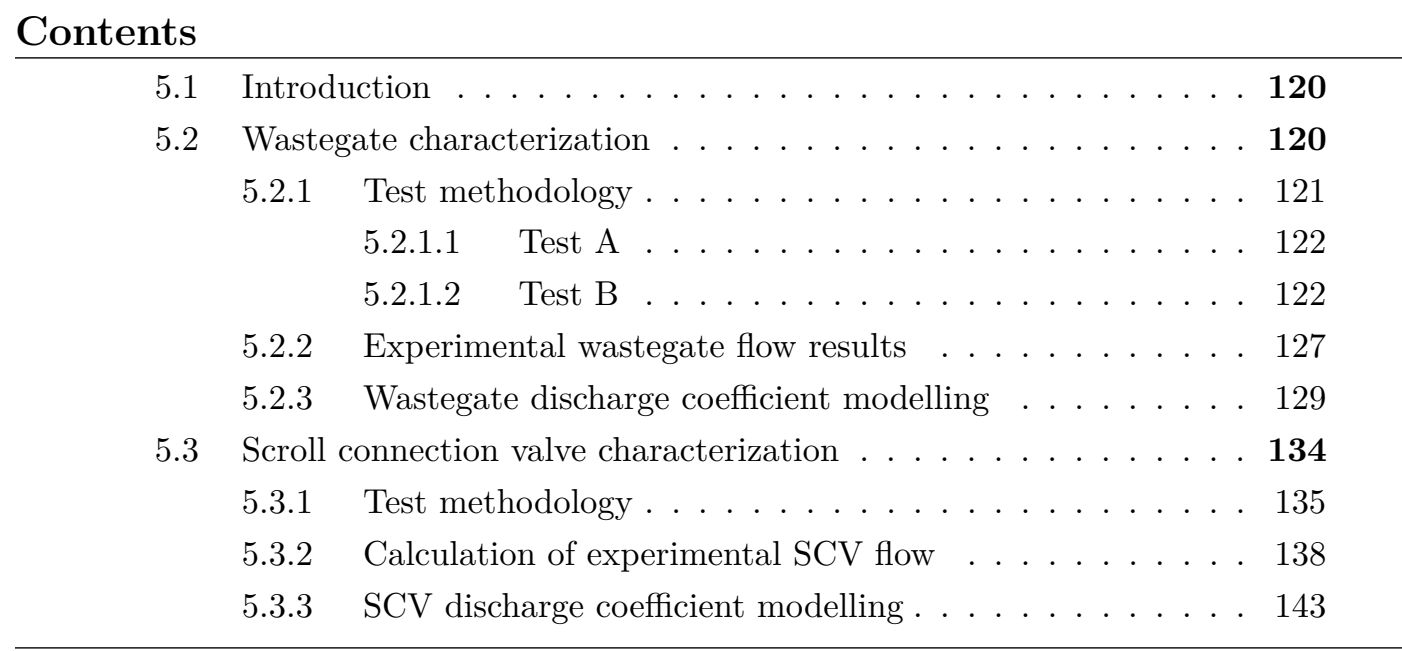




\subsection{Introduction}

In this chapter, a methodology which has been developed to characterize the discharge coefficient of an electronic wastegate valve and a scroll connection valve (SCV) in the double-entry turbines is described. It is useful in one-dimensional modelling for estimating the gas flow through the wastegate and SCV. When the SCV is present in a turbine, the flow can communicate between the turbine branches before going into the rotor. Usually, the scroll connection valve can be found in a dual-volute turbine. The advantage of having this valve is that it will allow the dual-volute turbines to work as a single entry turbine. Firstly, the test method and modelling of the wastegate discharge coefficient will be presented. Later, the characterization of scroll connection valve will be shown.

The works, analysis and ideas described in this chapter were the origins of publication number [1] from the publications list of the author of this thesis. In the sake of readiness and to protect the thesis writing style the publication number [1] from author's list of publications have not been specifically cited every time that ideas, figures or discussions contained in it are bring to this chapter again. This disclaimer corrects, compensates and justify this fact; being the Ph.D. candidate and the works of his thesis the origin of the innovation component in the publication number [1] listed in the referred section.

\subsection{Wastegate characterization}

The characterization of wastegate valve is done for two turbine types: one is twinentry (T\#4TER) and another dual-volute (T\#2DVR). In the case of T\#4TER turbine firstly, the turbine and compressor maps were measured at full admission conditions with two different turbine inlet temperature levels (moderate and high) in hot exposed (by allowing internal and external heat transfers). Later, for the points corresponds to those equivalent to engine full load conditions, the wastegate discharge coefficient characterization is performed with temperature levels similar to the engine exhaust. Whereas, T\#2DVR was characterized at adiabatic conditions for selected expansion ratios that work in a safe zone of the compressor map. The expansion ratios are selected from the full admission map of T\#2DVR turbine, which was already tested in the gas stand as detailed in chapter 3. It is worth highlighting that the procedure for characterizing the wastegate valve is done when the turbine is working at full admission conditions. As the flow conditions are equal in both turbine inlets, the turbine map is represented as similar to the single entry turbine, i.e., using the Equations from 2.11 to 2.12 that are shown in chapter 2 . 


\subsubsection{Test methodology}

The tests to characterize turbocharger wastegate valve were performed in a hot and high flow gas stand that detailed in the subsection 3.2.1 of chapter 3 and the same is shown in Figure 5.1. For characterizing the discharge coefficient of the wastegate experimentally, two tests were performed, Test A and Test B as outlined in the Figure 5.2 where the temperature, pressure, and mass flow measurement points are represented. Both tests are performed in full admission conditions, and all the inlet and outlet parameters were registered.

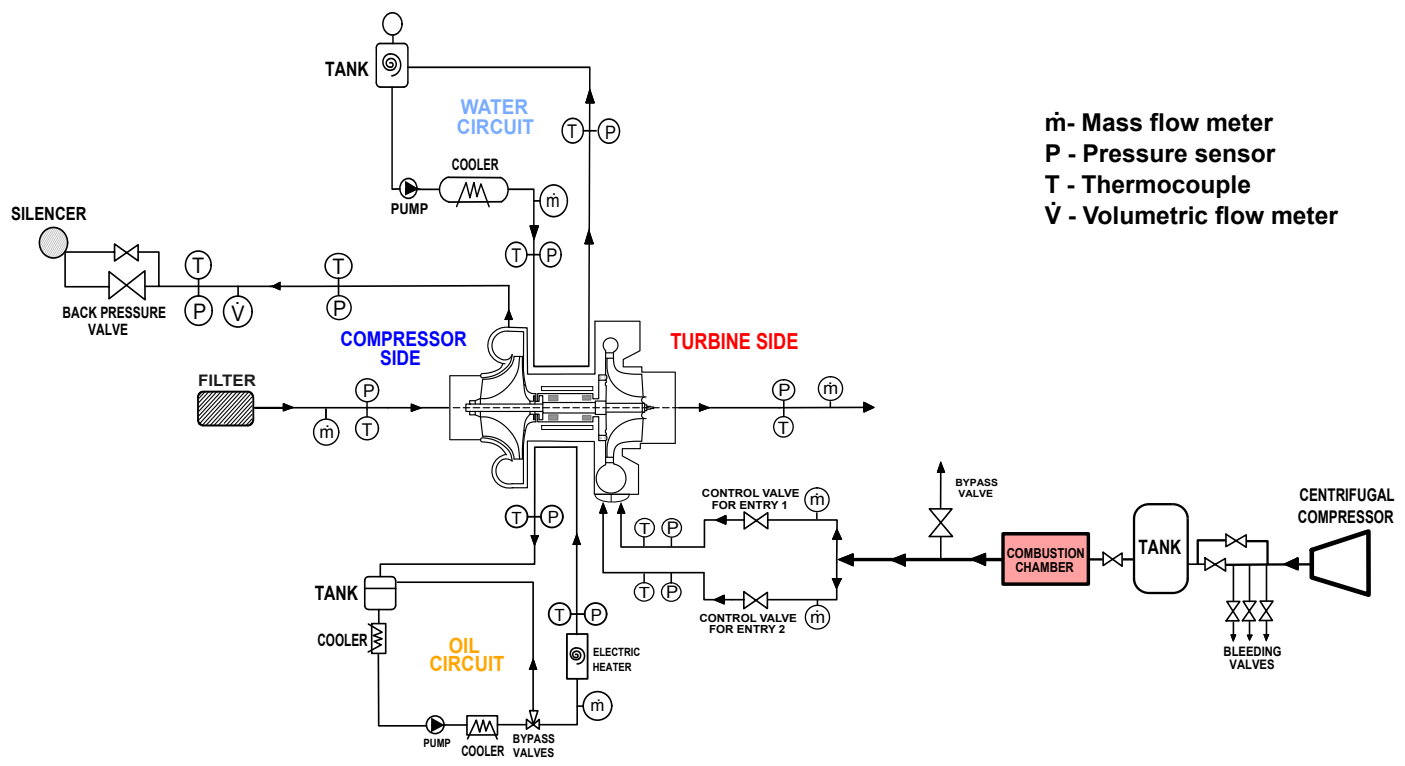

Figure 5.1: Schematic layout of turbocharger gas stand

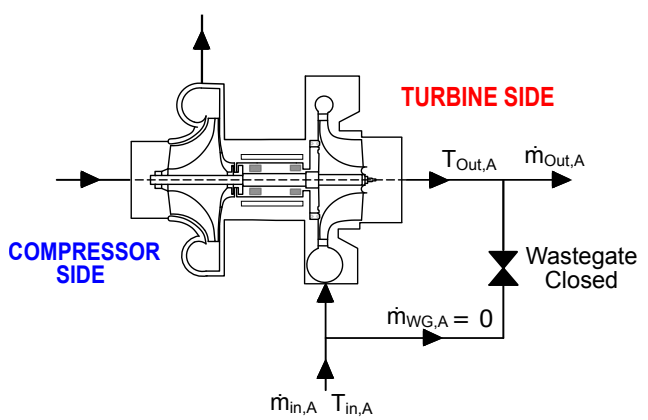

Test A with wastegate mechanically closed

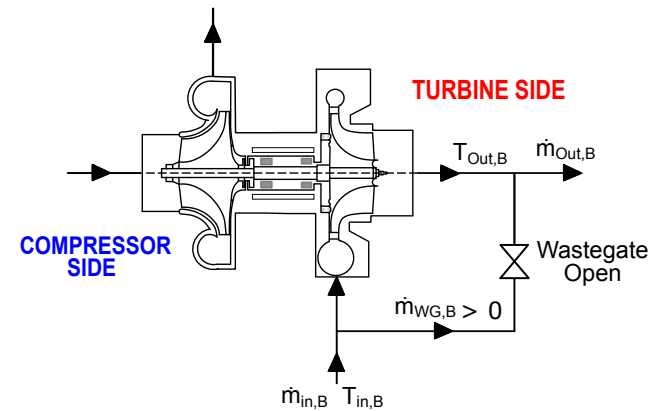

Test B with wastegate opened

Figure 5.2: Test method for characterizing wastegate valve experimentally in a gas stand 


\subsubsection{Test A}

In test $\mathrm{A}$, the turbocharger is characterized in a closed position of the wastegate valve. In order to ensure there is no leakage of mass flow from the wastegate side, the valve is closed with a mechanical stopper. For every tested point in test A, the important parameter like pressure, temperature, the mass flow of turbine and compressor and turbocharger speed were registered. The results of test A are shown in the Figure 5.3 for both turbine types. Figure 5.3(a) shows the map of T\#4TER turbine where it was obtained using two different turbine inlet temperatures levels (high, filled points and moderate, non-filled points). In this way, more extended maps were measured, compared to the traditional procedures with only one turbine inlet temperature. Filled and non-filled points are superimposed in Figure 5.3(a) showing that $T_{03}$ does not affect the reduced mass flow characteristic lines, as expected. This fact confirms that the experimental data of T\#4TER is coherent. Figure 5.3(b) shows the map of T\#2DVR turbine, which was tested in adiabatic conditions. It is worth highlighting that, in Figure 5.3(b), the mass flow values are normalized with the maximum mass flow value when the wastegate is opened. In both figures, the grey circled points represents the points that were given/selected for the characterization of the wastegate discharge coefficient.
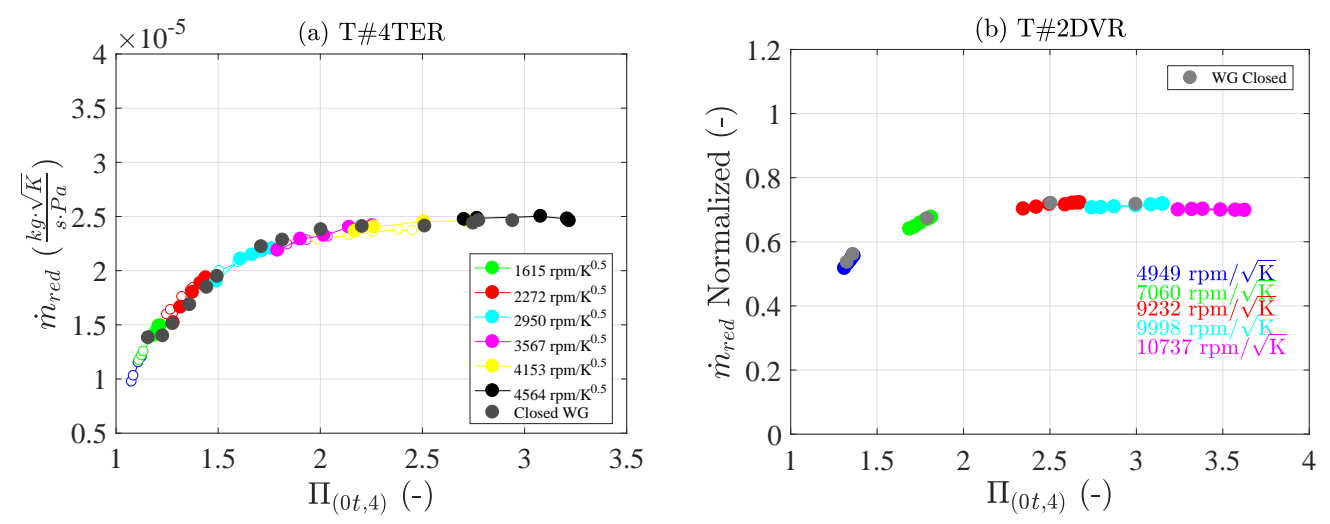

Figure 5.3: Turbine map with the points that measured when the wastegate valve is mechanically closed. (a) Twin-entry turbine with a given engine full load points, (b) Dual-volute turbine for a selected expansion ratios.

\subsubsection{Test B}

In Test B, the grey points are shown in Figure 5.3 were measured without the mechanical stopper and, by opening the wastegate valve at different positions going from $0 \%$ to $100 \%$. For doing this, a control system has been implemented to set the position of wastegate valve from the control room. Normally, the wastegate turbochargers are equipped with an electromechanical device which will be mounted on 
the compressor housing and connected to the wastegate valve by a lever/rod (see Figure 5.4). This device consists of a stepper motor with multistage spur gears and a rotary shaft. In engines, it directly communicates with the electronic control unit (ECU) and command the position of wastegate valve according to the engine speed, throttle positions and other sensor detection signals. For characterizing the wastegate turbocharger in a gas stand, the position of wastegate valve can be set by sending a signal (in the form of current to the stepper motor) from one of three computers from the control room. During the tests of T\#4TER turbine, it was observed that the stepper motor did not follow the same trend every time. That is when a signal sent by the computer to close the wastegate valve, the position of the valve is different from $0 \%$. Furthermore, the value was changed on each different day. Moreover, a variation of the signal at $0 \%$ opening brings out the variations in subsequent opening degrees for the whole scale. That is, comparing the different tests, the same signal (by the computer) corresponded to different opening positions of the wastegate valve. Therefore, to compare the different test results of T\#4TER turbine, a stepper motor calibration curve was required. Whereas, in the case of T\#2DVR turbine tests, the stepper motor followed the same trend all the time and tests were performed in cold conditions.

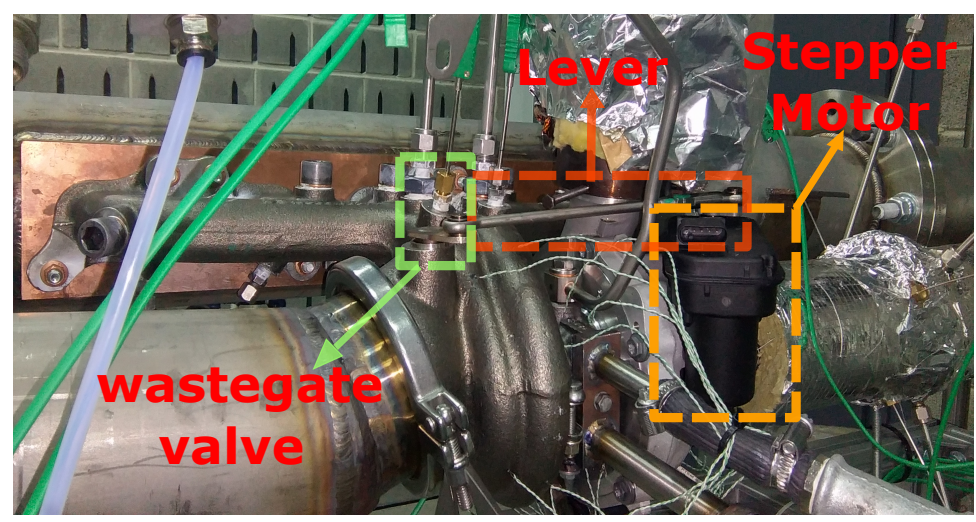

Figure 5.4: Electronic wastegate actuator

\section{Stepper motor calibration for T\#4TER turbine}

In order to compare the different test results of T\#4TER turbine, the stepper motor calibration curve was built. This curve shows the correlation between the voltage supplied to the motor and the percentage of wastegate valve opening. The main hypothesis followed to build the calibration curve was that it should be linear, thus requiring only a couple of values to calculate the correlation. One of the values corresponding to the maximum wastegate valve opening position, which is $0.25 \mathrm{~V}$ for $100 \%$, and another is the value corresponding to the wastegate valve closed position which is $0 \%$. The valve closed position is found by repeating one of the measured points 
when the wastegate valve is mechanically closed, that is, the latter taken as reference conditions. As the points corresponding to the maximum opening and closing known for each day, the calibration curve can be calculated as shown in Figure 5.5; and, it is straight line because the hypothesis consists of a linear correlation. Several calibration lines were obtained due to the inconsistency in calibration every day. The main difference can be found when cold turbo conditions are compared to the hot turbo conditions.

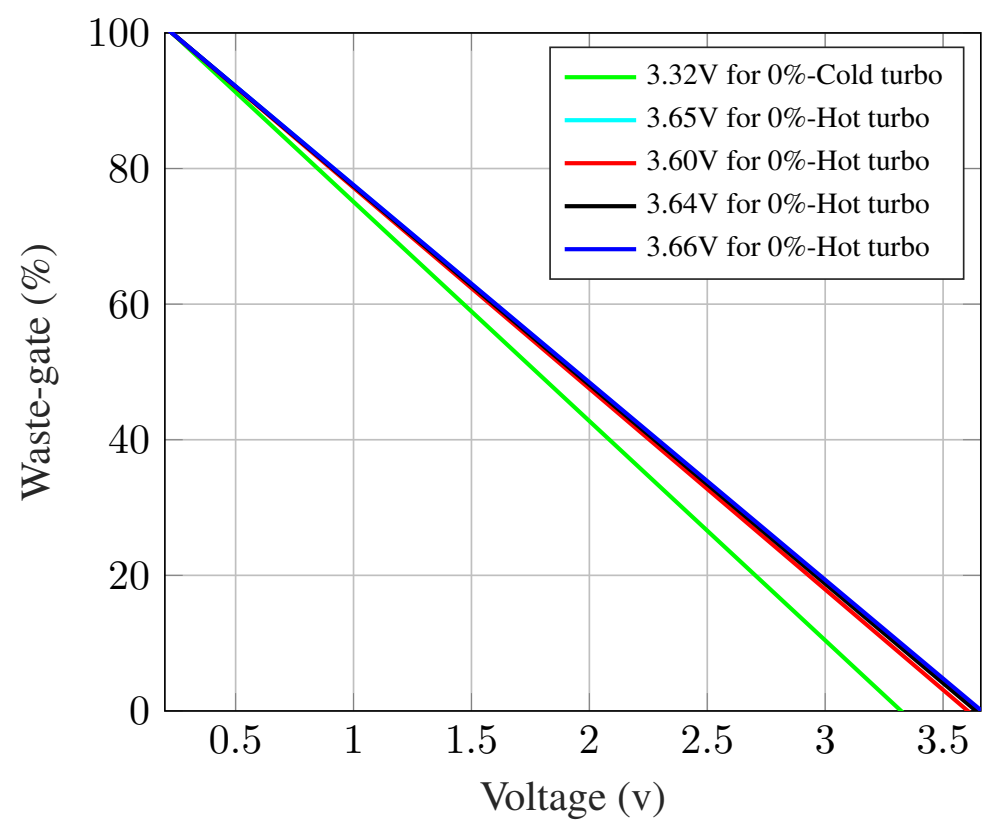

Figure 5.5: T\#4TER turbine stepper motor calibration curve for different tests

Figure 5.6, shows the current intensity for each expansion ratio, corresponding to the points close to those of real wastegate closed position for T\#4TER. From Figure 5.6 it can be observed that the electrical signal that needed to close the wastegate valve grows with the reduced turbine speed and with the increase in expansion ratio. Its maximum value has been measured for the maximum shaft speed and maximum expansion ratio. Higher current values are not attempted due to the limitations of the stepper motor and could also damage the engine. That is one of the reasons why it is not possible to completely close the wastegate valve with the stepper engine at higher expansion ratios for T\#4TER turbine. This will be shown further in Figure 5.10.

Once the stepper motor was calibrated, test B was performed in the gas stand for the given engine full load points. Each point is tested at different wastegate valve opening positions using the stepper motor. In test $\mathrm{B}$, for each opening of the wastegate valve, the test was carried out by adjusting the same turbocharger speed, expansion ratio and inlet temperature of the turbine, same as in test A. In this way, for each full load operating point even after opening the wastegate valve, 


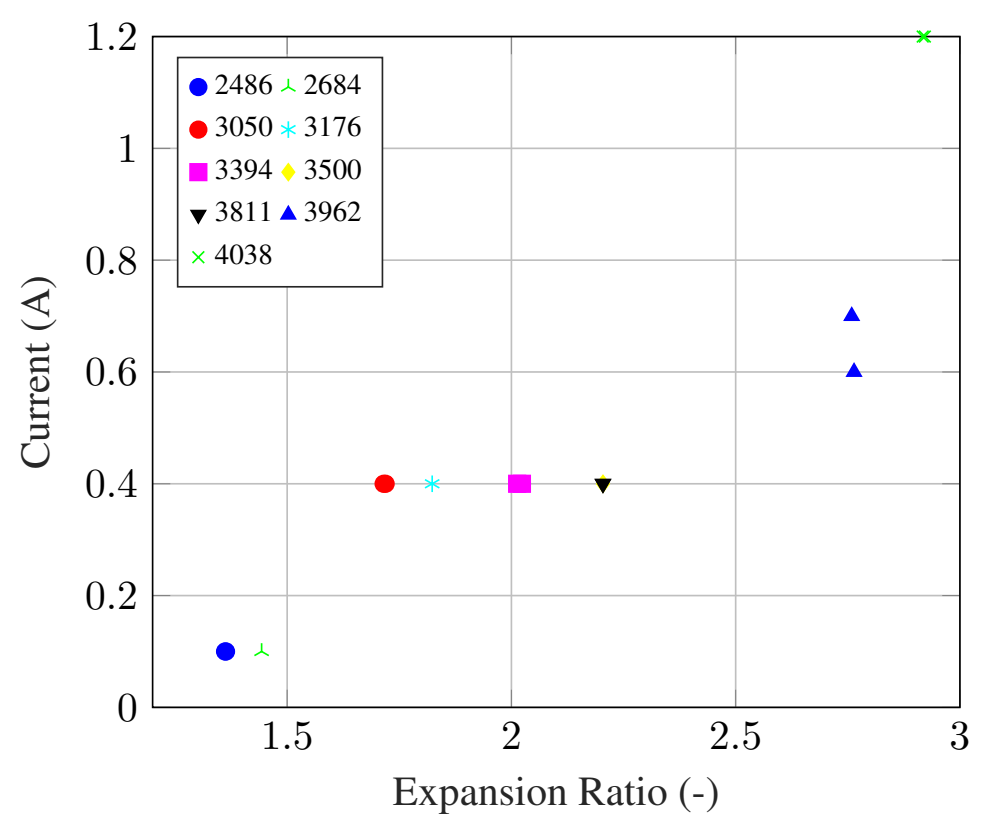

Figure 5.6: T\#4TER turbine stepper motor current intensity for the points near the wastegate valve closing position

turbine operative condition will be same as in test A. The same testing procedure was carried out for dual-volute turbine (T\#2DVR) in cold conditions. By maintaining the turbine conditions same in both tests, the turbocharger compressor power is not different for all the tested wastegate closed and different opening points. The same can be observed in the Figure 5.7, showing the compressor map of both twin-entry and dual-volute turbines. It can be observed that, in both test $\mathrm{A}$ and $\mathrm{B}$, the compressor pressure ratio and corrected mass flow values are very similar.

From both tests, now actual mass flow through the wastegate in test B can be calculated. That is, by making the difference between measured turbine mass flow from test B and test A as shown in the Equation 5.1

$$
\dot{m}_{\text {actual }}^{W G}=\dot{m}_{B}-\dot{m}_{A}
$$

where $\dot{m}_{A}$ and $\dot{m}_{B}$ are the turbine mass flows measured in the test A and test B. The mass flow measurements of the turbine in test A represents the real mass flow that is used for producing the turbine power (see Figure 5.8(a)); as the wastegate valve is mechanically closed. Whereas in the case of test $\mathrm{B}$, the mass flow measurements at the turbine outlet will be the sum of flow going to the turbine rotor plus the flow escaping through the wastegate section as shown in Figure 5.8(b). Figure 5.9 shows the turbine map measured with several wastegate openings, in which the grey diamond points corresponds to the total reduced mass flow measured in test B and 

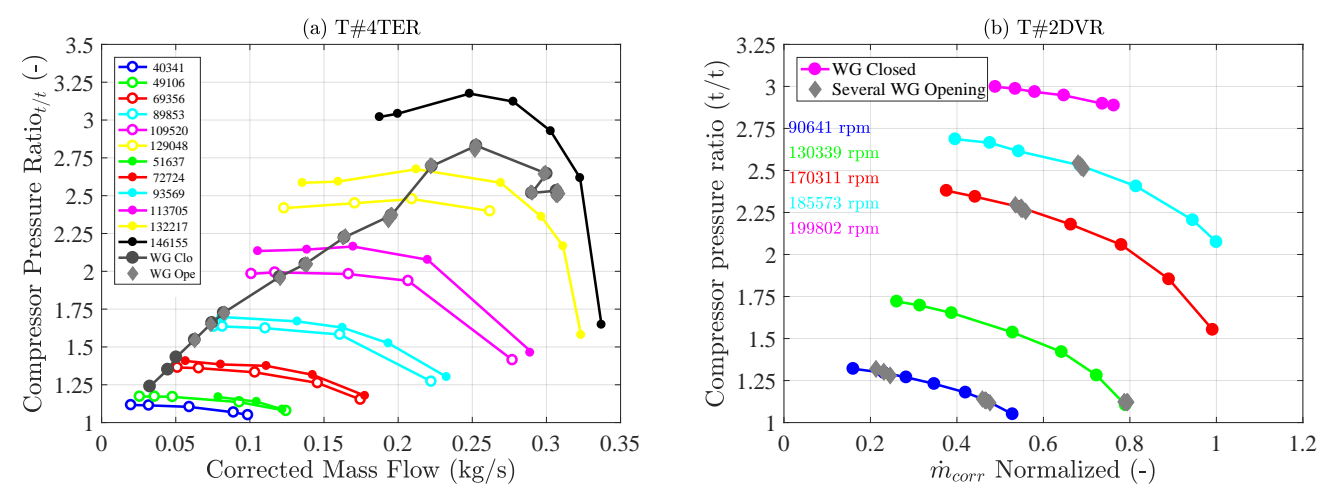

Figure 5.7: Compressor map with the points tested at wastegate closed and at different opening positions. (a) T\#4TER compressor map with the given engine full load points, (b) T\#2DVR compressor map with the selected expansion ratios.

the grey circled points represents the reduced mass flow measured in test A. Thus, using the Equation 5.1 and by doing the two different tests, the actual mass flow or reduced mass flow through the wastegate can be estimated experimentally for any type of double-entry turbine. As mentioned before, both turbines were tested in full admission conditions. Therefore, the turbine maps were represented as single turbine by using the equations 2.11 and 2.12. Its is worth highlighting that the measurement data of T\#2DVR turbine were normalized by taking the maximum experimental value from both tests.

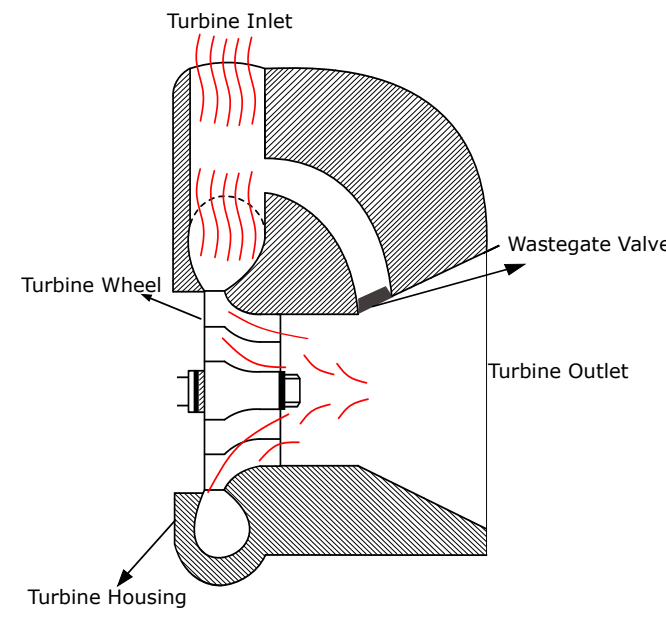

(a) Wastegate Valve Closed

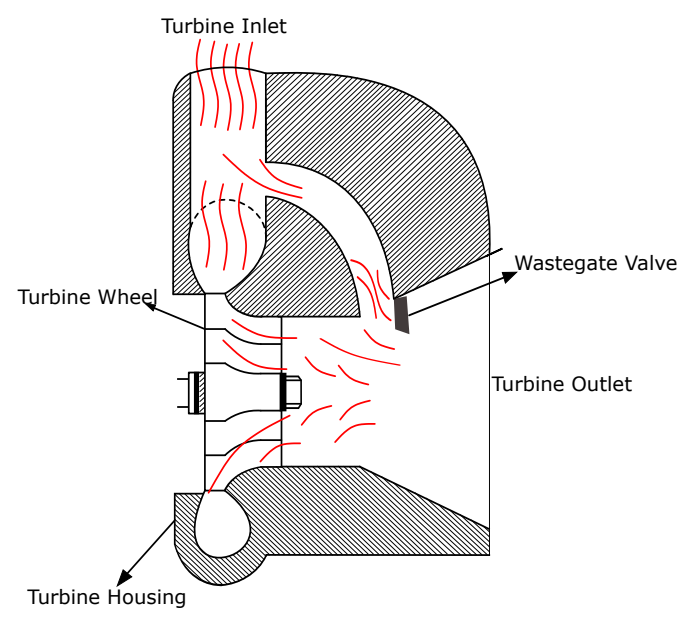

(b) Wastegate Valve Opened

Figure 5.8: Turbine mass flow diagram of an internal wastegate with the wastegate valve in the closed and open position. 

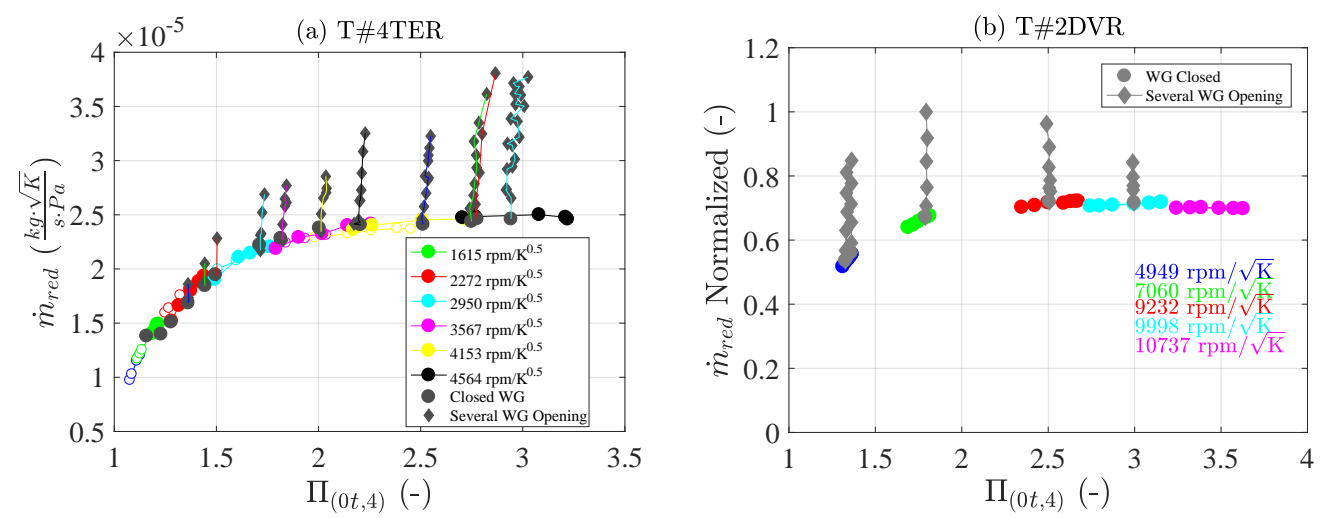

Figure 5.9: Turbine map with points tested when the wastegate is mechanically closed and with the several wastegate openings. (a) T\#4TER turbine map with the given engine full load points, (b) T\#2DVR turbine map with the selected expansion ratios.

\subsubsection{Experimental wastegate flow results}

Figure 5.10 shows the reduced mass flow through the wastegate estimated by using the Equation 5.1 for a series of expansion ratios that were tested on the respective turbine types. Point of each series expresses the difference between the flow outgoing from the turbine to wastegate closed with the two methods: one with the mechanically closed and another with the stepper motor. Therefore, a zero wastegate flow is expected, as shown in the Figure 5.10.
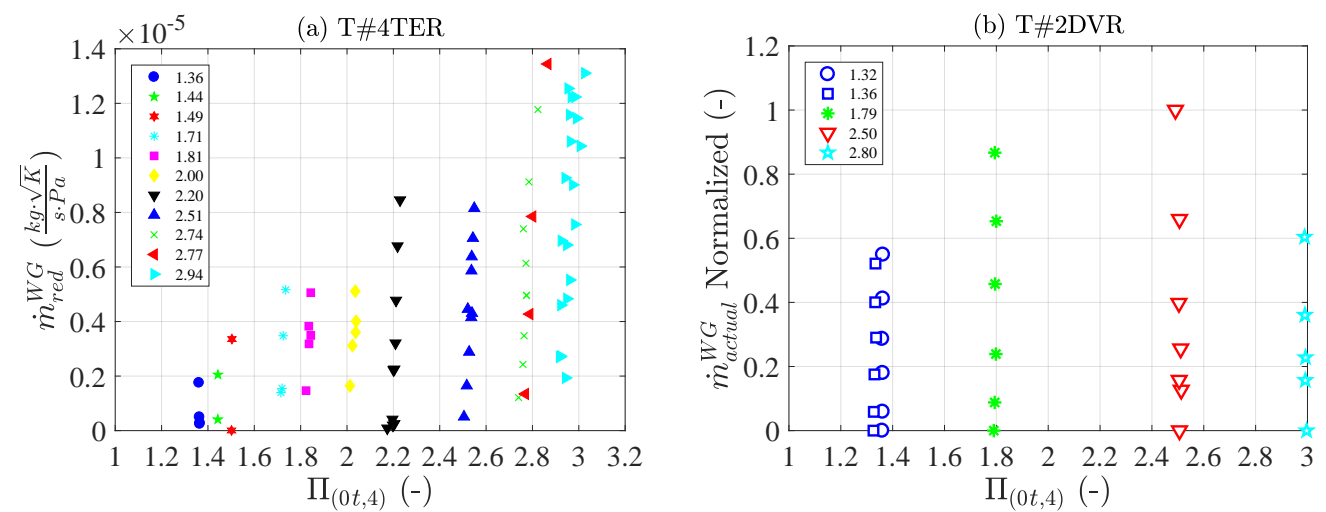

Figure 5.10: Experimentally estimated reduced mass flow through the wastegate section

(legends refers to closed wastegate expansion ratio), (a) Wastegate flow of T\#4TER turbine, (b) Wastegate flow of T\#2DVR turbine (mass flow values are normalized with the maximum experimental WG flow)

From Figure 5.10(a) it can be noted that the series has an expansion ratio of 2.4 and higher, zero wastegate mass flow is not achievable but only a value greater than zero. It means that, even after sending the close signal to the motor, the 
total flow rate of the gas that is measured at the turbine is greater than the one acquired with the wastegate locked with a mechanical stopper. Apparently, despite the sent signal to the stepper motor, it does not close the valve entirely. The cause of this phenomenon lies in the fact that these points, the pressure values of gases were relatively high and exerted an action that is opposed to close the valve. For avoiding these, very high intensity of current should be sent to the motor, with the risk of burning the system (see Figure 5.6). Therefore, it is expected that at high expansion ratios which are typical of full load engine operation or rated power, the wastegate can show leakages and that should be considered when the engine control is designed. In the case of T\#2DVR turbine (Figure 5.10 (b)) the wastegate valve is totally closed by the stepper motor for all the selected expansion ratios. Nevertheless, this turbine was tested in cold conditions and further it is smaller as compared to the T\#4TER turbine. Moreover, from Figure 5.10, it can be concluded that, irrespective of turbine expansion ratio, the temperature levels at the turbine inlet can affect more the wastegate valve mechanism to be closed perfectly.
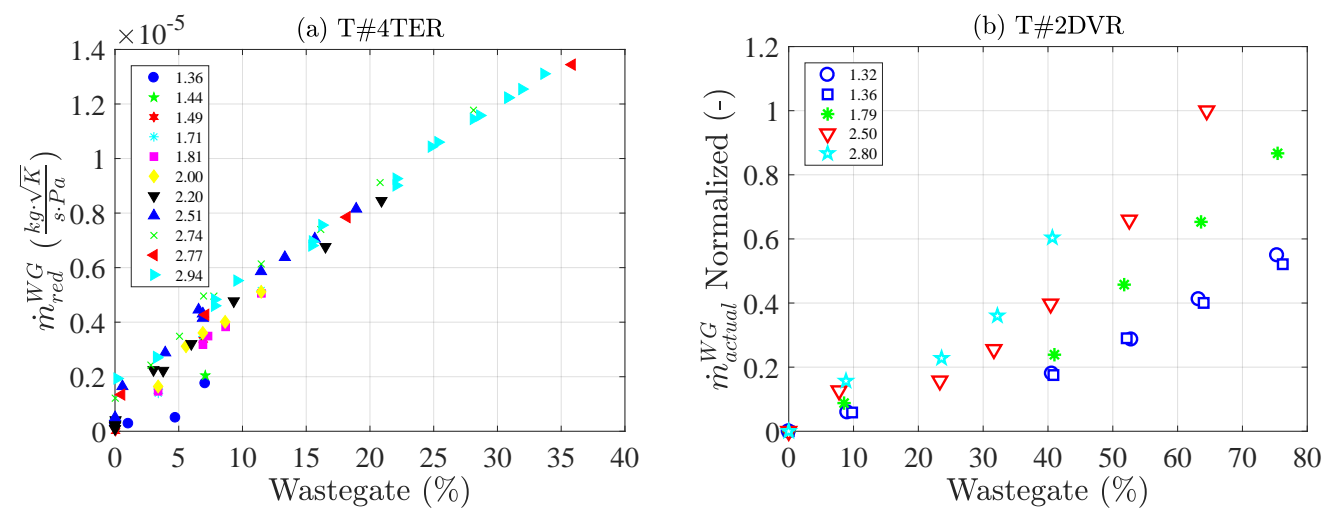

Figure 5.11: Reduced mass flow in the wastegate section with the wastegate opening percentage (legends refers to closed wastegate expansion ratio), (a) T\#4TER turbine results. (b) T\#2DVR turbine results (mass flow values are normalized with the maximum experimental WG flow).

Figure 5.11 shows the mass flow through the wastegate as a function of the commanded wastegate opening percentage for the tested turbine types. In the case of T\#4TER turbine (Figure 5.11(a)), it is already found that for higher expansion ratios, although the wastegate corresponds to close position ( $\mathrm{WG}=0 \%$ ), practically the valve is still open. Therefore, there is a leak of mass flow through the wastegate. From Figure 5.11, it can also be observed that there are also points at the lowest expansion ratio, for which, despite the opening of the wastegate valve (WG $>0 \%$ ) the mass flow is not passing through the wastegate section. This is because the pressure difference is too small to keep the mass flow in motion when the wastegate is opened. So, the flow through the wastegate section is dependent not only on the wastegate opening position but also on the expansion ratio. 


\subsubsection{Wastegate discharge coefficient modelling}

The discharge coefficient is a measure of how close the actual mass flow rate across the wastegate compared to the calculated ideal mass flow rate. It is used to obtain the effective flow area $\left(A_{e f f}\right)$ of the actual bypass throat area as shown in Equation 5.2

$$
A_{e f f}=C_{d}^{m e a} \cdot A_{r e f}
$$

The reference area $\left(A_{\text {ref }}\right)$ is chosen to be the wastegate throat cross-sectional area that can be estimated with the known diameter of the wastegate section as shown in Equation 5.3

$$
A_{\text {ref }}=\frac{\pi \cdot D_{W G}^{2}}{4}
$$

The geometric area of the wastegate valve was directly measured from the turbocharger unit at a maximum opening of the valve. In order to compute the discharge coefficient, the ideal mass flow rate through the wastegate reference area must be determined, which assumes a perfect gas undergoing a steady isentropic compressible flow in one dimension.

$$
\begin{gathered}
\frac{p_{0}}{p}=\left(\frac{T_{0}}{T}\right)^{\gamma /(\gamma-1)} \\
T_{0}=T\left(1+\frac{\gamma-1}{2} M^{2}\right)
\end{gathered}
$$

Using the isentropic relation equation 5.4 and 5.5, the ratio of total and static pressure as a function of Mach number, $M$, can be expressed as shown in Equation 5.6

$$
\frac{p_{0}}{p}=\left(1+\frac{\gamma-1}{2} M^{2}\right)^{\gamma /(\gamma-1)}
$$

where the Mach number is calculated by below equation

$$
M=\frac{u}{\sqrt{\gamma R T}}
$$

Next, the continuity equation is entreated along with the perfect gas equation of 
state to solve for mass flow rate according to

$$
\dot{m}_{\text {ideal }}=\rho A_{\text {ref }} u=\rho A_{\text {ref }} M \sqrt{\frac{\gamma}{R T}}
$$

Combining equations 5.4 to 5.8 gives the unchoked ideal mass flow rate through the wastegate as shown in Equation 5.9

$$
\frac{\dot{m}_{i d e a l} \sqrt{T_{0 t}}}{p_{0 t}}=A_{\text {ref }} \sqrt{\frac{\gamma}{R}}\left(\frac{1}{\Pi_{(0 t, 4)}}\right)^{\frac{1}{\gamma}} \sqrt{\frac{2}{\gamma-1}\left(1-\left(\frac{1}{\Pi_{(0 t, 4)}}\right)^{\frac{\gamma-1}{\gamma}}\right)}
$$

where $A_{\text {ref }}$ is the area referenced to a diameter of the wastegate (Equation 5.3). The static pressure at the wastegate throat is assumed to be equal to turbine static pressure at downstream the turbine, that is, $p_{4}$ is equal to $p_{\text {throat }} . T_{03}$ is the upstream turbine temperature, $R$ is the gas constant, $\gamma$ is the ratio of specific heats of the air and $\Pi_{(03,4)}$ is the ratio between the turbines total pressure at upstream to static pressure at downstream. Finally, the ideal wastegate reduced mass flow rate is compared to the actual wastegate reduced mass flow rate, which is measured on the turbocharger gas stand. This yields to the calculation of experimental discharge coefficient (see Equation 5.10).

$$
C_{d}^{m e a}=\frac{\dot{m}_{\text {actual }}^{*}}{\dot{m}_{\text {ideal }}^{*}}
$$

Since the turbine upstream temperature and pressure, downstream pressure and air mass flow were measured, discharge coefficient was calculated as the ratio between the experimental and theoretical flow rate as explained above and, the same shown in Equation 5.10. Its performance is qualitatively quite as expected: increasing with the percentage of the opening valve, and at the same, it is increasing with the expansion ratio. The result of the wastegate discharge coefficient for measured points of both turbine types was shown in Figure 5.12. 

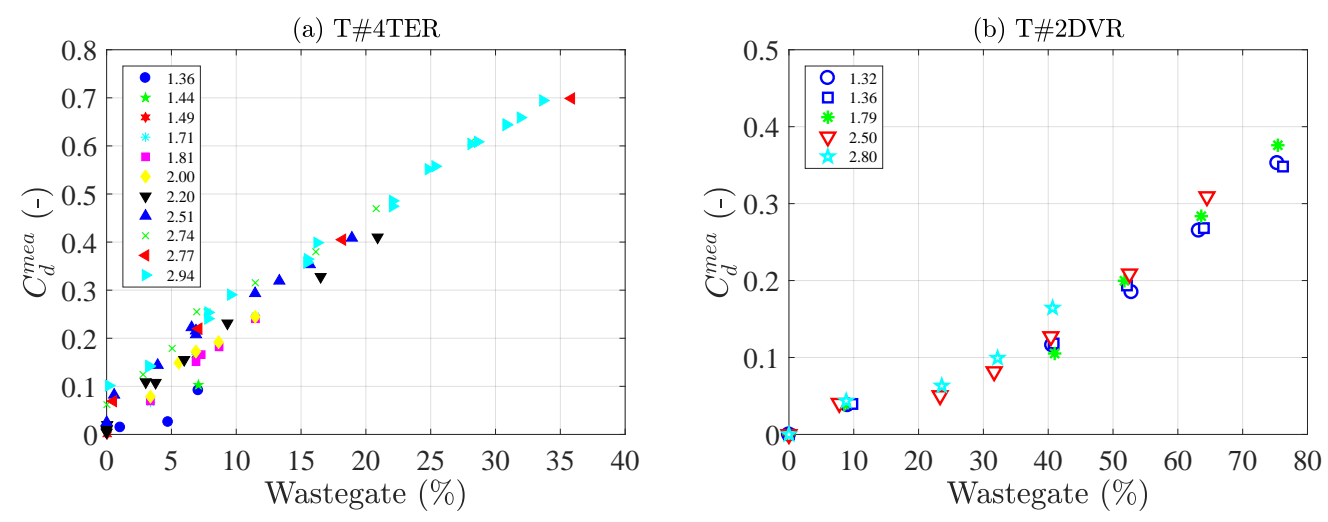

Figure 5.12: Experimental discharge coefficient values with the wastegate opening percentage (legends refers to closed wastegate expansion ratio), (a) T\#4TER turbine results, (b) T\#2DVR turbine results (actual discharge coefficient values are shown instead of normalized)

From Figure 5.12(a), it is important to remark that the discharge coefficient is not so totally linear with the wastegate opening from $0 \%$ to $15 \%$. However, from Figure 5.11(a) it is concluded that there is more dispersion in mass flow at these low wastegate openings ( $0 \%$ to $15 \%)$. Further, at higher turbine expansion ratios, the wastegate was left open. But, the discharge coefficient has a linear relation at higher wastegate openings, that is, from $15 \%$ to $40 \%$. At lower wastegate openings, there is more dispersion and some dependence on expansion ratio. So, the wastegate discharge coefficient is also related to the width of the turbulent boundary layer [82]. Whereas, observing the experimental discharge coefficient values of T\#2DVR turbine (Figure 5.12(b)), the trend shows similar to the beginning of a sigmoid curve. For low wastegate openings ( $0 \%$ to $20 \%$ ), the change in discharge coefficient values is very small. Later, it shows a linear trend with the opening of a wastegate valve from $20 \%$ to $80 \%$. From $80 \%$ to $100 \%$ of wastegate position, it can be expected that change in discharge coefficient values will be small enough as compared to other wastegate positions shown in Figure 5.12(b), as the change in effective area will be smaller.

In order to obtain an expression that can be used for predicting the discharge coefficient of a wastegate valve for a variety of operating conditions at which an engine works, a polynomial equation form is used. The equation is formed as a function of expansion ratio and wastegate valve opening as shown in the Equation 5.11 for T\#4TER turbine and Equation 5.12 for T\#2DVR turbine. Using the experimental data, an empirical model is fitted until the Root Mean Square Error (RMSE) is minimized between the experimental and modelled discharge coefficient. Table 5.1 shows the model coefficients and the statistics numbers that represent the quality of 
model fitting for both turbine types.

$$
\begin{gathered}
C_{d}^{\operatorname{modTE}}=a \cdot \Pi_{t / s}+b \cdot\left(\frac{\% W G}{100}\right)+c \cdot \Pi_{t / s} \cdot\left(\frac{\% W G}{100}\right) \\
C_{d}^{\bmod D V}=a \cdot \Pi_{t / s}\left(\frac{\% W G}{100}\right)+b \cdot\left(\frac{\% W G}{100}\right)^{2}+c \cdot \tan \left(\frac{\% W G}{100}\right)
\end{gathered}
$$

Table 5.1: Quality of wastegate discharge coefficient model fitting for T\#2DVR and T\#4TER turbines

\begin{tabular}{ccccc}
\hline \multirow{2}{*}{ Coefficients } & \multicolumn{2}{c}{ T\#2DVR } & \multicolumn{2}{c}{ T\#4TER } \\
\cline { 2 - 5 } & Estimation & $p$-value & Estimation & $p$-value \\
\hline$a$ & 0.0426 & $3.694 \times 10^{-10}$ & 0.0245 & $2.027 \times 10^{-8}$ \\
$b$ & 0.461 & 0.0108 & 1.132 & $1.228 \times 10^{-15}$ \\
$c$ & 0.0526 & $1.693 \times 10^{-8}$ & 0.259 & $4.108 \times 10^{-10}$ \\
\hline SSE & 0.0052 & 0.0528 \\
$\mathrm{R}^{2}$ & 0.9872 & 0.9837 \\
Adjusted $\mathrm{R}^{2}$ & 0.9863 & 0.9833 \\
RMSE & 0.0132 & 0.0253 \\
\hline
\end{tabular}

Figure 5.13, shows the empirical model prediction of the wastegate discharge coefficient with respect to the wastegate opening position and turbine expansion ratio. By this empirical model, it is easy to estimate the mass flow through wastegate using the predicted discharge coefficient in Equation 5.13. This model can be used in 1D simulations in order to improve the predictions of overall engine performance. It can be observed that the wastegate valve position has been divided by 100 to obtain fitting coefficients with an order of magnitude around unity.

$$
\dot{m}_{\text {actual }}^{*}=C_{d}^{m o d} \cdot \dot{m}_{\text {ideal }}^{*}
$$


(a) T\#4TER

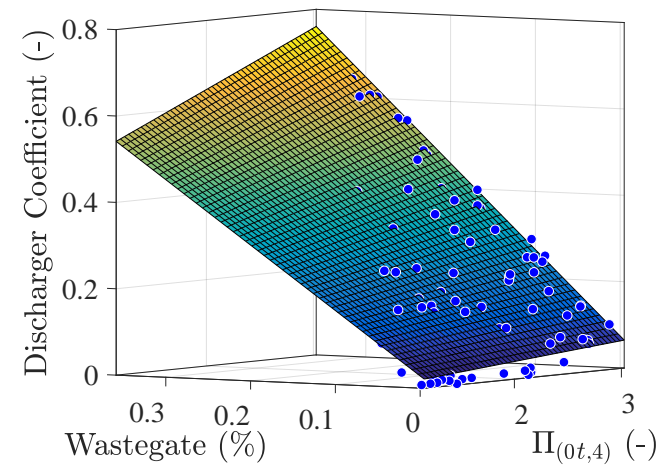

(b) T\#2DVR

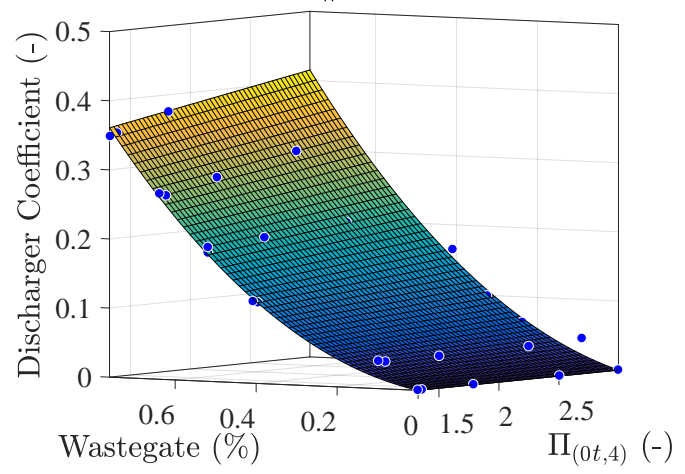

Figure 5.13: Wastegate valve discharge coefficient modelled as a function of turbine expansion ratio and wastegate opening position. (a) T\#4TER turbine predictions. (b) T\#2DVR turbine predictions.
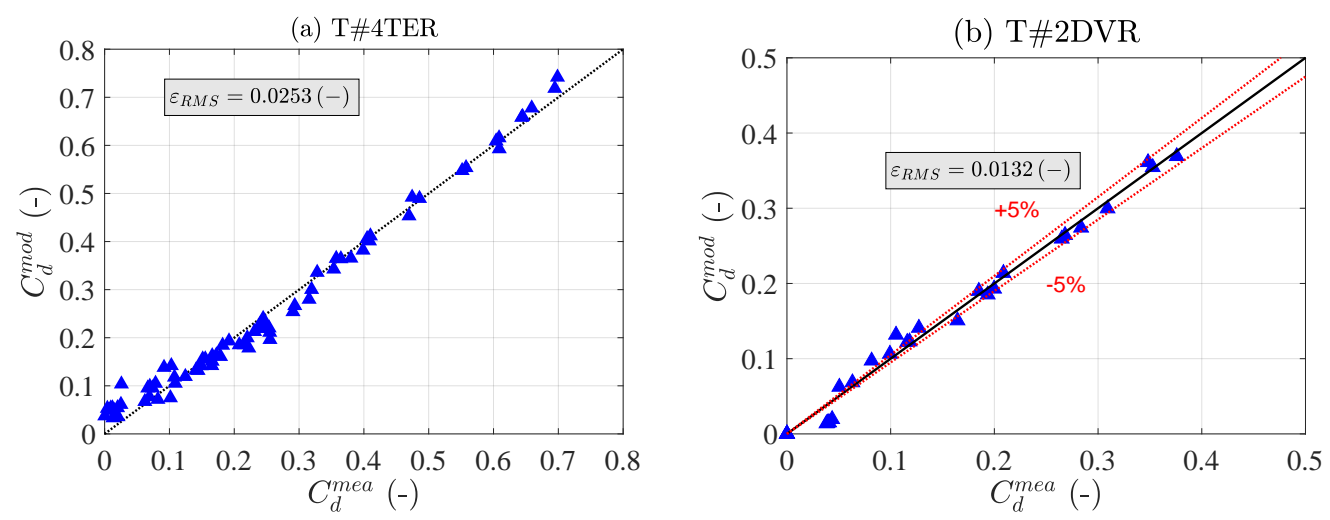

Figure 5.14: Modelled discharge coefficient against measured discharge coefficient

Figure 5.14 shows the result of the model predicted discharge coefficient compared with the discharge coefficient calculated from the measured points. As shown, predicted wastegate discharge coefficient values are well agreed with the measured one. A root mean square error of 0.0253 was observed for T\#4TER turbine (Figure 5.14 (a)); this is about $3.6 \%$ of the maximum measured discharge coefficient $\left(C_{d}^{\text {mea }}=0.7\right)$ at $35 \%$ wastegate opening, which is the maximum practical wastegate opening for the tested turbocharger. Whereas, in the case of T\#2DVR turbine (Figure 5.14(b)), predicted discharge coefficient values from the model showed few deviations as compared to the experimental values, mainly at lower wastegate openings (between $5 \%$ to $20 \%$ ). However, at the rest of the openings, the error between the modelled and experiments are in between the $\pm 5 \%$. The direct relation between the wastegate displacement, turbine expansion ratio, and discharge factor makes it possible to estimate the wastegate flow using Equation 5.13.

Figure 5.15 shows the modelled reduced mass flow through the wastegate valve, 

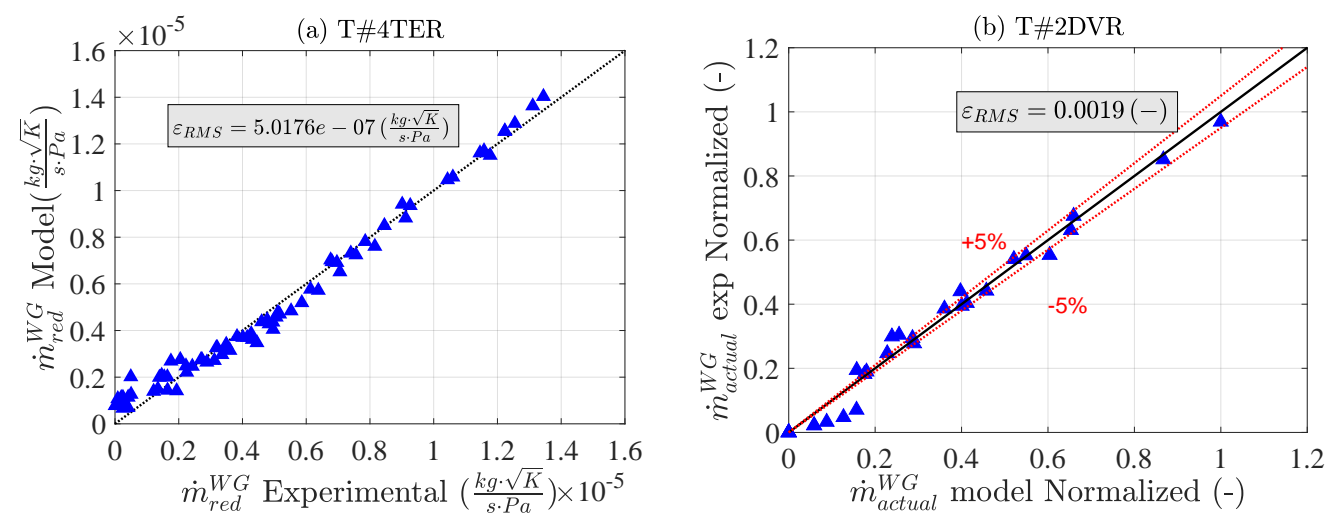

Figure 5.15: Modelled wastegate reduced mass flow against measured wastegate reduced mass flow

which is calculated from Equation 5.13 and is compared to the experimental wastegate reduced mass flow. Observing the results of T\#4TER turbine (Figure 5.15(a)), an root mean square error of $5.0176 \mathrm{e}-07 \frac{\mathrm{kg} \cdot \sqrt{K}}{\mathrm{~s} \cdot \mathrm{Pa}}$ in the reduced mass flow prediction was observed, which represents a $3.77 \%$ of maximum wastegate measured reduced mass flow. Therefore, this simple empirical model can be used in a whole engine simulation models for predicting the mass flows with lesser errors. Moreover, by comparing with the maximum turbine reduced mass flow, $\varepsilon_{R M S}$ is a bit less than $2.08 \%$, and this is the relevant turbine mass flow since it is directly proportional to turbine power. In the case of T\#2DVR turbine, the predictions of wastegate reduced mass flow shown in the same level of errors as discharge coefficient values that observed in the Figure 5.14(b). Further, observing the $\varepsilon_{R M S}$, the model produces an error not more than $2 \%$. The few discrepancies found in mass flow predictions comes from the overall quality of the discharge coefficient fitting at lower wastegate positions, as it can be observed in the Figure 5.13(b)

\subsection{Scroll connection valve characterization}

Turbocharger manufacturers are also implementing some new concepts in dual-volute turbines to be more efficient at different engine load conditions. One of them is placing a Scroll Connection Valve (SCV) before the turbine tongue, as shown in Figure 5.16. When the SCV is open, the flow can travel from long volute to short volute and vice versa. It is dependent on the pulse that comes from the engine cylinders in their respective volute inlets. In order to estimate the mass flow passing through an SCV in one-dimensional engine calculations, a model that predicts the discharge coefficient of the same is needed. The scroll connection valve is present in a dual-volute mixed inflow turbine (T\#3DVM) that used in this thesis. Therefore, T\#3DVM turbine is tested in hot conditions in steady flow rate with several opening positions of the 
SCV. Later, an empirical model has been correlated to able to predict the discharge coefficient of SCV.

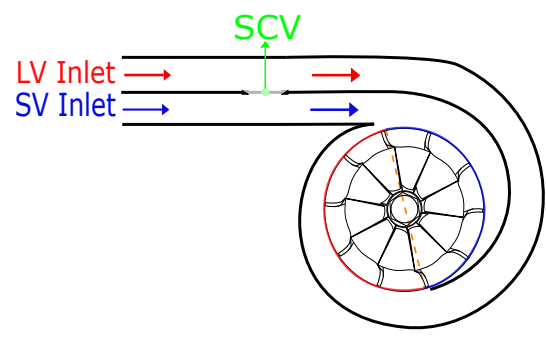

(a) SCV Closed

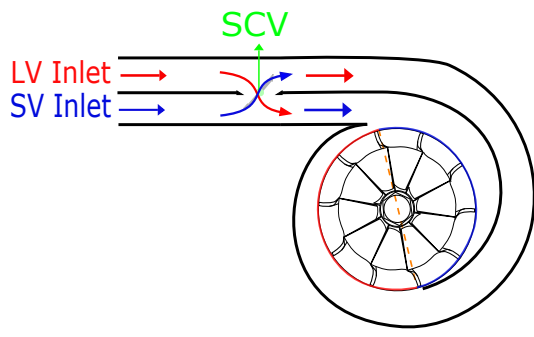

(b) SCV Opened

Figure 5.16: Exhaust mass flow conditions in a dual-volute turbine when the scroll connection valve is present.

\subsubsection{Test methodology}

For characterizing the discharge coefficient of SCV experimentally, two tests were performed, which are similar to the wastegate characterization. But, these two tests were carried out with partial admission map of each turbine volute (MFR 0 and 1). Testing each turbine volute separately, it makes easy to deduce the flow that passes from long volute to short volute and vice versa. Figure 5.17 shows the outline of two tests (Test A and Test B), and the main variables like temperature, pressure, and mass flow rate, measurement points are represented. It is worth highlighting that each turbine volute is tested twice for selected turbine expansion ratios, where the compressor operates within the surge and choke conditions. Further, the wastegate valve is also totally closed. The expansion ratio values were selected from the partial admission maps of Figure 3.22(e) and (f); which were tested in the gas stand as described in section 3.5. It is worth highlighting that, while measuring the T\#3DVM turbocharger maps, both the wastegate and SCV valves are totally closed. In the gas stand, SCV is controlled by passing a signal from the control system in a form of current to the stepper motor that came with the turbocharger unit.

Firstly, Test A was performed with the SCV closed and passing the mass flow through only in the long volute branch (MFR 1). Measurements of the turbine inlet and outlet air mass flow, pressure, temperature and turbocharger speed were registered. Secondly, Test B was performed with the same partial admission map of MFR 1 but with the opening of SCV. Test B was carried out by adjusting the same expansion ratio and inlet temperature of the long volute branch as in the test $\mathrm{A}$ for each opening of the SCV. It is worth noting from the Figure 5.17 that, the pressure and temperature sensors in the short volute branch are located after the control valve, which is closed fully. Therefore, it is possible to measure these variables in both long (where is actual flow is given) and short volute (flow that comes from long to short 


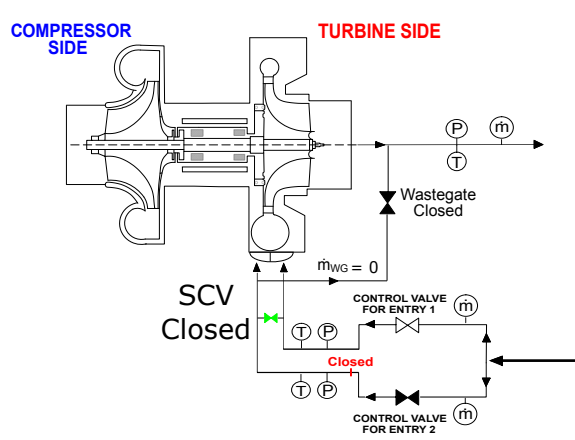

Test A

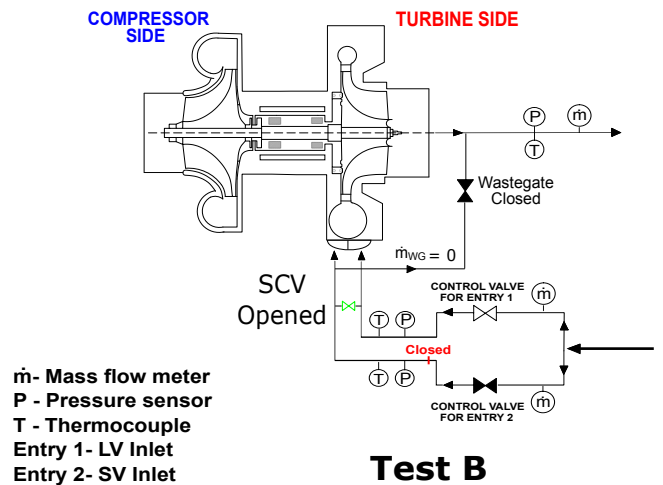

Figure 5.17: Test methodology for characterizing the scroll connection valve in partial admission maps.

through the SCV) branches. Similarly, both tests A and B were repeated with the partial admission map of MFR 0; i.e., passing all the flow through the short volute branch with SCV closed and opened positions. Figure 5.18 shows the turbine map of T\#3DVM that tested in the gas stand with the SCV closed and opened position of both branches. It should be noted that, in the following figures, the mass flow data were normalized with the highest flow found in Test B from both partial admission tests.
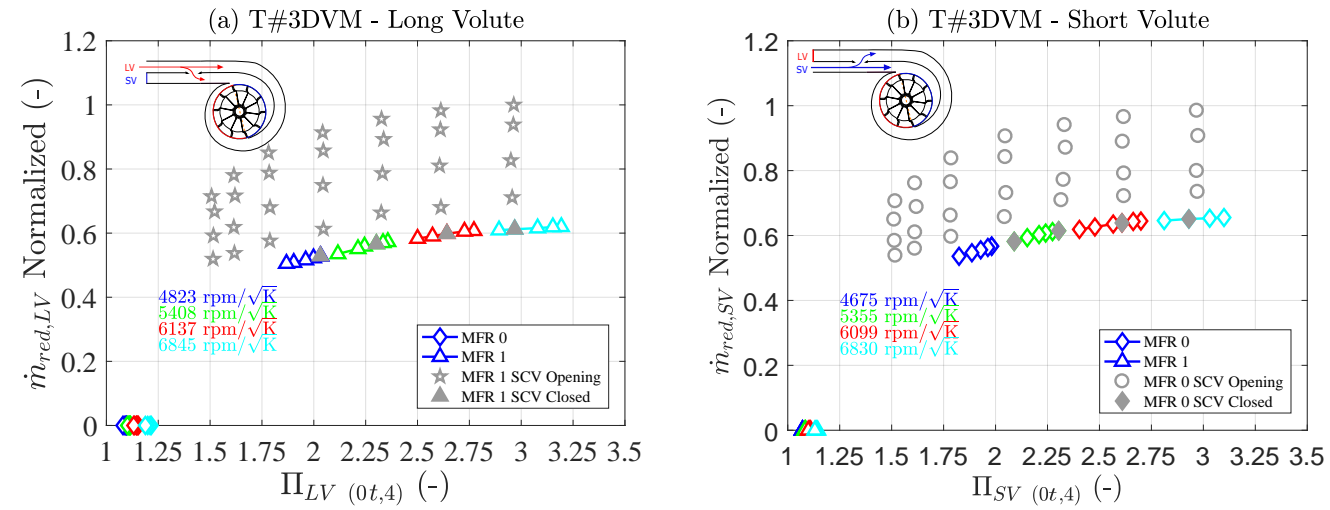

Figure 5.18: T\#3DVM Partial admission maps tested with SCV closed and with different SCV openings. (a) SCV opening when all flow is in long volute branch, (b) SCV opening when all the flow is in short volute branch.

It is worth highlighting that the positions of back pressure valve at the downstream of the turbocharger compressor are kept similar in both tests and for all the expansion ratios tested. For recovering the same expansion ratio as with the SCV closed, more energy at the turbine inlet is needed. So, keeping the back pressure valve at a fixed position ensures that the compressor always works in safe conditions even when the flow in a turbine is increased. Figure 5.19 shows the results of compressor maps of 
T\#3DVM turbine that tested in both tests. From Figure 5.19 it can be observed that for the same expansion ratio point and at an increasing SCV opening, the conditions in the compressor map are different. This is due to a change in the efficiency of the turbine as SCV opens. The change in the compressor map is linear with the SCV opening of the same expansion ratio. This is because of the fixed back pressure valve position. The same results can be observed (see Figure 5.19) in the compressor map when characterizing the SCV with partial admission map of MFR 0 (flow passing through a short volute branch).
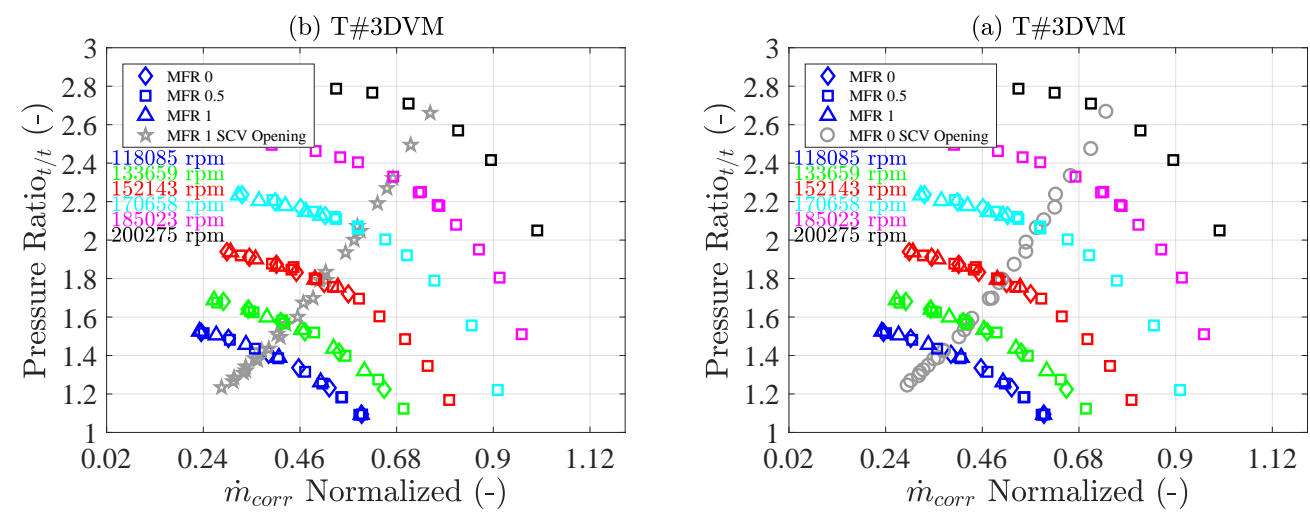

Figure 5.19: T\#3DVM compressor map with SCV closed and with different SCV opening tested points. (a) SCV opening when all flow is in long volute branch, (b) SCV opening when all the flow is in short volute branch.

In the case of wastegate characterization, by doing two different tests for the same expansion ratio and making the difference in between them. The mass flow through wastegate is easily estimated as described in section 5.2 . When the wastegate valve is opened, some amount of flow is bypassed to downstream the turbine such that it does not flow across the turbine wheel. This small amount of potential energy is not used by the turbine to generate power. However, in the case of SCV, when it opens, the flow that passes through the SCV segment goes into the turbine wheel. In test $\mathrm{B}$, for recovering the same expansion ratio than the SCV closed condition; more mass flow is given to the active branch (active branch refers to the flow passing through long or short volute). But, the measured mass flow $\left(\dot{m}_{B}\right)$ going through the active branch in test B is false; as some of the mass flow diverts through the SCV and goes to the turbine wheel. Thus, there is a change in turbocharger speed for every SCV opening. The same can be observed in the Figure 5.19: for the same expansion ratio value and at different SCV opening positions, the compressor corrected speed is changing. This indicates that the efficiency of the active branch varies when the SCV is opened, in spite of, keeping the turbine inlet temperature and expansion ratio values similar in the active branch for both tests.

Further, the turbine operates in an MFR other than the partial admission conditions, because the amount of flow escapes through SCV segment goes into the other 
branch. Thus, the flow admission conditions at the turbine inlets changes, therefore the MFR, according to its definition (Equation 3.5) shown in chapter 3. Thereby, making the difference of measured mass flow in test A and B as shown in Equation 5.14 (index $k$ refers to the direction of SCV flow), cannot offers the accurate SCV flow that is passing through the segment. So, the question remains, how to estimate the SCV flow accurately?

$$
\dot{m}_{e x p, k}^{S C V}=\dot{m}_{B}-\dot{m}_{A}
$$

\subsubsection{Calculation of experimental SCV flow}

In order to estimate the SCV flow with the experimental data, the efficiency of the active branch in both tests (A and B) should be similar. Nevertheless, efficiency is changing when the SCV is opening. This is due to the increment of turbocharger speed with the given mass flow in the active branch for recovering the same expansion ratio than the SCV closed. But, if there is a way to recalculate the right SCV closed mass flow in the active branch for the corresponding SCV opened turbocharger speed. Then the operating conditions of the active branch with the new SCV closed mass flow will be similar to test B situations. As the expansion ratio, turbine inlet temperature and turbocharger speed are now similar; therefore, the efficiency of the active branch will be the same. Eventually, the actual flow through the SCV segment can be estimated by making the difference between SCV opened $\left(\dot{m}_{B}\right)$ and new SCV closed $\left(\dot{m}_{A}^{N e w}\right)$ flows. The same is shown in the Equation 5.15 and the index $k$ refers to the direction of SCV flow.

$$
\dot{m}_{e x p, k}^{S C V}=\dot{m}_{B}-\dot{m}_{A}^{N e w}
$$

For calculating the new SCV closed mass flow, the reduced mass flow and efficiency models of double-entry turbines that are described in the chapter 4 is used. It is worth highlighting that both models consider two entries as two individual turbines. Moreover, it takes into account each entry with their respective turbine inlet conditions, like expansion ratio, inlet temperature, reduced turbine speed and blade speed ratios. Therefore, firstly the double-entry turbine model was fitted with the individual turbine maps data of T\#3DVM turbocharger. The turbine was tested with the three different MFRs (0, 0.5 and 1$)$ with SCV and wastegate totally closed (as shown in Figure 3.22 (e) and (f)). Once, the model is fitted to the map data, the active branch SCV opened test conditions like expansion ratio, turbine inlet temperature, reduced turbine speed and MFR were given as input to the fitted model. Using these variables as input new SCV closed mass flow can be estimated. Finally, using the Equation 5.15, the actual and accurate SCV flow that is going through the segment can be calculated. Figure 5.20 shows the procedure for calculating the exact 
SCV flow using the double-entry turbine models and experimental information.

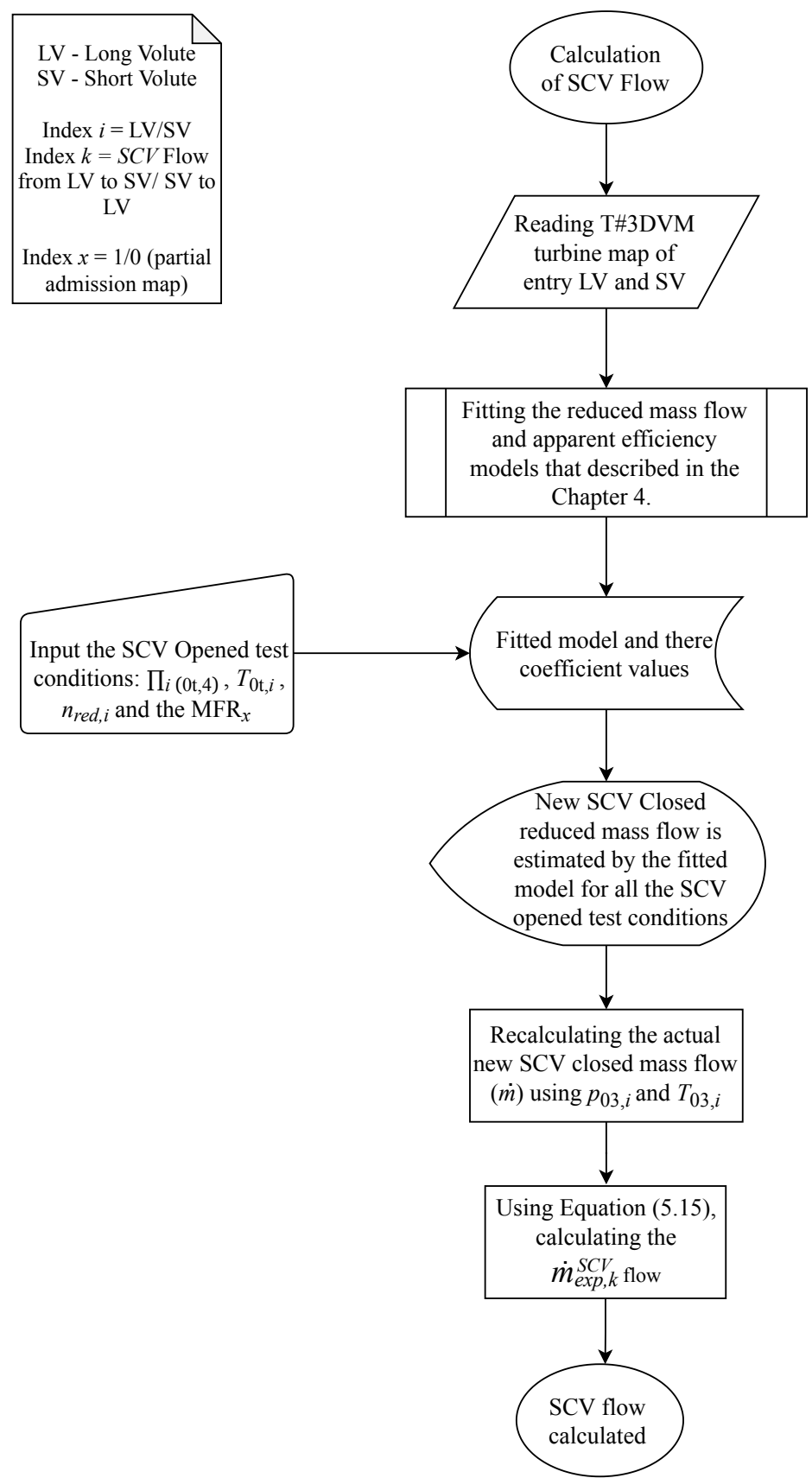

Figure 5.20: Showing the procedure for estimating the new SCV closed mass flow with the SCV opened test conditions. Once it is estimated, the actual SCV flow can be calculated. 
Figure 5.21 shows the long volute partial admission turbine map with new SCV closed mass flows for all the expansion ratios and SCV opening positions. It can be observed that the differences between the new and old SCV closed mass flow values are smaller in the case of small SCV openings (Figure 5.21(a) and (b)). It is due to change in reduced turbine speeds for these SCV openings is small, as shown in Figure 5.22. However, some notable variation in mass flows can be remarked when the SCV is adjacent to complete opened positions, as it is observed in Figure 5.21(c) and (d). Similar results can be followed when all the flow is in the short volute branch; as shown Figure 5.23. One can say that these small variations in mass flows are not that important. Nevertheless, the deviations are shown in the Figures 5.21 and 5.23 here are in reduced numbers. Further, the turbine inlet temperature and expansion ratio values were kept similar in both tests. Nevertheless, the disparity of mass flow in real numbers will be very significant. Moreover, in modelling a discharge coefficient of SCV, real mass flow values were used. Consequently, having an accurate SCV flow estimations from the experimental data can lead to have an good discharge coefficient model.

\section{(a) T\#3DVM - Long Volute}

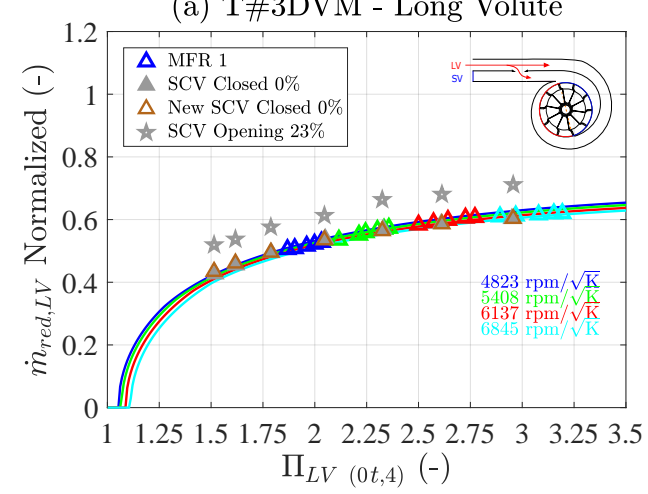

(c) T\#3DVM - Long Volute

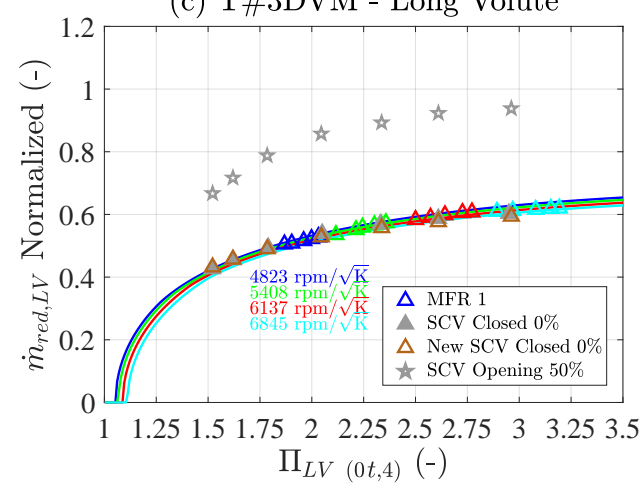

(b) T\#3DVM - Long Volute

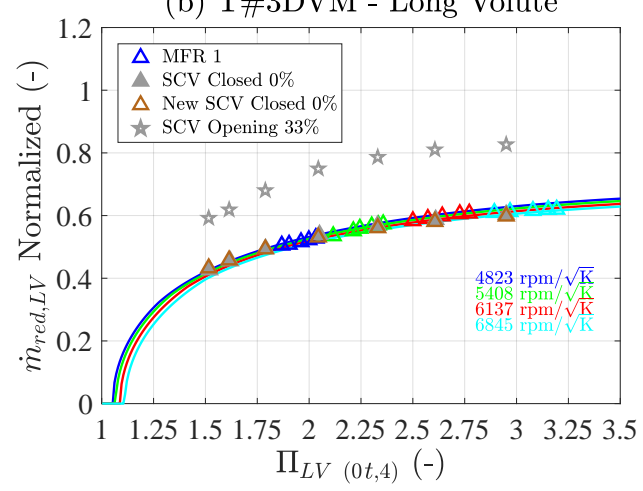

(d) T\#3DVM - Long Volute

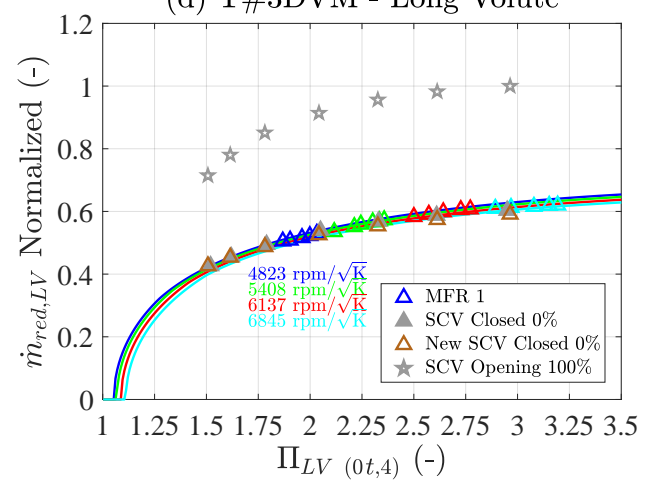

Figure 5.21: Extrapolated T\#3DVM turbine map when main flow is passed through the long volute branch. Showing with new and old SCV closed mass flows for comparison. 

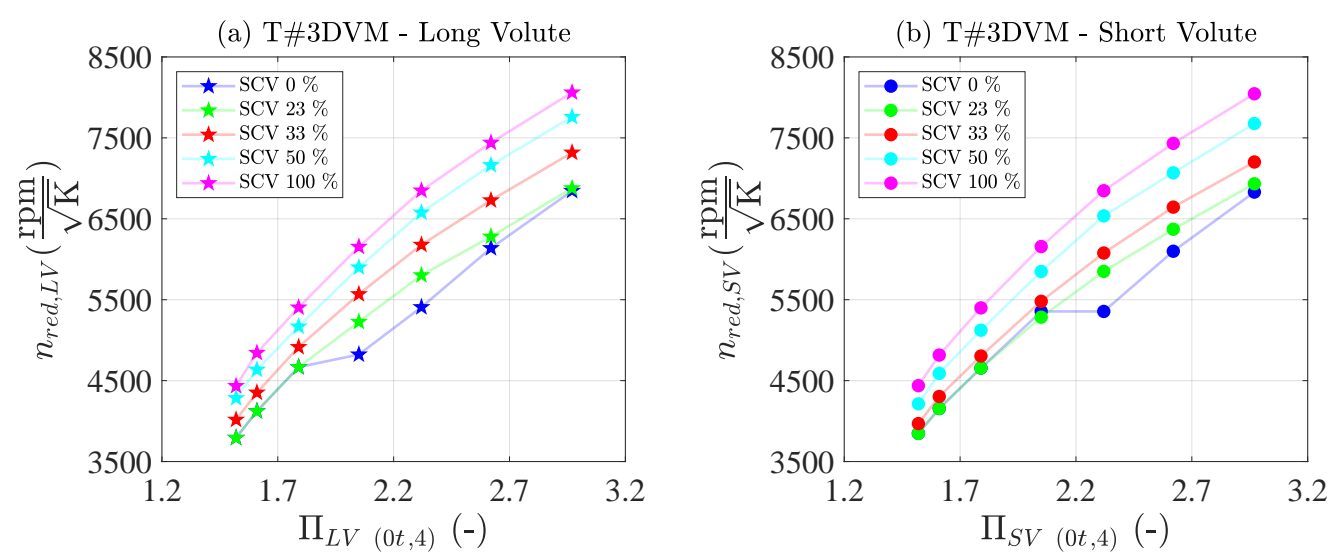

Figure 5.22: Variation in turbine reduced speed for recovering the same expansion ratio in the active branch when the SCV is opened. Left: Long volute branch testing with SCV opening. Right: Short volute branch testing with SCV opening.

(a) T\#3DVM - Short Volute

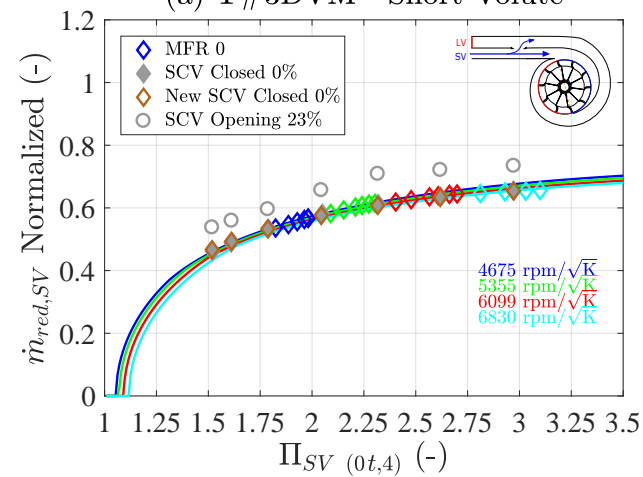

(c) T\#3DVM - Short Volute

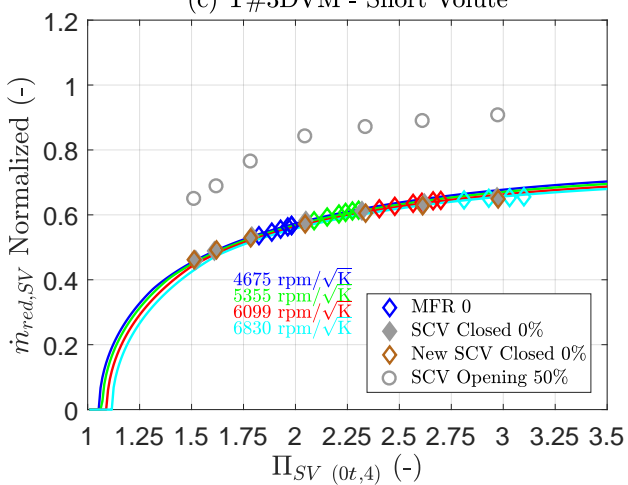

(b) T\#3DVM - Short Volute

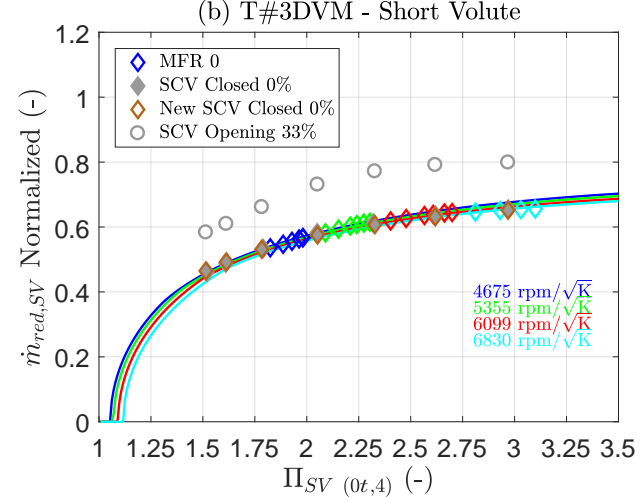

(d) T\#3DVM - Short Volute

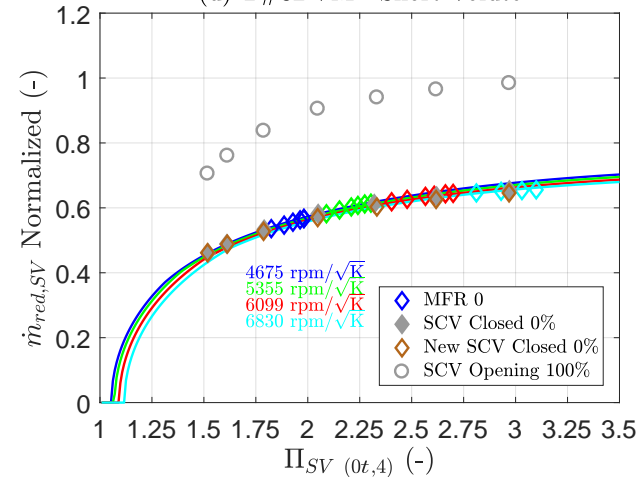

Figure 5.23: Extrapolated T\#3DVM turbine map when main flow is passed through the short volute branch. Showing with new and old SCV closed mass flows and there difference.

Figure 5.24 shows the results of flow that passed through the SCV segment for 
different SCV openings. As mentioned before, the flow can travel from long to short and vice versa depending on the flow situations in their respective volute branches. The first point of each series in Figure 5.24 expresses the difference between the flow measured in the turbine with the SCV closed in two test methods. Therefore, it is expected to be zero. For other SCV positions, the SCV flow is well coherent with there respective SCV openings and an expansion ratio of the turbine. It is worth highlighting that, testing both volutes individually with the same SCV positions and expansion ratio values, the SCV flow going from long to short is higher and from short to long is lower. It is because the pressure drop across the SCV section is different from long to short and vice versa. This indicates that flow motion in each direction dependent on the scroll pressure ratio; it is further discussed in the Figure 5.27. The reason for the different pressure drop at the SCV section lies in the shape of turbine volutes and its designs.

(a) T\#3DVM

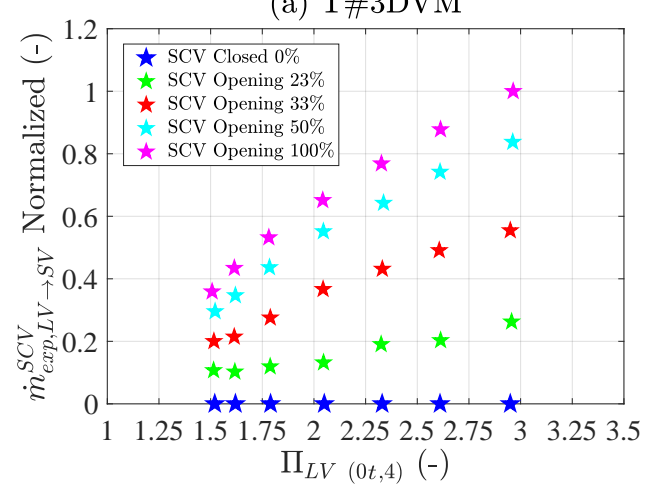

(b) T\#3DVM

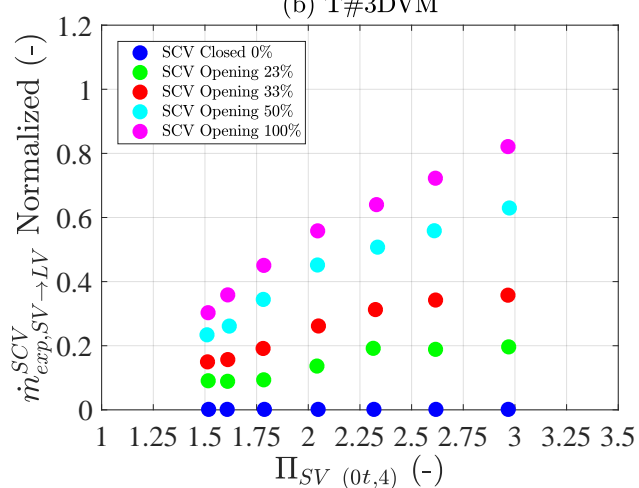

Figure 5.24: Actual mass flow through the SCV segment at different SCV openings. (a) Long volute branch testing with SCV opening, (b) Short volute branch testing with SCV opening.

When the scroll connection valve is opened, the flow capacity of the turbine changes from partial to unequal or full admission conditions. And which is entirely dependent on the flow in each direction and SCV position. Once, the SCV flow is estimated with the procedure shown in Figure 5.20, the real MFR of the turbine working condition can be calculated using the equations 5.16 and 5.17.

$$
\begin{aligned}
& M F R=\frac{\dot{m}_{L V}}{\dot{m}_{L V}+\dot{m}_{L V \rightarrow S V}^{S C V}} \\
& M F R=\frac{\dot{m}_{L V \leftarrow S V}^{S C V}}{\dot{m}_{L V \leftarrow S V}^{S C V}+\dot{m}_{S V}}
\end{aligned}
$$

Figure 5.25 shows the results of a change in mass flow ratio conditions for both tested volutes. From Figure 5.25 (a), it can be ascertained that MFR shows decreasing values from 1 to 0.7 when the SCV flow crossed from long to short volute 
branch (Equation 5.16). In the case when SCV flow moves from short to long volute, the MFR shows an increasing value from 0 to 0.27 (Equation 5.17) as shown in Figure 5.25(b). For both tested volutes, flow situation in the turbine never arrived at the full admission state even with the fully SCV opened position. Furthermore, it is essential to know the real MFR conditions in the turbine, when an SCV valve is present. As the efficiency of the turbine changes with MFR as detailed in the chapter 3.
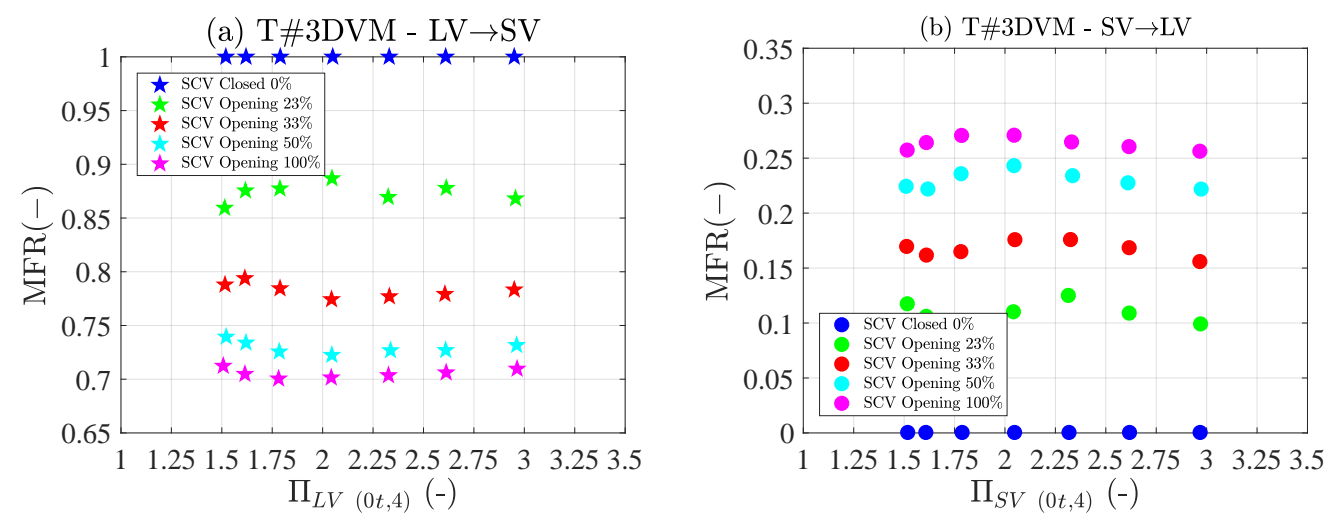

Figure 5.25: Change in MFR with respective to the SCV opening and the flow direction to each side through SCV. Left: Main flow in the long volute branch with SCV opening.

Right: Main flow in the short volute branch with SCV opening.

\subsubsection{SCV discharge coefficient modelling}

As detailed in section 5.2, the unchoked ideal mass flow rate through an orifice can be calculated using the gas properties, inlet conditions and pressure ratio between the inlet and outlet of the orifice. Accordingly, Equation 5.18 is used for calculating the ideal mass flow rate through the SCV segment at a given constant area $\left(A_{\text {geom }}^{S C V}\right)$. Index $k$ and $i$ represents the direction of SCV flow and the turbine inlet volutes.

$$
\dot{m}_{i d e a l, k}^{S C V}=\frac{p_{0 t, i}}{\sqrt{T_{0 t, i}}} A_{\text {geom }}^{S C V} \sqrt{\frac{\gamma}{R}}\left(\frac{1}{\Pi_{(t / s), k}^{S P R}}\right)^{\frac{1}{\gamma}} \sqrt{\frac{2}{\gamma-1}\left(1-\left(\frac{1}{\Pi_{(t / s), k}^{S P R}}\right)^{\frac{\gamma-1}{\gamma}}\right)}
$$

When the SCV is opened, the flow communicates between the branches. Therefore, the static pressure at the SCV segment is assumed to be equal to static turbine pressure measured in the non-active branch (see Figure 5.17). Consequently, the Scroll Pressure Ratio (SPR) between the branches can be determined, and it is represented as $\Pi_{(t / s), k}^{S P R}$. It is calculated by doing the ratio of total pressure in the active branch to the static pressure in the non-active branch (active branch refers to volute 
with the flow, and non-active branch refers to volute with no flow). It is worth noting that, when the SCV flow is passing from long to short volute, the SPR is calculated using the Equation 5.19 and, in the other flow direction using the Equation 5.20. Whereas, $T_{03, i}$ and $p_{03, i}$ is the turbine inlet temperature and pressure in the active branch.

$$
\begin{aligned}
& \Pi_{(t / s) L V \rightarrow S V}^{S P R}=\frac{p_{0 t, L V}}{p_{0, S V}} \\
& \Pi_{(t / s) L V \leftarrow S V}^{S P R}=\frac{p_{0 t, S V}}{p_{0, L V}}
\end{aligned}
$$

Figure 5.26 shows the relation of SCV mass flow estimated against the ideal SCV mass flow given by Equation 5.18 for different opening positions of the valve. On the one hand, the ideal mass flow rate rises with increasing scroll pressure ratio $\left(\Pi_{(t / s)}^{S P R_{k}}\right)$ for all the SCV openings as expected. On the other hand, it can be perceived that, at higher SCV openings (50\% and 100\%), the estimated ideal mass flow rates are lower than the SCV openings below $50 \%$. This is due to the difference in scroll pressure ratio values for each measured point is low (further shown in Figure 5.27). Moreover, a single discharge coefficient value for both flow directions at a given SCV position cannot be fitted. Since, the effective section for each flow direction is non-identical; as, there is a change in SCV flow depending on its direction. The experimental discharge coefficient values can be estimated by doing the ratio between the experimental and ideal SCV flow, as shown in Equation 5.21.

$$
C_{d, e x p}^{S C V, k}=\frac{\dot{m}_{e x p, k}^{S C V}}{\dot{m}_{i d e a l, k}^{S C V}}
$$

(a) T\#3DVM

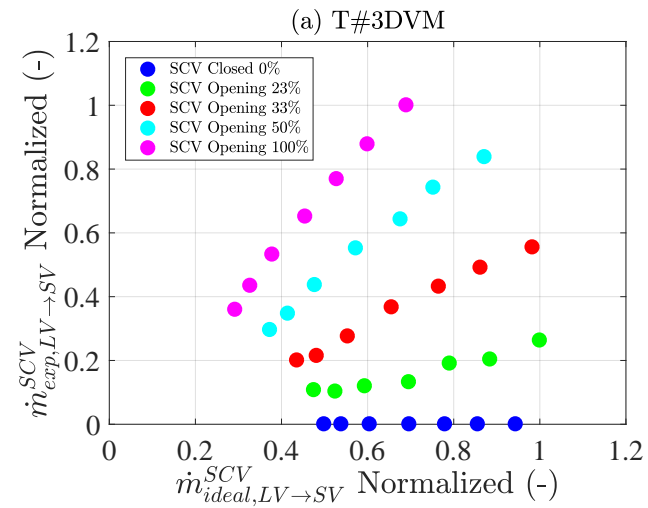

(b) T\#3DVM

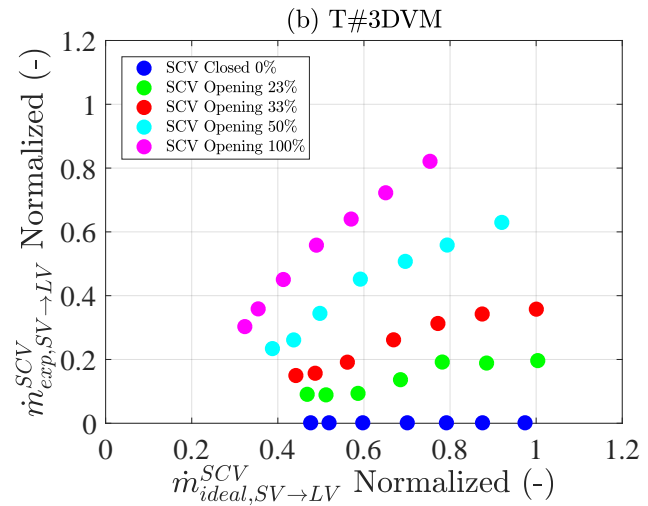

Figure 5.26: Actual mass flow against the ideal mass flow for SCV valve. (a) SCV flow LV to SV. (b) SCV flow SV to LV. 
Figure 5.27 shows the results of the SCV valve discharge coefficient for measured points. It is important to remark that for same SCV position having the entire flow in long or short volute, the change in discharge coefficient is as expected. Moreover, it is very dependent on the scroll pressure of the turbine (see equations 5.19 and 5.20). It can be observed that, when the SCV valve is at $23 \%$ and below, the discharge coefficient values showed a linear trend with scroll pressure ratios values in both the flow directions. But, for above $23 \%$ of SCV openings, the trend is not so linear. Looking at the 50\% and 100\% SCV openings in the Figure 5.27, as the scroll pressure ratio increases (it increases with the expansion ratio of the turbine) at a point, the discharge coefficient values are decreasing. This is due to the estimated ideal mass rate through the Equation 5.9 for this SCV position shows a lower value than the other SCV position; as the difference in scroll pressure ratio for each measured point is low compared to the other SCV openings.
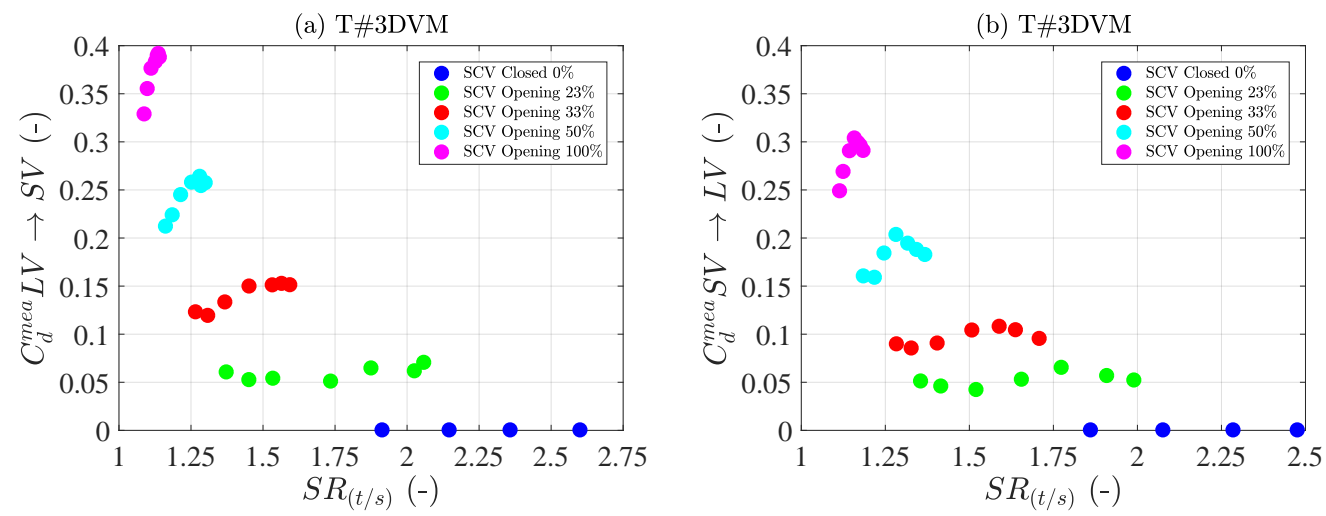

Figure 5.2\%: Variation of SCV discharge coefficient values with scroll pressure ratio. (a) Flow passing from LV to SV, (b) Flow passing from SV to LV.

In order to obtain an expression that can be used for predicting a variety of operating condition at which the engine works, an expression with the sum of sinus and exponential terms is used as a function of SCV opening and scroll pressure ratio as shown in the Equation 5.22. The same equation is used for predicting the discharge coefficient values in each flow direction and, it is fitted using all the measured points of each volute individually. This way, the coefficient values found during the fitting procedure are different, as shown in Table 5.2 with there significance. It is worth to be noted that, the SCV opening positions have been divided by 100 in order to obtain the fitting coefficient values with the orders of magnitude around unity.

$$
C_{d, \text { mod }}^{S C V, k}=a+\left[b \cdot\left(\sin \left(c \cdot\left(\frac{\% S C V, k}{100}\right)+d\right)^{2}\right)\right]+\exp \left(e \cdot\left(\Pi_{(t / s), k}^{S P R}-1\right)^{f}\right)
$$


Table 5.2: Quality of scroll connection valve discharge coefficient model fitting for T\#3DVM turbine

\begin{tabular}{ccc}
\hline \multirow{2}{*}{ Coefficients } & SCV flow LV to SV & SCV flow SV to LV \\
\cline { 2 - 3 } & Estimation & Estimation \\
\hline$a$ & -0.5050 & -0.6528 \\
$b$ & -0.4887 & -0.3435 \\
$c$ & 1.7785 & 1.7105 \\
$d$ & -1.5905 & -1.5683 \\
$e$ & -0.0059 & -0.0014 \\
$f$ & -1.3677 & -1.9237 \\
\hline SSE & 0.0090 & 0.0017 \\
R $^{2}$ & 0.9857 & 0.9952 \\
Adjusted R & 0.9861 & 0.9953 \\
RMSE & 0.0083 & 0.00699 \\
\hline
\end{tabular}

(a) Discharger Coefficient LV $\rightarrow \mathrm{SV}$

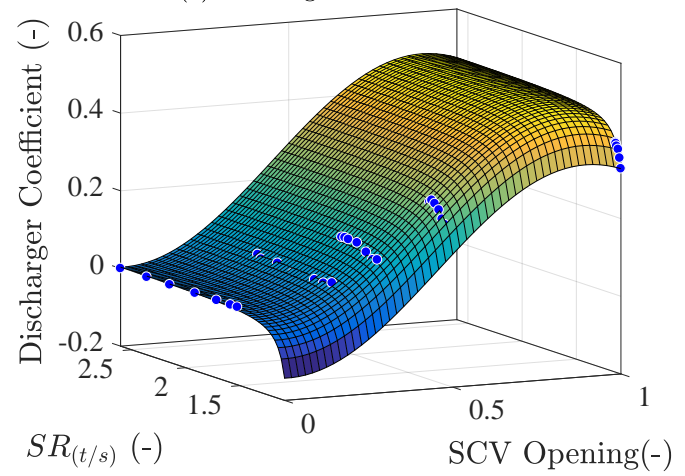

(b) Discharger Coefficient SV $\rightarrow \mathrm{LV}$

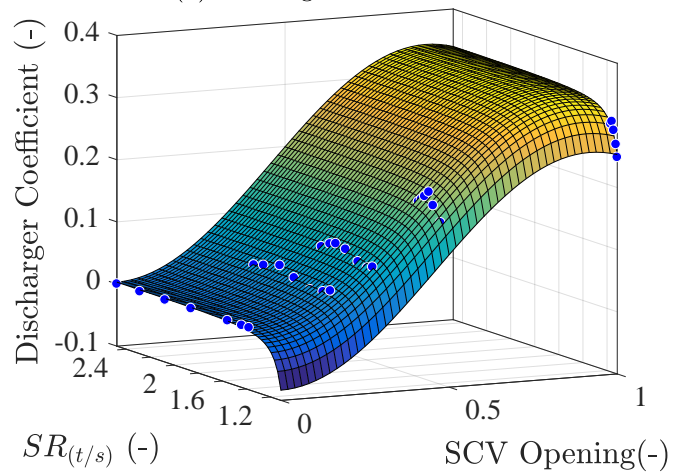

Figure 5.28: Modelled discharge coefficient of scroll connection valve. (a) For flow passing from LV to SV. (b) For flow passing from SV to LV.

Figure 5.28, shows the empirical model results that are fitted as a function of SCV opening and scroll pressure ratio. This model can be easily implemented in the one-dimensional engine simulations to improve the predictions of overall engine performance. In Figure 5.29, the modelled discharge coefficient values were compared against the calculated discharge coefficient values from the measured points. As shown, the predicted SCV discharge coefficient values for each flow direction agreed well with the measured ones. A root mean square error of 0.00833 for SCV flow LV to SV and 0.00699 for SCV flow SV to LV was observed. The relation between scroll connection valve opening and scroll pressure ratio makes it possible to model the accurate predictions of the SCV valve discharge coefficient.

Once the empirical model fitted, it is easy to determine the mass flow through the SCV going from LV to SV and SV to LV. That is, by using the respective predicted 

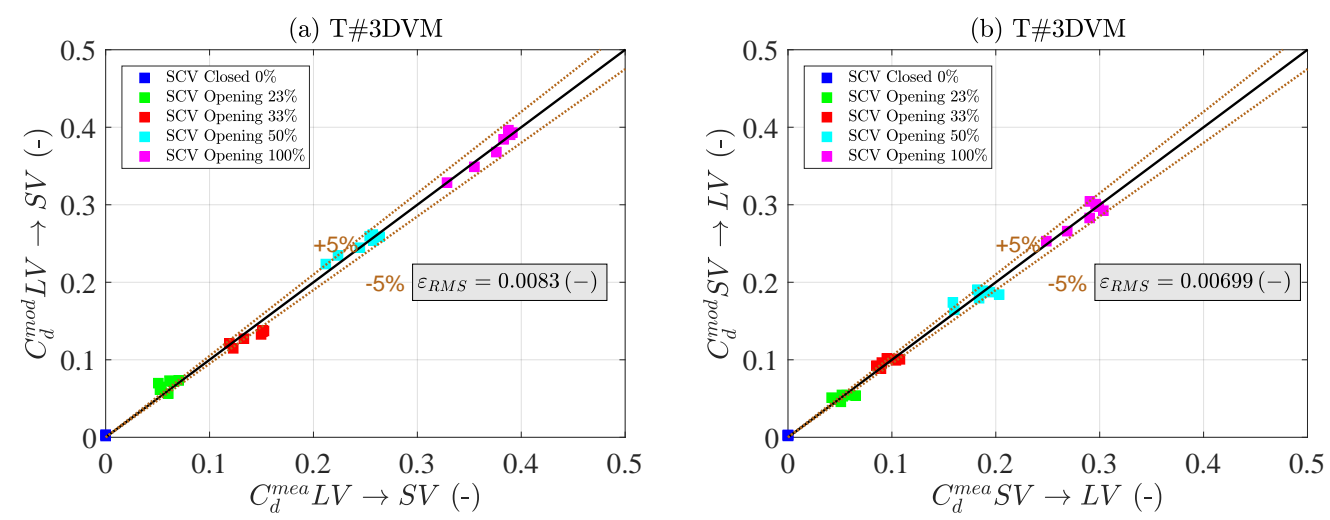

Figure 5.29: Comparison of modelled discharge coefficient values against experimental discharge coefficient values in all the SCV openings. (a) For flow passing from LV to SV. (b)

For flow passing from SV to LV.

discharge coefficient values from the Equation 5.22 into Equation 5.23. Figure 5.30 shows the modelled reduced mass flow through the SCV compared to experimental SCV reduced mass flows in each direction. A root mean square error of 0.0008989 for $\mathrm{LV}$ to $\mathrm{SV}$ and 0.0006877 for $\mathrm{SV}$ to $\mathrm{LV}$ in the reduced mass flow prediction is observed. Few discrepancies are found in the prediction of reduced mass flow at low SCV openings as compared to higher SCV openings. However, many of the points are within the range of $\pm 5 \%$. Therefore, this empirical model can be acceptable to be used in whole one-dimensional turbocharged engine simulations.

$$
\dot{m}_{e x p, k}^{S C V}=C_{d, \bmod }^{S C V, k} \cdot \dot{m}_{i d e a l, k}^{S C V}
$$
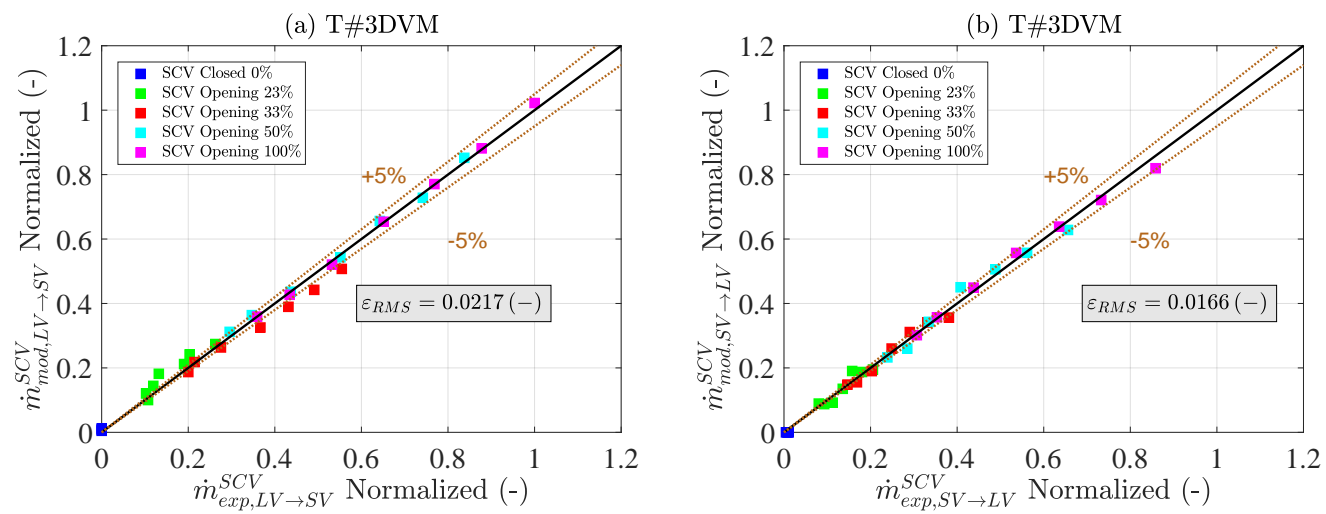

Figure 5.30: Comparison of modelled SCV reduced mass flow values against experimental $\mathrm{SCV}$ reduced mass flow values in all the SCV openings. Left: For flow passing from LV to SV. Right: For flow passing from SV to LV. 



\section{Chapter 6}

\section{Heat transfer characterization}

\section{Contents}

6.1 Introduction . . . . . . . . . . . . . . . . 150

6.2 Refined thermohydraulic tests . . . . . . . . . . . 150

6.3 General correlation for heat transfer . . . . . . . . . . . . . . 154

6.3.1 Capacitance modelling . . . . . . . . . . . . . . . 155

6.3.2 Conductive conductances modelling . . . . . . . . . . . . 159

6.4 Model validation using gas stand simulations . . . . . . . . . . . 162

6.4.1 Model Results . . . . . . . . . . . . . . . . . . . . . 163

6.5 Heat transfer in double entry turbines . . . . . . . . . . . 165 


\subsection{Introduction}

In this chapter, refined thermohydraulic tests were performed using the T\#4TER turbocharger to determine the metal properties like metal conductance and thermal capacitance. The sophisticated tests were performed by following the methodology described in [19]. Following this test, a general correlation model which determines the metal properties of the turbocharger has been updated with CMT turbocharger database. Also with new material properties which are more consistent enough for both diesel and petrol turbochargers. In the case of capacitance determination for each node, the model of Serrano et al. [65] has been used and has been refitted with the new database and material properties. Whereas, for conductance determination, the model of Serrano et al. [65] has been refined with a new definition for estimating the contact area between the two metal nodes. Further, both models were validated using the experimental data from thermohydraulic tests and also by performing the hot exposed gas stand simulation in one-dimensional software. Finally, the adaptation of the heat transfer model that is explained in the chapter 2 to double-entry turbochargers is discussed.

The works, analysis and ideas described in this chapter were the origins of publication number [2] from the publications list of the author of this thesis. In the sake of readiness and to protect the thesis writing style the publication number [2] from author's list of publications have not been specifically cited every time that ideas, figures or discussions contained in it are bring to this chapter again. This disclaimer corrects, compensates and justify this fact; being the Ph.D. candidate and the works of his thesis the origin of the innovation component in the publication number [2] listed in the referred section.

\subsection{Refined thermohydraulic tests}

The test rig and test methodology were already described in Chapter 2, and here, the test results are shown and explained. A typical trend of temperature evolution at each plane node during the entire test is shown in the Figure 6.1. It is divided into three stages. The first stage shows the sudden increase of all the wall temperatures due to hot oil which starts to circulate through the component. Before the first stage, all the wall temperatures were equal to the ambient temperature. In the second stage, stabilization is required until all the wall temperature are lower than $0.01^{\circ} \mathrm{C}$ per minute, as shown in the Figure 6.2. The end of this one coincides with the steadystate test; then the measurement is performed. The last stage shows the discharge of the hot oil from the hot circuit, in which transient state test temperatures are acquired. Both steady and transient were repeated twice: once passing the turbine element with hot oil and the compressor element with the cold oil, vice versa. 


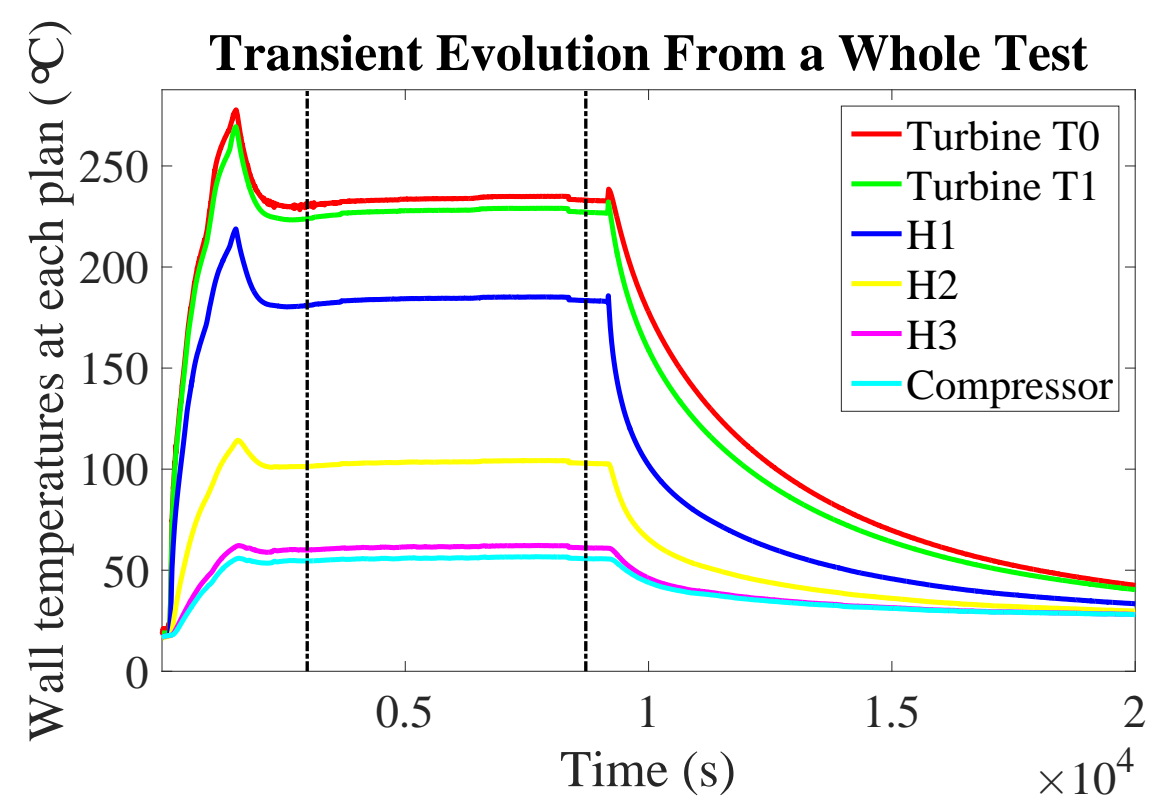

Figure 6.1: Node temperature evolution in whole test, both steady and transient test are performed at once.

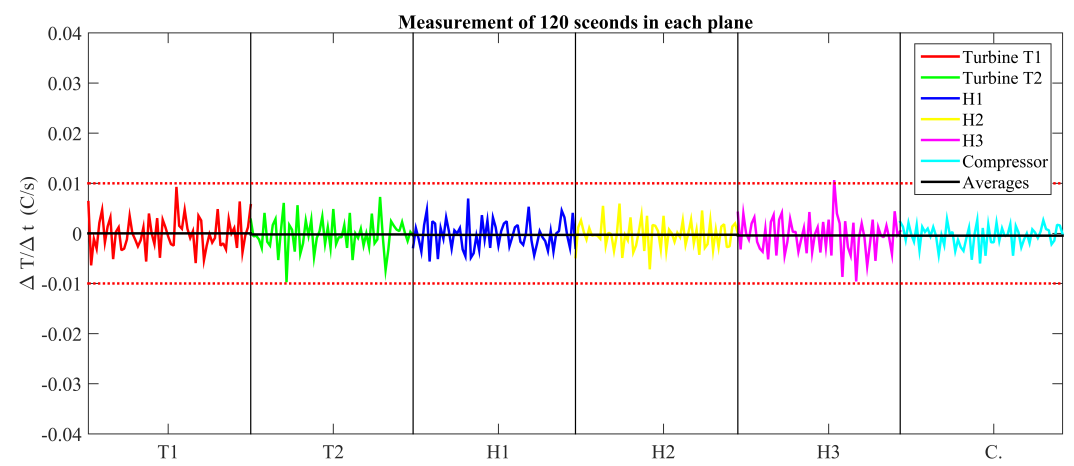

Figure 6.2: A criteria of lower variation than $0.01^{\circ} \mathrm{C}$ per minute in turbocharger wall temperature is used to ensure that steady state conditions are achieved.

Figure 6.3 shows the arrangement of $\mathrm{K}$ type thermocouples on the turbocharger surface. At each plane, three thermocouples were installed at different azimuthal locations in order ensure that circumferential temperatures were neglected compared to axial temperature distribution [19].

Both steady and transient experiments were performed with two inlet temperatures $\left(10^{\circ} \mathrm{C}\right.$ and $\left.20^{\circ} \mathrm{C}\right)$ in the cold circuit and constant inlet temperature of around $300^{\circ} \mathrm{C}$ in the hot circuit. Further, a constant oil mass flow rate of $25 \mathrm{~kg} / \mathrm{h}$ is maintained in both channels in a radial direction. Measurements with different inlet tempera- 


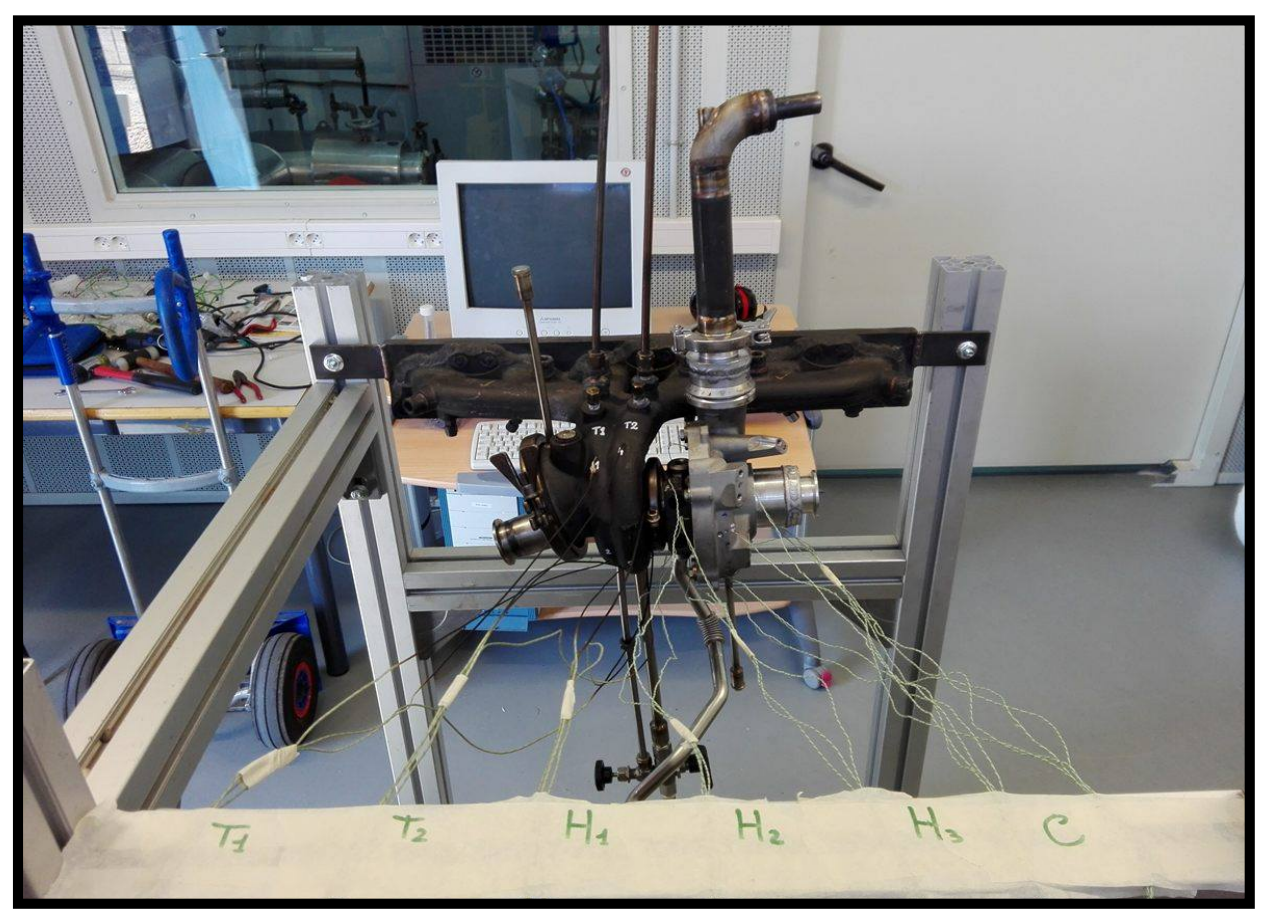

Figure 6.3: Showing thermocouple instrumentation on the turbocharger wall

tures at the cold circuit were verified by checking the averaged node temperatures, as shown in Figure 6.4. It is worth highlighting that the hot or cold oil at the turbine is passed into both inlets of the turbine. Accordingly, the node temperatures T0 and T1 in the Figure 6.4 representing the Shroud and Hub branches of the turbine are at similar temperatures in two different tests. Figure 6.4 (a) shows the evolution of node temperature when the hot oil is passed through the turbine and Figure 6.4 (b) shows the node temperatures when the hot oil is passed through the compressor.

In the steady-state test, all the heat released by the hot circuit is absorbed by the cold circuit since the turbocharger is thermally insulated. The temperature difference between the inlet and outlet of the turbine and compressor is due to only the heat transfer between them. Since the shaft is blocked, the system is externally insulated, and the fluid is incompressible [19]. The amount of this heat is determined through the mass flow measurements, oil temperature measurements, and the specific heat capacity of oil as shown in the equations 6.1 and 6.2 respectively.

$$
\begin{gathered}
\dot{Q}_{h o t}=\dot{m}_{h o t} \cdot c_{o i l} \cdot\left(T_{i n}^{h o t}-T_{o u t}^{h o t}\right) \\
\dot{Q}_{c o l d}=\dot{m}_{c o l d} \cdot c_{o i l} \cdot\left(T_{o u t}^{c o l d}-T_{i n}^{c o l d}\right)
\end{gathered}
$$


(a)

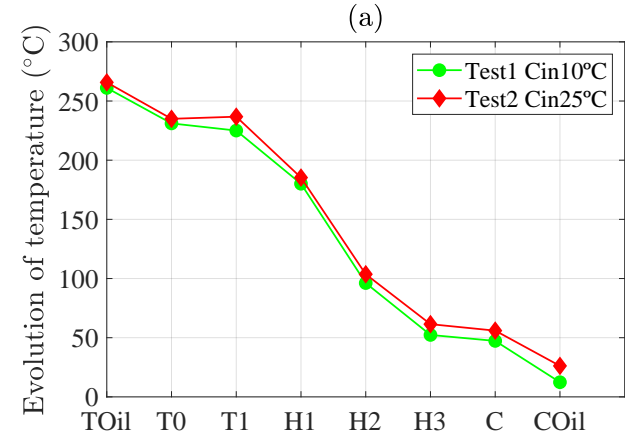

(b)

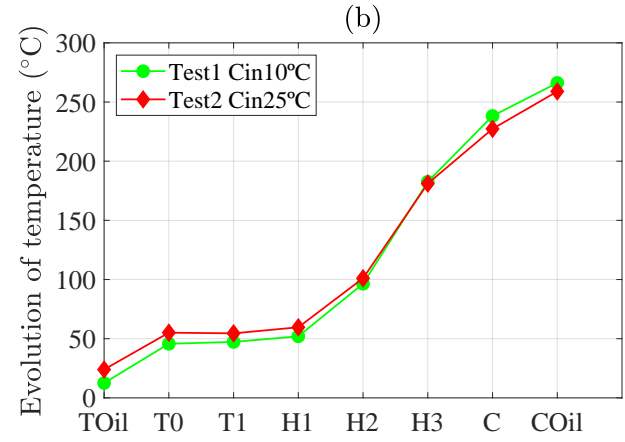

Figure 6.4: Node temperatures from steady state tests; (a) When the hot oil in turbine element; (b) When the hot oil in compressor element.

By keeping the constant mass flow in both circuits, the temperature difference between the inlet and outlet is increased and very low unbalance of heat is achieved in steady-state tests. From Figure 6.5, it can be observed that the possibility of achieving low unbalance of heat is with an inlet temperature of $10^{\circ} \mathrm{C}$ in the cold circuit. As it is taking all heat coming from the hot circuit through the housing without any loss to ambient. Therefore, it can be concluded that the obtained conductive conductance from these tests is reliable enough for estimating the heat transfer of this turbocharger.

(a)

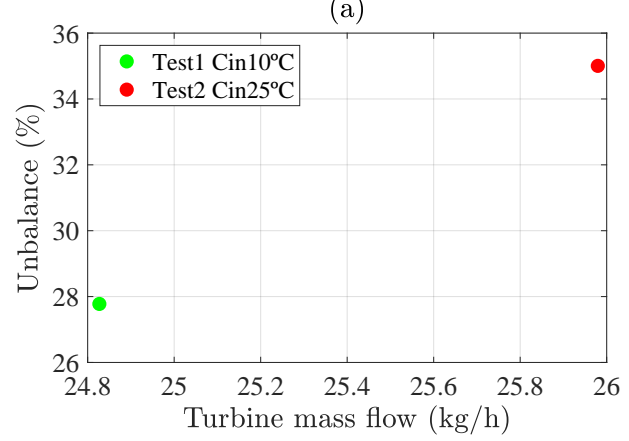

(b)

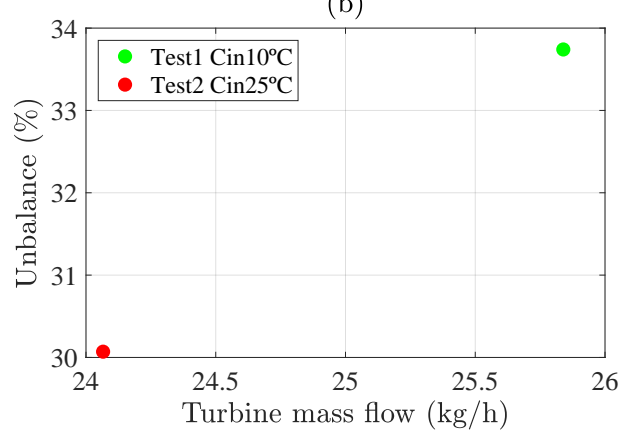

Figure 6.5: Heat unbalance in steady state tests; (a) When the hot oil in turbine element; (b) When the hot oil in compressor element.

Once the steady-state experiment is done, the hot oil is discharged from the hot circuit, and all the measurement variables recorded until they are stabilized. The data from this test are used for the determination of thermal capacitance.

Conductive conductances between the nodes $\mathrm{H} 1$ and $\mathrm{H} 3\left(K_{H 1 / H 2}\right.$ and $\left.K_{H 2 / H 3}\right)$ is estimated by using the Fourier's law Equation 6.3 with steady-state experimental data. Because, the case temperatures are acquired, and heat flux is the enthalpy drop of the oil, as equations 6.1 and 6.2 suggests. Regarding conductances $K_{T / H 1}$ and $K_{H 3 / C}$ are not easy to obtain because the fluid passing through the turbine and 
compressor exchanges heat by convection with the nodes $\mathrm{H} 1$ and $\mathrm{H} 3$ respectively. Because, in the thermohydraulic test bench, the residence time of the oil in both hot and cold circuits is high enough to allow convective heat transfer. Therefore, there is a convective heat transfer from hot oil to node $\mathrm{H} 1$ and from node $\mathrm{H} 3$ to cold oil. To properly determine the conductive conductance between the nodes T/H1 and $\mathrm{H} 3 / \mathrm{C}$, the convective heat transfer coefficient between oil/H1 and H3/oil should be known. However, this heat transfer disappears during the transient tests; since no oil is passed through one of the elements. As described in [19], by applying the law of energy conservation equation to metal node T, H1, H2, H3 and $\mathrm{C}$ for the transient state, it is possible to determine all the capacitance $\left(C_{T}, C_{H 1}, C_{H 2}, C_{H 3}\right.$ and $\left.C_{C}\right)$ and conductive conductance of $K_{T / H 1}$ and $K_{H 3 / C}$ values. Table 6.1 , shows the averaged values of conductance and capacitance of T\#4TER turbocharger obtained from the different tests that were performed in the thermohydraulic test bench.

$$
\dot{Q}_{i, j}^{C o n d}=K_{i, j} \cdot\left(T_{i}-T_{j}\right)
$$

Table 6.1: Conductance and capacitance results from the thermohydraulic test bench

\begin{tabular}{cc}
\hline Turbo & T\#4TER \\
\hline Conductances $\left[W K^{-1}\right]$ & \\
$K_{T / H 1}$ & 6.780 \\
$K_{H 1 / H 2}$ & 2.749 \\
$K_{H 2 / H 3}$ & 3.509 \\
$K_{H 3 / C}$ & 12.834 \\
Capacitances $\left[J K^{-1}\right]$ & \\
$C_{T}$ & 782.729 \\
$C_{H 1}$ & 305.108 \\
$C_{H 2}$ & 150.121 \\
$C_{H 3}$ & 202.564 \\
$C_{C}$ & 580.907 \\
\hline
\end{tabular}

\subsection{General correlation for heat transfer}

Conductive conductance and thermal capacitance properties are essential parameters to characterize the heat transfer model. To be able to use the lumped model described in the subsection 2.6.2, the user needs to provide these properties for each node. Therefore, a theoretical method to find the metal properties is necessary so that the thermohydraulic test can be avoided. In this point of view, Serrano et al. [65] described a model that divides the turbocharger corresponding to the lumped model 
shown in Figure 2.2. It divides the turbocharger into different nodal planes. Further, turbocharger geometry is simplified; it is considered as a series of cylinders and cone frustums coupled together [65].

In the thesis work, the model of Serrano et al. [65] has been used for estimating the thermal capacitance of the turbocharger for each node. For estimating conductive conductances, a different approach has been considered. In this regards, a precise definition for calculating the area with contact surface between the two metal nodes has been developed. Both capacitances and conductances models are fitted with various set of turbochargers. In which, they have different external and rotor geometries, as shown in Table 6.2. The turbocharger T\#13 is used to check the ability of the correlations for predicting the capacitance and conductance. As this turbocharger was tested in the thermohydraulic test bench. In the subsection 6.3.1, a summary of capacitance modelling has been described. Later the development of conductance modelling has been explained in subsection 6.3.2.

\subsubsection{Capacitance modelling}

The capacitance of each node can be calculated from the product of the mass of the node and the specific heat capacity of the corresponding node, whose different material properties for each turbocharger component are listed in Table 6.3. When the information of the material is not available, the properties that are shown in Table 6.3 gives a good approximation of material properties.

The division of turbocharger into different nodes does not allow to use the below expression directly.

$$
C_{i}=m_{i} \cdot c_{i}=\rho \cdot V_{i} \cdot c_{i}
$$

As the central housing is divided into three nodes, and the contribution of different turbocharger components on some of the nodes is significant. So, Serrano et al. [65] assumed direct contribution from each node to other adjacent nodes. Equation 6.4 has been modified with some fitting constants $(\alpha, \beta, \gamma$ and $\epsilon)$ to use directly without any adjacent node contribution. Fitting constants are obtained with the selected turbochargers shown in Table 6.4, and the constraints of the constants have been chosen so that they should be neither more than unity nor less than zero. In the case of central node $\mathrm{H} 2$, to avoid zero contribution $\beta+\gamma$ must be less than one.

$$
\begin{aligned}
& C_{T}=\alpha \cdot m_{T} \cdot c_{T} \\
& C_{H 1}=(1-\alpha) \cdot m_{T} \cdot c_{T}+\beta \cdot m_{H} \cdot c_{H} \\
& C_{H 2}=(1-\beta-\gamma) \cdot m_{H} \cdot c_{H} \\
& C_{H 3}=(1-\epsilon) \cdot m_{C} \cdot c_{C}+\gamma \cdot m_{H} \cdot c_{H} \\
& C_{C}=\epsilon \cdot m_{C} \cdot c_{C}
\end{aligned}
$$




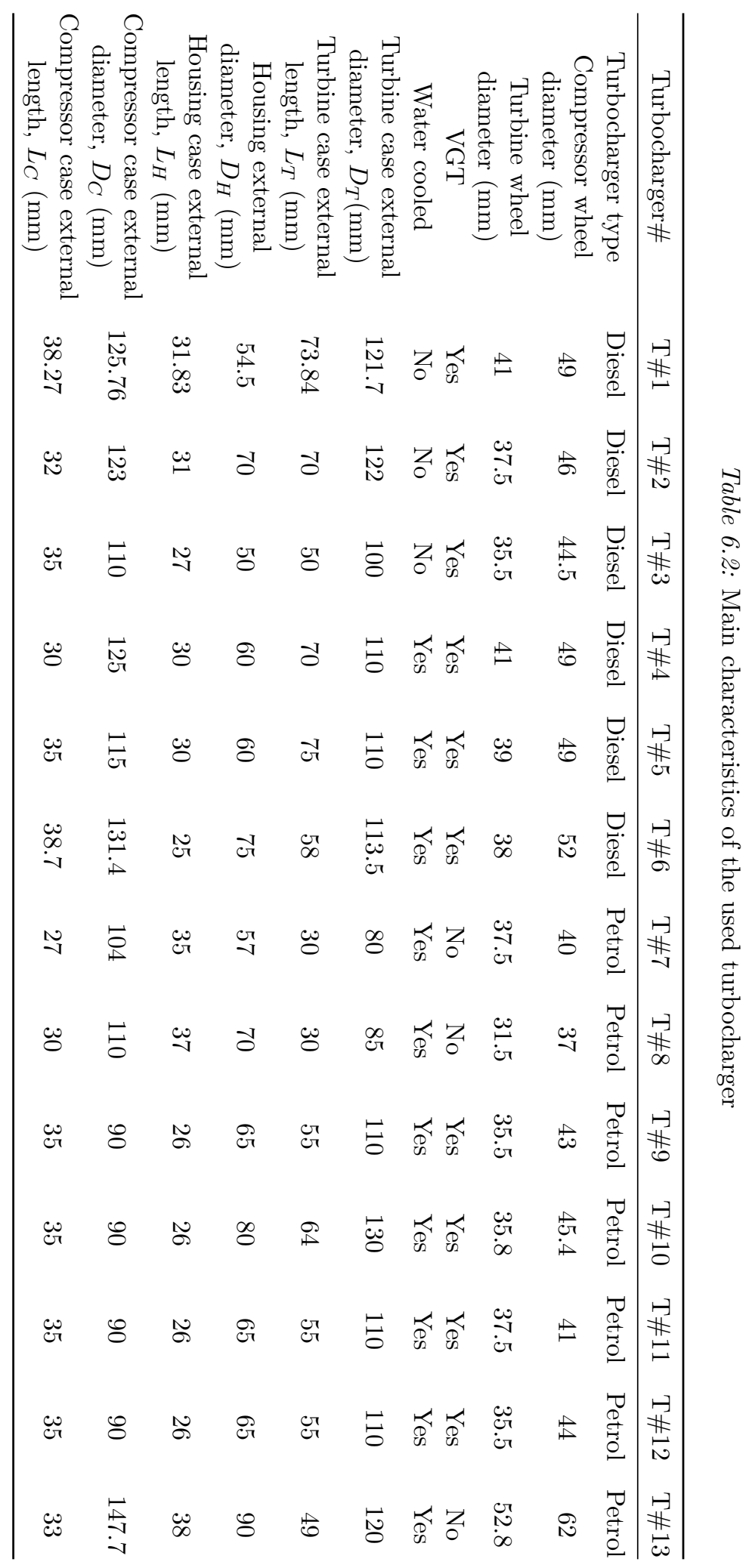


Table 6.3: Turbocharger material properties

\begin{tabular}{ccccc}
\hline Parts & Material & $\begin{array}{c}\text { Specific Heat } \\
\left(\mathrm{JKg}^{-1} \mathrm{~K}^{-1}\right)\end{array}$ & $\begin{array}{c}\text { Density } \\
\left(\mathrm{Kgm}^{-3}\right)\end{array}$ & $\begin{array}{c}\text { Conductivity } \\
\left(\mathrm{Wm}^{-1} \mathrm{~K}^{-1}\right)\end{array}$ \\
\hline Turbine & SIMO Iron Casting & 580 & 7200 & 36.60 \\
Housing & FT200 Iran Casting & 460 & 7200 & 47.50 \\
Compressor & Aluminum & 921 & 2690 & 190 \\
\hline
\end{tabular}

$$
\begin{array}{r}
0 \leq \alpha, \beta, \gamma, \epsilon \leq 1 \\
0<\beta+\gamma<1
\end{array}
$$

Aforementioned, turbocharger geometry is hard to acquire and sometimes it is not possible to calculate. So, to calculate the mass of each node, a simplified method is applied, as mentioned by Serrano et al [65]. This method has a few assumptions that need to be accepted. A cone frustum is considered for housing and cylinders for the turbine and compressor. To correct these simplifications three fitting constants are used. These fitting constants $\left(\theta_{i}\right)$ will take care of geometry because they are not solid cylinders and cone frustum, fluid is passing through the turbocharger.

$$
\begin{aligned}
& m_{H}=\theta_{H} \cdot \rho_{H} \cdot \frac{\pi}{12} \cdot L_{H} \cdot\left(D_{T}^{2}+D_{C}^{2}+D_{T} \cdot D_{C}\right) \\
& m_{T}=\theta_{T} \cdot \rho_{T} \cdot\left(\frac{\pi \cdot D_{T}^{2}}{4} \cdot L_{T}\right) \\
& m_{C}=\theta_{C} \cdot \rho_{C} \cdot\left(\frac{\pi \cdot D_{C}^{2}}{4} \cdot L_{C}\right)
\end{aligned}
$$

Summarizing, all the constants which are four for the capacitance $(\alpha, \beta, \gamma$ and $\epsilon)$ and three for the geometry $\left(\theta_{T}, \theta_{H}, \theta_{C}\right)$ are fitted with the experimental data of selected turbochargers. These data are obtained from individual tests performed in thermohydraulic test bench $[64,19]$. The non-linear regression technique was used to find out the unknown parameters by minimizing the root mean square error between the measured and the modelled capacitance. Table 6.4 shows the model fitting constants. Analyzing them, one can generally conclude that turbine case mass has an influence of $20 \%$ in node $\mathrm{H} 1$ capacitance but bearing housing mass just $46 \%$. Furthermore, the compressor mass does not have any influence in the capacitance of bearing housing node $\mathrm{H} 3$, being influenced just by the $38 \%$ of the bearing housing mass. 
Table 6.4: Capacitance model fitting values

\begin{tabular}{cc}
\hline Coefficients & Estimation \\
\hline$\alpha$ & 0.7978 \\
$\beta$ & 0.4576 \\
$\gamma$ & 0.3826 \\
$\epsilon$ & 0.9750 \\
$\theta_{T}$ & 0.5827 \\
$\theta_{H}$ & 0.4969 \\
$\theta_{C}$ & 0.6257 \\
\hline
\end{tabular}

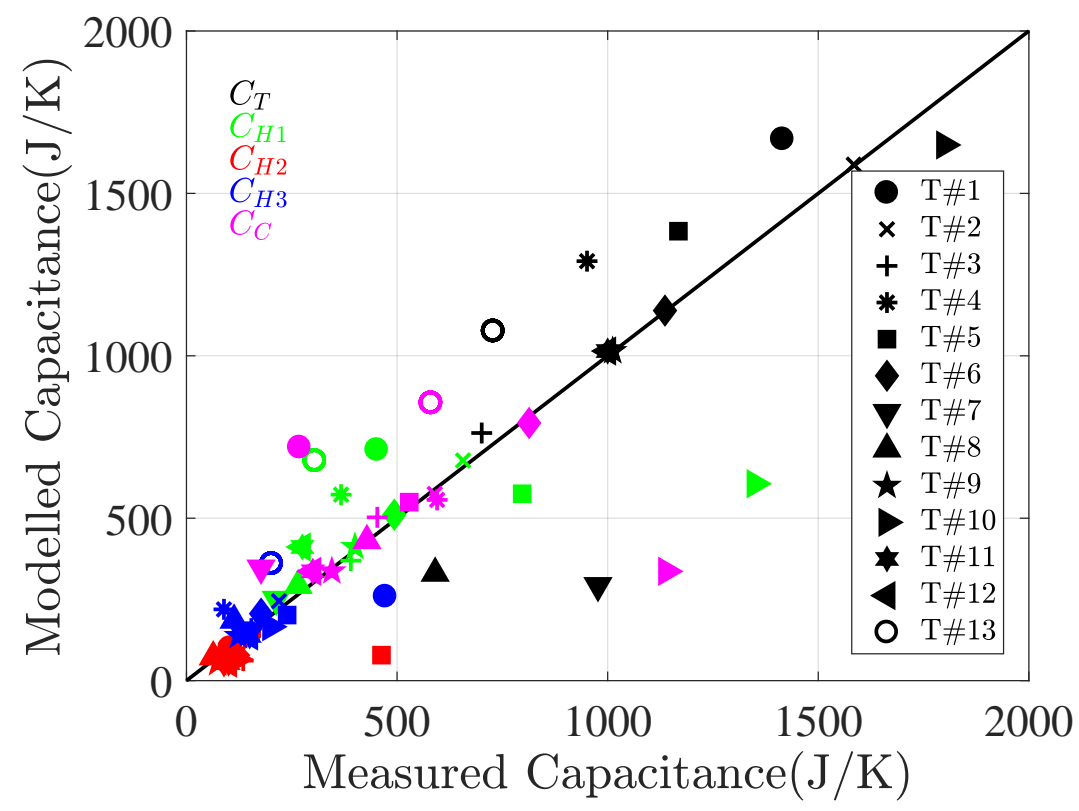

Figure 6.6: Capacitance fitting for different turbochargers

Concerning the mass approximation, one can conclude that just about $50 \%$ of the mass of a cone frustum represents the bearing housing mass. Considering the uncertainties in the experimental data and the inherent simplifications of Equation 6.7; the agreement between modeled and measured data is acceptable as it can be observed in Figure 6.6. Turbocharger T\#13 which is equal to T\#4TER in Table 1.1 (circle non-filled points in Figure 6.6) is used for the validation of the capacitance model, and its data has not been used in the minimization procedure. The modelled capacitance of this turbocharger shows good accordance with measured values. 


\subsubsection{Conductive conductances modelling}

The conductive conductance between two adjacent planes is a function of material conductivity, the width of each plane and a contact area between them [65] as defined in Equation 6.8.

$$
K_{i, j}=\frac{k_{i} \cdot k_{j}}{e_{i} \cdot k_{j}+e_{j} \cdot k_{i}} \cdot A_{i, j}
$$

For calculating the conductive conductance, it is necessary to know some information like material conductivity $\left(k_{i}\right.$ and $k_{j}$ ) at corresponding node, the equivalent width $\left(e_{i}\right.$ and $\left.e_{j}\right)$ between the planes and the contact area $\left(A_{i, j}\right)$. Table 6.3 shows the material conductivity of the turbocharger. The width of each plane is measured externally for turbine and compressor. As the housing is divided into three parts as shown in Figure 6.7 where the turbine and compressor casing corresponds to a node in the model. The fitting constants used for distributing the capacitance of housing nodes can be used for correct division of the housing length as shown in equation Equation 6.9 [65].

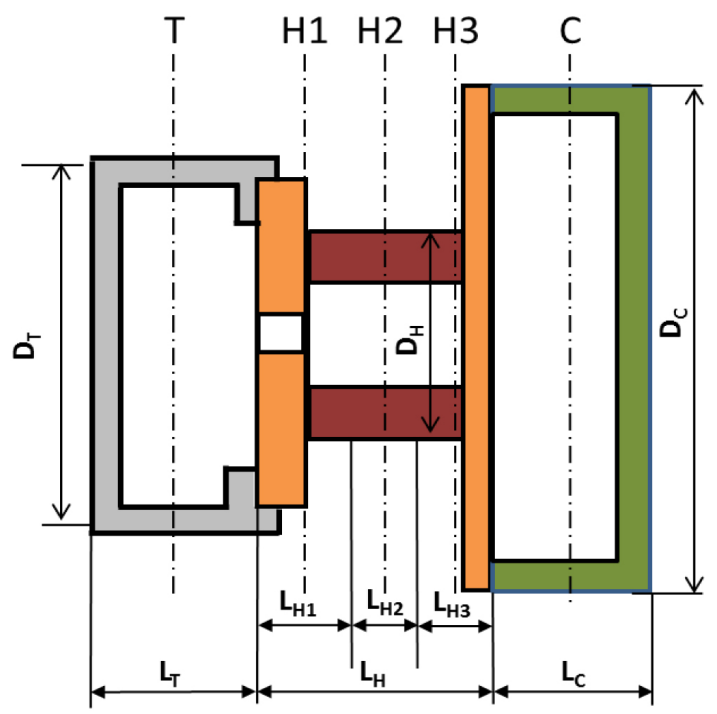

Figure 6.7: Simplification of turbocharger geometry [65]

$$
\begin{aligned}
& L_{H 1}=\beta \cdot L_{H} \\
& L_{H 2}=(1-\beta-\gamma) \cdot L_{H} \\
& L_{H 3}=\gamma \cdot L_{H}
\end{aligned}
$$

Finally, to find the conductive conductance from Equation 6.8, the contact area 
between the planes is needed. It cannot be calculated directly from the external geometry values, which are shown in Table 6.2. The flow passages of different fluids inside the turbocharger do not allow the simplification of contact areas to circumference areas. To the assumption of cone frustum and cylinders for turbo parts, the effective area is considered as circumferential with the contact width between the two metal nodes as shown in Equation 6.10; it is deduced according to the Figure 6.8.

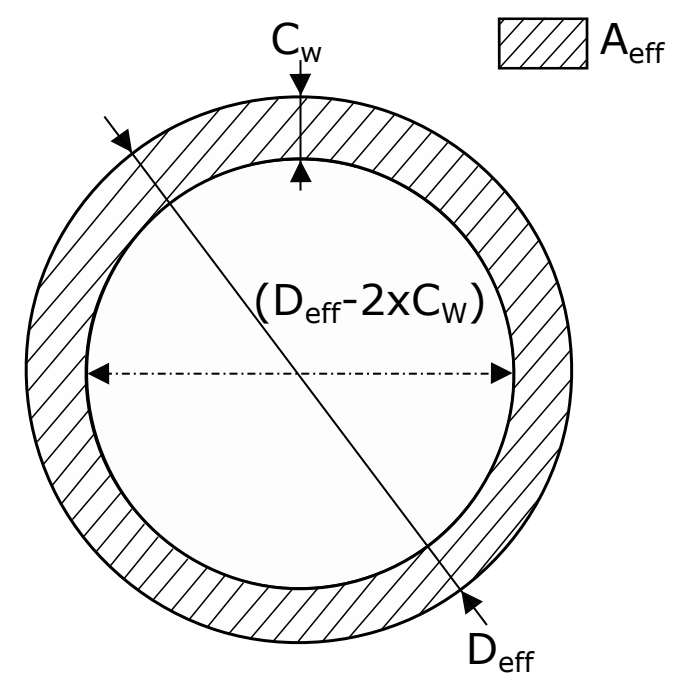

Figure 6.8: Estimating an effective area where contact is taking place between the two metal nodes

$$
A_{e f f}^{i, j}=\pi \cdot\left(D_{e f f}^{i, j}-C_{w}^{i, j}\right) \times C_{w}^{i, j}
$$

However, the measurement of effective contact width $\left(C_{w}\right)$ between the planes is unable to be determined from a turbocharger unit. Therefore, an expression with the average effective diameter $\left(D_{e f f}\right)$ between the two nodes have been considered by introducing three fitting parameters as shown in the Equation 6.11

$$
C_{w}^{i, j}=a_{i} \cdot\left(D_{e f f}^{i, j}\right)^{b_{i}}-c_{i}
$$

From Table 6.2, it can be observed that the external geometries of different turbochargers are not so uniform. Furthermore, the effective contact area of non-water cooled turbocharger should be different from those water-cooled; this is due to the additional water passages inside the housing. Considering these details, the effective diameter is modelled based on the power function, as shown in Equation 6.12, which has two fitting constants $\left(d_{i}\right.$ and $\left.e_{i}\right)$. This way, the effective diameter from smaller 
to bigger size turbochargers can be estimated.

$$
D_{e f f}^{i, j}=d_{i} \cdot\left(D_{m}^{i, j}\right)^{e_{i}}
$$

The mean diameter $\left(D_{m}^{i, j}\right)$ is taken as the average diameter between the corresponding turbocharger external geometry (turbine, housing, or compressor) as shown in Equation 6.13

$$
D_{m}^{i, j}=\frac{D_{i}+D_{j}}{2}
$$

It is worth to be noted that, the diameters of nodes $H 1$ and $H 3$ are considered as the average between the turbine, housing and compressor, housing diameters respectively as shown in the Equation 6.14

$$
D_{H 1}=\frac{D_{T}+D_{H}}{2} ; D_{H 3}=\frac{D_{H}+D_{C}}{2}
$$

This way, for calculating conductances $K_{T / H 1}$ and $K_{H 1 / H 2}$, diameters of turbine and housing are involved. While for $K_{H 2 / H 3}$ and $K_{H 3 / C}$, the diameters of compressor and housing are used. In summary, five fitting constants $\left(a_{i}, b_{i}, c_{i}, d_{i}\right.$, and $\left.e_{i}\right)$ have been fitted for each conductive conductance, using an optimization tool that minimizes the root mean square difference between modelled and measured conductances. The obtained fitting constants for different conductance are shown in Table 6.5. Figure 6.9 shows the results of the fitting with acceptable accuracy. The model is also validated with the turbocharger T\#13 (circle non-filled points in Figure Figure 6.9). The estimated conductance for this turbocharger shows good accordance with the measured values from the thermohydraulic test bench (section 6.2).

Table 6.5: Convective conductance model fitting parameter values.

\begin{tabular}{ccccc}
\hline Coefficients & $K_{T / H 1}$ & $K_{H 1 / H 2}$ & $K_{H 2 / H 3}$ & $K_{H 3 / C}$ \\
\hline$a_{i}$ & 1.4552 & 3.0227 & 4.5659 & 0.4529 \\
$b_{i}$ & 0.0197 & 0.0400 & 0.0199 & 0.0399 \\
$c_{i}$ & 1.3314 & 2.6608 & 4.2643 & 0.3778 \\
$d_{i}$ & 1.1156 & 0.5943 & 0.3017 & 1.1489 \\
$e_{i}$ & 1.1833 & 0.6977 & 0.6445 & 1.4686 \\
\hline
\end{tabular}




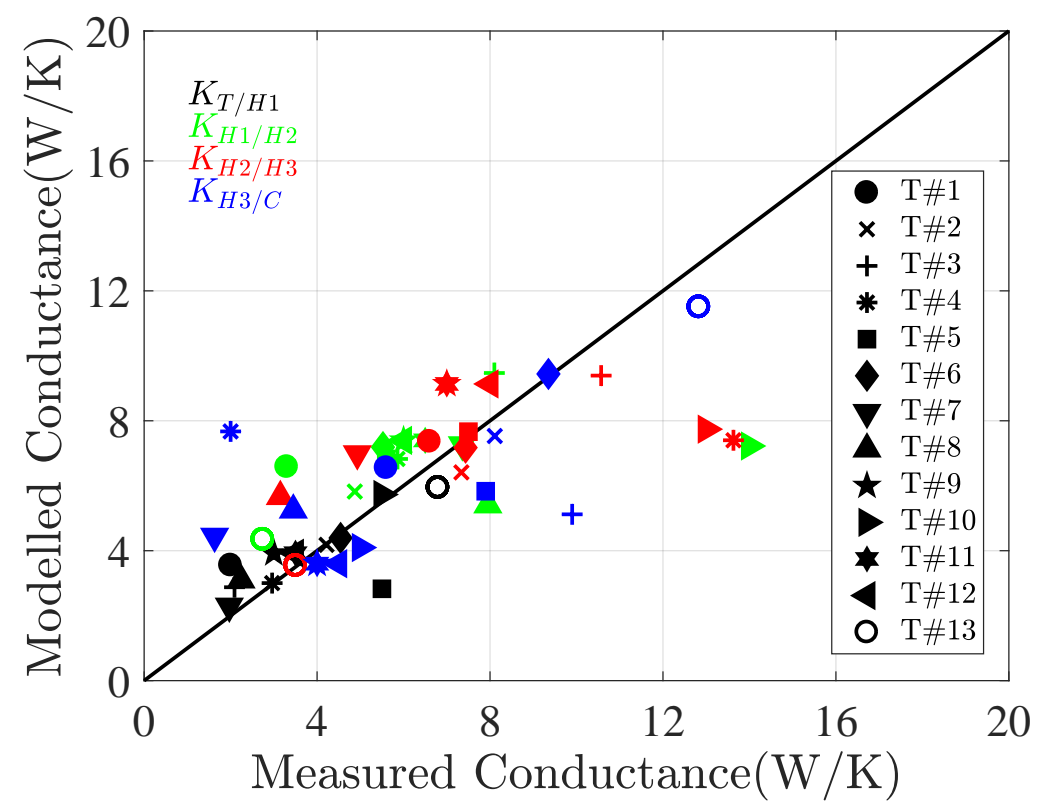

Figure 6.9: Conductive conductance fitting for different turbochargers

\subsection{Model validation using gas stand simulations}

The general correlation model developed in the section 6.3 was coupled with the thermal and mechanical losses models of $[19,20,83]$ and, all of them were integrated into the 1D GT-Power simulation software using an external library named as CMTTCM. In GT-Power, a gas stand simulation has been performed; for this, the hot exposed tests of T\#4TER experimental data were used. It is worth highlighting that, the experiments with T\#4TER were done at the full admission conditions; therefore, the maps were considered as single entry turbine. This way, it is possible to simulate the turbocharger behaviour using CMT-TCM with all the mentioned models coupled. The main variables affected by the usage of CMT-TCM are turbine and compressor outlet temperatures. Therefore, the output from this were compared with the experimental data. To highlight the importance of heat transfer in a turbocharger system, another configuration of gas stand simulation were launched. That is, without considering the additional heat transfer and mechanical losses given by the thermal model and the general correlation described in the section 6.3 ; this model is named as GT-TCM. It is worth highlighting that, the CMT-TCM model works using adiabatic maps. These maps can be obtained directly from the special measurements on the gas stand or from hot maps by removing the heat transfer (both internal and external) $[19,20]$ and mechanical losses [83] in a reverse way. This method is described by Reyes in [19] and most recently by Serrano et al. [2] with a sensibility study by changing the values of assumed parameters that are not included in the map data. 


\subsubsection{Model Results}

In Figure 6.10, a comparison of normalized surface temperatures at each metal node from CMT-TCM and experimental is shown. It can be observed that the CMT-TCM model estimates the surface temperature distribution similar to the experimental data. This is a good indicator of proper heat fluxes estimation from fluids to metal and also the heat conduction from metal to metal. It is worth highlighting that a GT-TCM model is a map-based approach without any consideration of heat transfers in the turbocharger system. Therefore, surface temperatures at each metal nodes are not provided by this model. Consequently, in Figure 6.10, the results from CMTTCM are only compared with the experimental data.

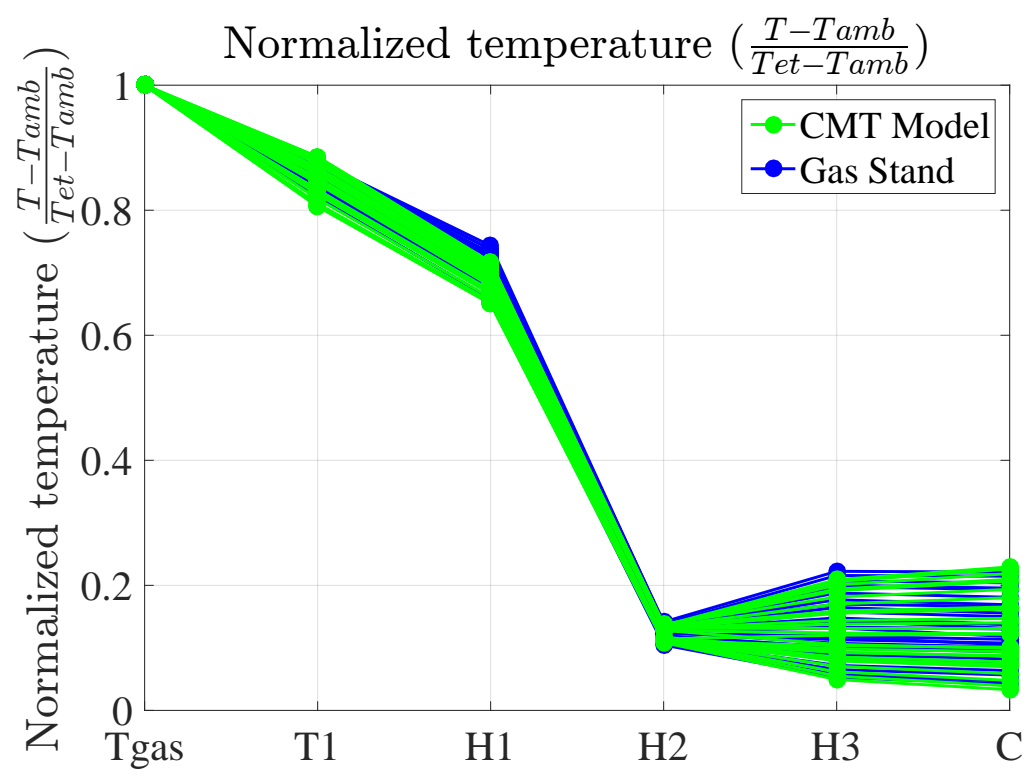

Figure 6.10: Comparison of normalized node temperature distribution between the experimental and CMT-TCM model output

The turbine and compressor outlet temperatures from both CMT-TCM and GTTCM were compared with experimental data, as shown in the Figure 6.11. From Figure 6.11(a), it can be observed that the predictions of compressor outlet temperatures from CMT-TCM are well ordered with the experimental data as compared to GT-TCM. At low turbo speeds, the predictions of both models are equally good. This can be explained due to the fact that, during the gas stand measurements of turbocharger $\mathrm{T} \# 13$ in hot conditions, the bearing housing was cooled with water at $50^{\circ} \mathrm{C}$. Therefore, with this so active water cooling, the compressor maps measured in lower speeds shows similar to the aerodynamic efficiency. However, at higher turbocharge speeds, the compressor works at higher loads, and there will be external heat losses from a compressor to the ambient, or towards the water cooling. Accordingly, some 
dispersion in the predictions of GT-TCM at higher speeds is observed.

Turbine outlet temperature predictions are shown in Figure 6.11(b); CMT-TCM shows a difference of $10^{\circ} \mathrm{C}$ with the experimental data in all the speeds. Whereas GT-TCM predictions are always higher, this is due to the impact of not considering the heat transfer effects in the turbine. Furthermore, the effective turbine efficiency (ETE) from hot steady tests are always combined with the heat transfer and mechanical friction losses [2]. This definition is commonly used by turbocharger manufacturers to represent the turbine efficiency maps. Therefore, the outlet temperatures of the compressor and turbine in one-dimensional calculations can be determined adequately using a thermal model and the adiabatic maps.
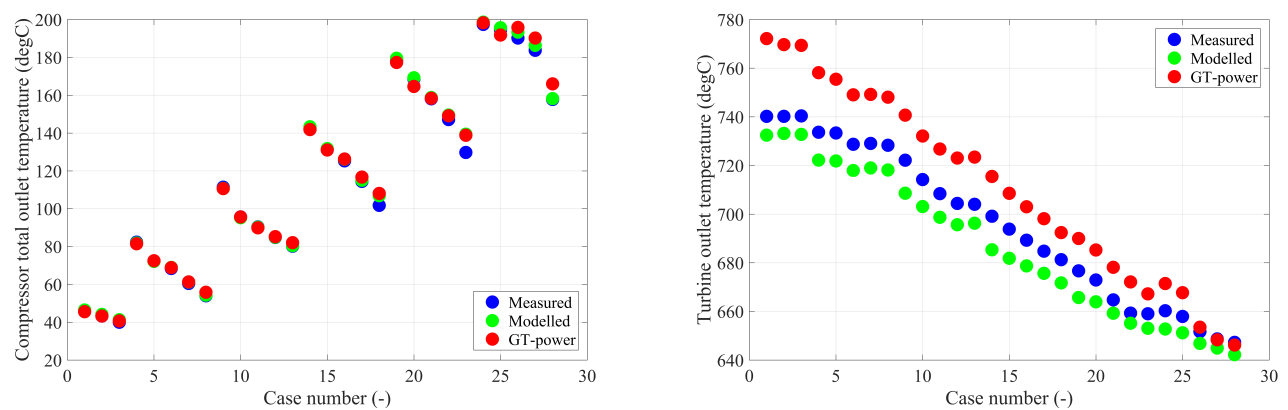

Figure 6.11: Predictions of outlet temperatures from both CMT-TCM and GT-TCM models; Left: Comparison of compressor outlet temperature; Right: Comparison of turbine outlet temperature.

In order to validate the capacitance model (subsection 6.3.1), thermal transient tests were performed in the gas stand using the T\#4TER turbocharger. Thermal transient tests were performed by imposing a minimum and maximum value of temperatures that can be achieved by the combustion chamber (see subsection 3.2.3) at the turbine inlet. During the transient tests, the entire evolution of all the turbocharger variables was recorded until stabilization. This transient test is simulated with CMT-TCM and GT-TCM models. Figure 6.12 shows the evolution of turbine and compressor outlet temperatures from both models compared to the experimental. It can be observed that the evolution of temperature decrement and increment were well predicted by the CMT-TCM model. This indicates that capacitance values provided by the model described in subsection 6.3.1 is sufficient for predicting the outlet temperatures of the turbine and compressor in transient operations. 

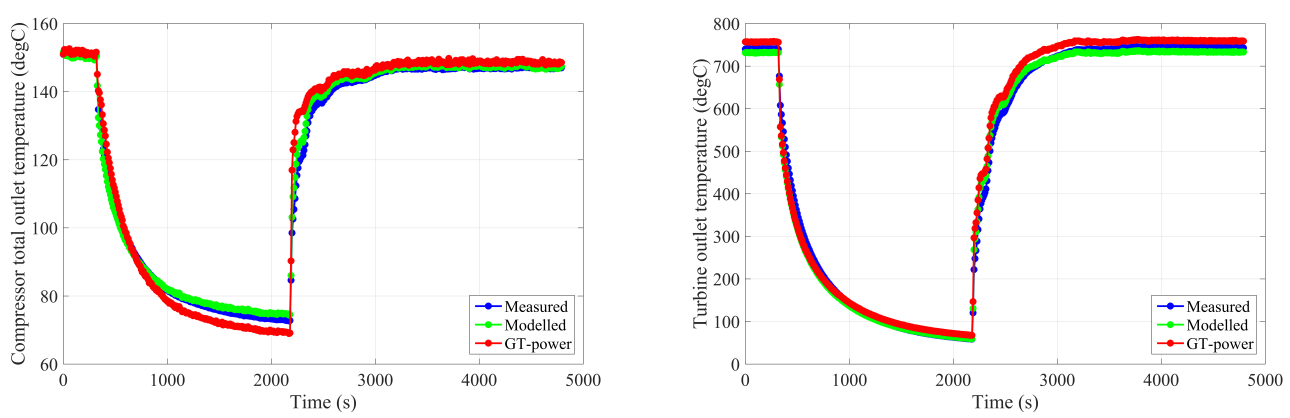

Figure 6.12: Predictions of outlet temperatures from both CMT-TCM and GT-TCM models; Left: Comparison of compressor outlet temperature; Right: Comparison of turbine outlet temperature.

\subsection{Heat transfer in double entry turbines}

Heat transfer in double-entry turbines is also an important factor to be considered to predict its behaviour in one-dimensional calculations accurately. However, in this thesis, the characterization of heat transfer in double-entry turbines was not the primary motive. The developed double-entry turbocharger model will be validated using the whole engine model; therefore, a first approximation of heat transfer in this type of turbocharger was calculated in a similar way to the single entry turbocharger thermal model based on the electric analogy as described in [19].

As it was described in [19], the thermal model based on the electric analogy has four working fluid nodes named as Gas, Air, Oil, and W. The exhaust gases that enter the turbine are referred to as node Gas; this node is characterized by the measured inlet temperature of the turbine $\left(T_{T \text { Gas }}\right)$. The adiabatically compressed air at the outlet diffuser of the compressor $\left(T_{T A i r}\right)$ is called node Air. Whereas, nodes $O i l$ and $W$ are characterized by lube oil $\left(T_{O I}\right)$ and coolant liquid inlet $\left(T_{W I}\right)$. Further, there are five metal nodes: the turbine and compressor casings are simplified by one node each ( $T$ and $C$ ) and, the rest three nodes ( $H 1, H 2$, and $H 3)$ are considered for the shaft housing.

For estimating the internal heat fluxes from fluid nodes to the metal nodes, the model uses the internal convection correlations which are already developed through the procedures described in [19]. In the case of radiation and external convection (natural or forced) with ambient, the correlation that is described in [20] will be used. For conduction heat transfer from one metal node to the other metal node and, also to consider the thermal inertia at each node, the model developed in the section 6.3 will be used. To close the model, friction losses in the lube oil are added as a power source [83]. This way, single entry turbochargers are thermally characterized during engine simulations, allowing the calculation of heat transfers in the system, as shown in Figure 6.13. It is worth highlighting that the entire heat transfer between the 
turbine gas $\left(T_{T \text { Gas }}\right)$ and metal node $T$ are considered in the turbine volute. Whereas, in the case of a compressor, heat transfer between compressed air $\left(T_{T A i r}\right)$ and metal node $C$ is considered in the outlet of the compressor rotor.

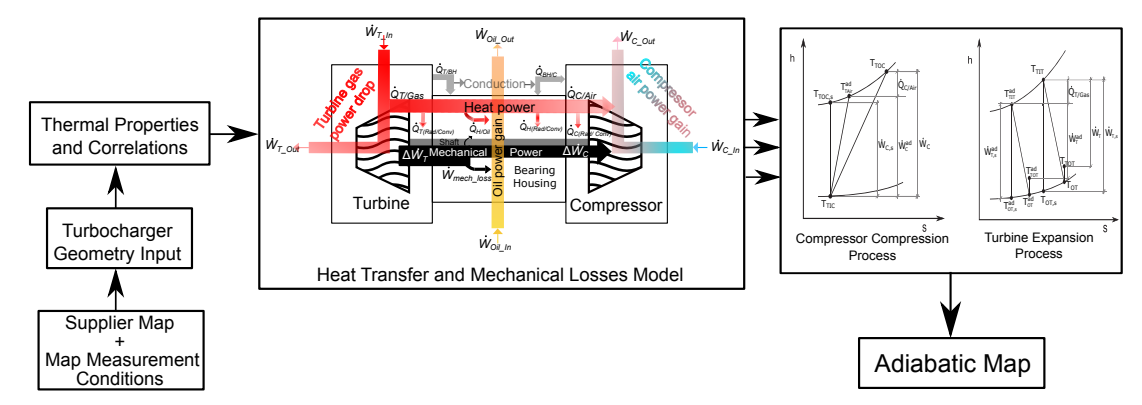

Figure 6.13: Overview of the calculation methodology of heat transfer in a turbocharger to obtain the adiabatic efficiency.

Nevertheless, in double-entry turbochargers, the turbine casing have two entries in which one entry $(\mathrm{Sh} / \mathrm{LV})$ will be far away from the shaft housing and highly exposed to the ambient, while the other entry (H/SV) will be close to the turbine backplate (i.e. node $H 1$ ). In partial and unequal admission conditions, there will be a temperature difference in both entries. Accordingly, the heat transfer from each entry will be different. In order to characterize the heat transfer problems in double-entry turbochargers, a specific experimental work and model correlation are needed. As mentioned before, the characterization of heat transfer in this type of turbochargers was not the main motive of the thesis. Therefore, the thermal model based on the electric analogy with all the previously mentioned correlations was applied to doubleentry turbochargers in the engine simulations.

Figure 6.14 shows an overview of heat transfer calculation in double-entry turbochargers. During the engine simulations, the double-entry turbocharger model reads the mass flow rate, temperature and other parameters from each turbine branch. While estimating the heat transfer, the mass flow and temperature conditions from each turbine entry are considered as total mass flow, and mass averaged temperature. This way, there will be now four fluid nodes and five metal nodes, as mentioned before. Therefore, the heat transfer system can be solved in a similar way to the single entry turbocharger method. Once, the temperatures of each metal node are estimated by the model, the internal heat fluxes from each turbine branch are reestimated. That is, using the individual turbine inlet temperatures, the estimated average metal node $T$ temperature by the model and, convection correlation from Gas to $T$, as shown in Equation 6.15 ( $k$ represents the generic term for each branch). Whereas, for conduction between the metals and external heat losses, the model carries out the calculations similar to the single entry turbine. Only the internal heat fluxes from each branch were able to be calculated. Therefore, the adiabatic total in- 


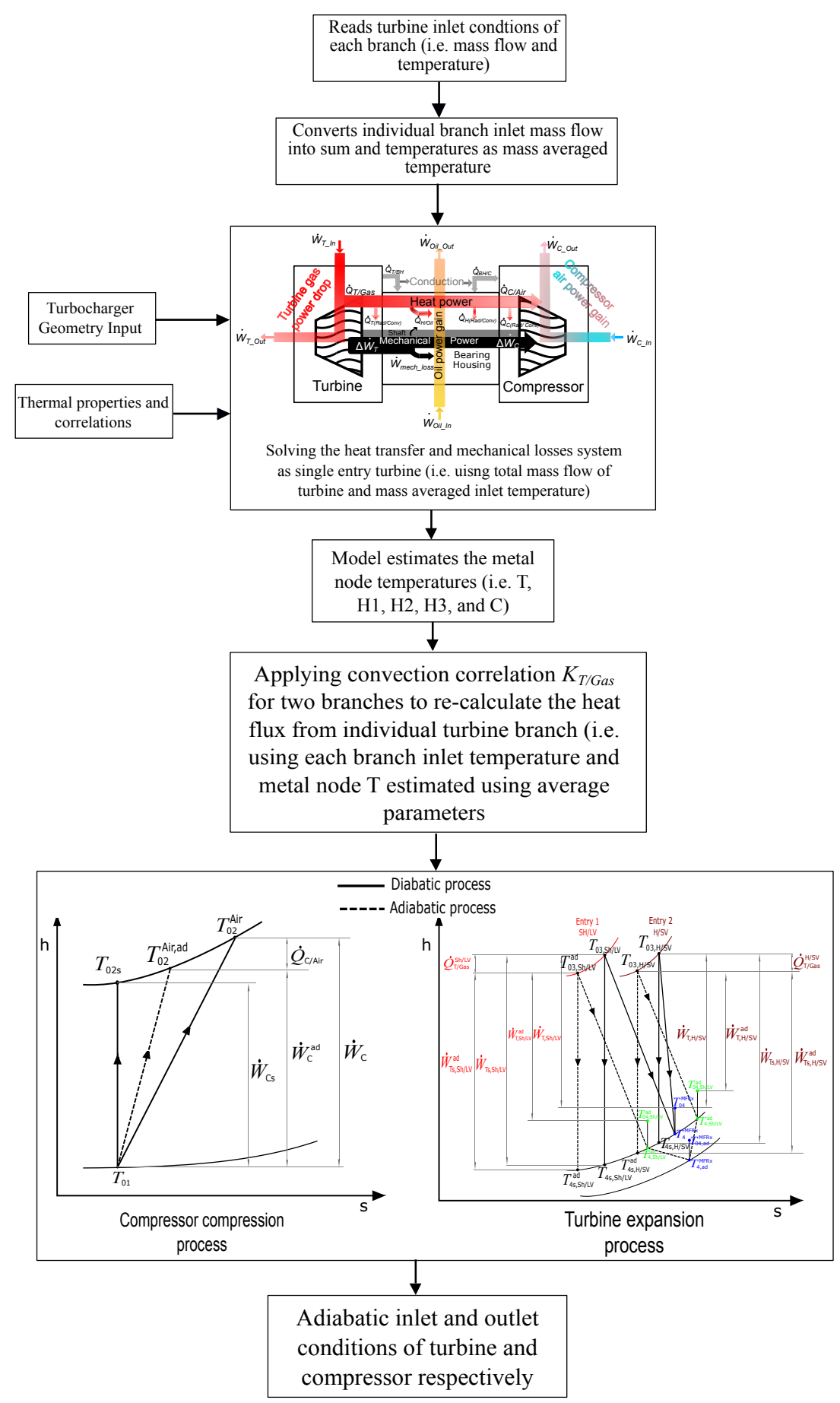

Figure 6.14: Overview of estimating the heat transfer in double entry turbines during the engine calculations. 
let temperature in each branch of the turbine after subtracting the heat transfer that takes from the turbine side can be estimated using the Equation 6.16. Nevertheless, the sum of the heat fluxes of the individual turbine inlets and the total turbine heat flux from Gas to $T$ used to compute the temperature of node $T$ may be different, so a final correction is used. Each of the individual turbine inlet heat fluxes is multiplied by the ratio between the total turbine heat flux from Gas to $T$ computed with the total mass flow and mass averaged temperature and the sum of the individual turbine inlet heat fluxes. This way, the individual heat fluxes are consistent with the overall one used for computing the temperatures of the metal nodes. For the compressor side, Equation 6.17 provides the adiabatic temperature at the outlet.

$$
\begin{gathered}
\dot{Q}_{T / G a s}^{k}=K_{T / G a s} \cdot\left(T_{0 t}^{k}-T_{i}\right) \\
T_{0 t}^{a d, k}=T_{\text {Gas }}^{k}-\frac{\dot{Q}_{T / G a s}^{k}}{\dot{m}_{T}^{k} \cdot c_{p, T}^{k}} \\
T_{02}^{A i r, a d}=T_{02}^{A i r}+\frac{\dot{Q}_{C / A i r}}{\dot{m}_{C} \cdot c_{p, C}}
\end{gathered}
$$




\section{Chapter 7}

\section{Double entry turbine model validation in $1 \mathrm{D}$ simulation software}

\begin{tabular}{|c|c|c|c|}
\hline \multicolumn{4}{|l|}{ Contents } \\
\hline 7.1 & \multicolumn{2}{|c|}{ Introduction } & 170 \\
\hline 7.2 & \multicolumn{2}{|c|}{ Double entry turbine model quasi-steady adaptation } & 170 \\
\hline 7.3 & \multicolumn{2}{|c|}{ Double entry turbine modelling as a lookup table in GT-Power } & 171 \\
\hline \multirow[t]{2}{*}{7.4} & \multicolumn{2}{|c|}{ Models validation in gas stand condition } & 173 \\
\hline & 7.4 .1 & Simulation results... & 176 \\
\hline \multirow[t]{6}{*}{7.5} & \multicolumn{2}{|c|}{ Models validation with engine conditions } & 182 \\
\hline & \multirow[t]{2}{*}{7.5 .1} & Experimental campaign . . . . & 183 \\
\hline & & 7.5.1.1 Test Methodology and results & 186 \\
\hline & 7.5 .2 & Engine Model Calibration . . . . . . . & 187 \\
\hline & 7.5 .3 & SCV opened simulation $\ldots \ldots \ldots \ldots \ldots \ldots$ & 191 \\
\hline & 7.5 .4 & SCV closed simulation $\ldots \ldots \ldots \ldots \ldots \ldots$ & 199 \\
\hline
\end{tabular}




\subsection{Introduction}

The objective of this chapter is to present the performance and potential of the doubleentry turbocharger model incorporated in the one-dimensional simulation software like GT-POWER. The results using the externally programmed library containing the models described in chapter 4 (named as CMT-DETCM) are compared with the standard 1D simulations in which the double-entry turbine is modelled as a lookup method (named as GT-DETCM). The simplified procedure for modelling doubleentry turbines as a standard lookup table is further discussed in this chapter. The validation of both CMT-DETCM and GT-DETCM models were performed in two different ways. Firstly, only a gas stand steady flow conditions were used. In this way, the main problems causing an engine model validation can be decoupled. They can check the predictions of the turbocharger itself. For this, the experimental data of T\#2DVR turbocharger, which was tested in quasi-adiabatic conditions were used. Secondly, both turbocharger models were coupled with the whole 1D calibrated engine model. Turbocharger T\#3DVM was tested with a gasoline engine at full load points. A brief of testing T\#3DVM turbocharger with an engine and the 1D whole engine model calibration procedure were also presented. It is worth to note that, in both validation cases, adiabatic maps of turbine and compressor were used.

\subsection{Double entry turbine model quasi-steady adaptation}

After the theoretical development of double-entry turbine model that is shown in chapter 4 , they were integrated into a one-dimensional gas dynamic simulation tool called VEMOD [84]. This tool is developed in CMT-Motores Térmicos. The complete adaptation of double-entry turbine model to be used in a quasi-steady way were detailed in [85]. The double-entry turbine model considers pressure pulses and reflections during the full engine simulation. They are solved by Euler's classical governing equations using a finite-volume approach and computed using a Godunov scheme [85]. Figure 7.1 shows the double-entry turbine model computational domain. The flow in stations C, D and E is determined using a technique described in [44]; to split the expansion ratio in the turbine between stator and rotor representing nozzles (stations $\mathrm{C}$ and $\mathrm{E}$ respectively). Further, heat transfer effects are taken into consideration as an energy source term, by changing the temperature of flow when it is passing between station $\mathrm{C}$ and $\mathrm{E}$. An extra energy sink term is introduced in the vaneless space $\mathrm{D}$ equal to the power output of the turbine at each time step. The extrapolated turbine maps (chapter 4) were used to compute the stator (C) and rotor (E) nozzles using the techniques as described in [44] and, the efficiency is quasi-steadily obtained as represented in the chapter 4. Gas dynamic effects on the compressor side are modelled using two volumes and connecting tube as described in [86]. All the model information were transferred into GT-Power software by creating an external library 
link. By using this library link, the CMT-DETCM model can be able to simulate in the GT-power software as a gas stand or just coupling to the engine model.

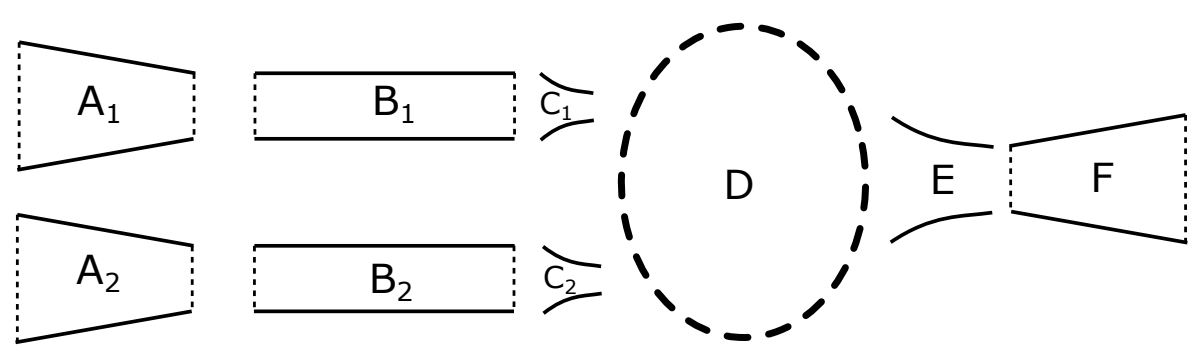
A Turbine Tongue
B Turbine Volute
D Vanless Space and Rotor Equivalent Volume
C Stator Nozzle
E Rotor Nozzle
F Turbine Diffuser
Index 1 and 2 represents entries

Figure 7.1: Double entry turbine model computational domain

\subsection{Double entry turbine modelling as a lookup table in GT-Power}

This section will describe the double-entry turbine modelling procedure in GT-Power. The approach for modelling is using a standard lookup method in GT-Power. It is done similar to the model described in the chapter 4 . That is, each turbine entry is considered as an individual turbine with their respective reduced mass flow and efficiency map data. In the double-entry turbine, the outlet of a is common for both inlets. Accordingly, a sub-volume is added downstream of the turbine part. In which all the mass flow rate coming from the individual turbine entries will be added, and pressure and temperature will be mass averaged. This way, it is simplified that two individual turbines work in parallel with their respective inlet conditions and later mixed at the outlet. The estimated torque of two turbines is added together, and the sum is applied to the main shaft that connects to the compressor side. Figure 7.2 shows the overview of double-entry turbine compound which has been developed in this thesis for the commercial code GT-Power.

When the double-entry turbines work with an engine, the flow conditions between the entries vary instantaneously and are always out of phase. To decide the amount of mass flow going into each branch of the turbine, a mass flow ratio (MFR) definition that described in chapter 3 is used and the same is shown in the Equation 7.1.

$$
M F R=\frac{\dot{m}_{S h / L V}}{\dot{m}_{S h / L V}+\dot{m}_{H / S V}}
$$




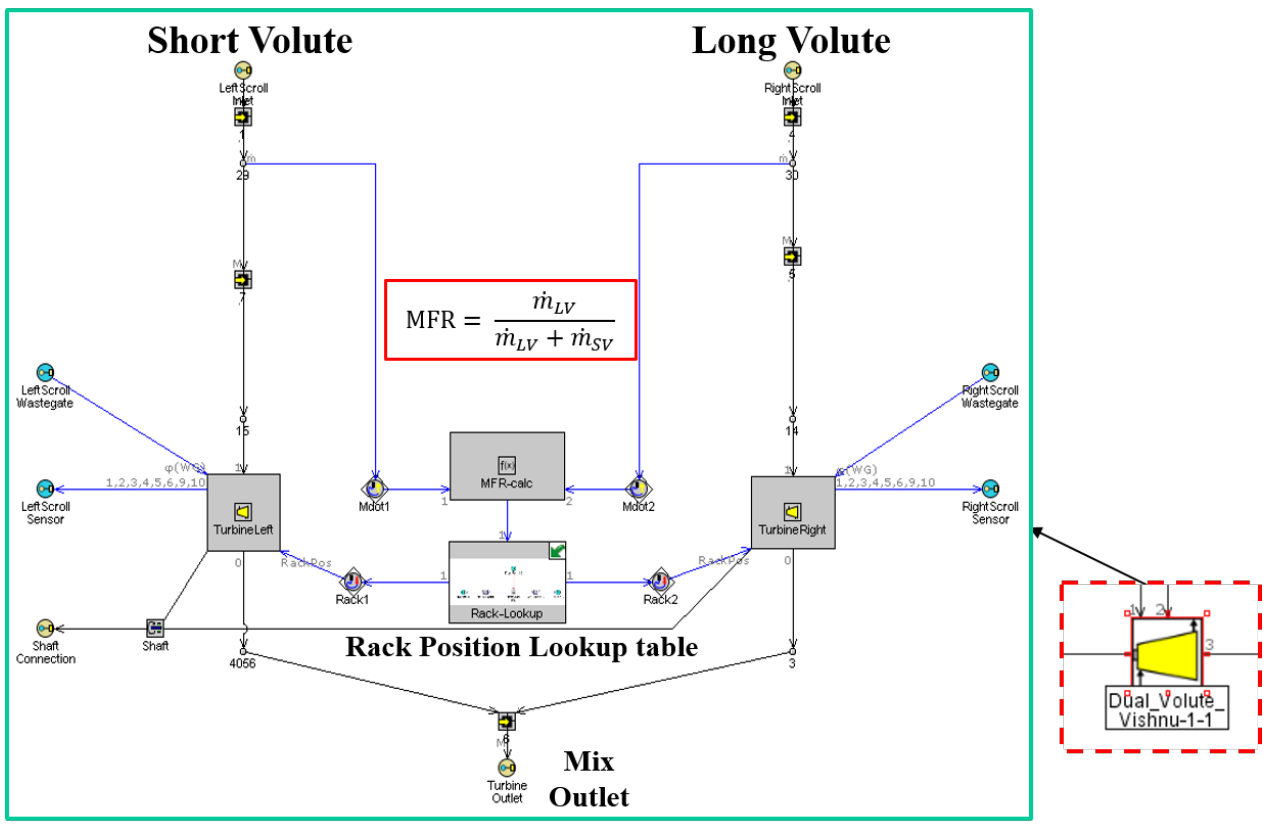

Figure 7.2: Concept of modelling double entry turbine using look up table method in GT-power

Aforementioned in chapter 3, reduced mass flow and efficiency of each entry showed an explicit dependency of flow behaviour with the MFR variation. That is, for an increasing MFR, the turbine mass flow parameter of the entry 1 (Shroud/Long Volute) increases and the entry 2 (Hub/Short Volute) decreases. Further, turbine maps of each entry look similar to single entry VGT turbine maps. So, each MFR map is designated with a dummy VGT position in each turbine part. Accordingly, a rack position lookup table is created for each turbine as a function of MFR and dummy VGT, as shown in Figure 7.3. The process starts with the calculation of MFR from inlets and based on the MFR value; the rack position lookup table will adjust a dummy VGT position in both turbines. Based on the dummy VGT position, the mass flow rate and efficiency are looked up in their respective turbines map data (like a VGT turbine model). For example: when the MFR $=0.6$, it means that $60 \%$ of flow is in turbine entry 1 and the $40 \%$ of flow is in turbine entry 2. Accordingly, the rack positions will be set in each turbine. It is worth to noted that MFR and rack position is not fixed but, they vary instantaneously depending on the flow conditions at the turbine inlets.

In a turbocharger, heat transfer between the elements is of high importance. Thus, heat transfers between the turbine, compressor, oil, water coolant and towards ambient are needed for the proper estimation of turbine and compressor outlet temperatures as shown in chapter 6 . However, a proper heat transfer characterization in double-entry turbines was not performed in this thesis. But, small modifications were done to approximate the heat transfer in the double-entry turbine, as discussed in the 

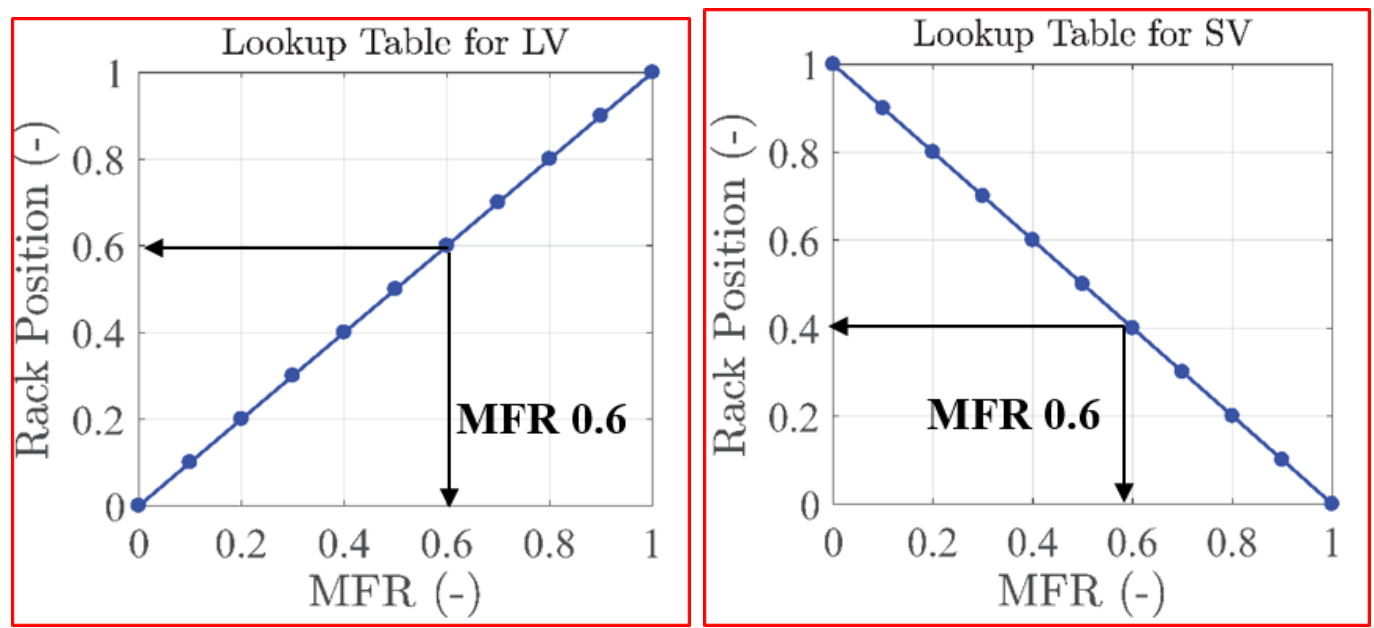

Figure 7.3: Dummy VGT position as a function of MFR

section 6.5 of a chapter 6 . This way, the CMT-DETCM model calculates an appropriate heat transfer from the turbine side to all the other elements in a simulation. Whereas, GT-DETCM model considers only the measured turbocharger maps and the output variables from this model are estimated without any consideration of heat transfer effects. Therefore, to compare the outcome results of both CMT-DETCM and GT-DETCM models, only adiabatic maps of turbine and compressor were used in all the simulations. The objective was only to check the errors of the models with as simple as possible operating conditions. After all, the one-dimensional turbocharger models are mainly structured to perform simulations using adiabatic maps and efficiencies as a source term.

\subsection{Models validation in gas stand condition}

For newly developed one-dimensional turbocharger models, it is necessary to check their prediction ability for different turbocharger variables measured with the steady flow at the gas stand. This kind of verification has the advantage compared to a whole 1D engine model simulations. That is, eliminating the specious effects due to the complex pulsating flow aspects in the engine and the calibration of the engine model. Therefore, in this section, both CMT-DETCM and GT-DETCM were validated using gas stand experimental data of T\#2DVR turbocharger. Figure 7.4 shows the outline of a turbocharger gas stand setup created in the GT-Power platform. For a given turbocharger, the geometry values like inlet and outlet diameters of the compressor and turbine and their wheel dimensions will help to simulate the behaviour of the models correctly. It can be observed that the CMT-DETCM model has the advantage of taking the turbocharger oil and coolant inlet conditions in order 
to calculate the mechanical losses and heat transfer effects, in addition to the test cell ambient conditions were also considered for the calculation of external heat transfers. These advantages are not available in the GT-DETCM models. Aforementioned, both models were simulated with the adiabatic maps in the turbine and compressor.

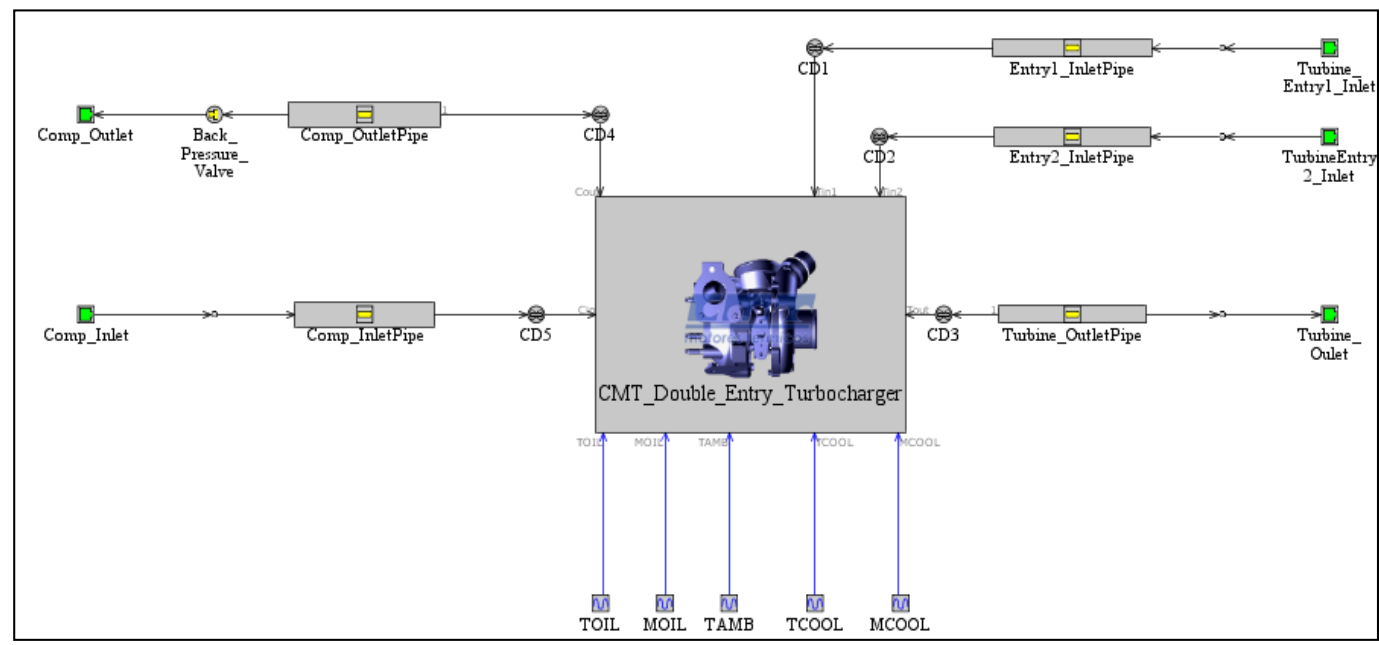

(a) CMT Double Entry Turbocharger Model

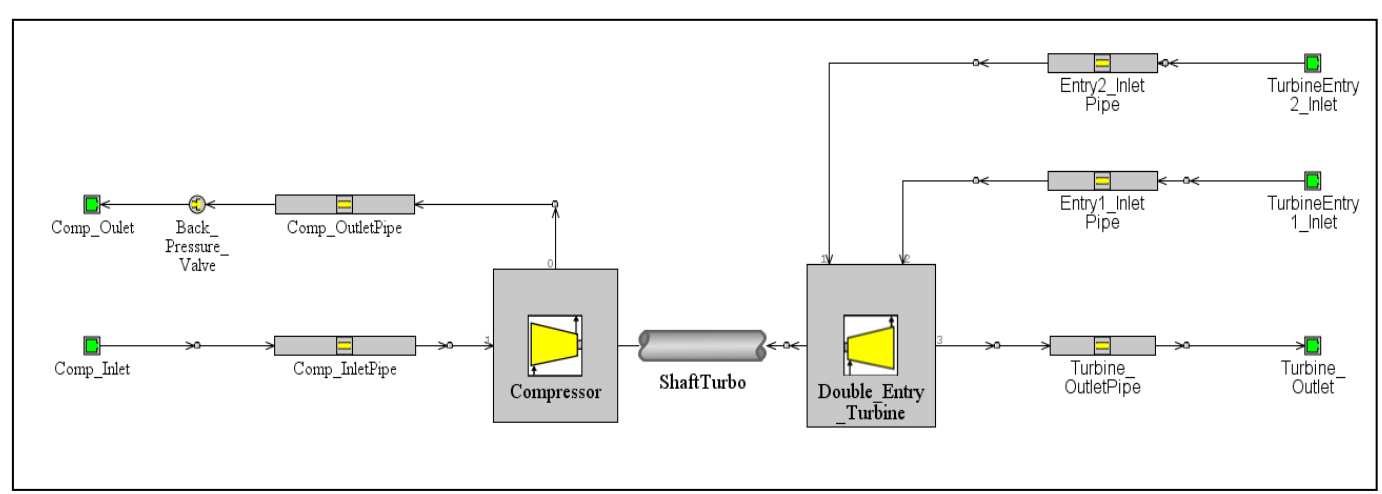

(b) GT Double Entry Turbocharger Model

Figure 7.4: Double entry turbocharger model as gas stand in GT-Power simulation software

In order to perform the simulations using the gas stand model, the following variables are imposed as boundary conditions:

- Compressor inlet temperature and pressure.

- Back-pressure valve position in the compressor outlet line.

- Turbine inlet temperature and pressure in both entries. 
- Turbine outlet pressure.

- Oil inlet mass flow rate and temperature.

- Water inlet mass flow rate and temperature (if exists, but in the current simulations, these variables were not imposed; as the tests are done in quasi-adiabatic conditions and water cooling is not used.

- Ambient temperature.

Using the above inputs in both CMT-DETCM and GT-DETCM models, the following variables are calculated by the models and are compared against the experimental data.

- Compressor outlet pressure.

- Compressor outlet temperature.

- Turbine outlet temperature mixed and individual (i.e., outlet temperature from each entry before mixing).

- Compressor and turbine mass flow rates.

- Turbocharger speed.

It is worth to be noted that, while simulating the CMT-DETCM model, quasiadiabatic test conditions were given as boundary conditions. In this way, CMTDETCM model estimates the heat transfer using all the thermal correlation and with the procedure that explained in the section 6.5 of chapter 6 and it will be added to the adiabatized maps. Accordingly, the results from this model were compared with the quasi-adiabatic test conditions. In the case of GT-DETCM model simulations, at the turbine side, adiabatic inlet temperature as a boundary was provided at each branch. Since the heat transfer in the turbine is always considered before expansion, these temperatures were estimated using the constant heat transfer rate given by the power-based adiabatization process. Doing this, the model should give the outlet temperatures similar to the experimental. Whereas, at the compressor side, the boundary conditions are given similar to the CMT-DETCM. Since the heat transfer in compressor takes place after the air is compressed, therefore, the compressor outlet temperature from GT-DETCM model will be in the adiabatic state. Accordingly, the model output results were compared with the experimental adiabatic temperature. This temperature is also calculated using the constant heat transfer rate given by the power-based adiabatized process of compressor map. 


\subsubsection{Simulation results}

In this section, the gas stand simulation results from CMT-DETCM and GT-DETCM are presented. In all the following figures, the continuous line shows the perfect correlation between the model and experimental values. Whereas, the separated lines shows the $\pm 5 \%$ deviation from perfect correlation line. In Figure 7.5, the gas stand simulation results of CMT-DETCM model is presented. To be sure that the mass flow in each turbine branch is well predicted, the mass flow ratio (MFR) is evaluated. In Figure 7.5(a), the experimental MFR values are compared with the model predictions. It can be observed that all the MFRs at different turbocharger speeds agree well with the experimental points. This indicates that the mass flow split in each branch is consistent with the boundary conditions of each branch, that is different inlet pressure and temperature for each branch and the same outlet pressure. However, small discrepancies are found in the case of MFR 0.22 predictions; this could be due to the uncertainty of experimental measurements with the low flow branch. In Figure 7.5 (b) it can be observed that the simulated turbocharger speeds match the experimental results in good quality. The prediction of turbocharger rotational speed highlights a good approximation of friction losses and the overall power balance. Consequently, the mass flow in the compressor and turbine are well predicted as it can be seen in Figure 7.5 (c) and (d). It is worth highlighting that the mass flow values of compressor and turbine were normalized with the maximum experimental value of the respective mass flow parameter.

Similar results can be found with GT-DETCM turbocharger model, and its predictions were shown with the experimental values in Figure 7.6. The prediction of turbocharger speeds with GT-DETCM model is high for some points; mostly at the higher speeds (Figure 7.6(b)). This is due to friction losses are not estimated by this model. In GT-Power software, the turbocharger mechanical efficiency is usually preset to a value of 1 , assuming that there are no friction losses [87]. But, in reality, there will be few losses due to the rolling friction [83]. However, the majority of the points (turbo speeds) predicted by GT-TCM are within the $\pm 5 \%$ level of error.

Figure 7.7 shows the predictions of compressor and turbine outlet temperatures for various turbocharger speeds from CMT-DETCM model. In the case of the compressor (Figure 7.7(a)), the difference between experimental and model predictions is high at higher turbocharger speeds. This could be due to the impact of heat transfer phenomena in CMT-DETCM model; as the heat transfer calculations were done similar to the single entry turbine as described in the section 6.5 of chapter 6 ; therefore, some discrepancies are observed. Nevertheless, the model gives an error within $\pm 5 \%$ in all the MFR and turbocharger speeds. Similar results can be seen in the mixed turbine outlet temperature predictions (Figure 7.7(b)). Better quality of the predictions was observed in the case of unequal admission conditions in all the turbocharger speeds. Small discrepancies are found in the partial admission predictions at higher turbocharger speeds. This discrepancy can come from the overall fitting 
(a) T\#2DVR
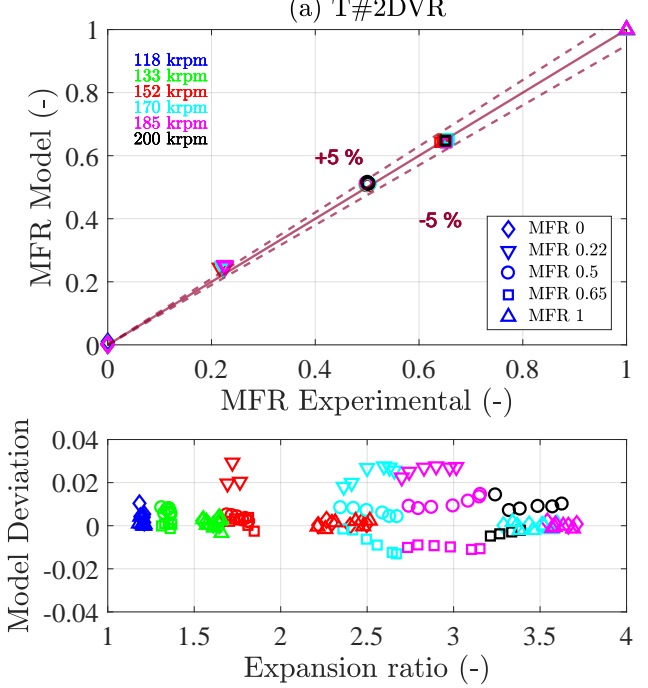

(c) T\#2DVR
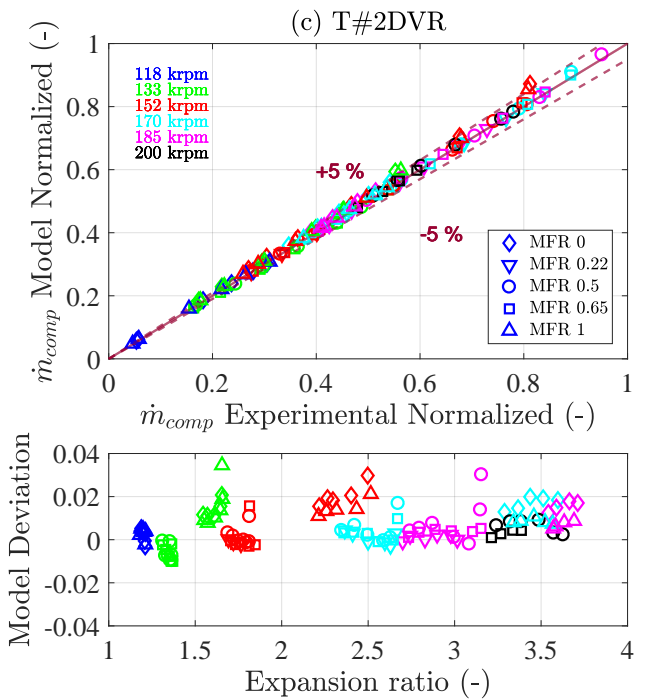

(b) T\#2DVR
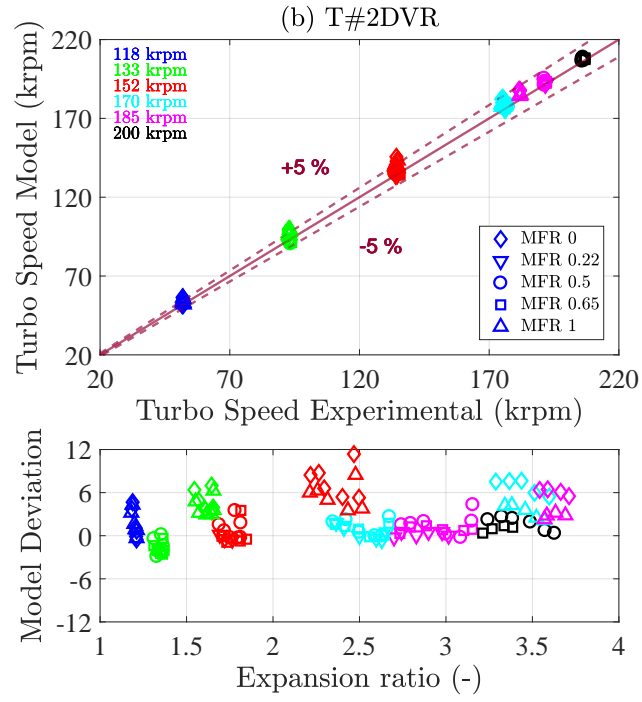

(d) T\#2DVR
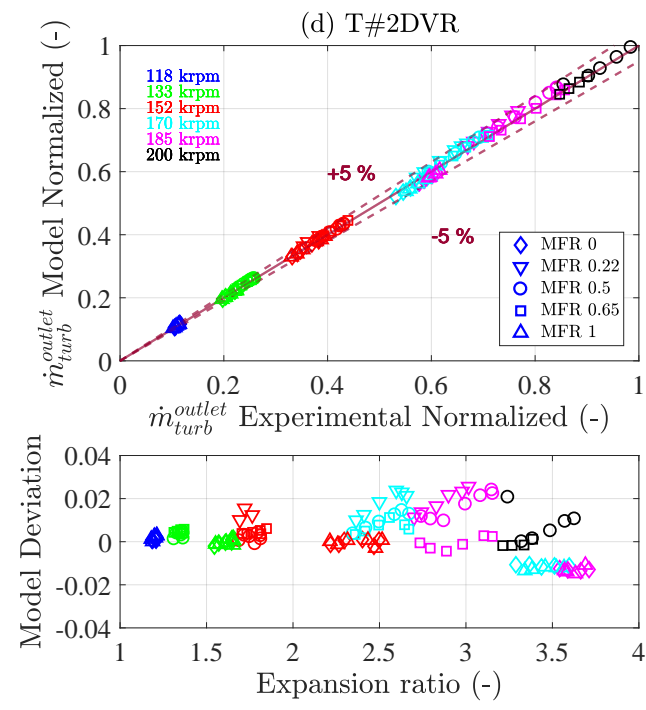

Figure 7.5: Gas stand simulation validation parameters; CMT-DETCM versus experimental values of T\#2DVR dual volute turbocharger; (a) Mass flow ratio, (b) Turbocharger speed, (c) Compressor mass flow and (d) Turbine outlet mass flow. 
(a) T\#2DVR
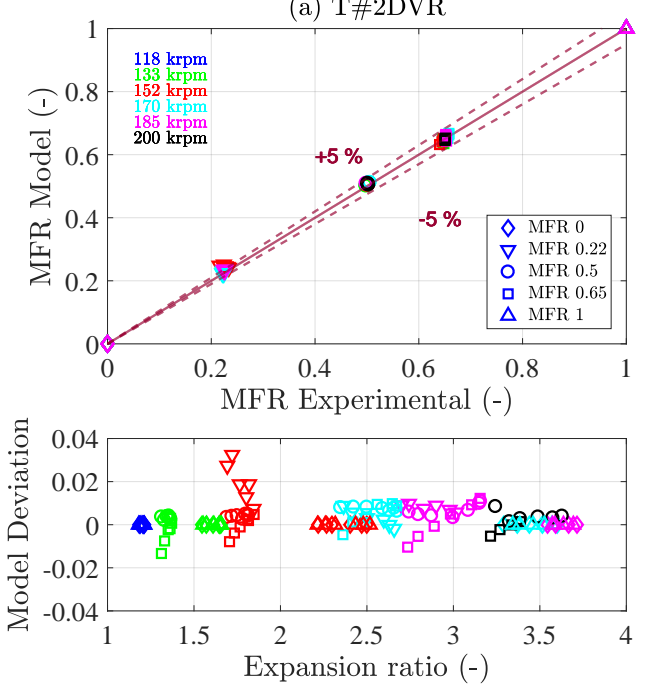

(c) T\#2DVR
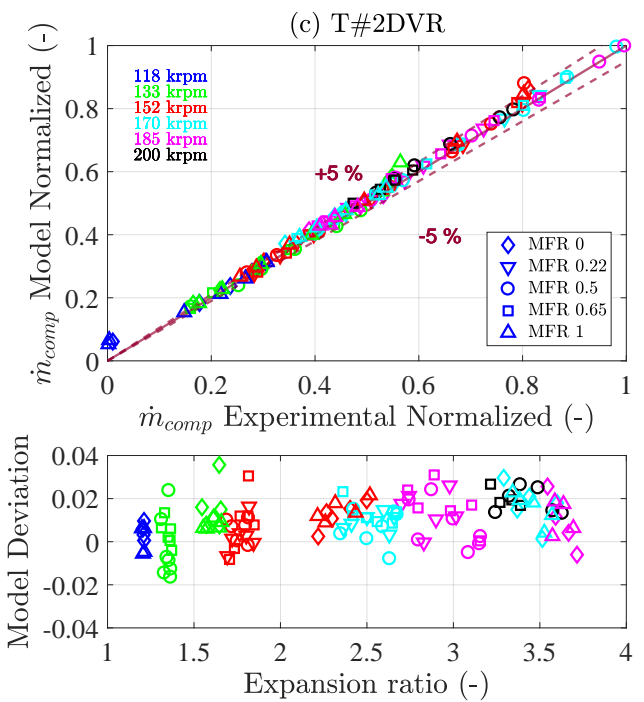

(b) T\#2DVR
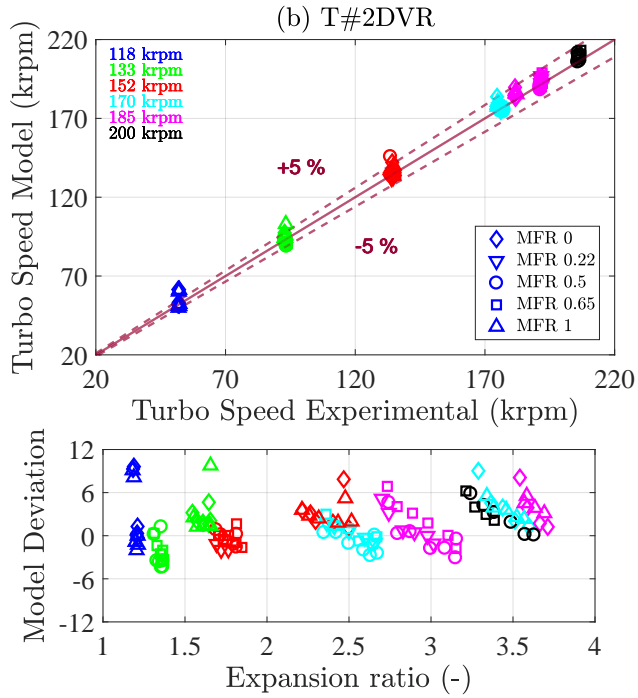

(d) T\#2DVR
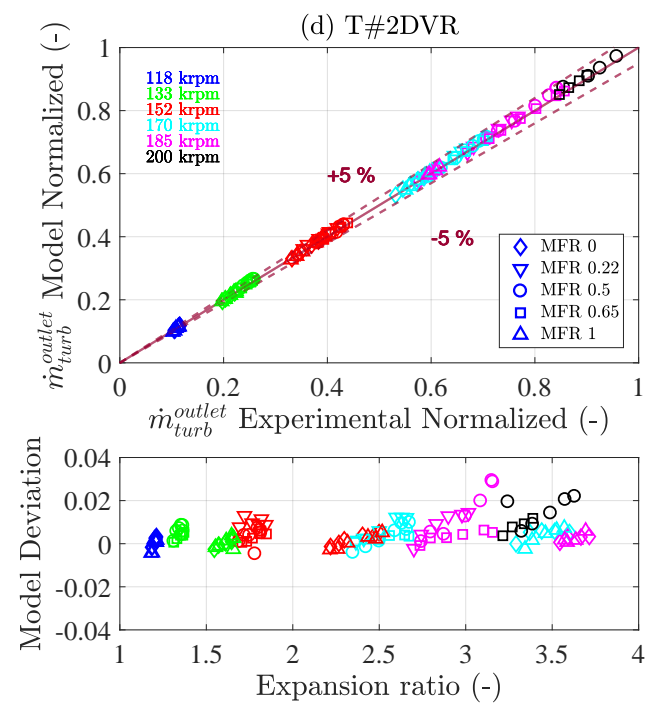

Figure 7.6: Gas stand simulation validation parameters; GT-DETCM versus experimental values of T\#2DVR dual volute turbocharger. (a) Mass flow ratio, (b) Turbocharger speed, (c) Compressor mass flow and (d) Turbine outlet mass flow. 
of the efficiency maps in the model. Nevertheless, the difference between model and experimental values are not more than 6 Kelvin.

(a) T\#2DVR
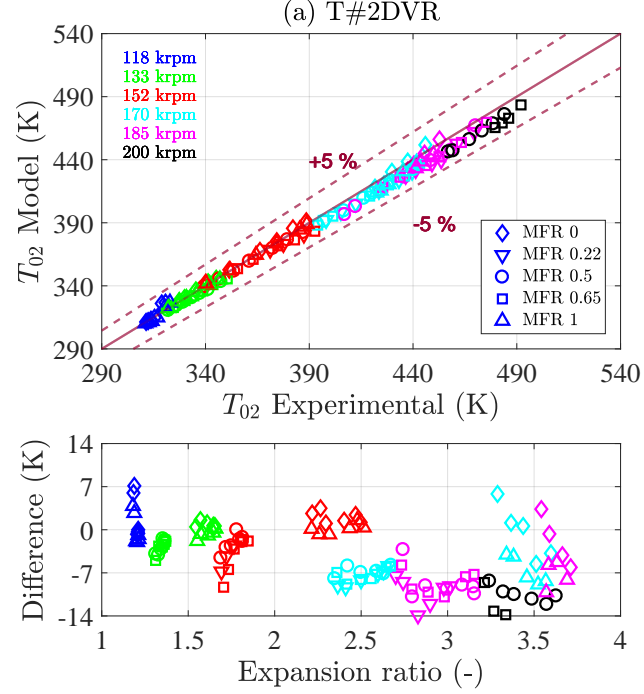

(b) T\#2DVR
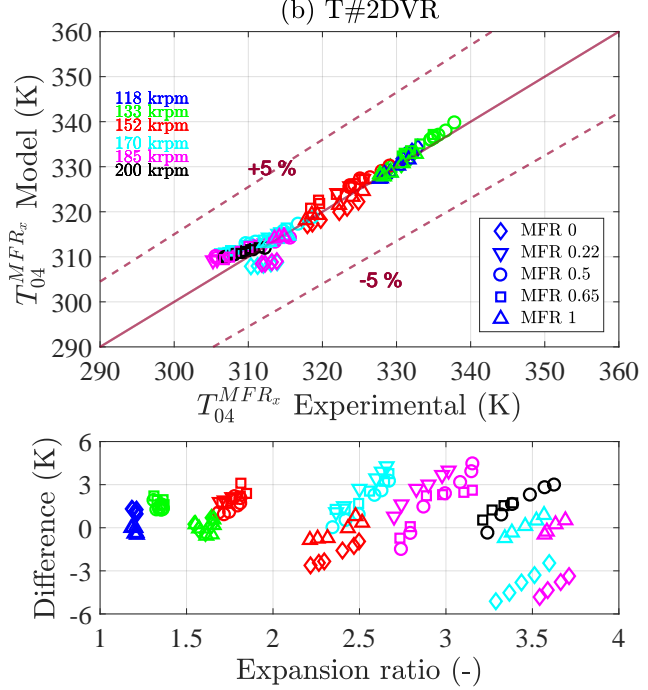

Figure 7.7: Gas stand simulation validation parameters; CMT-DETCM versus experimental values of T\#2DVR dual volute turbocharger; (a) Compressor outlet temperature, (b) Mixed turbine outlet temperature

In Figure 7.8, the predictions of compressor and turbine outlet temperatures from GT-DETCM model is shown with experimental data points. The difference between measured and model-predicted temperatures are lower in both compressor and turbine cases. On the one hand, the compressor outlet temperature predictions are slightly high (around $7 \mathrm{~K}$ ) at higher turbocharger speeds (Figure 7.8(a)). These small discrepancies can be due to the deviation in the compressor outlet pressure predictions by the model as compared to experiments. On the other hand, the prediction of mixed turbine outlet temperature is similar to the experimental values in all the MFRs and turbocharger speeds (Figure 7.8(b)). Nevertheless, the predicted temperatures for both compressor and turbine are within $\pm 5 \%$ in all the MFRs. Therefore, it can be concluded that the double-entry turbine model developed in this thesis is working quasi-steadily without producing any significant errors. Further, it can be used with a whole $1 \mathrm{D}$ engine model for predicting the engine performance variables.

It is worth highlighting that the double-entry turbine model developed in this thesis has the capability of estimating the actual turbine efficiencies of each branch (see Equation 4.37 and Equation 4.38). Therefore, it is possible to extract the information about actual turbine outlet temperatures in each branch from CMT-DETCM model, as shown in Figure 7.9. However, this information is not available from the gas stand at full/unequal admission conditions. Therefore, the results of each branch outlet from the model at these conditions were compared to each other. In order to 
(a) T\#2DVR
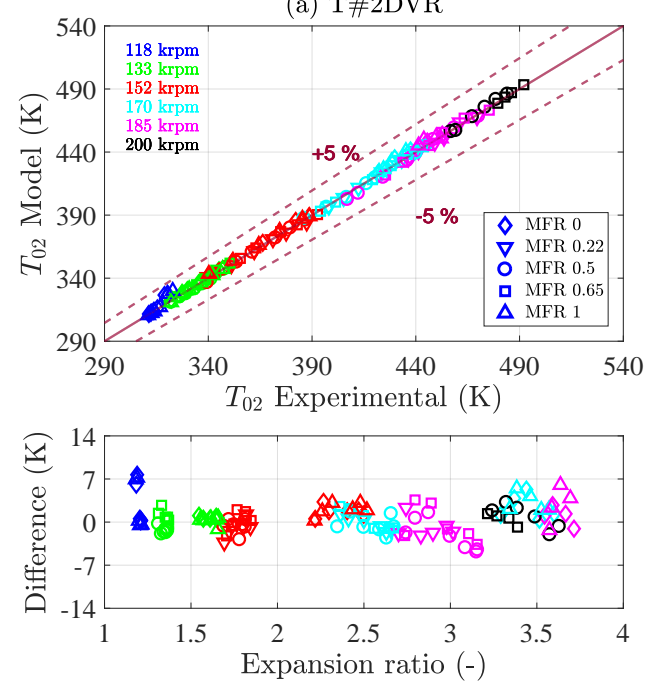

(b) T\#2DVR
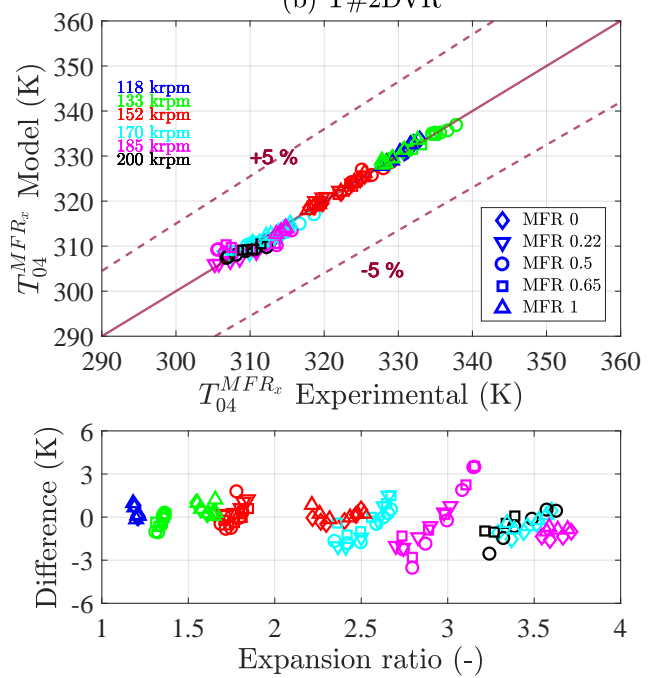

Figure 7.8: Gas stand simulation validation parameters; GT-DETCM versus experimental values of T\#2DVR dual volute turbocharger; (a) Compressor outlet temperature, (b) Mixed turbine outlet temperature

(a) T\#2DVR - Unequal
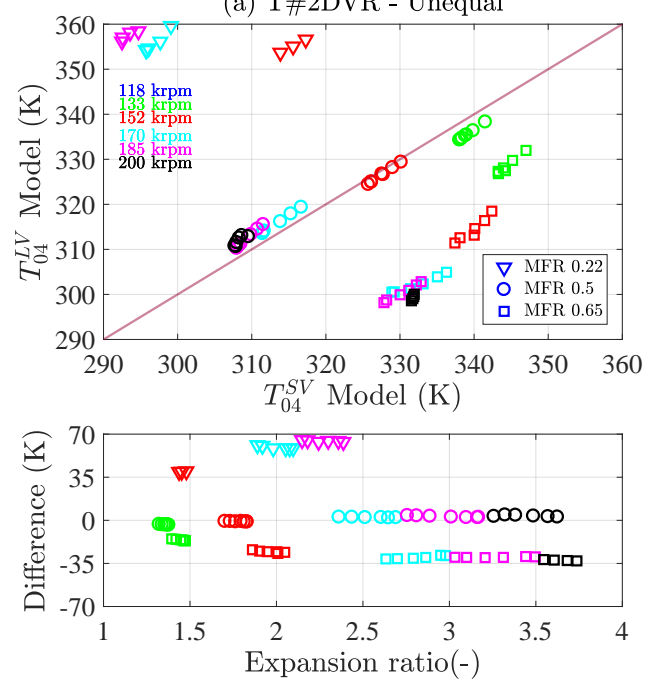

(b) T\#2DVR - Partial
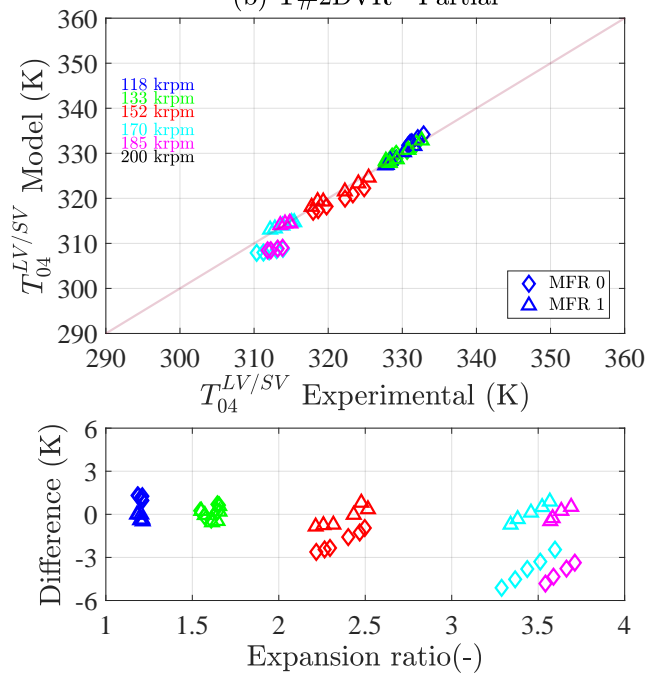

Figure 7.9: T\#2DVR turbine outlet estimation in each branch using CMT-DETCM turbo model (a) Outlet temperatures in full and unequal admissions, (b) Outlet temperatures in partial admission conditions 
understand the results better, the difference in temperatures between each branch was plotted against the expansion ratio. In the case of partial admission conditions, the outlet temperatures from each branch are due to expansion of that branch. Therefore, the results from the model were compared against the experimental data. According to the hypothesis of model development, the predictions of temperatures at full $(\mathrm{MFR}=0.5)$ and partial $(\mathrm{MFR}=0$ and 1$)$ admission conditions should be similar to the temperatures measured in the gas stand. Because energy extraction from each branch in this admission conditions are reasonably similar and the same can be observed in the Figure 7.9 (a) and (b). In the case of MFR $=0.22$, most of the energy extraction is done by the short volute branch due to a higher percentage $(80 \%)$ of mass flow in SV than LV. Consequently, the turbine outlet temperature values of a long volute branch are higher and closer to turbine inlet temperatures (see Figure 7.9(a)). Further, the same can be observed at MFR $=0.65$ in short volute branch (see Figure 7.9(a)); in this case, 60\% of mass flow is in the LV and $40 \%$ in SV. Accordingly, each turbine branch extracts the energy and model calculates the outlet temperatures. These individual temperatures are mixed at the end to estimate the mixed turbine outlet temperatures that are similar to the gas stand measured values, as it is shown in Figure 7.7(b).

Even though, the GT-DETCM was modelled as two individual turbines. This model is also capable of calculating the individual branch turbine outlet temperatures before mixing into the common sub-volume element. Figure 7.10 (a) and (b) shows the turbine outlet temperatures from each branch at full, unequal and partial admission conditions. From Figure 7.10 (a), it can be seen that there is not much difference between the actual temperature values from each branch. Because the GT-DETCM model works as a map lookup table, and the turbine efficiency maps provided to the model are in the form of apparent efficiencies values; which is not the real efficiency of each branch. As the apparent efficiencies are estimated using the individual turbine inlet and the mixed outlet temperature that is measured in the gas stand as it is discussed in the chapter 3. Therefore, GT-DETCM model estimates the outlet temperature of each branch by using the apparent efficiency values. As a result, they are similar to the mixed turbine outlet temperature values, as shown Figure 7.8(b). Therefore, the lookup table model (GT-DETCM) is not as physical to the CMT-DETCM model.

Since the GT-DETCM model is purely based on the apparent efficiencies of each branch, which is not real and also it does not consider any heat transfers. Besides, the energy extraction from each branch will be the same because of having the same extrapolated apparent efficiencies in every branch. Accordingly, even in unequal admission condition, the predicted temperatures from each branch are similar. Whereas, CMT-DETCM model is calibrated using the relation between 'apparent efficiency' $\left(\eta_{M F R_{x}}\right)$ which is able to be measured and the actual efficiencies $\left(\eta_{L V_{x}}\right)$ and $\left(\eta_{S V_{x}}\right)$ which is able to be calculated with the physical principle that is shown in equations 4.37 and 4.38. These equations are the merit functions to calibrate the 
(a) T\#2DVR - Unequal
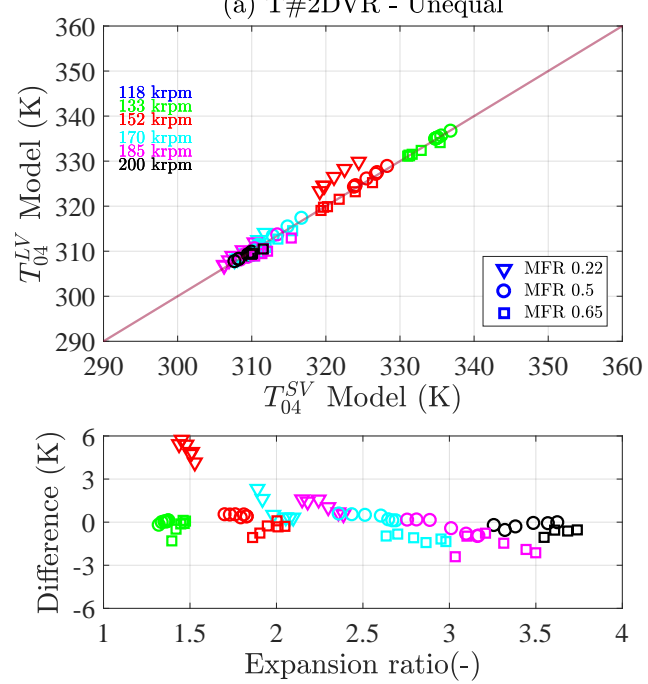

(b) T\#2DVR - Partial
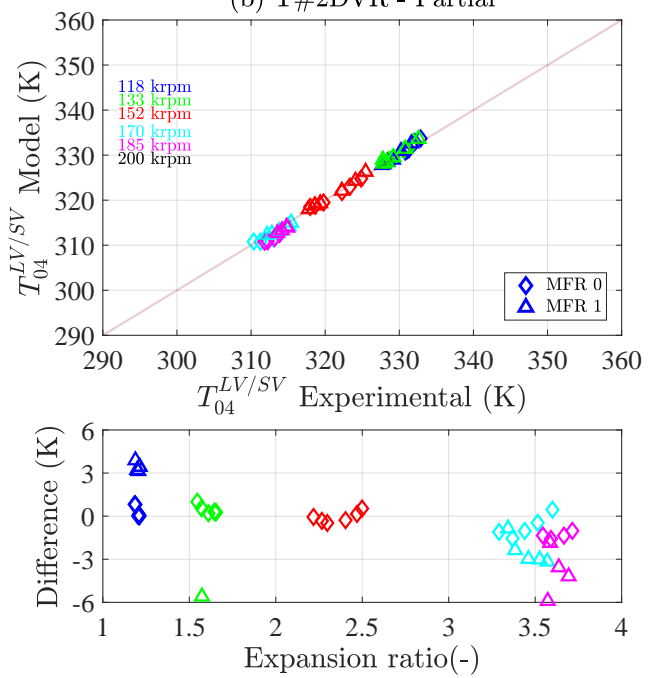

Figure 7.10: T\#2DVR turbine outlet estimation in each branch using GT-DETCM turbo model (a) Outlet temperatures in full and unequal admissions, (b) Outlet temperatures in partial admission conditions

model coefficients with the experimentally obtained 'apparent efficiency' $\left(\eta_{M F R_{x}}\right)$ but with the ultimate purpose of calibrating the model for calculating the physical 'actual efficiencies' $\left(\eta_{L V_{x}}\right.$ and $\left.\eta_{S V}\right)$.

Nevertheless, GT-DETCM model can be used with the 1D whole engine simulations with an adiabatic or adiabatized maps [2] as input, since the model is able to simulate the double-entry turbocharger behaviour similar to the measured data in the gas stand.

\subsection{Models validation with engine conditions}

The whole engine modelling work has been divided into three parts. In the first, the experimental works carried out with a dual-volute mixed flow turbine (T\#3DVM) with a gasoline engine in a test rig will be discussed. Then, the one-dimensional engine model calibration procedure will be explained. Finally, engine simulations with the proposed CMT-DETCM and GT-DETCM turbocharger models have been performed to see the model prediction with engine conditions. As the GT-DETCM model does not take into account any heat transfer effects and mechanical losses in the turbocharger. Therefore, the engine simulations were performed using the adiabatized maps of T\#3DVM turbocharger that shown in the chapter 3. 


\subsubsection{Experimental campaign}

The test bench used to validate the proposed models in a real application is a standard test rig which is designed by CMT-Motores Térmicos to study the internal combustion engines up to $200 \mathrm{~kW}$ of power. The facility allows to control and rate the engine performance in steady and transient conditions. Figure 7.11 shows, the scheme of the engine test facility and the sensor instrumentation.

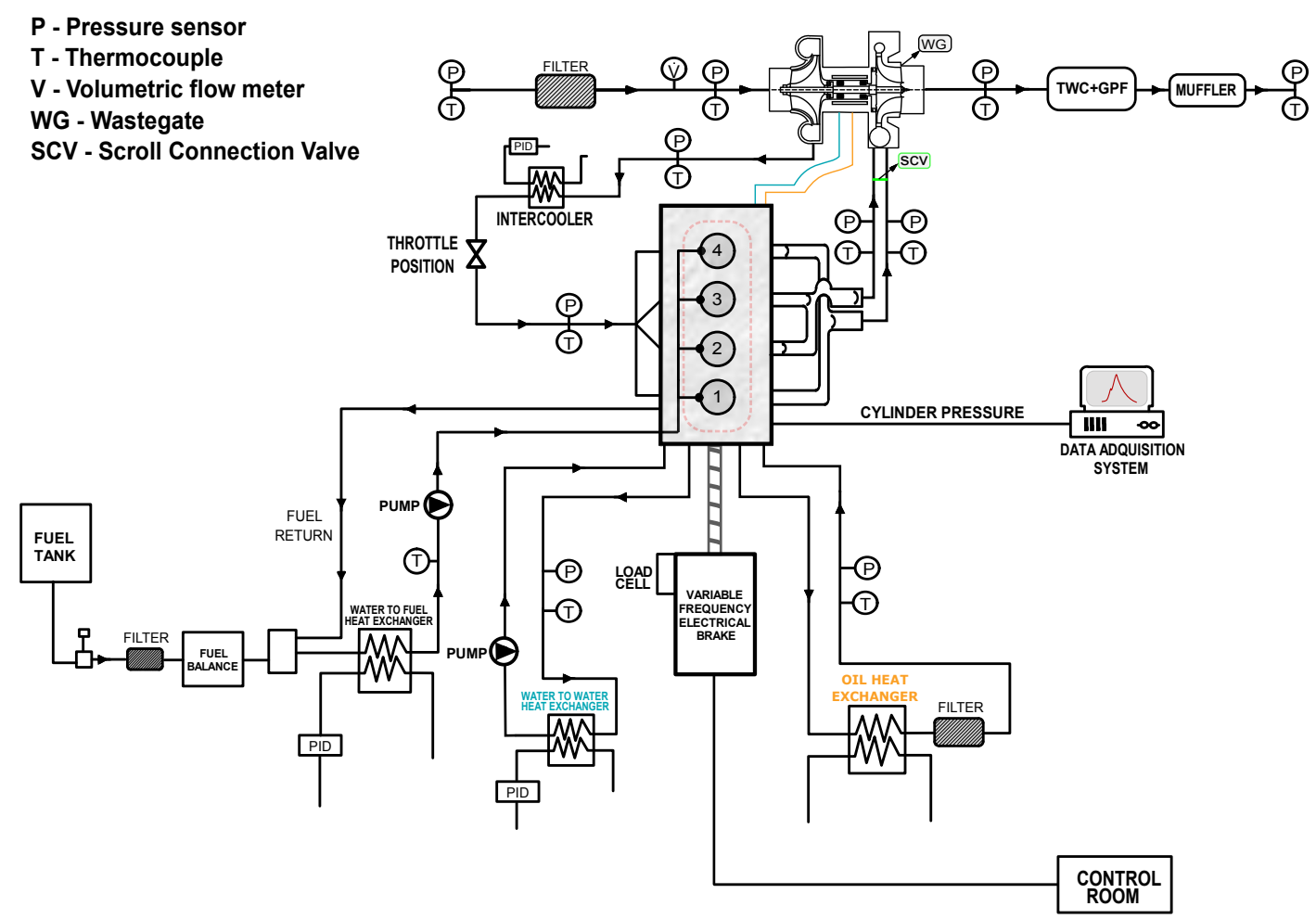

Figure 7.11: The layout of the engine test cell and the sensor instrumentation

The engine is coupled with the asynchronous dynamometer(APA). It is fixed to the testbed using metallic beams joined by screw or welding. The construction of the bed is designed in a way that it prevents longitudinal movement of the engine and makes easier the alignment with the dynamometer. The engine speed and load rate are controlled by the automatic acceleration system called throttle and the dynamometer. It is introduced into the control and data acquisition system called PUMA. The dynamometer offers essential resistance torque for the engine to test different rates of loads. The heat generated by the engine was controlled by the mean of water cooling systems. The thermal state of the different fluids like water cooling, air intake, fuel and oil is controlled by the heat exchange systems. The mass flow rate of the coolant is adjusted by an electric valve commanded by a PID controller. 
A turbocharged spark-ignition internal combustion engine with a dual-volute mixed flow turbine (T\#3DVM) was mounted on the facility. It is a four-cylinder engine with a displacement of 1.3 liters and has a variable valve timing (VVT), direct injection system and Euro 6 calibration. Some of the main technical characteristics of this engine are presented on Table 7.1.

Table 7.1: Engine main specifications

\begin{tabular}{cc}
\hline Parameters & Values \\
\hline Engine type & Gasoline spark ignition \\
Engine displacement $\left[\mathrm{cm}^{3}\right]$ & 1332.24 \\
Bore $[\mathrm{mm}]$ & 72.2 \\
Stroke $[\mathrm{mm}]$ & 81.35 \\
Number of cylinders & 4 in line \\
Valves & 4 valves per cylinder (VVT) \\
Compression ratio & 9.6 \\
\hline
\end{tabular}

The test facility is controlled automatically by a control system called PUMA V5. This system allows the acquisition of a set of variables that characterize the behaviour of the different systems of the engine. The sensors acquired by this system are within a frequency of 10 to $20 \mathrm{~Hz}$. In order to record the instantaneous measurements at the intake manifold and in the cylinders, Kistler type 4045A and AVL ZI33 sensors were used. The AVL sensor ZI33 is a spark plug with an integrated pressure sensor. In an engine test bench, the instantaneous pressure installation at the exhaust manifold is more restrictive due to the design and alignment position of the dual-volute turbine with the engine. Further, there were no pressure probes on the turbine as well. Therefore, a low-speed piezoresistive pressure sensor of Kistler type 4262A was installed. For temperature measurements, either K type thermocouples or thermoresistances Pt100 are used.

It is worth highlighting that, on the exhaust manifold, two pressure and temperature measurements were acquired instead of one as shown in Figure 7.12; due to both branches. Two of the sensors record the pressure and temperatures coming from cylinder 2 and 3, which is connected to the Long Volute branch, as shown in Figure 7.13 and, the other two are for the cylinder 1 and 4, which is connected to the Short Volute branch, as shown in Figure 7.13. This way, it is possible to measure the variables in both branches and helps in the validation of the models. The fuel flow rate is measured using an AVL fuel balance system (AVL733S). The torque of the engine is measured by means of load cell coupled to the dynamometer. The crankshaft rotation angle and engine speed are measured by optical angular encoder and Kistler 2613B sensor. Finally, the Electronic Control Unit (ECU) calculates some variables depending on the engine working conditions. These variables are measured by specific control software called INCA V5. 


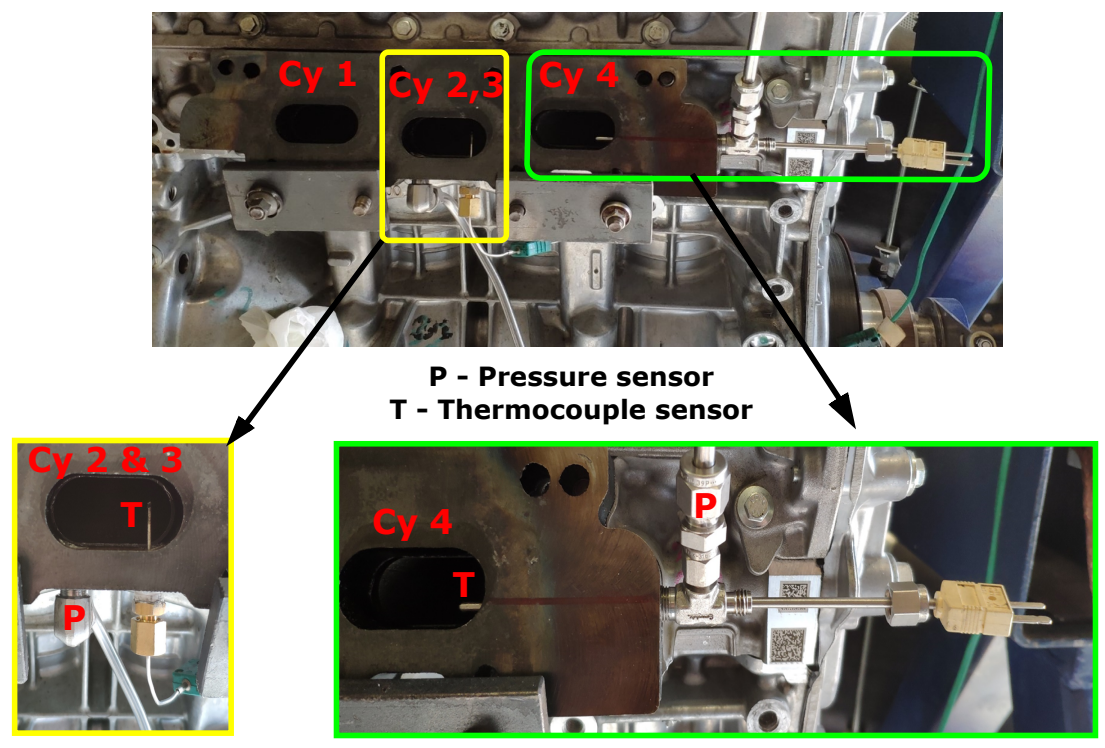

Figure 7.12: Pressure and temperature sensor instrumentation on the exhaust line of the engine.

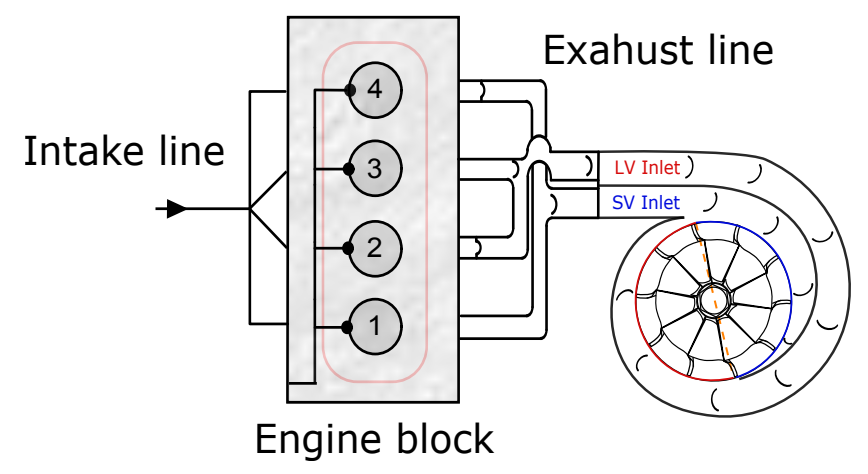

Figure 7.13: Dual volute turbine connection with the engine cylinders. 


\subsubsection{Test Methodology and results}

Turbocharger T\#3DVM is a wastegate turbine and have a special valve called scroll connection valve (SCV) for communicating the flows between branches. Both wastegate and SCV are controlled with a cylindrical type of valve which is connected to a stepper motor. When the cylindrical valve rotates, it has four different functions:

- Wastegate can be open totally without opening the SCV.

- Both wastegate and SCV are closed.

- SCV can be open with wastegate closed.

- Both SCV and wastegate are opened.

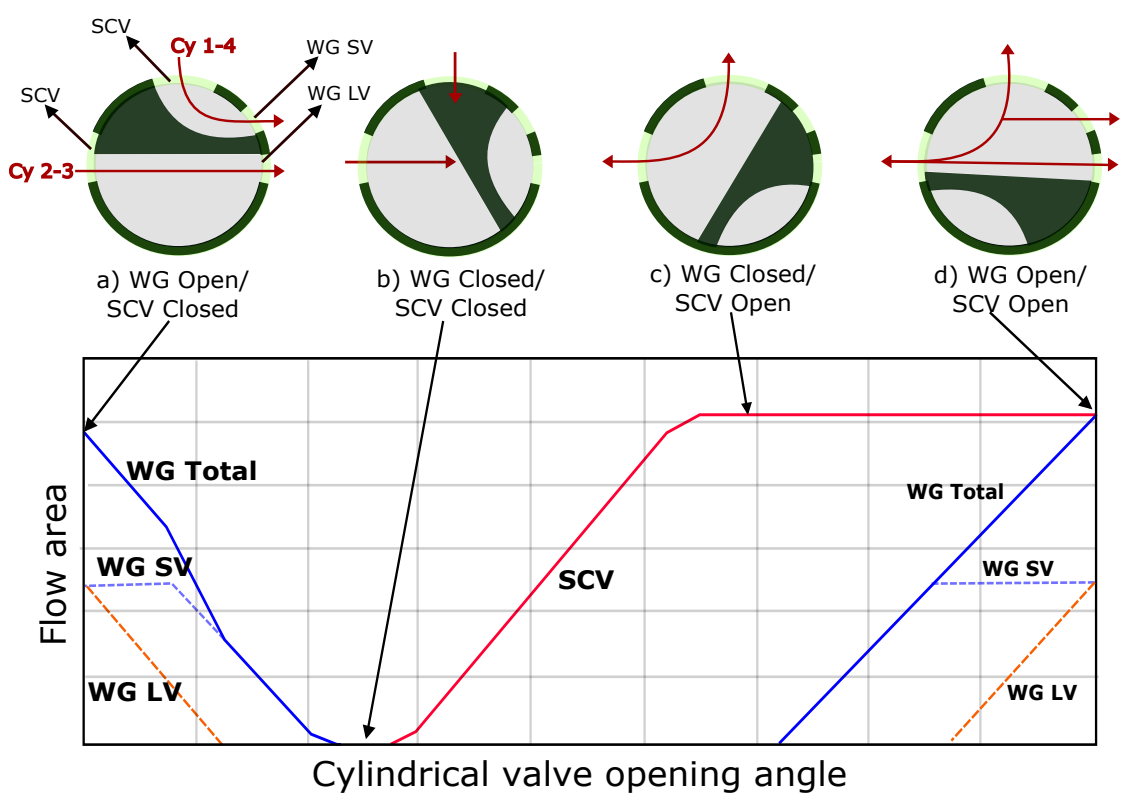

Figure 7.14: Wastegate and SCV flow areas with the cylindrical valve opening.

The function of the cylindrical valve is shown in the Figure 7.14. The position of this valve was controlled externally using a PXI ${ }^{T M}$ system from National Instruments. Nine steady-state engine full load points at different speeds have been measured with T\#3DVM turbocharger. Each full load point were tested twice by keeping SCV totally closed (function (a) in Figure 7.14) and with SCV totally open (function (c) in Figure 7.14). The target was to reach the maximum engine performance for each full load point in both the tests. While testing, the spark advance was optimized to keep the knock under control. This is done by using the knock detection and 
combustion diagnosis software which is designed by CMT-Motores Térmicos based on the in-cylinder pressure analysis, as it is described in [88]. The ECU controls Airto-Fuel Ratio (AFR) based on the estimation of turbine inlet temperature to protect the overheating of the turbine. Engine and turbocharger working limits provided by the manufacturers were kept under control. In the following figures, the test results of the engine are shown.

Figure 7.15 shows the test results of the engine at full loads for both configurations (i.e. SCV closed and open) is plotted against the engine speeds. From Figure 7.15, the following conclusions can be obtained:

- Comparing the torque of engine in both configurations (i.e. SCV closed and open), the benefits are observed for only SCV closed tests, although at very low engine speeds (up to $1500 \mathrm{rpm}$ ). Later on, a significant increase of backpressure in the long volute, which corresponds to the cylinder 2 and 3 limits the torque potential. The same results can be seen in the turbocharger speeds.

- At intermediate speeds, the SCV open configuration is affected by the compressor outlet temperature limitation. SCV closed configuration was not fully taken to the limit due to the significant turbine inlet pressure increase and led to lower torque potential.

- In SCV closed configuration test, the turbine inlet pressure in both volutes is unequal. This means that the wastegate flow area of each branch (see Figure 7.14) is different when the SCV is closed. But, when the SCV is open, the pressure levels in both volutes are similar due to the wastegate flow area is same for both branches (see Figure 7.14). Further, there is a communication of flows between the branches. It is worth highlighting that the turbine inlet pressure of LV and SV measured in both test configuration is the average of 2 pulses. Moreover, the turbine inlet temperature measured in each branch comes from the energy of 2 cylinders instead of 4 .

- The measured temperature in short volute is much lower than long volute. In general, the temperature difference between the branches could not be much higher, as seen in the experiments. The problem of measuring the low temperature in the short volute can be due to the position of a thermocouple sensor in cylinder 4 (see Figure 7.12).

\subsubsection{Engine Model Calibration}

In order to assess any turbocharger model with a one-dimensional engine model in GT-power; firstly, the engine modelling uncertainties have to be corrected in advance. An error in the engine torque during the GT-power simulations could be due to various incorrectly modelled sources like combustion, heat transfer in the cylinders during 
(a) T\#3DVM

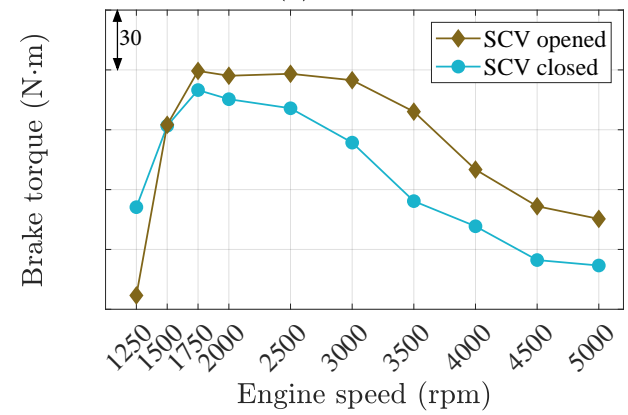

(c) T\#3DVM

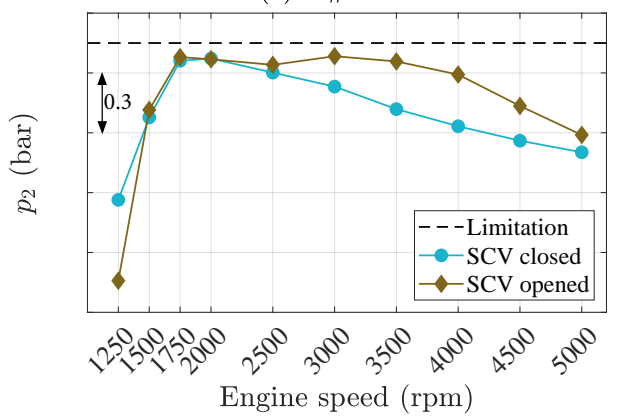

(e) T\#3DVM

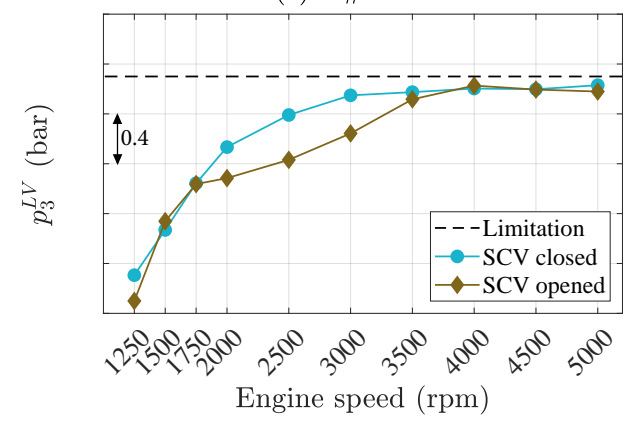

(g) T\#3DVM

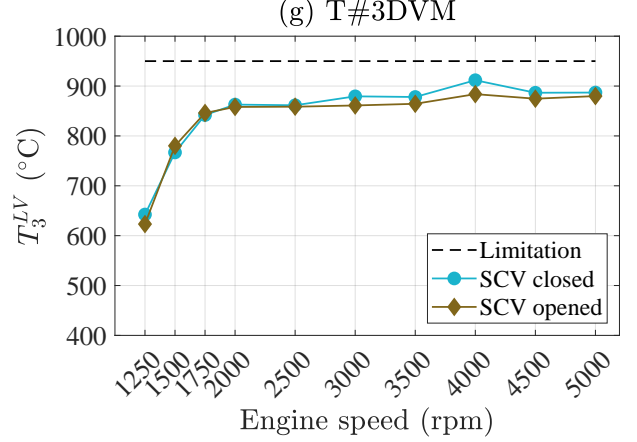

(b) T\#3DVM

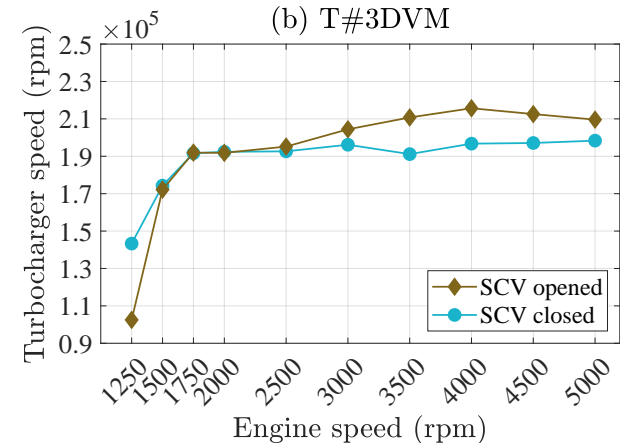

(d) T\#3DVM

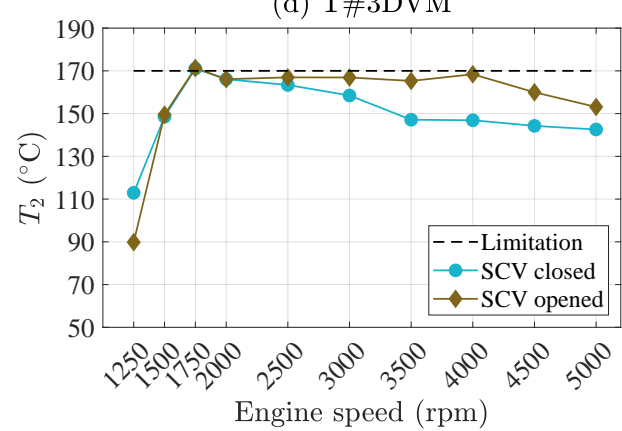

(f) T\#3DVM

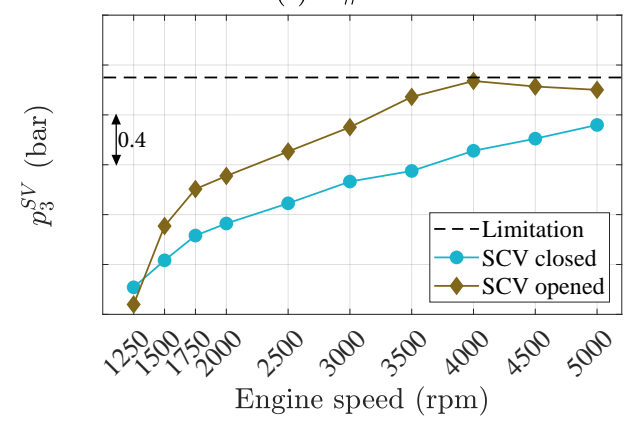

(h) T\#3DVM

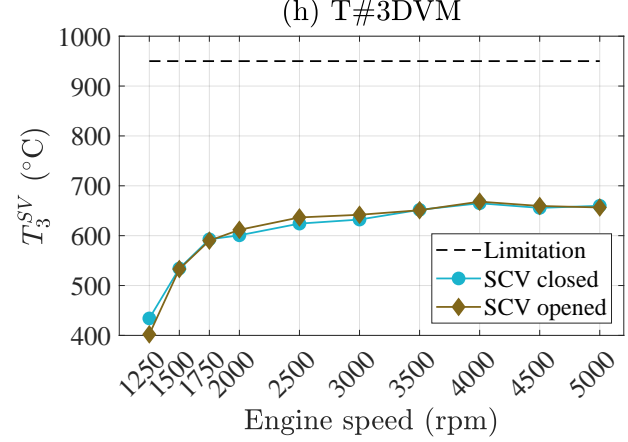

Figure 7.15: Dual volute mixed flow turbine engine test results at full load steady conditions. 
the combustion phase, prediction of engine mechanical losses and backpressure. Further, engine air mass flow error can be caused due to the errors in the volumetric efficiency. In addition, if the engine model is calibrated with a given turbocharger coupled, the errors in the turbocharger maps could also appear and impact the outcome of the calibration. By considering all these factors, the 1D engine model was calibrated beforehand with physical parameters as described in [89, 90]; using a VGT turbocharger unit that was tested with the same engine at full loads curves. The calibration procedure that described in $[89,90]$ is detailed below:

- During the virtual engine calibration, the important parameters like air-to-fuel ratio, intake and exhaust valves opening timings of the engine and test cell conditions were imposed to the experimental ones.

- The compressor inlet pressure $\left(p_{1}\right)$ and temperature $\left(T_{1}\right)$ conditions are reached by imposing the ambient and controlling the pressure drop in the air filter by means of a friction multiplier.

- The turbocharger is decoupled to separate the compressor and turbine powers and, they are connected to individual shafts. This enables to control the intake and exhaust conditions of the cylinders at the same time. On the one hand, the intake manifold pressure $\left(p_{2}^{\prime}\right)$ is fitted by controlling the compressor speed using a PID controller. On the other hand, the turbine shaft is fixed by imposing the experimental value of turbocharger speed and the turbine inlet pressure $\left(p_{3}\right)$ is adjusted by the rack position. This way, the impact of the turbocharger uncertainties on the engine response is avoided during the fitting.

- To achieve the intake manifold temperature $\left(T_{2}^{\prime}\right)$ similar to the experimental ones; the heat transfer multipliers are introduced in the water charge-air-cooler (WCAC) system pipe.

- Regarding the combustion analysis, a Wiebe function is implemented. The main variables require to use this function are the combustion phase at $50 \%$ of the crank angle (CA50) and the combustion duration which is estimated as the difference between the CA90 and CA10. The values of these variables are obtained by using the GT-power three pressure analysis (TPA) template.

- Overall cylinder heat transfer multiplier is used to fit engine volumetric efficiency. In doing this, it is essential to have the intake and exhaust boundary conditions equal to the experimental ones. This is achieved by the turbocharger decoupling method, as explained.

- Once the engine air mass flow and combustion process are fitted, the exhaust manifold heat transfer multipliers were used to fit the exhaust temperature $\left(T_{3}\right)$.

- Regarding the turbine outlet temperature $\left(T_{4}\right)$ and turbine back-pressure $\left(p_{4}\right)$, they are adjusted by using the heat transfer multipliers in the turbine diffuser 
pipe (a pipe that represents turbine volute equivalent surface) and by modifying the discharge coefficient at the outlet of the after-treatment system respectively.

- In respect of torque, friction mean effective pressure was calculated by making the difference between the indicated mean effective pressure (which is an output of the combustion process analysis) and the brake mean effective pressure (measured experimentally). These values are used to calibrate the engine friction model.

In summary, each heat transfer multipliers and discharge coefficient values were correlated with a dependent variable like air mass flow rate and the engine speeds. The obtained correlations were kept constant and validated by simulating full load curves obtained by the other VGT/WG turbocharger units, which were tested with the same 1.3 liters gasoline engine [89]. Therefore, the same fitted engine model is used for simulating the full load working points obtained with the dual-volute mixed flow turbine (T\#3DVM) that shown in the subsubsection 7.5.1.1.

It is worth noting that, in an engine, the scavenging process with double-entry turbines will be different than with single entry WG/VGT turbines. This is due to the separation of pressure interferences between the cylinders. Further, it should be noted that, while simulating the single entry turbines, the exhaust manifold is considered as one single element as shown in the Figure 7.16 (left) and accordingly, the exhaust heat transfer multipliers were found for this element during the engine fitting procedure [89, 90]. However, simulation with the dual-volute turbine was performed with separated exhaust manifold according to the turbo setup with the engine in a test cell. That is long volute branch connected to cylinders 2 and 3 and the short volute branch to cylinders 1 and 4 as shown in the Figure 7.16 (right). The exhaust heat transfer multipliers found for single entry turbines were applied to the separated manifold without any changes. Moreover, the pressure losses in the exhaust manifold could also be different. Considering these facts, and using an engine model that was previously fitted with a VGT/WG turbines to simulate with dual-volute turbine; there will be some discrepancy in the results. However, the study was mainly focused on the turbocharging system; therefore, the idea was not to re-calibrate the fitted engine model again with a dual-volute turbine. Therefore, the fitted engine model without any modification in the correlation is connected to CMT-DETCM and GT-DETCM turbo models. To perform the engine simulations and to check their predictions in the engine conditions.

It is worth highlighting that the heat transfer multipliers downstream the turbine was not used during engine simulations with CMT-DETCM model. Since the heat transfer problems are solved by CMT-DETCM model to give the turbine outlet temperature similar to experiments. 

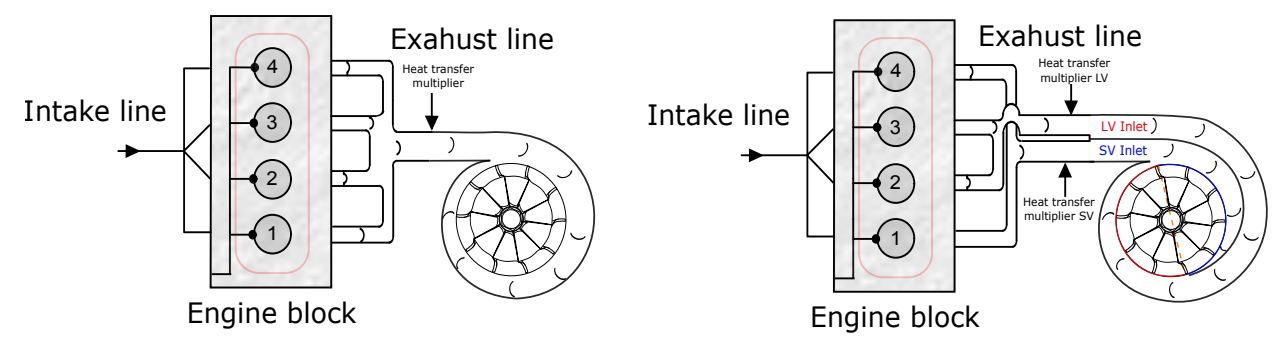

Figure 7.16: Exhaust manifold heat transfer multipliers in single entry and dual volute turbines during the engine simulations; Left: Single entry; Right: Dual-Volute.

\subsubsection{SCV opened simulation}

In this section, SCV opened 1D engine simulation results performed with CMTDETCM, and GT-DETCM turbo model will be discussed. From the engine test results shown in Figure 7.15, it can be observed that, when the scroll connection valve is opened, the pressure levels in both volutes are similar due to the fact that, the wastegate flow area in each branch is the same. Further, the flow from one branch to another can communicate depending on the pulse in each branch. For the communications of flows from long to short volute and vice versa, the SCV valve discharge coefficient models that developed in the section 5.3 of chapter 5 is used respectively. For a wastegate turbocharger, in 1D engine calculations, a wastegate model is necessary to control the boost pressure and also to predict the upstream turbine pressure accurately. The wastegate models can be in the form of discharge coefficient, as shown in chapter 5 . However, the wastegate discharge coefficient characterization for T\#3DVM turbine was not performed in this thesis. Therefore, for estimating the discharge coefficient of a wastegate in the engine calculations, the wastegate correlation of T\#2DVR dual-volute turbine developed in the section 5.2 of chapter 5 is used. This correlation is depended on the wastegate position and average expansion ratio of the whole turbine as discussed in the chapter 5. When the SCV is totally open, the wastegate flow area is the same in both long and short volute branches. Therefore, only one discharger coefficient for both branches is considered. In order to perform the simulation with these two discharge coefficient models (WG and SCV), a control system has been designed in the GT-power, as shown in the Figure 7.17.

From Figure 7.17, it can be observed that, when the SCV is opened, the wastegate flow area of each branch is the same. Moreover, the total flow area is linearly increasing with the cylindrical valve opening (highlighted with a green circle). Therefore to use the T\#2DVR turbine wastegate correlation in the simulation, a PID controller is utilized to target the experimental boost pressure value $\left(p_{2}^{\prime}\right)$. This PID generates an output signal value from 0 to 1 . Accordingly, a wastegate lookup table is created, as shown in the Figure 7.17. During the engine simulation, at every time step, the PID sends a signal (between 0 to 1 ) to the wastegate lookup table. Based on the 
value of the signal, the lookup table gives a position of the wastegate valve. Finally, using this wastegate position and an average expansion ratio of the whole turbine at the current time step are passed to the wastegate correlation of T\#2DVR turbine to calculate the discharge coefficient.

Regarding the SCV valve, the tests were done with opened position; therefore, the valve position is fixed to $100 \%$ during the entire simulation for all engine speeds. However, the SCV model depends on the scroll pressure ratio value, and it is different when the flow goes from branch to branch (i.e. LV to SV and vice versa) as discussed in the chapter 5. Therefore, at every time step, the scroll pressure ratio across the SCV section is passed to the correlations for estimating a discharge coefficient values for each flow direction. The designed control system continues until the PID reaches the target value (i.e., $\left.p_{2}^{\prime}\right)$ ), and all the other steady-state variables are converged. The working of this control system during the simulation is shown in the Figure 7.18.
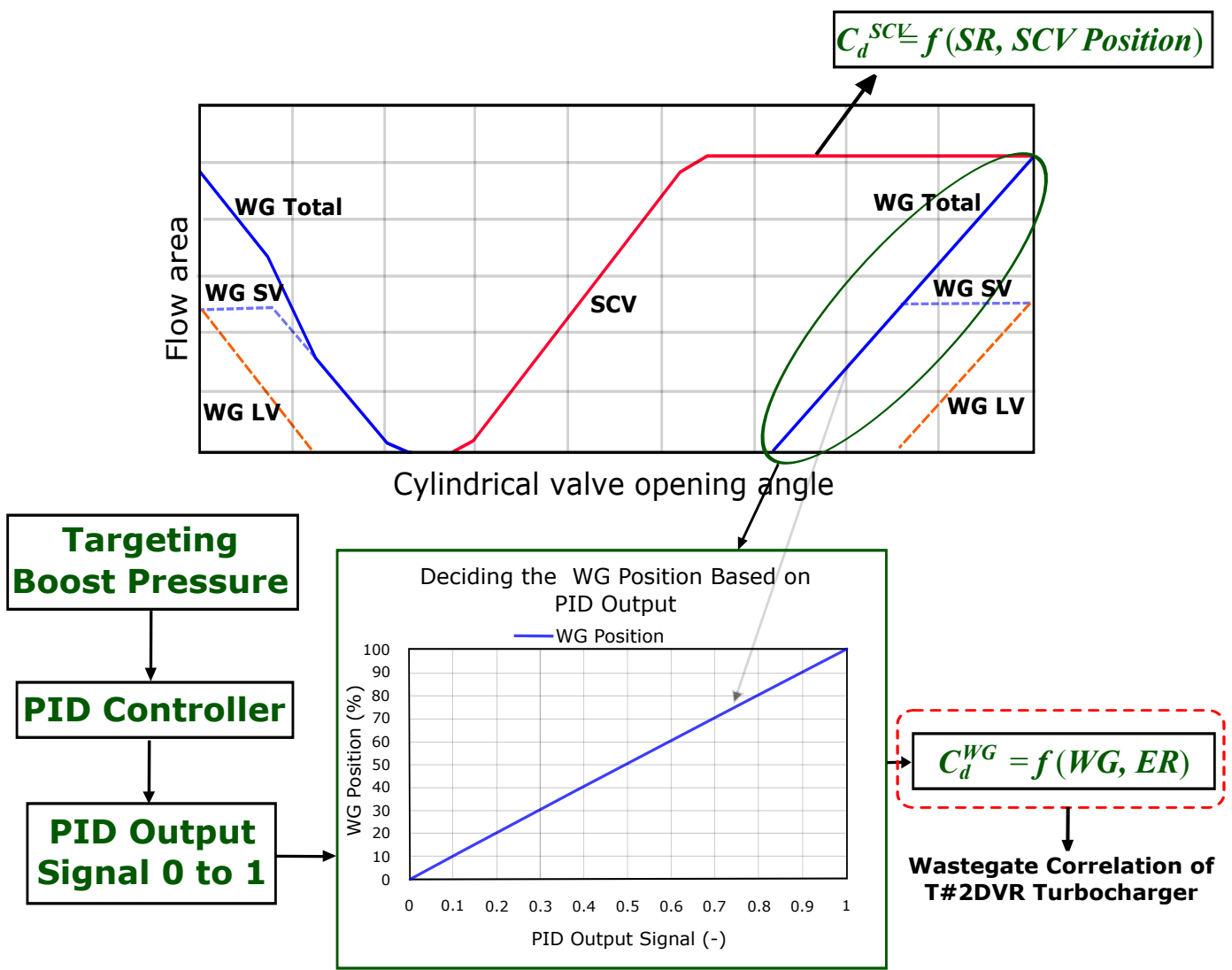

Figure 7.17: Methodology for simulating when the wastegate flow area is the same 


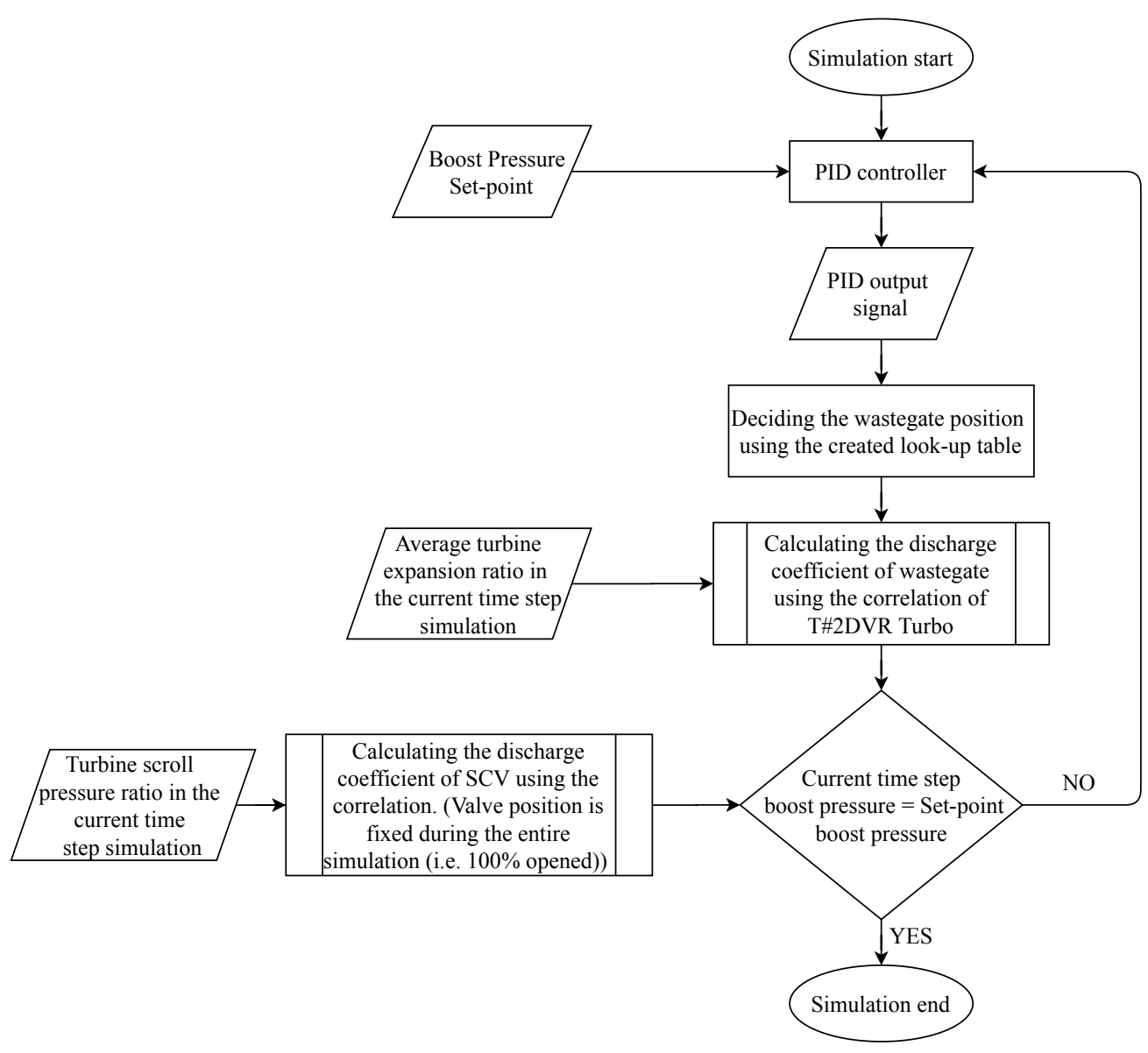

Figure 7.18: Working of the designed control system when the wastegate flow area is same and the SCV valve is opened.

Figure 7.19 shows result of manifold boost pressure $\left(p_{2}^{\prime}\right)$ in the engine simulations. It can be observed that the error between the experimental and model are very low. This confirms that the control design developed with the T\#2DVR wastegate correlation is efficient enough to reach the target values. From Figure 7.20 (a) and (b) it can be observed that the outcome of wastegate position and discharge coefficient values for both CMT-DETCM and GT-DETCM are very similar. Furthermore, the wastegate position at every engine speed estimated by the control system from both models is very close to the experimental values, as shown in Figure 7.20(a). These indicate that the turbine upstream pressure values in the simulation are also well predicted; the results are shown in the following figures. From Figure 7.20 (a), one can notice that, at low engine speed (1250 rpm), both CMT and GT models indicates that the wastegate should be opened in order to reach the experimental boost pressure value. One possible reason could be, small leakages from the cylindrical valve during the experiments even when the wastegate is closed totally and, the model discovers 
these.
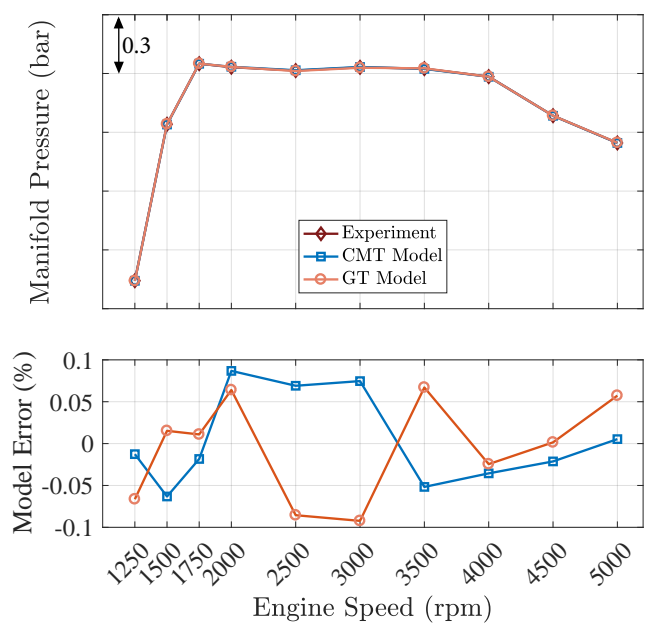

Figure 7.19: SCV opened inlet manifold boost pressure from the PID controller

(a)

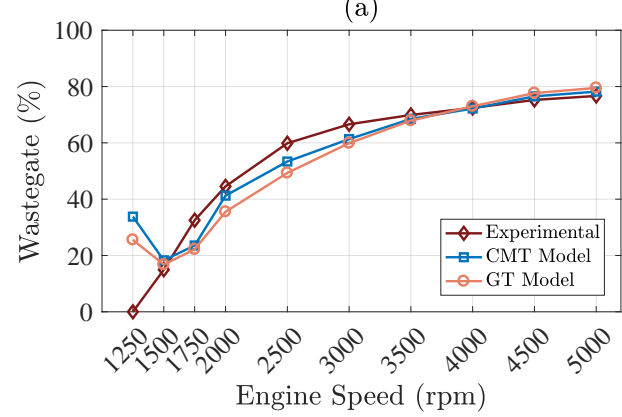

(b)

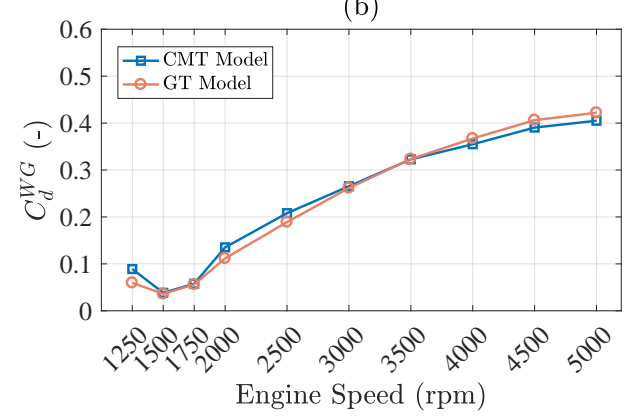

Figure 7.20: CMT-DETCM and GT-DETCM model wastegate positions and the estimated discharge coefficient values.

Figure 7.21 shows the position of scroll connection valve and the estimated discharge coefficient values for each flow direction. Aforementioned, the SCV opened tests were performed with totally opened valve and accordingly, in the simulation, the valve is fixed to $100 \%$ for all engine speeds as shown in the Figure 7.21. As discussed in the chapter 5, the flow passing from long to short and vice versa for the same operating point in the turbine is different. Due to the pressure drop across the SCV section is not the same in each flow direction. Consequently, the discharge coefficient values are different when the flow is moving from SV to LV and LV to SV in the simulations, as shown in Figure 7.21 (b). 

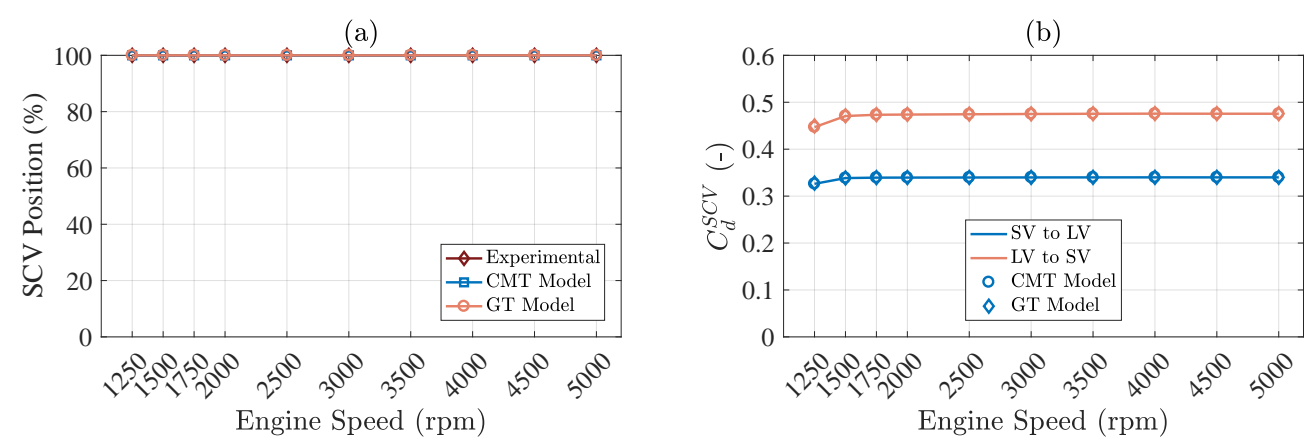

Figure 7.21: CMT-DETCM and GT-DETCM models SCV positions and the estimated discharge coefficient values.

In order to compare the compressor and turbine performances, the turbocharger rotational speed has to be close to the experimental one. From Figure 7.22(a), it can be perceived that the simulated turbocharger speeds are not that far from the experimental results. Better predictions were observed at the low engine speeds but, at high engine speeds, both models are slightly underestimated. Nevertheless, the error is not above $\pm 5 \%$ from the experimental value. The well predicted rotational speed highlights a reasonable estimation of friction losses and overall power balance. It is worth highlighting that the mechanical efficiency of the shaft estimated from CMT-DETCM model was used in the simulations with GT-DETCM model. This way, the GT model also considers the friction losses in the turbocharger shaft.

Since the wastegate position is changed until the manifold boost pressure $\left(p_{2}^{\prime}\right)$ is achieved. Therefore, compressor outlet temperature $\left(T_{2}\right)$ will be the primary variable to validate the compressor performance and heat transfer. As it can be seen in Figure 7.22(b), the compressor outlet temperatures from the models are always underestimated in all the engine speeds, an average of $15^{\circ} \mathrm{C}$ lower compared to the experimental. However, the compressor outlet pressure from the models agrees well with the experimental points as it is shown in Figure 7.22(c). So the difference in the outlet temperature predictions is mainly related to the heat transfer problems. Eventually, any temperature error from the compressor outlet is corrected by the WCAC heat transfer multipliers. Figure 7.22(d) shows the results of the inlet manifold temperature compared to the experimental data, and it can be observed that the maximum error is around $9^{\circ} \mathrm{C}$.

Suppose the engine boundary conditions of the model are well fitted with the experiments that are, inlet manifold $\left(p_{2}^{\prime}\right)$ and exhaust manifold pressures $\left(p_{3}^{L V / S V}\right)$. The air mass flow produced by the model should correspond to the experimental. From Figure 7.23(a) it can be observed that the air mass flow predictions are similar to the experimental results in both CMT and GT simulations. However, at engine speeds of 2000 and $2500 \mathrm{rpms}$, the relative error is above $5 \%$. The points with the low error indicate that the engine is operating close to the experimental conditions. 

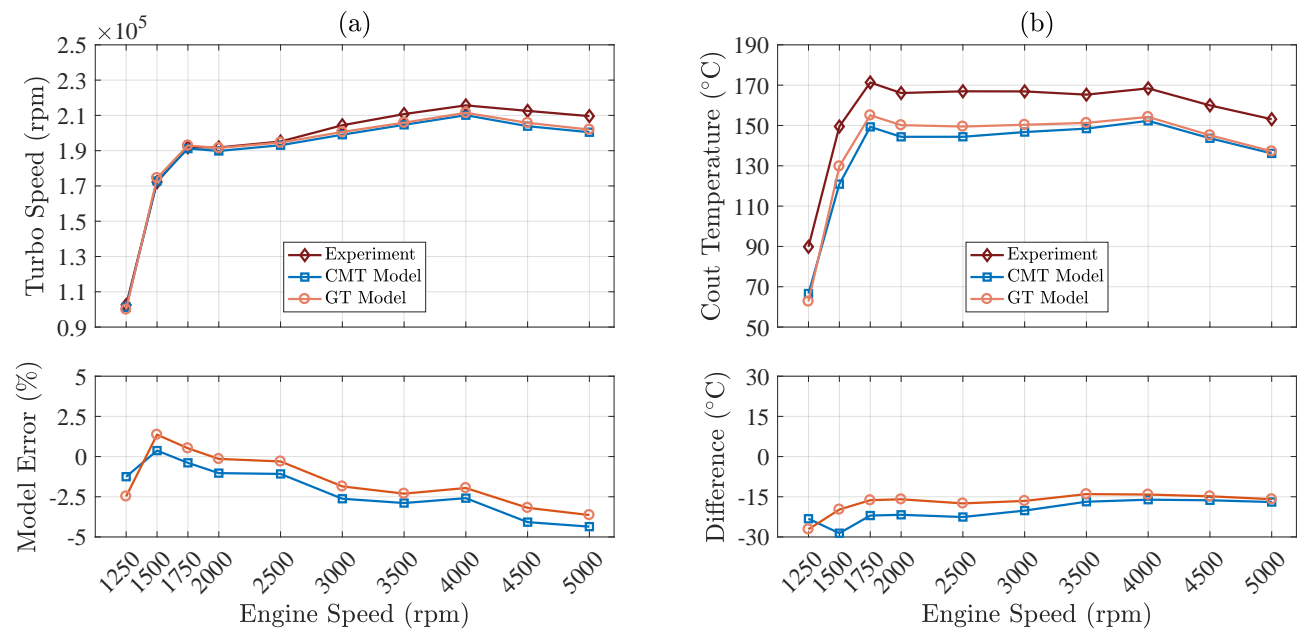

(c)
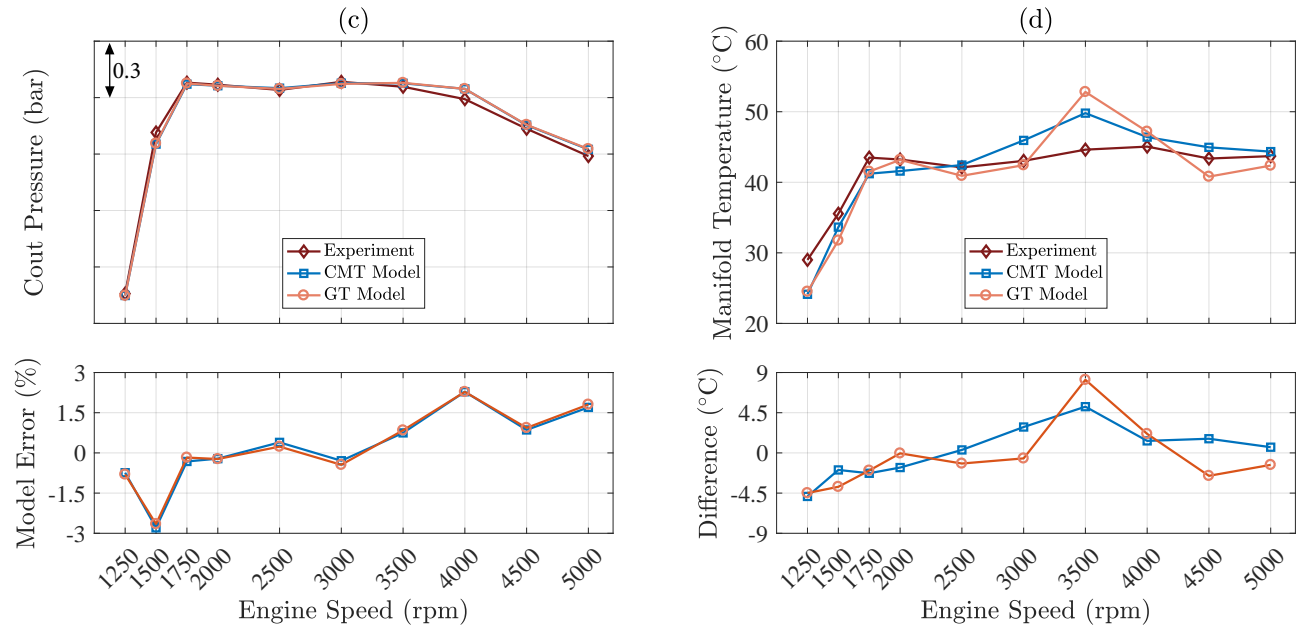

Figure 7.22: Validation parameters of SCV opened simulation; Outputs from CMT-DETCM and GT-DETCM were compared against the experimental data at different engine speeds. (a) Turbocharger speed; (b) Compressor outlet temperature; (c) Compressor outlet pressure; (d) Inlet manifold temperature.

Figure 7.23(b) reveals the fact that, if the model well predicts engine air mass flow, then the torque shows no significant mismatch against the experimental data. The small disagreement between the model and experimental torque values can come from the modelling of friction mean effective pressure (FMEP) and combustion.

Figure 7.23(c) and (d) shows the upstream turbine pressure in both long and short volute branches. It is evident that the mean pressure values with CMT-DETCM model agree well with the experimental data in both turbine branches. That indicates the backpressure from the turbine to the engine is also well captured. In 
case of simulations with GT-DETCM model, the turbine inlet pressure is slightly over predicted in both branches in 2000 engine rpms and above. In order to check the accuracy of gas dynamics in the model, instantaneous turbine upstream pressure values are needed. However, from the experiments, the instantaneous pressure data were not available due to the space issues of placing the instantaneous sensors at the turbine inlet. Therefore, the validation of instantaneous pressure evolution from the engine is still pending, and this will be the scope of future work.

(a)
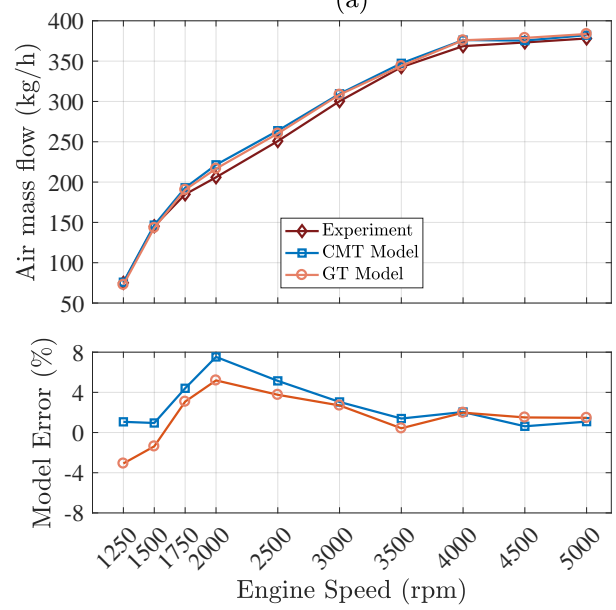

(c)
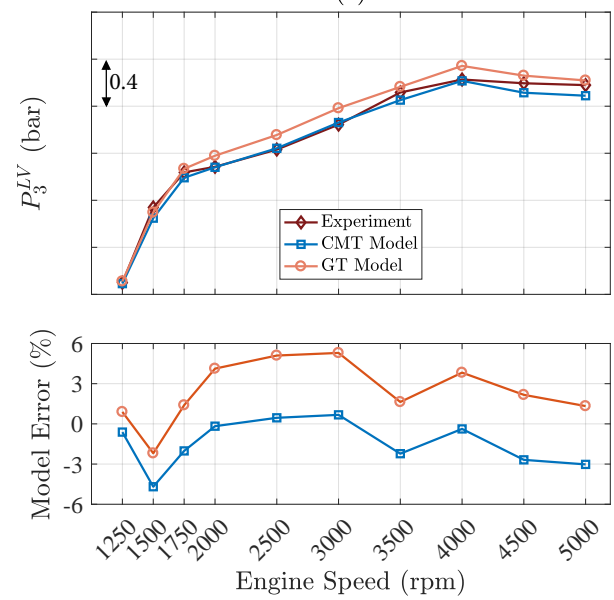

(b)
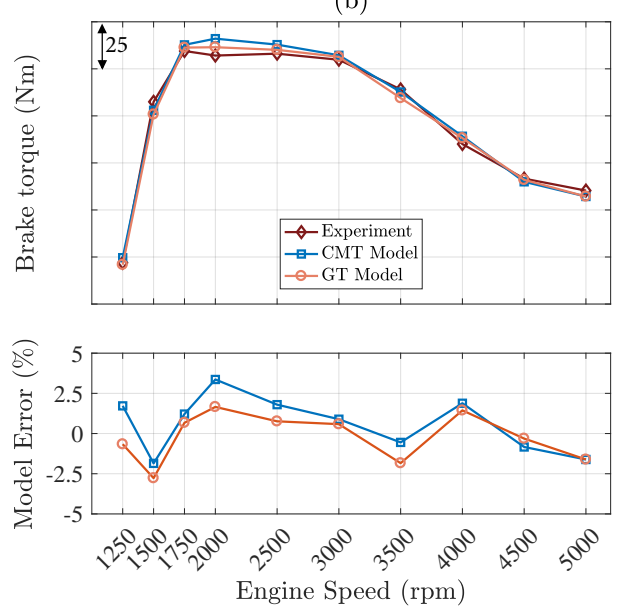

(d)
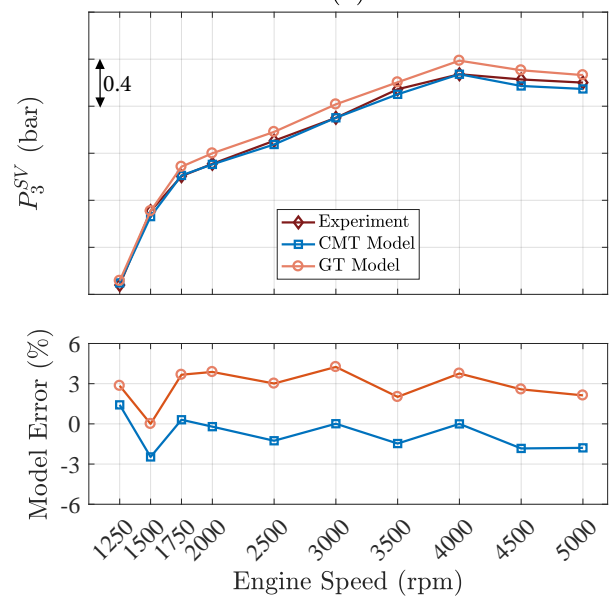

Figure 7.23: Validation parameters of SCV opened simulation; Outputs from CMT-DETCM ans GT-DETCM were compared against the experimental data at different engine speeds. (a) Air mass flow; (b) Brake torque; (c) Turbine inlet pressure in LV; (d)

Turbine inlet pressure in SV.

Figure 7.24(a) and (b) shows the results of turbine inlet temperatures at long and short volute branches. On the one hand, the difference between the model 
(a)
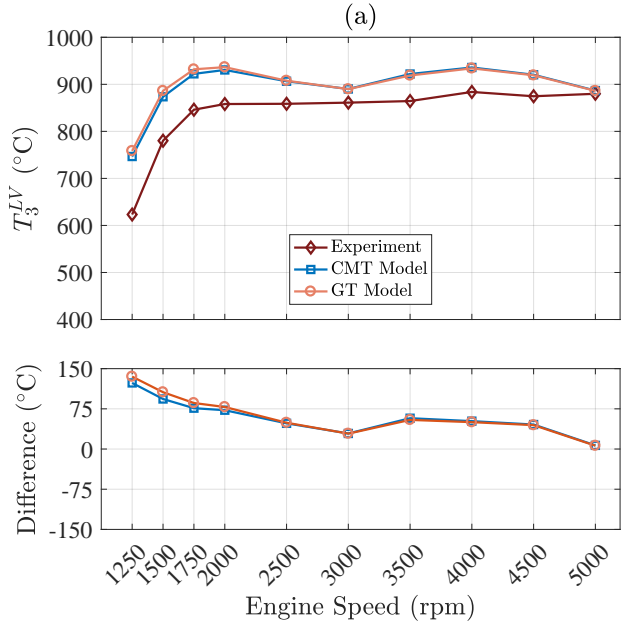

(c)
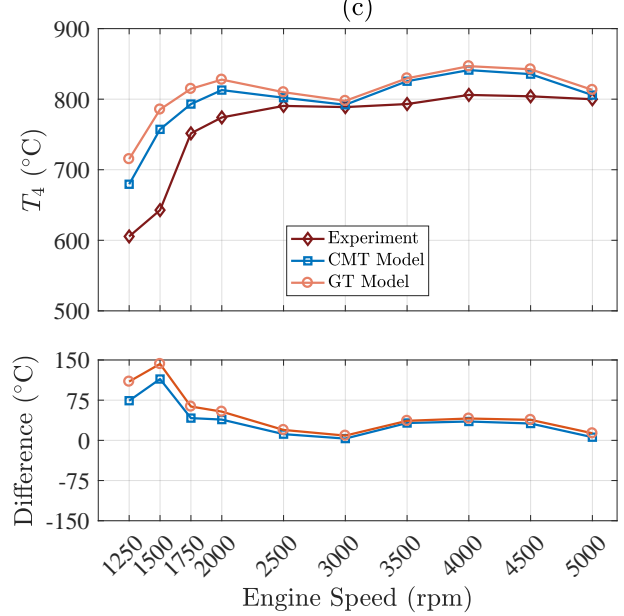

(b)
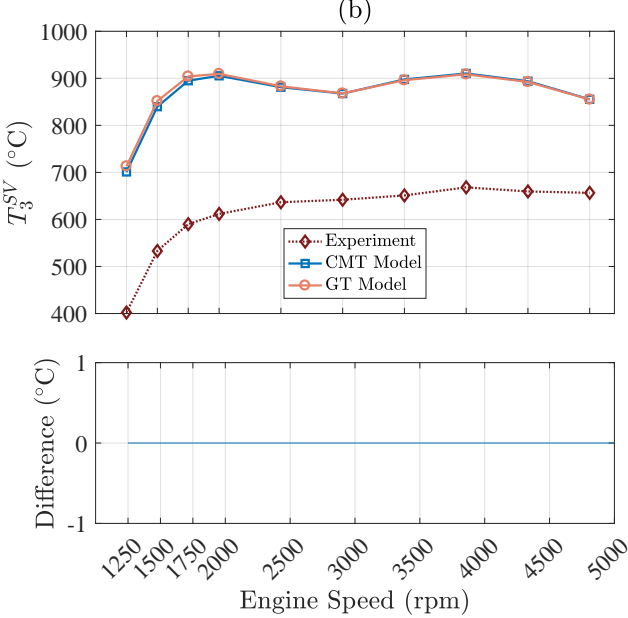

(d)
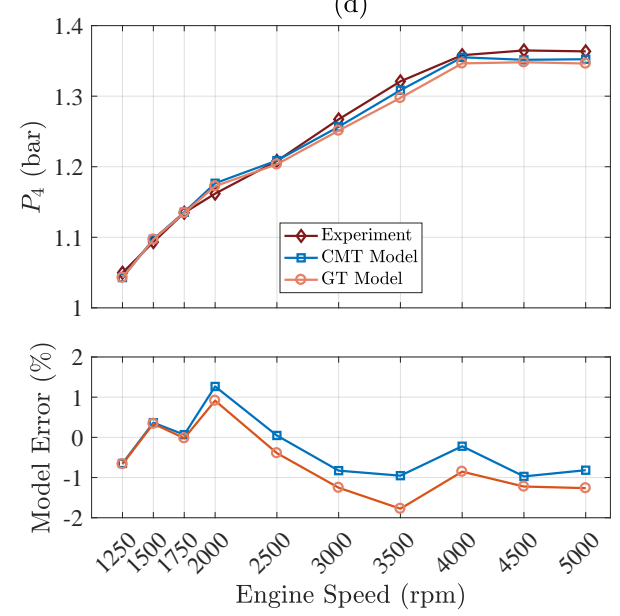

Figure 7.24: Validation parameters of SCV opened simulation; Outputs from CMT-DETCM and GT-DETCM were compared against the experimental data at different engine speeds. (a) Turbine inlet temperature LV; (b) Turbine inlet temperature SV; (c) Turbine outlet temperature; (d) Turbine outlet pressure. 
estimated and experimental temperatures in the long volute branch is higher at low engine speeds (i.e. 1250 to $2000 \mathrm{rpms}$ ). On the other hand, the difference is lower in the rest of the engine speeds. The discrepancy in temperature predictions can be from the usage of exhaust heat transfer multiplier correlation that is found for single entry VGT/WG turbines. As mentioned before, these multipliers are obtained by considering the exhaust manifold as one single component. Whereas, in simulating the dual-volute turbine, the exhaust manifold is separated and connected to each turbine branch. Therefore, the scavenging process is different than the single entry turbines and can affect the combustion calculations in the model. Eventually, the exhaust manifold multipliers can change.

Figure 7.24(b) shows the results of temperatures in the short volute branch. Aforementioned, the temperature values for this branch from the experimental are reasonably lower when compared with the long volute temperature values. This is more likely due to an error in the sensor installation or a problem with the sensor itself. Since, the model shows the temperature levels in the short volute branch is similar to the long volute branch. The difference between them is in a range of $30-40^{\circ}$, which is reasonable value as compared to the difference found in the experiments [36]. Figure 7.24(c) and (d) shows the results of temperature and pressure at the turbine outlet. The outlet temperatures from the model are higher than the experimental. This is due to having higher temperatures than required at the inlet of the turbine. Figure 7.24 (d) shows after-treatment back-pressure to the turbine from both CMT and GT models and, they are in good agreement with the experimental values.

One can assume that, if the model well predicts the turbine upstream and downstream conditions; this indicates that the experimental running point is correctly found inside the turbine maps. From Figure 7.24 (a) and (c) it can be observed that, at 3000 and 5000 engine rpms, the turbine inlet and outlet conditions are similar to the experimental values. Furthermore, all the other validation variables like boost pressure, engine air mass flow and torque are reasonably well agreed to the experimental values.

\subsubsection{SCV closed simulation}

In this section, SCV closed 1D engine simulation results performed with CMTDETCM, and GT-DETCM turbo models will be discussed. From engine test results (Figure 7.15), it can be observed that, when the scroll connection valve is closed, the turbine inlet pressures in the long and short volute are different; due to the fact that at this condition the wastegate flow area of each volute branch is unequal with the opening of the cylindrical valve. In order to capture this unequal effect in the $1 \mathrm{D}$ engine simulations, two different wastegate discharge coefficients value one for each branch is essential. However, the wastegate characterization of T\#3DVM turbine was not performed in this thesis. Further, there was no detailed geometrical/discharge co- 
efficient information provided by the turbocharger manufacturer, except the function of the cylindrical valve shown in the Figure 7.14. Therefore, in order to estimate the discharge coefficient of wastegate in each branch, the wastegate correlation developed for T\#2DVR turbocharger was used.

Nevertheless, this correlation is dependent on the position of wastegate and expansion ratio of the turbine, as shown in the chapter 5. Further, even from the engine tests, a total wastegate position was the only information that was able to record from the PXI ${ }^{\mathrm{TM}}$ system. Therefore, considering all these aspects, a control system has been designed in the GT-power using the cylindrical valve information provided by the turbocharger manufacturer. Figure 7.25 shows the control system methodology for simulating when the wastegate flow area is unequal.

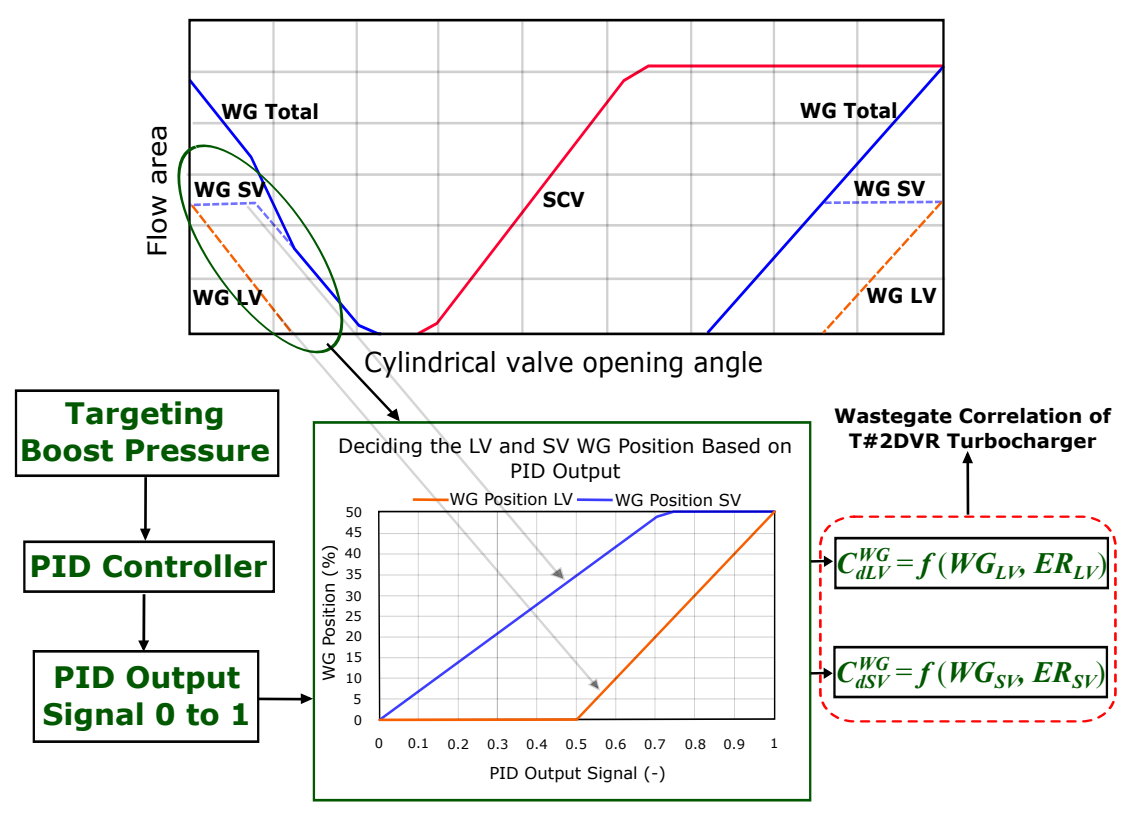

Figure 7.25: Methodology for simulating when the wastegate flow area is unequal with cylindrical opening angle

From Figure 7.25, it can be observed that, when the SCV is closed, the wastegate flow area of each branch is unequal with respect to the cylindrical valve opening angle (highlighted with a green circle). Similarly, a wastegate lookup table is created with the unequal wastegate positions for each branch, as shown in the Figure 7.25. In order to decide the long and short volute wastegate positions, the PID controller output signal is used. This PID controller targets the experimental boost pressure value at the inlet manifold $\left(p_{2}^{\prime}\right)$. Once the wastegate position and expansion ratio of each branch is known, using the T\#2DVR wastegate correlation, the discharge coefficient of each branch is estimated. This continues until the PID reaches the target value, 
and all the other steady-state variables are converged. The procedure of the designed control system during the simulation is shown in the Figure 7.26.

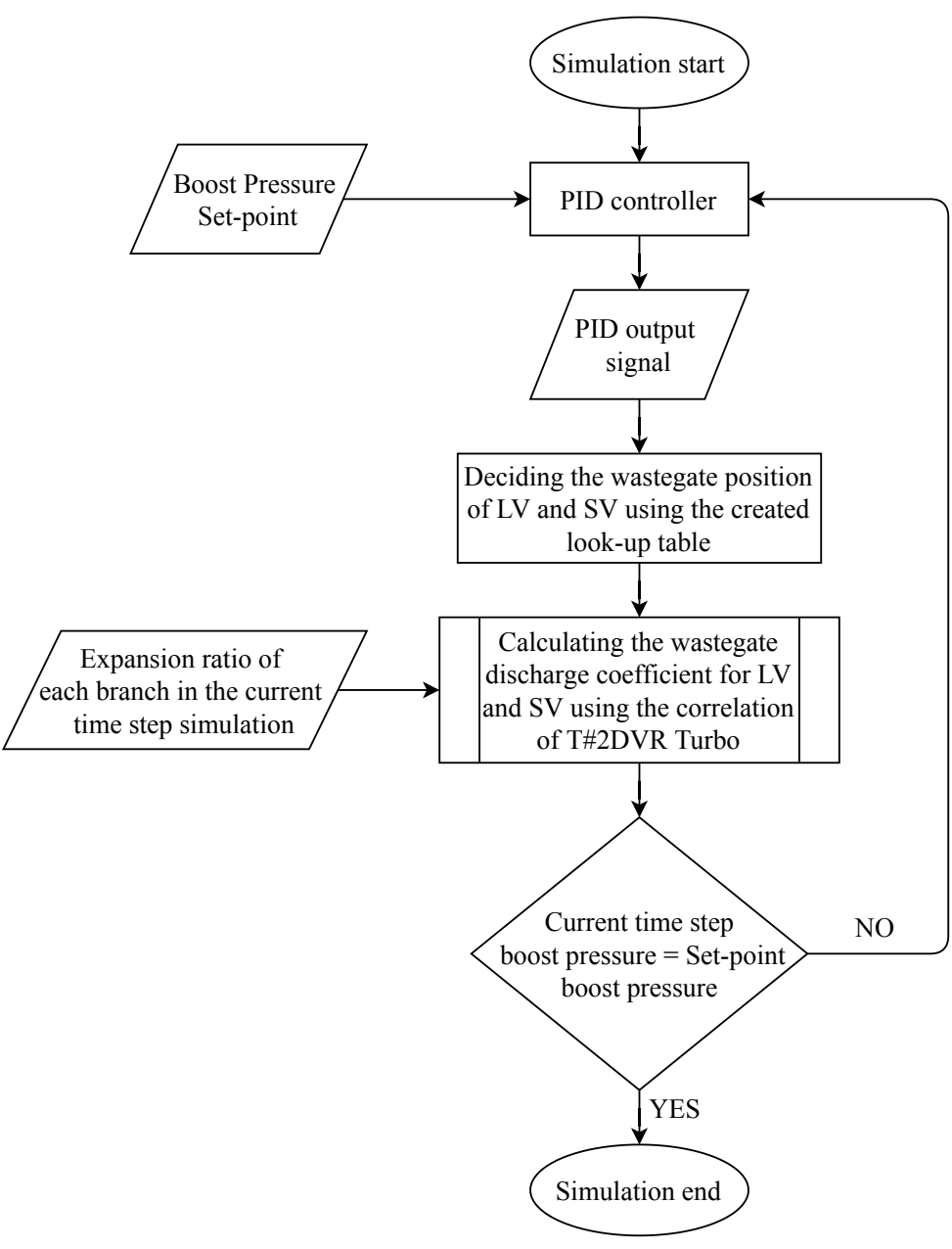

Figure 7.26: Working of the designed control system when the wastegate flow area is unequal and the SCV is closed

Figure 7.27 shows the manifold boost pressure $\left(p_{2}^{\prime}\right)$ values from engine simulations compared against the experimental. It can be observed that the control design able to reach the experimental target boost pressure with minute errors.

Figure 7.28 shows the results of the wastegate position and the discharge coefficient values of each branch with both CMT-DETCM and GT-DETCM engine simulations. It can be noted that the overall wastegate position values from the CMT-DETCM model are very close to the experimental values. However, at low engine speeds 1250 and $1500 \mathrm{rpms}$, for reaching the experimental boost pressure, the model shows that the wastegate should be opened. This could be due to the possibil- 

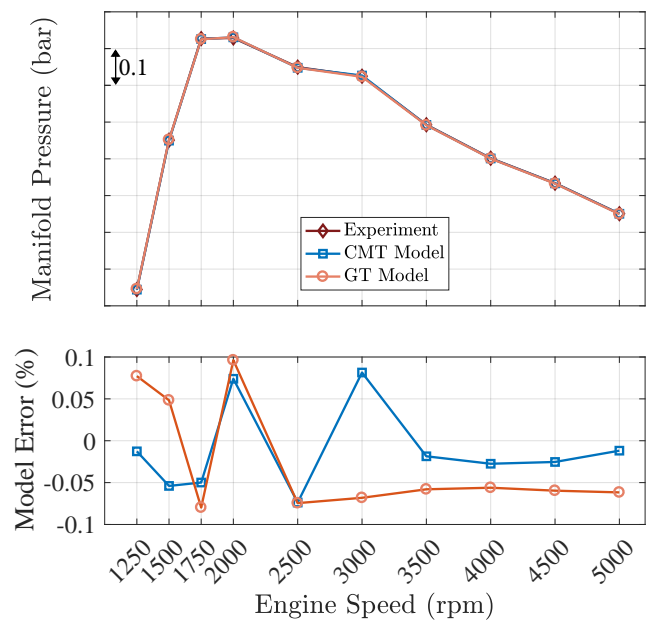

Figure 7.27: Inlet manifold boost pressure from the PID controller

(a)

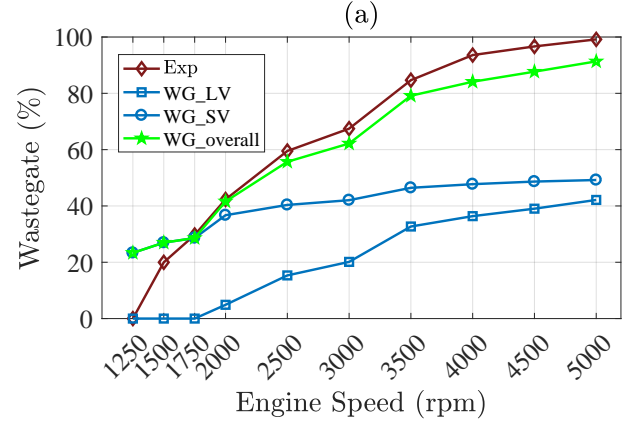

(c)

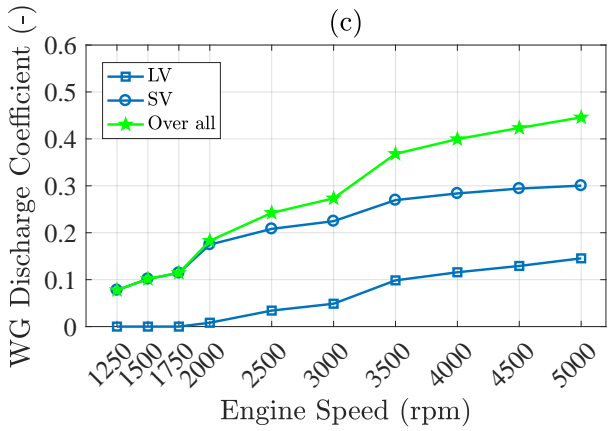

(b)

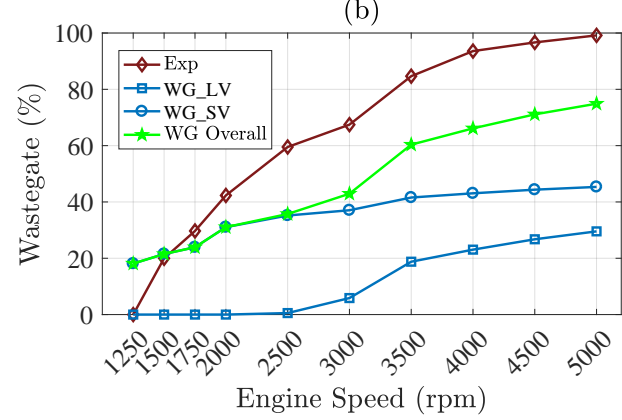

(d)

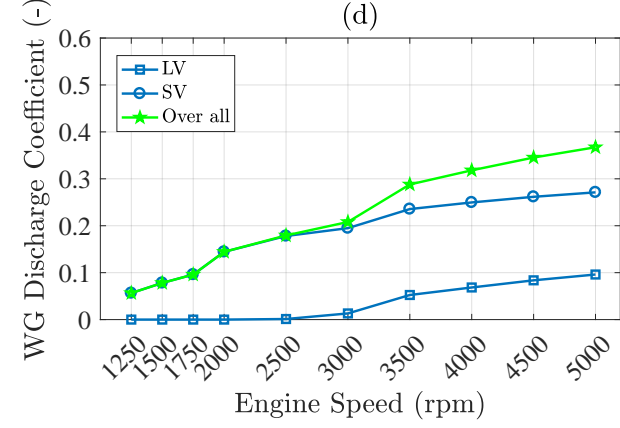

Figure 7.28: CMT-DETCM and GT-DETCM models wastegate positions and there discharge coefficient values when the SCV is closed. (a) and (c) are the results from CMT-DETCM model; (b) and (d) are the results from GT-DETCM model. 
ity of small leakages from the cylindrical valve at this speeds during the experimental campaign and were not able to be measured. Although, the PXI ${ }^{\mathrm{TM}}$ system showed that the wastegate is closed totally at the low engine speeds (1250 and $1500 \mathrm{rpms}$ ).

From Figure 7.28(c) and (d), it can be observed that the wastegate discharge coefficient values of long and short volutes are different as expected. It is worth highlighting that the change in the discharge coefficient is not only dependent on the wastegate position. But, also on another important parameter which is the expansion ratio of the turbine; as discussed in the section 5.2 of chapter 5 . As the T\#2DVR wastegate correlation is applied to long and short volute branches. The outcome of the correlation will be different because of the different wastegate and expansion ratio values of each branch, respectively.

From Figure 7.29(a), it can be observed that the simulated turbocharger speeds are very close to the experimental results in good quality (up to $3500 \mathrm{rpms}$ ). However, at high engine speeds (above $3500 \mathrm{rpms}$ ), the simulated turbocharger speeds were moderately underestimated. However, the error from both models is not above $\pm 4 \%$ from the experimental value. Figure 7.29(b) shows the outcomes of compressor outlet temperatures from the models. It can be observed that the outlet temperature values from both CMT and GT models are low in an average of $10^{\circ} \mathrm{C}$ when compared to the experimental. As mentioned before, this difference could be due to heat transfer problems. The compressor outlet pressure values are similar to the experimental results, and it can be observed in the Figure 7.29 (c) with $\pm 3 \%$ of error in relative terms. Nevertheless, the discrepancy of temperature from the compressor outlet is corrected by the WCAC heat transfer multiplier to reach the experimental inlet manifold temperatures and to have better combustion calculations. Figure 7.29 (d) shows the results of inlet manifold temperatures.

From Figure 7.30(a) it can be observed that the air mass flow values predicted by the model are slightly higher than the experimental. The relatively large percentage error at low engine speeds is due to the small absolute values. The impact in air mass flow comes from the exhaust manifold pressures (i.e. at each turbine inlets). As they are not predicted according to the experiments as shown in Figure 7.30 (c) and (d). This error comes from not knowing the exact wastegate flow area of each branch. Since the wastegate opening of each branch significantly influences the upstream turbine pressure. However, the designed control system shown in the Figure 7.26 is able to catch the non-identical pressure levels in each branch. But, with some differences when compared to the experimental values. On the one hand, this difference could come from the error of using the wastegate correlation from another turbocharger and applying the same to both branches. On the other hand, it could also be due to not knowing the exact geometrical sections of each wastegate branch. Figure 7.30(b) reveals the fact that, if there are some errors in the air mass flow predictions, then the torque shows a considerable mismatch against the experimental data. Nevertheless, the error in torque from the model is not more than $\pm 5 \%$ to 

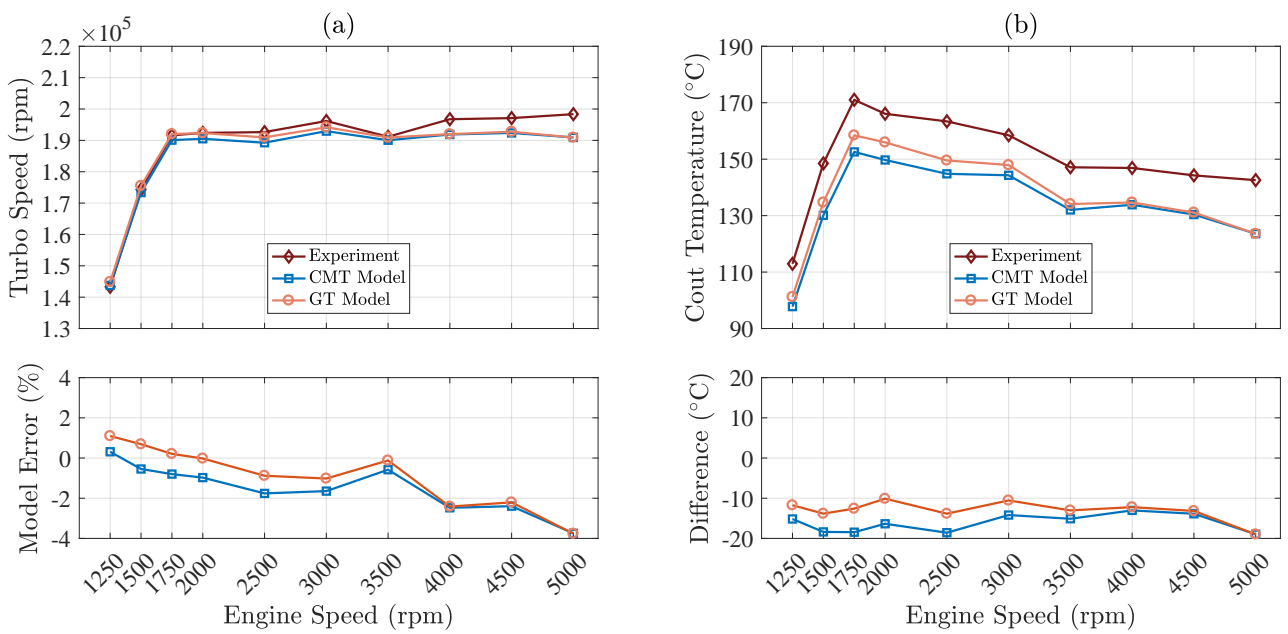

(c)
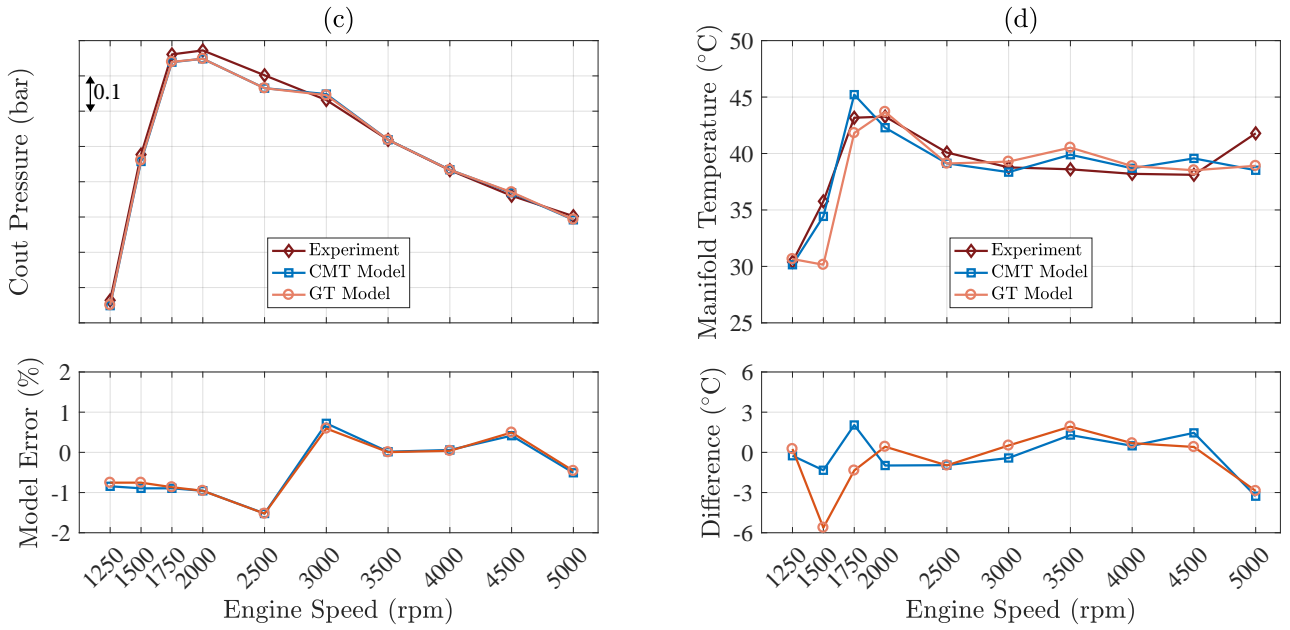

Figure 7.29: Validation parameters of SCV closed simulations; Outputs from CMT-DETCM and GT-DETCM were compared against the experimental data at different engine speeds. (a) Turbocharger speed; (b) Compressor outlet temperature; (c) Compressor outlet pressure; (d) Inlet manifold temperature. 
(a)
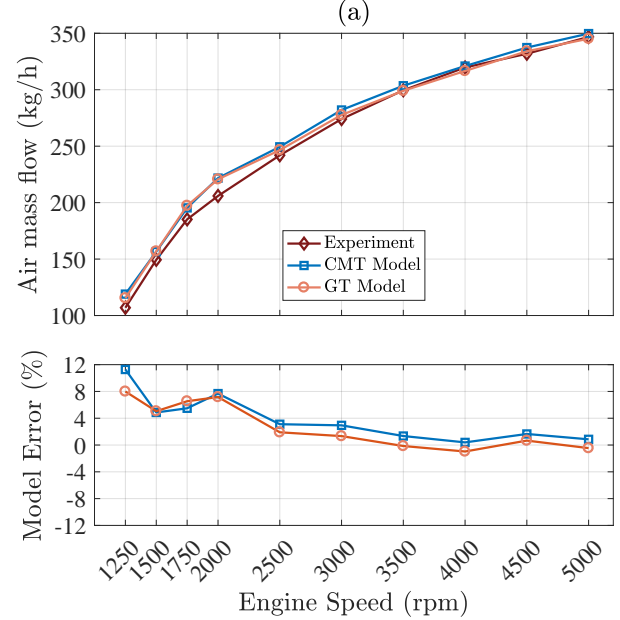

(c)
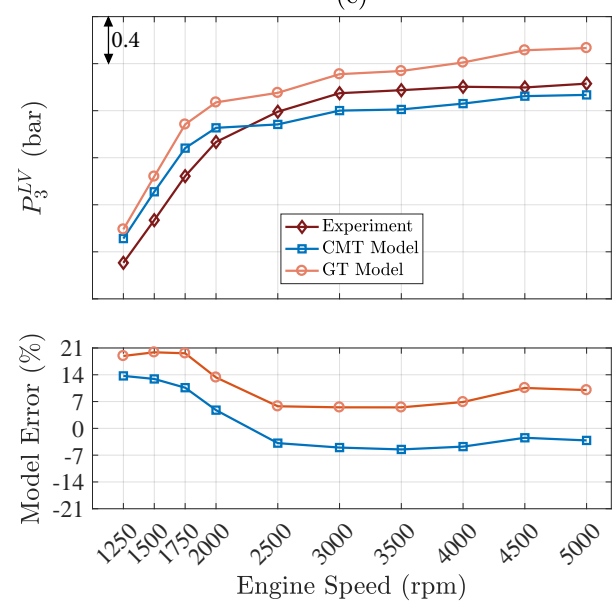

(b)
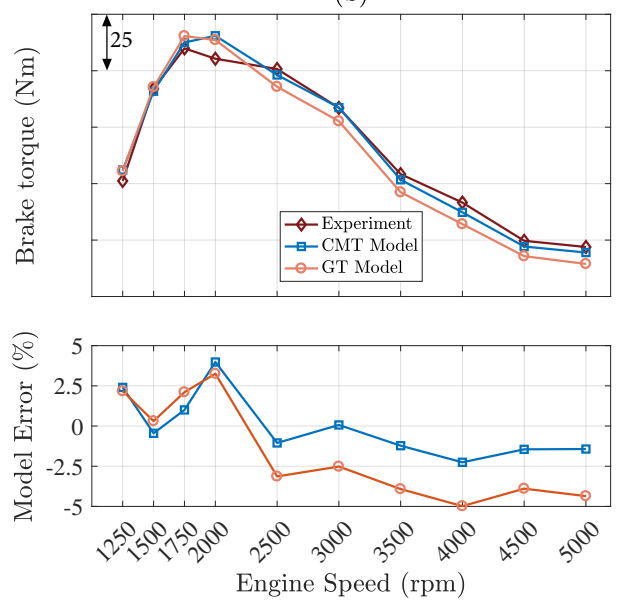

(d)
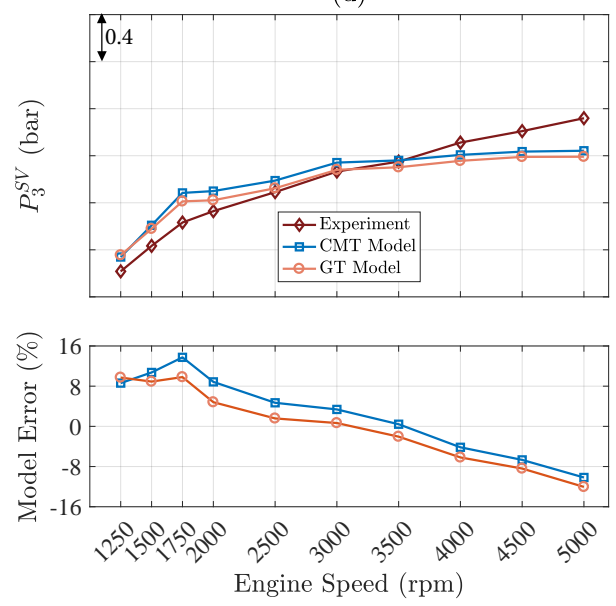

Figure 7.30: Validation parameters of SCV closed simulation; Outputs from CMT-DETCM ang GT-DETCM were compared against the experimental data at different engine speeds.

(a) Air mass flow; (b) Brake torque; (c) Turbine inlet pressure in LV; (d) Turbine inlet pressure in SV. 
experimental.
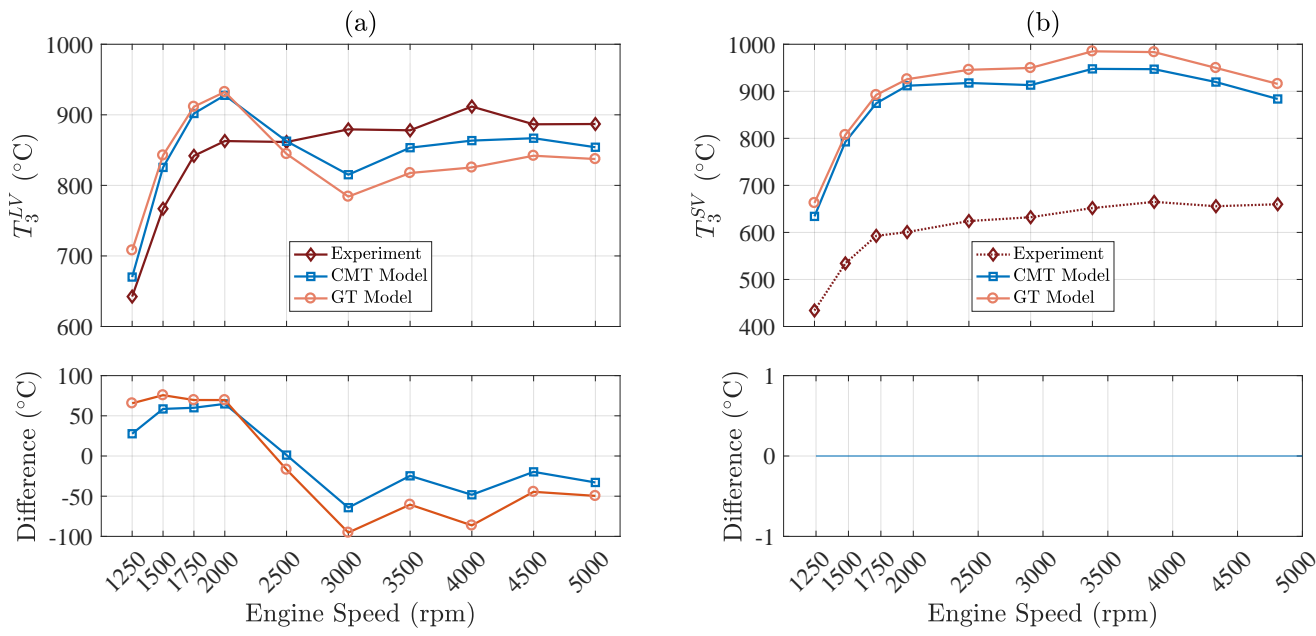

(c)

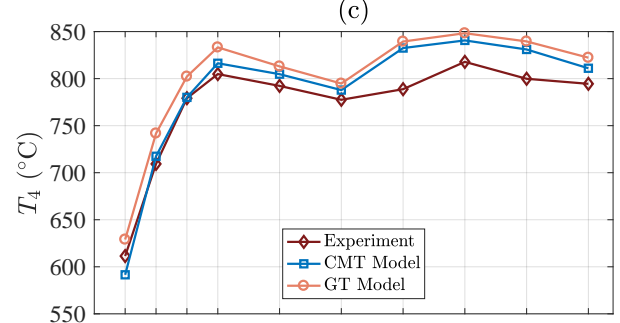

(d)
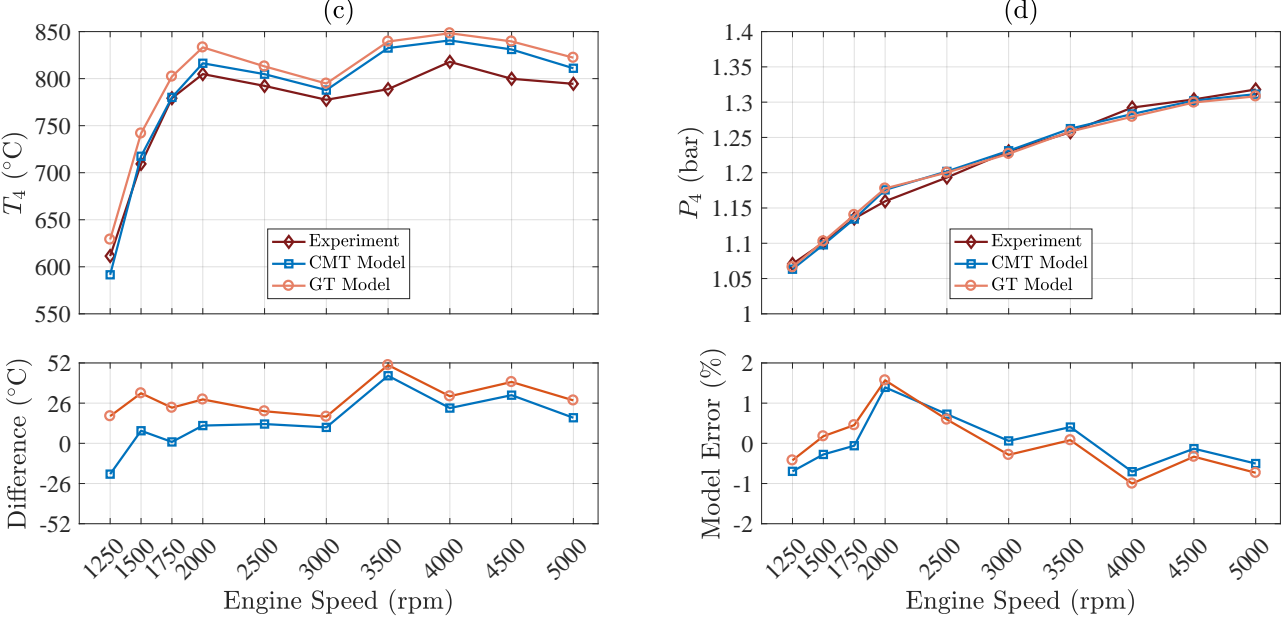

Figure 7.31: Validation parameters of SCV closed simulation; Outputs from CMT-DETCM ang GT-DETCM were compared against the experimental data at different engine speeds.

(a) Turbine inlet temperature LV; (b) Turbine inlet temperature SV; (c) Turbine outlet temperature; (d) Turbine outlet pressure.

Figure 7.31 shows the results of the turbine inlet and outlet temperatures and backpressure to the turbine. From Figure 7.31(a), it can be observed that the temperature predictions from models in the long volute branch are overestimated for 1250 to 2000 engine rpms and underestimated from 3000 to 5000 engine rpms. As mentioned before, the discrepancy in temperature predictions can come from the usage of exhaust heat transfer multiplier correlation that is found for single entry VGT/WG turbines. The difference between the model predictions and experimental are not 
more than $100^{\circ} \mathrm{C}$. It is worth noting that, the exhaust gas temperature prediction in long volute using the CMT-DETCM model are not that far away from the experimental values as compared to the GT-DETCEM model. Figure 7.31(b) shows the results of temperatures in the short volute branch. Aforementioned, the temperature values for this branch from the experimental are lower than the long volute temperature. This could be due to the incorrect positioning of the temperature sensor.

Nevertheless, the predictions of temperatures from the model in the short volute branch are in the same range of values with the long volute branch obtained from the experiments. The difference between them is not huge, as seen in the experimental results. The errors in mixed turbine outlet temperature are directly attributed to the errors in the turbine inlet temperatures. However, the big difference of $52^{\circ} \mathrm{C}$ is observed only at $3500 \mathrm{rpm}$. In other engine speeds the average difference between the experimental and CMT-DETCM is around $11^{\circ} \mathrm{C}$ and with GT-DETCM around $26^{\circ} \mathrm{C}$ were observed. The model after-treatment backpressure to the turbine is in good accordance with experiments, as shown in the Figure 7.31

In summary, without any recalibration of the engine model and also with no further changes in the turbocharger models. The discrepancies between the model and experimental are reasonable. One can notice that, at $2500 \mathrm{rpm}$ in all the validation parameters, the engine model produces results similar to experimental data. A good reproduction of turbine inlet pressure and temperature together with a wellestimated turbine outlet temperature indicates that the experimental running point and wastegate opening of the turbine has been correctly found inside the turbine maps. 



\section{Chapter 8}

\section{Conclusions and future works}

\section{Contents}

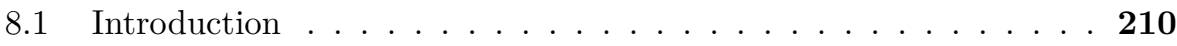

8.2 Main conclusions . . . . . . . . . . . . . . . . . . . 210

8.2.1 Experimental characterization . . . . . . . . . . . . 211

8.2.1.1 Double-entry turbines . . . . . . . . . . . . . . 211

8.2.1.2 Wastegate and scroll connection valve . . . . . . 212

8.2.1.3 Heat transfer properties determination . . . . . 213

8.2 .2 Modelling . . . . . . . . . . . . . . . . . . . . . . 213

8.2.2.1 Double-entry turbines . . . . . . . . . . . . . 213

8.2.2.2 Wastegate and scroll connection valve . . . . . 215

8.2.2.3 Heat transfer properties determination . . . . . 216

8.2.3 Validation in 1D simulation software . . . . . . . . . 216

8.2.3.1 Heat transfer properties . . . . . . . . . 216

8.2.3.2 Gas stand conditions . . . . . . . . . . . . . 217

8.2.3.3 Engine conditions . . . . . . . . . . . . . 217

8.3 Future works . . . . . . . . . . . . . . . . . 218

8.3.1 Experimental . . . . . . . . . . . . . . . . . 218

8.3 .2 Modelling . . . . . . . . . . . . . . . . . . . . 218

8.3 .3 Heat transfer . . . . . . . . . . . . . . . . . . . . . . 219 


\subsection{Introduction}

The present thesis mainly focused on the experimental characterization and development of a mass flow-oriented model for double-entry radial inflow turbine under any unequal and partial admission conditions. This includes the extrapolation models of reduced mass flow and adiabatic efficiency. Furthermore, the thesis also focused on the modelling of discharge coefficient prediction for a wastegate and, scroll connection valve. The scroll connection valve allows the flows to communicate between the branches of double-entry turbines. For developing each model, different experimental works have been carried out in this thesis. The models have been developed to be used coupled to one-dimensional codes and have been validated using this type of simulation. In order to validate all the models working coupled together, engine full load tests have been performed with a dual volute mixed turbine. All the sub-models were integrated into the GT-power and performed the simulations with the whole engine 1D model.

\subsection{Main conclusions}

The main conclusions of the thesis can be divided into three major categories: experimental characterization, modelling, and validation using one-dimensional software.

The first category includes a simple way of testing the double-entry turbines and method of estimating the mass flow through a wastegate and scroll connection valve (SCV) in the gas stand, respectively. Further, it also includes a refined test methodology for estimating the conductance and capacitance of a turbocharger with a very low unbalance of heat.

The second category includes the double-entry turbine extrapolation models, wastegate and SCV discharge coefficient predictions, and generalized heat properties estimation for automotive turbochargers.

The final one deals with the double-entry turbine model validation using the gas stand and engine test conditions in a commercial 1D software. Further, it also includes the validation of heat transfer properties simulating the hot steady and transient tests as a gas stand. 


\subsubsection{Experimental characterization}

\subsubsection{Double-entry turbines}

A simple and representative turbine testing procedure for double-entry turbines in the gas stand was developed as discussed in the chapter 3 . The flow conditions like full, unequal and partial admissions were discriminated by a parameter called MFR (mass flow ratio). This parameter is calculated by using the actual mass flow in each turbine inlet to the total mass flow in the whole turbine. Testing the doubleentry turbines with this parameter allows using the standard gas stand with small modifications. That is just adding the two mass flow meters and control valves for measuring and controlling the amount of flow going into each turbine inlet, respectively. The total inlet mass flow to the turbine in each different admission condition is changed accordingly to obtain the same corrected speed and operating range of the turbocharger compressor. This way, the efficiency of the turbine can be analyzed at different flow admission conditions. Further, testing the double-entry turbines with the actual mass flow ratio parameter (MFR), showed that it also takes into account the variations of pressure and temperature levels in each turbine branch depending on flow conditions in the same. At every MFR in full, unequal and partial admission conditions, the amount of mass flow rate going into the two branches, by adding them, is kept quite similar. This offered interesting insights into the problem. Like, the significant differences are found between the partial and rest of the flow admission conditions concerning the total mass flow rate in the turbine for obtaining the same turbocharger speed. Moreover, the test results in all MFRs showed that the compressor power could have a significant difference when it observed for the same isentropic power in the turbine.

The measurements highlighted that, analyzing the double-entry turbines as a single global turbine gives few lights to the problem of obtaining meaningful characteristic maps. Therefore, a way of handling the performance maps of the double-entry turbines is proposed. That is, to consider each turbine inlet individually with their respective inlet conditions to calculate the characteristics curves. By this, the importance of flow conditions become clear under different admission situations. Moreover, the flow characteristic maps with the proposed methodology for different constant MFR looks very similar to conventional single entry VGT turbine maps. These gave the idea that the mass flow maps of double-entry turbines can be extrapolated safely by the same procedures as used for single entry VGT turbine maps.

The total-to-static turbine efficiency is also estimated for each turbine branch independently. That is, by considering the respective turbine inlet temperature and the mix of temperature which is available at the outlet of a turbine. This way, the obtained apparent efficiency maps were linked to the mass flow parameter maps. Furthermore, the suggested turbine apparent efficiency of two branches confirms that it is dependent on the flow condition of each branch. 
Considering both reduced mass flow and apparent efficiency maps like two independent VGTs, but discriminating with MFR instead of with VGT position. This way, it makes the optimal use of experimental data measured on the gas stand. Further, it showed the advantages to use the current VGT turbine models as two individual turbines to be able to extrapolate the reduced mass flow and efficiency maps of each branch up to off-design conditions.

By using the proposed way of handling the maps of double-entry turbines, the data in these maps can be used as the merit function to predict the behaviour of every turbine branch in all tested MFR and BSR conditions. Alternatively, one can use the proposed arrangement for a quasi-steady interpolation of turbine efficiency and mass flow parameter as a function of instantaneous mass flow ratio and expansion ratio or BSR when trying to model double-entry turbines.

\subsubsection{Wastegate and scroll connection valve}

An experimental methodology has been developed to make possible a proper characterization of discharge coefficient of a wastegate valve and scroll connection valve (SCV) integrated with a double-entry turbine turbocharger as discussed in chapter 5 .

In the case of wastegate flow estimation, two different tests are carried out in full admission condition. Firstly, with the valve closed mechanically and secondly, with the valve partially opened. In these two different tests, it is important to ensure that the turbine inlet temperature, expansion ratio and turbocharger speed are maintained similar. Further, the back-pressure valves at the compressor side are kept the same as well. By doing this, it will guarantee that the turbine operative condition will be similar when the wastegate is closed and opened. In the end, by simply making the difference between two tests, mass flow through the wastegate is easily calculated from the experiments. Subsequently, experimental discharge coefficient was evaluated by doing ratio between the actual to ideal wastegate flows.

In the case of SCV flow estimation, the test methodology followed is similar to the wastegate characterization. But, the two tests are performed with a partial admission map of each turbine branch (MFR 0 and 1). Testing each turbine branch individually makes it easy to estimate the flow that is passing in each direction (i.e. from entry 1 to entry 2 and vice versa). When the SCV is opened and recovering the same test conditions than when the SCV is closed, it was found that the turbocharger speed changes. Further, the flow conditions in the turbine changed from partial to unequal admission. Accordingly, there is a change in efficiency and turbocharger speed. Thus, for estimating the SCV flow, an indirect approach was followed. Which is, re-calculating the SCV closed mass flow in the active branch (refers to the branch where the flow is given) with the corresponding SCV opened turbocharger speed, expansion ratio and turbine inlet temperature. This is done by using the double- 
entry turbine model described in the chapter 4 . Therefore, the operative conditions of the active branch with the new SCV closed mass flow will be similar to the SCV opened tests. Eventually, making the difference between them, the mass flow through the SCV segment is estimated, and the discharge coefficient in each flow direction is evaluated.

\subsubsection{Heat transfer properties determination}

Refined tests in thermo-hydraulic test bench were carried out for determining the conductance and thermal capacitances of a petrol turbocharger (T\#4TER) in chapter 6. During the tests, the turbocharger is entirely thermal insulated, to avoid the heat transfer towards the environment and, the shaft is blocked to prevent mechanical power transfer between the turbine and compressor. No oil or water coolant was supplied to the corresponding lube ports. The experiments were performed with two inlet temperatures $\left(10^{\circ} \mathrm{C}\right.$ and $\left.25^{\circ} \mathrm{C}\right)$ in the cold circuit and constant inlet temperature of around $300^{\circ} \mathrm{C}$ in the hot circuit. By maintaining the similar flows in both circuits, the temperature difference between the inlet and outlet is increased. Also, very low unbalance of heat is achieved in different steady-state tests. With a proper combination of steady and transient tests and using the measured metal temperatures at each node, the conductances and thermal capacitances were obtained.

\subsubsection{Modelling}

\subsubsection{Double-entry turbines}

In chapter 4, a model for extrapolating the performance maps of twin-entry and dualvolute radial inflow turbines in terms of different flow admission conditions, rotational speed and blade speed ratios is developed. For the development of the model, both double-entry turbines were tested at different flow admission conditions as described in chapter 3. Systematizing the performance maps of this type of turbines gave the ability to model any double-entry turbine as if it is formed of two VGTs. Further, made it possible to extrapolate the maps to off-design conditions.

For predicting the reduced mass flow parameter of double-entry turbines, VGT single entry turbine model was adapted to work for double entry turbines. The approach was based on considering two turbine entries as two separate equivalent nozzles. Therefore, each turbine entry is considered as it is a VGT; with its own set of maps depending on MFR instead of the VGT position. The stator and rotor geometrical areas were defined precisely for each entry of the turbine. Finally, the values of the effective area of two nozzles were adjusted using the 14 coefficients and available turbine map data. That is, seven coefficients are fitted independently using the turbine map corresponding to Entry 1, and the other seven are adjusted using 
the turbine map corresponding to Entry 2. Once the effective equivalent nozzle area of each turbine entry is known, the reduced mass flow parameter in both entries are calculated using the expression of the flow through an orifice with an isentropic expansion.

By assuming each turbine inlet being an individual turbine, the apparent efficiencies are computed using the respective branch turbine inlet temperature and mixed temperature available at the outlet of the turbine. For predicting the apparent efficiencies of each branch, it was analyzed that the double-entry turbines cannot be modelled as two individual turbines always working with the extreme flow conditions (i.e. $\mathrm{MFR}=0$ and MFR $=1$ ). In other words, the mixed turbine outlet temperature that measured in the gas stand in full and unequal admission conditions cannot be predicted just by mass averaging the individual outlet temperatures calculated from partial admission efficiency in each turbine. It was observed that following such a wrong hypothesis, the prediction of mixed outlet temperatures are always higher when compared to the experimental values. Further, it was noted that the error showed a nearly symmetrical trend with the distance between the actual $\mathrm{MFR}_{x}$ and MFR $=0.5$. Therefore, to correct this error in the efficiency model, the based VGT efficiency model has been refined using twin-entry turbocharger experimental data. Eventually, a new corrected mixed-flow efficiency model has been developed in this work, and that model uses 11 calibration coefficients to fit the efficiencies of both turbine branches. The model has been validated using the dual-volute radial inflow, and mixed flow turbines data of each volute and the results are in accordance with the experimental information

Further, the model shows good agreement with the experimental data even when it is calibrated with only full admission $(\mathrm{MFR}=0.5)$ and the two partial admissions $(\mathrm{MFR}=0$ and $\mathrm{MFR}=1)$ maps. Moreover, it is able to reproduce the unequal admission flow performance maps for both twin-entry and dual-volute turbines.

As mentioned before, for predicting the reduced mass flow parameters. The model needs 7 calibration constants for each turbine entry, which are fitted using a set of available turbine map data. Whereas, in the case of apparent efficiency model, it uses 11 calibration coefficients to the efficiencies of both branches. Despite the 25 needed constants ( 7 for reduced mass flow of each entry and 11 for efficiency model), only 14 testing points at 3 MFRs are needed to calibrate both reduced mass flow and efficiency models of double-entry turbines. Even reduced mass flow and efficiency models are separate. Their experimental data are obtained at the same time when testing partial admission conditions. Even when testing full or unequal admission conditions, both turbine branches are also measured at the same time.

After fitting the calibration coefficients of a double-entry turbine, for extrapolation purpose, a system with the reduced mass flow and apparent efficiency of both individual turbine branches must be solved together. Since the mass flow and apparent efficiency variables of each branch are interconnected. Both reduced mass 
flow and efficiency models can extrapolate beyond the normal turbine map measured range in various mass flow ratios, reduced turbine speed, and blade speed ratio for each turbine branch.

One of the main advantages of the model is that it can be used for both twinentry and dual-volute turbines, just giving attention to the geometrical simplifications while fitting type of turbine. It is essential to have standard turbine maps measured in almost adiabatic conditions (or adiabatized) and at the three most comfortable testing conditions, i.e., full admission $(\mathrm{MFR}=0.5)$ by blowing to both branches with same flow conditions and the partial admission conditions $(\mathrm{MFR}=0$ and $=1)$ by blocking the alternative branch. Nevertheless, having a larger number of data points, better the fitting results.

The model is sensitive to differences in total temperature and total pressure at each branch entry, as shown by Equations 4.39 and 4.40. Therefore, this magnitude can be different at every entry during testing campaigns, making them simpler campaigns. Finally, this sensitivity makes the model ideal to be used quasi-steadily for predicting turbine efficiency or mass flow parameter at 1D gas-dynamic software with the staggered pulsating flow at each turbine branch.

In this thesis, an approach of modelling the double-entry turbine as a map lookup table in the commercial code GT-Power has been discussed (in chapter 7). The model is simplified as two individual VGT turbines working in parallel with their respective inlet conditions and later mixed at the outlet. In each turbine, respective performance maps of reduced mass flow and efficiency measured at different mass flow admission conditions are stored with dummy VGT positions.

For deciding the VGT position in both turbines, a VGT look-up table as a function of MFR is created. The model can easily be used by imposing the respective turbine branch reduced mass flow and efficiency maps measured at different MFRs.

\subsubsection{Wastegate and scroll connection valve}

In one-dimensional engine calculations, to predict the mass flow through a wastegate or scroll connection valve(SCV), a discharge coefficient of that section is necessary. Therefore based on the experimental data, a simple empirical correlation is proposed in the chapter 5 for estimating a discharge coefficient of the valves.

For the wastegate discharge coefficient modelling, from the experiments, it was observed that the mass flow through the wastegate not only depends on the wastegate opening but also on the expansion ratio of the turbine. Accordingly, an empirical correlation is formed as a function of the wastegate valve position and the expansion ratio. The error of mass flow predictions using this function is small when compared with the relevant magnitude of turbine power. This correlation can be easily imple- 
mented in the $1 \mathrm{D}$ engine simulations for predicting the mass flow through a wastegate in any operative conditions of the engine.

Regarding the scroll connection valve, from experiments, it was noted that the SCV flow in each direction (Entry 1 to Entry 2 and vice versa) is not equal for same SCV openings. This is because the pressure drop across the SCV segment is different when the SCV flow is going in different directions. Based on this, two discharge coefficients were modelled as a function of scroll pressure ratio and SCV openings. The error in mass flow prediction in each direction using these models is small when compared with the experimental results. Further, this model can be easily used in the 1D engine calculations to see the amount of SCV flow passing from one entry to another depending on the pulse in each turbine entry that is coming from the consecutive engine cylinders.

\subsubsection{Heat transfer properties determination}

A general procedure for estimating the conductance and thermal capacitance for a different size of an automotive turbocharger was presented. These parameters are main to characterize the heat transfer model. The methodology is based on the material properties and simple geometrical information of the turbocharger as entry data to the model. In this present thesis, for estimating the conductance, a specific definition for calculating the area with contact width between the two metal nodes has been proposed in chapter 6 . Whereas, in the case of capacitance, the model proposed by has been refitted and updated their constants. Both models (conductance and capacitance) are fitted with twelve variety of turbochargers that have different external geometries and including water-cooled and non-water cooled. The model additionally validated with the turbocharger T\#13, which is T\#4TER and not used in the fitting procedure. Both model results showed considerable accordance with the measured values obtained from the thermo-hydraulic test rig.

\subsubsection{Validation in 1D simulation software}

\subsubsection{Heat transfer properties}

The conductance and capacitance model was coupled to the 1D heat transfer turbocharger model and performed gas stand simulation as discussed in the chapter 6 . A turbocharger (T\#4TER) not used in the model fitting procedure was simulated using the fitting constants found based on the twelve turbochargers. The simulation results showed better predictions in both compressor and turbine outlet temperatures when compared to the original model without heat transfer effects in the turbocharger system. The metal node temperatures from the $1 \mathrm{D}$ heat transfer turbocharger model showed good agreement with metal temperatures measured in the gas stand. 
Further, the ability to predict the temperatures in transient operation has also been checked, and the results are in acceptable quality. These results confirm that the methodology can easily be used for other different turbochargers for predicting the heat transfer properties.

\subsubsection{Gas stand conditions}

Initial validation was performed by simulating both CMT-DETCM and GT-DETCM turbocharger models with different steady flow admission conditions measured in the gas stand at a quasi adiabatic state as discussed in the chapter 7 . The simulation results showed that the outcomes of the double-entry turbocharger model are in good agreement with the experimental data in terms of mass flow predictions in each branch of the turbine, compressor mass flow, and turbocharger speeds. Some discrepancy is found in the prediction of compressor and turbine outlet temperatures. This is due to not taking into account the proper heat transfer characterization in double-entry turbines

\subsubsection{Engine conditions}

Both CMT-DETCM and GT-DETCM models were simulated using the whole onedimensional calibrated engine model at full load curves in two different configurations, as discussed in the chapter 7. One full and two partial admission maps obtained from the gas stand (chapter 3) were used in the turbocharger model while simulating. The engine model was configured to converge on the experimental intake manifold pressure as a target for each speed by using the in-built turbocharger wastegate controller. The results from both models and two different configuration simulations were compared with the corresponding experimental data from an engine test bench. The intake manifold pressure from the simulations confirms that the specified performance target was able to be met with the designed controller. The main findings of the simulations are that the engine brake torque and air mass flow were able to reach the experimental values with a maximum relative error of $3 \%$ and $8 \%$ when the wastegate flow area is equal. Even when the wastegate flow area is unequal, the model is sensitive enough to reproduce the engine torque and mass flow with an relative error of $5 \%$ and $12 \%$ in comparison to the experimental values. Further, in comparison to the other measured engine test parameters like intake manifold temperature, turbocharger speed, turbine upstream and downstream pressures showed the satisfactory validation of the engine model. The engine simulation results show the importance of having systematized performance maps for double-entry turbines as two individual turbines and modelling them accordingly. Doing this, the turbine model able to catch all the flow situations coming from the engine in each branch and is able to reproduce the engine performance similar to experimental values. 


\subsection{Future works}

Further research is needed to continue improving the double-entry turbocharger system in terms of compactness and reliability. The following aspects should be studied:

\subsubsection{Experimental}

When double-entry turbines work with an engine, at the partial admission conditions, the branch with no flow is not physically closed like the test performed in the gas stand. By reviewing the low-pressure traces at the turbine inlets of double-entry turbine obtained from an engine test cell; and comparing the pressure levels and ratios with those measured in the gas stand. Doing this, it could be reasoned that the gas stand measured turbine flow at partial admission condition does not represent the limit of flow behaviour. If the branch pressure ratio exceeds the boundaries of the partial admission at a given turbine pressure ratio that is MFR $<0$ and MFR $>1$, the interaction between the turbine scroll occurs. This means that a part of the mass flow going through the turbine branch with greater pressure levels does not arrive at the turbine wheel, but recirculates into the following branch in front of the wheel. This phenomenon is generally described as back-flow/cross-flow [33]. If one can quantify the impact of cross-flow through experimental works or CFD simulations, it will provide better insights for improving the 1D double-entry turbine model and also helps in obtaining the accurate predictions in the $1 \mathrm{D}$ engine simulation process.

\subsubsection{Modelling}

Performing the CFD simulations with double-entry turbines at different flow admission conditions allows a better understanding of the phenomena occurring in this kind of turbines. The flow of each branch can be tracked in the stator, vaneless space, and along with the turbine wheel. Tracking the degree of mixing of the flow from each branch along with the rotor, one can double-check the validity of the proposed model; that is, considering two entries as two individual VGTs working in parallel.

Additionally, one can analyze the type of different losses in the double-entry turbines for advancing the extrapolation models to be more physically based.

Furthermore, having the experimental data at off-design measurements can be used for the validation. 


\subsubsection{Heat transfer}

Heat transfer in double-entry turbines is also an important parameter to be considered in the one-dimensional modelling for predicting the accurate outlet temperatures of the turbine and compressor, respectively. In double-entry turbochargers, the turbine entries Shroud and Long Volute are far away from the shaft housing and largely exposed to the ambient. Whereas, Hub and Short Volute entries are close to the turbine backplate (i.e. node H1). In unequal and partial admission conditions, there will always be a temperature difference in both entries due to the imbalance of flows coming to the engine cylinders. Therefore, if the different thermal parameters representing the system can be identified, a proper representation of the heat transfer process taking place in the double-entry turbocharger should be possible. The single entry turbocharger heat transfer model that described in [19, 20] can be easily adapted to double-entry turbines by adding an extra gas node $\left(T_{\text {gas }}\right)$ and metal node $(T)$; to take into account the heat exchange from the additional entry (for example $\mathrm{Sh} / \mathrm{LV}$ ) as a first approximation. However, some specific heat transfer experiments with high temperatures at the turbine inlets in different flow admission conditions would be required for quantifying and validating the models. 



\section{Global bibliography}

[1] J. R. Serrano, F. J. Arnau, T. Andrés, and V. Samala. "Experimental procedure for the characterization of turbocharger's waste-gate discharge coefficient". Advances in Mechanical Engineering 9.(10) (2017). ISSN: 16878140. DOI: $10.1177 / 1687814017728242$ (cit. on pp. xii, 120).

[2] J. R. Serrano, P. Olmeda, F. J. Arnau, and V. Samala. "A holistic methodology to correct heat transfer and bearing friction losses from hot turbocharger maps in order to obtain adiabatic efficiency of the turbomachinery". International Journal of Engine Research (2019). ISSN: 20413149. DOI: 10.1177/ 1468087419834194 (cit. on pp. xii, 100, 150, 162, 164, 182).

[3] J. R. Serrano, F. J. Arnau, L. M. Gracía-Cuevas, V. Samala, and L. Smith. "Experimental approach for the characterization and performance analysis of twin entry radial-inflow turbines in a gas stand and with different flow admission conditions". Applied Thermal Engineering (2019), p. 113737. ISSN: 1359-4311. DOI: 10.1016/J . APPLTHERMALENG . 2019.113737 (cit. on pp. xii, 36).

[4] J. R. Serrano, F. J. Arnau, L. M. García-Cuevas, and V. Samala. "A Robust Adiabatic Model for a Quasi-Steady Prediction of Far-Off Non-Measured Performance in Vaneless Twin-Entry or Dual-Volute Radial Turbines". Applied Sciences 10.(6) (2020), p. 1955. DOI: 10.3390/app10061955 (cit. on pp. xii, $72)$.

[5] J. R. Serrano, F. J. Arnau, L. M. García-Cuevas, and V. Samala. "An experimental and modelling strategy for obtaining complete characteristic maps of twin-entry and dual-volute radial inflow turbines". In: Turbocharging Seminar 2019, Harbin , China. Nov. 10 (cit. on p. xii).

[6] J. R. Serrano, F. J. Arnau, L. M. García-Cuevas, V. Samala, G. Stephane, and B. Samuel. "An experimental and modelling strategy for obtaining complete characteristic maps of dual-volute radial inflow turbines". In: ASME Turbo Expo 2020, London, England. Accepted for Publication (Recommended to Journal). June 2020 (cit. on p. xii).

[7] G. Haq and M. Weiss. "CO2 labelling of passenger cars in Europe: Status, challenges, and future prospects". Energy Policy 95 (2016), 324-335. DOI: 10.1016/j.enpol.2016.04.043 (cit. on p. 2). 
[8] S. Wang, F. Zhao, Z. Liu, and H. Hao. "Heuristic method for automakers' technological strategy making towards fuel economy regulations based on genetic algorithm: A China's case under corporate average fuel consumption regulation". Applied Energy 204 (2017). cited By 6, pp. 544-559. DOI: 10.1016/j . apenergy.2017.07.076 (cit. on p. 2).

[9] M. Romare and L. Dahllöf. "The Life Cycle Energy Consumption and Greenhouse Gas Emissions from Lithium-Ion Batteries". IVL Swedish Environmental Research Institute (2017), pp. 8-42. DOI: 978-91-88319-60-9 (cit. on p. 2).

[10] J. R. Serrano. "Imagining the Future of the Internal Combustion Engine for Ground Transport in the Current Context". Applied Sciences 7 (10 2017), p. 1001. DOI: 10.3390/app7101001 (cit. on p. 2).

[11] J. Heywood, D. MacKenzie, I. B. Akerlind, P. Bastani, I. Berry, K. Bhatt, and S. Zoepf. On the Road toward 2050: Potential for Substantial Reductions in Light-Duty Vehicle Energy Use and Greenhouse Gas Emissions. 2015 (cit. on p. 2).

[12] G. Kalghatgi. "Is it really the end of internal combustion engines and petroleum in transport?" Applied Energy 225 (2018), pp. 965 -974. ISSN: 0306-2619. DOI: 10.1016/j . apenergy.2018.05.076 (cit. on p. 2).

[13] R. W. Kruiswyk. "The role of turbocompound in the era of emissions reduction." 10th International Conference on Turbochargers and Turbocharging (2012), 269-280. DOI: 10.1533/9780857096135.5.269 (cit. on p. 3).

[14] K. Kobayashi, T. Sako, Y. Sakaguchi], S. Morimoto, S. Kanematsu, K. Suzuki, T. Nakazono, and H. Ohtsubo. "Development of HCCI natural gas engines". Journal of Natural Gas Science and Engineering 3.(5) (2011). International Gas Research Conference 2011, pp. 651 -656. ISSN: 1875-5100. DOI: https: //doi.org/10.1016/j.jngse.2011.07.002 (cit. on p. 3).

[15] B. Hu, C. Copeland, P. Lu, S. Akehurst, C. Brace, J. Turner, A. Romagnoli, and R. Martinez-Botas. A New De-throttling Concept in a Twin-Charged Gasoline Engine System. 2015. DOI: https://doi.org/10.4271/2015-01-1258 (cit. on p. 3).

[16] M. Yang, K. Deng, R. Martines-Botas, and W. Zhuge. "An investigation on unsteadiness of a mixed-flow turbine under pulsating conditions". Energy Conversion and Management 110 (2016), pp. 51-58. ISSN: 0196-8904. DOI: 10 . 1016/J . ENCONMAN. 2015.12.007 (cit. on p. 3).

[17] Z. Ding, W. Zhuge, Y. Zhang, H. Chen, R. Martinez-Botas, and M. Yang. "A one-dimensional unsteady performance model for turbocharger turbines". Energy 132 (2017), pp. 341 -355. ISSN: 0360-5442. DOI: https://doi.org/ 10.1016/j . energy . 2017 .04 . 154. URL: http://www . sciencedirect. com/ science/article/pii/S0360544217307302 (cit. on p. 4). 
[18] N. Watson and M. Janota. Turbocharging the Internal Combustion Engine. Macmillan, 1982. ISBN: 9780333242902. DOI: 10.1007/978-1-349-04024-7 (cit. on pp. 4, 11, 19, 22, 25, 68, 86).

[19] M. A. Reyes Belmonte. "Contribution to the Experimental Characterization and 1-D Modelling of Turbochargers for IC Engines". PhD thesis. Valencia (Spain): Universitat Politècnica de València, 2013. DOI: 10 . 4995/Thesis / 10251/34777. URL: https://riunet.upv.es/handle/10251/34777 (cit. on pp. 4, 7, 19, 21, 27, 28, 32, 150-152, 154, 157, 162, 165, 219).

[20] A. Dombrovsky. "Synthesis of the 1D modelling of turbochargers and its effects on engine performance prediction". PhD thesis. Valencia (Spain): Universitat Politècnica de València, 2017. DOI: 10 .4995/Thesis/10251/82307. URL: https://riunet . upv.es/handle/10251/82307 (cit. on pp. 4, 21, 92, 162, $165,219)$.

[21] L. B. Inhestern. "Measurement, Simulation, and 1D-Modeling of Turbocharger Radial Turbines at Design and Extreme Off-Design Conditions". PhD thesis. Valencia (Spain): Universitat Politècnica de València, 2019. DOI: 10 .4995/ Thesis/10251/119989. URL: https://riunet.upv.es/handle/10251/119989 (cit. on p. 4).

[22] D. Zhu and X. Zheng. "Asymmetric twin-scroll turbocharging in diesel engines for energy and emission improvement". Energy 141 (2017), pp. $702-714$. ISSN: 0360-5442. DOI: $10.1016 /$ j.energy . 2017.07 .173 (cit. on p. 10).

[23] F. Pischinger and A. Wünsche. "The characteristic behaviour of radial turbines and its influence on the turbocharging process". In: Proceedings of the CIMAC Conference. Tokyo, Japan, 1977 (cit. on pp. 10, 15, 16).

[24] A. Romagnoli, C. D. Copeland, R. F. Martinez-Botas, M. Seiler, S. Rajoo, and A. Costall. "Comparison Between the Steady Performance of Double-Entry and Twin-Entry Turbocharger Turbines". Journal of Turbomachinery 135 (1 2012). DOI: $10.1115 / 1.4006566$ (cit. on pp. 10, 20).

[25] M. Cerdoun and A. Ghenaiet. "Characterization of a twin-entry radial turbine under pulsatile flow condition". International Journal of Rotating Machinery (2016). DOI: 10.1155/2016/4618298 (cit. on p. 11).

[26] N. Winkler, H.-E. Ångström, and U Olofsson. "Instantaneous On-Engine TwinEntry Turbine Efficiency Calculations on a Diesel Engine" (2005). DOI: 10 . 4271/2005-01-3887 (cit. on p. 11).

[27] A. Romagnoli, R. F. Martinez-Botas, and S. Rajoo. "Steady state performance evaluation of variable geometry twin-entry turbine". International Journal of Heat and Fluid Flow 32 (2 2011), 477-489. DOI: 10.1016/j.ijheatfluidflow. 2010.12.002 (cit. on pp. 11, 16). 
[28] N. A. Schorn. "The Radial Turbine for Small Turbocharger Applications: Evolution and Analytical Methods for Twin-Entry Turbine Turbochargers". SAE International Journal of Engines 7.(3) (2014), pp. 1422-1442. ISSN: 19463944. DOI: $10.4271 / 2014-01-1647$ (cit. on pp. 11, 47).

[29] D. Lückmann, T. Uhlmann, H. Kindl, and S. Pischinger. "Separation in Double Entry Turbine Housings at Boosted Gasoline Engines". MTZ worldwide 74.(10) (2013), pp. 4-9. DOI: 10.1007/s38313-013-0094-4 (cit. on pp. 12, 47).

[30] N. Brinkert, S. Sumser, S. Weber, K. Fieweger, A. Schulz, and H. J. Bauer. "Understanding The Twin Scroll Turbine: Flow Similarity". Journal of Turbomachinery 135.(2) (2012). ISSN: 0889504X. DOI: 10.1115/1.4006607 (cit. on pp. $12,45,51,56)$.

[31] A. Romagnoli, C. D. Copeland, R. F. Martinez-Botas, M. Seiler, S. Rajoo, and A. Costall. "Comparison Between the Steady Performance of Double-Entry and Twin-Entry Turbocharger Turbines". Journal of Turbomachinery 135 (1 2012). DOI: 10.1115/1.4006566 (cit. on pp. 14, 56).

[32] D. Japikse and N. C. Baines. Introduction to turbomachinery. Concepts ETI, Inc. and Oxford University Press, 1997. ISBN: 0-933283-10-5 (cit. on pp. 14, $60)$.

[33] D. Lückmann, M. Stadermann, R. Aymanns, and S. Pischinger. "Investigation of cross flow in double entry turbocharger turbines". In: Proceedings of the ASME Turbo Expo. Vol. 8. American Society of Mechanical Engineers (ASME), 2016. ISBN: 9780791849866. DOI: 10.1115/GT2016-57190 (cit. on pp. 14, 218).

[34] C. D. Copeland, P. J. Newton, R. Martinez-Botas, and M. Seiler. "The effect of unequal admission on the performance and loss generation in a doubleentry turbocharger turbine". Journal of Turbomachinery 134.(2) (2011). ISSN: 0889504X. DOI: 10.1115/1.4003226 (cit. on p. 14).

[35] H. Mai and A. Kaufmann. "Methodology to Evaluate the Characteristics of a Twin-scroll Turbocharger with Various Approaches for the Computation of Thermodynamic Properties". In: Turbo Expo: Power for Land, Sea, and Air. Volume 8: Microturbines, Turbochargers and Small Turbomachines; Steam Turbines: V008T26A007. ASME. 2017. DOI: 10.1115/GT2017-63462 (cit. on pp. 14, 15).

[36] A. Dale and N. Watson. "Vaneless radial turbocharger turbine performance". In: Proceedings of the IMechE. C110/86. Jan. 1986, pp. 65-76 (cit. on pp. 16, 199).

[37] M. Capobianco and A. Gambarotta. "Performance of a twin-entry automotive turbocharger turbine". In: ASME Energy-Sources Technology Conference and Exhibition. paper 93-ICE-2. Houston, 1993 (cit. on p. 16). 
[38] A. Hajilouy-Benisi, M. Rad, and M. R. Shahhosseini. "Flow and performance characteristics of twin-entry radial turbine under full and extreme partial admission conditions". Archive of Applied Mechanics 79 (12 2009), 1127-1143. DOI: $10.1007 / \mathrm{s} 00419-008-0295-5$ (cit. on p. 16).

[39] S. Rajoo, A. Romagnoli, and R. F. Martinez-Botas. "Unsteady performance analysis of a twin-entry variable geometry turbocharger turbine" (2012) (cit. on p. 16).

[40] M. S. Chiong, S. Rajoo, A. Ramagnoli, A. W. Costall, and R. F. MartinezBotas. "One-dimensional pulse-flow modeling of a twin-scroll turbine". Energy 115 (2016), pp. 1291-304. DOI: 10.1016/j .energy.2016.09.041 (cit. on pp. 16, 20).

[41] X. Fang and Q. Dai. "Modeling of turbine mass flow rate performances using the Taylor expansion". Applied Thermal Engineering 30.(13) (2010), pp. 1824 -1831. ISSN: 1359-4311. DOI: 10.1016/j . applthermaleng.2010.04.016 (cit. on p. 17).

[42] A. Romagnoli and R. Martinez-Botas. "Performance prediction of a nozzled and nozzleless mixed-flow turbine in steady conditions". International Journal of Mechanical Sciences 53.(8) (2011), pp. 557 -574. ISSN: 0020-7403. DOI: 10.1016/j.ijmecsci.2011.05.003 (cit. on pp. 17, 21).

[43] M. Chiong, S. Rajoo, A. Romagnoli, A. Costall, and R. Martinez-Botas. "Integration of meanline and one-dimensional methods for prediction of pulsating performance of a turbocharger turbine". Energy Conversion and Management 81 (2014), pp. 270 -281. ISSN: 0196-8904. DOI: 10.1016/j . enconman. 2014. 01.043 (cit. on p. 17).

[44] J. Serrano, F. Arnau, V. Dolz, A. Tiseira, and C. Cervelló. "A model of turbocharger radial turbines appropriate to be used in zero- and one-dimensional gas dynamics codes for internal combustion engines modelling". Energy Conversion and Management 49.(12) (2008), pp. 3729 -3745. ISSN: 0196-8904. DOI: doi.org/10.1016/j.enconman.2008.06.031 (cit. on pp. 17, 170).

[45] J. R. Serrano, F. J. Arnau, L. M. García-Cuevas, A. Dombrovsky, and H. Tartoussi. "Development and validation of a radial turbine efficiency and mass flow model at design and off-design conditions". Energy Conversion and Management 128 (2016), pp. 281 -293. ISSN: 0196-8904. DOI: 10.1016/j.enconman. 2016.09 .032 (cit. on pp. 17, 24).

[46] J. R. Serrano, L. M. Arnau Martínez Francisco José García-Cuevas, and L. B. Inhestern. "An innovative losses model for efficiency map fitting of vaneless and variable vaned radial turbines extrapolating towards extreme off-design conditions". Energy (2019). ISSN: 0360-5442. DOI: 10.1016/j.energy . 2019. 05.062 (cit. on p. 17). 
[47] X. Fang, Q. Dai, Y. Yin, and Y. Xu. "A compact and accurate empirical model for turbine mass flow characteristics". Energy 35.(12) (2010), pp. 4819-4823. ISSN: 03605442. DOI: 10.1016/j .energy.2010.09.006 (cit. on pp. 17, 18).

[48] X. Fang and Y. Xu. "Development of an empirical model of turbine efficiency using the Taylor expansion and regression analysis". Energy 36.(5) (2011), pp. 2937-2942. ISSN: 03605442. DOI: 10.1016/j.energy.2011.02.036 (cit. on pp. 17, 18).

[49] L. Eriksson, L. Nielsen, J. BrugÅrd, J. Bergström, F. Pettersson, and P. Andersson. "Modeling of a turbocharged SI engine". Annual Reviews in Control 26 I.(1) (2002), pp. 129-137. ISSN: 13675788. DOI: 10 . 1016/S13675788(02)80022-0 (cit. on p. 17).

[50] L. Eriksson. "Modeling and Control of Turbocharged SI and DI Engines". Oil and Gas Science and Technology - Revue de l'IFP 62 (July 2007). DOI: 10.2516/ogst: 2007042 (cit. on p. 17).

[51] F. Payri, J. Benajes, and M. Reyes. "Modelling of supercharger turbines in internal-combustion engines". International Journal of Mechanical Sciences 38.(8-9) (1996), pp. 853-869. ISSN: 00207403. DOI: 10.1016/0020-7403(95) 00105-0 (cit. on p. 19).

[52] J. P. Jensen, A. F. Kristensen, S. C. Sorenson, N. Houbak, and E. Hendricks. "Mean value modeling of a small turbocharged diesel engine". In: SAE Technical Papers. SAE International, 1991. DOI: $10.4271 / 910070$ (cit. on p. 19).

[53] J. R. Serrano, B. Pla, R. Gozalbo, and D. Ospina. "Estimation of the extended turbine maps for a radial inflow turbine". In: SAE Technical Papers. SAE International, 2010. DOI: 10.4271/2010-01-1234 (cit. on p. 19).

[54] J. R. Serrano, F. J. Arnau, V. Dolz, A. Tiseira, and C. Cervelló. "A model of turbocharger radial turbines appropriate to be used in zero- and one-dimensional gas dynamics codes for internal combustion engines modelling". Energy Conversion and Management 49.(12) (2008), pp. 3729-3745. ISSN: 01968904. DOI: 10.1016/j. enconman.2008.06.031 (cit. on p. 19).

[55] M. Chiong, S. Rajoo, R. Martinez-Botas, and A. Costall. "Engine turbocharger performance prediction: One-dimensional modeling of a twin entry turbine". Energy Conversion and Management 57 (2012), pp. 68 -78. ISSN: 0196-8904. DOI: $10.1016 / j$.enconman.2011.12.001 (cit. on p. 20).

[56] A. Hajilouy Benisi, M. Rad, and M. Reza Shahhosseini. "Modeling of TwinEntry Radial Turbine Performance Characteristics Based on Experimental Investigation Under Full and Partial Admission Conditions". Transaction B: Mechanical Engineering 16 (Sept. 2009) (cit. on pp. 20, 66).

[57] A. W. Costall, R. M. McDavid, R. F. Martinez-Botas, and N. C. Baines. "Pulse Performance Modeling of a Twin Entry Turbocharger Turbine Under Full and Unequal Admission". Journal of Turbomachinery 133.(2) (Oct. 2010). 021005. ISSN: 0889-504X. DOI: 10.1115/1.4000566 (cit. on p. 20). 
[58] P. Newton, A. Romagnoli, R. Martinez-Botas, C. Copeland, and M. Seiler. "A method of map extrapolation for unequal and partial admission in a double entry turbine". English. Journal of Turbomachinery: Transactions of the ASME 136.(6) (2013). ISSN: 0889-504X. DOI: 10.1115/1.4025763 (cit. on p. 20).

[59] C. Fredriksson, X. Qiu, N. C. Baines, M. Müller, N. Brinkert, and C. Gutmann. "Meanline Modeling of Radial Inflow Turbine With Twin-Entry Scroll". In: Proceedings of the ASME Turbo Expo. Vol. 5. June 2012. DOI: 10.1115/GT201269018 (cit. on p. 21).

[60] J. Macek, Z. Zak, and O. Vitek. "Physical Model of a Twin-scroll Turbine with Unsteady Flow". In: SAE 2015 World Congress and Exhibition. SAE International, 2015. DOI: 10.4271/2015-01-1718 (cit. on p. 21).

[61] T. Palenschat, M. Mueller, S. Rajoo, M. S. Chiong, P. Newton, R. MartinezBotas, and F. X. Tan. "Steady-State Experimental and Meanline Study of an Asymmetric Twin-Scroll Turbine at Full and Unequal and Partial Admission Conditions". In: SAE Technical Paper. SAE International, Apr. 2018. DOI: 10.4271/2018-01-0971 (cit. on p. 21).

[62] F. Payri, J. R. Serrano, P. Fajardo, M. A. Reyes-Belmonte, and R. GozalboBelles. "A physically based methodology to extrapolate performance maps of radial turbines". Energy Conversion and Management 55 (2012), pp. 149-163. ISSN: 01968904. DOI: $10.1016 / \mathrm{j}$. enconman . 2011 . 11 .003. URL: http : //dx.doi.org/10.1016/j.enconman.2011.11.003 (cit. on pp. 22, 23, 72, $92)$.

[63] J. R. Serrano, F. J. Arnau, L. M. García-Cuevas, A. Dombrovsky, and H. Tartoussi. "Development and validation of a radial turbine efficiency and mass flow model at design and off-design conditions". Energy Conversion and Management 128 (2016). DOI: 10.1016/j . enconman.2016.09.032 (cit. on pp. 22-26, $69,72,74,78,79,85)$.

[64] J. R. Serrano, P. Olmeda, A. Páez, and F. Vidal. "An experimental procedure to determine heat transfer properties of turbochargers". Measurement Science and Technology 21.(3) (2010). DOI: 10.1088/0957-0233/21/3/035109 (cit. on pp. 28, 157).

[65] J. R. Serrano, P. Olmeda, F. Arnau, and A. Dombrovsky. "General Procedure for the Determination of Heat Transfer Properties in Small Automotive Turbochargers". SAE International Journal of Engines 8.(1) (2014), pp. 30-41. DOI: $10.4271 / 2014-01-2857$ (cit. on pp. $28,150,154,155,157,159$ ).

[66] J. R. Serrano, P. Olmeda, F. J. Arnau, M. A. Reyes-Belmonte, and H. Tartoussi. "A study on the internal convection in small turbochargers. Proposal of heat transfer convective coefficients". Applied Thermal Engineering 89 (2015), pp. 587-599. DOI: $10.1016 / \mathrm{j}$. applthermaleng. 2015.06.053 (cit. on pp. 28, 29). 
[67] F. Payri, P. Olmeda, F. J. Arnau, A. Dombrovsky, and L. Smith. "External heat losses in small turbochargers: Model and experiments". Energy 71 (2014), pp. 534-546. DOI: $10.1016 /$ j.energy . 2014.04 .096 (cit. on pp. 29, 30).

[68] J. R. Serrano, P. Olmeda, A. Tiseira, L. M. García-Cuevas, and A. Lefebvre. "Theoretical and experimental study of mechanical losses in automotive turbochargers". Energy 55 (2013), pp. 888-898. DOI: 10.1016/j . energy. 2013. 04.042 (cit. on pp. 30, 31).

[69] Supercharger Testing Standard. SAE J1723. Society of Automotive Engineers, 1995 (cit. on pp. 41, 42).

[70] Turbocharger Gas Stand Test Code. SAE J1826. Society of Automotive Engineers, 1995 (cit. on pp. 41, 42).

[71] R Zimmermann, R Baar, and C Biet. "Determination of the isentropic turbine efficiency due to adiabatic measurements and the validation of the conditions via a new criterion". Proceedings of the Institution of Mechanical Engineers, Part C: Journal of Mechanical Engineering Science 232.(24) (2018), pp. 44854494. DOI: $10.1177 / 0954406216670683$ (cit. on pp. $45,52,54$ ).

[72] B Savic, R Zimmermann, B Jander, and R Baar. "New Phenomenological and Power-Based Approach for Determining the Heat Flows of a Turbocharger directly from hot gas test data". 12th European Conference on turbomachinery, ETC, Stockholm, Sweden (2017), pp. 1-12 (cit. on pp. 45, 54).

[73] F. Payri, J. Serrano, P. Olmeda, A. Páez, and F. Vidal. "Experimental Methodology to Characterize Mechanical Losses in Small Turbochargers". In: vol. 5. Jan. 2010, pp. 413-424. ISBN: 9780791844007. DOI: 10.1115/GT2010-22815 (cit. on p. 53).

[74] Evaluation of measurement data - Guide to the expression of uncertainty in measurement. JCGM 100:2008. Joint Comittee for Guides in Metrology, 2008 (cit. on p. 55).

[75] P. Olmeda, A. Tiseira, V. Dolz, and L. M. García-Cuevas. "Uncertainties in power computations in a turbocharger test bench". Measurement 59 (2015), pp. 363 -371. ISSN: 0263-2241. DOI: 10.1016/j.measurement. 2014.09.055 (cit. on p. 55).

[76] J. R. Serrano, A. Tiseira, L. M. García-Cuevas, L. B. Inhestern, and H Tartoussi. "Radial turbine performance measurement under extreme off-design conditions". Energy 125 (2017), 72-84. DOI: 10.1016/j.energy.2017.02.118 (cit. on p. 62).

[77] R. Baar, C. Biet, V. Boxberger, H. Mai, and R. Zimmermann. "New evaluation of turbocharger components based on turbine outlet temperature measurements in adiabatic conditions". In: 15th international symposium on transport phenomena and dynamics of rotating machinery. ISROMAC-15, Honolulu, USA. 2014 (cit. on pp. 62, 63). 
[78] B. Lüddecke, D. Filsinger, and J. Ehrhard. "On Mixed Flow Turbines for Automotive Turbocharger Applications". International Journal of Rotating Machinery 2012 (Aug. 2012). DOI: 10.1155/2012/589720 (cit. on p. 65).

[79] Y. Xue, M. Yang, R. F. Martinez-Botas, A. Romagnoli, and K. Deng. "Loss analysis of a mix-flow turbine with nozzled twin-entry volute at different admissions". Energy 166 (2019), pp. 775 -788. ISSN: 0360-5442. DOI: https : //doi.org/10.1016/j.energy.2018.10.075 (cit. on p. 92).

[80] J. R. Serrano, R. Navarro, L. M. García-Cuevas, and L. Inhestern. "Turbocharger turbine rotor tip leakage loss and mass flow model valid up to extreme off-design conditions with high blade to jet speed ratio". Energy 147 (Mar. 2018), pp. 1299-1310. DOI: 10.1016/j.energy.2018.01.083 (cit. on p. 92).

[81] F. E. Harrell. "Ordinal Logistic Regression". In: Regression Modeling Strategies: With Applications to Linear Models, Logistic Regression, and Survival Analysis. New York, NY: Springer New York, 2001, pp. 331-343. ISBN: 978-1-4757-3462-1. DOI: $10.1007 / 978-1-4757-3462-1 \_13$ (cit. on p. 106).

[82] F. H. CLAUSER. "Turbulent Boundary Layers in Adverse Pressure Gradients". Journal of the Aeronautical Sciences 21.(2) (1954), pp. 91-108. DOI: 10.2514/ 8.2938 (cit. on p. 131).

[83] J. R. Serrano, P. Olmeda, A. Tiseira, L. M. García-Cuevas, and A. Lefebvre. "Theoretical and experimental study of mechanical losses in automotive turbochargers". Energy 55 (2013), pp. 888 -898. ISSN: 0360-5442. DOI: doi.org/ 10.1016/j.energy.2013.04.042 (cit. on pp. 162, 165, 176).

[84] J. Martin, F. Arnau, P. Piqueras, and A. Auñon. "Development of an Integrated Virtual Engine Model to Simulate New Standard Testing Cycles". In: SAE Technical Papers. Vol. 2018-April. SAE International, 2018. DOI: 10.4271/ 2018-01-1413 (cit. on p. 170).

[85] J. R. Serrano, F. J. Arnau, L. M. García-Cuevas, P. Soler, and R. Cheung. "Experimental validation of a one-dimensional twin-entry radial turbine model under non-linear pulse conditions". International Journal of Engine Research 0.(0) (0), p. 1468087419869157. DOI: $10.1177 / 1468087419869157$ (cit. on p. 170).

[86] J. Galindo, R. Navarro, L. M. García-Cuevas, D. Tarí, H. Tartoussi, and S. Guilain. "A zonal approach for estimating pressure ratio at compressor extreme off-design conditions". International Journal of Engine Research 20.(4) (2019), pp. 393-404. DOI: 10.1177/1468087418754899 (cit. on p. 170).

[87] GT-Power Users Manual, Version 2017. Gamma Technologies (cit. on p. 176).

[88] B. Pla, J. D. la Morena, P. Bares, and I. Jiménez. "Cycle-to-cycle combustion variability modelling in spark ignited engines for control purposes". International Journal of Engine Research 21.(8) (2020), pp. 1398-1411. DOI: 10.1177/1468087419885754 (cit. on p. 187). 
[89] J. R. Serrano, F. Arnau, J. De La Morena, G. Alejandro, G. Stephane, and B. Samuel. "A methodology to Calibrate Gas-Dynamic Models of Turbocharged Petrol Engines with Variable Geometry Turbines and with Focus on Dynamics Prediction during Tip-in Load Transient Tests". In: ASME Turbo Expo 2020, London, England. Accepted for Publication. June 2020 (cit. on pp. 189, 190).

[90] J. Serrano, H. Climent, R. Navarro, and D. González-Domínguez. "Methodology to Standardize and Improve the Calibration Process of a 1D Model of a GTDI Engine". In: SAE Technical Paper. SAE International, Apr. 2020. DOI: 10 . 4271/2020-01-1008. URL: https://doi.org/10.4271/2020-01-1008 (cit. on pp. 189, 190). 
पीएचडी, एक ऐसी यात्रा है, जिसमें तुम खुद को खो देते हो, किन्तु जब खुड्से मिलते हो, तो जीदेगी की सुक्रिया करते हो।

Birlipta 
